APERFEIÇOAMENTO DE TESTES ESTATÍSTICOS EM VÁRIAS FAMÍLIAS DE DISTRIBUIÇÕES

\author{
Miguel Angel Uribe Opazo
}

TESE APRESENTADA

$\mathrm{AO}$

INSTITUTO DE MATEMÁTICA E ESTATÍSTICA

DA

UNIVERSIDADE DE SÃO PAULO

PARA

OBTENÇÃO DO GRAU

$\mathrm{DE}$

DOUTOR EM ESTATÍSTICA

Área de Concentração: Estatística

Orientadora: Profa. Dra. Silvia L. P. Ferrari 


\title{
APERFEIÇOAMENTO DE TESTES ESTATÍSTICOS EM VÁRIAS FAMÍLIAS DE DISTRIBUIÇÕES
}

\author{
Este exemplar corresponde à redação final \\ da tese devidamente corrigida e defendida \\ por Miguel Angel Uribe Opazo e aprovada \\ pela comissão julgadora.
}

São Paulo, 26 junho de 1997.

Banca examinadora:

- Profạ. Dr a . Silvia Lopes de Paula Ferrari (Orientadora) - IME - USP

- Prof a. Dra a . Denise Aparecida Botter - IME - USP

- Prof․ Dr ‥ Gauss Moutinho Cordeiro - UFPE

- Profo. Drํ. Klaus Leite Pinto Vasconcellos -UPPE

- Profo. Dr ‥ Enrico Colosimo-UFMG. 


\section{RESUMO}

Nesta tese desenvolvemos diversos resultados sobre correções de Bartlett para a estatística da razão de verossimilhança e correções tipo-Bartlett para a estatística escore em vários modelos.

A primeira parte da tese é dedicada ao problema de estimação e teste de hipóteses em modelos de regressão linear com uma distribuição simétrica para os erros. Ênfase é dada ao desenvolvimento de correções de Bartlett e tipo-Bartlett nestes modelos. Assim, estendemos o artigo de Ferrari e Arellano-Valle (1996), que é restrito só aos modelos com erros distribuídos como $t$-Student, a uma classe bem mais ampla de distribuições. Incluímos também um estudo do vício dos estimadores de máxima verossimilhança e do poder de três testes clássicos.

$\mathrm{Na}$ segunda parte da tese, obtemos correções tipo-Bartlett para a estatística escore em modelos exponenciais não lineares, estendendo o artigo de Ferrari e Cordeiro (1996) para modelos com dispersão desconhecida.

Finalmente, obtemos correções tipo-Bartlett para a estatística escore em modelos da família exponencial uniparamétrica e biparamétrica. 


\section{ABSTRACT}

In this thesis, we develop several results concerning Bartlett corrections to the likelihood ratio statistic and Bartlett-type corrections to the score statistic in a number of models.

The first part of the thesis is dedicated to the problem of estimation and hypothesis testing in linear regression models with a symmetric distribution for the errors. Emphasis is given to the development of Bartlett and Bartlett-type corrections in such models. Thus, we extend the paper by Ferrari and Arellano-Valle (1996), which is only restricted to Student- $t$ distributed errors, to a much wider class of distributions. We also include a study of the bias of maximum likelihood estimators and the power of three classic tests.

In the second part of the thesis, we obtain Bartlett-type corrections to the score statistic in exponential family non-linear models. We extend the paper by Ferrari and Cordeiro (1996) for models with unknown dispersion.

Finally, we obtain Bartlett-type corrections to score tests in one- and two-parameter exponential family models. 
A mis padres Angel y Eliana.

A mi esposa Estanis.

A mis hijos Guilherme (in memoria) $y$ Andreia. 


\section{AGRADECIMENTOS}

A Deus, que concebeu a vida e a inspiração necessária para chegar ao final deste trabalho.

À minha orientadora, Professora Silvia Lopes de Paula Ferrari, pela confiança que em mim depositou e pelo constante apoio durante o desenvolvimento deste trabalho.

Aos Professores Gauss M. Cordeiro, Francisco Cribari-Neto e Heleno Bolfarine, pelo apoio com que me brindaram.

Aos Professores do Departamento de Estatística do IME-USP, pelo apoio, amizade e confiança.

A meus colegas: Antonio, Claudia, Filidor, Manuel, Manoel, Loreta, Oscar, Patrícia e Thelma, pela amizade, companheirismo e boa disposição que sempre tiveram comigo.

Aos meus familiares, que sempre me incentivaram a continuar, principalmente nos momentos difíceis, e que souberam compreender os momentos em que fiquei ausente do seu convívio.

À Universidade Estadual do Oeste do Paraná e à Universidade de São Paulo, pela oportunidade de continuar minha formação.

Ao Laboratório de Computação Científica Avançada - LCCA da USP.

A CAPES, pelo apoio financeiro que permitiu realizar meus estudos.

Ao Walter, por sua paciência e notável eficiência na digitação desta tese.

$\mathrm{Y}$ en especial, a mi esposa Estanis y mi hija Andreia que las Amo Mucho.

A todos, obrigado por tudo! 


\section{Índice}

1 Alguns resultados assintóticos de segunda ordem para testes de hipóteses e estimadores de máxima verossimilhança 1

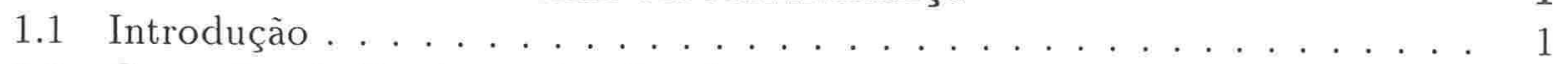

1.2 Correções de Bartlett e tipo Bartlett . . . . . . . . . . . . . . . . . . 2

1.2 .1 Uma breve revisão . . . . . . . . . . . . . . . . . . . . 2

1.2 .2 Desenvolvimento de correções . . . . . . . . . . . . . . . 3

1.3 Expansão para o vício de estimadores de máxima verossimilhança . . . . . 8

1.4 Poderes de três testes clássicos . . . . . . . . . . . . . . . . . . . . . . . . . . .

1.5 Objetivo do trabalho . . . . . . . . . . . . . . . . . . 10

2 Inferência em modelos de regressão linear usando uma família simétrica 12

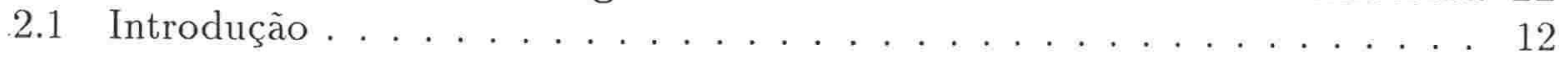

2.2 Distribuições simétricas . . . . . . . . . . . . . . . . . . . . . . . . . . . . . . . . . . . . .

2.3 Modelos de regressão linear . . . . . . . . . . . . . . . . . . . . . 27

2.4 Estimação por mínimos quadrados . . . . . . . . . . . . . . . . . . . . . . . . . . . . . . . . . . . . . .

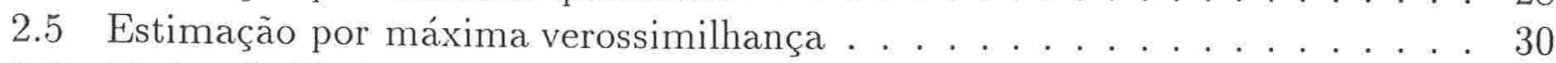

2.6 Testes de hipóteses . . . . . . . . . . . . . . . . . . . . . . . 43

2.6.1 Testes de hipóteses sobre o parâmetro $\beta \operatorname{com} \phi$ conhecido . . . . . . 43

2.6.2 Testes de hipóteses sobre o parâmetro $\beta$ com $\phi$ desconhecido . . . . 45

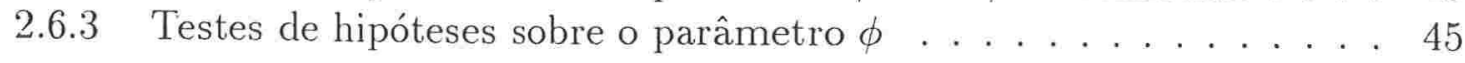

3 Aperfeiçoamento de testes em modelos de regressão usando uma família simétrica

3.1 Introdução . . . . . . . . . . . . . . . . . . . . . . . . . 47

3.2 Fator de correção de Bartlett para a estatística da razão de verossimilhança 48

3.2.1 Testes de hipóteses sobre o parâmetro $\beta \operatorname{com} \phi$ conhecido . . . . . . 48

3.2.2 Testes de hipóteses sobre o parâmetro $\beta \operatorname{com} \phi$ desconhecido . . . . 54

3.2 .3 Testes de hipóteses sobre o parâmetro $\phi \ldots . . . . . . . . . .59$

3.3 Fator de correção tipo Bartlett para a estatística escore . . . . . . . . . 62

3.3.1 Testes de hipóteses sobre o parâmetro $\beta \operatorname{com} \phi$ conhecido . . . . . . 62

3.3.2 Testes de hipóteses sobre o parâmetro $\beta \mathrm{com} \phi$ desconhecido . . . . 66

3.3.3 Testes de hipóteses sobre o parâmetro $\phi \ldots . . . . . . . . . .70$

3.4 Alguns estudos de simulação . . . . . . . . . . . . . . . . . . 73 
3.5 Aplicações . . . . . . . . . . . . . . . . . . . . . . . . . 87

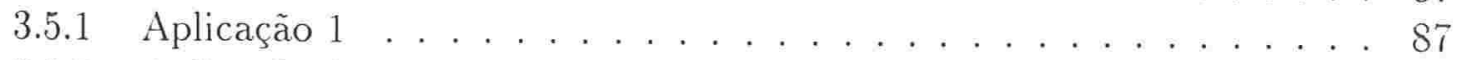

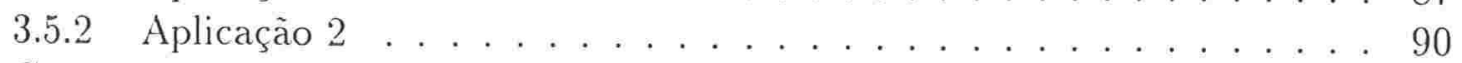

3.6 Consideraçōes finais . . . . . . . . . . . . . . . . . . . . . . 93

4 Vício de estimadores de máxima verossimilhança e poder de testes em modelos de regressão linear usando uma família simétrica $\quad 94$

4.1 Introdução . . . . . . . . . . . . . . . . . . . . . . . . . . . 94

4.2 Vício de ordem $n^{-1}$ para os estimadores de máxima verossimilhança nos modelos simétricos . . . . . . . . . . . . . . . . . . . . . 94

4.3 Poderes de três testes clássicos em modelos simétricos . . . . . . . . . . . 98

4.3.1 Testes de hipóteses sobre o parâmetro $\beta \operatorname{com} \phi$ conhecido . . . . . . . 98

4.3.2 Teste de hipóteses sobre o parâmetro $\beta \operatorname{com} \phi$ desconhecido . . . . 99

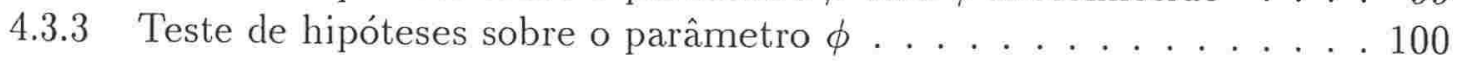

4.3.4 Comparações entre funções de poder . . . . . . . . . . . . . 106

5 Aperfeiçoamento de testes escore em modelos não lineares da família exponencial

5.1 Introdução . . . . . . . . . . . . . . . . . . . . . 110

5.2 Desenvolvimento da correção . . . . . . . . . . . . . . . . . . . . . . . . . . . . . . . 111

5.3 Alguns estudos de simulação . . . . . . . . . . . . . . . . . . . . . . . . . . . . . . . . . . . . . . . . . 117

5.4 Uma aplicação . . . . . . . . . . . . . . . . . . . . . . . 117

6 Aperfeiçoamento de testes escore para modelos da família exponencial uniparamétrica

6.1 Introdução . . . . . . . . . . . . . . . . . . . . . . . . . 119

6.2 Correção para o teste escore . . . . . . . . . . . . . . . . . . . . . . . . . . . . 120

6.3 Casos especiais . . . . . . . . . . . . . . . . . . . . . . . . . . . . . . . . . . . . . . . . . . . . . . . . . . . . 132

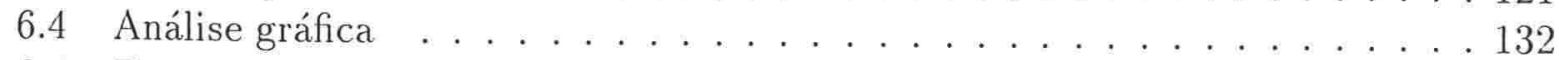

6.5 Família exponencial natural . . . . . . . . . . . . . . . . . 138

7 Aperfeiçoamento de testes escore para modelos da família exponencial biparamétrica

7.1 Introdução. . . . . . . . . . . . . . 142

7.1 Introdução . . . . . . . . . . . . . . . . . . . . . . 142

7.2 Correção em modelos com parâmetros globalmente ortogonais . . . . . . . 144

7.3 Casos especiais . . . . . . . . . . . . . . . . . . . . . . . . . . . . . . . . . . . . . . . . . . . . . . . .

7.4 Aplicações . . . . . . . . . . . . . . . . . . . . . . . . . . . . . . . . 148

7.5 Construção de parâmetros globalmente ortogonais . . . . . . . . . . . . 156

Conclusões 163

$\begin{array}{ll}\text { Apêndices } & 165\end{array}$ 
A

A.'1 Obtenção de alguns cumulantes conjuntos de derivadas do logaritmo da função de verossimilhança em modelos simétricos . . . . . . . . . . . 166

A.2 Obtenção de $A_{1}, A_{2}$ e $A_{3}$ para o teste de hipóteses $H_{0}: \beta_{1}=\beta_{1}^{(0)}$ versus $H_{1}: \beta_{1} \neq \beta_{1}^{(0)}$ em modelos simétricos com o conhecido . . . . . . . . . 168

B

B.1 Obtenção de alguns cumulantes conjuntos de derivadas do logaritmo da função de verossimilhança em modelos simétricos . . . . . . . . . . . . . 170

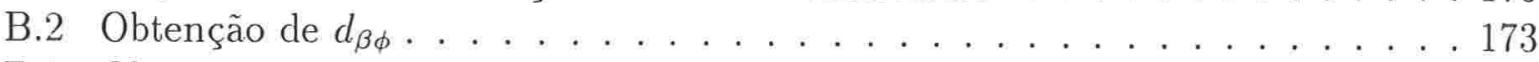

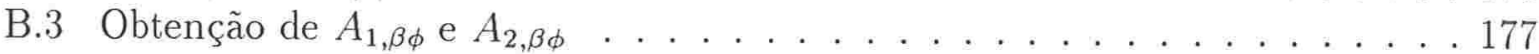

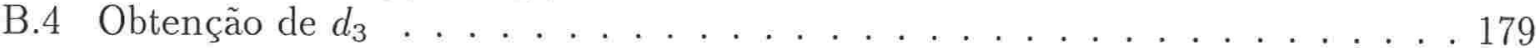

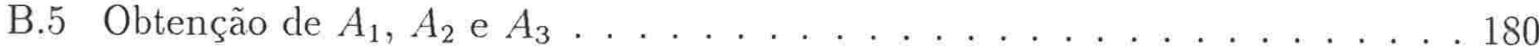

C

C.1 Distribuição normal . .

C.2 Distribuição de Cauchy . . . . . . . . . . . . . . . . . . . . . . . 182

C.3 Distribuição t-Student . . . . . . . . . . . . . . . . . . . . . 183

C.4 Distribuição $t$-Student generalizada . . . . . . . . . . . . . . . 184

C.5 Distribuição Logística I . . . . . . . . . . . . . . . . . . . . . . . . 186

C.6 Distribuição Logística II . . . . . . . . . . . . . . . . . . . . . . . 187

C.7 Distribuição Logística Generalizada . . . . . . . . . . . . . . . . . . . 188

C.8 Exponencial potência . . . . . . . . . . . . . . . . . 190

D 192

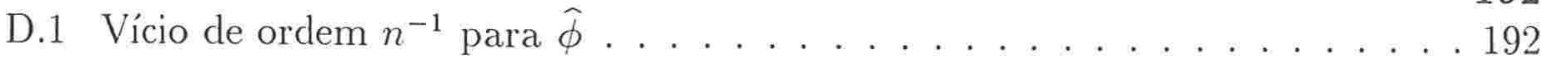

D.2 Comparações entre funções de poder . . . . . . . . . . . . . . . . . . 193

E 197

E.1 Obtenção de alguns cumulantes conjuntos de derivadas do logaritmo da função de verossimilhança em modelos não lineares da família exponencial . 197

E.2 Obtenção de $A_{1, \beta \phi}$ e $A_{2, \beta \phi}$ para o teste das hipóteses $H_{0}: \beta_{1}=\beta_{1}^{(0)}$ versus $H_{1}: \beta_{1} \neq \beta_{1}^{(0)}$ em modelos não lineares de família exponencial . . . . . . 200

F

F.1 Obtenção de alguns cumulantes conjuntos de derivadas do logaritmo da função de verossimilhança em modelos exponenciais biparamétricos . . . . 203 


\section{Capítulo 1}

\section{Alguns resultados assintóticos de segunda ordem para testes de hipóteses e estimadores de máxima verossimilhança}

\subsection{Introdução}

Neste capítulo apresentamos alguns resultados assintóticos de segunda ordem para testes de razão de verossimilhança, de Wald e escore (ou multiplicador de Lagrange) e para o vício de estimadores de máxima verossimilhança. A Seção 1.2 é dedicada a uma breve revisão da literatura acerca de expansões assintóticas até ordem $n^{-1}$ da função de distribuição da estatística da razão de verossimilhança e escore, onde $n$ é o tamanho da amostra. Em todo o texto, "até ordem $n^{-k}$ " significa que, na expansão assintótica sendo considerada, termos de ordens inferiores a $n^{-k}$ são desprezadas. Apresentamos também resultados que possibilitam construir estatísticas aperfeiçoadas que têm distribuição $\chi^{2}$ até ordem $n^{-1}$ através do uso da chamadas correções de Bartlett e tipo Bartlett. Ênfase é dada aos testes da razão de verossimilhança e escore. A revisão da literatura feita aqui é restrita somente aos principais artigos que fornecem resultados relevantes para o desenvolvimento de nosso trabalho. Uma revisão recente, bastante ampla, é feita por Cribari-Neto e Cordeiro (1997); vide também Jensen (1993). Na Seção 1.3, apresentamos expansão para o vício de estimadores de máxima verossimilhança até ordem $n^{-1}$ e mostramos como esta expansão pode ser usada para modificar tais estimadores com o objetivo de torná-los não viciados até esta ordem. Finalmente, na Seção 1.4, apresentamos propriedades de segunda ordem da função de poder dos testes da razão de verossimilhança, de Wald e escore. 


\subsection{Correções de Bartlett e tipo Bartlett}

\subsubsection{Uma breve revisão}

Em problemas de testes de hipóteses envolvendos grandes amostras, é muito comum o uso dos testes da razão de verossimilhança, de Wald e escore. Em geral. é difícil determinar a distribuição exata das estatísticas destes testes, razão pela qual estes testes são freqüentemente baseados em resultados assintóticos. Como se sabe, estas estatísticas têm, sob a hipótese nula, uma distribuição $\chi_{q}^{2}$ assintoticamente, em problemas regulares. Aqui $q$ representa a diferença entre as dimensões dos espaços paramétricos sob as hipóteses alternativa e nula. No entanto, a aproximação por $\chi^{2}$ pode não ser boa quando o tamanho da amostra não for suficientemente grande. Sendo assim, é desejável verificar a possibilidade de se construir estatísticas modificadas que tenham distribuições que convirjam "mais rapidamente" para $\chi^{2}$ do que as estatísticas usuais. Com o objetivo de melhorar a qualidade da aproximação pela qui-quadrado para a distribuição da estatística da razão de verossimilhança $(R V)$ sob a hipótese nula, Bartlett (1937) dá início à idéia de aperfeiçoamento dos testes. Bartlett partiu do fato que, sob uma hipótese nula composta (ou simples), o valor esperado da estatística da razão de verossimilhança é $E(R V)=q\{1+d\}+O\left(n^{-2}\right)$, onde $n$ é o tamanho da amostra, $d$ é uma quantidade de ordem $n^{-1}$ que pode depender de parâmetros (e, neste caso, pode ser estimada, consistentemente, sob a hipótese nula) e $q$ foi definida acima. Então, a estatística corrigida $R V^{*}=R V(1-d)$ (ou, equivalentemente, $R V^{\prime *}=R V(1+d)^{-1}$ ) tem um valor esperado mais próximo do valor esperado da distribuição qui-quadrado de referência do que a estatística não corrigida $R V$. Este fator de correção $(1-d)$, que "aperfeiçoa" a estatística da razão de verossimilhança, passou a ser amplamente conhecido como correção de Bartlett. A importância do aperfeiçoamento da estatística da razão de verossimilhança pelo fator de correção de Bartlett vem do fato de que, sob a hipótese nula, a distribuição de $R V^{*}$ se aproxima melhor da distribuição $\lambda_{q}^{2}$ do que a estatística $R V$. Em problemas regulares, para o teste de uma hipótese nula composta qualquer, Lawley (1956) desenvolveu uma fórmula geral para obter $d$ que é função de vários cumulantes das quatro primeiras derivadas do logaritmo da função de verossimilhança e mostrou que a estatística corrigida $R V^{*}$ tem todos os momentos coincidindo com os da distribuição $\chi^{2}$ de referência até ordem $n^{-1}$.

Uma contribuição à teoria de aperfeiçoamento da estatística da razão de verossimilhança foi dada por Hayakawa (1977), ao obter uma expansão assintótica até ordem $n^{-1}$ para a função distribuição da estatística $R V$, sob uma hipótese nula composta. Os resultados de Hayakawa apresentam um erro que foi, posteriormente, corrigido por Chesher e Smith (1995) (vide também Harris, 1986, Cordeiro, 1987, e Hayakawa, 1987).

Diversos trabalhos foram desenvolvidos nos últimos anos apresentando correçôes de Bartlett para a estatística da razão de verossimilhança em problemas específicos e em modelos amplos. Em particular, correçòes de Bartlett para modelos lineares generalizados foram obtidas por Cordeiro (1983, 1985, 1987) e para modelos não-lineares na família exponencial com parâmetro de dispersào conhecido foram obtidas por Cordeiro e Paula (1989). Para a classe dos modelos de dispersào proposto por Jørgensen (1983, 1987b) 
correções de Bartlett foram obtidas por Cordeiro, Paula e Botter (1994). Em modelos discretos, Williams (1976), Cordeiro (1983) e McCullagh (1985) obtêm as correções para modelos log-lineares. Testes da razão de verossimilhança aperfeiçoados em modelos de regressão logística foram considerados por McCullagh (1985) e Moulton, Weissfeld e Laurent (1993). Correções de Bartlett na família exponencial uniparamétrica foram obtidas por Cordeiro, Cribari-Neto, Aubin e Ferrari (1995) e em modelos de regressão com erros t-Student foram obtidos por Ferrari e Arellano-Valle (1996). Correções similares para modelos lineares normais heterocedásticos e em alguns modelos de regressão multivariada foram obtidas por Cribari-Neto e Ferrari (1995a) e Cribari-Neto e Zarkos (1995), respectivamente. Por outro lado, Barndorff-Nielsen e Cox (1984) apresentam um método indireto de cálculo das correções de Bartlett em modelos paramétricos. Barndorff-Nielsen e Blaesild (1986) desenvolvem um método alternativo para obtenção de correções de Bartlett e McCullagh e Cox (1986) apresentam fórmulas alternativas para obter as correções.

Posteriormente à obtenção do fator de correção de Bartlett para a estatística da razão de verossimilhança, foram obtidas correções similiares para outros testes estatísticos que convergem para uma distribuição $\chi^{2}$. Tais correções são chamadas correções tipo Bartlett (uma revisão da literatura pode ser encontrada em Cribari-Neto e Cordeiro, 1997).

Cordeiro e Ferrari (1991) encontram uma estatística escore aperfeiçoada que tem distribuição qui-quadrado até ordem $n^{-1}$. A estatística modificada é obtida através da multiplicação da estatística escore por um fator de correção tipo Bartlett que envolve um polinômio de segundo grau na própria estatística. Os resultados são válidos para problemas que envolvem parâmetros uni e multidimensionais, para hipóteses simples e compostas e são mais gerais que os trabalhos de Chandra e Mukerjee (1991) e Mukerjee (1992). Ferrari e Cordeiro (1994) desenvolveram fórmulas matricias para o cálculo da correção da estatística escore no caso do teste de uma hipótese nula geral simples ou composta e para problemas com parâmetros globalmente ortogonais. Fórmulas matriciais para os fatores de correção de testes escore na classe dos modelos lineares generalizados foram obtidas por Cordeiro, Ferrari e Paula (1993) para o caso em que o parâmetro de dispersão é conhecido e por Cribari-Neto e Ferrari (1995b) para o caso oposto. Testes escore para modelos lineares heterocedásticos foram aperfeiçoados por Cribari-Neto e Ferrari (1995a). Similarmente, correções para testes escore em alguns modelos de regressão multivariada foram obtidos por Cribari-Neto e Zarkos (1995). Correção tipo Bartlett para o teste escore de heterocedasticidade em modelos normais lineares foi obtida por Cribari-Neto e Ferrari (1995c). Correções para testes escore em modelos de regressão com erros independentes tendo distribuição $t$-Student foram desenvolvidas por Ferrari e Arellano-Valle (1996).

A seguir apresentamos a correção de Bartlet para o teste da razão verossimilhança segundo o método de Lawley (1956) e a correção tipo Bartlett para o teste escore sob o enfoque de Cordeiro e Ferrari (1991).

\subsubsection{Desenvolvimento de correções}

Consideremos $y_{1}, \ldots, y_{n} n$ variáveis aleatórias independentes com distribuição contínua. Seja $L(\beta)$ o logaritmo da função de verossimilhança total, dados $y_{1}, \ldots, y_{n}$, que depende 
de um vetor de parâmetros desconhecidos $\beta=\left(\beta_{1}^{\top}, \beta_{2}^{\top}\right)^{\top}$ com $p$ componentes, sendo $\beta_{1}=\left(\beta_{1}, \ldots, \beta_{q}\right)^{\top}$ o vetor parâmetros de interesse e $\beta_{2}=\left(\beta_{q+1}, \ldots, \beta_{p}\right)^{\top}$ o vetor de dimensão $(p-q)$ de parâmetros de pertubação. Assumimos que $L(\beta)$ é regular com respeito às derivadas em relação aos componentes de $\beta$ até quarta ordem. Detalhes sobre condições de regularidade podem ser encontrados nos trabalhos de Jensen (1993), Chandra (1985) e Cox e Hinkley (1974, Capítulo 9).

Consideramos o teste da hipótese nula $H_{0}: \beta_{1}=\beta_{1}^{(0)}$ contra a alternativa $H_{1}: \beta_{1} \neq$ $\beta_{1}^{(0)}$, onde $\beta_{1}^{(0)}$ é um vetor fixado de dimensão $q$. Denotamos por $\widehat{\beta}=\left(\widehat{\beta}_{1}^{\top}, \widehat{\beta}_{2}^{\top}\right)^{\top}$ o estimador de máxima verossimilhança irrestrito de $\beta$ e por $\widetilde{\beta}_{2}$, o estimador de máxima verossimilhança de $\beta_{2}$ sob $H_{0}$. Funções avaliadas em $\tilde{\beta}=\left(\beta_{1}^{(0) \top}, \tilde{\beta}_{2}^{\top}\right)^{\top}$ serão denotadas com um til. Seja

$$
U=U(\beta)=\frac{\partial L(\beta)}{\partial \beta}=\left(U_{1}^{\top}\left(\beta_{1}, \beta_{2}\right), U_{2}^{\top}\left(\beta_{1}, \beta_{2}\right)\right)^{\top},
$$

a função escore total para $\beta$ sub-dividida da mesma maneira que $\beta$. A partição de $\beta=$ $\left(\beta_{1}^{\top}, \beta_{2}^{\top}\right)^{\top}$ conduz também as seguintes partições

$$
K=\left(\begin{array}{ll}
K_{11} & K_{12} \\
K_{21} & K_{22}
\end{array}\right) \text { e } K^{-1}=\left(\begin{array}{ll}
K^{11} & K^{12} \\
K^{21} & K^{22}
\end{array}\right)
$$

onde $K=E\left(U U^{\top}\right)$ é a matriz de informação total de Fisher que supomos ser positiva definida e $K^{-1}$ é sua inversa. A estatística da razão de verossimilhança para o teste da hipótese $H_{0}$ é dada por

$$
R V=2\left\{L\left(\widehat{\beta}_{1}, \widehat{\beta}_{2}\right)-L\left(\beta_{1}^{(0)}, \tilde{\beta}_{2}\right)\right\} .
$$

Em problemas regulares, $R V$ tem distribuição assintótica $\chi_{q}^{2}$ central sob a hipótese nula $H_{0}$.

A estatística escore $S_{R}\left(\right.$ Rao, 1947) para testar $H_{0}$ contra $H_{1}$ é da forma

$$
S_{R}=\widetilde{U}^{\top} \widetilde{K^{-1}} \tilde{U}
$$

onde $U$ e $K^{-1}$ são definidas em (1.1) e (1.2), respectivamente. Cox e Hinkley (1974, Seção 9.3) expressam $S_{R}$ como

$$
S_{R}=\widetilde{U}_{1}^{\top} \widetilde{K}^{11} \widetilde{U}_{1},
$$

onde $K^{11}=K^{11}\left(\beta_{1}, \beta_{2}\right)$ é a matriz de covariância assintótica de $\widehat{\beta}_{1}$ obtida de (1.2) e $U_{1}=U_{1}\left(\beta_{1}, \beta_{2}\right)$ é definida em (1.1). Em grandes amostras, satisfeitas certas condições de regularidade, a estatística $S_{R}$ tem distribuição assintótica $\chi_{q}^{2}$ sob $H_{0}$. A vantagem de se trabalhar com a estatística escore em lugar da estatística de verossimilhança é que ela não depende de estimação sob a hipótese alternativa.

$\operatorname{Sejam} U_{r}=\partial L(\beta) / \partial \beta_{r}, U_{r s}=\partial^{2} L(\beta) / \partial \beta_{r} \partial \beta_{s}, U_{r s t}=\partial^{3} L(\beta) / \partial \beta_{r} \partial \beta_{s} \partial \beta_{t}$, as derivadas do logaritmo da funçào de verossimilhança total com respeito aos componentes do vetor $\beta$, onde todos os índices variam pelos inteiros $1, \ldots, p$. Consideremos a notação tensorial 
para os cumulantes conjuntos de derivadas do logaritmo da função de verossimilhança (Lawley 1956; Hayakawa, 1977, McCullagh, 1984, 1987): $\kappa_{r s}=E\left(U_{r s}\right), \kappa_{r s t}=E\left(U_{r s t}\right)$, $\kappa_{r s t u}=E\left(U_{r s t u}\right), \kappa_{r, s}=E\left(U_{r} U_{s}\right), \kappa_{r, s t}=E\left(U_{r} U_{s t}\right), \kappa_{r s, t u}=E\left(U_{r s} U_{t u}\right)-\kappa_{r s} \kappa_{t u}, \kappa_{r, s, t u}=$ $\left.E\left(U_{r} U_{s} U_{t u}\right)-\kappa_{r, s} \kappa_{t u}\right)$ e $\kappa_{r, s, t, u}=E\left(U_{r} U_{s} U_{t} U_{u}\right)-\kappa_{r, s} \kappa_{t, u}-\kappa_{r, t} \kappa_{s, u}-\kappa_{r, u} \kappa_{s, t}$. As derivadas dos cumulantes em relação aos componentes do vetor $\beta$ são denotadas por $\kappa_{r s}^{(t)}=\partial \kappa_{r s} / \partial \beta_{t}$, $\kappa_{r s}^{(t u)}=\partial^{2} \kappa_{r s} / \partial \beta_{t} \partial \beta_{u}, \kappa_{r s t}^{(u)}=\partial \kappa_{r s t} / \partial \beta_{u}$, etc. Todos os $\kappa$ 's se referem a um total sobre a amostra e são, em geral, de ordem $n$. A matriz de informação total de Fisher para $\beta$ tem elementos $\kappa_{r, s}=-\kappa_{r s}$, sendo $\kappa^{r, s}=-\kappa^{r s}$ os correspondentes elementos de sua inversa.

Os cumulantes satisfazem relações (Bartlett, 1953a,b; Lawley, 1956), denominadas identidades de Bartlett, que facilitam seus cálculos. Algumas delas são as seguintes: $\kappa_{r, s}=-\kappa_{r s}, \kappa_{r s}^{(t)}+\kappa_{r, s, t}+\kappa_{r, s t}+\kappa_{s, r t}=0, \kappa_{r s}^{(t)}=\kappa_{r s t}+\kappa_{r s, t}, \kappa_{r s t}^{(u)}=\kappa_{r s t u}+\kappa_{r s t, u}$, $\kappa_{r, s, t}=-\kappa_{r s t}-\sum_{(3)} \kappa_{r, s t}, \kappa_{r, s, t}=2 \kappa_{r s t}-\sum_{(3)} \kappa_{r s}^{(t)}, \kappa_{r, s, t, u}=-3 \kappa_{r s t u}+2 \sum_{(4)} \kappa_{r s t}^{(u)}-\sum_{(6)} \kappa_{r s}^{(t u)}+$ $\sum_{(3)} \kappa_{r s, t u}$ e $\kappa_{r, s, t u}=\kappa_{r s t u}-\kappa_{r t u}^{(s)}-\kappa_{s t u}^{(r)}-\kappa_{t u}^{(r s)}-\kappa_{r s, t u}$, onde $\sum_{(k)}$ denota o somatório que varre todas as $k$ permutações de índices.

A expansão assintótica para a função distribuição acumulada de $R V$, sob uma hipótese nula composta e até ordem $n^{-1}$, obtida por Hayakawa (1977), com a devida correção de Chesher e Smith (1995), é da forma

$$
F_{R V}(x)=G_{q}(x)+\frac{a_{1}}{24}\left\{G_{q+2}(x)-G_{q}(x)\right\},
$$

onde $a_{1}$ é uma função de ordem $n^{-1}$ de cumulantes das quatro primeiras derivadas do logaritmo da função de verossimilhança e $G_{r}(x)$ é a função distribuição acumulada de uma variável aleatória qui-quadrado com $r$ graus de liberdade.

A estatística da razão de verossimilhança $R V$ definida em (1.3) pode ser reescrita como

$$
R V=2\left\{\left(L\left(\widehat{\beta}_{1}, \widehat{\beta}_{2}\right)-L\left(\beta_{1}, \beta_{2}\right)\right)-\left(L\left(\beta_{1}^{(0)}, \widetilde{\beta}_{2}\right)-L\left(\beta_{1}, \beta_{2}\right)\right)\right\} .
$$

Segundo Lawley (1956), sob $H_{0}$, o valor esperado da estatística da razão de verossimilhança em (1.6) tem a forma

$$
E(R V)=q+\varepsilon_{p}-\varepsilon_{p-q}+O\left(n^{-2}\right),
$$

onde $\varepsilon_{q}$ e $\varepsilon_{p-q}$ são de ordem $n^{-1}$, e são dados por

$$
\begin{aligned}
\varepsilon_{p} & =\sum^{\prime}\left(\ell_{r s t u}-\ell_{\text {rstuvw }}\right), \\
\varepsilon_{p-q} & =\sum^{\prime \prime}\left(\ell_{r s t u}-\ell_{r s t u v w}\right),
\end{aligned}
$$

com os índices $r, s, t, u, v, w$ referindo-se aos componentes do vetor $\beta$ e onde

$$
\begin{aligned}
\ell_{r s t u}= & \kappa^{r s} \kappa^{t u}\left(\frac{1}{4} \kappa_{r s t u}-\kappa_{r s t}^{(u)}+\kappa_{r t}^{(s u)}\right), \\
\ell_{r s t u v w}= & \kappa^{r s} \kappa^{t u} \kappa^{v w}\left\{\kappa_{r t v}\left(\kappa_{s u w} / 6-\kappa_{s w}^{(u)}\right)\right. \\
& \left.+\kappa_{r t u}\left(\kappa_{\text {svw }} / 4-\kappa_{s w}^{(v)}\right)+\kappa_{r t}^{(v)} \kappa_{s w}^{(u)}+\kappa_{r t}^{(u)} \kappa_{s w}^{(v)}\right\},
\end{aligned}
$$


sendo que $\sum^{\prime}$ denota o somatório sobre todos os componentes de $\beta$, isto é, sobre os $p$ parâmetros e $\sum^{\prime \prime}$ denota o somatório apenas sobre os componentes do vetor $\beta_{2}$, isto é, sobre $p-q$ parâmetros.

Como mencionamos anteriormente, podemos melhorar a aproximação da estatística $R V$ pela distribuição $\chi_{q}^{2}$ substituindo $R V$ pela estatística $R V^{*}=R V(1-d)$, onde $(1-d)$ é o fator de correção de Bartlett, sendo que $q d$ é o termo de ordem $n^{-1}$ de expansão de $E(R V)$ sob $H_{0}$. Assim, temos

$$
d=\frac{\varepsilon_{p}-\varepsilon_{p-q}}{q}
$$

e a estatística corrigida $R V^{*}$ tem distribuição $\chi_{q}^{2}$ até ordem $n^{-1}$ sob $H_{0}$. No caso em que o fator de correção de Bartlett depende de parâmetros desconhecidos, estes devem ser substituídos por seus respectivos estimadores de máxima verossimilhança sob $H_{0}$. Assim, a estatística resultante $R V^{*}=R V(1-\tilde{d})$ ainda tem distribuição $\lambda_{q}^{2}$ até ordem $n^{-1}$ sob $H_{0}$, isto é, a substituição dos parâmetros desconhecidos por valores estimados não afeta a ordem do erro da aproximação da distribuição da estatística corrigida pela distribuição $\chi_{q}^{2}$ (Lawley, 1956, e Cordeiro e Ferrari, 1991).

Relacionando os resultados de Lawley (1956), Hayakawa (1977) e Chesher e Smith (1995) (vide, também, Cordeiro, 1987), temos que em (1.5) $a_{1}$ tem a forma

$$
a_{1}=\varepsilon_{p}-\varepsilon_{p-q} .
$$

Harris (1985) obteve uma expansão assintótica até ordem $n^{-1}$ para a distribuição da estatística escore $S_{R}$ para testar a hipótese nula $H_{0}: \beta_{1}=\beta_{1}^{(0)}$ contra a alternativa $H_{1}: \beta_{1} \neq \beta_{1}^{(0)}$. Harris (1985) mostrou que até ordem $n^{-1}$ e sob $H_{0}$, a função distribuição acumulada de $S_{R}$ pode ser escrita como

$$
\begin{aligned}
F_{S_{R}}(x)= & G_{q}(x)+\frac{1}{24}\left\{A_{3} G_{q+6}(x)+\left(A_{2}-3 A_{3}\right) G_{q+4}(x)\right. \\
& \left.+\left(3 A_{3}-2 A_{2}+A_{1}\right) G_{q+2}(x)+\left(A_{2}-A_{4}-A_{3}\right) G_{q}(x)\right\},
\end{aligned}
$$

onde os $A$ 's são de ordem $n^{-1}$ e são dados por

$$
\begin{aligned}
A_{1}= & 3 \sum^{\prime}\left(\kappa_{j j j k}+2 \kappa_{i, j k}\right)\left(\kappa_{r s t}+2 \kappa_{r s, t}\right) a_{i j} a_{s t} m_{k r}-6 \sum^{\prime}\left(\kappa_{i j k}+2 \kappa_{i j j k}\right) \kappa_{r, s, t} a_{i j} a_{k r} m_{s t} \\
+ & 6 \sum^{\prime}\left(\kappa_{i / j k}-\kappa_{i / j, k}\right)\left(\kappa_{r s t}+2 \kappa_{r s, t}\right) a_{j s} a_{k t} m_{i r}-6 \sum^{\prime}\left(\kappa_{i, j, k, r}+\kappa_{i, j, k r}\right) a_{k r} m_{i j}, \\
A_{2}= & -3 \sum^{\prime} \kappa_{i, j, k} \kappa_{r, s, t} a_{k r} m_{i j} m_{s t}+6 \sum^{\prime}\left(\kappa_{i j k}+2 \kappa_{i, j k}\right) \kappa_{r, s, t} a_{i j} m_{k r} m_{s t} \\
& -6 \sum^{\prime} \kappa_{i, j, k} \kappa_{r, s, t} a_{k t} m_{k r} m_{j s}+3 \sum^{\prime} \kappa_{i, j, k, r} m_{i j} m_{k r} \\
A_{3}= & 3 \sum^{\prime} \kappa_{i, j, k} \kappa_{r, s, t} m_{i j} m_{k r} m_{s t}+2 \sum^{\prime} \kappa_{i, j, k} \kappa_{r, s, t} m_{i r} m_{j s} m_{k t},
\end{aligned}
$$

sendo $a_{i j}$ e $m_{i j}$ os elementos $(i, j)$ das matrizes

$$
A=\left(\begin{array}{cc}
0 & 0 \\
0 & K_{22}^{-1}
\end{array}\right), \quad M=K^{-1}-A
$$


respectivamente. Como antes, $\sum^{\prime}$ indica o somatório sobre todos os parâmetros $\beta$ 's e $G_{r}(x)$ denota a função distribuição acumulada de uma variável qui-quadrado com $r$ graus de liberdade. Harris (1985) também mostrou que os primeiros três momentos da estatística escore, sob $H_{0}$, são dados por

$$
\begin{aligned}
\mu_{1}^{\prime}\left(S_{R}\right) & =q+\frac{A_{1}}{12}, \\
\mu_{2}^{\prime}\left(S_{R}\right) & =q(q+2)+\frac{A_{1}(q+2)+2 A_{2}}{6}, \\
\mu_{3}^{\prime}\left(S_{R}\right) & =q(q+2)(q+4)+\frac{A_{1}(q+2)(q+4)+4 A_{2}(q+4)+8 A_{3}}{4},
\end{aligned}
$$

até ordem $n^{-1}$. Como se sabe, $\mu_{1}^{\prime}\left(\chi_{q}^{2}\right)=q, \mu_{2}^{\prime}\left(\chi_{q}^{2}\right)=q(q+2)$ e $\mu_{3}^{\prime}\left(\chi_{q}^{2}\right)=q(q+2)(q+4)$ e, conseqüentemente, se determinamos $A_{1}, A_{2}$ e $A_{3}$, podemos usar as expressões (1.17) para encontrarmos os três primeiros momentos da estatística escore até ordem $n^{-1}$ e comparálos com os momentos da variável aleatória $\chi_{q}^{2}$, que é a base de nossa aproximação de primeira ordem.

Com base na expansão (1.12), Cordeiro e Ferrari (1991) desenvolvem uma estatística escore aperfeiçoada por um fator de correção tipo Bartlett que tem distribuição $\chi_{q}^{2}$ até ordem $n^{-1}$ sob $H_{0}$. Ela tem a forma

$$
S_{R}^{*}=S_{R}\left\{1-\left(c+b S_{R}+a S_{R}^{2}\right)\right\},
$$

onde

$$
a=\frac{A_{3}}{12 q(q+2)(q+4)}, \quad b=\frac{A_{2}-2 A_{3}}{12 q(q+2)}, \quad c=\frac{A_{1}-A_{2}+A_{3}}{12 q} .
$$

Caso os A's envolvam parâmetros desconhecidos, eles podem ser substituídos por estimadores de máxima verossimilhança sob $H_{0}$ e isto não afeta a ordem de aproximação da correção. Notemos que a correção em (1.18) é uma função da estatística não corrigida e, portanto, esta correção não é uma correção de Bartlett no sentido clássico. No entanto, dada sua semelhança com a correção de Bartlett, este termo é chamado de correção tipo Bartlett.

Cordeiro e Ferrari (1991) desenvolveram também fatores de correção tipo Bartlett para uma classe ampla de estatísticas que têm distribuição assintótica $\chi_{q}^{2}$. sob a hipótese nula (as estatísticas da razão de verossimilhança, de Wald e escore são casos particulares). Sob certas condições de regularidade, Chandra (1985) mostra que é possível escrever a função de distribuição de tais estatísticas (denotadas aqui por $S$ ) na forma

$$
P[S \leq x]=G_{q}(x)+\sum_{j=1}^{k} a_{j} G_{q+2 j}(x)+o\left(n^{-1}\right), \quad \text { onde } \sum_{j=1}^{k} a_{j}=0 .
$$

Notamos que o termo de ordem $n^{-1}$ da expansão da função distribuição de $S$ escrito como uma combinação linear finita de qui-quadrados centrais com coeficientes $a_{i}$ 's que são de 
ordem $n^{-1}$. Para $S_{R}, k=3$ e para $R V, k=1$. Cordeiro e Ferrari (1991) mostram que a estatística modificada

$$
S^{*}=S\left\{1-2 \sum_{i=1}^{k}\left(\sum_{j=1}^{k} a_{j}\right)\left(\mu_{i}^{\prime}\right)^{-1} S^{i-1}\right\},
$$

onde $\mu_{i}^{\prime}=2^{i} \Gamma(i+q / 2) / \Gamma(q / 2)$ é o $i$-ésimo momento de uma distribuição $\chi_{q}^{2}$ e $\Gamma(\cdot)$ é a função gama, tem distribuição $\chi_{q}^{2}$ até ordem $n^{-1}$, sob a hipótese nula.

\subsection{Expansão para o vício de estimadores de máxima verossimilhança}

Nesta seção, nossa atenção está dirigida a uma correção para o vício de estimadores de máxima verossimilhança. A metodologia para encontrar este vício segue do trabalho de Cox e Snell (1968), onde eles mostram que para observações independentes, mas não necessariamente identicamente distribuídas, o vício de ordem $n^{-1}$ de $\widehat{\beta}_{r}$, para $r=1,2, \ldots, p$, é dado por

$$
B_{\widehat{\beta}_{r}}(\beta)=E\left(\widehat{\beta}_{r}-\beta_{r}\right)=\sum^{\prime} \kappa^{r i} \kappa^{j l}\left(\frac{1}{2} \kappa_{i j l}+\kappa_{i j, l}\right),
$$

onde $\kappa^{r i}, \kappa^{j l}, \kappa_{i j l}$ e $\kappa_{i j, l}$ são definidos na Seção 1.2 e o somatório $\sum^{\prime}$ é tomado sobre todos os $p$ parâmetros $\beta_{1}, \ldots, \beta_{p}$.

No lado direito da equação (1.20), estimadores consistentes dos parâmetros $\beta_{1}, \ldots, \beta_{p}$ podem ser inseridos para definir os estimadores de máxima verossimilhança corrigidos $\widetilde{\beta}_{r}=\widehat{\beta}_{r}-B_{\widehat{\beta}_{r}}(\widehat{\beta})$, para $r=1,2, \ldots, p$. Os estimadores corrigidos $\widetilde{\beta}_{r}$ 's têm vícios de ordem $n^{-2}$.

Cordeiro e McCullagh (1991) usam (1.20) para obter fórmulas gerais para o vício de segunda ordem de estimadores de máxima verossimilhança em modelos lineares generalizados. Cordeiro (1993) obtém o vício em modelos de regressão normal heteroscedásticos, Cordeiro e Klein (1994), em modelos ARMA e Aubin e Cordeiro (1997), em modelos de regressão normal linear com matriz de covariância desconhecida. Cribari-Neto, Botter, Cordeiro e Ferrari (1995) e Ferrari, Botter, Cordeiro e Cribari-Neto (1996) obtêm redução de vício de segunda e terceira ordem em modelos uniparamétricos. Resultados de simulação para correção de vício são dados por Cordeiro e Cribari-Neto (1993).

\subsection{Poderes de três testes clássicọ}

As estatísticas da razão de verossimilhança, de Wald e escore, que chamamos agora de $S_{1}, S_{2}$ e $S_{3}$, respectivamente, têm a mesma distribuição assintótica, não só sob $H_{0}$, mas também sob uma seqüência de hipóteses alternativas de Pitman, convergindo para a hipótese nula à taxa $n^{-1 / 2}$, onde $n$ é o tamanho da amostra (Cox e Hinkley, 1974, Seção 9.3). As alternativas de Pitman à hipótese nula, $H_{0}: \beta_{1}=\beta_{1}^{(0)}$, são definidas como a 
seqüência de hipóteses alternativas contíguas, $H_{n}: \beta_{1}=\beta_{1}^{(0)}+\zeta$, onde $\zeta=\left(\zeta_{1}, \ldots, \zeta_{q}\right)^{\top}$, $\operatorname{com} \zeta_{i}=\beta_{i}-\beta_{i}^{(0)}$ suposto ser de ordem $n^{-1 / 2}$. Uim critério de escolha entre as três estatísticas pode se basear na comparação dos poderes dos testes sob esta seqüencia de alternativas até ordem $n^{-1 / 2}$. As hipóteses alternativas contíguas refletem o interesse no estudo do poder de testes para valores do parâmetro próximos daquele estabelecido sob a hipótese nula, ou seja, na região em que o poder não é grande (Cox e Hinkley, 1974, p.318). Hayakawa (1975) e Harris e Peers (1980) desenvolveram uma expansão assintótica para o poder dos testes da razão de verossimilhança, de Wald e escore para uma seqüência de hipóteses alternativas contíguas de Pitman. Denotando por $\bar{P}_{1}, \bar{P}_{2}$ e $\bar{P}_{3}$ as respectivas funções de poder dos testes da razão de verossimilhança, de Wald e escore, temos, para a seqüência de alternativas $H_{n}$ e sob certas condições de regularidade,

$$
\bar{P}_{i}=\bar{P}_{i}\left(x_{\alpha}\right)=1-P\left(S_{i} \leq x_{\alpha}\right), \quad i=1,2,3,
$$

onde

$$
P\left(S_{i} \leq x_{\alpha}\right)=G_{q, \lambda}\left(x_{\alpha}\right)+\sum_{j=0}^{3} b_{i j} G_{q+2 j, \lambda}\left(x_{\alpha}\right)+o\left(n^{-1 / 2}\right),
$$

com o valor $x_{\alpha}$ obtido de $P\left(\chi_{q}^{2} \leq x_{\alpha}\right)=1-\alpha$ e $G_{r, \lambda}\left(x_{\alpha}\right)=P\left(\chi_{r, \lambda}^{\prime 2} \leq x_{\alpha}\right)$, sendo que $\chi_{r, \lambda}^{\prime 2}$ representa uma variável aleatória qui-quadrado não central com $r$ graus de liberdade $\mathrm{e}$ parâmetro de não centralidade $\lambda$. Aqui, $\lambda=\gamma^{\top} K \gamma$ com

$$
\gamma=\left(\begin{array}{c}
I_{q} \\
-K_{22}^{-1} K_{22}
\end{array}\right) \zeta
$$

sendo que $I_{q}$ denota uma matriz identidade de dimensão $q \times q$ e as matrizes $K, K_{21}$ e $K_{22}$ são definidas em (1.2). As quantidades $b_{i j}, i=1,2,3$, e $j=0,1,2,3$, de ordem $n^{-1 / 2}$, são dadas por

$$
\begin{aligned}
& b_{11}=\frac{1}{6} \sum_{r, s, t=1}^{p}\left\{\left(\kappa_{r s t}-2 \kappa_{r, s, t}\right) \gamma_{r} \gamma_{s} \gamma_{t}+3(\underbrace{\kappa_{r s t}+2 \kappa_{r, s t}}) a_{r s} \gamma_{t}\right\} \\
& -\frac{1}{2} \sum_{r=1}^{q} \sum_{s, t=1}^{p}\left(\kappa_{r s t}+\kappa_{r, s t}\right) \zeta_{r} \gamma_{s} \gamma_{t} \\
& b_{12}=\frac{1}{6} \sum_{r, s, t=1}^{p} \kappa_{r, s, t} \gamma_{r} \gamma_{s} \gamma_{t} \\
& b_{13}=0 \\
& b_{21}=\frac{1}{2} \sum_{r, s, t=1}^{p}\left\{(\underbrace{\kappa_{r s t}+2 \kappa_{r, s t}}) \gamma_{r} \gamma_{s} \gamma_{t}-2 \kappa_{r, s t} m_{r s s} \gamma_{t}+(\underbrace{\kappa_{r s t}+2 \kappa_{r, s t}}) \kappa^{r, s} \gamma_{t}\right\} \\
& -\frac{1}{2} \sum_{r=1}^{q} \sum_{s, t=1}^{p}\left(\kappa_{r s t}+\kappa_{r, s t}\right) \zeta_{r} \gamma_{s} \gamma_{t} \\
& b_{22}=-\frac{1}{2} \sum_{r, s, t=1}^{p}\left(\kappa_{r, s t} \gamma_{r} \gamma_{s} \gamma_{t}+\kappa_{r s t} m_{r s} \gamma_{t}\right) \text {, }
\end{aligned}
$$




$$
\begin{aligned}
& b_{23}=-\frac{1}{6} \sum_{r, s, t=1}^{p} \kappa_{r s t} \gamma_{i r} \gamma_{s} \gamma_{t}, \\
& b_{31}=\frac{1}{6} \sum_{r, s, t=1}^{p}\left\{(\overbrace{\kappa_{r s t}-2 \kappa_{r, s, t}}) \gamma_{r} \gamma_{s} \gamma_{t}-3 \kappa_{r, s, t} m_{r s} \gamma_{t}+3(\underbrace{\kappa_{r s t}+2 \kappa_{r, s t}}) a_{r s} \gamma_{t}\right\} \\
& -\frac{1}{2} \sum_{r=1}^{q} \sum_{s, t=1}^{p}(\underbrace{\left.\kappa_{r s t}+\kappa_{r, s t}\right) \zeta_{r}} \gamma_{t} \gamma_{s} \\
& b_{32}=\frac{1}{2} \sum_{r, s, t=1}^{p} \kappa_{r, s, t} m_{r s} \gamma_{t} \text {, } \\
& b_{13}=\frac{1}{6} \sum_{r, s, t=1}^{p} \kappa_{r, s, t} \gamma_{r} \gamma_{s} \gamma_{t} \text {. }
\end{aligned}
$$

Os coeficientes $b_{i 0}$ são obtidos de $b_{i 0}=-\left(b_{i 1}+b_{i 2}+b_{i 3}\right)$, para $i=1,2,3$. Aqui, $\gamma_{i}$ é o $i$-ésimo elemento do vetor $\gamma$ definido em (1.23) e $a_{i j}$ e $m_{i j}$ representam os elementos $(i, j)$ das matrizes $A$ e $M$ dadas em (1.16), respectivamente. Nas expressões (1.24) - (1.26) todas as quantidades, exceto $\zeta$, são avaliadas sob a hipótese nula $H_{0}$.

Podemos verificar que, sob a hipótese nula $H_{0}: \beta_{1}=\beta_{1}^{(0)}$, os três testes apresentam igual probabilidade de erro tipo I até ordem $n^{-1 / 2}$ e das expansões em (1.22), verificamos que, sob alternativas contíguas, os três testes têm o mesmo poder até primeira ordem. Assim, faz sentido comparar as funções de poder dos três testes até ordem $n^{-1 / 2}$. Para fazer estas comparações, denotaremos por $P_{i}$, para $i=1,2,3$, a soma das parcelas de ordem um e $n^{-1 / 2}$ de $\bar{P}_{i}$ definida em $(1.21)$, ou seja, as expansões do poder truncadas desconsiderando os erros de ordem $o\left(n^{-1 / 2}\right)$. Estas comparações podem identificar regiões do espaço paramétrico onde um teste é mais poderoso que os demais, até ordem $n^{-1 / 2}$.

Cordeiro, Botter e Ferrari (1994) e Ferrari, Botter e Cribari-Neto (1997) obtêm expansões assintóticas para a função de poder dos três testes em modelos lineares generalizados com dispersão conhecida e desconhecida, respectivamente. Self e Mauritsen (1988), Self, Mauritsen e Ohara (1992) também apresentam estudos de poder dos testes escore e razão de verossimilhança, respectivamente, na classe dos modelos lineares generalizados.

Para finalizar esta seção, cabe um comentário sobre os poderes dos testes da razão de verossimilhança e escore modificados (vide Seção 1.2). Ferrari (1991) mostra que, até ordem $n^{-1 / 2}$, os poderes destes testes coincidem com os de suas respectivas versões não modificadas. Sendo assim, as correções para os testes têm o efeito de "melhorar" a qualidade das aproximações das estatísticas por qui-quadrado sob a hipótese nula sem com isto alterar os poderes dos testes até ordem $n^{-1 / 2}$.

\subsection{Objetivo do trabalho}

O principal objetivo deste trabalho é desenvolver o aperfeiçoamento das estatísticas da razão de verossimilhança e escore para diferentes famílias de distribuiçōes, utilizando os resultados de Lawley (1956) e Cordeiro e Ferrari (1991), respectivamente. As expressōes resultantes são bastante simples e de fácil aplicação. 
Este trabalho está organizado em sete capítulos e seis apêndices. O Capitulo 2 é dedicado à inferência estatística em modelos de regressào com erros tendo uma distribuiçào simétrica. Estabeleceremos algumas propriedades e resultados para modelos simétricos em geral.

No Capítulo 3, deduzimos fórmulas simples e de fácil aplicaçào para os fatores de correção dos testes da razão de verossimilhança e escore sobre parâmetros de regressão em modelos simétricos considerando os casos em que o parâmetro de escala é conhecido e desconhecido. Desenvolvemos também fatores de correçào para testes do parâmetro de escala. Finalmente, incluímos um estudo de simulaçào e aplicaçòes dos resultados obtidos.

O Capítulo 4 trata do estudo do vício até ordem $n^{-1}$ de estimadores de máxima verossimilhança em diversos modelos simétricos. Comparamos, através do erro quadrático médio, o estimador de máxima verossimilhança usual e o corrigido até ordem $n^{-1}$. Também estudamos o poder dos testes da razào de verossimilhança, Wald e escore, até ordem $n^{-1 / 2}$, discutindo os resultados obtidos.

No Capítulo 5, desenvolvemos testes escore aperfeiçoados para os modelos não lineares da família exponencial considerando o caso do parâmetro de dispersão ser desconhecido, apresentamos um estudo de simulação e uma aplicação. Este resultado estende aquele apresentado por Ferrari e Cordeiro (1996).

No Capítulo 6, desenvolvemos correções tipo Bartlett para testes escore na família exponencial uniparamétrica e aplicamos os resultados a muitos casos especiais. Apresentamos algumas aproximações das correçòes quando elas envolvem funções matemáticas complicadas.

Finalmente, no Capítulo 7 , apresentamos a correção tipo Bartlett de teste escore para modelos da família exponencial biparamétrica. 


\section{Capítulo 2}

\section{Inferência em modelos de regressão linear usando uma família simétrica}

\subsection{Introdução}

A análise de regressão linear é uma técnica estatística muito utilizada na análise de dados e é usada, freqüentemente, nas diversas áreas do conhecimento. A distribuição normal é útil em muitos casos e grande parte da inferência estatística para variáveis aleatórias contínuas tem sido desenvolvida sob suposição de normalidade. Porém, inferência baseada na distribuição normal é vulnerável à presença de dados aberrantes ("outliers") que provêm de distribuições com caudas mais pesadas do que as da distribuição normal. Neste sentido, alguns modelos simétricos alternativos ao normal têm sido utilizados em análise de regressão. Lange, Little e Taylor (1989), por exemplo, propõem o modelo $t$-Student como uma extensão paramétrica robusta do modelo normal. Little (1988) utiliza a distribuição normal contaminada, Taylor (1992) faz uma extensão robusta da teoria de regressão normal utilizando a família de densidades exponencial potência supondo que os erros têm uma distribuição com um parâmetro extra de forma. Albert, Delampady e Polasek (1991) consideram a família de distribuições potência estendida estudando propriedades robustas no contexto da estimação dos parâmetros de regressào linear. Recentemente, Arellano-Valle (1994) apresenta vários resultados sobre regressão usando a distribuição $t$-Student.

Em nosso trabalho, consideramos a família de distribuições simétricas com suporte na reta real. Estas são invariantes com respeito a transformações lineares e formam uma classe geral de distribuições com a mesma simetria que a distribuição normal padrão. As famílias de distribuições $t$-Student, $t$-Student generalizada, Kotz. Kotz generalizada, normal, exponencial potência, logística generalizada, dentre outras, definidas a seguir na Seção 2.2, são elementos particulares desta classe.

Na Seção 2.2, apresentamos a definição e algumas propriedades básicas das distribuiçòes simétricas que serào utilizadas nas seções seguintes. Na Seçào 2.3. apresentamos modelos de regressão linear usando a família de distribuições simétricas. Mostraremos, nas Seções 2.4 e 2.j), alguns métodos de estimação dos parâmetros envolvidos no modelo e algumas propriedades dos estimadores, estendendo. assim, o trabalho de Arellano-Valle 
(1994). Finalmente. na Seçào 2.6. apresentamos os testes de razào de verossimilhança. Wald e escore sobre os parâmetros do modelo.

\subsection{Distribuições simétricas}

Dizemos que a variável aleatória $y$ tem distribuiçào simétrica, com suporte em $\mathbb{R}$, com parâmetro de locação $\mu \in \mathbb{R}$ e de escala $\phi>0$, se sua funçào densidade é da forma

$$
\pi(y ; \mu, \phi)=\frac{1}{\phi} h\left(\left(\frac{y-\mu}{\phi}\right)^{2}\right), y \in \mathbb{R},
$$

para alguma função $h$ (chamada função geradora de densidades), com $h(u)>0$, para $u>0$, e $\int_{0}^{\infty} u^{-1 / 2} h(u) \mathrm{d} u=1$. Esta condição garante que $\pi(\cdot ; \mu, \phi)$ é de fato uma funçào densidade. As famílias de distribuições normal, normal contaminada, $t$-Student, $t$-Student generalizada, logística generalizada, Kotz, Kotz generalizada e exponencial potência, dentre outras, pertencem a esta classe de distribuições como será mostrado neste capítulo. Cabe ressaltar que algumas das propriedades clássicas das distribuições normais são válidas para todas as distribuições simétricas como veremos a seguir.

Se $y$ tem uma distribuição simétrica com parâmetros $\mu$ e $\phi$ escrevemos $y \sim S\left(\mu, o^{2}\right)$.

Se $y \sim S\left(\mu, \phi^{2}\right)$, então a função característica de $y, \iota_{y}(t)=E\left(e^{i t y}\right)$ tem a forma $\psi_{y}(t)=e^{i t \mu} \varphi\left(t^{2} \phi^{2}\right), t \in \mathbb{R}$, para alguma função $\varphi, \operatorname{com} \varphi(u) \in \mathbb{R}$, para $u>0$. É fácil ver, entào, que $a+b y \sim S\left(a+b \mu, b^{2} \phi^{2}\right)$, onde $a, b \in \mathbb{R}$, com $b \neq 0$, isto é, a distribuiçào de qualquer transformação linear de uma variável aleatória com distribuição simétrica é também simétrica. Em particular, se $y \sim S\left(\mu, \phi^{2}\right)$, então $z=(y-\mu) / \phi \sim S(0,1)$, com função densidade $\pi(z)=\pi(z ; 0,1)=h\left(z^{2}\right), z \in \mathbb{R}$.

Desde que existam, $E(y)=\mu$ e $\operatorname{Var}(y)=\xi \phi^{2}$, para alguma constante $\xi>0$. Em termos da função característica, esta constante é dada por $\xi=-2 \varphi^{\prime}(0)$, onde $\varphi^{\prime}(0)=$ $\mathrm{d} \varphi(u) /\left.\mathrm{d} u\right|_{u=0}$, e não depende dos parâmetros $\mu$ e $\phi$ (Fang, Kotz e Ng, 1990, p.43). Kelker (1970) nota que, se $u^{-\frac{1}{2}(k+1)} h(u)$ é integrável, então o $k$-ésimo momento de $y$ existe.

Considerando o caso em que $y \sim S(0,1)$ e seus momentos existam. Berkane e Bentler (1986) mostram que a função característica de $y$ pode ser expandida como

$$
\psi_{y}(t)=\sum_{k=0}^{\infty} i^{k} \mu_{k}^{\prime} \frac{t^{k}}{k !},
$$

onde $\mu_{k}^{\prime}=E\left(y^{k}\right)=i^{-k} \psi_{y}^{l^{(k)}}(0)$, sendo que $\psi_{y}^{(k)}(0)$ denota a $k$-ésima derivada de $\psi_{y}(t)$ avaliada em $t=0$. Então, $\mu_{k}^{\prime}=0$ para $k$ ímpar e para $k=2 m, m=1,2, \ldots$, vale a relação

$$
\mu_{2 m}^{\prime}=\frac{(2 m) !}{2^{m} m !}\left(\mu_{2}^{\prime}\right)^{m}(k(m)+1) .
$$

onde

$$
h(m)=\frac{\varphi^{(m)}(0)}{\left(\varphi^{(1)}(0)\right)^{m}}-1 .
$$


sendo $\varphi^{(r)}(0)$ a $r$-ésima derivada derivada da função $\varphi$. avaliada em zero. Os coeficientes $k(m) . m=1,2 \ldots$ são chamados de parâmetros de momentos e generalizam o coeficiente de curtose $\gamma_{2}=3(k(2)+1)$ de uma distribuição $S\left(\mu, O^{2}\right)$ (Muirhead, 1982).

Devemos observar que a família de distribuiçòes simétricas coincide com a classe de distribuiçòes elípticas univariadas segundo Cambanis. Huang e Simons (1981). Como se sabe, a partir do trabalho de Kelker (1970), vários artigos foram publicados sobre as distribuições elípticas univariadas e multivariadas, tendo importante papel na teoria estatística e suas aplicações. Desde então. estas distribuições têm sido objeto de diversos estudos sobre suas propriedades e aplicações. Trabalhos mais recentes das propriedades das distribuições elípticas univariadas podem ser encontrados em Berkane e Bentler (1986), Muirhead (1980. 1982), Rao (1990), Cambanis, Huang e Simons (1981), Anderson e Fang (1987). Um desenvolvimento resumido e bem organizado das distribuiçôes elípticas encontra-se em Fang, Kotz e Ng (1990), Fang e Zhang (1990) e Arellano-Valle (1994, Cap.2).

A seguir, apresentamos algumas distribuições simétricas com suporte em $y \in \mathbb{R}$ (vide também Fang, Kotz e Ng, 1990, (ap.2). Apresentamos também gráficos comparando algumas distribuições com a distribuição normal. Em todos os gráficos a linha pontilhada corresponde à distribuição normal padrão. Para todas as distribuições consideradas, o parâmetro de locação é fixado em $\mu=0$ e o de escala em $\phi=1$.

\section{(i) Distribuição normal}

A distribuição normal é considerada a mais importante das distribuições em estatística devido às suas inúmeras aplicações e serve como uma excelente aproximação para uma grande classe de distribuiçòes.

Os resultados obtidos acima mostram que várias das propriedades clássicas da distribuição normal são válidas para todas as distribuições simétricas. Porém, a distribuição normal possui várias propriedades que permitem caracterizá-la dentro da classe das distribuições simétricas nas chamadas distribuições normais compostas. Alguns resultados neste sentido são resumidos em Muirhead (1982), Devlin, Gnanadesikan e Kettenring (1976).

Se $y \sim S\left(\mu, \phi^{2}\right)$ e a função geradora de densidades $h(\cdot)$ é da forma

$$
h(u)=\frac{1}{\sqrt{2 \pi}} \exp \{-u / 2\}, u>0
$$

então $y$ tem uma distribuição normal e escrevemos $y \sim N\left(\mu, \phi^{2}\right)$, com a função característica da forma

$$
\psi_{y}(t)=\epsilon^{i t \mu} \exp \left\{-\frac{1}{2} t^{2} \phi^{2}\right\}, t \in \mathbb{R} .
$$

Se $y \sim N\left(\mu, \phi^{2}\right)$, entào $E(y)=\mu$. $\operatorname{Var}(y)=\phi^{2}$, os momentos centrais de ordem $r$ são

$$
\mu_{r}=E\left((y-\mu)^{r}\right)= \begin{cases}0, & r \text { impar } \\ \phi^{r} r ! /\left(2^{r / 2}(r / 2) !\right), & r \text { par }\end{cases}
$$

e. conseqüentemente, o coeficiente de curtose é $\gamma_{2}=3$. 


\section{(ii) Distribuição de Cauchy}

Uma variável aleatória $y \sim S^{\prime}\left(\mu . \circ^{2}\right)$ tem uma distribuiçào de Cauchy se a funçào geradora de densidades $h(\cdot)$ tem a forma

$$
h(u)=\frac{1}{\pi}(1+u)^{-1}, u>0,
$$

e denotamos por $y \sim C\left(\mu, \phi^{2}\right)$. A funçào característica é da forma

$$
\psi_{y}(t)=E\left[\epsilon^{i t y}\right]=\exp \{i t \mu-|t| \phi\}, \quad t \in \mathbb{R} .
$$

Devemos enfatizar o fato que os momentos e cumulantes da distribuição de Cauchy não existem. Ela tem mediana e moda iguais a $\mu$, os quartis superior e inferior são $\mu \pm \phi$. A função densidade tem pontos de inflexão em $\mu \pm \sqrt{3} \phi$ e pode-se demostrar que os valores da função distribuição acumulada nos pontos de inflexão são 0.273 e 0.727 , que podem ser compararados com os correspondentes valores 0.159 e 0.841 da distribuição normal. A mais notável diferença entre as distribuições normal e de Cauchy é que esta última tem caudas mais pesadas (vide Figura 2.1).

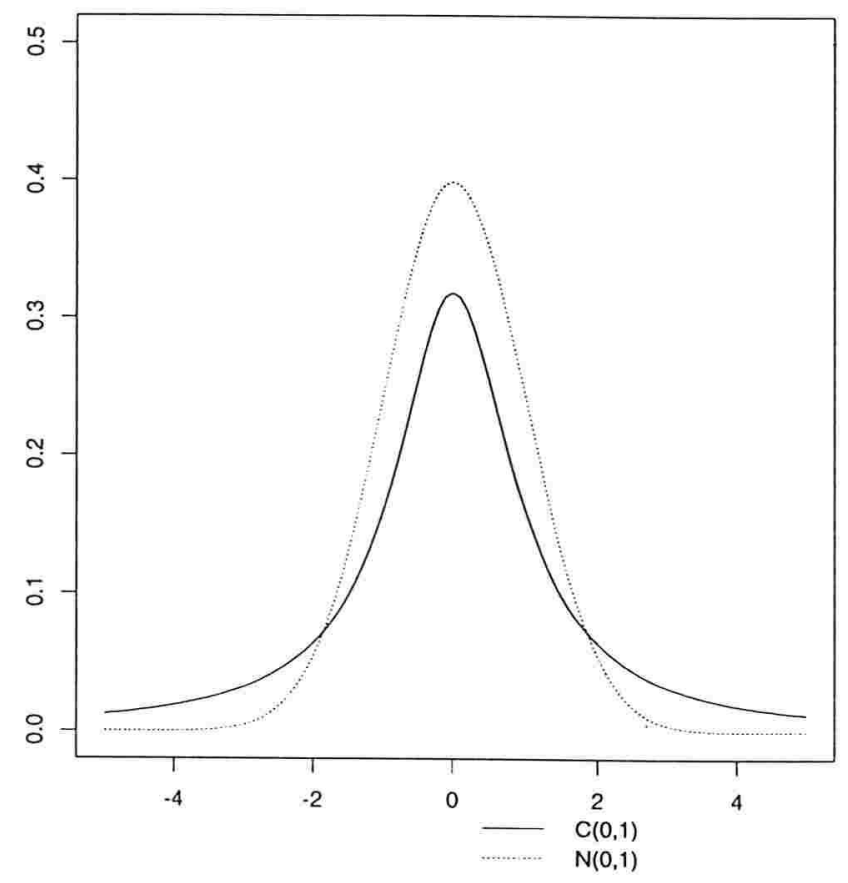

Figura 2.1: Gráficos de funçôes densidade Cauchy e normal.

Se $y_{1}, y_{2}, \ldots, y_{n}$ são variáveis aleatórias independentes onde $y_{j} \sim C\left(\mu_{j}, \phi_{j}^{2}\right)$. então $S=\sum_{j=1}^{n} y_{j}$ tem distribuição de Cauchy com parâmetros de locação $\mu=\sum_{j=1}^{n} \mu_{j}$ e escala $\phi=\sum_{j=1}^{n} \phi_{j}$. Um resultado mais geral é que para $a_{j} \neq 0, \sum_{j=1}^{n} a_{j} y_{j}$ tem uma distribuição de Cauchy com parâmetros de locaçào $\sum_{j=1}^{n} a_{j} \mu_{j}$ e escala $\sum_{j=1}^{n}\left|a_{j}\right| o_{j}$. Em particular, se 
$y_{1}, \ldots, y_{n}$ são variáveis aleatórias i.i.d. com $y_{j} \sim C\left(\mu \cdot o^{2}\right)$. então

$$
\bar{y}=n^{-1} \sum_{j=1}^{n} y_{j} \sim C\left(\mu \cdot o^{2}\right) \text {. }
$$

A distribuição padronizada de Cauchy $\operatorname{com} \mu=0$ e $\phi^{2}=1$ é uma distribuição $t$-Student central com um grau de liberdade. Uma forma de gerar observações de uma distribuição de Cauchy com parâmetros $\left(\mu, \phi^{2}\right)$ é definir y pela relação $y=\mu+\phi N_{1} / N_{2}$. onde $N_{i} \sim N(0,1)$, para $i=1,2$, e são independentes.

\section{(iii) Distribuição $t$-Student}

Uma variável aleatória $y \sim S\left(\mu, \phi^{2}\right)$ tem uma distribuição $t$-Student com $\nu$ graus de liberdade se a função geradora de densidades $h(\cdot)$ tem a forma

$$
h(u)=\frac{\nu^{\nu / 2}}{B(1 / 2, \nu / 2)}[\nu+u]^{-\frac{\nu+1}{2}}, \nu>0, u>0,
$$

onde $B(\cdot, \cdot)$ denota a função beta, e denotamos por $y \sim t\left(\mu, \phi^{2}, \nu\right)$. Assim, a funçào densidade de $y$ é obtida a partir de $(2.1)$ e da função $h(\cdot)$ acima. Sua função característica é dada por Fang. Kotz e $\mathrm{Ng}$ (1990, p.87).

Se $y$ é definido por $y=v^{1 / 2} z$, onde $v \sim G I(\nu / 2, \nu / 2)$ (gama inversa), $\nu>0$, e $z \sim N(0,1)$ são independentes, então $y \sim t(0,1, \nu)$.

Se $y \sim t(0,1, \nu)$, então temos as seguintes propriedades:

(a) para $\nu>r$, os momentos de ordem $r$ existem e têm a seguinte forma

$$
E\left(y^{r}\right)= \begin{cases}\nu^{r / 2} \Gamma\left(\frac{r+1}{2}\right) \Gamma\left(\frac{\nu-r}{2}\right) /\left(\Gamma\left(\frac{1}{2}\right) \Gamma\left(\frac{\nu}{2}\right)\right), & r \text { par } \\ 0, & r \text { impar. }\end{cases}
$$

Assim, $E(y)=0$, se $\nu>1$ e $\operatorname{Var}(y)=\nu /(\nu-2)$, se $\nu>2$. Se $r \geq \nu$, e $r$ é par, o momento de ordem $r$ é infinito;

(b) o desvio médio tem a forma

$$
E(|y|)=\frac{\nu^{1 / 2} \Gamma\left(\frac{\nu-1}{2}\right)}{\Gamma(1 / 2) \Gamma(\nu / 2)}
$$

(c) o coeficiente de curtose é $\gamma_{2}=3+6 /(\nu-4)$, para $\nu>4$, sendo este coeficiente maior que o coeficiente de curtose da distribuição normal:

(d) $y^{2} \sim F_{(1, \nu)}$, isto é, $y^{2}$ tem distribuição $F$ com 1 e $\nu$ graus de liberdade;

(e) se $w=(\nu+1) /\left(\nu+y^{2}\right)$. entào

$$
E\left(y^{2 k} u^{l}\right)=\frac{\left(-\frac{\nu+1}{2}\right)^{l}}{\nu^{l-k}} \frac{B((2 k+1) / 2 \cdot(\nu+2(1-k)) / 2)}{B(1 / 2, \nu / 2)},
$$

para $l=0.1,2 \ldots$ e $k=1.2 \ldots$; 
(f) a função densidade de $y$ tem pontos de inflexão em $\pm(\nu /(\nu+2))^{1 / 2}$ :

(g) a variável aleatória $u=\left(1+\nu / y^{2}\right)^{-1}$ tem distribuiçào beta com parâmetros $a=1 / 2$ e $b=\nu / 2($ Manoukian, 198.5, p.41);

(h) $(y \mid v=\nu) \sim N(0, \nu)$;

(i) $(v \mid y=y) \sim G I\left((\nu+1) / 2,\left(\nu+y^{2}\right) / 2\right)$.

Note-se que a distribuição $t$-Student com parâmetros $\left(\mu, \phi^{2}, \nu\right)$ tende a uma distribuição normal com média $\mu$ e variância $\phi^{2}$ quando $\nu \rightarrow \infty$. Para $\nu=1 . y \sim C\left(\mu, \phi^{2}\right)$.

A distribuição $t$-Student é utilizada para estudar o comportamento de dados que provêm de uma distribuição com caudas mais pesadas que a distribuição normal, permitindo reduzir a influência de observaçòes aberrantes. Lange. Little e Taylor (1989) propõem o modelo $t$-Student como uma extensão paramétrica robusta do modelo normal, já que a distribuição $t$-Student permite ajustar a curtose da distribuição dos dados através do parâmetro $\nu$.

Na Figura 2.2, observamos a forma da distribuição $t$-Student para vários valores de $\nu$.
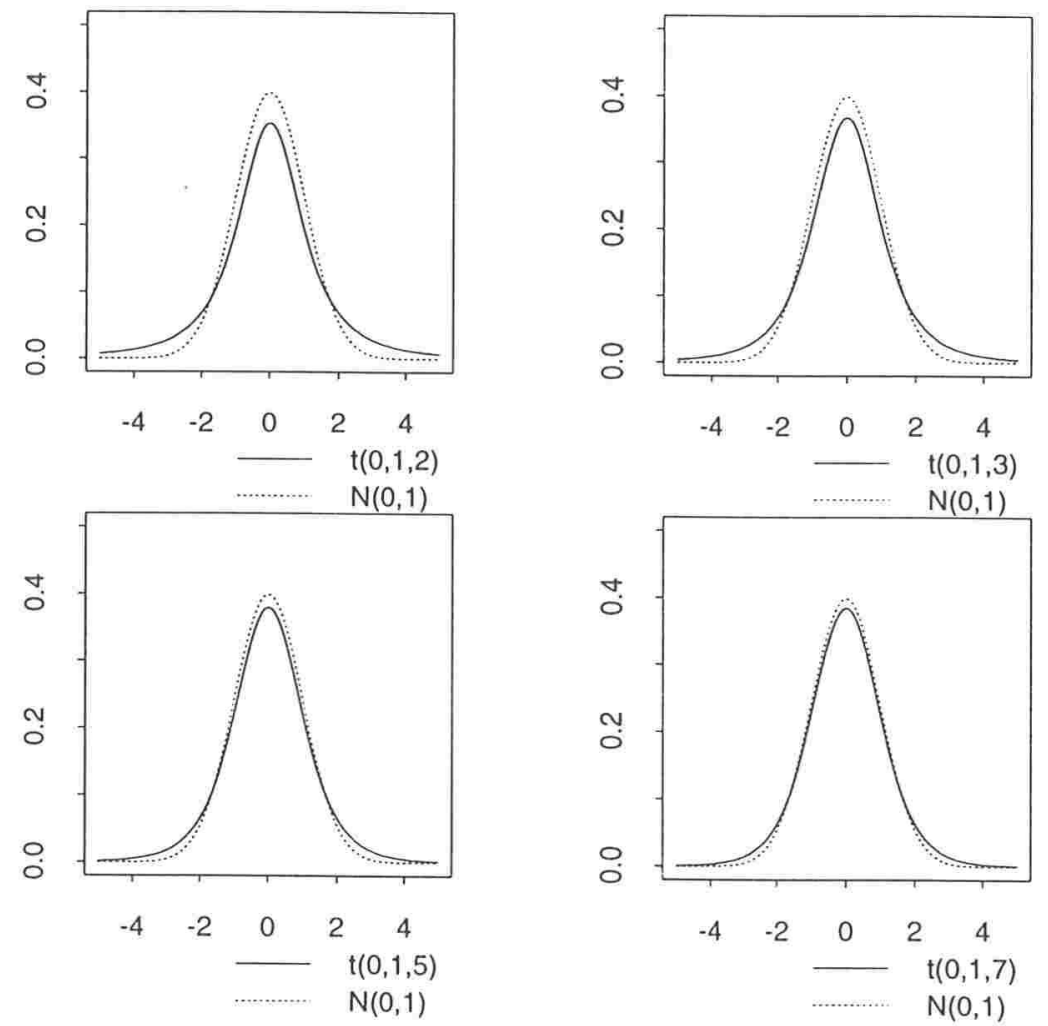

Figura 2.2: Gráficos de funçòes densidade t-Student e normal. 
(iv) $t$-Student generalizada forma

Se uma variável aleatória $y \sim S\left(\mu, O^{2}\right)$ e a funçào geradora de densidades $h(\cdot)$ tem a

$$
h(u)=\frac{s^{r / 2}}{B(1 / 2 \cdot r / 2)}(s+u)^{-\frac{r+1}{2}}, s, r>0, u>0 .
$$

então $y$ tem uma distribuiçào $t$-Student generalizada e escrevemos $y \sim t G\left(\mu, o^{2}, s, r\right)$ (Dickey, 1967). A distribuiçào $t$-Student generalizada tem como casos particulares as distribuições $t$-Student $(s=r=\nu)$ e Cauchy $(s=r=1)$.

Uma parametrização alternativa desta distribuição é obtida colocando na função geradora de densidades acima $c=\sqrt{s}$ e $m=(r+1) / 2$, com $m>1 / 2$. Neste caso, a distribuição é chamada Pearson VII (Fang, Kotz e Ng, 1990).

Suponhamos que $(y \mid v=\nu) \sim N\left(\mu, \nu \dot{o}^{2}\right)$ para $v \sim G I(r / 2, s / 2)$, onde $s>0$ e $r>0$ podem ser não inteiros. Temos as seguintes propriedades:

(a) $y \sim t G\left(\mu, \phi^{2}, s, r\right)$;

(b) $E(y)=\mu$, para $r>1$, $\operatorname{Var}(y)=(s /(r-2)) \phi^{2}$, para $r>2$, e o coeficiente de curtose é $\gamma_{2}=3+6 /(r-4)$. para $r>4$, que é maior que o coeficiente de curtose da distribuição normal, e nào depende do valor do parâmetro $s$;

(c) $(v \mid y=y) \sim G I\left((r+1) / 2,\left(s+z^{2}\right) / 2\right)$, onde $z^{2}=(y-\mu)^{2} / \phi^{2}$;

(d) $u^{2}=r z^{2} / s \sim F_{(1, r)}$;

(e) se $w=(r+1) /\left(s+z^{2}\right)$, entào

$$
E\left(z^{2 k} w^{l}\right)=\frac{\left(-\frac{r+1}{2}\right)^{l}}{s^{l-k}} \frac{1}{B(1 / 2, r / 2)} B((2 k+1) / 2,(r+2(l-k)) / 2),
$$

para $l=0,1,2, \ldots$ e $k=1,2 \ldots$;

(f) $s$ e $r$ têm as seguintes relaçòes com o coeficiente de curtose e o segundo momento central (Johnson e Kotz, 1970b, p.116):

$$
r=\frac{2\left(2 \gamma_{2}-3\right)}{\gamma_{2}-3} \quad \text { e } \quad s=\frac{2 \mu_{2} \gamma_{2}}{\gamma_{2}-3}
$$

(g) os parâmetros $\mu$ e $\phi$ não necessariamente admitem uma interpretação real em termos dos momentos. O l-ésimo momento existe se e somente se $r>l$;

(h) se definimos $y=v^{-1 / 2} z$, $\operatorname{com} z$ e $v$ variáveis aleatórias independentes, onde $z \sim$ $N(0,1)$ e $v \sim G I(r / 2, s / 2)$. então $y \sim t G(0,1, s, r)$.

Esta última propriedade é importante para gerar observaçòes de uma distribuição $t$ generalizada e também para utilização do algoritmo EM para estimação por máxima verossimilhança de parâmetros de modelos de regressào com distribuição $t$-Student generalizada para os erros (vide Seçào 2.5). 
Quando $s=r$ a distribuiçào $t$-Student generalizada coincide com a distribuição $t$ Student com parâmetros de locação $\mu$. escala $\phi$ e $r$ graus de liberdade. Na Figura 2.3. observamos a forma da distribuiçào $t$-Student generalizada para vários valores de $r$ e $s$.
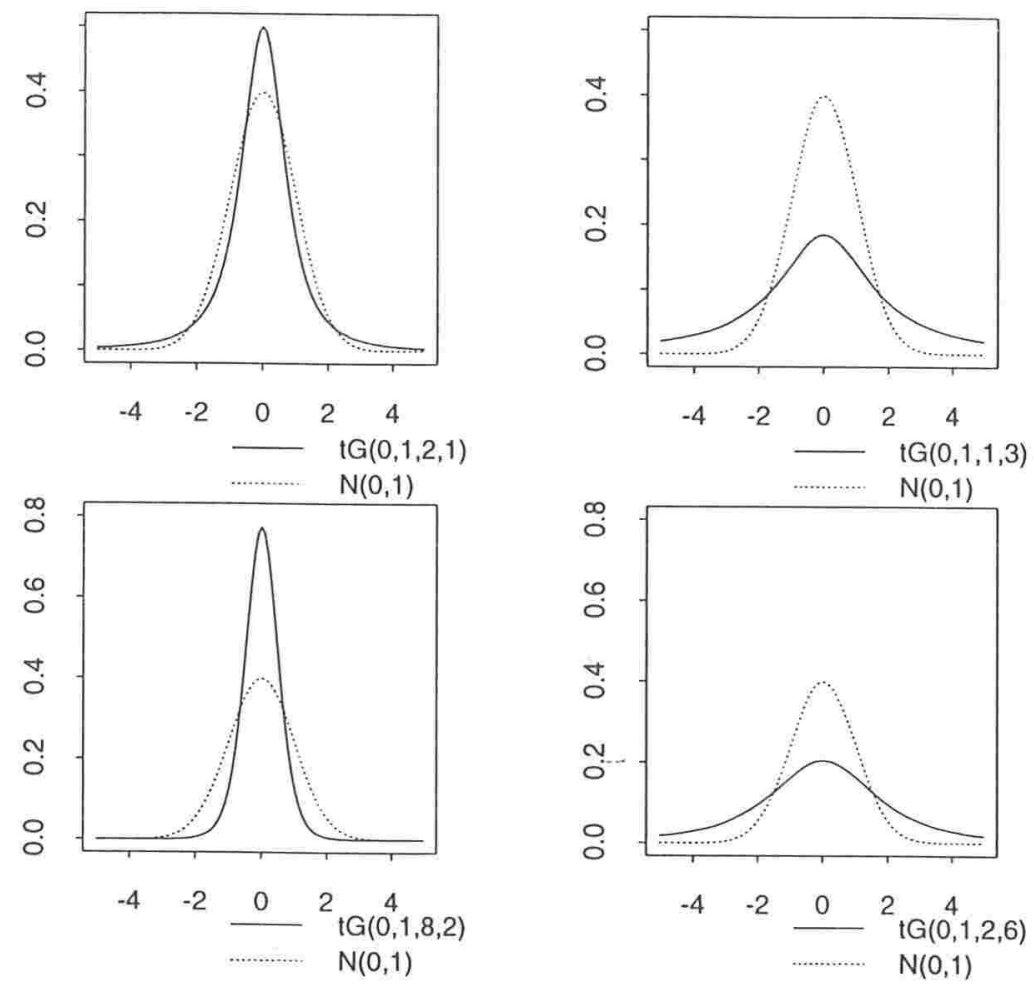

Figura 2.3: Gráficos de funções densidade t-Student generalizada e normal.

(v) Distribuição logística $I^{1}(($ mplumint udot mo $O X)$

Se uma variável aleatória $y \sim S\left(\mu, \phi^{2}\right)$ e a função geradora de densidades $h(\cdot)$ tem a forma (Fang, Kotz, NG, 1990)

$$
h(u)=c \frac{e^{-u}}{\left(1+e^{-u}\right)^{2}}, \quad u>0,
$$

onde $c$ é a constante de normalização obtida de $\int_{0}^{\infty} u^{-1 / 2} h(u) \mathrm{d} u=1$, ou seja, $c \approx$ 1,484300029 , então $y$ tem distribuição logística I e denotamos por $y \sim L I\left(\mu, \phi^{2}\right)$. Te$\operatorname{mos} E(y)=\mu, \operatorname{Var}(Y) \approx 0,79569 \phi^{2}$ e coeficiente de curtose $\gamma_{2} \approx 2.385165$. Salienta-se que a curtose desta distribuição é menor do que da distribuição normal.

Se $v=\left(e^{-z^{2}}-1\right) /\left(1+\epsilon^{-z^{2}}\right)$. com $z^{2}=(y-\mu)^{2} / \phi^{2}$, entào

$$
E\left(z^{2 r} v^{l}\right)=\frac{c}{2}(-1)^{l} \int_{0}^{1}\left[\log \left(\frac{1+s}{1-s}\right)\right]^{r-1 / 2} s^{l} \mathrm{~d} s, \quad l=0.1 .2, \ldots \quad \text { e } \quad r=1,2, \ldots
$$

\footnotetext{
${ }^{1}$ As distribuiçōes em (v) e (vi) são usualmente chamadas de logística. Por este motivo, as distinguimos usando a nomenclatura logística I e logística II.
} 
Na Figura 2.4, observamos a forma achatada no patamar da distribuiçào logística I que difere da distribuição normal.

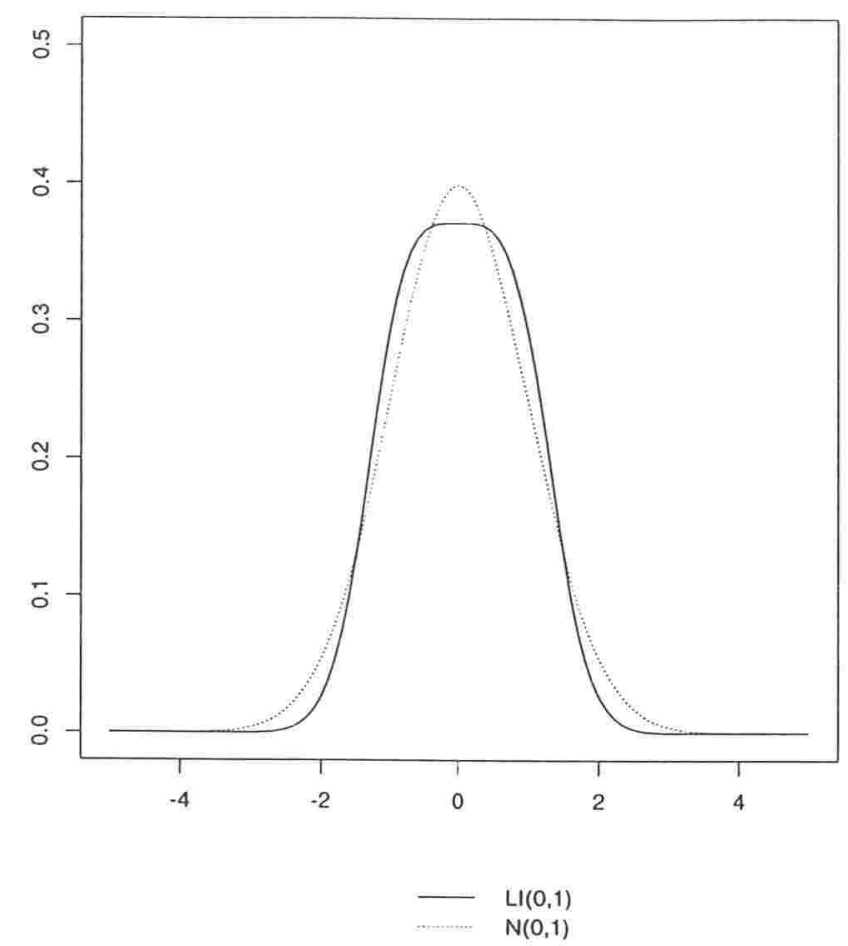

Figura 2.4: Gráficos de funções densidade logística I e normal.

\section{(vi) Distribuição logística II}

Uma variável aleatória $y \sim S\left(\mu, \phi^{2}\right)$ tem uma distribuição logística II se a função geradora de densidades $h(\cdot)$ tem a forma

$$
h(u)=\frac{\epsilon^{-u^{1 / 2}}}{\left(1+\epsilon^{-u^{1 / 2}}\right)^{2}}, \quad u>0,
$$

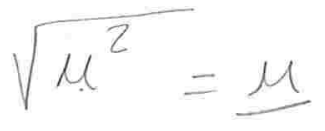

e denotamos por $y \sim L I I\left(\mu, \phi^{2}\right)$. Assim, a função densidade de $y$ é obtida a partir de (2.1) e da função $h(\cdot)$ acima. Sua função característica tem a forma $\psi_{y}(t)=2\left(e^{i t \mu} \pi \phi t\right) /\left(e^{\pi \phi t}-\right.$ $\left.e^{-\pi \phi t}\right), t \in \mathbb{R}$.

Se $y \sim L I I\left(\mu, \phi^{2}\right)$, então $E(y)=\mu$, $\operatorname{Var}(y)=\pi^{2} \phi^{2} / 3$, o coeficiente de curtose é $\gamma_{2}=4.2$, que é maior que as curtoses das distribuições normal e logística I, e $y$ tem mediana e moda iguais a $\mu$. A forma da distribuição logística II é similar à da normal (vide Figura 2.5).

Para gerar observações de uma distribuição logística II com parâmetros de locaçào $\mu$ e escala $\phi$ definimos y pela relação

$$
y=\mu+\phi \log \frac{u}{1-u}
$$


onde $u \sim$ Uniforme $(0,1)$ (vide Hastings e Peacock. 1975).

A função distribuição acumulada da distribuiçào logística II é utilizada para representar curvas de crescimento, por exemplo. em economia e demografia (Johnson e Kotz, 1970b). Na Figura 2.5 observamos a forma da distribuiçào logística II e constatamos que ela tem caudas mais pesadas do que a distribuição normal.

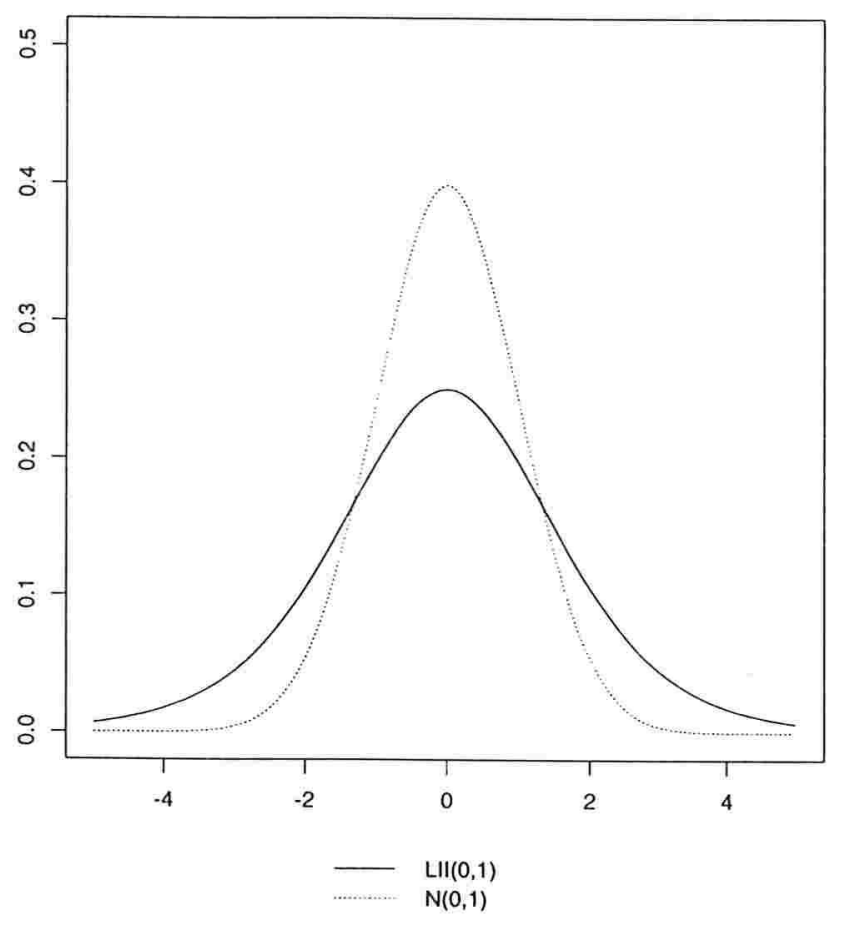

Figura 2.5: Gráficos de funções densidade logística II e normal.

\section{(vii) Distribuição logística generalizada}

Uma variável aleatória $y \sim S\left(\mu, \phi^{2}\right)$ tem uma distribuição logística generalizada se a função geradora de densidades é da forma

$$
h(u)=\frac{\alpha}{B(m, m)}\left[\frac{e^{-\alpha \sqrt{u}}}{\left(1+e^{-\alpha \sqrt{u}}\right)^{2}}\right]^{m} . m>0, u>0,
$$

onde $\alpha=\alpha(m) \operatorname{com} \alpha(\cdot)$ definida em $\mathbb{R}^{+}$e $\alpha(m)>0$, para $m>0$, e $B(\cdot, \cdot)$ é a função beta, e denotamos $y \sim L G\left(\mu, \phi^{2}, m\right)$. Assim, a função densidade de $y$ é obtida a partir de $(2.1)$ e da função $h(\cdot)$ acima. Esta distribuição pertence à classe de distribuições de Perks (vide Johnson e Kotz, 1970b). Se $\alpha(m)=1$. para todo $m>0$ e $m=1$, temos a distribuição logística II.

A distribuição logística generalizada aparece no trabalho de Gumbel (1944), para uma particular função $a(\cdot)$ como a distribuição da $m$-ésima amplitude média (média entre o maior e o menor valor em uma amostra aleatória de tamanho $n$ ) para uma classe de distribuições simétricas. Se $y \sim L G\left(\mu \cdot \phi^{2} . m\right)$, entào $E(y)=\mu \cdot \operatorname{Var}(y)=2 \psi^{\prime}(m) \phi^{2} / a(m)$ 
e o coeficiente de curtose é dado por

$$
\hat{i}_{2}=3+\frac{\psi^{\prime \prime \prime}(m)}{2 \psi^{\prime}(m)^{2}},
$$

onde $\psi^{\prime}(\cdot)$ e $\psi^{\prime \prime \prime}(\cdot)$ são a primeira e terceira derivada da função digama. Quando $m \rightarrow \infty$, $\gamma_{2} \rightarrow 3$, ou seja, $\hat{1}_{2}$ converge para a curtose da distribuição normal. Para todo $m>0$, temos $\gamma_{2}>3$ (vide Tabela 2.1).

Tabela 2.1: Coeficiente de curtose da distribuição logística generalizada

\begin{tabular}{ccccccccccccccccc}
\hline$m$ & 0,1 & 0,2 & 0.3 & 0.4 & 0.5 & $0, \bar{\tau}$ & 0,9 & 1,0 & 1,2 & 1,6 & 2,0 & 3,0 & 4,0 & 6,0 & 10,0 & 100,0 \\
\hline$\gamma_{2}$ & 5.92 & 5.72 & 5.48 & 5.23 & 5,00 & 4,61 & 4,32 & 4,2 & 4,01 & 3.75 & 3,66 & 3,38 & 3,28 & 3.17 & 3,10 & 3,01 \\
\hline
\end{tabular}

Na Figura 2.6. observamos a forma da distribuição logística generalizada para o caso em que $\alpha(m)=\sqrt{2 \psi^{\prime}(m)}$, considerando vários valores de $m$. A função $\alpha(\cdot)$ foi escolhida de tal forma que $\operatorname{Var}(y)=\phi^{2}$ (independentemente de $m$ ).
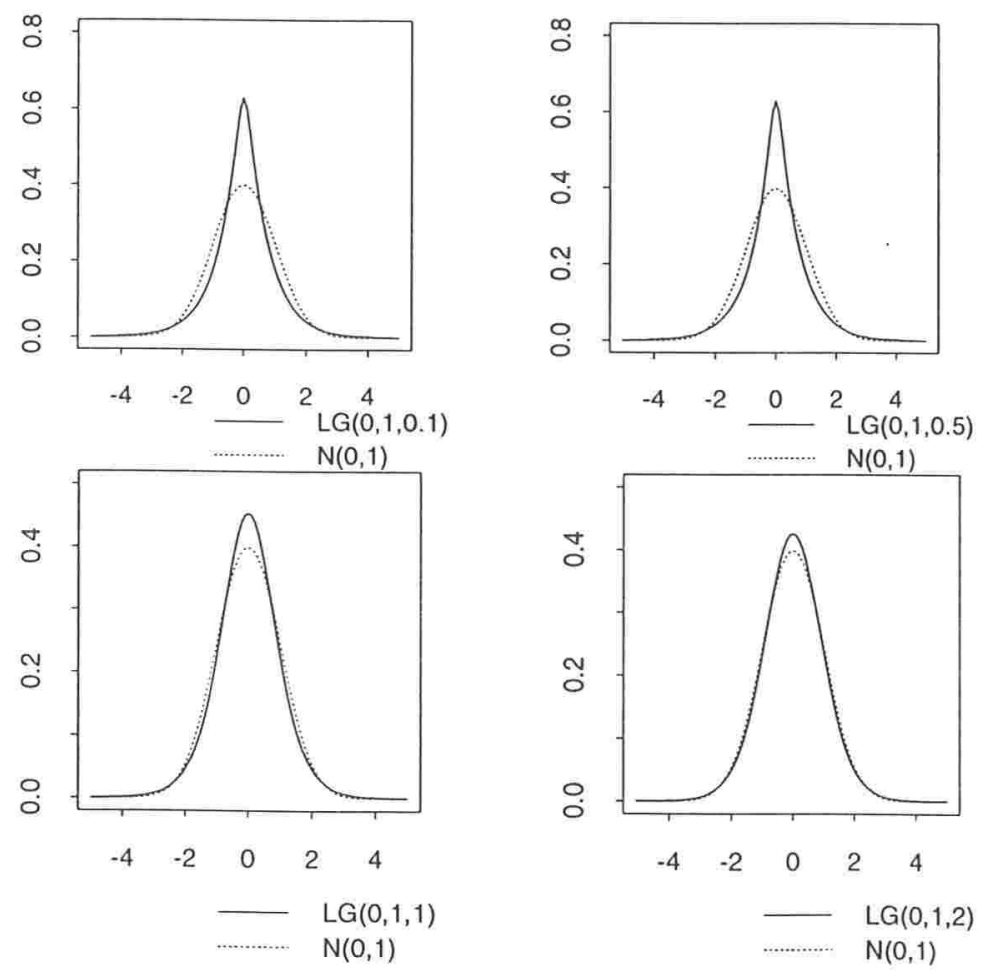

Figura 2.6: Gráficos de funçòes densidade logística generalizada e normal. 


\section{(viii) Distribuição de Kotz}

Uma variável aleatória $y \sim S\left(\mu . \phi^{2}\right)$ tem distribuiçào de Kiotz (Kiotz, 197j) se a função geradora de densidades $h(\cdot)$ tem a forma

$$
h(u)=\frac{r^{(2 N-1) / 2}}{\Gamma\left(\frac{2 N-1}{2}\right)} u^{N-1} \exp \{-r u\}, r>0, N \geq 1 . u>0 .
$$

e denotamos por $y \sim K\left(\mu, \phi^{2}, N, r\right)$. Desta maneira, a função densidade de $y$ é obtida de (2.1) e da função $h(\cdot)$.

Se $N=1$, a distribuição de Kotz se reduz a uma distribuição normal de média $\mu$ e variância $\phi^{2} /(2 r)$. Por outro lado, se $N>1$, a distribuição é bimodal com modas em $y=\mu \pm \sqrt{(N-1) / r} \phi$.

Se $y \sim K\left(\mu, \phi^{2}, N, r\right)$, entào $E(y)=\mu$, $\operatorname{Var}(y)=\{(2 N-1) /(2 r)\} \phi^{2}$, e o coeficiente de curtose é dado por $\gamma_{2}=(2 N+1) /(2 N-1)$ e os momentos centrais de ordem $2 m$ sào da forma

$$
\mu_{2 m}=E\left((y-\mu)^{2 m}\right)=\frac{\Gamma\left(\frac{2 m+2 N-1}{2}\right)}{r^{m} \Gamma\left(\frac{2 N-1}{2}\right)} \phi^{2 m}, m>0 .
$$

Além disto, a distribuiçào da variável $z^{2}=(y-\mu)^{2} / \phi^{2}$ é $\operatorname{Gama}((2 N-1) / 2, r)$. Em particular, se $N=1$ e $r=1 / 2, z^{2} \sim \chi_{(1)}^{2}$.

\section{(ix) Distribuição de Kotz generalizada}

Se $y \sim S\left(\mu, \phi^{2}\right)$ e a função geradora de densidades é da forma

$$
h(u)=\frac{s r^{(2 N-1) /(2 s)}}{\Gamma\left(\frac{2 N-1}{2 s}\right)} u^{N-1} \exp \left\{-r u^{s}\right\}, r, s>0, N \geq 1, u>0,
$$

então $y$ tem uma distribuição de Kotz generalizada e escrevemos $y \sim K G\left(\mu, \phi^{2}, N, r, s\right)$.

Quando $s=1$, esta distribuição se reduz à de $\operatorname{Kotz} K\left(\mu, \phi^{2}, N, r\right)$ e quando $N=1$, $s=1$ e $r=1 / 2$, se reduz a uma normal $N\left(\mu, \phi^{2}\right)$. Por outro lado, se $N=1, r=1 / 2 \mathrm{e}$ $s=1 /(1+k)$, a distribuição coincide com a distribuição exponencial potência que será definida abaixo.

Se $y \sim K G\left(\mu, \phi^{2}, N, r, s\right)$, então

$$
E(y)=\mu, \quad \operatorname{Var}(y)=\frac{\Gamma((2 N+1) / 2 s)}{r^{1 / s} \Gamma((2 N-1) / 2 s)} \phi^{2} .
$$

O coeficiente de curtose é dado por

$$
\gamma_{2}=\frac{\Gamma((2 N-1) / 2 s) \Gamma((2 N+3) / 2 s)}{\Gamma((2 N+1) / 2 s)^{2}}
$$

e os momentos centrais de ordem $2 m$ sào dados por

$$
\mu_{2 m}=E\left((y-\mu)^{2 m}\right)=\frac{\Gamma((2 N+2 m-1) / 2 s)}{r^{m / s} \Gamma((2 N-1) / 2 s)} \phi^{2 m} \cdot m>0 .
$$



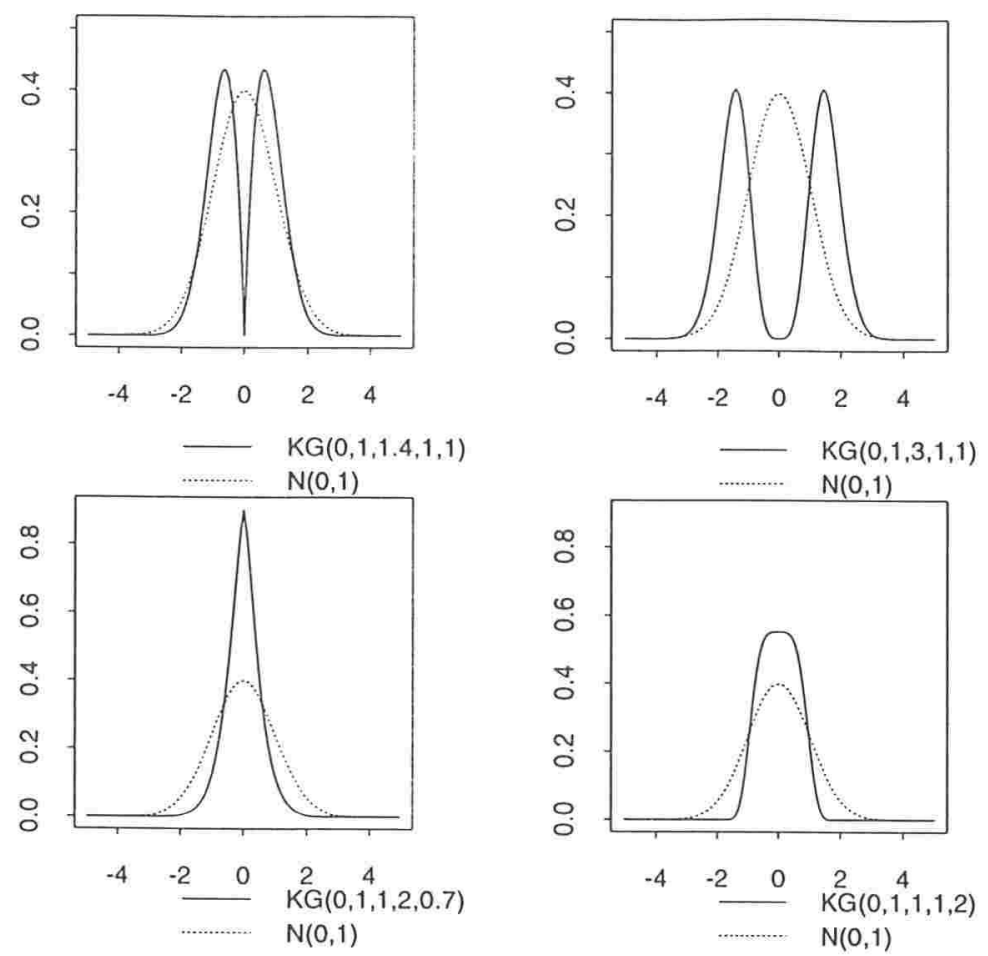

Figura 2.7: Gráficos de funções densidade Kiotz generalizada e normal.

Na Figura 2.7, observamos a forma da distribuição de Kotz generalizada para vários valores de $N, s$ e $r$. A distribuição apresenta uma forma bimodal quando $N>1$.

\section{(x) Distribuição normal contaminada}

Uma variável aleatória $y \sim S^{\prime}\left(\mu, \phi^{2}\right)$ tem uma distribuição normal contaminada se a função geradora de densidades $h(\cdot)$ tem a forma

$$
\begin{aligned}
h(u)= & (1-\varepsilon) \frac{1}{\sqrt{2 \pi}} \exp \{-u / 2\}+\varepsilon \frac{1}{\sqrt{2 \pi} \sigma} \exp \left\{-u /\left(2 \sigma^{2}\right)\right\} \\
& u>0, \quad \sigma>0, \quad 0 \leq \varepsilon \leq 1,
\end{aligned}
$$

e denotamos $y \sim N C\left(\mu, \phi^{2}, \varepsilon, \sigma^{2}\right)$. Temos $E(y)=\mu, \operatorname{Var}(y)=\left(1+\varepsilon\left(\sigma^{2}-1\right)\right) \phi^{2}$ e o coeficiente de curtose (Berkane e Bentler. 1986) é

$$
\gamma_{2}=3 \frac{\left(1+\varepsilon\left(\sigma^{4}-1\right)\right)}{\left(1+\varepsilon\left(\sigma^{2}-1\right)\right)^{2}} .
$$

A distribuição normal contaminada é utilizada também para estudar populaçòes de caudas mais pesadas do que as da distribuição normal. Little (1988) usa esta distribuição incorporando parâmetros adicionais que permitem ajustar a curtose da distribuição dos dados. 
(xi) Exponencial dupla

Se $y \sim S\left(\mu, \phi^{2}\right)$ e a funçào geradora de densidades $h(\cdot)$ é da forma

$$
h(u)=\frac{1}{2} \exp \{-\sqrt{u}\}, u>0
$$

então $y$ tem uma distribuiçào exponencial dupla (ou Laplace) e escrevemos $y \sim E D\left(\mu, \phi^{2}\right)$. Sua função característica tem a forma

$$
\psi_{y}(t)=\frac{e^{i t \mu}}{1+t^{2} \phi^{2}}, \quad t \in \mathbb{R}
$$

Se $z \sim E D(0,1)$, os momentos $\mu_{r}^{\prime}$ têm a forma

$$
\mu_{r}^{\prime}=E\left(z^{r}\right)= \begin{cases}0 & , \text { se } r \text { é ímpar, } \\ r ! & , \text { se } r \text { é par. }\end{cases}
$$

Logo, $y=\mu+\phi z \sim E D\left(\mu, \phi^{2}\right) \operatorname{com} E(y)=\mu$, $\operatorname{Var}(y)=2 \phi^{2}$, mediana e moda iguais a $\mu$ e coeficiente de curtose $\gamma_{2}=6$, que é maior que o coeficiente de curtose da distribuição normal. Os quartis superior e inferior são $\mu \pm 0,534 \phi$, enquanto que, para a distribuição normal, os correspondentes quartis são $\mu \pm 0,674 \phi$.

A distribuição exponencial dupla surge da diferença de duas variáveis aleatórias independentes com distribuição exponencial.

\section{(xii) Exponencial potência}

A variável aleatória $y \sim S\left(\mu, \phi^{2}\right)$ tem distribuição exponencial potência (Box e Tiao, 1973, Cap. 3) com parâmetro de locação $\mu$, escala $\phi$ e parâmetro de forma $k$ se sua função geradora de densidades é dada por

$$
h(u)=C(k) \exp \left\{-\frac{1}{2} u^{1 /(1+k)}\right\},-1<k \leq 1, u>0 .
$$

onde $C(k)^{-1}=\Gamma\left(1+\frac{1+k}{2}\right) 2^{1+(1+k) / 2}$, e denotamos $y \sim E P\left(\mu, \phi^{2}, k\right)$, sendo

$$
E(y)=\mu, \quad \operatorname{Var}(y)=2^{(1+k)}\left\{\frac{\Gamma\left(\frac{3(1+k)}{2}\right)}{\Gamma\left(\frac{1+k}{2}\right)}\right\} \phi^{2} .
$$

O coeficiente de curtose é dado por

$$
\gamma_{2}=\frac{\Gamma\left(\frac{5}{2}(1+k)\right) \Gamma\left(\frac{1+k}{2}\right)}{\Gamma\left(\frac{3}{2}(1+k)\right)^{2}} .
$$

Para $k>0$, temos $\gamma_{2}<3$, ou seja, a distribuição é leptocúrtica e. para $k<0$, temos $\gamma_{2}>3$ e, portanto, a distribuição é platicúrtica. O parâmetro $k$ pode ser considerado como uma medida de curtose, um indicador de não-normalidade. Em particular. se $k=0$, a distribuição é normal e quando $k=1$, a distribuição é exponencial dupla. Se $k$ tende a -1 , a distribuição tende a uma distribuiçào uniforme no intervalo $(\mu-\sqrt{3} \phi, \mu+\sqrt{3} \phi)$. 
Para gerar observaçòes de uma distribuiçào exponencial potência $(0.1 . k)$ definimos y pela relaçào

$$
y=(2 w)^{1 / r} v
$$

onde $v \sim \operatorname{Uniforme}(-1,1)$. $w \sim \operatorname{Gama}(1+1 / r, 1)$ e $r=2 /(1+k)$ (vide Devroye, 1986, p.174-175).

Esta família de distribuições foi estudada por Taylor (1992) considerando um modelo de regressão linear e supondo que a distribuição dos erros tem um parâmetro extra de forma.

Na Figura 2.8, observamos a forma da distribuição exponencial potência para vários valores de $k$.
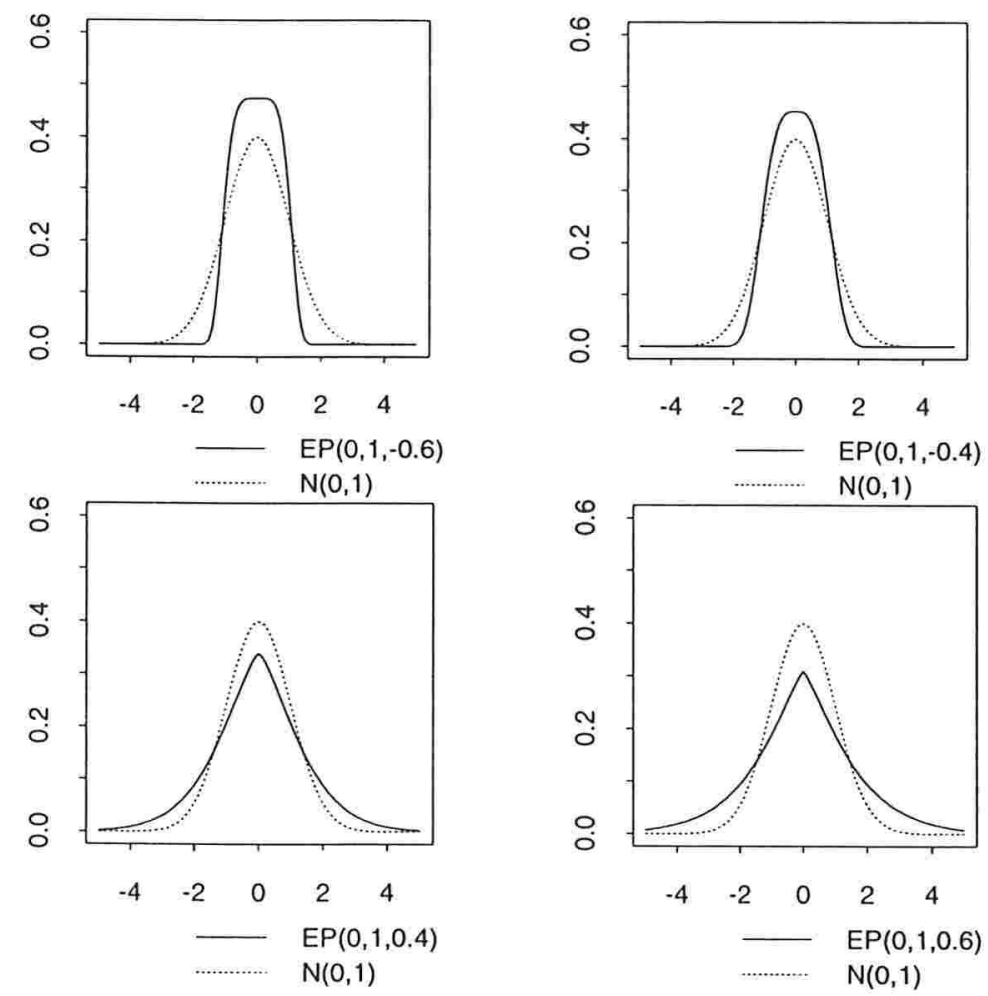

Figura 2.8: Gráficos de funções densidade exponencial potência e normal.

\section{(xiii) Potência estendida}

Uma variável aleatória $y \sim S\left(\mu, \dot{\phi}^{2}\right)$ pertence à família potência estendida se sua função geradora de densidades $h(\cdot)$ é da forma

$$
h(u)=K(c \cdot \lambda) \exp \left\{-\frac{1}{2} c \rho_{\lambda}\left(1+\frac{u}{c-1}\right)\right\},
$$


onde $K(c, \lambda)$ é uma constante normalizadora, $c>1, \lambda \geq 0, u>0 \mathrm{e}$

$$
\rho_{\lambda}(v)= \begin{cases}\frac{v^{\lambda}-1}{\lambda}, & \text { se } \lambda>0, \\ \lim _{\lambda \rightarrow 0} \frac{v^{\lambda}-1}{\lambda}=\log v, & \text { se } \lambda=0 .\end{cases}
$$

Esta família de distribuições inclui algumas distribuições na família de locação escala. Quando $\lambda=1$, a função densidade é a de uma distribuição normal com média $\mu$ e variância $((c-1) / c) \phi^{2}$. Se $\lambda=0$, a função densidade é de uma distribuição $t$-Student $\operatorname{com} c-1$ graus de liberdade, parâmetro de locação $\mu$ e escala $\phi$. Quando $\lambda=1 / 2$, a função densidade é similar à da exponencial dupla. Se $\lambda>0$, os momentos $E\left(y^{k}\right)$ existem para todo $k \geq 0$. Esta família de distribuições é estudada por Albert, Delampady e Polasek (1991).

Nas seções seguintes, estudaremos o problema de estimação e testes de hipóteses em modelos de regressão assumindo uma distribuição simétrica para os erros. A idéia é desenvolver resultados gerais, válidos para todos os modelos simétricos em problemas regulares, e apresentar, como casos particulares, os resultados para algumas distribuições simétricas descritas acima.

\subsection{Modelos de regressão linear}

Consideremos $n$ variáveis aleatórias contínuas independentes $y_{1}, \ldots, y_{n}$, todas com distribuição tendo suporte na reta real. Assumimos que cada $y_{l}$ tem função densidade na forma (2.1) com parâmetro de locação $\mu_{l} \in \mathbb{R}$ e de escala $\phi>0$, comum para todas as observações. Consideremos ainda que o vetor $\mu=\left(\mu_{1}, \ldots, \mu_{n}\right)^{\top}$ tem uma estrutura linear especificada por

$$
\mu=X \beta,
$$

onde $X$ é uma matriz $n \times p$, não estocástica, de posto $p(p<n)$ e $\beta=\left(\beta_{1}, \ldots, \beta_{p}\right)^{\top}$ é um conjunto de parâmetros de regressão desconhecidos a serem estimados.

Evidentemente, o modelo definido por (2.1) e (2.2) pode ser escrito como

$$
Y=X \beta+\phi \zeta
$$

onde $Y=\left(y_{1}, \ldots, y_{n}\right)^{\top}, X$ e $\beta$ estão definidos acima, $\phi>0$ e $\zeta=\left(z_{1}, \ldots, z_{n}\right)^{\top}$ é um vetor de variáveis aleatórias independentes e identicamente distribuídas com a função densidade

$$
\pi\left(z_{l}\right)=h\left(z_{l}^{2}\right), \quad z_{l} \in \mathbb{R},
$$

onde $h(\cdot)$ é a função geradora de densidades, definida na Seção 2.2. Em outras palavras, se $x_{1}^{\top}, \ldots, x_{n}^{\top}$ denotam as linhas da matrix $\mathrm{X}$, estamos assumindo que $y_{1}, \ldots, y_{n}$ são variáveis aleatórias independentes e $y_{l} \sim S\left(x_{l}^{\top} \beta, \phi^{2}\right)$ para $l=1, \ldots, n$.

Evidentemente, a classe de modelos aqui definida engloba os modelos de regressão linear com erros independentes e normalmente distribuídos, com a vantagem de que se permite que a distribuição dos erros, embora simétrica, tenha, por exemplo, caudas mais 
pesadas do que a da distribuição normal padrão. Como visto na seção anterior, é possível se tomar uma distribuição para os erros com curtose tanto maior quanto menor do que a da curva normal. Uma desvantagem de se trabalhar com distribuições simétricas, que não sejam a normal, é a de que, em geral, algoritmos iterativos são necessários para se obter as estimativas de máxima verossimilhança para os parâmetros do modelo. No entanto, é claro que a estimação por mínimos quadrados não vai requerer tais algoritmos como será visto na seção seguinte, mas com a desvantagem dos estimadores serem vulneráveis a observações extremas ("outliers").

\subsection{Estimação por mínimos quadrados}

Consideremos o modelo de regressão linear definido na Seção 2.3. O método de mínimos quadrados para estimar $\beta$ consiste em minimizar a soma de quadrados residual

$$
\zeta^{\top} \zeta=\phi^{-2}(Y-X \beta)^{\top}(Y-X \beta)
$$

onde $\zeta=\left(z_{1}, \ldots, z_{n}\right)^{\top}$, com $z_{l}=\left(y_{l}-x_{l}^{\top} \beta\right) / \phi$. É bem conhecido o fato de que o estimador de mínimos quadrados de $\beta$, ou seja, aquele que minimiza $\zeta^{\top} \zeta$, é dado por

$$
\widehat{\beta}_{M Q}=\left(X^{\top} X^{\top}\right)^{-1} X^{\top} Y
$$

independentemente da distribuição assumida para $y_{l}, l=1, \ldots, n$.

$\mathrm{O}$ estimador $\widehat{\beta}_{M Q}$ é linear em $Y$ e é muito simples de ser calculado. Para se verificar outras propriedades de $\widehat{\beta}_{M Q}$, é necessário lembrar que, se os dois primeiros momentos de $z_{l}$, para $l=1, \ldots, n$, existem, então $E\left(z_{l}\right)=0$ e $\operatorname{Var}\left(z_{l}\right)=\xi<\infty$, onde $\xi>0$ é definido na Seção 2.2. Assim, pode-se mostrar que, se tais momentos existem, $\widehat{\beta}_{M Q}$ é um estimador não viciado de $\beta, \operatorname{Var}\left(\widehat{\beta}_{M Q}\right)=\xi \phi^{2}\left(X^{\top} X\right)^{-1}$ e $\widehat{\beta}_{M Q}$ é o estimador da variância mínima na classe dos estimadores lineares e não viciados (BLUE) de $\beta$.

Se, além de assumir a existência dos dois primeiros momentos de $z_{l}$, assumimos que

$$
\begin{gathered}
\max _{1 \leq k \leq n} x_{k}^{\top}\left(X^{\top} X\right)^{-1} x_{k} \rightarrow 0, \text { quando } n \rightarrow \infty, \\
\lim _{n \rightarrow \infty} n^{-1}\left(X^{\top} X\right)=\Omega,
\end{gathered}
$$

com $\Omega$ uma matriz $p \times p$ finita e não-singular, então (Sen e Singer, 1993, p.280): $\sqrt{n}\left(\widehat{\beta}_{M Q}-\right.$ $\beta$ ) converge em distribuição para $U \sim N_{p}\left(0, \xi \phi^{2} \Omega^{-1}\right)$. Isto é, $\widehat{\beta}_{M Q}$ tem distribuição assintótica normal $p$-variada com média $\beta$ e matriz de covariância $\xi \phi^{2}\left(X^{\top} X\right)^{-1}$. Assim, comparando com o modelo de regressão linear normal, o estimador de mínimos quadrados de $\beta$ tem variância assintótica que é multiplicada pela quantidade $\xi$. Na Tabela 2.2 são apresentados os valores de $\xi$ para cada distribuição estudada. Notamos que no caso normal este valor é igual a 1 . sendo maior que os valores das distribuições logística I, logística generalizada com $m \geq 3, t$-Student generalizada com $r>s+2$, Kotz com $\frac{1}{2}<N<r+\frac{1}{2}$, Kotz generalizada com $r>[\Gamma((2 N+1) / 2 s) / \Gamma((2 N-1) / 2 s)]^{s}$ e normal contaminada com $\gamma\left(\sigma^{2}-1\right)<0$. 
Tabela 2.2: Valor de $\xi$ para algumas distribuições simétricas

\begin{tabular}{|c|c|}
\hline DISTRIBUIÇÃO & $\xi$ \\
\hline Normal & 1 \\
\hline$t$-Student & $\frac{\nu}{\nu-2}, \quad \nu>2$ \\
\hline$t$-Student generalizada & $\frac{s}{r-2}, \quad s>0, r>2$ \\
\hline Logística I & 0,79569 \\
\hline Logística II & $\pi^{2} / 3$ \\
\hline Logística generalizada & $2 \psi^{\prime}(m)$ \\
\hline Kotz & $(2 N-1) / 2 r, \quad N \geq 1, r>0$ \\
\hline Kotz-generalizada & $\frac{\Gamma\left(\frac{2 N+1}{2 s}\right)}{\Gamma\left(\frac{2 N-1}{2 s}\right)} r^{-1 / s}, \quad r, s>0, N \geq 1$ \\
\hline Normal contaminada & $1+\gamma\left(\sigma^{2}-1\right), \quad 0 \leq \gamma \leq 1, \sigma>0$ \\
\hline Exponencial dupla & 2 \\
\hline Exponencial potência & $2^{(1+k)} \frac{\Gamma\left(\frac{3}{2}(k+1)\right)}{\Gamma\left(\frac{k+1}{2}\right)}$ \\
\hline
\end{tabular}

Nota: $\psi^{\prime}(\cdot)$ é a primeira derivada da função digama.

Seja $\hat{e}_{M Q}=Y-X \widehat{\beta}_{M Q}$ o vetor resíduos. Então, usando o fato de que $\widehat{e}_{M Q}=(I-Z) Y$, onde $I$ é uma matriz identidade $n \times n$ e $Z=X\left(X^{\top} X\right)^{-1} X^{\top}$ é uma matriz $n \times n$, e notando que $I-Z$ e $Z$ são matrizes idempotentes de postos $n-p$ e $p$, respectivamente, o erro quadrático médio de y é $E Q M=S Q R /(n-p)$, onde $S Q R=\widehat{e}_{M Q}^{\top} \widehat{e}_{M Q}$ é a soma dos quadrados dos resíduos. Se $E\left(z_{l}\right)=0$ e $\operatorname{Var}\left(z_{l}\right)=\xi<\infty, \xi>0$, é bem conhecido o fato de que $E Q M$ é um estimador não viciado e consistente de $\xi \phi^{2}$ (vide Arnold, 1981, p.148). Desta forma, o estimador de $\phi^{2}$ tem a forma $\widehat{\phi}_{M \dot{Q}}^{2}=E Q M / \xi$.

Cabe mencionar que se as observações são independentes e possuem distribuições normais então a regressão por mínimos quadrado é ótima e fornece estimadores de máxima 
verossimilhança para os parâmetros do modelo. Entretanto, a regressão por mínimos quadrados está longe de ser ótima em diversas situações, especialmente quando a distribuição das observações tem caudas alongadas como, por exemplo, as distribuições Cauchy, $t$-Student e Logística II. Além disto, valores aberrantes associados a valores extremos das variáveis regressoras podem tornar inadequadas as estimativas de mínimos quadrados. Diversos métodos e procedimentos robustos de regressão têm sido desenvolvidos para superar algumas dificuldades da regressão de mínimos quadrados, em particular, para acomodar a possibilidade da presença de valores aberrantes nos dados. Entre os métodos alternativos robustos à regressão de mínimos quadrados temos a estimação por máxima verossimilhança que fornece pesos diferenciados a valores aberrantes nas estimativas dos parâmetros de interesse. A estimação $L_{1}$ (vide Narula e Stangenhaus, 1988), que também obtém estimativas de regressão robustas que são resistentes a valores aberrantes (Hampet, 1971), tem como idéia básica minimizar a soma de valores ábsolutos dos resíduos, isto é,

$$
\operatorname{minimizar} \sum_{i=1}^{n}\left|z_{i}\right| \text {, }
$$

onde $z_{i}=\left(y_{i}-x_{i}^{\top} \beta\right) / \phi$, sujeito a $(2.1)$ e (2.2).

Outro método de estimação robusta é obtido através dos $M$-estimadores propostos por Huber (1973). São dados como solução do seguinte problema de minimização:

$$
\operatorname{minimizar} \sum_{i=1}^{n} \rho\left(z_{i}\right) \text {, }
$$

onde $\rho(\cdot)$ é uma função simétrica e tem um único mínimo em zero. Os estimadores de mínimos quadrados e os estimadores $L_{1}$ são casos particulares dos $M$-estimadores onde $\rho\left(z_{i}\right)=z_{i}^{2}$ e $\rho\left(z_{i}\right)=\left|z_{i}\right|$, respectivamente. Se consideramos $\rho\left(z_{i}\right)=\log \pi\left(y_{i} ; \mu_{i}, \phi\right)$, sujeito a (2.1) e (2.2), e os $M$-estimadores coincidem com os estimadores de máxima verossimilhança obtidos na seção seguinte. Outras formas para a função $\rho$ podem ser consideradas (vide Bustos, 1981).

\subsection{Estimação por máxima verossimilhança}

Seja $L(\theta)$, onde $\theta=\left(\beta^{\top}, \phi\right)^{\top}$, o logaritmo da função verossimilhança do modelo de regressão definido em $(2.1)$ e $(2.2)$, dados $y_{1}, \ldots, y_{n}$. Temos

$$
L(\theta)=-n \log \phi+\sum_{l=1}^{n} \log h\left(\left(\frac{y_{l}-x_{l}^{\top} \beta}{\phi}\right)^{2}\right),
$$

ou seja,

$$
L(\theta)=-n \log \phi+\sum_{l=1}^{n} t\left(z_{l}\right)
$$

onde

$$
t\left(z_{l}\right)=\log h\left(z_{l}^{2}\right)
$$


com

$$
z=\left(y_{l}-x_{l}^{\top} \beta\right) / \phi
$$

representando o erro "padronizado" relativo à l-ésima observação.

Para a obtençào de estimadores de máxima verossimilhança. bem como para a obtenção das estatísticas de testes (Seçào 2.6) e de suas respectivas correçòes de Bartlett ou tipo Bartlett (Capítulo 3) será necessário obter derivadas do logaritmo da função de verossimilhança com relaçào aos parâmetros desconhecidos e alguns momentos destas derivadas. Assumimos, portanto, no que segue, que tais derivadas e momentos existem. Assim, os modelos simétricos que não satisfazem esta condição estão excluídos do nosso estudo. Por exemplo, para a distribuição exponencial dupla, a primeira derivada de $L(\theta)$ com repeito a $\beta$ não existe para todo $\beta \in \mathbb{R}^{p} \mathrm{e}$, portanto, não será considerada aqui. Em outros casos, as derivadas de $L(\theta)$ existem para todos os valores dos parâmetros desconhecidos no espaço paramétrico, somente se o parâmetro extra conhecido (por exemplo. o parâmetro $k$ da distribuição exponencial potência) pertence a um determinado intervalo. Este intervalo será indicado à medida que os resultados forem sendo apresentados.

A primeira derivada $\partial L(\theta) / \partial \theta$ do logaritmo da função de verossimilhança dado èm (2.5) é obtida de

$$
\frac{\partial L(\theta)}{\partial \beta_{r}}=-\frac{1}{\phi} \sum_{l=1}^{n} t_{l}^{(1)} x_{l r},
$$

$\mathrm{e}$

$$
\frac{\partial L(\theta)}{\partial \phi}=-\frac{n}{\phi}-\frac{1}{\phi} \sum_{l=1}^{n} t_{l}^{(1)} z_{l},
$$

onde

$$
t_{l}^{(1)}=\frac{\mathrm{d}}{\mathrm{d} z_{l}} t\left(z_{l}\right), \quad l=1, \ldots, n
$$

assumindo que esta derivada exista para todo $z_{l} \in \mathbb{R}$. Como $t_{l}^{(1)}=-z_{l} w_{l}$, onde

$$
w_{l}=w_{l}(\theta)=-2 \frac{\mathrm{d}}{\mathrm{d} u} \log h(u),\left.\right|_{u=y^{2}}
$$

a função escore total de $\theta, U=U(\theta)$, tem a forma $U=\left(U_{\beta}^{\top}, U_{\phi}\right)^{\top}=\left(\partial L(\theta) / \partial \beta_{1}, \ldots\right.$, $\left.\partial L(\theta) / \partial \beta_{p}, \partial L(\theta) / \partial \phi\right)^{\top}$, com

$$
U_{\beta}=\frac{1}{\phi^{2}} X^{\top} W(Y-X \beta)
$$

e

$$
U_{\phi}=-\frac{n}{\phi}+\frac{1}{\phi^{3}}(Y-X \beta)^{\top} W(Y-X \beta)
$$

sendo $W=W(\theta)=\operatorname{Diag}\left\{w_{1} \ldots, w_{n}\right\}$.

Consideramos aqui o método de máxima verossimilhança para estimar os parâmetros $\beta_{1} \ldots, \beta_{p}$ e $\phi$, e, portanto. os parâmetros de locação $\mu_{1}, \ldots, \mu_{n}$. O estimador de máxima

$$
U_{\phi}=\frac{1}{\phi}\left[\frac{1}{\phi^{2}}(y-X \beta)^{\top} W(Y-X \beta)-m\right]
$$


verossimilhança (EMV) $\hat{\theta}$ de $\theta$ é soluçào do sistema de equaçòes daclo por

$$
\left.\frac{\partial L(\theta)}{\partial \beta_{r}}\right|_{\theta=\hat{\theta}}=0 . r=1 \ldots p . \quad \text { e }\left.\frac{\partial L(\theta)}{\partial o}\right|_{\theta=\hat{\theta}}=0 .
$$

Estas equações podem ser escritas como

$$
\sum_{l=1}^{n}\left(y_{l}-x_{l}^{\top} \hat{\beta}\right) \hat{u}_{l} x_{l r}=0
$$

$\mathrm{e}$

$$
\sum_{l=1}^{n}\left(y_{l}-x_{l}^{\top} \widehat{\beta}\right)^{2} \widehat{w}_{l}-n \widehat{\phi}^{2}=0
$$

É fácil ver que as equações acima podem ser expressas de forma equivalente como

$$
\widehat{\beta}=\left(X^{\top} \widehat{W} X^{\top}\right)^{-1} X^{\top} \widehat{W} Y
$$

e

$$
\widehat{\phi}^{2}=\frac{1}{n} \widehat{\epsilon}^{\top} \widehat{W} \widehat{\epsilon}
$$

respectivamente, onde $\widehat{W}=W(\widehat{\theta})$ e $\widehat{\epsilon}=(Y-\mathrm{X} \widehat{\beta})$ é o vetor de resíduos.

No estudo das propriedades dos EMV's de $\theta$, observamos que a contribuição de cada observação nas equações de verossimilhança dadas em (2.10) e (2.11) envolve o fator $w_{l}$ definido em (2.7) que pode ser interpretado como o peso da l-ésima observação nas estimativas de $\beta$ e $\phi$. Na Tabela 2.3, mostramos o valor de $w$ para algumas distribuiçòes estudadas na Seção 2.2 e na Figura 2.9 apresentamos gráficos de $w$ versus $u\left(u=z^{2}\right)$.

Da Tabela 2.3, observamos que no modelo normal todas as observaçòes têm o mesmo peso $(w=1)$ na estimativa dos parâmetros $\beta$ e $\phi$. Na distribuição $t$-Student, qualquer que seja o valor de $0<\nu<\infty$. tem-se que o peso $w_{l}$ é uma função decrescente em $\tilde{z}_{l}^{2}=\left(y_{l}-x_{l}^{\top} \beta\right)^{2} / \phi^{2}$. Conseqüentemente, os EMV's de $\beta$ e $\phi$ sào robustos no sentido de que observaçòes com um valor $z_{l}^{2}$ grande, digamos $z_{l}^{2}>1$. têm um peso $w_{l}<1$. que decresce quando $z_{l}^{2}$ cresce. Na distribuição $t$-Student generalizada, o peso também é uma funçào decrescente na distância $z_{l}^{2}$. Além disto. é fácil ver também que $w_{l}<1$ se $\tilde{z}_{l}^{2}>1+r-s, w_{l}=1$ se $z_{l}^{2}=1+r-s$, e $w_{l}>1$ se $z_{l}^{2}<1+r-s$. Na distribuição logística I, temos $w_{l}<2$ e os pesos $w_{l}$ são funções crescentes em $z_{l}^{2}$ devido à característica de que esta distribuição tem caudas curtas. Nas distribuiçòes logística II e logística generalizada. os pesos $w_{l}$ sàos funçòes decrescentes em $|z l|$. Na distribuiçào exponencial potência, $w_{l}<1$ se $: z_{l}^{2}>(1+k)^{-(1+k) / k}$. 
Tabela 2.3: Valor de $w$ para algumas distribuiçòes simétricas

\begin{tabular}{|c|c|}
\hline DISTRIBUIÇAO & $w$ \\
\hline Normal & $1 / y$ \\
\hline Cauchy & $2 /\left(1+z^{2}\right)$ \\
\hline$t$-Student & $(\nu+1) /\left(\nu+z^{2}\right)$ \\
\hline$t$-Student generalizada & $(r+1) /\left(s+z^{2}\right)$ \\
\hline Logística I & $\frac{2\left(1-e^{-z^{2}}\right)}{1+e^{-z^{2}}}=2 \tanh \left(z^{2} / 2\right)$ \\
\hline Logística II & $\left(e^{|z|}-1\right) /\left(|z|\left(1+e^{|z|}\right)\right)$ \\
\hline Logística generalizada & $\alpha m\left(e^{\alpha|z|}-1\right) /\left(|z|\left(1+e^{\alpha|z|}\right)\right)$ \\
\hline Normal contaminada & $f_{1}\left(z^{2}\right) / f_{0}\left(z^{2}\right)$ \\
\hline \multicolumn{2}{|c|}{ onde $f_{i}\left(z^{2}\right)=(1-\varepsilon) e^{-z^{2} / 2}+\varepsilon\left(\sigma^{2}\right)^{-1 / 2-i} e^{-z^{2} / 2 \sigma^{2}}, i=0,1$} \\
\hline Exponencial potência & $1 /\left[(1+k)\left(z^{2}\right)^{k /(1+k)}\right]$, para $-1<k<1$. \\
\hline
\end{tabular}


$\mathrm{C}(0,1)$

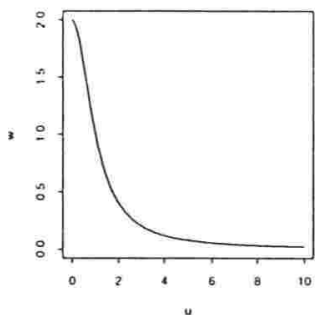

Lᄂ(1)

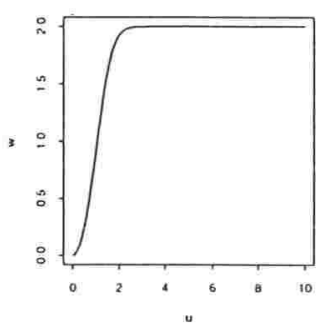

$E P(0,1,-0.6)$

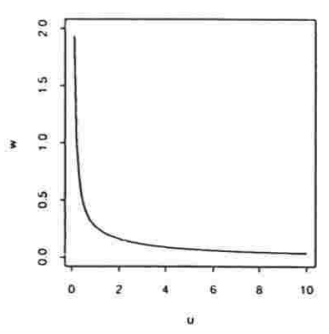

$t(0,1,4)$

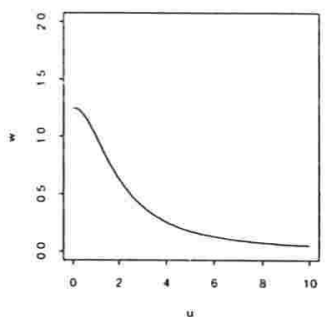

LIII $(0.1)$

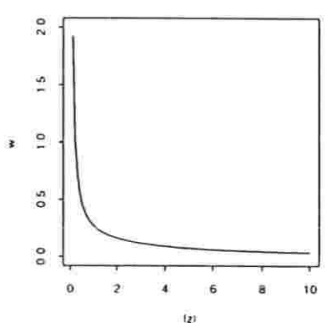

$\mathrm{IG}(0,1,3.2)$

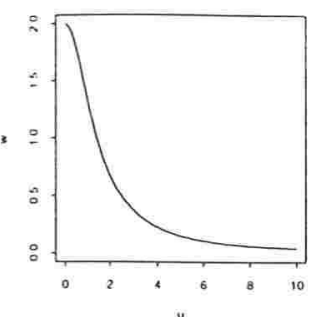

LG(0.1.0.5)

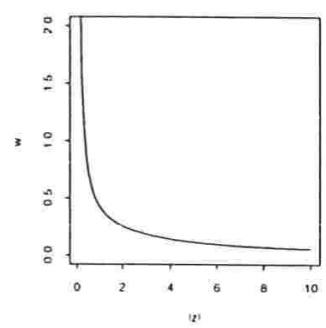

Figura 2.9: Gráficos da função w para diversas distribuições

Observemos que as equações em (2.10) e (2.11) são não lineares, exceto para o modelo normal $\left(w_{l}=1, l=1, \ldots, n\right)$ e, portanto, não podem ser resolvidas explicitamente. Desta maneira, as equações dadas acima devem ser resolvidas iterativamente usando, por exemplo, o algoritmo de Newton-Raphson, de scoring ou o algoritmo EM. Os algoritmos de Newton-Raphson e scoring envolvem, respectivamente, as matrizes de informação observada e esperada que obtemos a seguir.

As segundas derivadas do logaritmo da função de verossimilhança têm as formas

$$
\begin{aligned}
\frac{\partial^{2} L(\theta)}{\partial \beta_{r} \partial \beta_{s}} & =\frac{1}{\phi^{2}} \sum_{l=1}^{n} t_{l}^{(2)} x_{l r} x_{l s}, r, s=1, \ldots, p, \\
\frac{\partial^{2} L(\theta)}{\partial \phi^{2}} & =\frac{1}{\phi^{2}} \sum_{l=1}^{n}\left(1+2 t_{l}^{(1)} z_{l}+t_{l}^{(2)} \tilde{z}_{l}^{2}\right), \\
\frac{\partial^{2} L(\theta)}{\partial \beta_{r} \partial \phi} & =\frac{1}{\phi^{2}} \sum_{l=1}^{n}\left(t_{l}^{(2)} z_{l}+t_{l}^{(1)}\right) x_{l}, r=1, \ldots, p .
\end{aligned}
$$


Assumindo que a função de verossimilhança é regular. isto é, quando é permitida a troca de derivadas da funçào de verossimilhança com respeito aos parâmetros com integrais sobre o espaço amostral. e das propriedades das distribuições simétricas, tem-se

$$
\begin{aligned}
E\left[\frac{\partial^{2} L(\theta)}{\partial \beta_{r} \partial \beta_{s}}\right] & =-E\left[\frac{\partial L(\theta)}{\partial \beta_{r}} \frac{\partial L(\theta)}{\partial \beta_{s}}\right]=-\frac{\delta_{(2.0 .0 .0 .0)}}{\phi^{2}} \sum_{l=1}^{n} x_{l r} x_{l s}, \text { para } r, s=1,2, \ldots, p ; \\
E\left[\frac{\partial^{2} L(\theta)}{\partial \phi^{2}}\right] & =-E\left[\frac{\partial L(\theta)}{\partial \phi} \frac{\partial L(\theta)}{\partial \phi}\right]=-\frac{n}{\phi^{2}}\left(\delta_{(2.0 .0 .0 .2)}-1\right), \quad K_{\phi, \phi}=\frac{m}{\phi^{2}}\left(\delta_{z 000 z^{-1}}\right) \\
E\left[\frac{\partial^{2} L(\theta)}{\partial \beta_{r} \partial \phi}\right] & =0, r=1, \ldots, \phi p,
\end{aligned}
$$

\section{$K_{\phi, \phi}=\frac{n}{\phi^{2}}\left(\delta_{20002^{-1}}\right)$}

para $a, b, c, d, e=0,1,2,3,4, \operatorname{com} t^{(r)}=\partial^{r} t(z) / \partial z^{r}\left(\delta_{(2,0,0,0,0)}\right.$ e $\delta_{(2,0,0,0,2)}$ para várias distribuições podem ser encontrados no Apêndice C). Assim, a matriz de informação é bloco diagonal e tem a forma

$$
K^{*}=K(\theta)=\operatorname{Diag}\left\{K_{\beta, \beta}, \kappa_{\phi, \phi}\right\},
$$

com

$$
K_{\beta, \beta}=\frac{\delta_{(2,0,0,0,0)}}{\phi^{2}} X^{\top} X
$$

$\mathrm{e}$

$$
K_{\phi, \phi}=\kappa_{\phi, \phi}=\frac{n}{\phi^{2}}\left(\delta_{(2,0,0.0,2)}-1\right) .
$$

$\mathrm{O}$ fato de que $\beta$ e $\phi$ são globalmente ortogonais, no sentido de que a matriz de informação $K$, em (2.14), é bloco diagonal nas correspondentes submatrizes $K_{\beta, \beta}$ e $K_{\phi, \phi}$, implica que os EMV's $\hat{\beta}$ e $\widehat{\phi}$ são assintoticamente não correlacionados. Além disto, se o logaritmo da função de verossimilhança definida em (2.4) verifica as condições de regularidade sob as quais o EMV de $\theta=\left(\beta^{\top}, \phi\right)^{\top}$ é assintoticamente normal, então, sob as condições dadas em (2.3). tem-se também que

$$
\left(\hat{\beta}^{\top}, \widehat{\phi}\right)^{\top} \sim A N_{p+1}\left(\left(\beta^{\top}, \phi\right)^{\top}, K^{-1}\right),
$$

onde $K^{-1}=\operatorname{Diag}\left\{K_{\beta, \beta}^{-1}, K_{\phi, \phi}^{-1}\right\}, \operatorname{com} K_{\beta, \beta}$ e $K_{\phi, \phi}$ definidos em (2.15) e (2.16), respectivamente. Em outras palavras, tem-se que

$$
\widehat{\beta} \sim A N_{p}\left(\beta, K_{\beta, 3}^{-1}\right) \text { e } \widehat{\phi} \sim A N\left(\phi, K_{\phi, \phi}^{-1}\right) .
$$

e são assintoticamente independentes.

A seguir. apresentamos alguns algoritmos iterativos para a obtençào dos estimadores de máxima verossimilhança. 


\section{(a) Algoritmo de Newton-Raphson}

Sejam $\theta^{(0)}$ a estimativa inicial de $\theta$. e $\theta^{(l)}$ a estimativa na l-ésima iteraçào. Segundo o algoritmo de Newton-Raphson. a estimativa de $\theta$ na iteração $l+1$ é dada por (vide Little e Rubin, 1987, p.128)

$$
\theta^{(l+1)}=\theta^{(l)}-\left(\frac{\partial^{2} L\left(\theta^{(l)}\right)}{\partial \theta \partial \theta^{\top}}\right)^{-1} U^{(l)},
$$

onde $U^{(l)}=U\left(\theta^{(l)}\right)=\left(U_{3}^{(l)}, L_{o}^{(l)}\right)$ e $\partial^{2} L\left(\theta^{(l)}\right) / \partial \theta \partial \theta^{\top}$ são o vetor escore e a matriz de informação observada na l-ésima iteraçào. e são obtidas de (2.8). (2.9) e (2.12), respectivamente.

A partir de uma estimativa inicial $\theta^{(0)}$ (por exemplo, $\theta^{(0)}=\hat{\theta}_{M Q}$ ). o algoritmo vai atualizando a estimativa de $\theta$ até obter convergência, o que não é garantido.

\section{(b) Algoritmo scoring de Fisher}

Uma variação do algoritmo de Newton-Raphson definido em (a) é o algoritmo scoring de Fisher, no qual a matriz de informação observada é substituída pela matriz de informação (esperada) de Fisher (vide Little e Rubin, 1987, p.128). Assim, a estimativa de $\theta$ na $(l+1)$-ésima iteraçào é determinada pela equaçào

$$
\theta^{(l+1)}=\theta^{(l)}+\left(K^{(l)}\right)^{-1} U^{(l)}
$$

onde $K^{(l)}=K^{\prime}\left(\theta^{(l)}\right)$ e $U^{(l)}=U\left(\theta^{(l)}\right)=\left(U_{\beta}^{(l)}, U_{\phi}^{(l)}\right)$.

Como a matriz de informação para os parâmetros $\beta$ e $\phi$ é bloco diagonal (vide (2.14)), a equação acima pode ser escrita como

$$
\begin{aligned}
\beta^{(l+1)} & =\beta^{(l)}+\left(K_{\beta, \beta}^{(l)}\right)^{-1} U_{\beta}^{(l)} \\
& =\beta^{(l)}+\frac{1}{\delta(2,0,0,0,0)}\left(X^{\top} X^{\top}\right)^{-1} \cdot X^{\top} \underbrace{W^{(l)}\left(Y-X \beta^{(l)}\right)},
\end{aligned}
$$

e

$$
\begin{aligned}
\phi^{(l+1)} & =\phi^{(l)}+\left(K_{\phi, \phi}^{-(l)}\right)^{-1} U_{\phi}^{(l)} \\
& =\phi^{(l)}+\frac{1}{\phi^{(l)}\left(\delta_{(2,0,0,0,2)}-1\right)}\left\{\frac{1}{n}\left(Y-X \beta^{(l)}\right)^{\top} W^{(l)}\left(Y-X \beta^{(l)}\right)-\phi^{(l) 2}\right\} .
\end{aligned}
$$

As equações (2.18) e (2.19) são usadas quando os demais parâmetros do modelo, se houver, são considerados conhecidos (por exemplo, o número de graus de liberdade $\nu$ na distribuição $t$ ). Quando há ainda outros parâmetros desconhecidos, além de o e $\beta$, é necessário obter a matriz de informação para todos os parâmetros desconhecidos. Outra alternativa, em geral mais simples. é repetir o processo iterativo para uma gama de valores para o(s) parâmetro(s) extra(s) e escolher aquele(s) que produz(em) o maior valor para a verossimilhança.

Na Tabela 2.3 e no Apêndice $\mathrm{C}$ são dados, respectivamente, os valores para os elementos da matriz $W$ e os $\delta$ s necessários aos algoritmos para algumas distribuiçòes. 
Para as distribuições $t$-Student e $t$-Student generalizada nào é difícil incorporar os parâmetros extras no método scoring ou Fisher. Vejamos:

(i) $t$-Student: $y \sim t\left(x_{l}^{\top} \beta, \phi^{2} \cdot \nu\right), \nu>0$.

Se consideramos o parâmetro $\nu$ desconhecido. o EMV de $\theta=\left(\beta^{\top}, \phi, \nu\right)^{\top}$, digamos $\hat{\theta}=\left(\hat{\beta}^{\top}, \widehat{\phi}, \widehat{\nu}\right)^{\top}$ é solução do sistema de equações $(2.10),(2.11) \mathrm{e}$

$$
\left.\frac{\partial L(\theta)}{\partial \nu}\right|_{\theta=\widehat{\theta}}=\frac{n}{2}\left\{\log \left(\frac{\hat{\nu}}{\hat{\nu}+1}\right)+\psi\left(\frac{\hat{\nu}+1}{2}\right)-\psi\left(\frac{\hat{\nu}}{2}\right)+1+\log \hat{\bar{w}}_{g}-\widehat{\bar{w}}_{a}\right\}=0,
$$

onde $\psi(z)=\mathrm{d} \log \Gamma(z) / \mathrm{d} z$ é a função digama,

$$
\bar{w}_{g}=\left(\prod_{l=1}^{n} w_{l}\right)^{1 / n}, \quad \bar{w}_{a}=n^{-1} \sum_{l=1}^{n} w_{l} .
$$

Escrevendo $\theta=\left(\beta^{\top}, \delta^{\top}\right)^{\top}$, onde $\delta=(\phi, \nu)^{\top}$, a implementação do algoritmo scoring envolve a função escore $U=\left(U_{1}^{\top}, U_{2}^{\top}\right)^{\top}, \operatorname{com} U_{1}=\partial L(\theta) / \partial \beta$ e $U_{2}=(\partial L(\theta) / \partial \phi, \partial L(\theta) / \partial \nu)^{\top}$ e a matriz de informação $K^{\prime}=K^{\prime}(\theta)$ que é bloco diagonal entre os parâmetros $\beta$ e $\delta$, isto é,

$$
K^{\prime}=\operatorname{Diag}\left\{K_{\beta, \beta}, K_{\delta, \delta}\right\},
$$

onde $K_{\beta, \beta}$ é definida em (2.15) e

$$
K_{\delta, \delta}=\left(\begin{array}{cc}
K_{\phi, \phi} & K_{\phi, \nu} \\
K_{\nu, \phi}^{*} & K_{\nu, \nu}^{*}
\end{array}\right)
$$

com

$$
\begin{aligned}
K_{\phi, \phi} & =\frac{2 \nu n}{(\nu+3) \phi^{2}}, \quad K_{\phi, \nu}=K_{\nu, \phi}=-2 n /(\phi(\nu+1)(\nu+3)) \\
\mathrm{e} \quad K_{\nu, \nu} & =-\frac{n}{2}\left\{\frac{1}{2}\left[\psi^{\prime}\left(\frac{\nu+1}{2}\right)-\psi^{\prime}(\nu / 2)\right]+\frac{(\nu+5)}{\nu(\nu+1)(\nu+3)}\right\},
\end{aligned}
$$

sendo que $\psi^{\prime}(\cdot)$ denota a primeira derivada da função digama.

Seja $\theta^{(l)}=\left(\beta^{(l) \top}, \phi^{(l)}, \nu^{(l)}\right)^{\top}$ a estimativa de $\theta$ na l-ésima iteração. A equação (2.17) pode ser decomposta em (2.18) e

$$
\delta^{(l+1)}=\delta^{(l)}+\left(K_{\delta, \delta}^{(l)}\right)^{-1} U_{2}^{(l)} .
$$

O valor inicial $\theta^{(0)}=\left(\beta^{(0) \top}, \phi^{(0)}, \nu^{(0)}\right)^{\top}$ pode ser escolhido tomando, por exemplo, $\beta^{(0)}=$ $\widehat{\beta}_{M Q}, \phi^{(0)}=\widehat{\phi}_{M Q}$ e $\nu^{(0)}=2\left(2 \hat{\gamma}_{2}-3\right) /\left(\hat{\gamma}_{2}-3\right)$, onde $\widehat{\gamma}_{2}$ é o coeficiente de curtose amostral, que é um estimador consistente de $\nu$ (Arellano-Valle, 1994, p.112).

(ii) $t$-Student generalizada: $y_{l} \sim t G\left(x_{l}^{\top} \beta, \phi^{2}, s, r\right), s, r>0$. 
Se consideramos os parâmetros s e $r$ desconhecidos. o EMV de $\theta=\left(\beta^{\top} . \phi, s, r\right)^{\top}$. digamos $\hat{\theta}=\left(\widehat{\beta}^{\top} \cdot \hat{\delta}^{\top}\right)^{\top} \cdot \operatorname{com} \widehat{\delta}=(\hat{\phi} \cdot \widehat{s} \cdot \widehat{r})^{\top}$. é soluçào das equações (2.10). (2.11). com $\widehat{w}_{l}=(\widehat{r}+1) /\left(\widehat{s}+\widehat{z}_{i}^{2}\right) \cdot l=1, \ldots, n$, e juntamente com as equaçòes

$$
\begin{aligned}
& \left.\frac{\partial L(\theta)}{\partial s}\right|_{\theta=\widehat{\theta}}=\frac{n}{2 \widehat{s}}-\frac{1}{2} n \hat{\bar{w}}_{a}=0 \\
& \left.\frac{\partial L(\theta)}{\partial r}\right|_{\theta=\widehat{\theta}}=\frac{n}{2}\left\{\log \left(\frac{\widehat{s}}{\widehat{r}+1}\right)+\psi\left(\frac{\widehat{r}+1}{2}\right)-\psi\left(\frac{\widehat{r}}{2}\right)+\log \widehat{\bar{w}}_{g}\right\}=0,
\end{aligned}
$$

onde $\bar{w}_{g}$ e $\bar{w}_{a}$ estão definidos em (2.20).

Definimos a função escore $U=\left(U_{1}^{\top}, U_{2}^{\top}\right)^{\top}, \operatorname{com} U_{1}=\partial L(\theta) / \partial \beta$ e $U_{2}=(\partial L(\theta) / \partial \phi$, $\partial L(\theta) / \partial s, \partial L(\theta) / \partial r)^{\top}$. A matriz de informação $K^{\top}=\operatorname{Diag}\left\{K_{\beta, \beta}, K_{\delta, \delta}\right\}$ é o bloco diagonal entre o vetor de parâmetros de regressào $\beta$ e $(\phi, s, r)^{\top}$. onde $K_{\beta, \beta}^{-}$é definida em $(2.15)$ e

$$
K_{\delta, \delta}=\left(\begin{array}{lll}
K_{\phi, \phi} & K_{\phi, s} & K_{\phi, r} \\
\kappa_{s, \phi} & \kappa_{s, s} & K_{s, r}^{\prime} \\
K_{r, \phi} & K_{r, s} & K_{r, r}
\end{array}\right) \text {, }
$$

com

$$
\begin{aligned}
& K_{\phi, \phi}=\frac{2 r n}{(r+3) \phi^{2}}, \quad K_{s, s}=\frac{n r}{2 s^{2}}\left(\frac{2 r+5}{r+3}\right) \\
& K_{r, r}=\frac{n}{4}\left\{\psi^{\prime}\left(\frac{r}{2}\right)-\psi^{\prime}\left(\frac{r+1}{2}\right)\right\}, \quad K_{\phi, s}=\frac{n r}{\phi s(r+3)}, \\
& K_{\phi, r}=-\frac{n}{\phi(r+1)} \text { e } K_{r, s}=-\frac{(2 r+1) n}{2 s(r+1)} .
\end{aligned}
$$

Seja $\theta^{(l)}=\left(\beta^{(l) \top}, \phi^{(l)}, s^{(l)}, r^{(l)}\right)^{\top}$ a estimativa de $\theta$ na l-ésima iteração. A equação $(2.17)$ pode ser decomposta em (2.18) e

$$
\delta^{(l+1)}=\delta^{(l)}+\left(K_{\delta, \delta}^{(l)}\right)^{-1} U_{2}^{(l)} .
$$

O valor inicial $\theta^{(0)}=\left(\beta^{(0) \top}, \phi^{(0)}, s^{(0)}, r^{(0)}\right)^{\top}$ pode ser escolhido tomando, por exemplo, $\beta^{(0)}=\hat{\beta}_{M Q}, \phi^{(0)}=\hat{\phi}_{M Q}, r^{(0)}=2\left(2 \hat{\gamma}_{2}-3\right) /\left(\hat{\gamma}_{2}-3\right)$ e $s^{(0)}=\left(2 \hat{\mu}_{2} \hat{\gamma}_{2}\right) /\left(\hat{\gamma}_{2}-3\right)$, onde $\widehat{\gamma}_{2}$ é o coeficiente de curtose amostral e $\widehat{\mu}_{2}$ é o segundo momento central amostral. Se $s$ é conhecido, tem-se $\left.\delta=(\phi, r)^{\top}, U_{2}=(\partial L(\theta) / \partial \phi), \partial L(\theta) / \partial r\right)^{\top} \mathrm{e}$

$$
K^{\prime}(\delta)=\left(\begin{array}{cc}
K_{\phi, \phi} & K_{\phi, r} \\
K_{r, \phi}^{*} & K_{r, r}^{r}
\end{array}\right)
$$

Substituindo em (2.17). obtemos iterativamente o EMV de $\delta$. Em forma análoga se procede se $r$ é conhecido.

\section{(c) Algoritmo EM}

O algoritmo EM é um algoritmo muito versátil para a estimaçào por máxima verossimilhança (Dempster. Laird e Rubin. 197T, vide também Little e Rubin. 1987. e Lange. 
Little e Taylor, 1989). Este algoritmo aumenta os dados $Y=\left(y_{1}, \ldots, y_{n}\right)^{\top}$ considerando dados hipotéticos adicionais. $V=\left(v_{1} \ldots v_{n}\right)^{\top}$, de tal forma que o EMV de $\theta$ baseado no logaritmo da função de verossimilhança $L_{Y *} \cdot(\theta)$ dos dados aumentados, $Y^{*}=\left(Y^{\top} \cdot V^{\top}\right)^{\top}$, é fácil de ser obtido. Dada a estimativa $\theta^{(l)}$ de $\theta$ na $l$-ésima iteração, então a $(l+1)$-ésima iteração do algoritmo EM consiste no passo E (esperança) e no passo M (maximização). $\mathrm{O}$ passo E determina o valor esperado de $L_{Y^{*}}(\theta)$ com respeito à distribuição condicional de $V$, dados $Y$ e $\theta^{(l)}$. O passo $M$ maximiza a funçào resultante com respeito a $\theta$ e obtém a nova estimativa $\theta^{(l+1)}$ de $\theta$. Sob condições adequadas de regularidade, cada iteração do algoritmo EM incrementa o logaritmo da função observada $L(\theta \mid Y)$, isto é, $L\left(\theta^{(l)} \mid Y\right) \leq L\left(\theta^{(l+1)} \mid Y\right)$. Em particular, o algoritmo EM é útil quando o passo $\mathrm{M}$ não é iterativo. Quando o passo M é iterativo, pode-se usar o algoritmo ECM (Meng e Rubin, 1993) que decompõe o passo $\mathrm{M}$ em duas ou mais sub-etapas não iterativas.

A seguir, apresentamos aplicações do algoritmo EM às distribuições $t$-Student e $t$ Student generalizada. Em ambos os casos, $y_{1}, \ldots, y_{n}$ são consideradas variáveis aleatórias independentes.

(1) $t$-Student: $y_{l} \sim t\left(x_{l}^{\top} \beta, \phi^{2}, \nu\right), \nu>0$ conhecido.

Lange, Little e Taylor (1989) estudam o algoritmo EM para adaptá-lo a modelos de regressão onde os erros têm distribuição $t$-Student (vide também Arellano-Valle. 1994).

Considerem-se dados hipotéticos adicionais $V=\left(v_{1}, \ldots, v_{n}\right)^{\top}$, independentes e identicamente distribuídos com $v_{i} \sim G I(\nu / 2, \nu / 2)$, onde $G I(a, b)$ denota a distribuição gama inversa com parâmetros $a$ e $b$ e função densidade

$$
p_{v}(v)=\frac{b^{a}}{\Gamma(a)}\left(\frac{1}{v}\right)^{a+1} \exp \{-b / v\}, \quad v>0 .
$$

A função de verossimilhança do par $Y^{*}=\left(Y^{\top}, V^{\top}\right)^{\top}$ é dada por

$$
f_{Y *}(Y, V \mid \beta, \phi, \nu)=\prod_{i=1}^{n} f_{y_{i} \mid v_{i}=v_{i}}\left(y_{i} \mid \beta, \phi\right) f_{v_{i}}\left(v_{i} \mid \nu\right) \text {. }
$$

Como $\left(y_{i} \mid v_{i}=v_{i}\right) \stackrel{\text { ind }}{\sim} N\left(x_{i}^{\top} \beta, v_{i} \phi^{2}\right)$, a log-verossimilhança de $\theta=\left(\beta^{\top}, \phi\right)$, dados $y_{1}^{\times}, \ldots, y_{2 n}^{\times}$ tem a forma

$$
\begin{aligned}
L_{Y^{*}}(\theta)= & -n \log \phi+n\left(\frac{\nu}{2} \log \left(\frac{\nu}{2}\right)-\log \Gamma\left(\frac{\nu}{2}\right)-\frac{1}{2} \log 2 \pi\right) \\
& -\sum_{i=1}^{n}\left\{\frac{1}{2 v_{i}}\left(z_{i}^{2}+\nu\right)-\frac{(\nu+3)}{2} \sum_{i=1}^{n} \log v_{i}\right\} .
\end{aligned}
$$

Como $\left(v_{i} \mid y_{i}=y_{i}\right) \stackrel{\text { ind }}{\sim} G I\left((\nu+1) / 2,\left(\nu+z_{i}^{2}\right) / 2\right)$, temos $^{2}$

$$
\left(v_{i}^{-1} \mid y_{i}=y_{i}\right) \stackrel{\mathrm{d}}{=} \frac{w_{i} t}{(\nu+1)} \text {, onde } t \sim \chi_{\nu+1}^{2} \text {. }
$$

\footnotetext{
${ }^{2} \mathrm{~A}$ notaçào $x \stackrel{\mathrm{d}}{=} y$ indica que as variáveis aleatórias $x$ e $y$ sào identicamente distribuídas.
} 
No passo E da iteração $(l+1)$ do algoritmo EM, tem-se

$$
\begin{aligned}
L_{E}(\theta)= & E\left[L_{\left.Y \cdot(\theta) \mid Y=y, \theta^{(l)}\right]}=-\frac{1}{2 \phi^{2}}(Y-X \beta)^{\top} W^{(l)}\left(Y-X^{\prime} \beta\right)-n \log \phi\right. \\
& +n\left\{\frac{\nu}{2}\left(\log \left(\frac{\nu}{2}\right)-\bar{w}_{a}^{(l)}\right)-\log \Gamma\left(\frac{\nu}{2}\right)-\frac{1}{2} \log 2 \pi\right. \\
& \left.+\left(\frac{\nu+3}{2}\right)\left(\psi\left(\frac{\nu^{(l)}+1}{2}\right)-\log \left(\frac{\nu^{(l)}+1}{2}\right)+\log \bar{w}_{g}^{(l)}\right)\right\},
\end{aligned}
$$

onde $\bar{w}_{g}$ e $\bar{w}_{a}$ estào definidas em $(2.20)$ e $y=\left(y_{1}, \ldots, y_{n}\right)^{\top}$.

$\mathrm{O}$ algoritmo $\mathrm{EM}$ procede com o passo $\mathrm{M}$ no qual a função $L_{E}(\theta)$ é maximizada com respeito a $\theta$. Derivando $L_{E}(\theta)$ com respeito a $\beta$ e $\phi$ temos

$$
\frac{\partial L_{E}(\theta)}{\partial \beta_{r}}=\frac{1}{\phi^{2}} \sum_{i=1}^{n}\left(y_{i}-x_{i}^{\top} \beta\right) w_{i}^{(l)} x_{i r}, r=1 \ldots, n,
$$

e

$$
\frac{\partial L_{E}(\theta)}{\partial \phi}=\frac{1}{\phi^{3}} \sum_{i=1}^{n}\left(y_{i}-x_{i}^{\top} \beta\right)^{2} w_{i}^{(l)}-\frac{n}{o} .
$$

Agora, igualando a zero estas derivadas temos que $\beta^{(l+1)}$ e $\phi^{(l+1)^{2}}$ são dados em forma matricial por

$$
\begin{aligned}
\beta^{(l+1)} & =\left(X^{\top} W^{(l)} X\right)^{-1} X^{\top} W^{(l)} Y \\
\phi^{(l+1)^{2}} & =\frac{1}{n}\left(Y-X^{\top} \beta^{(l+1)}\right)^{\top} W^{(l)}\left(Y-X^{\top} \beta^{(l+1)}\right) .
\end{aligned}
$$

A conveniência do algoritmo EM é particularmente clara quando $\nu$ é conhecido, uma vez que o algoritmo se reduz a avaliar iterativamente as estimativas de mínimos quadrados ponderados dadas em (2.21) e (2.22). Para $\nu$ desconhecido. um procedimento sugerido por Lange, Little e Taylor (1989, p.892) é utilizar $\nu=4$ para amostras pequenas em lugar de estimar este parâmetro ou também considerar uma gama de valores para $\nu$, aplicar o algoritmo EM para cada um destes valores e escolher aquela estimativa de $\theta$ que maximiza a verossimilhança de $Y$. Alternativamente, o algoritmo EM pode ser aplicado para estimar $\nu$ simultaneamente $\operatorname{com} \beta$ e $\phi$. Dadas as estimativas na l-ésima iteração $\theta^{(l)}=\left(\beta^{(l) \top}, \phi^{(l)}, \nu^{(l)}\right)^{\top}$, no passo E tem-se $w_{i}^{(l)}=\left(\nu^{(l)}+1\right) /\left(\nu^{(l)}+\widehat{z}_{i}^{(l) 2}\right)$. Maximizando $L_{E}(\theta)$ com respeito a $\nu, \nu^{(l+1)}$ é dado por $\partial L_{E}(\theta) / \partial \nu=0$, isto é,

$$
\psi\left(\frac{\nu^{(l+1)}}{2}\right)-\log \left(\frac{\nu^{(l+1)}}{2}\right)=\bar{w}_{a}^{(l)}-\log \bar{w}_{g}^{(l)} .
$$

(2) $t$-Student generalizada $y_{l} \sim t G\left(x_{i}^{\top} \beta, \phi^{2}, s, r\right), r . s>0$ conhecidos.

Seja $V=\left(v_{1}, \ldots, v_{n}\right)^{\top}$ o vetor de dados hipotéticos adicionais independentes e identicamente distribuídos. com $\imath_{l} \sim G I(r / 2, s / 2)$. onde $G I(a . b)$ denota a distribuição gama 
inversa com parâmetros $a$ e $b$. A função de verossimilhança de $Y^{*}=\left(Y^{\top}, V^{\top}\right)^{\top}$ é dada por

$$
f_{Y} \cdot=\left(Y, V^{\prime} \mid \beta, o, s, r\right)=\prod_{i=1}^{n} f_{y_{i} \mid v_{i}=v_{i}}\left(y_{i} \mid, 3, \phi\right) f_{v_{t}}\left(v_{i} \mid s, r\right) .
$$

Como $\left(y_{i} \mid v_{i}=v_{i}\right) \stackrel{\text { ind }}{\sim} N\left(x_{i}^{\top} \beta, v_{i} \phi^{2}\right)$, o logaritmo da função de verossimilhança de $\theta$, dado $Y^{*}$, pode ser escrito como

$$
L_{Y} \cdot(\theta)=\sum_{i=1}^{n} \log f_{y_{i} \mid v_{i}=v_{i}}\left(y_{i} \mid v_{i}, \beta, \phi\right)+\sum_{i=1}^{n} \log f_{v_{i}}\left(v_{i} \mid s, r\right),
$$

de onde segue que

$$
\begin{aligned}
L_{Y *}(\theta)= & \sum_{i=1}^{n}\left(-\frac{1}{2 v_{i}}\left(z_{i}^{2}+s\right)\right)-\left(\frac{r+3}{2}\right) \sum_{i} \log v_{i}-n \log \phi \\
& +n\left(\frac{r}{2} \log \left(\frac{s}{2}\right)-\log \Gamma\left(\frac{r}{2}\right)-\frac{1}{2} \log 2 \pi\right),
\end{aligned}
$$

onde $\theta=\left(\beta^{\top}, \phi\right)^{\top}$ e $z_{i}=z_{i}(\theta)$ está definida em (2.6).

Uma vez que $\left(v_{i} \mid y_{i}=y_{i}\right) \stackrel{\text { ind }}{\sim} G I\left((r+1) / 2,\left(s+z_{i}^{2}\right) / 2\right)$ e aplicando o mesmo argumento utilizado por Arellano-Valle (1994, p.119) para a distribuição $t$-Student, tem-se

$$
E\left(v_{i}^{-1} \mid y_{i}=y_{i}\right)=\frac{r+1}{s+z_{i}^{2}}=w_{i}, \quad i=1, \ldots, n
$$

Como $\left(v_{i}^{-1} \mid y_{i}=y_{i}\right) \stackrel{\mathrm{d}}{=}\left(w_{i} t\right) /(r+1)$, para $i=1, \ldots, n$, onde $t \sim \chi_{r+1}^{2}$, usando $E(\log t / 2)=$ $\psi((r+1) / 2)$ tem-se

$$
-E\left(\log v_{i} \mid y_{i}=y_{i}\right)=E\left(\log v_{i}^{-1} \mid y_{i}=y_{i}\right)=-\log w_{i}+\log \left(\frac{r+1}{2}\right)-\psi\left(\frac{r+1}{2}\right) .
$$

Assim, no passo E da iteração $(l+1)$ do algoritmo EM, tem-se

$$
\begin{aligned}
L_{E}(\theta)= & E\left(L_{Y * *}(\theta ; Y, V) \mid Y=y, \theta^{(l)}\right) \\
= & -\frac{1}{2} \sum_{i=1}^{n} z_{i}^{2} w_{i}^{(l)}-\frac{s}{2} \sum_{i=1}^{n} w_{i}^{(l)}-n \log \phi \\
& +\left(\frac{r+3}{2}\right) \sum_{i=1}^{n} \log w_{i}^{(l)}+n\left\{\frac{r}{2} \log \left(\frac{s}{2}\right)-\log \Gamma\left(\frac{r}{2}\right)\right. \\
& \left.-\frac{1}{2} \log 2 \pi+\left(\frac{r+3}{2}\right) \psi\left(\frac{r+1}{2}\right)-\left(\frac{r+3}{2}\right) \log \left(\frac{r+1}{2}\right)\right\},
\end{aligned}
$$

ou, ainda,

$$
\begin{aligned}
L_{E}(\theta)= & -\frac{1}{2 \phi^{2}}(Y-X \beta)^{\top} W^{(l)}(Y-X \beta) \\
& +n\left\{\frac{r+3}{2}\left(\log \bar{w}_{g}^{(l)}+\psi\left(\frac{r+1}{2}\right)\right)-\log \left(\frac{r+1}{2}\right)\right. \\
& \left.+\frac{r}{2} \log \left(\frac{s}{2}\right)-\log \Gamma\left(\frac{r}{2}\right)-\log \phi-\frac{1}{2} \log 2 \pi-\frac{s}{2} \bar{w}_{a}^{(l)}\right\} .
\end{aligned}
$$


Agora o algoritmo EM procede com o passo M. no qual a funçào $L_{E}(\theta)$ é maximizada com respeito a $\theta$. Derivando $L_{E}(\theta)$ com respeito a $\beta$ e $o$ temos

$$
\begin{aligned}
\frac{\partial L_{E}(\theta)}{\partial \beta_{r}} & =-\frac{1}{2} \sum_{i=1}^{n} 2 z_{i} u_{i}^{(l)}\left(-x_{i r} / \phi\right) \\
& =\frac{1}{o^{2}} \sum_{i=1}^{n}\left(y_{i}-x_{i}^{\top} \beta\right) w_{i}^{(l)} x_{i r}
\end{aligned}
$$

e

$$
\begin{aligned}
\frac{\partial L_{E}(\theta)}{\partial \phi} & =-\frac{n}{\phi}-\frac{1}{2} \sum_{i=1}^{n} 2 z_{i} w_{i}^{(l)}\left(-z_{i} / \phi\right) \\
& =\frac{1}{\phi^{3}} \sum_{i=1}^{n}\left(y_{i}-x_{i}^{\top} \beta\right)^{2} w_{i}^{(l)}-\frac{n}{\phi}
\end{aligned}
$$

Agora, igualando a zero estas derivadas. temos que $\beta^{(l+1)}$ e $\phi^{(l+1)^{2}}$ são dados por

$$
\begin{aligned}
\beta^{(l+1)} & =\left(X^{\top} W^{(l)} X^{r}\right)^{-1} X^{\top} W^{(l)} Y, \\
\phi^{(l+1)^{2}} & =\frac{1}{n}\left(Y-X \beta^{(l+1)}\right)^{\top} W^{(l)}\left(Y-X \beta^{(l+1)}\right) .
\end{aligned}
$$

Como na distribuição $t$-Student, quando $r$ e $s$ são conhecidos o algoritmo EM reduz-se a avaliar iterativamente as estimativas de mínimos quadrados ponderados dadas em (2.23). Quando $r$ e $s$ são desconhecidos, um procedimento para estimar um dos parâmetors é considerar um parâmetro conhecido, digamos $r$ (ou $s$ ) e considerar uma gama de valores do outro parâmetro, aplicar o algoritmo EM para cada um destes valores e escolher aquela estimativa de $\theta$ que maximiza a verossimilhança de $Y$ e, em seguida, escolher um outro valor do parâmetro desconhecido $r$ (ou $s$ ) e uma gama de valores do parâmetro $s$ (ou $r$ ), seguir o procedimento e, ao final, considerar o par $(s, r)$ que maximize a verossimilhança de $Y$. Alternativamente, o algoritmo EM pode ser aplicado para estimar $s$ (ou $r$ ) simultaneamente com $\beta^{\top}$ e $\phi$ considerando $r$ (ous $s$ ) conhecido. Dadas as estimativas de $\theta$ na l-ésima iteração, $\theta^{(l)}=\left(\beta^{(l) \top}, \phi^{(l)}, s^{(l)}\right)^{\top}$, no passo E temos $w_{i}^{(l)}=(r+1) /\left(s^{(l)}+z_{i}^{(l) 2}\right) \mathrm{e}$ maximizando $L_{E}(\theta)$ com respeito a $s$, tem-se

$$
\frac{\partial L_{E}(\theta)}{\partial s}=\frac{n}{2}\left(\frac{r}{s}-\bar{w}_{a}^{(l)}\right)=0
$$

Assim, $s^{(l+1)}$ é solução da equação

$$
s^{(l+1)}=\frac{r}{\bar{w}_{a}^{(l)}} .
$$

Logo, se consideramos $r$ desconhecido, usando o procedimento de Lange. Little e Taylor (1989) e considerando uma gama de valores para $r$. podemos utilizar o algoritmo E.I para cada um destes valores e escolher "aquela estimativa de $\theta$ que maximiza a verossimilhança de $Y$. 


\subsection{Testes de hipóteses}

\subsubsection{Testes de hipóteses sobre o parâmetro 3 com $o$ conhecido}

Consideremos $n$ variáveis aleatórias independentes $y_{1}, \ldots y_{n}$, onde cada $y_{l}$ tem funçào densidade na família simétrica da forma (2.1). Assumimos que o parâmetro de escala $\phi$ é conhecido e o vetor de parâmetros de locação $\mu$ está relacionado $\operatorname{com} \beta$ através de (2.2). Seja $L(\beta)$ o logaritmo da função de verossimilhança total para o parâmetro $\beta$, dados $y_{1}, \ldots, y_{n}$. dado em (2.4). Assumimos que a função $L(\beta)$ é regular com respeito às derivadas em relação aos componentes de $\beta$ até quarta ordem. Da definição de $\delta_{(a, b, c, d, \epsilon)}$ em (2.13), temos que $-\phi^{-1} \delta_{(1,0,0,0,0)}, \phi^{-2} \delta_{(0,1,0,0,0)},-\phi^{-3} \delta_{(0,0.1,0,0)}$ e $\phi^{-4} \delta_{(0,0,0,1,0)}$ são valores esperados da $i$-ésima derivada do logaritmo da função de verossimilhança com respeito a $\beta\left(\right.$ se $\left.\mu=1_{n} \beta\right)$. para $i=1,2,3,4$, respectivamente. Pelas condições de regularidade, $\delta_{(1,0,0,0,0)}=0$ e $\delta_{(0,1,0,0,0)}=-\delta_{(2,0,0,0,0)}$. Como veremos no Capítulo 3 , a função $\delta_{(a, b, c, d, \epsilon)}$ desempenha um papel importante no cálculo das correçôes de Bartlett para a estatística da razão de verossimilhança e tipo Bartlett para a estatística escore na família de distribuições simétricas.

Consideremos o problema de testar a hipótese nula simples ou composta $H_{0}: \beta_{1}=\beta_{1}^{(0)}$ versus a hipótese alternativa composta $H_{1}: \beta_{1} \neq \beta_{1}^{(0)}$, onde o vetor de parâmetros desconhecidos $\beta$ com $p$ componentes é partido como $\beta=\left(\beta_{1}^{\top}, \beta_{2}^{\top}\right)^{\top}$, sendo $\beta_{1}=\left(\beta_{1}, \ldots, \beta_{q}\right)^{\top}$ o vetor de parâmetros de interesse, $\beta_{2}=\left(\beta_{q+1}, \ldots, \beta_{p}\right)^{\top}$ o vetor de dimensão $(p-q)$ de parâmetros de perturbação e $\beta_{1}^{(0)}$ um vetor especificado de dimensão $q(q \leq p)$. Assumimos que a matriz $X^{\top}=\left(X_{1} X_{2}\right)$ é partida de acordo com a partição $\left(\beta_{1}^{\top}, \beta_{2}^{\top}\right)^{\top}$ de $\beta$. Denotamos por $\widehat{\beta}=\left(\widehat{\beta}_{1}^{\top}, \widehat{\beta}_{2}^{\top}\right)^{\top}$ o estimador de máxima verossimilhança irrestrito de $\beta$ e por $\tilde{\beta}_{2}$ o estimador de máxima verossimilhança de $\beta_{2}$ sob $H_{0}$. Escrevemos as funções avaliadas em $\beta=\left(\beta^{(0) \top}, \widetilde{\beta}_{2}^{\top}\right)^{\top}$ com um til.

A função escore total para $\beta$ dada em (2.8) pode ser partida da mesma maneira que $\beta$ como

$$
U_{1}=U_{1}\left(\beta_{1}, \beta_{2}\right)=\frac{1}{\phi^{2}} X_{1}^{\top} W(Y-X \beta) \text { e } U_{2}=U_{2}\left(\beta_{1}, \beta_{2}\right)=\frac{1}{\phi^{\top}{ }_{2}^{\top} W(Y-X \beta),} \text { eros! }
$$

onde os elementos da matriz diagonal $W$ estão definidos em (2.7). A matriz de informação total de Fisher $K^{*}=K(\beta)$ dada em (2.15) pode ser dividida segundo a partição de $\beta$ como

$$
\Pi=\left(\begin{array}{ll}
K_{11} & K_{12} \\
K_{21} & K_{22}
\end{array}\right)
$$

onde

$$
\begin{aligned}
& K_{11}=\frac{\delta_{(2.0 .0,0,0)}}{\phi^{2}} X_{1}^{\top} X_{1}, \quad K_{22}=\frac{\delta_{(2.0 .0 .0 .0)}}{0^{2}} X_{2}^{\top} X_{2} \\
& K_{12}=K_{21}=\frac{\delta_{(2,0.0 .0 .0)}}{\phi^{2}} X_{1}^{\top} X_{2} .
\end{aligned}
$$


Assim, a matriz inversa de $I$ tem a forma

$$
K^{r-1}=\frac{\phi^{2}}{\delta_{(2,0.0 .0,0)}}\left(X^{\top} X^{\prime}\right)^{-1},
$$

e também pode ser dividida segundo a partição de $\beta$ em submatrizes como

$$
K^{\prime-1}=\left(\begin{array}{ll}
K^{\prime 11} & K^{-12} \\
K^{21} & K^{-22}
\end{array}\right)
$$

Utilizando a fórmula de inversa de matrizes simétricas divididas (Rao, 1973, p.33), após alguma álgebra, chega-se a

$$
\begin{aligned}
& K^{11}=\frac{\phi^{2}}{\delta_{(2,0,0,0,0)}}\left(R^{\top} R\right)^{-1}, \quad K^{12}=K^{-21 \top}=-\frac{\phi^{2}}{\delta_{(2,0,0,0,0)}}\left(R^{\top} R\right)^{-1} C^{\top}, \\
& K^{22}=\frac{\phi^{2}}{\delta_{(2,0,0,0,0)}}\left\{\left(X_{2}^{\top} X_{2}\right)^{-1}+C\left(R^{\top} R\right)^{-1} C^{\top}\right\},
\end{aligned}
$$

onde $R=X_{1}-X_{2} C$, com $C=\left(X_{2}^{\top} X_{2}\right)^{-1} X_{2}^{\top} X_{1}$ representando uma matriz $(p-q) \times q$ cujas colunas são vetores dos coeficientes de uma regressão linear das colunas de $X_{1}$ sobre a matriz modelo $\hat{X}_{2}$.

Na maioria das situações práticas, para testar a hipótese nula $H_{0}$ versus a alternativa $H_{1}$, o teste baseia-se numa das três estatisticas seguintes: razão de verossimilhança $(R V)$, escore $\left(S_{R}\right)$ e Wald $\left(W_{a}\right)$, que em problemas regulares e sob a hipótese nula, têm uma distribuição assintótica $\chi^{2} \operatorname{com} q$ graus de liberdade, onde $q$ é a diferença entre as dimensões dos espaços paramétricos sob a hipótese alternativa e nula.

A estatística da razão de verossimilhança para testar $H_{0}$ versus $H_{1}$, definida em (1.3) tem a forma

$$
R V=2 \sum_{l=1}^{n} \log \left\{\frac{h\left(\hat{\tilde{z}}_{l}^{2}\right)}{h\left(\widetilde{z}_{l}^{2}\right)}\right\},
$$

onde $z$ está definido em (2.6). Por outro lado, a estatística escore $S_{R}$ para testar $H_{0}$ versus $H_{1}$ definida em (1.4) tem a forma

$$
S_{R}=\tilde{s}^{\top} X_{1}\left(R^{\top} R\right)^{-1} \cdot X_{1}^{\top} \tilde{s}
$$

onde $s=\left(s_{1}, \ldots, s_{n}\right)^{\top}$, com

$$
s_{l}=z_{l} w_{l} / \delta_{(2,0,0,0,0)}^{1 / 2} .
$$

Finalmente, a estatística de Wald para testar $H_{0}$ versus $H_{1}$, é dada por

$$
W_{a}=\left(\widehat{\beta}_{1}-\beta_{1}^{(0)}\right)^{\top}\left(\widehat{K}^{11}\right)^{-1}\left(\widehat{\beta}_{1}-\beta_{1}^{(0)}\right) .
$$

onde (2.30) pode ser expressa como

$$
W_{a}=\delta_{(2,0,0,0,0)} 0^{-2}\left(\hat{\beta}_{1}-\beta_{1}^{(0)}\right)^{\top}\left(R^{\top} R\right)\left(\hat{\beta}_{1}-\beta_{1}^{(0)}\right) .
$$


O teste escore pode ter grande vantagem computacional sobre os testes da razào de verossimilhança e de Wald. Enquanto o teste da razào de verossimilhança envolve estimativas de parâmetros sob as hipóteses alternativa e nula e o teste de Wald envolve estimativas só sob o modelo irrestrito, o teste escore requer estimação somente sob a hipótese nula. Assim, se, por exemplo. a hipótese nula for simples $\left(H_{0}: \beta=\beta^{(0)}\right.$, com $\phi$ conhecido) o teste escore não envolve nenhuma estimação.

\subsubsection{Testes de hipóteses sobre o parâmetro $\beta \operatorname{com} \phi$ desconhe- cido}

$\mathrm{Na}$ seção anterior, apresentamos testes de hipóteses para os parâmetros de regressão de modelos simétricos supondo que o parâmetro $\phi$ é conhecido e igual para todas as observações. Nesta seção eliminamos a suposição de que $\phi$ é conhecido.

Consideremos a hipótese nula $H_{0}: \beta_{1}=\beta_{1}^{(0)}$ versus a alternativa $H_{1}: \beta_{1} \neq \beta_{1}^{(0)} \operatorname{com} \phi$ e $\beta_{2}$ representando os parâmetros de perturbação e $\beta_{1}^{(0)}$ é um vetor fixado de dimensão $q$ $(q \leq p)$. A estatística da razão de verossimilhança para testar $H_{0}$ é dada por

$$
R V=2\left\{L\left(\widehat{\beta}_{1}, \widehat{\beta}_{2}, \widehat{\phi}\right)-L\left(\beta_{1}^{(0)}, \widetilde{\beta}_{2}, \tilde{\phi}\right)\right\},
$$

onde $\widehat{\beta}_{1}, \widehat{\beta}_{2}$ e $\hat{\phi}$ são as estimativas de máxima verossimilhança irrestritas de $\beta_{1}, \beta_{2}$ e $\phi$, respectivamente, enquanto que $\widetilde{\beta}_{2}$ e $\widetilde{\phi}$ são as estimativas de máxima verossimilhança sob $H_{0}$. Desta forma, $R V$ tem a forma

$$
R V=2\left\{\sum_{l=1}^{n} \log \left[\frac{h\left(\widehat{\tilde{z}}_{l}^{2}\right)}{h\left(\tilde{\tilde{z}}_{l}^{2}\right)}\right]+n \log \left(\frac{\tilde{\phi}}{\widehat{\phi}}\right)\right\} .
$$

Por outro lado, a estatística escore $S_{R}$, definida em (1.4) para testar $H_{0}$ é escrita como

$$
S_{R}=\widetilde{s}^{\top} X_{1}\left(R^{\top} R\right)^{-1} X_{1}^{\top} \tilde{s}
$$

onde $\tilde{s}$ é o vetor definido em $(2.29)$, avaliado em $\theta^{(0)}=\left(\beta_{1}^{(0) \top}, \tilde{\beta}_{2}^{\top}, \widetilde{\phi}\right)^{\top}$. Finalmente, a estatística de Wald para testar $H_{0}$ tem a forma

$$
W_{a}=\delta_{(2,0,0,0,0)} \hat{\phi}^{-2}\left(\widehat{\beta}_{1}-\beta_{1}^{(0)}\right)^{\top}\left(R^{\top} R\right)\left(\widehat{\beta}_{1}-\beta_{1}^{(0)}\right) .
$$

Em problemas regulares, estas três estatísticas têm distribuição assintótica $\chi^{2} \operatorname{com} q$ graus de liberdade.

\subsubsection{Testes de hipóteses sobre o parâmetro $\phi$}

Consideremos agora a hipótese nula $H_{0}: \phi=\phi^{(0)}$ contra a hipótese alternativa $H_{1}: \phi \neq$ $\phi^{(0)}, \operatorname{com} \beta$ representando o parâmetro de perturbação e $\phi^{(0)}$ é um valor fixo positivo. A estatística da razão de verossimilhança para testar $H_{0}$ contra $H_{1}$ é dada por

$$
R V=2\left\{L(\widehat{\beta}, \hat{\phi})-L\left(\tilde{\beta}, \phi^{(0)}\right)\right\},
$$


e tem a forma

$$
R V=2\left\{\sum_{l=1}^{n} \log \left[\frac{h\left(\hat{\tilde{z}}_{l}^{2}\right)}{h\left(\tilde{\tilde{z}}_{l}^{2}\right)}\right]+n \log \left(\frac{\phi^{(0)}}{\hat{o}}\right)\right\},
$$

onde $\tilde{\beta}$ é o estimador de máxima verossimilhança de $\beta$ sob $H_{0}$. Por outro lado, a estatística escore $S_{R}$ para testar $H_{0}$ tem a forma

$$
S_{R}=n^{-1}\left(\delta_{(2,0,0,0,2)}-1\right)^{-1}\left(\sum_{l=1}^{n} z_{l}^{2} \tilde{w}_{l}-n\right)^{2} .
$$

Finalmente, a estatística de Wald para testar $H_{0}$ é dada por

$$
W_{a}=n\left(\delta_{(2,0,0,0,2)}-1\right)\left(\frac{\widehat{\phi}-\phi^{(0)}}{\widehat{\phi}}\right)^{2} .
$$

Em problemas regulares, as três estatísticas, sob a hipótese nula, têm distribuição assintótica qui-quadrado com um grau de liberdade. 


\section{Capítulo 3}

\section{Aperfeiçoamento de testes em modelos de regressão usando uma família simétrica}

\subsection{Introdução}

Nesté capítulo obtemos os fatores de correção de Bartlett para a estatística da razão de verossimilhança e tipo Bartlett para a estatística escore em forma geral e fechada na ampla classe dos modelos de regressão simétricos definidos no Capítulo 2, considerando que o parâmetro de locação está relacionado com um vetor de parâmetros desconhecidos através da relação dada em (2.2) e considerando os casos em que o parâmetro de escala é ou não conhecido. A suposição importante que devemos ter para obter estas correções é que o logaritmo da função de verossimilhança é regular com respeito às derivadas em relação aos parâmetros até quarta ordem (Jensen, 1993).

Recentemente, DiCiccio (1986) estuda um aperfeiçoamento da estatística da razão de verossimilhança para os modelos de locação baseado em inferência condicional. Ferrari e Arellano-Valle (1996) estudam aperfeiçoamentos para os testes da razão de verossimilhança e escore para os modelos de regressão linear com erros independentes tendo uma distribuição $t$-Student. Eles mostram por simulação, que o desempenho dos testes da razão de verossimilhança e escore modificados têm melhor desempenho que os testes da razão de verossimilhança e escore usuais em algumas situações particulares.

Com base no trabalho de Lawley (1956), obtemos na Seção 3.2 o fator de correção de Bartlett para a estatística da razão de verossimilhança. Na Seção 3.3 obtemos, a partir do trabalho de Cordeiro e Ferrari (1991), a correção tipo Bartlett para a estatística escore. Na Seção 3.4, apresentamos estudos de simulação com o intuito de verificar a eficácia das correções. Finalmente, na Seção 3.5 apresentamos aplicaçòes dos resultados. Cabe ressaltar que os parâmetros extras de cada distribuição pertencente à família simétrica são considerados conhecidos. 


\subsection{Fator de correção de Bartlett para a estatística da razão de verossimilhança}

Os testes da razão de verossimilhança aperfeiçoados apresentados no Capítulo 1 dependem da quantidade $d$ definida em (1.11). que é uma função aparentemente complicada de cumulantes conjuntos $\kappa$ 's de derivadas do logaritmo da função de verossimilhança total. O objetivo desta seção é apresentar $d$ em forma simples e de fácil cálculo para os testes de razão de verossimilhança discutidos no capítulo anterior. Uma propriedade fundamental dos modelos simétricos, assim como dos modelos lineares generalizados e dos modelos de dispersão (Jørgensen, 1987a) é que os cumulantes envolvidos são invariantes sob permutação de parâmetros de regressão. Isto conduz a uma simplificação considerável na obtenção de $d \mathrm{e}$, conseqüentemente, na estatística da razão de verossimilhança aperfeiçoada.

\subsubsection{Testes de hipóteses sobre o parâmetro $\beta \operatorname{com} \phi$ conhecido}

$\mathrm{O}$ aperfeiçoamento do teste da razão de verossimilhança para a hipótese $H_{0}: \beta_{1}=\beta_{1}^{(0)}$ contra $H_{1}: \beta_{1} \neq \beta_{1}^{(0)}$ considerando $\phi$ conhecido é baseado no desenvolvimento das fórmulas definidas em (1.8) - (1.10) para obter a constante $d$.

Sejam $\Sigma^{\prime}$ e $\Sigma$ os somatórios sobre todos os $\beta$ 's e sobre os dados, respectivamente. Usamos a notação $\partial \mu_{l} / \partial \beta_{r}=x_{l r}=(r)_{l},\left(\partial^{2} \mu_{l} / \partial \beta_{r} \partial \beta_{s}\right)=x_{l r} x_{l s}=(r, s)_{l}=(r)_{l}(s)_{l}$, $\left(\partial^{3} \mu_{l} / \partial \beta_{r} \partial \beta_{s} \partial \beta_{t}\right)=x_{l r} x_{l s} x_{l t}=(r, s, t)_{l}=(r)_{l}(s)_{l}(t)_{l}$, etc. Assumindo que o logaritmo da função de verossimilhança é regular com respeito a derivadas até segunda ordem, temos os seguintes cumulantes para os modelos simétricos (Apêndice A).

$$
\begin{aligned}
& \downarrow_{\kappa_{r s}}=\frac{\delta_{(0,1,0,0,0)}}{\phi^{2}} \sum(r, s)_{i}, \quad \kappa_{r, s}=\frac{\delta_{(2,0,0,0,0)}}{\phi^{2}} \sum(r, s)_{l} \\
& V_{\kappa_{r s t u}}=\frac{\delta_{(0,0,0,1,0)}}{\phi^{4}} \sum(r, s, t, u)_{l}, \\
& \sqrt{\kappa_{r, s, t, u}}=\frac{1}{\phi^{4}}\left(\delta_{(4,0,0,0,0)}-3 \delta_{(2,0,0,0,0)}^{2}\right) \sum(r, s, t, u)_{l}, \\
& V_{\kappa_{r, s, t u}}=\frac{1}{\phi^{4}}\left(\delta_{(2,1,0,0,0)}+\delta_{(2,0,0,0,0)}^{2}\right) \sum(r, s, t, u)_{l}, \\
& V_{\kappa_{r s}^{(t)}}=\kappa_{r s}^{(t u)}=\kappa_{r s t}^{(u)}=0, \quad \kappa_{r s t}=\kappa_{r, s t}=\kappa_{r, s, t}=\kappa_{r s, t}=0,
\end{aligned}
$$

onde $\delta_{(a, b, c, d, e)}$, para $a, b, c, d, e=0,1,2,3,4$ são definidos em (2.13). Mostra-se facilmente que $\kappa_{r, s, t, u}+3 \kappa_{r, s, t u}=0$. Cabe ressaltar que $\kappa_{r s}^{(t)} \cdot \kappa_{r s}^{(t u)}$ e $\kappa_{r s t}^{(u)}$ são iguais a zero devido à estrutura linear da relação do vetor $\mu$ com o parâmetro $\beta$ dada em $(2.2)$, e $\kappa_{r s t}, \kappa_{r, s t}, \kappa_{s t, r}$. $\kappa_{r, s, t}$ são nulos devido à simetria dos modelos aqui considerados.

Duas propriedades importantes dos cumulantes nos modelos simétricos são dadas a seguir: primeiro, todos os cumulantes dependem somente de o e dos parâmetros considerados conhecidos de cada distribuiçào e não dependem de $\beta$ e, segundo. qualquer cumulante que envolva um número ímpar de componentes do parâmetro $\beta$ é nulo. 
Assumindo que o parâmetro de escala é conhecido, a matriz de informaçào total de Fisher para 3 na classe dos modelos simétricos é dada em (2.15). Definimos

$$
Z=\left\{\tilde{\sim}_{l m}\right\}=X^{\prime}\left(X^{\top} X^{\top}\right)^{-1} \cdot X^{\top}, \quad Z_{2}=\left\{\tilde{z}_{2 l m}\right\}=X_{2}\left(X_{2}^{\top} X_{2}\right)^{-1} \cdot X_{2}^{\top},
$$

as matrìzes $n \times n$ de postos $p$ e $(p-q)$, respectivamente. Se $p=q$ define-se $Z_{2}=0$, onde 0 denota uma matriz nula $n \times n$. É importante observar que as matrizes $Z \phi^{2} \delta_{(2,0,0,0,0)}^{-1} \mathrm{e}$ $Z_{2} \phi^{2} \delta_{(2,0,0,0,0)}^{-1}$ sào as estruturas de covariância assintótica de $X \widehat{\beta}$ e $X_{2} \widetilde{\beta}_{2}$. respectivamente. Definimos ainda

$$
Z_{d}=\operatorname{Diag}\left\{z_{11}, \ldots, z_{n n}\right\} \quad \text { e } Z_{2 d}=\operatorname{Diag}\left\{z_{211}, \ldots, z_{2 n n}\right\},
$$

representando as matrizes com elementos diagonais de $Z$ e $Z_{2}$, respectivamente. Nota-se que $-\sum^{\prime}(r)_{l}(s)_{m} r^{r s}$ é o elemento $(l, m)$ da matriz $Z \phi^{2} \delta_{(2,0,0,0,0)}^{-1}$.

Expressões simples para obter $d$ dada em (1.11) podem ser obtidas substituindo os $k$ 's nas expressões (1.9) - (1.10) e efetuando as somas sobre a amostra depois de avaliar as somas sobre os parâmetros. Desta maneira, para obter a parcela $\sum{ }^{\prime} \ell_{r s t u}$ de $\varepsilon_{p}$, substituimos $\kappa_{r s t u}, \kappa_{r s t}^{(u)}$ e $\kappa_{r s}^{(u)}$ na expressào de $\ell_{r s t u}$ dada em (1.9) e obtemos

$$
\sum{ }^{\prime} C_{r s t u}=\frac{\delta_{(0,0,0,1,0)}}{4 \phi^{4}} \sum{ }^{\prime} \kappa^{r s} \kappa_{i}{ }^{t u} \sum(r, s, t, u)_{l}
$$

Invertendo a ordem das somas e rearranjando os termos obtemos

$$
\sum{ }^{\prime} \ell_{r s t u}=\frac{\delta_{(0.0,0.1 .0)}}{4 \phi^{4}} \sum\left(-\sum{ }^{\prime}(r)_{l}(s)_{l} \kappa^{r s}\right)\left(-\sum{ }^{\prime}(t)_{l}(u)_{l} \kappa^{t u}\right)
$$

ou ainda

$$
\sum^{\prime} \ell_{r s t u}=\frac{\delta_{(0,0.0 .1,0)}}{4 \phi^{4}} \sum\left(z_{l l} \phi^{2} \delta_{(2,0,0,0,0)}^{-1}\right)\left(z \| l \phi^{2} \delta_{(2,0,0,0,0)}^{-1}\right)=\frac{\delta_{(0,0,0.1,0)}}{4 \delta_{(2,0,0,0,0)}^{2}} \sum z_{l l}^{2} .
$$

Finalmente, temos a expressão para $\sum{ }^{\prime} \ell_{r s t u}$ da forma

$$
\sum{ }^{\prime} \ell_{r s t u}=\frac{\delta_{(0,0,0,1,0)}}{4 \delta_{(2,0,0,0,0)}^{2}} \operatorname{tr}\left(Z_{d} Z_{d}\right)
$$

onde tr denota traço. A parcela restante de $\varepsilon_{p}$, isto é, $\sum^{\prime} \ell_{\text {rstuvw }}$ se anula devido ao fato de que os cumulantes da expressão de $\ell_{\text {rstuvw }}$ dados em (1.10) são todos iguais a zero. A expressão de $\varepsilon_{p}$ resultante é, portanto,

$$
\Sigma_{p}=\frac{\delta_{(0,0,0,1,0)}}{4 \delta_{(2,0,0,0,0)}^{2}} \operatorname{tr}\left(Z_{d} Z_{d}\right)
$$

De forma análoga. temos

$$
\Sigma_{p-q}=\frac{\delta_{(0,0,0,1,0)}}{4 \delta_{(2,0,0,0,0)}^{2}} \operatorname{tr}\left(Z_{2 d} Z_{2 d}\right)
$$


O fator de correção de Bartlett para o teste da razào de verossimilhança discutido na Seção 1.2 é obtido de (1.11) com as quantidades $\Sigma_{p}$ e $\Sigma_{p-q}$ dadas acima. Assim.

$$
d=\frac{d_{0}}{n q} h_{0}\left(X_{1}, X_{2}\right),
$$

onde

$$
h_{0}\left(X_{1}, X_{2}\right)=\rho_{Z Z}-\rho_{Z_{2} Z_{2}}
$$

$\operatorname{com} \rho_{Z_{2} Z_{2}}=n \operatorname{tr}\left(Z_{2 d} Z_{2 d}\right), \rho_{Z Z}=n \operatorname{tr}\left(Z_{d} Z_{d}\right) \mathrm{e}$

$$
d_{0}=\frac{\delta_{(0,0,0,1,0)}}{4 \delta_{(2,0,0,0,0)}^{2}} .
$$

Note-se que a expressão de $d$ é muito simples, não depende de estimação de parâmetros desconhecidos e é decomposta em dois fatores, um que só depende da distribuição assumida para os dados (através de $d_{0}$ que é função dos $\delta$ 's) e a outra que só depende da matriz modelo $\mathrm{X}$ (através das matrizes de projeção $Z$ e $Z_{2}$ definidas em (3.1)).

A expansão assintótica da função distribuição da estatística da razão de verossimilhança $R V$ dada em (2.27), sob a hipótese nula $H_{0}$, tem a forma

$$
F_{R V}(x)=G_{q}(x)+\frac{1}{24 n} d_{0} h_{0}\left(X_{1}, X_{2}\right)\left\{G_{q+2}(x)-G_{q}(x)\right\}
$$

até ordem $n^{-1}$. Logo, de (3.5) podemos obter a expressão assintótica da função densidade de $R V$, sob $H_{0}$, e até ordem $n^{-1}$, como

$$
f_{R V}(x)=g_{q}(x)+\frac{d_{0}}{n} h_{0}\left(X_{1}, X_{2}\right)\left(g_{q+2}(x)-g_{q}(x)\right),
$$

onde $g_{r}(x)$ é a densidade de uma variável aleatória $\lambda_{r}^{2}$. Usando a relação de recorrência $g_{r+2}(x)=g_{r}(x) x / r$, segue que

$$
f_{R V}(x)=g_{q}(x)\left(1+\frac{d_{0}}{n} h_{0}\left(X_{1}, X_{2}\right)\left(\frac{x}{q}-1\right)\right),
$$

até ordem $n^{-1}$.

Assim, para uma dada estrutura do modelo (matriz X) e uma dada hipótese nula, obtemos a quantidade $h_{0}\left(X_{1}, X_{2}\right)$ e podemos avaliar a influência da distribuição dos dados na qualidade da aproximação da distribuição da estatística $R V$ por qui-quadrado através da quantidade $d_{0}$. Analogamente, dada uma distribuição para os dados, obtemos a quantidade $d_{0}$ e podemos avaliar a influência da estrutura do modelo e da hipótese nula na qualidade desta aproximação através da quantidade $h_{0}\left(X_{1}, X_{2}\right)$.

Fornecemos a seguir algumas aplicações da fórmula (3.4) a diversas distribuições na família simétrica definida em (2.1) e (2.2), assumindo conhecido o parâmetro de escala (no Apêndice C estào os $\delta$ 's para cada distribuição). 
(i) Normal: $y_{l} \sim N\left(x_{l}^{\top} \beta, \phi^{2}\right)$ :

$$
d_{0}=0
$$

(ii) Cauchy: $y_{l} \sim C\left(x_{l}^{\top} \beta, \phi^{2}\right)$;

$$
d_{0}=\frac{3}{4}
$$

(iii) $t$-Student: $y_{l} \sim t\left(x_{l}^{\top} \beta, \dot{\phi}^{2}, \nu\right), \nu>0$ conhecido;

$$
d_{0}=\frac{3(\nu+2)(\nu+3)^{2}}{2 \nu(\nu+1)(\nu+5)(\nu+7)} .
$$

(iv) $t$-Student generalizada: $y_{l} \sim t G\left(x_{l}^{\top} \beta, \phi^{2}, s, r\right), r, s>0$ conhecidos;

$$
d_{0}=\frac{3(r+2)(r+3)^{2}}{2 r(r+1)(r+5)(r+7)} .
$$

(v) Logística I: $y_{l} \sim L I\left(x_{l}^{\top} \beta, \phi^{2}\right)$;

$$
d_{0} \approx 0,4879224
$$

(vi) Logística II: $y_{l} \sim \operatorname{LII}\left(x_{l}^{\top} \beta, \phi^{2}\right)$;

$$
d_{0}=\frac{3}{20}
$$

(vii) Logística generalizada: $y_{l} \sim L G\left(x_{l}^{\top} \beta, \phi^{2}, m\right), m>0$ conhecido;

$$
d_{0}=\frac{(2 m+1)}{4 m(2 m+3)} .
$$

(viii) Exponencial potência: $y_{l} \sim E P\left(x_{l}^{\top} \beta, \phi^{2}, k\right),-1<k<-1 / 2$ conhecido;

$$
d_{0}=k(1-k) \frac{\Gamma\left(\frac{1-3 k}{2}\right) \Gamma\left(\frac{1+k}{2}\right)}{8 \Gamma\left(\frac{3-k}{2}\right)^{2}} .
$$

Cabe ressaltar que a correção para a estatística da razão de verossimilhança no modelo t-Student coincide. como esperado, com o resultado obtido por Ferrari e Arellano-Valle (1996).

Note-se que o coeficiente $d_{0}$ não depende de 3 e $\phi$, mas depende dos parâmetros considerados conhecidos em cada distribuição. Por exemplo, no caso da distribuição $t$-Student. depende de $\nu$ através de funções que são definidas para todo $\nu>0$ e que se anula quando $\nu \rightarrow \infty$ (modelo normal), pois para o modelo de regressào normal, a estatística $R V$ tem distribuiçào $\chi_{q}^{2}$ exata sob a hipótese nula. Cabe mencionar que este 
resultado coincide com o de DiCiccio (19S6. p.11) que trabalha com uma família de locaçào $t$-Student com um único parâmetro desconhecido. No caso do modelo $t$-Student generalizada, $d_{0}$ depende da constante $r$ (que está relacionada com a curtose) através de funções que sào definidas para $r>0$ e. como na distribuição $t$-Student, se anula quando $r \rightarrow \infty$ (modelo normal). Para a distribuição logística generalizada, o coeficiente $d_{0}$ depende da constante conhecida $m$ e se anulando quando $m \rightarrow \infty$. No caso do modelo exponencial potência, o coeficiente $d_{0}$ depende da constante $k$ através de funçòes que são definidas para $-1<k<-1 / 2$.

Examinaremos a seguir algumas estruturas lineares especiais que induzem a simplificaçòes na fórmula (3.2).

\section{(1) Caso uniparamétrico}

Consideremos $\beta$ um parâmetro unidimensional. O interesse é testar a hipótese nula. simples $H_{0}: \beta=\beta^{(0)}$ contra a alternativa composta $H_{1}: \beta \neq \beta^{(0)}$.

(a) Seja $\mu_{l}=\beta, l=1 \ldots n$. De (3.3) é fácil ver que $h_{0}\left(X_{1}, X_{2}\right)=1$ e. assim, $d=d_{0} / n$.

(b) Seja $\mu=X \beta$, onde $X=\left(x_{1}, \ldots, x_{n}\right)^{\top}$. De (3.3) tem-se $h_{0}\left(X_{1}, X_{2}\right)=\bar{s}_{4} / \bar{s}_{2}^{2}$, onde $\bar{s}_{a}=\sum_{i=1}^{n} x_{i}^{a} / n$. Conseqüentemente, o coeficiente $d$ é escrito como $d=d_{0} \bar{s}_{4} /\left(n \bar{s}_{2}^{2}\right)$.

\section{(2) Hipótese nula simples}

Consideremos que $\mu$ tem a estrutura linear definida em (2.2). O interesse é testar a hipótese nula simples $H_{0}: \beta=\beta^{(0)}$ contra a alternativa composta $H_{0}: \beta \neq \beta^{(0)}(p=q)$. Tem-se $Z_{2}=0$, o que conduz a $h_{0}\left(X_{1}, X_{2}\right)=\rho Z Z$.

Assim, o coeficiente da correçào de Bartlett $d$ é escrito como $d=d_{0} \rho_{Z Z} /(n p)$.

\section{(3) Modelo de classificação a um fator}

Consideremos $p \geq 2$ populações, cada uma tendo densidade da forma (2.1). Suponhamos que amostras aleatórias independentes de tamanhos $n_{1}, \ldots, n_{p}\left(n_{i} \geq 1, i=1, \ldots, p\right)$ foram retiradas dessas populações. Suponhamos também que $\phi$ é conhecido e o mesmo para as $p$ populações. Assumimos a estrutura linear $\mu_{i}=\beta+\beta_{i}, i=1, \ldots, p$, $\operatorname{com} \beta_{p}=0$. Notemos que $\beta_{i}$ é o efeito da $i$-ésima população na variável resposta $y=\left(y_{11}, \ldots, y_{1 n_{1}}, \ldots, y_{p 1}, \ldots, y_{p n_{p}}\right)^{\top}$. Denotaremos as médias amostrais por $\bar{y}_{1}, \ldots, \bar{y}_{p}$, o número total de observações por $n=\sum_{i=1}^{p} n_{i}$ e a média geral por $\bar{y}=\sum_{i=1}^{p} n_{i} \bar{y}_{i} / n$.

A estatística da razão de verossimilhança para testar a homogeneidade dos parâmetros de locação das $p$ populações, isto é, testar $H_{0}: \beta_{1}=\cdots=\beta_{p-1}=0$ contra a alternativa $H_{1}$ : pelo menos um $\beta_{i}, i=1 \ldots, p-1$, não é nulo, é obtido de (1.3). e tem a forma

$$
R V=2\left\{L\left(\widehat{\beta}_{1}, \widehat{\beta}_{2}, \ldots, \widehat{\beta}_{p-1}, \widehat{\beta}\right)-L(0, \ldots, 0 . \widetilde{\beta})\right\} .
$$

onde $L(\cdot)$ é o logaritmo da função de verossimilhança. Assintoticamente, $R V$ tem distribuição $\chi_{p-1}^{2}$ sob a hipótese nula. 
Para obter $d$ precisamos encontrar $Z$ e $Z_{2}$ definidas em (3.1). que sào funçòes das matrizes $\mathrm{X}^{\prime}$ e $\mathrm{X}_{2}$. de dimensòes $n \times p$ e $n \times 1$. respectivamente. De

$$
X=\left(\begin{array}{ccccc}
1_{n_{1} \times 1} & 0_{n_{1} \times 1} & \cdots & 0_{n_{1} \times 1} & 1_{n_{1} \times 1} \\
0_{n_{2} \times 1} & 1_{n_{2} \times 1} & & 0_{n_{2} \times 1} & 1_{n_{2} \times 1} \\
\vdots & & & & \vdots \\
0_{n_{p-1} \times 1} & 0_{n_{p-1} \times 1} & & 1_{n_{p-1} \times 1} & 1_{n_{p-1} \times 1} \\
0_{n_{p} \times 1} & 0_{n_{p} \times 1} & \cdots & 0_{n_{p} \times 1} & 1_{n_{p} \times 1}
\end{array}\right) \text {, }
$$

encontramos

$$
X^{\top} \mathrm{X}^{\prime}=\left(\begin{array}{ccccc}
n_{1} & 0 & \cdots & 0 & n_{1} \\
0 & n_{2} & \cdots & 0 & n_{2} \\
\vdots & & \ddots & & \vdots \\
0 & 0 & \cdots & n_{p-1} & n_{p-1} \\
n_{1} & n_{2} & \cdots & n_{p-1} & n_{p}
\end{array}\right)
$$

De $X_{2}=1_{n}$, temos $X_{2}^{\top} X_{2}^{\prime}=n$. A matriz inversa de $X^{\top} X^{\prime}$ segue da fórmula de inversas de matrizes divididas apresentada em (2.26). Encontramos

$$
Z_{d}=\operatorname{Diag}\left\{n_{1}^{-1}, \ldots, n_{1}^{-1}, \ldots, n_{p}^{-1}, \ldots, n_{p}^{-1}\right\},
$$

e $Z_{2 d}=n^{-1} I_{n}$. Assim, das matrizes $Z_{d}$ e $Z_{2 d}$ verifica-se que

$$
\rho_{Z Z}=n \operatorname{tr}\left(Z_{d} Z_{d}\right)=n \sum_{i=1}^{p} n_{i}^{-1} \quad \text { e } \quad \rho_{Z_{2} Z_{2}}=n \operatorname{tr}\left(Z_{2_{d}} Z_{2_{d}}\right)=1 .
$$

Logo, $h_{0}\left(X_{1}, X_{2}\right)=n \sum_{i=1}^{p}\left(1 / n_{i}\right)-1$ e, portanto, o coeficiente da correçào de Bartlett é escrito como $d=d_{0} /[n(p-1)]\left(n \sum_{i=1}^{p} n_{i}^{-1}-1\right)$.

No caso balanceado, ou seja, se $n_{1}=n_{2}=\cdots=k$ e $n=p k, d=d_{0}(p+1) /(p k)$.

\section{(4) Modelo de regressão linear simples}

Consideramos aqui um modelo simétrico definido em (2.1) com a estrutura para cada $\mu_{l}$ da forma $\mu_{l}=\alpha+\beta x_{l}, l=1, \ldots, n$, onde $x_{l}$ denota o valor da variável explicativa para a l-ésima observação. A estatística da razão de verossimilhança para testar $H_{0}: \beta=\beta^{(0)}$ contra $H_{1}: \beta \neq \beta^{(0)}$ vem de $(2.27)$ e tem a forma

$$
R V=2\left\{\sum_{l=1}^{n} \log \frac{h\left(\left(\frac{y_{l}-\left(\hat{\alpha}+\widehat{\beta} x_{l}\right)}{\phi}\right)^{2}\right)}{h\left(\left(\frac{y_{l}-\left(\tilde{\alpha}+\beta^{(0)} x_{l}\right)}{\phi}\right)^{2}\right)}\right\} .
$$

O coeficiente da correção de Bartlett $(d)$ para este teste é obtido de (3.2), com

$$
h_{0}\left(X_{1}, X_{2}\right)=\frac{\bar{S}_{4}^{2}}{\bar{S}_{2}^{2}}+2 \text {. }
$$


onde

$$
\bar{S}_{a}=\sum_{i=1}^{n} \frac{\left(x_{i}-\bar{x}\right)^{a}}{n}, \quad \text { para } a=2,4 .
$$

Desta maneira, $d=d_{0}\left(\left(\bar{S}_{4} / \bar{S}_{2}^{2}\right)+2\right) / n$.

Observa-se então que a aproximação da distribuição da estatística da razão de verossimilhança por $x^{2}$ em (3.5) é particularmente sensível a mudanças na medida de curtose amostral $\left(\bar{S}_{4} / \bar{S}_{2}^{2}\right)$ de $x$.

(5) Modelo de regressão com dois parâmetros

Consideremos um modelo simétrico definido em (2.1) $\operatorname{com} \mu_{l}=\alpha u_{l}+\beta x_{l}, l=$ $1,2, \ldots, n$, onde $u_{l}$ e $x_{l}$ denotam os valores de duas variáveis auxiliares associadas à $l$ ésima observação. A estatística da razão de verossimilhança para testar $H_{0}: \beta=\beta^{(0)}$ versus $H_{1}: \beta \neq \beta^{(0)}$ pode ser obtida de $(2.27)$ e tem a forma

$$
R V=2\left\{\sum_{l=1}^{n} \log \frac{h\left(\left(\frac{y_{l}-\left(\hat{\alpha} u_{l}+\widehat{\beta} x_{l}\right)}{\circ}\right)^{2}\right)}{h\left(\left(\frac{y_{l}-\left(\tilde{\alpha} u_{l}+\beta^{(0)} x_{l}\right)}{\circ}\right)^{2}\right)}\right\} .
$$

Assintoticamente, $R V$ tem distribuição $\chi_{1}^{2}$ sob $H_{0}$. Neste caso, $h_{0}\left(X_{1}, X_{2}\right)$ tem a forma

$$
\begin{aligned}
h_{0}\left(X_{1}, X_{2}\right)= & n \Delta^{-2}\left(s_{40} s_{02}^{2}+s_{04} s_{20}^{2}+4 s_{22} s_{11}^{2}+2 s_{22} s_{20} s_{02}\right. \\
& \left.-4 s_{11} s_{02} s_{31}-4 s_{11} s_{20} s_{13}\right)-n s_{40} / s_{20}^{2},
\end{aligned}
$$

com $s_{a b}=\sum_{l=1}^{n} u_{l}^{a} x_{l}^{b}$ e $\Delta=s_{02} s_{20}-s_{11}^{2}$, para $a, b=0,1,2,3,4$. O coeficiente $d$ vem de (3.2) com $h_{0}\left(X_{1}, X_{2}\right)$ dado acima.

\subsubsection{Testes de hipóteses sobre o parâmetro $\beta$ com $\phi$ desconhe- cido}

Na Seção 3.2.1 trabalhamos com a classe dos modelos simétricos supondo o parâmetro de escala $\phi$ conhecido e igual para todas as observações. Nesta seção, eliminamos a suposição de que $\phi$ é conhecido e obtemos fatores de correção de Bartlett para a estatística da razão de verossimilhança associada ao teste da hipótese $H_{0}: \beta_{1}=\beta_{1}^{(0)}$ versus $H_{1}: \beta_{1} \neq \beta_{1}^{(0)}$, onde $\beta_{1}^{(0)}$ é um vetor especificado de dimensão $q(q \leq p), \beta_{2}=\left(\beta_{q+1}, \ldots, \beta_{p}\right)^{\top}$ e $\phi$ são parâmetros de perturbação. O logaritmo da função de verossimilhança $L=L(\beta, \phi)$ é agora função dos parâmetros $\beta$ e $\phi$, dados $y_{1}, \ldots, y_{n}$.

Introduzimos a seguinte notação para definir os cumulantes envolvendo o parâmetro $\phi$ : $\kappa_{\phi \phi}=E\left(\partial^{2} L / \partial \phi^{2}\right), \kappa_{\phi r}=E\left(\partial^{2} L / \partial \beta_{r} \partial \phi\right), \kappa_{\phi r s}=E\left(\partial^{3} L / \partial \beta_{r} \partial \beta_{s} \partial \phi\right), \kappa_{\phi \phi \phi}=E\left(\partial^{3} L / \partial \phi^{3}\right)$, etc. Assim, temos, além dos cumulantes definidos na Seção 3.2.1, os seguintes para os modelos simétricos (Apêndice B):

$$
\begin{aligned}
& \kappa_{\phi \phi}=\frac{n}{\phi^{2}}\left(\delta_{(0,1,0,0,2)}-1\right), \quad \Longrightarrow \quad K_{\phi \phi}^{-1}= \\
& 2-\delta_{20002} \\
& 54 \\
& \phi^{2} \\
& m\left(\delta(0,1,0,0,2)^{-1}\right) \\
& K_{\phi \pi}=K_{\Pi \phi} . \\
& K_{\phi M n}=K_{M \rightarrow \phi} \\
& =\frac{m}{\phi^{2}}\left(1-\delta_{20002}\right)
\end{aligned}
$$




$$
\begin{aligned}
& \kappa_{\phi, \phi}=\frac{n}{\bar{o}^{2}}\left(\delta_{(2,0,0,0,2)}-1\right), \\
& \kappa_{r \phi}=\kappa_{r \varphi}^{(\phi)}=\kappa_{r \phi}^{(s)}=\kappa_{\phi \phi}^{(r)}=0 . \\
& \kappa_{\phi r, \phi}=\kappa_{\phi \infty r r}=\kappa_{\phi \phi, r}=\kappa_{i, \phi, \phi}=\kappa_{i \phi \phi}^{(\phi)}=\kappa_{\phi \phi}^{(i j)}=0 \text {, } \\
& \kappa_{r s}^{(\phi)}=-\frac{2}{\phi^{3}} \delta_{(0,1,0,0,0)} \sum(r, s)_{l} \text {, } \\
& \left.\kappa_{\phi \phi}^{(\phi)}=-\frac{2 n}{0^{3}} \delta_{(0,1,0,0,2)}-1\right), \\
& \kappa_{i j \phi}=-\frac{1}{\phi^{3}}\left(\delta_{(0,0,1,0,1)}+2 \delta_{(0,1,0,0,0)}\right) \sum(i, j)_{l} \text {, } \\
& \pi^{\kappa_{i \phi, j}}=-\frac{1}{\phi^{3}}\left(\delta_{(1,1,0,0,1)}-\delta_{(0,1,0,0,0)}\right) \sum(i, j)_{l} \\
& =\frac{1}{\phi^{3}}\left(\delta_{(0,0.1,0,1)}+2 \delta_{(0,1,0,0,0)}\right) \sum(i, j)_{l}, \\
& \kappa_{i j, \phi}=\frac{1}{\phi^{3}} \delta_{(0,0,1,0,1)} \sum(i, j)_{l} \text {, } \\
& =-\frac{1}{\phi^{3}}\left(\delta_{(1,1,0,0,1)}+\delta_{(0,1,0,0,0)}\right) \sum(i, j)_{l}, \\
& \kappa_{i, j, \phi}=\frac{2}{\phi^{3}} \delta_{(1,1,0,0,1)} \sum(i, j)_{l} \\
& \kappa_{\phi \phi \phi}=-\frac{n}{\phi^{3}}\left(6 \delta_{(0,1,0,0,2)}+\delta_{(0,0,1,0,3)}-4\right) \text {, } \\
& \kappa_{\phi \phi, \phi}=\frac{n}{\phi^{3}}\left(4 \delta_{(0,1,0,0,2)}+\delta_{(0,0,1,0,3)}-2\right) \\
& \kappa_{\phi, \phi, \phi}=\frac{2 n}{\phi^{3}}\left(\delta_{(1,1,0,0,3)}+1\right) \text {, } \\
& \kappa_{i j}^{(\phi \phi)}=\frac{6}{\phi^{2}} \kappa_{i j}, \\
& \kappa_{\phi \phi \phi}^{(\phi)}=-\frac{3}{\phi} \kappa_{\phi \phi \phi}, \quad \kappa_{i j \phi}^{(\phi)}=-\frac{3}{\phi} \kappa_{i j \phi}, \quad \kappa_{\phi \phi}^{(\phi \phi)}=\frac{6}{\phi^{2}} \kappa_{\phi \phi},=-\frac{\sigma^{n}}{\phi^{4}}\left(\delta_{2 \operatorname{ccc} i}-1\right) \\
& \kappa_{\phi \phi \phi \phi)}=\frac{n}{\phi^{4}}\left(\delta_{\underline{(0,0,0,1,4)}}+12 \underline{\delta_{(0,0,1,0,3)}}+36 \delta_{(0,1,0,0,2)}-18\right), \\
& \kappa_{i j \phi \phi}^{\prod 1}=\frac{1}{\phi^{4}}\left(\delta_{(0,0,0,1,2)}-6 \delta_{(1,1,0,0,1)}\right) \sum(i, j)_{l}, \\
& \rtimes^{\kappa_{\dot{r}, s \phi \phi}}=\frac{1}{\phi^{4}}\left(\delta_{(1,0,1,0,2)}+4 \delta_{(1,1,0,0,1)}+2 \delta_{(2,0,0,0,0)}\right) \sum(r, s)_{l}, \\
& \kappa_{r s, \phi, \phi}=\frac{1}{\phi^{4}}\left(2 \delta_{(1,1,0,0,1)}+\delta_{(0,1,0,0,2)} \delta_{(0,1,0,0,0)}+\delta_{(2,1,0,0,2)}\right) \sum(r . s)_{l}, \\
& \kappa_{i \phi, j \phi}=\frac{1}{o^{4}}(\delta_{(0,2,0,0,2)}+\underbrace{}_{(1,1,0,0,1)}-\delta_{(0,1,0,0,0)}) \sum(i, j)_{l}, \\
& -2 \delta 00101-2 \delta 01000-\delta 01000
\end{aligned}
$$




$$
\begin{aligned}
\kappa_{\phi, \phi, \phi, \phi} & =\frac{n}{\phi^{4}}\left(\delta_{(4,0,0.0 .4)}+4 \delta_{(3,0.0,0.3)}+12 \delta_{(2.0 .0 .0,2)}-3 \delta_{(2.0 .0 .0 .2)}^{2}-6\right), \\
\kappa_{i, j, \phi, \phi} & =\frac{1}{\phi^{4}}\left(2 \delta_{(3,0.0 .0 .1)}+\delta_{(4,0,0,0,2)}-\delta_{(0,1,0.0 .0)} \delta_{(0,1,0.0 .2)}\right) \sum(i, j)_{l}, \\
\kappa_{i, j, \phi \phi} & =\frac{1}{\phi^{4}}\left(2 \delta_{(3,0,0.0,1)}+\delta_{(2,1,0,0,2)}-2 \delta_{(0,1,0,0,0)}+\delta_{(0,1,0.0 .0)} \delta_{(0.1,0,0,2)}\right) \sum(i, j)_{l}
\end{aligned}
$$

e a relação entre os cumulantes: $\kappa_{\phi, \phi, \phi}=-\left(\kappa_{\phi \phi \phi}+3 \kappa_{\phi, \phi \phi}\right), \kappa_{\phi, \phi, \phi}=2 \kappa_{\phi \phi \phi}-3 \kappa_{\phi \phi}^{(\phi)} \mathrm{e}$ $\kappa_{i j \phi}=-\kappa_{i \phi, j}$.

Observamos que todos os cumulantes independem de $\beta$ e que os cumulantes relativos somente a $\phi$ não dependem da matriz $X$. Notamos também que o cumulante $\kappa_{r \phi}$ é nulo para $r=1, \ldots, p$. Isto demonstra que, nos modelos simétricos, $\beta$ e $\phi$ são parâmetros globalmente ortogonais segundo a definição de Cox e Reid (1987).

A estatística da razào de verossimilhança $R V$ para testar $H_{0}$ dada em (2.32) pode ser escrita como

$$
R V=2\left\{\left\{L\left(\widehat{\beta}_{1}, \widehat{\beta}_{2}, \widehat{\phi}\right)-L\left(\beta_{1}, \beta_{2}, \phi\right)\right\}-\left\{L\left(\beta_{1}^{(0)}, \widetilde{\beta}_{2}, \widetilde{\phi}\right)-L\left(\beta_{1}, \beta_{2}, \phi\right)\right\}\right\},
$$

onde $L$ é o logaritmo da função de verossimilhança. Do resultado (1.7), sob $H_{0}$ temos, segundo Lawley (1956), que

$$
2 E\left[L\left(\widehat{\beta}_{1}, \widehat{\beta}_{2}, \widehat{\phi}\right)-L\left(\beta_{1}, \beta_{2}, \phi\right)\right]=p+1+\varepsilon_{p+1}+O\left(n^{-2}\right)
$$

$\mathrm{e}$

$$
2 E\left[L\left(\widehat{\beta}_{1}^{(0)}, \widetilde{\beta}_{2}, \tilde{\phi}\right)-L\left(\beta_{1}, \beta_{2}, \phi\right)\right]=p-q+1+\varepsilon_{p-q+1}+O\left(n^{-2}\right),
$$

onde os termos $\varepsilon_{p+1}$ e $\varepsilon_{p+q-1}$. de ordem $n^{-1}$, são dados por

$$
\begin{aligned}
\varepsilon_{p+1} & =\sum^{\prime}\left(\ell_{r s t u}-\ell_{r s t u v w}\right)+\sum_{\beta, \phi}^{\prime}\left(\ell_{r s t u}-\ell_{r s t u v w}\right)+\left(\ell_{\phi \phi \emptyset}-\ell_{\phi \phi \phi \phi \phi}\right) \\
& =\varepsilon_{p}+\varepsilon_{p+1, \beta \phi}+\varepsilon_{\phi}, \\
\varepsilon_{p-q+1} & =\sum^{\prime \prime}\left(\ell_{r s t u}-\ell_{r s t u v w}\right)+\sum_{\beta, \phi}^{\prime \prime}\left(\ell_{r s t u}-\ell_{r s t u v w}\right)+\left(\ell_{\phi \phi \circ}-\ell_{\phi \phi \phi \phi \phi}\right) \\
& =\varepsilon_{p-q}+\varepsilon_{p-q+1, \beta \phi}+\varepsilon_{\phi},
\end{aligned}
$$

sendo que $\sum^{\prime}$ representa o somatório sobre todas as combinações dos parâmetros $\beta, \sum^{\prime \prime}$ representa o somatório sobre todas as combinações no conjunto $\left\{\beta_{q+1}, \ldots, \beta_{p}\right\}$, $\sum_{\beta, \phi}^{\prime}$ representa o somatório sobre todas as combinações dos parâmetros $\beta$ e $o$ ( $p+1$ parâmetros), mas com pelo menos um índice igual a $\phi$ e $\sum_{\beta, \phi}^{\prime \prime}$ representa o somatório sobre todas as combinações de parâmetros $\left\{\beta_{q+1}, \ldots, \beta_{p}, \phi\right\}$ com pelo menos um índice igual a $\phi(p-q+1$ parâmetros). Das equações (3.7) segue que, sob $H_{0}$, o valor esperado da estatística da razão de verossimilhança dada em (3.6) tem a forma

$$
\begin{aligned}
E(R V) & =q+\varepsilon_{p}+\varepsilon_{p+1, \beta \phi}-\varepsilon_{p-q}-\varepsilon_{p-q+1,3 \phi}+O\left(n^{-2}\right) \\
& =q\left\{1+d^{\times}\right\}+O\left(n^{-2}\right),
\end{aligned}
$$


onde

$$
d^{*}=\frac{\Sigma_{p}-\varepsilon_{p-q}}{q}+\frac{\Sigma_{p+1, B o}-\varepsilon_{p-q+1 . B \phi}}{q}
$$

é um termo de ordem $n^{-1}$. Assim, o fator de correção de Bartlett é da forma $\left(1-d^{*}\right)$. onde a expressào de $d^{\times}$dada acima pode ser escrita como

$$
d^{*}=d_{\beta}+d_{\beta \phi},
$$

onde $d_{\beta}$ tem a mesma expressão de $d$ considerando $\phi$ conhecido e apresentada em (3.2) e $d_{\beta \phi}$ representa a contribuiçào adicional devido ao fato de que $\phi$ é desconhecido e é da forma

$$
d_{\beta \phi}=\frac{\left(\varepsilon_{p+1, \beta \phi}-\varepsilon_{p-q+1, \beta \phi}\right)}{q},
$$

ou seja,

$$
d_{\beta \phi}=\frac{1}{q}\left\{\sum_{\beta, \phi}{ }^{\prime}\left(\ell_{r \text { stu }}-\ell_{\text {rstuvw }}\right)-\sum_{\beta, \phi}{ }^{\prime \prime}\left(\ell_{\text {rstu }}-\ell_{\text {rstuvw }}\right)\right\} .
$$

Devido à ortogonalidade global entre $\beta$ e $\phi$ e à estrutura linear definida em (2.2), $d_{\beta \phi}$ se reduz a (Apêndice $\mathrm{B})$

$$
d_{30}=\frac{1}{n}\left\{d_{1}+\left(\frac{2 p-q}{\not 2}\right) d_{2}\right\}
$$

onde

$$
\begin{aligned}
& d_{1}=-\frac{m_{3}}{2 m_{1}^{2}}\left\{m_{2}+m_{1}\left(2+m_{3}\right)\right\}-\frac{m_{4}}{2 m_{1}}, \\
& d_{2}=-\frac{m_{3}^{2}}{2 m_{1}},
\end{aligned}
$$

com

$$
\begin{aligned}
m_{1} & =\delta_{(0,1,0,0,2)}-1 \\
m_{2} & =4-\delta_{(0,0,1,0,3)}-6 \delta_{(0,1,0,0,2)}, \\
m_{3} & =\left(\delta_{(0,0,1,0,1)}+2 \delta_{(0,1,0,0,0)}\right) / \delta_{(2,0,0,0,0)} \\
v_{4} & =\left(\delta_{(0,0,0,1,2)}-6 \delta_{(1,1,0,0,1)}\right) / \delta_{(2,0,0,0,0)} .
\end{aligned}
$$

Observamos que o coeficiente $d_{\beta \phi}$ dado em (3.8), além de não depender dos parâmetros $\beta$ e $\phi$, também independe da estrutura da matriz modelo $X$. Finalmente, o coeficiente $d^{*}$ tem a expressão

$$
d^{\times}=\frac{1}{n}\left\{\frac{d_{0}}{q} h_{0}\left(X_{1}, X_{2}\right)+d_{1}+\left(\frac{2 p-q}{2}\right) d_{2}\right\}
$$

e a estatística da razào de verossimilhança modificada é clada por $R V^{\star *}=R V\left(1-d^{\star}\right)$. Enfatizamos que a fórmula (3.11) é uma combinação linear das quantidades $d_{0}, d_{1}$ e $d_{2}$, que dependem da distribuição assumida para os dados através dos $\delta$ 's. com coeficientes que dependem da matriz modelo $X$ (através de $h_{0}\left(X_{1}, X_{2}\right)$ ). do número de parâmetros de regressão $(p)$ e do número de parâmetros fixados em $H_{0}(q)$. 
Damos, agora. aplicações das fórmulas (3.9) e (3.10) a diversas distribuições simétricas (no Apêndice $\mathrm{C}$ estão os $\delta$ 's para cada distribuição). Cabe ressaltar que $d_{0}$ já foi dado anteriormente na Seção 3.2.1.

(i) Normal: $y_{l} \sim N\left(x_{l}^{\top} \beta, \phi^{2}\right)$;

$$
d_{1}=1 \quad \text { e } \quad d_{2}=1
$$

(ii) Cauchy: $y_{l} \sim C\left(x_{l}^{\top} \beta, \phi^{2}\right)$;

$$
d_{1}=\frac{1}{2} \quad \text { e } \quad d_{2}=1
$$

(iii) $t$-Student: $y_{l} \sim t\left(x_{l}^{\top} \beta, \phi^{2}, \nu\right), \nu>0$ conhecido;

$$
d_{1}=\frac{(\nu+2)(\nu+3)\left(\nu^{2}+9 \nu+2\right)}{\nu(\nu+5)^{2}(\nu+7)} \quad \mathrm{e} \quad d_{2}=\frac{(\nu+3)(\nu+2)^{2}}{\nu(\nu+5)^{2}}
$$

(iv) $t$-Student generalizada: $y_{l} \sim t G\left(x_{l}^{\top} \beta, \phi^{2}, s, r\right), s, r>0$ conhecidos;

$$
d_{1}=\frac{(r+2)(r+3)\left(r^{2}+9 r+2\right)}{r(r+5)^{2}(r+7)} \quad \text { e } \quad d_{2}=\frac{(r+3)(r+2)^{2}}{r(r+5)^{2}} .
$$

(v) Logística I: $y_{l} \sim \operatorname{LI}\left(x_{l}^{\top} \beta, \phi^{2}\right)$;

$$
d_{1} \approx 1,470555027 \text { e } \quad d_{2} \approx 1,362652631 .
$$

(vi) Logística II: $y_{l} \sim \operatorname{LII}\left(x_{l}^{\top} \beta, \phi^{2}\right)$;

$$
d_{1} \approx 0,985182 \text { e } \quad d_{2} \approx 0,786756 .
$$

(vii) Logística generalizada: $y_{l} \sim L G\left(x_{l}^{\top} \beta, \phi^{2}, m\right), m>0$ conhecido;

$$
\begin{aligned}
d_{1}= & \frac{(2 m+1)\left(4 m^{2}(m+1) \psi^{\prime}(m)+8 m^{2}-2 m-1\right)}{2(m+1)^{2}\left(2 m^{2} \psi^{\prime}(m)+2 m-1\right)^{2}} \\
& +\frac{(2 m+1)\left(m^{2} \psi^{\prime}(m)-6 m^{2}-3 m-1\right)}{m\left(2 m^{2} \psi^{\prime}(m)+2 m-1\right)}
\end{aligned}
$$

e

$$
d_{2}=\frac{(2 m+1)^{3}}{2\left(2 m^{2} \psi^{\prime}(m)+2 m-1\right)(m+1)^{2}} .
$$

Podemos observar que os coeficientes $d_{1}$ e $d_{2}$ envolvem funções poligama avaliadas no parâmetro $m$. Com a finalidade de simplificar o cálculo da correção de Bartlett neste caso, desenvolvemos aproximações simples para $d_{1}$ e $d_{2}$. Utilizando a expansão assintótica de $\psi^{\prime}(m)$ para valores grandes de $m$ (Abramowitz e Stegun, 1970, fórmula 6.4.12)

$$
\psi^{\prime}(m)=\frac{1}{m}+\frac{1}{2 m^{2}}+\frac{1}{6 m^{3}}-\frac{1}{30 m^{5}}+\frac{1}{42 m^{7}}+O\left(m^{-9}\right)
$$


obtemos

$$
\begin{aligned}
& d_{1}=1-\frac{1}{2 m}+\frac{1}{3 m^{2}}-\frac{1}{6 m^{3}}+O\left(m^{-4}\right), \\
& d_{2}=1-\frac{1}{2 m}+\frac{2}{3 m^{2}}+\frac{1}{6 m^{3}}+O\left(m^{-4}\right) .
\end{aligned}
$$

Para valores pequenos de $m$ podemos usar a expansào (Abramowitz e Stegun, 1970, fórmula 6.3.14)

$$
\begin{aligned}
\psi(m)= & -\frac{1}{m}-\gamma_{0}+\frac{\pi^{2}}{6} m-\zeta(3) m^{2}+\frac{\pi^{3}}{90} m^{3}-\zeta(5) m^{4} \\
& +\frac{\pi^{6}}{945} m^{5}-\zeta(7) m^{6}+\frac{\pi^{8}}{9450} m^{7}-\zeta(9) m^{8}+O\left(m^{9}\right),
\end{aligned}
$$

onde $\gamma_{0} \approx 0,57721566$ é a constante de Euler e $\zeta(\cdot)$ é a função zeta de Rie$\operatorname{mann}(\zeta(3) \approx 1,2020.56903 ; \zeta(5) \approx 1,036927755 ; \zeta(T) \approx 1,008349277$ e $\zeta(9) \approx$ $1,002008393)$. Então usando esta expansão obtemos

$$
\begin{aligned}
d_{1}= & \frac{1}{2}-\left(\frac{\pi^{2}}{18}+\frac{2}{3}\right) m-\left(\frac{2}{3} \zeta(3)+\frac{10}{27} \pi^{2}-\frac{11}{18}\right) m^{2} \\
& +\left(-\frac{1}{135} \pi^{4}+\frac{40}{9} \zeta(3)+\frac{65}{81} \pi^{2}+\frac{11}{27}\right) m^{3}+O\left(m^{4}\right), \\
d_{2}= & \frac{1}{2}+m-\left(\frac{1}{2}+\frac{\pi^{6}}{6}\right) m^{2}+2 \zeta(3) m^{3}+O\left(m^{4}\right) .
\end{aligned}
$$

(viii) Exponencial potência: $y_{l} \sim E P\left(x_{2}^{\top} \beta, \phi^{2}, k\right),-1<k<-1 / 2$, conhecido;

$$
\text { s }
$$

$$
d_{1}=d_{2}=\frac{1}{k+1} \text {. }
$$

Note-se que os resultados das distribuições $t$-Student só dependem do parâmetro $\nu$ (conhecido) e coincidem com os resultados de Ferrari e Arellano-Valle (1996). Quando $\nu \rightarrow$ $\infty, d_{1}$ e $d_{2}$ coincidem com os $d$ 's correspondentes da distribuição normal. Os resultados da distribuição $t$-Student generalizada só dependem de um dos parâmetros conhecidos $(r)$ e tem a mesma forma que a distribuição $t$-Student. Os resultados da distribuição exponencial potência dependem do parâmetro de curtose $k$ limitado em $-1<k<-1 / 2$. Já os resultados das distribuições normal, Cauchy, logísticas I e II são constantes fixas. A distribuição logística generalizada tem resultados que dependem do parâmetro $m$ e que convergem para aqueles da distribuição normal quando $m \rightarrow \infty$.

\subsubsection{Testes de hipóteses sobre o parâmetro $\phi$}

O objetivo desta seçào é encontrar o fator de correçào de Bartlett para a estatística da razão de verossimilhança do teste da hipótese nula composta $H_{0}: \dot{\phi}=\delta^{(0)}$ contra a 
alternativa composta $H_{1}: \emptyset \neq o^{(0)}$. onde $\beta$ é um vetor de parâmetros de perturbaçào e $\phi^{(0)}$ é um valor especificado para 0 .

A estatística da razào de verossimilhança $R V$ para testar $H_{0}$ definicla em (2.36) pode ser escrita como

$$
R V=2\left\{\{L(\widehat{3}, \widehat{o})-L(\beta, \phi)\}-\left\{L\left(\tilde{\beta}, \phi^{(0)}\right)-L(\beta, \phi)\right\}\right\} .
$$

Sob $H_{0}, R V$ tem distribuiçào $\lambda^{2}$ com um grau de liberdade. Mostramos, através dos resultados de Lawley (1956), que sob $H_{0}$,

$$
E(R V)=1+\varepsilon_{p+1}-\varepsilon_{p}+O\left(n^{-2}\right),
$$

onde $\varepsilon_{p}$ e $\varepsilon_{p+1}$ são definidas nas Seçòes 3.2 .1 e 3.2.2, respectivamente. Substituindo a decomposição de $\varepsilon_{p+1}$ dada em (3.7) temos, até ordem $n^{-1}$,

$$
E(R V)=1+\varepsilon_{\phi}+\varepsilon_{p+1, \beta \phi} .
$$

Mas, dos resultados do Apêndice B, temos $\varepsilon_{p+1, \beta \phi}=n^{-1}\left(d_{1} p+d_{2} p^{2} / 2\right)$. Agora, escrevendo $\varepsilon_{\phi}=d_{3} / n$, temos até ordem $n^{-1}$

$$
E(R V)=1+\frac{1}{n}\left(d_{1} p+\frac{d_{2}}{2} p^{2}+d_{3}\right),
$$

onde $d_{1}$ e $d_{2}$ são dados em (3.9) e (3.10) e

$$
\begin{aligned}
d_{3}= & \frac{1}{4\left(\delta_{(0.1,0,0,2)}-1\right)^{3}}\left\{( \delta _ { ( 0 , 1 , 0 , 0 , 2 ) } - 1 ) \left(\delta_{(0,0,0,1,4)}\right.\right. \\
& \left.+16 \delta_{(0,0,1,0,3)}+26\left(2 \delta_{(0,1,0,0,2)}-1\right)\right) \\
& \left.-\frac{5}{3}\left(6 \delta_{(0,1,0,0,2)}+\delta_{(0,0,1,0,3)}-4\right)^{2}\right\} .
\end{aligned}
$$

A obtenção de $d_{3}$ é feita no Apêndice B. Conseqüentemente, a estatística da razão de verossimilhança corrigida tem a forma

$$
R V^{*}=R V(1-d),
$$

onde

$$
d=\frac{1}{n}\left(d_{1} p+\frac{d_{2} p^{2}}{2}+d_{3}\right)
$$

A estatística corrigida $R V^{\star}$ tem distribuição $\chi_{1}^{2}$ até ordem $n^{-1}$. Notamos que a correção de Bartlett não depende da matriz modelo $X$.

Fazemos a seguir aplicaçào da fórmula (3.14) a diversas distribuições simétricas. Cabe ressaltar que $d_{1}$ e $d_{2}$ já foram dados anteriormente na Seção 3.2.2.

(i) Normal: $y_{l} \sim N\left(x_{l}^{\top} \beta, o^{2}\right)$ :

$$
d_{3}=1 / 3 .
$$


(ii) Cauchy: $y_{l} \sim C\left(x_{l}^{\top} \beta .0^{2}\right)$;

$$
d_{3}=1 / 4
$$

(iii) $t$-Student: $y_{l} \sim t\left(x_{l}^{\top} \beta, \sigma^{2}, \nu\right), \nu>0$ conhecido:

$$
d_{3}=\frac{(\nu+3)\left(\nu+14 \nu^{2}+14 \nu+25\right)}{3 \nu(\nu+5)^{2}(\nu+7)} .
$$

(iv) $t$-Student generalizada: $y_{l} \sim t G\left(x_{l}^{\top} \beta, \phi^{2}, s, r\right) . s, r>0$ conhecidos;

$$
d_{3}=\frac{(r+3)\left(r^{3}+14 r^{2}+14 r+25\right)}{3 r(r+5)^{2}(r+7)} .
$$

(v) Logística I: $y_{l} \sim \operatorname{LI}\left(x_{l}^{\top} \beta, \phi^{2}\right)$;

$$
d_{3} \approx 0,481968387 .
$$

(vi) Logística II: $y_{l} \sim \operatorname{LII}\left(x_{l}^{\top} \beta, \phi^{2}\right)$;

$$
d_{3} \approx 0,22795 .
$$

(vii) Logística generalizada: $y_{l} \sim L G\left(x_{l}^{\top} \beta, \phi^{2}, m\right), m>0$ conhecido;

$$
\begin{aligned}
d_{3}= & (2 m+1)\left(6 m^{3}(m+1) \psi^{\prime}(m)^{2}+m^{3}(m+1) \psi^{\prime \prime \prime}(m)-4 m\left(26 m^{3}+41 m^{2}\right.\right. \\
& \left.+15 m+3) \psi^{\prime}(m)-52 m^{2}(m+1)+21 m+9\right) /\left(2 ( 2 m + 3 ) ( m + 1 ) \left(2 m^{2} \psi^{\prime}(m)\right.\right. \\
& \left.+2 m-1)^{2}\right)+5(2 m+1)^{3}\left(3 m^{2} \psi^{\prime}(m)+2 m-1\right)^{2} \\
& /\left(3(m+1)^{2}\left(2 m^{2} \psi^{\prime}(m)+2 m-1\right)^{3}\right) .
\end{aligned}
$$

Utilizando a expressão (3.12), para $m$ grande obtemos

$$
d_{3}=\frac{1}{3}-\frac{7}{12 m^{2}}+\frac{13}{8 m^{3}}+O\left(m^{-4}\right)
$$

e, para $m$ pequeno, de (3.13) obtemos

$$
d_{3}=\frac{1}{6}-\frac{\pi^{2}}{3} m^{2}+\left(4 \zeta(3)+\frac{5 \pi^{2}}{3}+\frac{7}{180} \pi^{4}\right) m^{3}+O\left(m^{4}\right) .
$$

(viii) Exponencial potência: $y_{l} \sim E P\left(x_{l}^{\top} \beta, \phi^{2}, k\right),-1<k<-1 / 2$, conhecido;

$$
d_{3}=\frac{1}{3(k+1)} \text {. }
$$

As fórmulas simples de $d_{0}, d_{1}, d_{2}$ e $d_{3}$ dadas em (3.4), (3.9), (3.10) e (3.14) podem ser calculadas facilmente dada a distribuição assumida para os dados. A vantagem principal das expressões dos d’s está em conseguir obter fatores de correção de Bartlett em forma fechada e simples para diferentes testes de hipóteses de interesse prático. Cabe destacar que embora os $d$ 's sejam calculados facilmente para cada distribuição, aparentemente eles não têm interpretação alguma. Também observamos que eles nào dependem dos parâmetros $\beta$ e $\phi$ nem da matriz modelo $X$, sendo funções apenas dos $\delta$ 's obtidos a partir da distribuiçào assumida para os dados. 


\subsection{Fator de correção tipo Bartlett para a estatística escore}

Os testes escore aperfeiçoados pela correção tipo Bartlett desenvolvida por Cordeiro e Ferrari (1991) e apresentados no Capítulo 1 dependem das quantidades $A_{1}, A_{2}$ e $A_{3}$ expressas em (1.13) - (1.15) que são funções aparentemente complicadas dos cumulantes conjuntos $\kappa$ 's de derivadas do logaritmo da função de verossimilhança. O objetivo desta seção é apresentar $A_{1}, A_{2}$ e $A_{3}$ em forma simples e de fácil cálculo para os testes discutidos no capítulo anterior. Alguns dos cumulantes referentes aos modelos simétricos foram apresentados nas seções anteriores na obtenção do fator de correção de Bartlett para a estatística da razão de verossimilhança. Como mencionamos na seção anterior, propriedades fundamentais dos modelos simétricos, tais como sua estrutura linear que induz a todos os cumulantes envolvidos nos $A$ 's serem invariantes sob permutação de parâmetros, a simetria da distribuição e ortogonalidade dos parâmetros $\beta$ e $\phi$ que induzem a alguns cumulantes serem nulos, levam a uma simplificação considerável na obtençào dos $A$ 's e, conseqüentemente, na estatística escore aperfeiçoada.

\subsubsection{Testes de hipóteses sobre o parâmetro $\beta \operatorname{com} \phi$ conhecido}

Sejaim $Z$ e $Z_{2}$ as matrizes definidas em (3.1). Expressões simples para os $A$ 's podem ser obtidas substituindo os $\kappa$ 's obtidos na Seção 3.2 .1 nas expressões (1.13) - (1.15) e efetuando as somas sobre a amostra depois de avaliar as somas sobre os parâmetros. Fazendo isto, aparecem termos das formas $\sum^{\prime} a_{i j} x_{l i} x_{l j}$ e $\sum^{\prime} m_{i j} x_{l i} x_{l j}$, onde $a_{i j}$ e $m_{i j}$ são os elementos $(i, j)$ das matrizes $A$ e $M$ definidas em (1.16), e estes representam os elementos das matrizes $\left(\phi^{2} / \delta_{(2,0,0,0,0)}\right) Z_{2}$ e $\left(\phi^{2} / \delta_{(2,0,0,0,0)}\right)\left(Z-Z_{2}\right)$, respectivamente.

Devido à simetria dos modelos considerados, os $A$ 's reduzem-se a

$$
A_{1}=12 \sum^{\prime} \kappa_{i, j, k r} a_{k r} m_{i j}, \quad A_{2}=-9 \sum^{\prime} \kappa_{i, j, k r} m_{i j} m_{k r} \quad \text { e } \quad A_{3}=0 .
$$

Substituindo $\kappa_{i, j, k r}$ nas expressões de $A_{1}$ e $A_{2}$ dadas em (3.15), e após alguma álgebra (Apêndice A), as expressões resultantes são:

$$
\begin{aligned}
& A_{1}=12 \frac{b_{0}}{n}\left(\rho_{Z Z_{2}}-\rho_{Z_{2} Z_{2}}\right), \\
& A_{2}=-9 \frac{b_{0}}{n}\left(\rho_{Z Z}-2 \rho_{Z Z_{2}}+\rho_{Z_{2} Z_{2}}\right),
\end{aligned}
$$

onde

$$
b_{0}=\frac{\delta_{(2,1,0,0,0)}}{\delta_{(2,0,0,0,0)}^{2}}+1,
$$

$\rho_{Z Z}=n \operatorname{tr}\left(Z_{d} Z_{d}\right), \rho_{Z Z_{2}}=n \operatorname{tr}\left(Z_{d} Z_{2 d}\right)$ e $\rho_{Z_{2} Z_{2}}=n \operatorname{tr}\left(Z_{2 d}, Z_{2 d}\right)$.

Substituindo $A_{1}$ e $A_{2}$ dados em (3.16) e $A_{3}=0$ em $a, b$ e $c$. definidos em (1.19), obtemos a estatística escore aperfeiçoada do teste de $H_{0}: \beta_{1}=\beta_{1}^{(0)}$ contra $H_{1}: \beta_{1} \neq \beta_{1}^{(0)}$ 
(vide Seção 2.6.1). Esta estatística é dada por (1.18) com $a, b$ e $c$ dados por

$$
\begin{aligned}
& a=0 . \\
& b=-\frac{3 b_{0}}{4 n q(q+2)} h_{1}\left(X_{1}, X_{2}\right), \\
& c=\frac{b_{0}}{4 n q} h_{2}\left(X_{1}, X_{2}\right),
\end{aligned}
$$

onde

$$
h_{1}\left(X_{1}, X_{2}\right)=\rho_{Z Z}-2 \rho_{Z Z_{2}}+\rho_{Z_{2} Z_{2}}, \quad h_{2}\left(X_{1}, X_{2}\right)=3 \rho_{Z Z}-2 \rho_{Z Z_{2}}-\rho_{Z_{2} Z_{2}},
$$

Observamos que as fórmulas acima sào fáceis de serem utilizadas e envolvem operações muito simples com matrizes diagonais. Notamos também que as expressões de $b$ e $c(a=0)$ não dependem de parâmetros desconhecidos e são decompostas da mesma forma que a correção de Bartlett para a estatística da razão de verossimilhança em duas partes, uma dependendo da distribuição assumida para os dados através dos $\delta$ 's e outra dependendo da matriz modelo $X$ através de $Z$ e $Z_{2}$.

Podemos observar ainda que, como $a=0$, o fator de correção da estatística escore definida em (2.28), se reduz a $\left\{1-c-b S_{R}\right\}$. Note-se que os coeficientes $b$ e $c$ não dependem de $\beta$ e $\phi$ como mencionamos anteriormente, e em muitos casos dependem dos parâmetros conhecidos de cada distribuição. Por exemplo, na distribuição $t$-Student dependem de $\nu$ através de funçòes que são definidas para todo $\nu>0$, na distribuição $t$-Student generalizada dependem do parâmetro conhecido $r>0$ (que está relacionada com a curtose), e na distribuição exponencial potência dependem de $k$, que também está relacionado com a curtose.

Os valores de $b_{0}$ definidos em (3.17) sào apresentados para os seguintes casos particulares (no Apêndice C estão os $\delta$ 's para cada distribuição):

(i) Normal: $y_{l} \sim N\left(x_{l}^{\top} \beta, \phi^{2}\right)$;

$$
b_{0}=0 \cdot \quad V
$$

(ii) Cauchy: $y_{l} \sim C\left(x_{l}^{\top} \beta, \phi^{2}\right)$;

$$
b_{0}=1 / 2 .
$$

(iii) $t$-Student: $y_{l} \sim t\left(x_{l}^{\top} \beta, \phi^{2}, \nu\right), \nu>0$ conhecido;

$$
b_{0}=\frac{6\left((\nu+2)^{2}-5\right)}{\nu(\nu+5)(\nu+7)} .
$$

(iv) $t$-Student generalizada: $y_{l} \sim t G\left(x_{l}^{\top} \beta, \phi^{2}, s, r\right), s, r>0$ conhecidos;

$$
b_{0}=\frac{6\left((r+2)^{2}-5\right)}{r(r+5)(r+7)} .
$$


(v) Logística I: $y_{l} \sim \operatorname{LI}\left(x_{l}^{\top} \beta, \delta^{2}\right)$;

$$
b_{0} \approx-0,903461273 .
$$

(vi) Logística II: $y_{l} \sim \operatorname{LII}\left(x_{l}^{\top} \beta, \phi^{2}\right)$;

$$
b_{0}=2 / 5 .
$$

(vii) Logística generalizada: $y_{l} \sim L G^{\prime}\left(x_{l}^{\top} \beta, \phi^{2}, m\right), m>0$ conhecido;

$$
b_{0}=\frac{2}{2 m+3} \text {. }
$$

(viii) Exponencial potência: $y_{l} \sim E P\left(x_{1}^{\top} \beta, \phi^{2}, k\right),-1<k<-1 / 2$ conhecido;

$$
b_{0}=1-\frac{(1-k)}{2} \frac{\Gamma\left(\frac{k+1}{2}\right) \Gamma\left(\frac{3}{2}(1-k)\right)}{\Gamma\left(\frac{3-k}{2}\right)^{2}} . \quad \frac{3-3 k}{2}
$$

A seguir examinaremos alguns casos especiais definidos na Seção 3.2.1 que levam a simplificações nas fórmulas (3.18) - (3.19).

\section{(1) Caso uniparamétrico}

(i) Se $\mu_{l}=\beta, l=1, \ldots, n$, onde $\beta$ é unidimensional, temos $\rho_{Z Z}=1$ e $\rho_{Z Z_{2}}=\rho_{Z_{2} Z_{2}}=0$ e, conseqüentemente, $A_{1}=A_{3}=0$ e $A_{2}=-9 b_{0} / n$ e, portanto, $b=-b_{0} /(4 n) \mathrm{e}$ $c=3 b_{0} /(4 n)$.

(ii) Se $\mu_{l}=x_{l} \beta, l=1, \ldots, n$. temos $\rho_{Z Z_{2}}=\rho_{Z_{2} Z_{2}}=0$ e $\rho_{Z Z}=\bar{s}_{4} /\left(\bar{s}_{2}^{2}\right)$, onde $\bar{s}_{a}=$ $\sum x_{i}^{a} / n$, conseqüentemente, $A_{1}=A_{3}=0$ e $A_{2}=\left(-9 b_{0} / n\right)\left(\bar{s}_{4} / \bar{s}_{2}^{2}\right)$. Logo, temos de (3.18) - (3.19) que

$$
b=\left(-b_{0} / 4 n\right)\left(\bar{s}_{4} / \bar{s}_{2}^{2}\right) \text { e } c=\left(3 b_{0} / 4 n\right)\left(\bar{s}_{4} / \bar{s}_{2}^{2}\right) .
$$

(2) Hipótese nula simples

Considerando a hipótese de interesse $H_{0}: \beta=\beta^{(0)}$ contra $H_{1}: \beta \neq \beta^{(0)}$, temos que $\rho_{Z Z_{2}}=\rho_{Z_{2} Z_{2}}=0$ e, conseqüentemente, $A_{1}=A_{3}=0$ e $A_{2}=\left(-9 b_{0} / n\right) \rho_{Z Z}$. Logo temos

$$
b=-\frac{3 b_{0}}{4 n p(p+2)} \rho_{Z Z}, \quad c=\frac{3 b_{0}}{4 n p} \rho_{Z Z} .
$$


(3) Modelo de classificação a um fator

Considerando $p \geq 2$ populações e assumindo a estrutura linear $\mu_{i j}=\beta+\beta_{i}, i=$ $1, \ldots, p, j=1 \ldots, n_{i}, \operatorname{com} \beta_{p}=0$, os coeficientes $b$ e $c$ para corrigir a estatística escore do teste de homogeneidade dos parâmetros de locação das $p$ populações são

$$
\begin{aligned}
b & =-\frac{3 b_{0}}{4 n(p-1)(p+1)}\left\{n \sum_{i=1}^{p}\left(1 / n_{i}\right)-(2 p-1)\right\} \\
\mathrm{e} \quad c & =\frac{b_{0}}{4 n(p-1)}\left\{3 n \sum_{i=1}^{p}\left(1 / n_{i}\right)-(2 p+1)\right\} .
\end{aligned}
$$

Aqui, $\rho_{Z Z}=n \sum_{i=1}^{p}\left(1 / n_{i}\right), \rho_{Z Z_{2}}=p$ e $\rho_{Z_{2} Z_{2}}=1$.

\section{(4) Modelo de regressão linear simples}

Consideramos agora a seguinte estrutura linear para os parâmetros de locação: $\mu_{l}=$ $\alpha+\beta x_{l}, l=1, \ldots, n$. As fórmulas dos coeficientes $b$ e $c$ para corrigir a estatística escore do teste da hipótese $H_{0}: \beta=\beta^{(0)}$ contra $H_{1}: \beta \neq \beta^{(0)}$, dependem dos termos $\rho_{Z Z}=$ $\left(\bar{S}_{4} / \bar{S}_{2}^{2}\right)+3, \rho_{Z Z_{2}}=2$ e $\rho_{Z_{2} Z_{2}}=1$. Conseqüentemente, $A_{1}=12 b_{0} / n \cdot A_{2}=-9 b_{0}\left(\bar{S}_{4} / \bar{S}_{2}^{2}\right) / n$ e $A_{3}=0$. Substituindo estas expressões em (3.18) - (3.19), temos

$$
b=-\frac{b_{0}}{4 n} \frac{\bar{S}_{4}}{\bar{S}_{1}^{2}} \quad \text { e } \quad c=\frac{b_{0}}{4 n}\left(\frac{\bar{S}_{4}}{\bar{S}_{2}^{2}}+4\right),
$$

onde $\bar{S}_{a}=\left(\sum_{l}\left(x_{l}-\bar{x}\right)^{a}\right) / n$.

\section{(5) Modelo de regressão com dois parâmetros}

Se o parâmetro de locação $\mu_{l}$ tem a forma $\mu_{l}=\alpha u_{l}+\beta x_{l}, l=1, \ldots, n$, e estamos interessados no teste da hipótese $H_{0}: \beta=\beta^{(0)}$ contra $H_{1}: \beta \neq \beta^{(0)}$, os coeficientes do fator de correção da estatística escore definidos em (3.18) - (3.19) dependem de $\rho_{Z_{2}} Z_{2}, \rho_{Z Z}$ e $\rho_{Z Z_{2}}$ que são dados por

$$
\begin{aligned}
\rho_{Z_{2} Z_{2}} & =\frac{n \sum u_{i}^{4}}{\left(\sum u_{i}^{2}\right)^{2}}=\frac{n s_{40}}{s_{20}^{2}}, \\
\rho_{Z Z} & =n \Delta^{-2} \sum_{l}\left(u_{l}^{2} \sum_{i} x_{i}^{2}+x_{l}^{2} \sum_{i} u_{i}^{2}-2 u_{l} x_{l} \sum_{i} x_{i} u_{i}\right)^{2} \\
& =n \Delta^{-2}\left(s_{40} s_{02}^{2}+s_{04} s_{20}^{2}+2 s_{22} s_{20} s_{02}+4 s_{22} s_{11}^{2}-4 s_{31} s_{11} s_{02}-4 s_{13} s_{11} s_{20}\right), \\
\rho_{Z Z_{2}} & =n \Delta^{-1}\left(s_{20}\right)^{-1} \sum_{l}\left(u_{l}^{4} \sum_{i} x_{i}^{2}+u_{l}^{2} x_{l}^{2} \sum_{i} u_{i}^{2}-2 u_{l}^{3} x_{l} \sum_{i} x_{i} u_{i}\right) \\
& =n \Delta^{-1}\left(s_{20}\right)^{-1}\left(s_{40} s_{02}+s_{22} s_{20}-2 s_{31} s_{11}\right) .
\end{aligned}
$$

Os coeficientes $b$ e $c$ vêm de (3.18) - (3.19), com $\rho_{Z Z}, \rho_{Z_{2} Z_{2}}$ e $\rho_{Z Z_{2}}$ dados acima.

A expansão assintótica da função distribuição da estatística escore $S_{R}$ sob a hipótese nula dada em (1.12) para a família de distribuiçōes simétricas tem a forma

$$
F_{S_{R}}(x)=G_{q}(x)-\frac{b_{0}}{8}\left\{3 h_{1}\left(X_{1}, X_{2}\right) G_{q+4}(x)-2 h_{3}\left(X_{1}, X_{2}\right) G_{q+2}(x)+h_{2}\left(X_{1}, X_{2}\right) G_{q}(x)\right\},
$$


onde $h_{1}\left(X_{1}, X_{2}\right)$ e $h_{2}\left(X_{1}, X_{2}\right)$ são definidas em (3.20) e

$$
h_{3}\left(X_{1}, X_{2}\right)=3 \rho_{Z Z}-4 \rho_{Z Z_{2}}+\rho_{Z_{2} Z_{2}} \text {. }
$$

Usando a relaçào de recorrência $g_{m+2}(x)=x g_{m}(x) / m$, encontra-se, até ordem $n^{-1}$,

$$
f_{S_{R}}(x)=g_{q}(x)\left(1-\frac{b_{0}}{8 n} \frac{h_{2}\left(X_{1} . X_{2}\right)-2 h_{3}\left(X_{1}, X_{2}\right) x+3 h_{1}\left(X_{1}, X_{2}\right) x^{2}}{q(q+2)}\right) .
$$

Assim, para uma dada matriz modelo $X$ e uma dada hipótese nula, a função

$$
g_{q}(x)=\frac{h_{2}\left(X_{1}, X_{2}\right)-2 h_{3}\left(X_{1}, X_{2}\right) x+3 h_{1}\left(X_{1}, X_{2}\right) x^{2}}{q(q+2)}
$$

pode ser obtida e a influência da distribuição na qualidade da aproximação da estatística $S_{R}$ por qui-quadrado pode ser avaliada através de $b_{0}$. De forma semelhante, dada a distribuição dos dados a influência da matriz modelo e da hipótese nula na qualidade desta aproximação deve ser feita através do estudo da função $g$ definida acima, mas vista como função de $x, X_{1}, X_{2}$ e $q$. Uma forma mais rudimentar, porém mais simples, de avaliar a qualidade desta aproximação pode ser obtida dos três primeiros momentos de $S_{R}$, sob $H_{0}$, até ordem $n^{-1}$, dados por

$$
\begin{aligned}
& \mu_{1}^{\prime}\left(S_{R}\right)=q+\frac{b_{0}}{n}\left(\rho_{Z Z_{2}}-\rho_{Z_{2} Z_{2}}\right) \\
& \mu_{2}^{\prime}\left(S_{R}\right)=q(q+2)+\frac{b_{0}}{n}\left\{-3 \rho_{Z Z}+10 \rho_{Z Z_{2}}-7 \rho_{Z_{2} Z_{2}}+2 q\left(\rho_{Z Z_{2}}-\rho_{Z_{2} Z_{2}}\right)\right\} \\
& \mu_{3}^{\prime}\left(S_{R}\right)=q(q+2)(q+4)+\frac{12 b_{0}}{n}(q+4)\left\{2 q\left(\rho_{Z Z_{2}}-\rho_{Z_{2} Z_{2}}\right)+\left(\delta \rho_{Z Z_{2}}-3 \rho_{Z Z}-5 \rho_{Z_{2} Z_{2}}\right)\right\}
\end{aligned}
$$

(vide equação (1.17)).

\subsubsection{Testes de hipóteses sobre o parâmetro $\beta \operatorname{com} \phi$ desconhe- cido}

Consideremos a hipótese nula $H_{0}: \beta_{1}=\beta_{1}^{(0)}$ e a alternativa $H_{1}: \beta_{1} \neq \beta_{1}^{(0)}$, com $\beta_{2}$ e $\phi$ representando os parâmetros de perturbação e $\beta_{1}^{(0)}$ sendo um vetor de valores especificados de dimensão $q$. Nesta seção obtemos os coeficientes $A^{\prime}$ s usados na correçào da estatística escore definida em (2.34), assumindo $\phi$ desconhecido.

Seja $\theta=\left(\beta_{1}^{\top}, \beta_{2}^{\top}, \phi\right)^{\top}$ o vetor de $(p+1)$ parâmetros. É importante lembrar que devido ao fato de que os parâmetros $\beta$ e $\phi$ são globalmente ortogonais, a matriz de informação total de Fisher $K$ e sua inversa $K^{-1}$ sào bloco diagonais, isto é, sào da forma

$$
K=\left(\begin{array}{ccc}
K_{11} & K_{12} & 0 \\
K_{21} & K_{22} & 0 \\
0 & 0 & \kappa_{\phi, \phi}
\end{array}\right) \text { e } K^{-1}=\left(\begin{array}{ccc}
K^{-11} & K^{-12} & 0 \\
K^{21} & K^{-22} & 0 \\
0 & 0 & \kappa_{\phi, \phi}^{-1}
\end{array}\right) \text {. }
$$


onde os elementos da matriz $K^{\prime}$ e $K^{-1}$ são definidos em (2.25), (2.26) e (2.16). Conseqüentemente, definimos

e podemos escrever

$$
K_{\beta, \beta}^{-1}=\left(\begin{array}{cc}
K^{11} & K^{-12} \\
K^{21} & K^{22}
\end{array}\right), \quad A_{\beta}=\left(\begin{array}{cc}
0 & 0 \\
0 & K_{22}^{-1}
\end{array}\right)
$$

$$
A=\left(\begin{array}{cc}
A_{\beta} & 0 \\
0 & 0
\end{array}\right) \quad \text { e } M=\left(\begin{array}{cc}
M_{\beta} & 0 \\
0 & 0
\end{array}\right),
$$

onde $M_{\beta}=K_{\beta, 3}^{-1}-A_{\beta}$. Sejam $m_{r \phi}$ e $a_{r \phi}$ os $(r, p+1)$-ésimos elementos de $M$ e $A$, respectivamente. e $m_{\phi \phi}$ e $a_{\phi \phi}$ os $(p+1, p+1)$ elementos da matriz $M$ e 1 , respectivamente. Tem-se $m_{r \phi}=m_{o r}=m_{\phi \phi}=0$ e $a_{r \phi}=a_{\phi r}=0(r=1, \ldots, p)$ e $a_{\phi \phi}=r_{\phi, \phi}^{-1}$.

Para o parâmetro de dispersão desconhecido, Cribari-Neto é Ferrari (1995b) mostram que, em problemas regulares e, se $\beta$ e $\phi$ são globalmente ortogonais, os coeficientes $A$ 's podem ser escritos como $A_{1}=A_{1, \beta}+A_{1, \beta \phi}, A_{2}=A_{2, \beta}+A_{2, \beta \phi}$ e $A_{3}=A_{3, \beta}+A_{3, \beta \phi}$, onde $A_{1, \beta}, A_{2, \beta}$ e $A_{3, \beta}$ são as quantidades $A_{1}, A_{2}$ e $A_{3}$ obtidas supondo que $\phi$ é conhecido e $A_{1, \beta \phi}, A_{2, \beta \phi}$ e $A_{3.3 \phi}$ representam as contribuições adicionais devido ao fato de que $\phi$ é desconhecido.

Cribari-Neto e Ferrari (1995b) mostram que $A_{1, \beta \phi}$ e $A_{2, \beta \phi}$ são dados por

$$
\begin{aligned}
A_{1, \beta \phi}= & 3 \sum^{\prime}\left(\kappa_{\phi \phi k}+2 \kappa_{\phi, \phi k}\right)\left(\kappa_{r s t}+2 \kappa_{r s, t}\right) a_{\phi \phi} a_{s t} m_{k r} \\
& +3 \sum^{\prime}\left(\kappa_{i j k}+2 \kappa_{i, j k}\right)\left(\kappa_{r \phi \phi}+2 \kappa_{r \phi, \phi}\right) a_{i j} a_{\phi \phi} m_{k r} \\
& +3 \sum^{\prime}\left(\kappa_{\phi \phi k}+2 \kappa_{\phi, \phi k}\right)\left(\kappa_{r \phi \phi}+2 \kappa_{r \phi, \phi}\right) a_{\phi \phi}^{2} m_{k r} \\
& -6 \sum^{\prime}\left(\kappa_{\phi \phi k}+2 \kappa_{\phi, \phi k}\right) \kappa_{r, s, t} a_{\phi \phi} a_{k r} m_{s t} \\
\triangle & -6 \sum^{\prime}\left(\kappa_{i j \phi}+2 \kappa_{i, j \phi}\right) \kappa_{\phi, s, t} a_{i j} a_{\phi \phi} m_{s t} \\
\searrow & -6 \sum^{\prime}\left(\kappa_{\phi \phi \phi}+2 \kappa_{\phi, \phi \phi}\right) \kappa_{\phi, s, t} a_{\phi \phi}^{2} m_{s t} \\
\searrow & +6 \sum^{\prime}\left(\kappa_{i, \phi k}-\kappa_{i, \phi, k}\right)\left(\kappa_{r \phi t}+2 \kappa_{r \phi, t}\right) a_{\phi \phi} a_{k t} m_{i r} \\
\searrow & +6 \sum^{\prime}\left(\kappa_{i, j \phi}-\kappa_{i, j, \phi}\right)\left(\kappa_{r s \phi}+2 \kappa_{r s, \phi}\right) a_{j s} a_{\phi \phi} m_{i r} \\
& +6 \sum^{\prime}\left(\kappa_{i, \phi \phi}-2 \kappa_{i, \phi, \phi}\right)\left(\kappa_{r \phi \phi}+2 \kappa_{r \phi, \phi}\right) a_{\phi \phi}^{2} m_{i r} \\
\searrow & -6 \sum^{\prime}\left(\kappa_{i, j, \phi, \phi}+\kappa_{i, j, \phi \phi}\right) a_{\phi \phi} m_{i j}, \\
A_{2, \beta \phi} & -3 \sum^{\prime} \kappa_{i, j, \phi} \kappa_{\phi, s, t} a_{\phi \phi} m_{i j} m_{s t} \\
& +6 \sum^{\prime}\left(\kappa_{\phi \phi k}+2 \kappa_{\phi, \phi k}\right) \kappa_{r, s, t} a_{\phi \phi} m_{k r} m_{s t} \\
& -6 \sum^{\prime} \kappa_{i, j, \phi} \kappa_{r, s, \phi} a_{\phi \phi} m_{i r} m_{j s}, \\
A_{3, \beta \phi}= & 0 .
\end{aligned}
$$

A simetria do modelo (2.1) e a estrutura linear do vetor $\mu$ sào considerados para a redução das fórmulas $(3.21)$ e $(3.22) \mathrm{em}$

$$
A_{1, \beta \phi}=-6 \sum^{\prime} \kappa_{i, j \phi} \kappa_{\phi, s, t} a_{i j} a_{\phi \phi} m_{s t}
$$




$$
\begin{aligned}
& -6 \sum^{\prime}\left(\kappa_{\phi \phi \phi}+2 \kappa_{\phi, \phi \phi}\right) \kappa_{\phi, s, t} a_{\phi \phi}^{2} m_{s t} \\
& +6 \sum^{\prime}\left(\kappa_{i, \phi k}-\kappa_{i, \phi, k}\right) \kappa_{r \phi, t} a_{\phi \phi} a_{k, t} m_{i r} \\
& +6 \sum^{\prime}\left(\kappa_{i, j \phi}-\kappa_{i, j, \phi}\right)\left(\kappa_{r s}^{(\phi)}+\kappa_{r s, \phi}\right) a_{j s} a_{\phi \phi} m_{i r} \\
& -6 \sum^{\prime}\left(\kappa_{i, j, \phi, \phi}+\kappa_{i, j, \phi \phi}\right) a_{\phi \phi} m_{i j}, \\
A_{2, \beta \phi}= & -3 \sum^{\prime} \kappa_{i, j, \phi} \kappa_{\phi, s, t} a_{\phi \phi} m_{i j} m_{s t} \\
& -6 \sum^{\prime} \kappa_{i, j, \phi} \kappa_{r, s, \phi} a_{\phi \phi} m_{i r} m_{j s} .
\end{aligned}
$$

Usando os cumulantes dados na Seção $3.2 .2, A_{1, \beta \phi}$ e $A_{2, \beta \phi}$ são dados por (Apêndice $\mathrm{B}$ )

$$
A_{1, \beta \phi}=\frac{12 b_{1}}{n} q(p-q)-\frac{6 b_{2}}{n} q, \quad A_{2, \beta \phi}=-\frac{12 b_{3}}{n} q(q+2),
$$

onde $b_{1}, b_{2}$ e $b_{3}$ são definidos como

$$
\begin{aligned}
b_{1}= & \frac{\delta_{(1,1,0,0,1)}\left(\delta_{(1,1,0,0,1)}-\delta_{(0,1,0,0,0)}\right)}{\delta_{(2,0,0,0,0)}^{2}\left(\delta_{(2,0,0,0,2)}-1\right)} \\
b_{2}= & \left\{2 \delta_{(1,1,0,0,1)}\left(2 \delta_{(0,1,0,0,2)}+\delta_{(0,0,1,0,3)}\right)\right. \\
& \left.+\left(\delta_{(2,0,0,0,2)}-1\right)\left(4 \delta_{(3,0,0,0,1)}+\delta_{(4,0,0,0,2)}+\delta_{(2,1,0,0,2)}-2 \delta_{(0,1,0,0,0)}\right)\right\} \\
& \left\{\delta_{(2,0,0,0,0)}\left(\delta_{(2,0,0,0,2)}-1\right)^{2}\right\}^{-1} \\
b_{3}= & \frac{\delta_{(1,1,0,0,1)}^{2}}{\delta_{(2,0,0,0,0)}^{2}\left(\delta_{(2,0,0,0,2)}-1\right)} .
\end{aligned}
$$

É importante observar que $A_{1, \beta \phi}$ e $A_{2, \beta \phi}$, dados acima, são funções que dependem do número total de parâmetros $(p)$, do número de restrições $(q)$, dos $\delta$ 's, que por sua vez dependem da distribuição assumida para os dados, e do tamanho da amostra. Em particular, estes termos não dependem da matriz modelo $X$ (exceto pelo seu posto).

Assim, os coeficientes $A_{1}, A_{2}$ e $A_{3}$ usados na correção do teste escore nos modelos simétricos quando o parâmetro de escala $\phi$ é desconhecido, são

$$
\begin{aligned}
& A_{1}=\frac{12 b_{0}}{n}\left(\rho_{Z Z_{2}}-\rho_{Z_{2} Z_{2}}\right)+\frac{12 b_{1}}{n} q(p-q)-\frac{6 b_{2}}{n} q, \\
& A_{2}=-\frac{9 b_{0}}{n}\left(\rho_{Z Z}-2 \rho_{Z Z_{2}}+\rho_{Z_{2} Z_{2}}\right)-\frac{12 b_{3}}{n} q(q+2)
\end{aligned}
$$

e

$$
A_{3}=0 \text {. }
$$

Desta forma, temos

$$
a=0 \text {, }
$$




$$
\begin{aligned}
b & =-\frac{3 b_{0}}{4 n q(q+2)} h_{1}\left(X_{1} \cdot X_{2}\right)-\frac{b_{3}}{n}, \\
c & =\frac{b_{0}}{4 n q} h_{2}\left(X_{1}, X_{2}\right)+\frac{b_{1}}{n}(p-q)-\frac{b_{2}}{2 n}+\frac{b_{3}}{n}(q+2),
\end{aligned}
$$

onde as funções $h_{1}$ e $h_{2}$ são definidas em (3.20).

Damos agora aplicações de (3.23) - (3.25) a diversas distribuições simétricas (no Apêndice C estão os $\delta$ 's para cada distribuição).

(i) Normal: $y_{l} \sim N\left(x_{l}^{\top} \beta, \phi^{2}\right)$;

$$
b_{1}=1 . \quad b_{2}>0 \quad \text { e } \quad b_{3}=\frac{1}{2} .
$$

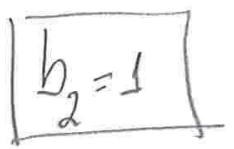

(ii) Cauchy: $y_{l} \sim C\left(x_{l}^{\top} \beta, \phi^{2}\right)$;

$$
b_{1}=0, \quad b_{2}=-1 \quad \text { e } \quad b_{3}=0 .
$$

(iii) $t$-Student: $y_{l} \sim t\left(x_{l}^{\top} \beta, \phi^{2}, \nu\right), \nu>0$ conhecido;

$$
\begin{aligned}
b_{1} & =\frac{(\nu-1)(\nu+2)(\nu+3)}{\nu(\nu+5)^{2}}, \quad b_{2}=-\frac{12(\nu+1)(\nu+2)(\nu+3)}{\nu(\nu+5)^{2}(\nu+7)} \\
\text { e } \quad b_{3} & =\frac{(\nu-1)^{2}(\nu+3)}{2 \nu(\nu+5)^{2}} .
\end{aligned}
$$

(iv) $t$-Student generalizada: $y_{l} \sim t G\left(x_{l}^{\top} \beta, \phi^{2}, s, r\right), s, r>0$ conhecidos;

$$
\begin{aligned}
b_{1} & =\frac{(r-1)(r+2)(r+3)}{r(r+5)^{2}}, \quad b_{2}=-\frac{12(r+1)(r+2)(r+3)}{r(r+5)^{2}(r+7)} \\
\mathrm{e} \quad b_{3} & =\frac{(r-1)^{2}(r+3)}{2 r(r+5)^{2}} .
\end{aligned}
$$

(v) Logística I: $y_{l} \sim L I\left(x_{l}^{\top} \beta, \phi^{2}\right)$;

$$
b_{1} \approx 1,77437106, \quad b_{2} \approx 0,569031708 \text { e } \quad b_{3} \approx 1,155242856 .
$$

(vi) Logística II: $y_{l} \sim \operatorname{LII}\left(x_{l}^{\top} \beta, \phi^{2}\right)$;

$$
b_{1} \approx 0,52446, \quad b_{2} \approx-0,58350 \text { e } b_{3} \approx 0,174816 .
$$

(vii) Logística generalizada: $y_{l} \sim \operatorname{LG}\left(x_{l}^{\top} \beta, \phi^{2}, m\right), m>0$;

$$
\begin{aligned}
b_{1}= & \frac{(2 m+1)^{2} m}{(m+1)^{2}\left(\psi^{\prime}(m)+2 m-1\right)}, \\
b_{2}= & \frac{-4 m(2 m+1)\left(2 m^{2}(m-1) \psi^{\prime}(m)-2 m+1\right)}{(m+1)^{2}\left(2 m^{2} \psi^{\prime}(m)+2 m-1\right)^{2}} \\
& +\frac{4(2 m+1)\left(m^{2}(m+1) \psi^{\prime}(m)-4 m-1\right)}{(2 m+3)(m+1)\left(2 m(m+1) \psi^{\prime}(m)-1\right)} \\
b_{3}= & \frac{(2 m+1) m^{2}}{(m+1)^{2}\left(2 m^{2} \psi^{\prime}(m)+2 m+1\right)},
\end{aligned}
$$


Utilizando-se a expansão (3.12) para $m$ grande. obtemos

$$
\begin{aligned}
& b_{1}=1-\frac{1}{m}+\frac{7}{6 m^{2}}-\frac{17}{12 m^{3}}+O\left(m^{-4}\right), \\
& b_{2}=-\frac{2}{m}+\frac{13}{3 m^{2}}-\frac{17}{2 m^{3}}+O\left(m^{-4}\right), \\
& b_{3}=\frac{1}{2}-\frac{3}{4 m}+\frac{23}{24 m^{2}}-\frac{19}{48 m^{3}}+O\left(m^{-4}\right) .
\end{aligned}
$$

E, para $m$ pequeno, de (3.13) obtemos

$$
\begin{aligned}
& b_{1}=m-\left(1+\frac{1}{3} \pi^{2}\right) m^{3}+O\left(m^{4}\right), \\
& b_{2}=-4 m+\left(\frac{20}{3}+\frac{2 \pi^{2}}{9}\right) m^{2}+\left(\frac{50 \pi^{2}}{27}-\frac{76}{9}-\frac{8}{3} \zeta(3)\right) m^{3}+O\left(m^{4}\right) \\
& b_{3}=m^{2}-2 m^{3}+O\left(m^{4}\right) .
\end{aligned}
$$

(viii) Exponencial potência: $y_{l} \sim E P\left(x_{l}^{\top} \beta, \phi^{2}, k\right),-1<k<-1 / 2$ conhecido;

$$
b_{1}=\frac{(1-k)}{(1+k)}, \quad b_{2}=\frac{2(k-1) k}{(1+k)}, \quad b_{3}=\frac{(1-k)^{2}}{2(1+k)} .
$$

\subsubsection{Testes de hipóteses sobre o parâmetro $\phi$}

O objetivo desta seção é encontrar o fator de correção tipo Bartlett para a estatística escore (dada em (2.32)) do teste da hipótese nula $H: \phi=\phi^{(0)}$ contra a alternativa $H_{1}: \phi \neq \phi^{(0)}$, onde $\beta$ é um vetor de parâmetros de perturbação e $\phi^{(0)}$ é um valor positivo especificado para $\phi$.

Ferrari e Cordeiro (1994) mostram que as quantidades $A_{1}, A_{2}$ e $A_{3}$ para a correção do teste escore de um parâmetro escalar, $\phi$ digamos, ortogonal a um vetor de parâmetros de perturbação, $\beta$ digamos, são dadas por

$$
\begin{aligned}
A_{1}= & \kappa_{\phi \phi}^{-1}\left\{6 \sum^{\prime}\left(\kappa_{\phi \phi}^{(t)}-2 \kappa_{j \phi \phi}\right)\left(\kappa_{i j s}-2 \kappa_{i j}^{(s)}\right) \kappa^{i j} \kappa^{s t}\right. \\
& -3\left(\sum^{\prime} \kappa_{i j \phi} \kappa^{i j}\right)^{2}-6 \sum^{\prime} \kappa_{j t \phi}\left(3 \kappa_{i s \phi}-2 \kappa_{i s}^{(\phi)}\right) \kappa^{i j} \kappa^{s t} \\
& \left.+6 \sum^{\prime}\left(2 \kappa_{i j \phi \phi}-4 \kappa_{i \phi \phi}^{(j)}-2 \kappa_{i j \phi}^{(\phi)}+\kappa_{\phi \phi}^{(i j)}-2 \kappa_{i \phi, j \phi}\right) \kappa^{i j}\right\} \\
A_{2}= & \kappa_{\phi \phi}^{-2}\left\{\sum^{\prime}\left\{9\left(2 \kappa_{i \phi \phi}-\kappa_{\phi \phi}^{(i)}\right)\left(2 \kappa_{j \phi \phi}-\kappa_{\phi \phi}^{(j)}\right)+6 \kappa_{\phi, \phi, \phi} \kappa_{i j \phi}\right\} \kappa^{i j}+3 \kappa_{\phi, \phi, \phi, \phi}\right\}, \\
A_{3}= & -\frac{5 \kappa_{\phi, \phi, \phi}^{2}}{\kappa_{\phi \phi}^{3}} .
\end{aligned}
$$

Devido à estrutura linear de $\mu$ e a simetria dos modelos aqui considerados, alguns cumulantes se anulam e as fórmulas acima reduzem-se a:

$$
A_{1}=\kappa_{\phi \infty}^{-1}\left\{-3\left(\sum^{\prime} \kappa_{i j \phi} \kappa^{i j}\right)^{2}-6 \sum^{\prime} \kappa_{j t \phi}\left(3 \kappa_{i s \phi}-2 \kappa_{i s}^{(\rho)}\right) \kappa^{i j} \kappa^{s t}\right.
$$




$$
\begin{aligned}
&\left.+12 \sum^{\prime}\left(\kappa_{i j \phi \phi}-\kappa_{i j \phi}^{(\phi)}-\kappa_{i \phi, j \phi}\right) \kappa^{i j}\right\}, \\
& A_{2}= \kappa_{\phi \phi}^{-2}\left\{\sum^{\prime}\left(6 \kappa_{\phi, \phi, \phi} \kappa_{i j \phi}\right) \kappa^{i j}+3 \kappa_{\phi, \phi . \phi, \phi}\right\}, \\
& A_{3}=-\frac{5 \kappa_{\phi, \phi, \phi}^{2}}{\kappa_{\phi \phi}^{3}} .
\end{aligned}
$$

Utilizando os cumulantes dados nas Seções 3.2 .1 e $3.2 .2, A_{1}, A_{2}$ e $A_{3}$ são dados por (Apêndice B):

$$
\begin{aligned}
A_{1}= & \frac{-3}{n \delta_{(2,0,0,0,0)}^{2}\left[\delta_{(0,1,0,0,2)}-1\right]}\left\{\left[\delta_{(0,0,1,0,1)}+2 \delta_{(0,1,0,0,0)}\right]^{2} p^{2}\right. \\
& +2\left[\delta_{(0,0,1,0,1)}+2 \delta_{(0,1,0,0,0)}\right]\left[3 \delta_{(0,0,1,0,1)}+2 \delta_{(0,1,0,0,0)}\right] p \\
& \left.-4 \delta_{(2,0,0,0,0)}\left[\delta_{(0,2,0,0,2)}-\delta_{(0,0,0,1,2)}+5 \delta_{(1,1,0,0,1)}+2 \delta_{(0,1,0,0,0)}\right] p\right\}, \\
A_{2}= & \frac{3}{n \delta_{(2,0,0,0,0)}\left[\delta_{(0,1,0,0,2)}-1\right]^{2}}\left\{4\left(1+\delta_{(1,1,0,0,3)}\right)\left(\delta_{(0,0,1,0,1)}+2 \delta_{(0,1,0,0,0)}\right) p\right. \\
& \left.+\delta_{(2,0,0,0,0)}\left(\delta_{(4,0,0,0,4)}+4 \delta_{(3,0,0,0,3)}+12 \delta_{(2,0,0,0,2)}-3 \delta_{(2,0,0,0,2)}^{2}-6\right)\right\}, \\
A_{3}= & \frac{-20\left(1+\delta_{(1,1,0,0,3)}\right)^{2}}{n\left(\delta_{(0,1,0,0,2)}-1\right)^{3}} .
\end{aligned}
$$

A estatística escore aperfeiçoada $S_{R}^{*}$ é obtida de (2.38) com $A_{1}, A_{2}$ e $A_{3}$ dados em (3.26) - (3.28). A estatística $S_{R}^{*}$ tem distribuição $\lambda_{1}^{2}$ até ordem $n^{-1}$.

Damos agora aplicações de (3.26) - (3.28) a algumas distribuições simétricas (no Apêndice C estão os $\delta$ 's para cada distribuição).

(i) Normal: $y_{l} \sim N\left(x_{l}^{\top} \beta, \phi^{2}\right)$;

$$
A_{1}=6 p(p-2) / n, \quad A_{2}=12(3-2 p) / n \quad \text { e } \quad A_{3}=40 / n .
$$

(ii) Cauchy: $y_{l} \sim C\left(x_{l}^{\top} \beta, \phi^{2}\right)$;

$$
A_{1}=\frac{6 p}{n}(p-1), \quad A_{2}=-\frac{9}{2 n} \quad \text { e } \quad A_{3}=0 .
$$

(iii) $t$-Student: $y_{l} \sim t\left(x_{l}^{\top} \beta, \phi^{2}, \nu\right), \nu>0$ conhecido;

$$
\begin{aligned}
& A_{1}=\frac{6 p(\nu+2)(\nu+3)}{n \nu(\nu+5)^{2}(\nu+7)}\left[(\nu+2)(\nu+7) p-2\left(\nu^{2}+3 \nu+8\right)\right], \\
& A_{2}=\frac{12}{n \nu(\nu+5)}\left[\frac{3\left(\nu^{3}-10 \nu+3\right)}{(\nu+7)}-\frac{2\left(\nu^{3}+4 \nu^{2}+\nu-6\right) p}{(\nu+5)}\right], \\
& A_{3}=\frac{40(\nu-1)^{2}(\nu+3)}{n \nu(\nu+5)^{2}} .
\end{aligned}
$$


(iv) $t$-Student generalizada: $y_{l} \sim t G^{\prime}\left(x_{l}^{\top} 3 . \delta^{2}\right.$.s.r). s. $r>0$ conhecidos:

$$
\begin{aligned}
& A_{1}=\frac{6 p(r+2)(r+3)}{n r(r+5)^{2}(r+\tau)}\left[(r+2)(r+7) p-2\left(r^{2}+3 r+\delta\right)\right] . \\
& A_{2}=\frac{12}{n r(r+5)}\left[\frac{3\left(r^{3}-10 r+3\right)}{(r+7)}-\frac{2 p(r-1)(r+2)(r+3)}{(r+5)}\right] \\
& A_{3}=\frac{40(r-1)^{2}(r+3)}{n r(r+5)^{2}} .
\end{aligned}
$$

(v) Logística I: $y_{l} \sim \operatorname{LI}\left(x_{l}^{\top} \beta, \phi^{2}\right)$;

$$
\begin{aligned}
& A_{1} \approx \frac{1}{n}\left(8,176132.57 p^{2}+63,04589499 p\right), \\
& A_{2} \approx \frac{1}{n}(28,57127439 p+32,85445997), \\
& A_{3} \approx \frac{5,513332236}{n} .
\end{aligned}
$$

(vi) Logística II: $y_{l} \sim \operatorname{LII}\left(x_{l}^{\top} \beta, \phi^{2}\right)$;

$$
\begin{aligned}
& A_{1} \approx \frac{1}{n}\left(4,77042 p^{2}-6,5061 p\right), \\
& A_{2} \approx \frac{1}{n}(14,2856-14,4802 p), \\
& A_{3} \approx \frac{18,5079}{n} .
\end{aligned}
$$

(vii) Logística generalizada: $y_{l} \sim \operatorname{LG}\left(x_{l}^{\top} \beta, \phi^{2}, m\right), m>0$ conhecido;

$$
\begin{aligned}
A_{1}= & \frac{3(2 m+1)}{n(m+1)\left(2 m^{2} \psi^{\prime}(m)+2 m-1\right)}\left\{\frac{(2 m+1)^{2}}{(m+1)} p^{2}+\frac{(2 m+1)^{2}(2 m-1)}{(m+1)} p\right. \\
& \left.-\frac{4}{(2 m+3)}\left(2 m^{2}(m+1) \psi^{\prime}(m)+6 m^{2}+m-2\right) p\right\} \\
A_{2}= & -12(2 m+1)^{2}\left(6 m^{3} \psi^{\prime}(m)+2 m^{2}-3 m+1\right) p /\left(n(m+1)^{2}\left(2 m^{2} \psi^{\prime}(m)+2 m-1\right)^{2}\right) \\
& +18\left(m^{4}(2 m+1)(m+1) \psi^{\prime \prime \prime}(m)+8 m^{5}(m+1) \psi^{\prime}(m)^{2}+8 m^{3}\left(6 m^{2}+m-2\right) \psi^{\prime}(m)\right. \\
& \left.+8 m^{4}-20 m^{3}+6 m+7 m-3\right) /\left(n(2 m+3)(m+1)\left(2 m^{2} \psi^{\prime}(m)+2 m-1\right)^{2}\right) \\
A_{3}= & \frac{20(2 m+1)\left(6 m^{3} \psi^{\prime}(m)+2 m^{2}-3 m+1\right)^{2}}{n(m+1)^{2}\left(2 m^{2} \psi^{\prime}(m)+2 m-1\right)^{3}}
\end{aligned}
$$

Utilizando a expansào (3.12) para $m$ grande obtemos

$$
A_{1}=\frac{1}{n}\left(6-\frac{3}{m}+\frac{4}{m^{2}}-\frac{5}{m^{3}}\right) p^{2}-\frac{6}{n}\left(2-\frac{3}{m}+\frac{6}{m^{2}}-\frac{32}{3 m^{3}}\right) p+O\left(m^{-4}\right)
$$




$$
\begin{aligned}
& A_{2}=-\frac{1}{n}\left(24-\frac{24}{m}+\frac{32}{m^{2}}-\frac{38}{m^{3}}\right) p-\left(36-\frac{72}{m}+\frac{135}{m^{2}}-\frac{441}{2 m^{3}}\right)+O\left(m^{-4}\right) . \\
& A_{3}=\frac{1}{n}\left(40-\frac{60}{m}+\frac{90}{m^{2}}-\frac{115}{m^{3}}\right)+O\left(m^{-4}\right) .
\end{aligned}
$$

e de (3.13) para $m$ pequeno obtemos uma aproximaçào numérica para $A_{1}, A_{2}$ e $A_{3}$ utilizando o MAPLEV (Abell e Baselton, 1994)

$$
\begin{aligned}
A_{1}= & \frac{1}{n}\left\{3 p(p-2)+6 p^{2} m+\frac{1}{2} p\left(6+2 \pi^{2}-9 p-3 p \pi^{2}\right) m^{2}\right. \\
& \left.+\frac{4 p}{9}\left(27 \zeta(3) p-7 \pi^{2}-18 \zeta(3)-21\right) m^{3}\right\}+O\left(m^{4}\right) \\
A_{2}= & -\frac{2}{n}\left\{3(2 p-3)+6 p m-2\left(3 p+2 p \pi^{2}-3 \pi^{2}\right) m^{2}\right. \\
& \left.+2\left(3 p+5 p \pi^{2}+24 \zeta(3)-6 \pi^{2}-36 \zeta(3)\right) m^{3}\right\}+O\left(m^{4}\right) \\
A_{3}= & \frac{20}{n}\left(1-\pi^{2} m^{2}+4\left(3 \zeta(3)+\pi^{2}\right) m^{3}\right)+O\left(m^{4}\right)
\end{aligned}
$$

(viii) Exponencial potência: $y_{l} \sim E P\left(x_{l}^{\top} \beta, \phi^{2}, k\right),-1<k<-1 / 2$ conhecido;

$$
\begin{aligned}
& A_{1}=\frac{6 p}{n(1+k)}\left\{p-2+2 k+7 k^{2}-k^{4}\right\} \\
& A_{2}=\frac{3}{n}\left\{-2[4-k(1+k)] p+\frac{1}{2(k+1)}\left[3+k^{3}+16 k^{2}+30 k+24\right]\right\}, \\
& A_{3}=\frac{5}{2 n}(4-k(1+k))^{2} .
\end{aligned}
$$

Devemos observar que as fórmulas dos coeficientes do fator de correção tipo Bartlett para a estatística escore, embora tenham aparência complicada, são simples e de fácil aplicação dada a distribuição assumida para os dados. Embora o cálculo dos coeficientes do fator de correção seja simples, eles são de difícil interpretação. Além disto, da mesma forma que os coeficientes do fator de correção de Bartlett, não dependem dos parâmetros $\beta$ e $\phi$ nem da matriz $X$, sendo apenas funções dos $\delta$ 's obtidos da distribuição assumida para os dados.

\subsection{Alguns estudos de simulação}

O objetivo desta seção é mostrar o desempenho dos testes baseados nas estatísticas $S_{R}^{\prime}, S_{R}^{\prime \times}$, $R V$ e $R V^{*}$ mediante um estudo de simulação em modelos de regressão múltipla supondo que $y$ tem as seguintes distribuições: Cauchy $\left(\mu, \phi^{2}\right)$ e $t$-Student $\left(\mu . \sigma^{2}, \nu\right)$ para $\nu=$ $2,3,4,5,6$ e 20. Em todos os casos consideramos o parâmetro de escala $\phi$ desconhecido.

Dez mil amostras foram geradas para diferentes valores de $n$ e distintos níveis nominais. Em cada caso o vetor de parâmetros de locação $\mu$ tem a forma $\mu=\mathrm{X} 3$, onde $\beta=$ $\left(\beta_{1}, \beta_{2}, \cdots, \beta_{7}\right)^{\top}$. Consideremos o problema de testar $H_{0}: \beta_{2}=0$ versus $H_{1}: \beta_{2} \neq 0$. Os 
valores das covariáveis foram selecionados como segue. $x_{1}$ é um vetor de uns, $x_{2}, x_{3} \cdot x_{4}, x_{5}$ e $x_{6}$ sào obtidos como amostras aleatórias das distribuiçòes normal padrào. uniforme no intervalo (0,1), Cauchy padrào. $F(8,6)$ e exponencial com média 8 , respectivamente. e $x_{7}$ tem os $n / 2$ primeiros componentes iguais a zero e os demais iguais a 1 . Todas as simulaçòes foram feitas no Laboratório de Computação Científica Avançada - LCCA da USP através de programas em FORTRAN e sub-rotinas do IMSL. As estimativas das probabilidades de rejeição de $H_{0}$ para os testes da razão de verossimilhança, escore e suas versões modificadas são dados nas Tabelas 3.1 - 3.12 para as diferentes situações.

Nas Tabelas 3.2 - 3.7 são apresentados os resultados relativos à distribuição $t$-Student. Para todos os valores de $n$ considerados, observamos que as taxas de rejeição do teste baseado em $S_{R}^{\star}$ são bem mais próximas dos correspondentes níveis nominais do que as do teste baseado em $S_{R}$. Para $n=10$, ou seja, para uma amostra bem pequena levando-se em conta que o modelo tem sete parâmetros desconhecidos sob a hipótese nula, o teste escore teve um desempenho bastante ruim. Mesmo assim, a correção conseguiu produzir um teste com taxas de rejeição um pouco mais próximas dos correspondentes níveis nominais. Sendo assim, conclui-se que o teste escore corrigido tem um desempenho melhor que o não corrigido. Os resultados referentes ao teste da razão de verossimilhança mostram também que o teste corrigido tende a ser bem melhor que o não corrigido, exceto se $n$ for muito pequeno $(n=10)$ e, concomitantemente, o valor de $\nu$ também for pequeno. Vale notar que, 'se $\nu$ não é muito pequeno, mesmo para $n=10$, o teste baseado em $R V^{*}$ tem um desempenho muito superior ao daquele baseado em $R V$. Tomemos, por exemplo, o caso em que $\nu=6$ e $n=10$ e consideremos o nível nominal de $10 \%$. Enquanto o teste baseado em $R V$ apresentou uma taxa de rejeição de $46,9 \%$, aquele baseado em $R V^{*}$ teve taxa de rejeição de $10,1 \%$, muito próximo, portanto, do nível nominal.

Os resultados de simulação descritos foram obtidos supondo que o parâmetro $\nu$ é conhecido. Na prática, este valor não é de conhecimento do pesquisador e, então, este pode ser fixado em algum valor plausível ou, se o tamanho da amostra não for muito pequeno, pode ser estimado com base nos dados. Como mencionado anteriormente, Lange, Little e Taylor (1989, p.892) sugerem utilizar $\nu=4 \mathrm{em}$ amostras pequenas em lugar de estimar este parâmetro. Sendo assim, realizamos outros estudos de simulação nos quais os dados foram gerados segundo distribuiçòes $t$-Student $\operatorname{com} \nu=2,3,5,6$ e 20, mas os testes corrigidos e não corrigidos foram calculados supondo que $\nu$ fosse igual a 4 . Os resultados são apresentados nas Tabelas 3.8 - 3.12. Nota-se que, na maior parte dos casos, o uso de $\nu=4$ em lugar do verdadeiro valor de $\nu$ não perturbou muito as taxas de rejeição dos testes não corrigidos. Em alguns casos nota-se até que os resultados obtidos com o valor $\nu=4$ são melhores do que os obtidos com o valor correto de $\nu$. Além disto, na maioria dos casos, os testes corrigidos tiveram desempenho melhor que os correspondentes não corrigidos.

Uma observação que se pode fazer em todas as simulações é que, conforme cresce o tamanho da amostra, os efeitos das correçòes vào se tornando cada vez menos expressivos. Isto, evidentemente, era de se esperar uma vez que, conforme $n$ cresce, as probabilidades $P\left(S_{R} \geq x_{\alpha}\right)$ e $P\left(R V \geq x_{\alpha}\right)$ vào se aproximando dos correspondentes niveis nominais e a necessidade de se fazerem correções vai diminuindo. 
A conclusão a que chegamos após a análise de todas as tabelas é a de que para a grande maioria dos casos simulados, há evidência da superioridade do desempenho dos testes corrigidos sobre os nào corrigidos.

Tabela 3.1: Valores simulados de $P\left(S_{R} \geq x_{\alpha}\right), P\left(S_{R}^{*} \geq x_{\alpha}\right)$, $P\left(R V \geq x_{\alpha}\right)$ e $P\left(R V^{*} \geq x_{\alpha}\right)$ (em porcentagem), sendo $y_{l} \sim \operatorname{Cauchy}\left(x_{l}^{\top} \beta, \phi^{2}\right), l=1,2 \ldots, n$.

\begin{tabular}{|c|c|c|c|c|c|}
\hline $\begin{array}{c}\text { Tamanho } \\
\text { da amostra } \\
n\end{array}$ & $\begin{array}{c}\text { Nível } \\
\text { nominal } \\
(\%)\end{array}$ & (1) & (2) & (3) & (4) \\
\hline \multirow{4}{*}{10} & 1,0 & 33,2 & 27.9 & 99,5 & 0,0 \\
\hline & 2,5 & 38,9 & 33.1 & 99,7 & 0,0 \\
\hline & 5,0 & 45,2 & 38,0 & 99,8 & 0,0 \\
\hline & 10,0 & 51,8 & 45.2 & 99,9 & 0,0 \\
\hline \multirow{4}{*}{20} & 1,0 & 5,1 & 4,0 & 11,9 & 0,0 \\
\hline & 2,5 & 8,6 & 6,5 & 18,3 & 1,4 \\
\hline & 5,0 & 12,9 & 10,3 & 25,0 & 5,7 \\
\hline & 10,0 & 19,6 & 15,9 & 33,8 & 21,2 \\
\hline \multirow{4}{*}{30} & 1,0 & 1,9 & 1,4 & 6,6 & 0,6 \\
\hline & 2,5 & 3,9 & 3,2 & 11,2 & 1,8 \\
\hline & 5,0 & 7,1 & 5,7 & 17,5 & 3,9 \\
\hline & 10,0 & 13,3 & 11,2 & 26,2 & 8,7 \\
\hline \multirow{4}{*}{40} & 1,0 & 2,5 & 2,1 & 4,4 & 1,0 \\
\hline & 2,5 & $4, \bar{\imath}$ & 4,1 & $\delta, 0$ & 2,3 \\
\hline & 5,0 & 7,9 & 6,9 & 12,8 & 4,8 \\
\hline & 10,0 & 14,1 & 12.5 & 20,6 & 9,9 \\
\hline \multirow{4}{*}{50} & 1,0 & 1,9 & $1, \pi$ & 3,0 & 1,0 \\
\hline & $2, \overline{5}$ & 3,9 & 3,4 & 6,2 & 2,3 \\
\hline & 5,0 & 6,8 & 6,1 & 10,3 & 4,9 \\
\hline & 10,0 & 12,5 & 11,4 & 17,5 & 9,7 \\
\hline \multirow{4}{*}{60} & 1,0 & 1,9 & 1,6 & 2,7 & 1,0 \\
\hline & 2,5 & 3,7 & 3,4 & 5,1 & 2.5 \\
\hline & 5.0 & 6,9 & 6.3 & 9,1 & 4.7 \\
\hline & 10,0 & 12,8 & 11.7 & 16,0 & 9.7 \\
\hline
\end{tabular}

Nota: (1), (2), (3) e (4) correspondem a $P\left(S_{R} \geq x_{\alpha}\right) . P\left(S_{R}^{\prime \times} \geq x_{\alpha}\right)$, $P\left(R V \geq x_{\alpha}\right)$ e $P\left(R V^{*} \geq x_{\alpha}\right)$, respectivamente. 
Tabela 3.2: Valores simulados de $P\left(S_{R} \geq x_{\alpha}\right)$, $P\left(S_{R}^{*} \geq x_{\alpha}\right)$. $P\left(R I^{*} \geq x_{a}\right)$ e $P\left(R V^{*} \geq x_{\alpha}\right)$ (em porcentagem), sendo $y_{l} \sim t-\operatorname{Student}\left(x_{l}^{\top} \beta, \phi^{2}, 2\right) . l=1,2, \ldots, n$.

\begin{tabular}{|c|c|c|c|c|c|}
\hline $\begin{array}{c}\text { Tamanho } \\
\text { da amostra } \\
n\end{array}$ & $\begin{array}{c}\text { Nivel } \\
\text { nominal } \\
(\%)\end{array}$ & (1) & (2) & (3) & (4) \\
\hline \multirow{4}{*}{10} & 1,0 & 12,9 & 5,9 & 62,4 & 0,0 \\
\hline & 2,5 & 20,2 & 9,9 & 75,6 & 0,0 \\
\hline & 5,0 & 27,6 & 14,9 & 89,4 & 0,0 \\
\hline & 10,0 & 37,6 & 23,1 & 96,1 & 0,0 \\
\hline \multirow{4}{*}{20} & 1,0 & 3,5 & 2,3 & 6,0 & 0,7 \\
\hline & 2,5 & 6,6 & 4,2 & 10,2 & 1,8 \\
\hline & 5,0 & 10,3 & 7,2 & 14,9 & 3,8 \\
\hline & 10,0 & 16,3 & 12,1 & 22,3 & $\$, 2$ \\
\hline \multirow{4}{*}{30} & 1,0 & 1,8 & 1,2 & 4,0 & 0,9 \\
\hline & 2,5 & 4,0 & 2,8 & 7,5 & 2,7 \\
\hline & 5,0 & 7,3 & 5,3 & 11,9 & 5,3 \\
\hline & 10,0 & 13,3 & 10,4 & 19,7 & 10,4 \\
\hline \multirow{4}{*}{40} & 1,0 & 1,8 & 1,3 & 2,6 & 0,7 \\
\hline & 2,5 & 4,0 & 3,1 & 5,4 & 2,3 \\
\hline & 5,0 & 7,3 & 5,9 & 8,9 & 4,8 \\
\hline & 10,0 & 13,3 & 11,1 & 15,7 & 9,6 \\
\hline \multirow{4}{*}{50} & 1,0 & 1,5 & 1,1 & 2,1 & 0,9 \\
\hline & 2,5 & 3,4 & 2,7 & 4,6 & 2,4 \\
\hline & 5,0 & 6,3 & 5,3 & 8,0 & 4,8 \\
\hline & 10,0 & 12,4 & 10,7 & 14,6 & 10,0 \\
\hline \multirow{4}{*}{60} & 1,0 & 1,6 & 1,3 & 1,8 & 0,9 \\
\hline & 2,5 & 3,6 & 3,0 & 4,0 & 2,4 \\
\hline & 5,0 & 6,6 & 5,7 & 7,2 & 4,8 \\
\hline & 10,0 & 12,3 & 11,0 & 13,2 & 10,0 \\
\hline
\end{tabular}

Nota: (1), (2), (3) e (4) correspondem a $P\left(S_{R} \geq x_{\alpha}\right), P\left(S_{R}^{*} \geq x_{\alpha}\right)$, $P\left(R V \geq x_{\alpha}\right)$ e $P\left(R V^{*} \geq x_{\alpha}\right)$, respectivamente. 
Tabela 3.3: Valores simulados de $P\left(S_{R} \geq x_{\alpha}\right)$. $P\left(S_{R}^{\prime *} \geq x_{\alpha}\right)$, $P\left(R V^{*} \geq x_{\alpha}\right)$ e $P\left(R V^{*} \geq x_{\alpha}\right)$ (em porcentagem). sendo $y_{l} \sim t-\operatorname{Student}\left(x_{l}^{\top} \beta, \phi^{2}, 3\right), l=1.2 \ldots, n$.

\begin{tabular}{|c|c|c|c|c|c|}
\hline $\begin{array}{c}\text { Tamanho } \\
\text { da amostra } \\
n\end{array}$ & $\begin{array}{c}\text { Nivel } \\
\text { nominal } \\
(\%)\end{array}$ & (1) & (2) & (3) & (4) \\
\hline \multirow{4}{*}{10} & 1,0 & 11,8 & 3,1 & 45,6 & 0,0 \\
\hline & 2,5 & 18,0 & 7,8 & 53,4 & 0,1 \\
\hline & 5,0 & 26,6 & 12,4 & 60,7 & 1,0 \\
\hline & 10,0 & 37,8 & 18,8 & 67,2 & 4,1 \\
\hline \multirow{4}{*}{20} & 1,0 & 3,7 & 2,0 & 4.9 & 0,8 \\
\hline & 2,5 & 6,8 & 4,2 & 8,8 & 2,0 \\
\hline & 5,0 & 10,7 & 7,2 & 13,2 & 4,5 \\
\hline & 10,0 & 17,4 & 12,1 & 20,2 & 9,1 \\
\hline \multirow{4}{*}{30} & 1,0 & 1,9 & 1,2 & 3,2 & 1,0 \\
\hline & 2,5 & 4,0 & 2,8 & 6,1 & 2,5 \\
\hline & 5,0 & 7,3 & 5,9 & 10,2 & 5,1 \\
\hline & 10,0 & 13,4 & 10,4 & 17,3 & 10,1 \\
\hline \multirow{4}{*}{40} & 1,0 & 1,9 & 1,2 & 2,3 & 0,9 \\
\hline & 2,5 & 4,0 & 3,0 & 4,7 & 2,4 \\
\hline & 5,0 & 7,7 & 5,7 & 8,6 & 4,9 \\
\hline & 10,0 & 13,5 & 11,1 & 14,7 & 10,2 \\
\hline \multirow{4}{*}{50} & 1,0 & 1,6 & 1,4 & 2,1 & 1,1 \\
\hline & 2,5 & 3,7 & 2,9 & 4,4 & 2,6 \\
\hline & 5,0 & 6,6 & 5,4 & 7,7 & 5,0 \\
\hline & 10,0 & 12,7 & 10,8 & 14,1 & 10,1 \\
\hline \multirow{4}{*}{60} & 1,0 & 1,7 & 1,3 & 1,8 & 1,1 \\
\hline & 2,5 & 3,5 & 2,9 & 4,0 & 2,6 \\
\hline & 5,0 & 6,9 & 5,6 & 7,2 & 5,0 \\
\hline & 10,0 & 12,4 & 10,9 & 13.0 & 10,2 \\
\hline
\end{tabular}

Nota: (1), (2), (3) e (4) correspondem a $P\left(S_{R} \geq x_{\alpha}\right), P\left(S_{R}^{*} \geq x_{\alpha}\right)$, $P\left(R V \geq x_{\alpha}\right)$ e $P\left(R V^{*} \geq x_{\alpha}\right)$, respectivamente. 
Tabela 3.4: Valores simulados de $P\left(S_{R} \geq x_{\alpha}\right)$. $P\left(S_{R}^{\star} \geq x_{\alpha}\right)$. $P\left(R V^{\circ} \geq x_{\alpha}\right)$ e $P\left(R V^{*} \geq x_{\alpha}\right)$ (em porcentagem). sendo $y_{l} \sim t-\operatorname{Student}\left(x_{l}^{\top} \beta, \phi^{2}, 4\right), l=1,2, \ldots n$.

\begin{tabular}{|c|c|c|c|c|c|}
\hline $\begin{array}{c}\text { Tamanho } \\
\text { da amostra } \\
n\end{array}$ & $\begin{array}{c}\text { Nivel } \\
\text { nominal } \\
(\%)\end{array}$ & (1) & (2) & (3) & $(4)$ \\
\hline \multirow{4}{*}{10} & 1,0 & 13,2 & 2,6 & 32,1 & 2,4 \\
\hline & 2,5 & 20,0 & 7,9 & 39,9 & 10,3 \\
\hline & 5,0 & 26,5 & 13,3 & 46,7 & 3,5 \\
\hline & 10,0 & 37,6 & 20,0 & 55,1 & 9,0 \\
\hline \multirow{4}{*}{20} & 1,0 & 3,1 & 1,7 & 4,2 & 0,9 \\
\hline & 2,5 & 5,9 & 3,4 & 7,4 & 2,1 \\
\hline & 5,0 & 10,0 & 5,9 & 11,7 & 4,3 \\
\hline & 10,0 & 16,2 & 11,0 & 18,6 & 8,5 \\
\hline \multirow{4}{*}{30} & 1,0 & 1,7 & 1,1 & 2,7 & 1,0 \\
\hline & 2,5 & 3,9 & 2,6 & 5,4 & 2,4 \\
\hline & 5,0 & 7,3 & 5,0 & 10,0 & 4,7 \\
\hline & 10,0 & 13,6 & 10,0 & 16,0 & 10,0 \\
\hline \multirow{4}{*}{40} & 1,0 & 2,0 & 1,4 & 2,6 & 1,3 \\
\hline & 2,5 & 4,3 & 2,6 & 4,3 & 2,9 \\
\hline & 5,0 & 7,5 & 5,4 & 8,4 & 5,3 \\
\hline & 10,0 & 13,7 & 10,4 & 15,0 & 10,3 \\
\hline \multirow{4}{*}{50} & 1,0 & 1,4 & 1,1 & 1,7 & 1,0 \\
\hline & 2,5 & 3,6 & 2,6 & 4,0 & 2,4 \\
\hline & 5,0 & 6,5 & 5,4 & 7,4 & 5,0 \\
\hline & 10,0 & 12,5 & 10,4 & 13,4 & 10,2 \\
\hline \multirow{4}{*}{60} & 1,0 & 1,5 & 1,1 & 1,6 & 1,0 \\
\hline & 2,5 & 3,6 & 2,9 & 3,8 & 2,6 \\
\hline & 5,0 & 6,5 & 5,3 & 6,6 & 4,9 \\
\hline & 10,0 & 11,9 & 10,3 & 12,4 & 9,8 \\
\hline
\end{tabular}

Nota: (1), (2), (3) e (4) correspondem a $P\left(S_{R} \geq x_{\alpha}\right), P\left(S_{R}^{\star *} \geq x_{\alpha}\right)$, $P\left(R V \geq x_{\alpha}\right)$ e $P\left(R V^{*} \geq x_{\alpha}\right)$, respectivamente. 
Tabela 3.5: Valores simulados de $P\left(S_{R} \geq x_{\alpha}\right), P\left(S_{R}^{\times} \geq x_{\alpha}\right)$, $P\left(R V \geq x_{\alpha}\right)$ e $P\left(R V^{*} \geq x_{\alpha}\right)$ (em porcentagem). sendo $y_{l} \sim t-\operatorname{Student}\left(x_{l}^{\top} \beta, o^{2}, 5\right) . l=1.2, \ldots n$.

\begin{tabular}{|c|c|c|c|c|c|}
\hline $\begin{array}{c}\text { Tamanho } \\
\text { da amostra } \\
n\end{array}$ & $\begin{array}{c}\text { Nivel } \\
\text { nominal } \\
(\%)\end{array}$ & (1) & (2) & (3) & (4) \\
\hline \multirow{4}{*}{10} & 1,0 & 13,4 & 2,9 & 28,3 & 0,6 \\
\hline & 2,5 & 21.0 & 7.6 & 35,1 & 2,3 \\
\hline & 5,0 & 27,7 & 13,1 & 41,8 & 4,9 \\
\hline & 10,0 & 36,7 & 20,6 & 50,1 & 10,7 \\
\hline \multirow{4}{*}{20} & 1,0 & 2,9 & 1,4 & 3,9 & 0,9 \\
\hline & 2,5 & 5,9 & 3,1 & 7,3 & 2,1 \\
\hline & 5,0 & 9,9 & 5,8 & 11,8 & 4,3 \\
\hline & 10,0 & $16, \pi$ & 11,0 & 18,7 & 9,0 \\
\hline \multirow{4}{*}{30} & 1,0 & 1,9 & 1,1 & 2,6 & 1,0 \\
\hline & 2,5 & 4,0 & 2,6 & 5,4 & 2,4 \\
\hline & 5,0 & $\$, 0$ & 5,2 & 9,7 & 4,9 \\
\hline & 10,0 & 14,9 & 10,9 & 16,6 & 10,3 \\
\hline \multirow{4}{*}{40} & 1,0 & 1,7 & 1,1 & 1,8 & 0,9 \\
\hline & 2,5 & 3,7 & 2,6 & 4,2 & 2,2 \\
\hline & 5,0 & 7,1 & 5,3 & 7,9 & 4,8 \\
\hline & 10,0 & 12,9 & 10,3 & 13,1 & 9,7 \\
\hline \multirow{4}{*}{50} & 1,0 & 1,5 & 1,2 & 1,7 & 1,0 \\
\hline & 2,5 & 3,5 & 2,6 & 3,9 & 2,2 \\
\hline & 5,0 & 6,5 & 5,2 & 7,2 & 4,8 \\
\hline & 10,0 & 12,5 & 10,7 & 13,1 & 9,9 \\
\hline \multirow{4}{*}{60} & 1,0 & 1,4 & 1,1 & 1,5 & 0,9 \\
\hline & 2,5 & 3,2 & 2,7 & 3,5 & 2,4 \\
\hline & 5,0 & 6,5 & 5,3 & 7,1 & 5,0 \\
\hline & 10,0 & 12,3 & 10,6 & 12,8 & 10,3 \\
\hline
\end{tabular}

Nota: (1), (2), (3) e (4) correspondem a $P\left(S_{R} \geq x_{\alpha}\right), P\left(S_{R}^{*} \geq x_{\alpha}\right)$, $P\left(R V \geq x_{\alpha}\right)$ e $P\left(R V^{*} \geq x_{\alpha}\right)$, respectivamente. 
Tabela 3.6: Valores simulados de $P\left(S_{R} \geq x_{\alpha}\right)$. $P\left(S_{R}^{*} \geq x_{\alpha}\right)$, $P\left(R V \geq x_{\alpha}\right)$ e $P\left(R V^{*} \geq x_{o}\right)$ (em porcentagem), sendo $y_{l} \sim t-\operatorname{Student}\left(x_{l}^{\top} \beta, \delta^{2}, 6\right) . l=1,2, \ldots, n$.

\begin{tabular}{|c|c|c|c|c|c|}
\hline $\begin{array}{c}\text { Tamanho } \\
\text { da amostra } \\
n\end{array}$ & $\begin{array}{c}\text { Nível } \\
\text { nominal } \\
(\%)\end{array}$ & (1) & $(2)$ & (3) & (4) \\
\hline \multirow{4}{*}{10} & 1,0 & 13,6 & 2,2 & 24,1 & 0,7 \\
\hline & 2,5 & 21,3 & 7,3 & 31,7 & 1,9 \\
\hline & 5,0 & 28,4 & 13,0 & 38,3 & 4,7 \\
\hline & 10,0 & 36,9 & 20,5 & 46,9 & 10,1 \\
\hline \multirow{4}{*}{20} & 1,0 & 2,7 & 1,2 & 3,8 & 0,7 \\
\hline & 2,5 & 5,7 & 2,9 & 7,5 & 2,1 \\
\hline & 5,0 & 10,0 & 5.6 & 11,5 & 4,3 \\
\hline & 10,0 & 16,9 & 10,9 & 18,8 & 9,0 \\
\hline \multirow{4}{*}{30} & 1,0 & 1,9 & 1,2 & 2,8 & 1,1 \\
\hline & 2,5 & 4,2 & 2,8 & 5,3 & 2,6 \\
\hline & 5,0 & 8,1 & 5,3 & 9,4 & 5,0 \\
\hline & 10,0 & 14,7 & 10,7 & 16,0 & 10,2 \\
\hline \multirow{4}{*}{40} & 1,0 & 1,6 & 1,0 & 2,0 & 0,9 \\
\hline & 2,5 & 3,6 & 2,6 & 4,3 & 2,3 \\
\hline & 5,0 & 6,7 & 4,9 & 7,5 & 4,7 \\
\hline & 10,0 & 12,9 & 10,3 & 13,8 & 9,7 \\
\hline \multirow{4}{*}{50} & 1,0 & 1,7 & 1,2 & 1,9 & 1,1 \\
\hline & 2,5 & 3,7 & 2,8 & 4,1 & 2,7 \\
\hline & 5,0 & 6,5 & 5,3 & 7,0 & 5,1 \\
\hline & 10,0 & 12,0 & 9,8 & 12,7 & 9,7 \\
\hline \multirow{4}{*}{60} & 1,0 & 1,5 & 1,1 & 1,7 & 1,0 \\
\hline & 2,5 & 3,4 & 2,8 & 3,7 & 1,9 \\
\hline & 5,0 & 6,5 & 5,3 & 7,0 & 4,7 \\
\hline & 10,0 & 12,5 & 10,7 & 12,9 & 10,1 \\
\hline
\end{tabular}

Nota: (1), (2), (3) e (4) correspondem a $P\left(S_{R} \geq x_{\alpha}\right), P\left(S_{R}^{*} \geq x_{\alpha}\right)$, $P\left(R V \geq x_{\alpha}\right)$ e $P\left(R V^{\star} \geq x_{\alpha}\right)$, respectivamente. 
Tabela 3.7: Valores simulados de $P\left(S_{R} \geq x_{\alpha}\right)$. $P\left(S_{R}^{* *} \geq x_{\alpha}\right)$, $P\left(R V \geq x_{\alpha}\right)$ e $P\left(R V^{*} \geq x_{\alpha}\right)$ (em porcentagem), sendo $y_{l} \sim t-\operatorname{Student}\left(x_{l}^{\top} \beta, \phi^{2}, 20\right), l=1,2 \ldots n$.

\begin{tabular}{|c|c|c|c|c|c|}
\hline $\begin{array}{c}\text { Tamanho } \\
\text { da amostra } \\
n\end{array}$ & $\begin{array}{l}\text { Nivel } \\
\text { nominal } \\
(\%)\end{array}$ & (1) & $(2)$ & (3) & (4) \\
\hline \multirow{4}{*}{10} & 1,0 & 11,1 & 1,2 & 18,8 & 1,0 \\
\hline & 2,5 & 19,1 & 5,3 & 25,1 & 2,6 \\
\hline & 5,0 & 27,2 & 10,2 & 31,7 & 4,8 \\
\hline & 10,0 & 36,9 & 17,1 & 39,7 & 10,1 \\
\hline \multirow{4}{*}{20} & 1,0 & 2,6 & 1,2 & 3,9 & 1,0 \\
\hline & 2,5 & 5,7 & 2,9 & 7,5 & 2,4 \\
\hline & 5,0 & 9,9 & 5,5 & 11,6 & 4,9 \\
\hline & 10,0 & 17,4 & 10,3 & 18,7 & 9,6 \\
\hline \multirow{4}{*}{30} & 1,0 & 1,7 & 1,0 & 2,4 & 0,9 \\
\hline & 2,5 & 4,2 & 2,5 & 5,1 & 2,3 \\
\hline & 5,0 & 8,1 & 5,2 & 8,9 & 4,9 \\
\hline & 10,0 & 14,6 & 10,4 & 15,5 & 10,0 \\
\hline \multirow{4}{*}{40} & 1,0 & 1,4 & 1,0 & 1,9 & 1,0 \\
\hline & 2,5 & 3,5 & 2,4 & 4,0 & 2,3 \\
\hline & 5,0 & 7,1 & 4,9 & 7,7 & 4,7 \\
\hline & 10,0 & 12,9 & 10,0 & 13,6 & 9,8 \\
\hline \multirow{4}{*}{50} & 1,0 & 1,3 & 1,0 & 1,5 & 1,0 \\
\hline & 2,5 & 3,1 & 2,4 & 3,5 & 2,3 \\
\hline & 5,0 & 6,3 & 4,7 & 6,7 & 4,7 \\
\hline & 10,0 & 12,0 & 9,8 & 12,4 & 9,7 \\
\hline \multirow{4}{*}{60} & 1,0 & 1,5 & 1,2 & 1,8 & 1,12 \\
\hline & 2,5 & 3,3 & 2,7 & 3,5 & 2,6 \\
\hline & 5,0 & 6,0 & 5,0 & 6,5 & 4,8 \\
\hline & 10,0 & 11,7 & 9,8 & 12,0 & 9,7 \\
\hline
\end{tabular}

Nota: (1), (2), (3) e (4) correspondem a $P\left(S_{R} \geq x_{\alpha}\right), P\left(S_{R}^{*} \geq x_{\alpha}\right)$, $P\left(R V \geq x_{\alpha}\right)$ e $P\left(R V^{*} \geq x_{\alpha}\right)$, respectivamente. 
Tabela 3.8: Valores simulados de $P\left(S_{R} \geq x_{\alpha}\right), \quad P\left(S_{R}^{\times} \geq x_{\alpha}\right)$, $P\left(R V \geq x_{\alpha}\right)$ e $P\left(R V^{*} \geq x_{\alpha}\right)$ (em porcentagem), sendo $y_{l} \sim t-\operatorname{Student}\left(x_{l}^{\top} \beta, \phi^{2}, 2\right), l=1,2, \ldots, n$, supondo $\nu=4$.

\begin{tabular}{|c|c|c|c|c|c|}
\hline $\begin{array}{c}\text { Tamanho } \\
\text { da amostra } \\
n\end{array}$ & $\begin{array}{c}\text { Nível } \\
\text { nominal } \\
(\%)\end{array}$ & (1) & (2) & (3) & (4) \\
\hline \multirow{4}{*}{10} & 1,0 & 12,7 & 2,3 & 31,9 & 0,2 \\
\hline & 2,5 & 19,9 & 7,1 & 39,2 & 1,2 \\
\hline & 5,0 & 26,8 & 12,8 & 45,9 & 3,3 \\
\hline & 10,0 & 37,2 & 19,9 & 53,4 & 8,7 \\
\hline \multirow{4}{*}{20} & 1,0 & 2,4 & 1,3 & 3,6 & 0,6 \\
\hline & 2,5 & 5,2 & 2,8 & 6,7 & 1,7 \\
\hline & 5,0 & 8,8 & 5,3 & 10,7 & 3,7 \\
\hline & 10,0 & 14,8 & 9,8 & 16,8 & 7,7 \\
\hline \multirow{4}{*}{30} & 1,0 & 1,3 & 0,7 & 2,1 & 0,8 \\
\hline & 2,5 & 3,5 & 2,1 & 4,7 & 1,8 \\
\hline & 5,0 & 6,6 & 4,5 & 8,2 & 4,0 \\
\hline & 10,0 & 12,2 & 9,2 & 14,3 & 8,5 \\
\hline \multirow{4}{*}{40} & 1,0 & 1,0 & 0,7 & 1,5 & 0,6 \\
\hline & 2,5 & 3,1 & 2,1 & 3,5 & 1,7 \\
\hline & 5,0 & 6,1 & 4,4 & 6,3 & 3,8 \\
\hline & 10,0 & 11,5 & 9,3 & 12,2 & 8,1 \\
\hline \multirow{4}{*}{50} & 1,0 & 1,2 & 0,8 & 1,3 & 0,6 \\
\hline & 2,5 & 2,8 & 2,1 & 3,0 & 1,8 \\
\hline & 5,0 & 5,6 & 4,3 & 5,8 & 3,8 \\
\hline & 10,0 & 11,0 & 9,2 & 11,3 & 8,2 \\
\hline \multirow{4}{*}{60} & 1,0 & 1,1 & 0,9 & 1,1 & 0,7 \\
\hline & 2,5 & 2,6 & 2,0 & 2,6 & 1,7 \\
\hline & 5,0 & 5,1 & 4,2 & 5,2 & 3,5 \\
\hline & 10,0 & 10,1 & 8,6 & 10,2 & 7,9 \\
\hline
\end{tabular}

Nota: (1), (2), (3) e (4) correspondem a $P\left(S_{R} \geq x_{\alpha}\right), P\left(S_{R}^{\times} \geq x_{\alpha}\right)$. $P\left(R V \geq x_{\alpha}\right)$ e $P\left(R V^{\star} \geq x_{\alpha}\right)$, respectivamente. 
Tabela 3.9: Valores simulados de $P\left(S_{R} \geq x_{\alpha}\right)$. $P\left(S_{R}^{\times} \geq x_{\alpha}\right)$, $P\left(R V \geq x_{\alpha}\right)$ e $P\left(R V^{\prime \times} \geq x_{\alpha}\right)$ (em porcentagem). sendo $y_{l} \sim t-\operatorname{Student}\left(x_{l}^{\top} \beta, O^{2} .3\right) . l=1,2 \ldots, n$. supondo $\nu=4$.

\begin{tabular}{|c|c|c|c|c|c|}
\hline $\begin{array}{c}\text { Tamanho } \\
\text { da amostra } \\
n\end{array}$ & $\begin{array}{c}\text { Nível } \\
\text { nominal } \\
(\%)\end{array}$ & (1) & (2) & (3) & (4) \\
\hline \multirow{4}{*}{10} & 1,0 & 13,2 & 2.7 & 32,3 & 0,3 \\
\hline & 2,5 & 20,0 & 7,7 & 40,5 & 1,4 \\
\hline & 5,0 & 27,3 & 13.3 & 47,1 & 3,8 \\
\hline & 10,0 & 38,1 & 20.0 & 55,0 & 9,3 \\
\hline \multirow{4}{*}{20} & 1,0 & 2,6 & 1,3 & 3,8 & 0,6 \\
\hline & 2,5 & 5,5 & 2,9 & 7,1 & 1,8 \\
\hline & 5,0 & 9,2 & 5,5 & 11,3 & 3,9 \\
\hline & 10,0 & 15,9 & 10.2 & 18,6 & 8,2 \\
\hline \multirow{4}{*}{30} & 1,0 & 1,6 & 0,9 & 2,6 & 0,7 \\
\hline & 2,5 & 3,9 & 2,4 & 5,8 & 2,3 \\
\hline & 5,0 & 7,6 & 5,1 & 9,8 & 5,0 \\
\hline & 10,0 & 13,9 & 10,4 & 16,3 & 10,1 \\
\hline \multirow{4}{*}{40} & 1,0 & 1,5 & 1,0 & 2,0 & 0,9 \\
\hline & 2,5 & 3,6 & 2.6 & 4,2 & 2,3 \\
\hline & 5,0 & 6,7 & 4,8 & 7,7 & 4,6 \\
\hline & 10,0 & 12,7 & 10.2 & 13,9 & 9,6 \\
\hline \multirow{4}{*}{50} & 1,0 & 1,4 & 1,1 & 1,6 & 0,9 \\
\hline & 2,5 & 3,3 & 2.6 & 3,7 & 2,2 \\
\hline & 5,0 & 6,4 & 4,9 & 6,7 & 4,8 \\
\hline & 10,0 & 11,5 & 10,2 & 12,2 & 9,2 \\
\hline \multirow{4}{*}{60} & 1,0 & 1,4 & 1,0 & 1,4 & 0,9 \\
\hline & 2,5 & 3,2 & 2,5 & 3,4 & 2,2 \\
\hline & 5,0 & 5,9 & 4,9 & 6,4 & 4,7 \\
\hline & 10,0 & 11,8 & 10.2 & 12,4 & 9,6 \\
\hline
\end{tabular}

Nota: (1), (2), (3) e (4) correspondem a $P\left(S_{R} \geq x_{\alpha}\right), P\left(S_{R}^{\times} \geq x_{\alpha}\right)$, $P\left(R V \geq x_{\alpha}\right)$ e $P\left(R V^{*} \geq x_{\alpha}\right)$, respectivamente. 
Tabela 3.10: Valores simulados de $P\left(S_{R} \geq x_{\alpha}\right)$. $P\left(S_{R}^{*} \geq x_{\alpha}\right)$. $P\left(R V^{\circ} \geq x_{0}\right)$ e $P\left(R V^{\star} \geq x_{0}\right)$ (em porcentagem), sendo $y_{l} \sim t-\operatorname{Student}\left(x_{l}^{\top} \beta, o^{2}, 5\right), l=1,2 \ldots, n$, supondo $\nu=4$.

\begin{tabular}{|c|c|c|c|c|c|}
\hline $\begin{array}{c}\text { Tamanho } \\
\text { da amostra } \\
n\end{array}$ & $\begin{array}{c}\text { Nível } \\
\text { nominal } \\
(\%)\end{array}$ & (1) & (2) & (3) & (4) \\
\hline \multirow{4}{*}{10} & 1,0 & 12,9 & 2,7 & 32,1 & 0,4 \\
\hline & 2,5 & 19,6 & 7,7 & 39,5 & 1,5 \\
\hline & 5,0 & 26,2 & 13,0 & 46,6 & 3,7 \\
\hline & 10,0 & 37,1 & 20,0 & 53,8 & 9,1 \\
\hline \multirow{4}{*}{20} & 1,0 & 3,3 & 1,7 & 4,3 & 0,9 \\
\hline & 2,5 & 6,5 & 3,6 & 8,1 & 2,1 \\
\hline & 5,0 & 10,5 & 6,5 & 12,7 & 4,4 \\
\hline & 10,0 & 17,2 & 11,6 & 20,0 & 9,4 \\
\hline \multirow{4}{*}{30} & 1,0 & 2.1 & 1,3 & 3,1 & 1,1 \\
\hline & 2,5 & 4,5 & 2,9 & 6,1 & 2,7 \\
\hline & 5,0 & 7,9 & 5,6 & 10,1 & 5,3 \\
\hline & 10,0 & 14,3 & 10,7 & 17,1 & 10,4 \\
\hline \multirow{4}{*}{40} & 1,0 & 1,8 & 1,3 & 2,3 & 1,1 \\
\hline & 2,5 & 4,1 & 3,1 & 4,8 & 2,6 \\
\hline & 5,0 & 7,6 & 5,8 & 8,4 & 5,3 \\
\hline & 10,0 & 13,8 & 11,1 & 14,8 & 10,4 \\
\hline \multirow{4}{*}{50} & 1,0 & 1,7 & 1,2 & 2,0 & 0,9 \\
\hline & 2,5 & 3,7 & 2,9 & 4,3 & 2,5 \\
\hline & 5,0 & 7,0 & 5,7 & 7,7 & 5,1 \\
\hline & 10,0 & 13,0 & 10,8 & 13,8 & 10,2 \\
\hline \multirow{4}{*}{60} & 1,0 & 1,4 & 1,1 & 1,6 & 0,9 \\
\hline & 2,5 & 3,5 & 2,8 & 3,8 & 2.5 \\
\hline & 5,0 & 6,6 & 5,5 & 6,9 & 5,1 \\
\hline & 10,0 & 12,3 & $10, \bar{T}$ & 12,8 & 10,1 \\
\hline
\end{tabular}

Nota: (1), (2), (3) e (4) correspondem a $P\left(S_{R} \geq x_{\alpha}\right), P\left(S_{R}^{\times} \geq x_{\alpha}\right)$, $P\left(R V \geq x_{\alpha}\right)$ e $P\left(R V^{\times} \geq x_{\alpha}\right)$, respectivamente. 
Tabela 3.11: Valores simulados de $P\left(S_{R}^{\prime} \geq x_{\alpha}\right) . P\left(S_{R}^{\times} \geq x_{\alpha}\right)$, $P\left(R V \geq x_{\alpha}\right)$ e $P\left(R V^{*} \geq x_{\alpha}\right)$ (em porcentagem). sendo $y_{l} \sim t-\operatorname{Student}\left(x_{l}^{\top} \beta, \phi^{2}, 6\right), l=1,2 \ldots, n$. supondo $\nu=4$.

\begin{tabular}{|c|c|c|c|c|c|}
\hline $\begin{array}{c}\text { Tamanho } \\
\text { da amostra } \\
n\end{array}$ & $\begin{array}{c}\text { Nivel } \\
\text { nominal } \\
(\%)\end{array}$ & (1) & (2) & $(3)$ & (4) \\
\hline \multirow{4}{*}{10} & 1,0 & 12,7 & 2,9 & 31,9 & 0,4 \\
\hline & 2,5 & 19,8 & 7,8 & 39,6 & 1,4 \\
\hline & 5,0 & 26,3 & 12,1 & 46,8 & 3,6 \\
\hline & 10,0 & 36,8 & 19,8 & 54,7 & 9,2 \\
\hline \multirow{4}{*}{20} & 1,0 & 3,5 & 1,9 & 4,5 & 0,9 \\
\hline & 2,5 & 6,5 & 3,8 & 8,2 & 2,3 \\
\hline & 5,0 & 10,8 & 6,6 & 12,5 & 4,7 \\
\hline & 10,0 & 17,3 & 11,9 & 19,8 & 9,4 \\
\hline \multirow{4}{*}{30} & 1,0 & 2.2 & 1,4 & 3,1 & 1,2 \\
\hline & 2,5 & 4,5 & 3,1 & 6,5 & 2,8 \\
\hline & 5,0 & 8,3 & 5,7 & 10,8 & 5,8 \\
\hline & 10,0 & 15,2 & 11,4 & 17,8 & 11,3 \\
\hline \multirow{4}{*}{40} & 1,0 & 2,0 & 1,5 & 2,4 & 1,2 \\
\hline & 2,5 & 4,2 & 3,1 & 4,9 & 2,6 \\
\hline & 5,0 & 7,7 & 5,9 & 8,8 & 5,4 \\
\hline & 10,0 & 14,1 & 11,4 & 15,3 & 10,9 \\
\hline \multirow{4}{*}{50} & 1,0 & 1,7 & 1,2 & 2,0 & 1,1 \\
\hline & 2,5 & 3,8 & 3,1 & 4,4 & 2,6 \\
\hline & 5,0 & 7,1 & 5,6 & 8,0 & 5,5 \\
\hline & 10,0 & 13,2 & 11,0 & 13,9 & 10,7 \\
\hline \multirow{4}{*}{60} & 1,0 & 1,7 & 1,3 & 1,9 & 1,2 \\
\hline & 2,5 & 3,9 & 3,1 & 4,3 & 2,8 \\
\hline & 5,0 & 7,0 & 5,9 & 7,5 & 5,5 \\
\hline & 10,0 & 13,3 & 11.2 & 13,6 & 10,7 \\
\hline
\end{tabular}

Nota: (1), (2), (3) e (4) correspondem a $P\left(S_{R} \geq x_{\alpha}\right), P\left(S_{R}^{\prime *} \geq x_{\alpha}\right)$, $P\left(R V \geq x_{\alpha}\right)$ e $P\left(R V^{*} \geq x_{\alpha}\right)$, respectivamente. 
Tabela 3.12: Valores simulados de $P\left(S_{R} \geq x_{\alpha}\right)$. $P\left(S_{R}^{\prime *} \geq x_{\alpha}\right)$, $P\left(R V \geq x_{\alpha}\right)$ e $P\left(R V^{*} \geq x_{\alpha}\right)$ (em porcentagem), sendo $y_{l} \sim t$ - Student $\left(x_{l}^{\top} \beta, \phi^{2}, 20\right), l=1,2, \ldots, n$. supondo $\nu=4$.

\begin{tabular}{|c|c|c|c|c|c|}
\hline $\begin{array}{c}\text { Tamanho } \\
\text { da amostra } \\
n\end{array}$ & $\begin{array}{c}\text { Nivel } \\
\text { nominal } \\
(\%)\end{array}$ & (1) & (2) & (3) & (4) \\
\hline \multirow{4}{*}{10} & 1,0 & 12,7 & 2,3 & 31,9 & 0,2 \\
\hline & 2,5 & 19,9 & 7,1 & 39,2 & 1,2 \\
\hline & 5,0 & 26,8 & 12,8 & 45,9 & 3,3 \\
\hline & 10,0 & 37,2 & 19,9 & 53,4 & 8,7 \\
\hline \multirow{4}{*}{20} & 1,0 & 3,7 & 2,1 & 4,8 & 1,1 \\
\hline & 2,5 & 6,7 & 4,1 & 8,4 & 2,7 \\
\hline & 5,0 & 11,0 & 6.8 & 12,6 & 4,8 \\
\hline & 10,0 & 17,2 & 11,9 & 19,4 & 9,7 \\
\hline \multirow{4}{*}{30} & 1,0 & 2.1 & 1,3 & 3,2 & 1,2 \\
\hline & 2,5 & 4,6 & 2,9 & 6,3 & 2,9 \\
\hline & 5,0 & 8,0 & 5,7 & 10,6 & 5,6 \\
\hline & 10,0 & 14,6 & 10,9 & 17,7 & 11,0 \\
\hline \multirow{4}{*}{40} & 1,0 & 2,4 & 1,8 & 2,8 & 1,4 \\
\hline & 2,5 & 5,0 & 3,6 & 5,8 & 3,3 \\
\hline & 5,0 & 8,6 & 6,7 & 9,7 & 6,3 \\
\hline & 10,0 & 14,7 & 11,9 & 16,3 & 11,7 \\
\hline \multirow{4}{*}{50} & 1,0 & 2,0 & 1,6 & 2,5 & 1,5 \\
\hline & 2,5 & 4,3 & 3,3 & 5,1 & 3,2 \\
\hline & 5,0 & 7,9 & 6,5 & 8,9 & 6,2 \\
\hline & 10,0 & 14,1 & 12,1 & 15,5 & 11,7 \\
\hline \multirow{4}{*}{60} & 1,0 & 1,7 & 1,4 & 2,0 & 1,3 \\
\hline & 2,5 & 3,8 & 3,1 & 4,6 & 2,9 \\
\hline & 5,0 & 7,4 & 6,2 & 8,2 & 6,1 \\
\hline & 10,0 & 13,4 & $11, \overline{1}$ & 14,4 & 11,4 \\
\hline
\end{tabular}

Nota: (1), (2), (3) e (4) correspondem a $P\left(S_{R} \geq x_{\alpha}\right) \cdot P\left(S_{R}^{*} \geq x_{\alpha}\right)$, $P\left(R V \geq x_{\alpha}\right)$ e $P\left(R V^{*} \geq x_{\alpha}\right)$, respectivamente. 


\subsection{Aplicações}

Nesta seção apresentamos aplicações de nossos resultados a dois conjuntos de dados reais. A primeira aplicaçào trata de um modelo de regressào simples e à segunda de um modelo de regressão múltipla.

\subsubsection{Aplicação 1}

Binkes e Dodge (1993, cap.1) analisam um conjunto de dados de 14 países das Américas do Norte e Central com populações acima de um milhão de habitantes no ano de 1985 . Para cada país, foram consideradas duas variáveis: a taxa de nascimento (número de nascimentos por 1.000 habitantes) e o percentual urbano (percentual da população que vive em cidades acima de 100.000 habitantes). A Tabela 3.13 mostra os dados.

Tabela 3.13: Taxas de nascimento e percentuais urbanos em 14 países das Américas do Norte e Central no ano de 1985.

\begin{tabular}{lcc}
\hline País & Taxa de nascimento & Percentual urbano \\
& $y_{l}$ & $x_{l}$ \\
\hline Canadá & 16,2 & 55,0 \\
Costa Rica & 30,5 & 27,3 \\
Cuba & 16,9 & 33,3 \\
República Dominicana & 33,1 & 37,1 \\
El Salvador & 40,2 & 11,5 \\
Estados Unidos & 16,0 & 56,5 \\
Guatemala & 38,4 & 14,2 \\
Haiti & 41,3 & 13,9 \\
Honduras & 43,9 & 19,0 \\
Jamaica & 28,3 & 33,1 \\
México & 33,9 & 43,2 \\
Nicarágua & 44,2 & 28,5 \\
Panamá & 28,0 & 37,7 \\
Trinidad Tobago & 24,6 & 6,8 \\
\hline
\end{tabular}

Fonte: Binkes e Dodge (1993).

Na Figura 3.1 apresentamos um diagrama de dispersão das duas variáveis estudadas. Nota-se que há indicação de uma relaçào linear entre as variáveis. A figura mostra também a reta de mínimos quadrados e fica claro que o ponto correspondente a Trinidad Tobago se destaca dos demais.

Para estes dados, consideramos um modelo linear da forma:

$$
y_{l}=\beta_{0}+\beta_{1} x_{l}+\phi z_{l}, \quad l=1, \ldots, 14,
$$

onde os erros $z$ são variáveis aleatórias independentes e identicamente distribuídas. Consideramos as seguintes distribuiçòes simétricas para os erros: normal padrào. $t$-Student 


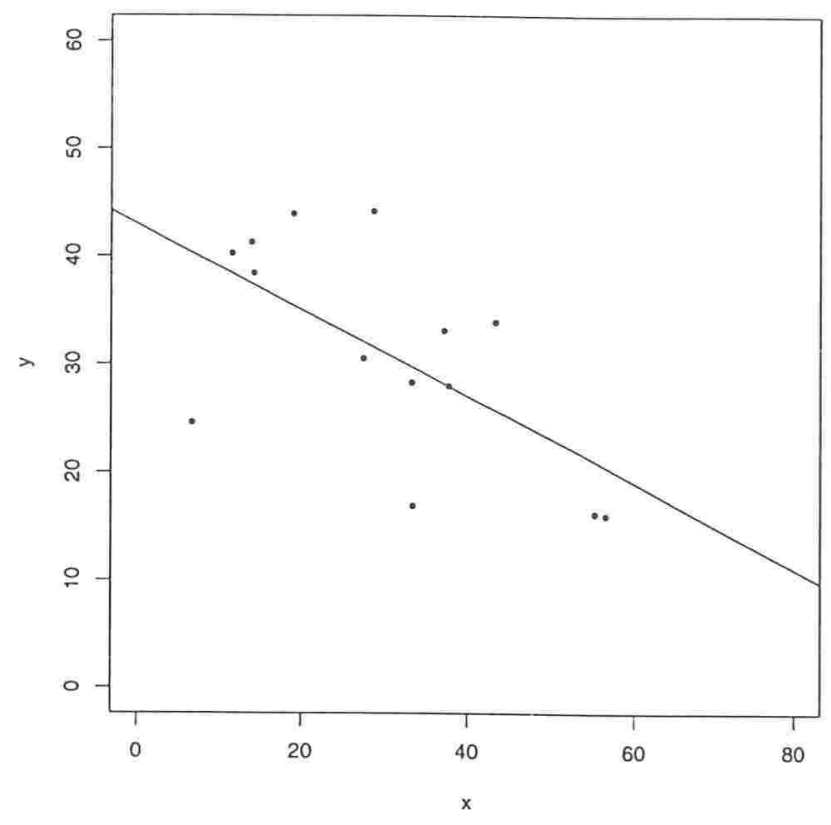

Figura 3.1: Diagrama de dispersão

com quatro graus de liberdade. exponencial potência $\operatorname{com} k=0,4$ e logística generalizada com $m=0,5\left(\alpha(m)=\left(2 \psi^{\prime}(m)\right)^{1 / 2}\right)$. A motivação para se utilizar os modelos com caudas mais pesadas do que as da distribuição normal vem do fato de haver ao menos uma observação aparentemente discrepante (Trinidad Tobago). Na Tabela 3.14 apresentamos as estimativas de máxima verossimilhança de $\beta_{0}, \beta_{1}$ e $\phi$ sob os diversos modelos adotados. Evidentemente, os resultados relativos ao modelo normal coincidem com as estimativas de mínimos quadrados.

Tabela 3.14: Estimativas de máxima verossimilhança para os parâmetros segundo os diversos modelos adotados

\begin{tabular}{lccc}
\hline & \multicolumn{3}{c}{ Estimativas } \\
Distribuição & $\widehat{\beta}_{0}$ & $\widehat{\beta}_{1}$ & $\hat{\phi}$ \\
\hline$N(0,1)$ & 42,991 & $-0,399$ & 7,549 \\
& $(4,485)$ & $(0,133)$ & $(1,426)$ \\
$t(0,1,4)$ & 46,432 & $-0,491$ & 5,758 \\
& $(4,048)$ & $(0.119)$ & $(1,439)$ \\
$E P(0,1,0.4)$ & 45,524 & $-0,481$ & 5,137 \\
& $(4,173)$ & $(0.123)$ & $(1,148)$ \\
$L G(0.1,0.5)$ & 46.303 & $-0,494$ & 7,525 \\
& $(4.025)$ & $(0.119)$ & $(1,810)$ \\
\hline
\end{tabular}

Nota: Entre parênteses são dados os desvios padrão dos estimadores. 
Tabela 3.15: Estimativas de máxima verossimilhança para os parâmetros segundo os diversos modelos adotados sem considerar o país de Trinidad Tobago

\begin{tabular}{lccc}
\hline & \multicolumn{3}{c}{ Estimativas } \\
Distribuição & $\hat{\beta}_{0}$ & $\widehat{\beta}_{1}$ & $\widehat{\phi}$ \\
\hline$N(0,1)$ & 48,972 & $-0,549$ & 6,043 \\
& $(4,110)$ & $(0,117)$ & $(1,185)$ \\
$t(0,1,4)$ & 48,719 & $-0,550$ & 4,638 \\
& $(3,733)$ & $(0,107)$ & $(1.203)$ \\
$E P(0,1,0.4)$ & 48,768 & $-0,562$ & 4,102 \\
& $(3,815)$ & $(0,109)$ & $(0,951)$ \\
$L G(0,1,0.5)$ & 48,542 & $-0,552$ & 6,021 \\
& $(3,687)$ & $(0,106)$ & $(1,503)$ \\
\hline
\end{tabular}

Nota: Entre parênteses são dados os desvios padrão dos estimadores.

Para avaliar a adequação do modelo normal, podemos comparar o modelo ajustado, isto é, $\widehat{y}_{l}=42,991-0,399 x_{l}$ com o modelo ajustado sem o ponto discrepante (Trinidad Tobago foi eliminado). Eliminando este ponto, e considerando somente os 13 pontos restantes, encontra-se o seguinte modelo ajustado: $\widehat{y}_{l}=48,972-0,549 x_{l}$, que é consideravelmente diferente da equação acima. Isto nos leva a crer que a suposição de normalidade não é adequada. Na Tabela 3.15 mostramos as estimativas de máxima verossimilhança dos parâmetros eliminando o país Trinidad Tobago e considerando os diversos modelos alternativos ao normal. Comparando as informaçôes desta tabela com as da Tabela 3.14, fica claro que a eliminação deste país tem bem menor influência nos ajustes se os modelos alternativos são utilizados em lugar do modelo normal. Nota-se também que, quando este país estava presente, os modelos alternativos levaram a equações parecidas entre si e razoavelmente diferentes daquela obtida com o modelo normal. Já, sem este país, todos os modelos levam a equações semelhantes. Esta análise indica que o modelo normal não é adequado ao problema e que os modelos alternativos são bem mais apropriados.

Na Tabela 3.16 apresentamos os valores das estatísticas escore e da razão de verossimilhança e de suas versões corrigidas para o teste de $H_{0}: \beta_{1}=0$ versus a alternativa $H_{1}: \beta_{1} \neq 0$. Para a distribuição exponencial potência, as estatísticas corrigidas não foram apresentadas, pois os resultados sobre correções para esta distribuição somente são válidos para $-1<k<-1 / 2$ e, neste estudo, consideramos $k=0,4$. Como era de se esperar, há diferenças entre os valores das estatísticas escore $S_{R}$ e da razão de verossimilhança $R V$ (sob todos os modelos considerados). No entanto, as diferenças entre os valores das correspondentes estatísticas corrigidas $\left(S_{R}^{*}\right.$ e $\left.R V^{*}\right)$ são bem menores. Além disto. todos os testes corrigidos levam a níveis descritivos menores do que $5 \%$, o que indica evidências contra a hipótese nula. 
Tabela 3.16: Valores das estatísticas $S_{R} . S_{R}^{\times}, R V$ e $R V^{*}$ para o teste $H_{0}: \beta_{1}=0$ versus $H_{1}: \beta_{1} \neq 0$ considerando diversos modelos.

\begin{tabular}{ccccc}
\hline Distribuição & $S_{R}$ & $S_{R}^{\times}$ & $R V$ & $R V^{\star}$ \\
\hline$N(0,1)$ & 5.402 & 5,479 & 6.825 & 5.606 \\
& $(0.020)$ & $(0,019)$ & $(0,009)$ & $(0.018)$ \\
$t(0,1,4)$ & 5.420 & 5,271 & 8,783 & 7.081 \\
& $(0,020)$ & $(0,022)$ & $(0,003)$ & $(0.008)$ \\
$L G(0,1,0.5)$ & 4,921 & 4,746 & 9,062 & 7.260 \\
& $(0.027)$ & $(0,029)$ & $(0,003)$ & $(0.007)$ \\
\hline
\end{tabular}

Nota: Entre parênteses são dados os níveis descritivos dos testes

\subsubsection{Aplicação 2}

Draper e Stoneman (1966) estudam a relação entre resistência $(y)$ de vigas de madeira e duas variáveis preditoras: peso específico $\left(x_{1}\right)$ e umidade $\left(x_{2}\right)$ das vigas. A Tabela 3.17 apresenta os dados referentes a 10 vigas.

Tabela 3.17: Dados em vigas de madeira

\begin{tabular}{cccc}
\hline Obs. & $\begin{array}{c}\text { Resistência } \\
\left(y_{l}\right)\end{array}$ & $\begin{array}{c}\text { Peso específico } \\
\left(x_{1 l}\right)\end{array}$ & $\begin{array}{c}\text { Umidade } \\
\left(x_{2 l}\right)\end{array}$ \\
\hline 1 & 11,14 & 0,499 & 11,1 \\
2 & 12,74 & 0,558 & 8,9 \\
3 & 13,13 & 0,604 & 8,8 \\
4 & 11,51 & 0,441 & 8,9 \\
5 & 12,38 & 0,550 & 8,8 \\
6 & 12,60 & 0,528 & 9,9 \\
7 & 11,13 & 0,418 & 10,7 \\
8 & 11,70 & 0,480 & 10,5 \\
9 & 11,02 & 0,406 & 10,5 \\
10 & 11,41 & 0,467 & 10,7 \\
\hline
\end{tabular}

Fonte: Draper e Stonemann (1966).

Para estes dados, Draper e Stoneman consideram o modelo de regressão linear múltipla da forma

$$
y_{l}=\beta_{0}+\beta_{1} x_{1 l}+\beta_{2} x_{2 l}+\phi z l \cdot l=1,2, \ldots, 10,
$$

onde os erros $\approx$ sào variáveis aleatórias independentes e identicamente distribuídas. Inicialmente, ajustamos o modelo assumindo uma distribuiçào normal para os erros. Um 
gráfico dos resíduos "estudentizados ${ }^{\prime} r_{\ell}=\left(y_{\ell}-x_{\ell}^{\top} \hat{\beta}\right) /\left(\hat{\phi} \sqrt{1-z_{\ell \ell}}\right)$. onde $z_{\ell \ell}$ é o $\ell$-ésimo elemento da diagonal da matriz de projeção $Z$ definida em (3.1). versus a ordem $\ell$ das observações é apresentado na Figura 3.2.

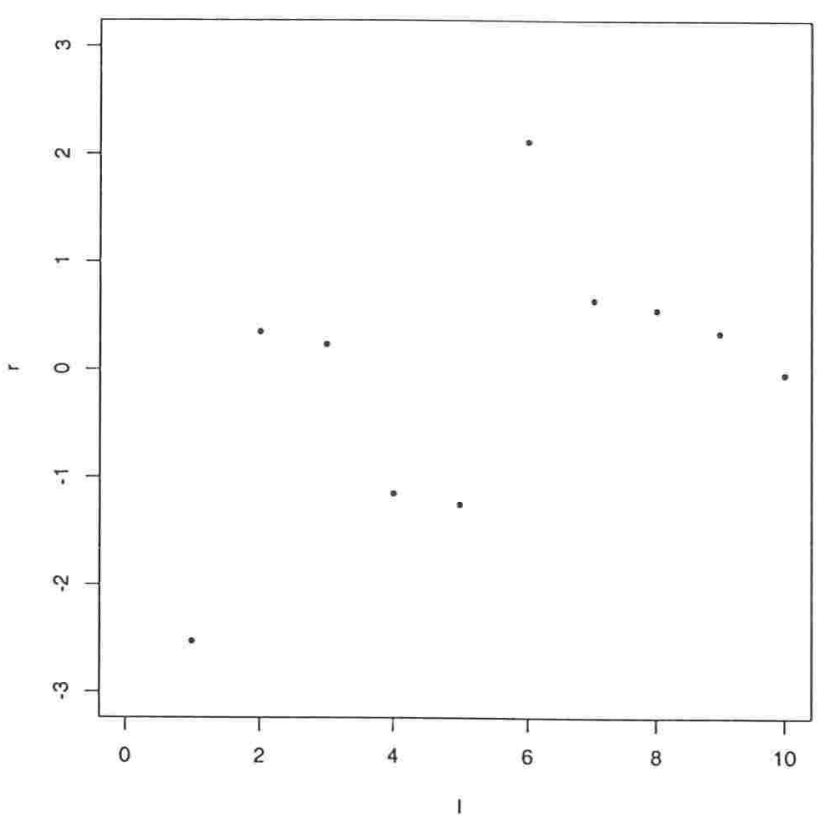

Figura 3.2: Gráfico dos resíduos "estudentizados"versus a ordem $\ell$ das observações.

Uma análise do gráfico indica que uma distribuição com caudas mais pesadas do que as da normal parece ser mais adequada. Em particular, há um ponto (primeira observação) bem discrepante dos demais. Ajustamos então algumas distribuições alternativas e apresentamos as estimativas dos parâmetros sob diversos modelos na Tabela 3.18.

Tabela 3.18: Estimativas de máxima verossimilhança dos parâmetros sob diversos modelos

\begin{tabular}{ccccc}
\hline Distribuição & $\widehat{\beta}_{0}$ & $\widehat{\beta}_{1}$ & $\widehat{\beta}_{2}$ & $\widehat{\phi}$ \\
\hline$N(0,1)$ & 10,302 & 8,493 & $-0,266$ & 0,230 \\
& $(1,583)$ & $(1,491)$ & $(0,103)$ & $(0,0514)$ \\
$t(0,1,4)$ & 9,068 & 9,231 & $-0,175$ & 0,159 \\
& $(1,295)$ & $(1,219)$ & $(0,084)$ & $(0,047)$ \\
$E P(0,1,0.5)$ & 9,368 & 9,095 & $-0,198$ & 0,138 \\
& $(1,682)$ & $(1,449)$ & $(0,113)$ & $(0,037)$ \\
$L G(0,1,0.4)$ & 9,104 & 9,226 & $-0,178$ & 0,217 \\
& $(1,345)$ & $(1.266)$ & $(0.087)$ & $(0.060)$ \\
\hline
\end{tabular}

Nota: Entre parênteses são dados os desvios padrão dos estimadores. 
Para avaliar a adequação dos modelos ajustados, retiramos a primeira observaçào (discrepante) e ajustamos novamente os diversos modelos. A Tabela 3.19 mostra os resultados. Notamos que. sob o modelo normal, a retirada da observaçào discrepante teve influência notável na reta de regressào enquanto que, sob os demais modelos, a influência é bem menos marcante. Isto indica que os modelos alternativos são mais adequados que o normal para este conjunto de dados.

Tabela 3.19: Estimativas de máxima verossimilhança para os parâmetros sob diversos modelos sem considerar a primeira observação

\begin{tabular}{ccccc}
\hline Distribuição & $\widehat{\beta}_{0}$ & $\widehat{\beta}_{1}$ & $\widehat{\beta}_{2}$ & $\widehat{\phi}$ \\
\hline$N(0,1)$ & 7,592 & 10,267 & $-0,073$ & 0,140 \\
& $(1,163)$ & $(1,002)$ & $(0,078)$ & $(0,032)$ \\
$t(0,1,4)$ & 8,148 & 9,833 & $-0,109$ & 0,106 \\
& $(1,042)$ & $(0,898)$ & $(0,070)$ & $(0,037)$ \\
$E P(0,1,0.5)$ & 8,028 & 9,862 & $-0,098$ & 0,087 \\
& $(1,275)$ & $(1,098)$ & $(0,085)$ & $(0,025)$ \\
$L G(0,1,0.4)$ & 8,139 & 9,844 & $-0,108$ & 0,135 \\
& $(0,986)$ & $(0,849)$ & $(0,066)$ & $(0,041)$ \\
\hline
\end{tabular}

Nota: Entre parênteses são dados os desvios padrão dos estimadores.

Tabela 3.20: Valores das estatísticas $S_{R}, S_{R}^{*}, R V$ e $R V^{*}$ para o teste de $H_{0}: \beta_{2}=0$ versus $\beta_{2} \neq 0$, sob diversos modelos

\begin{tabular}{ccccc}
\hline Distribuição & $S_{R}$ & $S_{R}^{*}$ & $R V$ & $R V^{*}$ \\
\hline$N(0,1)$ & 3,971 & 3,368 & 5,068 & 3,295 \\
& $(0,046)$ & $(0,067)$ & $(0,024)$ & $(0,047)$ \\
$t(0,1,4)$ & 0,665 & 0,474 & 3,205 & 1,955 \\
& $(0,414)$ & $(0,491)$ & $(0,073)$ & $(0,162)$ \\
$L G(0,1,0.4)$ & 2,946 & 2,437 & 3,666 & 2,139 \\
& $(0,086)$ & $(0,119)$ & $(0,056)$ & $(0,144)$ \\
\hline
\end{tabular}

Nota: Entre parênteses são dados os níveis descritivos dos testes. 
Tabela 3.21: Valores das estatísticas $S_{R}, S_{R}^{*}, R V$ e $R V^{\star *}$ para o teste de $H_{0}: \beta_{2}=0$ versus $H_{1}: \beta_{2} \neq 0$. sob diversos modelos sem considerar a primeira observaçào

\begin{tabular}{ccccc}
\hline Distribuição & $S_{R}$ & $S_{R}^{\times}$ & $R V$ & $R V^{\star}$ \\
\hline$N(0,1)$ & 0,737 & 0,481 & 0,769 & 0.470 \\
& $(0,391)$ & $(0,488)$ & $(0,381)$ & $(0,493)$ \\
$t(0,1,4)$ & 1,573 & 1,134 & 2,074 & 1,162 \\
& $(0,209)$ & $(0,286)$ & $(0,149)$ & $(0.281)$ \\
$L G(0,1,0.4)$ & 2,643 & 2,088 & 2,526 & 1,336 \\
& $(0,104)$ & $(0,148)$ & $(0,112)$ & $(0.247)$ \\
\hline
\end{tabular}

Nota: Entre parênteses são dados os níveis descritivos dos testes.

Nas Tabelas 3.20 e 3.21 apresentamos os valores das estatísticas escore, razão de verossimilhança e suas versões corrigidas para o teste de $H_{0}: \beta_{2}=0$ versus $H_{1}: \beta_{2} \neq 0$. Consideramos na Tabela 3.20 todas as observações e na Tabela 3.21 desconsideramos a primeira observação discrepante. Analisando estas duas tabelas podemos observar que o dado discrepante muda consideravelmente os níveis descritivos dos testes quando é utilizado o modelo normal. Já nos modelos alternativos, estas mudanças são bem menores. Em particular, se tomarmos um nível de significancia de $10 \%$, os testes corrigidos no modelo alternativo não levam a rejeição de $H_{0}$ tanto se o dado discrepante for incluído quanto se for excluído. Já, se o modelo normal for considerado, a hipótese $H_{0}$ é rejeitada a este nível se o ponto discrepante for incluído e não é rejeitado em caso contrário. Este exemplo mostra a robustez a observações discrepantes dos modelos simétricos estudados nos Capítulos 2 e 3.

\subsection{Considerações finais}

Finalizando este capítulo, ressaltamos que nosso objetivo foi encontrar expressões simples e de fácil aplicação prática para os coeficientes dos fatores de correção de Bartlett para a estatística da razão de verossimilhança e tipo Bartlett para a estatística escore nos modelos de regressão linear da família simétrica, associados a vários testes de hipóteses. Em todos os casos, consideramos os parâmetros adicionais de cada distribuição (como o número de graus de liberdade da distribuição $t$-Student por exemplo) como conhecidos, ou fixados em algum valor "plausível". Em trabalhos futuros, temos intenção de obter correções para os testes considerando estes parâmetros desconhecidos. Além disto, pretendemos realizar, futuramente, estudos de simulação para verificar o desempenho dos testes corrigidos assumindo outras estruturas para a matriz modelo $X$ que não a considerada neste trabalho e outras distribuições para os erros que não a t-Student. 


\section{Capítulo 4}

\section{Vício de estimadores de máxima verossimilhança e poder de testes em modelos de regressão linear usando uma família simétrica}

\subsection{Introdução}

O primeiro objetivo deste capítulo é obter fórmulas gerais para os vícios até ordem $n^{-1}$ dos estimadores de máxima verossimilhança de parâmetros em diversas distribuições nos modelos simétricos definidos em (2.1) e (2.2). Para obter estes vícios utilizaremos o trabalho desenvolvido por Cox e Snell (1968) que foi apresentado na Seção 1.3. O segundo objetivo é obter expansões até ordem $n^{-1 / 2}$ para as funções de poder de testes da razão de verossimilhança, de Wald e escore em modelos simétricos, sob uma seqüência de alternativas de Pitman, utilizando os trabalhos desenvolvidos por Hayakawa (1975) e Harris e Peers (1980) e apresentados na Seção 4.2. Na Seção 4.3, forneceremos fórmulas explícitas para a diferença entre os poderes de quaisquer dois destes testes e compararemos as funções de poder dos três testes.

\subsection{Vício de ordem $n^{-1}$ para os estimadores de máxi- ma verossimilhança nos modelos simétricos}

Sabe-se que os estimadores de máxima verossimilhança podem ser viciados e seus vícios podem ser consideráveis especialmente quando o tamanho da amostra, $n$, é pequeno ou quando a informação total de Fisher é pequena. Nesta seção. obtemos uma expressão fechada para o vício, até ordem $n^{-1}$. dos estimadores de máxima verossimilhança dos parâmetros nos modelos simétricos. Determinamos estimadores de máxima verossimilhança corrigidos pelo vício e comparamos estes estimadores com os de máxima verossimilhança usual em termos do erro quadrático médio até ordem $n^{-1}$. 
Sejam $y_{1}, \ldots y_{n} n$ variáveis aleatórias independentes, cada $y_{l}$ tendo uma função densidade definida em (2.1). com o vetor de parâmetros $\mu=\left(\mu_{1}, \ldots, \mu_{n}\right)^{\top}$ definido em (2.2). Seja $\theta=\left(\beta^{\top} . \phi\right)^{\top}$ o vetor de parâmetros do modelo. e seja $\hat{\theta}=\left(\hat{\beta}^{\top} . \hat{o}\right)^{\top}$ o estimador de máxima verossimilhança de $\theta$. Os estimadores $\widehat{\beta}$ e $\hat{o}$ podem ser obtidos numericamente através dos algoritmos iterativos definidos na Seção 2.5.

A expansão assintótica até ordem $n^{-1}$ do vício de $\hat{\theta}_{a}$, para $a=1,2 \ldots, p, p+1$, onde $\hat{\theta}_{a}$ representa o $a$-ésimo componente de $\hat{\theta}=\left(\widehat{\beta}^{\top}, \widehat{\phi}\right)^{\top}$ é dada por

$$
E\left(\hat{\theta}_{a}-\theta_{a}\right)=B_{\hat{\theta}_{a}}(\theta)+O\left(n^{-2}\right)
$$

onde $B_{\widehat{\theta}_{a}}(\theta)$ é o vício de ordem $n^{-1}$ para o estimador de máxima verossimilhança $\widehat{\theta}_{a}$, e é obtido da expressão geral de Cox e Snell (1968), apresentada na Seção 1.3. Os cumulantes envolvidos na obtenção do vício foram introduzidos no Capítulo 3.

Consideremos, primeiramente, o caso em que o parâmetro $\phi$ é conhecido. Nos modelos simétricos temos $\kappa_{r s}^{(t)}=\kappa_{r s t}=0 \mathrm{e}$, portanto, de $(1.20)$, temos que o vício $B_{\widehat{\beta}_{i}}(\beta)=0$, para $i=1, \ldots, p$. Em outras palavras, o vício de $\hat{\beta}$ para $i=1, \ldots, p$ é de ordem $n^{-2}$. Por outro lado, se $\phi$ é desconhecido, o vício de $\hat{\theta}_{a}$, para $a=1, \ldots, p$, é da forma

$$
B_{\widehat{\beta}_{a}}(\theta)=\sum^{\prime} \kappa^{a r} \kappa^{\phi \phi}\left(\kappa_{r \phi}^{(\phi)}-\frac{1}{2} \kappa_{r \phi \phi}\right),
$$

onde $\sum^{\prime}$ denota o somatório sobre todos os componentes do vetor de parâmetros $\beta$. Devido $\backslash$ ? à ortogonalidade global de $\beta$ e $\phi$ e a simetria da família em estudo, tem-se $\kappa_{r \phi}^{(\phi)}=\kappa_{r \phi \phi}=0$. Assim, o vício de ordem $n^{-1}$ de $\widehat{\beta}$ é zero, isto é, $B_{\widehat{\beta}_{a}}(\theta)=0$, para $a=1, \ldots, p$. Ou seja, mesmo sendo $\phi$ desconhecido, o vício de $\widehat{\beta}$ é de ordem $n^{-2}$. Este resultado não é óbvio já que o estimador de máxima verossimilhança de $\beta$ é obtido de uma equação não-linear.

O estimador de máxima verossimilhança do parâmetro de escala $\phi$ em modelos simétricos é solução de (2.11). Seja $B_{\widehat{\phi}}(\theta)$ o vício de ordem $n^{-1}$ de $\widehat{\phi}$. Da expressão geral de Cox e Snell (1968) apresentada em (1.20) para o vício de ordem $n^{-1}$ dos estimadores de máxima verossimilhança podemos escrever

$$
B_{\widehat{\phi}}(\theta)=-\frac{1}{2} \kappa^{\phi \phi} \sum^{\prime} \kappa^{r s} \kappa_{\phi r s}+\kappa^{\phi \phi 2}\left(\kappa_{\phi \phi}^{(\phi)}-\frac{1}{2} \kappa_{\phi \phi \phi}\right) .
$$

Substituindo os cumulantes obtidos na Seção 3.2 na equação acima, o vício de $\widehat{\phi}$, até ordem $n^{-1}$, é dado pela expressão simples (vide Apêndice D)

$$
B_{\widehat{\phi}}(\theta)=\frac{\phi}{n}\left(r_{1}-r_{2} p\right)
$$

onde

$$
\begin{aligned}
& r_{1}=\frac{\delta_{(0,0,1,0,3)}+2 \delta_{(0,1,0,0,2)}}{2\left(\delta_{(0,1,0,0,2)}-1\right)^{2}}, \\
& r_{2}=\frac{\delta_{(0,0,1,0,1)}+2 \delta_{(0.1 .0 .0 .0)}}{2\left(\delta_{(2,0,0,0,0)}\left(\delta_{(0,1,0,0,2)}-1\right)\right.},
\end{aligned}
$$




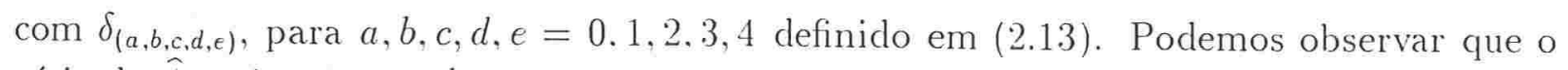
vício de $\hat{\phi}$, até ordem $n^{-1}$, é uma função que depende somente da distribuição assumida para os dados, através dos $\delta$ 's, e do número total de parâmetros $(p)$.

Podemos então definir um estimador de máxima verossimilhança corrigido pelo vício da forma

$$
\widehat{\phi}_{c}=\hat{\phi}-B_{\widehat{\phi}}(\hat{\theta})
$$

Assim,

$$
\widehat{\phi}_{c}=\widehat{\phi}\left\{1-\frac{1}{n}\left(r_{1}-r_{2} p\right)\right\} .
$$

É fácil ver que $\widehat{\phi}_{c}$ dado em (4.4) é um estimador não viciado de $\phi$ até ordem $n^{-1}$, isto é, $E\left(\widehat{\phi}_{c}-\phi\right)=O\left(n^{-2}\right)$.

No que segue, apresentamos os vícios do estimador de máxima verossimilhança $\widehat{\phi}$, até ordem $n^{-1}$, dado na equação (4.1) para diversas distribuições pertencentes à família simétrica.

(i) Normal: $y_{l} \sim N\left(x_{l}^{\top} \beta, \phi^{2}\right)$;

$$
B_{\widehat{\phi}}(\theta)=-\phi(1+2 p) /(4 n) .
$$

(ii) Cauchy: $y_{l} \sim C\left(x_{l}^{\top} \beta, \phi^{2}\right)$;

$$
B_{\widehat{\phi}}(\theta)=\phi(1-p) / n
$$

(iii) $t$-Student: $y_{l} \sim t\left(x_{l}^{\top} \beta, \phi^{2}, \nu\right), \nu>0$ conhecido;

$$
B_{\widehat{\phi}}(\theta)=-\frac{\phi(\nu+2)(\nu+3)}{4 n \nu(\nu+5)}\left(2 p+\frac{\nu-7}{\nu+2}\right) .
$$

(iv) $t$-Student generalizada: $y_{l} \sim t G\left(x_{l}^{\top} \beta, \phi^{2}, s, r\right), r, s>0$ conhecidos;

$$
B_{\widehat{\phi}}(\theta)=-\phi \frac{(r+2)(r+3)}{4 n r(r+5)}\left(2 p+\frac{r-7}{r+2}\right) .
$$

(v) Logística I: $y_{l} \sim \operatorname{LI}\left(x_{l}^{\top} \beta, \phi^{2}\right)$;

$$
B_{\widehat{\phi}}(\theta) \approx-\frac{\phi}{n}[0,475468 p+0,249724] .
$$

(vi) Logística II: $y_{l} \sim \operatorname{LII}\left(x_{l}^{\top} \beta, \phi^{2}\right)$;

$$
B_{\widehat{\phi}}(\theta) \approx-\frac{\phi}{n}[0,524449 p+0,05257] .
$$


(vii) Logística generalizada: $y_{l} \sim L G\left(x_{l}^{\top} \beta, \phi^{2} . m\right) . m>0$ conhecido;

$$
B_{\widehat{\phi}}(\theta)=-\frac{\phi(2 m+1)}{2 n(m+1)\left(2 m^{2} \psi^{\prime}(m+1)+2 m+1\right)}\left\{\frac{2 m^{2}(2 m+1) \psi^{\prime}(m+1)}{\left(2 m^{2} \psi^{\prime}(m+1)+2 m+1\right)}+(2 m+1) p\right\},
$$

onde $\psi^{\prime}(\cdot)$ é a primeira derivada da função digama. Utilizando a expansão dada em (3.12) para $m$ grande obtemos

$$
B_{\widehat{\phi}}(\theta)=-\frac{\phi}{n}\left[\left(\frac{1}{2}+\frac{1}{12 m^{2}}-\frac{1}{8 m^{3}}\right) p+\left(\frac{1}{4}-\frac{3}{8 m}+\frac{5}{8 m^{2}}-\frac{25}{96 m^{3}}\right)\right]+O\left(m^{-4}\right),
$$

e para $m$ pequeno de (3.13) obtemos

$$
\begin{aligned}
B_{\hat{\phi}}(\theta)= & -\frac{\phi}{n}\left[\left(\frac{1}{2}+\frac{m}{2}-\left(\frac{\pi^{2}}{6}+\frac{1}{2}\right) m^{2}+\left(\frac{1}{2}+2 \zeta(3)+\frac{\pi^{2}}{6}\right) m^{3}\right) p\right. \\
& \left.-\frac{\pi^{2}}{6} m^{2}+\left(2 \zeta(3)+\frac{5}{6} \pi^{2}\right) m^{3}\right]+O\left(m^{4}\right) .
\end{aligned}
$$

(viii) Exponencial potência: $y_{l} \sim E P\left(x_{l}^{\top} \beta, \phi^{2}, k\right),-1<k<-1 / 2$, conhecido;

$$
B_{\widehat{\phi}}(\theta)=-\frac{\phi}{4 n}(1-k+2 p) \text {. }
$$

Com a finalidade de comparar o estimador de máxima verossimilhança usual, $\widehat{\phi}$, e o corrigido, $\widehat{\phi}_{c}$, de $\phi$, até ordem $n^{-1}$, obtemos o erro quadrático médio de cada estimador. O erro quadrático médio do estimador corrigido $\widehat{\phi}_{c}$, até ordem $n^{-1}$, tem a forma

$$
\operatorname{EQM}\left(\widehat{\phi}_{c}\right)=\left\{1-\frac{2}{n}\left(r_{1}-r_{2} p\right)\right\} V(\widehat{\phi})+O\left(n^{-2}\right),
$$

onde $V(\widehat{\phi})$ é a variância do estimador $\widehat{\phi}$. E o erro quadrático médio de $\widehat{\phi}$, até ordem $n^{-1}$, é dado por

$$
\operatorname{EQM}(\widehat{\phi})=V(\widehat{\phi})+O\left(n^{-2}\right),
$$

e assim

$$
E Q M(\hat{\phi})-E Q M\left(\hat{\phi}_{c}\right)=\frac{2}{n}\left(r_{1}-r_{2} p\right) V(\hat{\phi})+O\left(n^{-2}\right) .
$$

A escolha entre $\hat{\phi}$ e $\widehat{\phi}_{c}$ pode ser baseada no sinal de $\left(r_{1}-r_{2} p\right)$, com $r_{1}$ e $r_{2}$ dados em (4.2) e (4.3), respectivamente. Uma análise da equação (4.5) mostra que para as distribuições normal, Cauchy $(p>1), t$-Student $(\nu \geq 1, p>1)$, $t$-Student generalizada $(r \geq 1$, $p>1)$, logística I e II, logística generalizada e exponencial potência $(-1<k<-1 / 2)$ temos $r_{1}-r_{2} p<0$, o que implica que $E Q M(\widehat{\phi})<E Q M\left(\widehat{\phi}_{c}\right)$, até ordem $n^{-1}$. Para a distribuição de Cauchy com $p=1$, temos que $E Q M(\widehat{\phi})=E Q M\left(\widehat{\phi}_{c}\right)$, até ordem $n^{-1}$.

É importante mencionar que a fórmula fechada do vício $B_{\hat{\phi}}(\theta)$ de segunda ordem é simples e nào depende da matriz modelo $X$ e sim da distribuição assumida para os dados. 
através dos $\delta$ 's, e do número de parâmetros do vetor $\beta$. Embora o cálculo de $B_{\widehat{\phi}}(\theta)$ seja fácil, ele tem difícil interpretação.

A validade da fórmula (4.1) pode ser comprovada no caso de $n$ variáveis aleatórias independentes e identicamente distribuídas, com distribuição normal de média desconhecida $\mu$ e variância desconhecida $\phi^{2}$. Neste caso, em (2.2), temos que a matriz $X=1_{n}$ e $\beta=\mu$ $(p=1)$. As estimativas de máxima verossimilhança de $\mu$ e $\phi^{2}$ são, respectivamente, $\widehat{\mu}=\bar{y}$ e $\widehat{\phi}^{2}=\sum\left(y_{i}-\bar{y}\right)^{2} / n$. Sabe-se que $\widehat{\mu} \sim N\left(\mu, \phi^{2} / n\right)$ e $\widehat{\phi}^{2} \sim \phi^{2} \chi_{(n-1)}^{2} / n$ e. conseqüentemente, $E(\widehat{\mu})=\mu$ e $E(\widehat{\phi})=(\phi / \sqrt{n}) 2^{1 / 2} \Gamma(n / 2) / \Gamma((n-1) / 2)$. Utilizando a expansão assintótica para a função gama para valores grandes do argumento (Abramowitz e Stegun, 1970, fórmula $6.1 .4 i$, p.25i) obtemos, até ordem $n^{-1}$,

$$
E(\widehat{o})=\phi\left\{1-\frac{3}{4 n}\right\}+O\left(n^{-2}\right)
$$

o que implica que $E(\widehat{\phi}-\phi)=-3 \phi /(4 n)+O\left(n^{-2}\right)$. Observa-se que este resultado coincide com o valor obtido para o vício do estimador do parâmetro $\phi$ até ordem $n^{-1}$, utilizando a expressão (4.1). Este resultado representa uma comprovação parcial da fórmula (4.1).

\subsection{Poderes de três testes clássicos em modelos si- métricos}

Nesta seção obtemos, usando os resultados de Hayakawa (1975) e Harris e Peers (1980) (vide Seção 1.4), expansões assintóticas até ordem $n^{-1 / 2}$ para as funções de poder dos testes da razão de verossimilhança, de Wald e escore em modelos simétricos, sob uma seqüência de hipóteses alternativas de Pitman. Estas expansões para as funções de poder dos três testes são feitas quando consideramos hipóteses sobre um subconjunto de parâmetros de regressão e sobre o parâmetro de escala do modelo.

\subsubsection{Testes de hipóteses sobre o parâmetro $\beta \operatorname{com} \phi$ conhecido}

Sejam $y_{1}, \ldots, y_{n} n$ variáveis aleatórias independentes, com cada $y_{l}$ tendo uma função densidade definida em (2.1) e o vetor de parâmetros $\mu=\left(\mu_{1}, \ldots, \mu_{n}\right)^{\top}$ estando relacionado com um vetor de parâmetros $\beta=\left(\beta_{1}, \ldots, \beta_{p}\right)^{\top}$ através da estrutura linear definida em (2.2).

Como na Seção 2.6.1, consideramos a partição $\beta=\left(\beta_{1}^{\top}, \beta_{2}^{\top}\right)^{\top}, \operatorname{com} \beta_{1}=\left(\beta_{i}, \ldots, \beta_{q}\right)^{\top}$ e $\beta_{2}=\left(\beta_{q+1}, \ldots, \beta_{p}\right)^{\top}$, que induz a partições adequadas da matriz . $X$, da função escore e da matriz de informação (vide Seção 2.5). Para testar a hipótese $H_{0}: \beta_{1}=\beta_{1}^{(0)}$ contra $H_{1}: \beta_{1} \neq \beta_{1}^{(0)}$, considerando 0 conhecido, podemos basear o teste nas estatísticas da razào de verossimilhança, de Wald e escore, definidas em (2.27), (2.28) e (2.31), e que chamaremos nesta seção de $S_{1}, S_{2}$ e $S_{3}$, respectivamente. Sabe-se que, para grandes amostras e em problemas regulares. a distribuiçào das três estatísticas, $S_{1}$. $S_{2}$ e $S_{3}$, sob $H_{0}$, é aproximadamente $\chi_{q}^{2}$ e, para uma seqüência de hipóteses de alternativas contíguas 
de Pitman convergindo para a hipótese nula à taxa de $n^{-1 / 2}$. ou seja, $H_{n}: \beta_{1}=\beta_{1}^{(0)}+\xi$. onde $\xi=\left(\xi_{1} \ldots \ldots \xi_{q}\right)^{\top} \operatorname{com} \xi_{i}=\beta_{i}-\beta_{i}^{(0)}$ suposto ser de ordem $n^{-1 / 2}$. é aproximadamente $\lambda_{q, \lambda}^{2}$, isto é, qui-quadrado não central com q graus de liberdade e um parâmetro de não centralidade $\lambda$ apropriado.

Substituindo nas equações (1.24) - (1.26) as expressòes dos cumulantes de derivadas do logaritmo da função de verossimilhança dos modelos simétricos obtidos na Seçào 3.2.1, encontramos que as quantidades $b_{i j}$ 's nos modelos simétricos sào nulos para $i=1,2,3 \mathrm{e}$ $j=0,1,2,3$. O parâmetro de nào-centralidade $\lambda$. de ordem um, tem a seguinte forma matricial

$$
\lambda=\frac{\delta_{(2,0,0,0,0)}}{\phi^{2}} \xi^{\top} R^{\top} R \xi
$$

onde a matriz $R$ é definida na Seção 2.6. Assim. as distribuições de $S_{1}, S_{2}$ e $S_{3}$ sob a seqüência de alternativas $H_{n}: \beta_{1}=\beta_{1}^{(0)}+\xi$ coincidem com a distribuição $\chi_{q, \lambda}^{2}$, até ordem $n^{-1 / 2}$, e com parâmetro de não-centralidade $\lambda$ dado em (4.6). Este resultado implica que $S_{1}, S_{2}$ e $S_{3}$ têm o mesmo poder até ordem $n^{-1 / 2}$.

\subsubsection{Teste de hipóteses sobre o parâmetro $\beta \operatorname{com} \phi$ desconhe- cido}

Consideramos nesta seção os testes da hipótese nula $H_{0}: \beta_{1}=\beta_{1}^{(0)}$ contra a alternativa $H_{1}: \beta_{1} \neq \beta_{1}^{(0)}$, onde $\beta_{1}^{(0)}$ é um vetor especificado de dimensão $q(q \leq p)$ e $\phi$ é desconhecido. Desenvolveremos expansões assintóticas para as funções distribuição de $S_{1}, S_{2}$ e $S_{3}$ definidas em (2.33) - (2.35), respectivamente, sob a seqüência de alternativas de Pitman.

A ortogonalidade global de $\beta$ e $\phi$ implica que a matriz de informação total de Fisher é bloco diagonal e tem a forma

$$
K=\left(\begin{array}{ccc}
K_{11} & K_{12} & 0 \\
K_{21} & K_{22} & 0 \\
0 & 0 & \kappa_{\phi, \phi}
\end{array}\right)=\left(\begin{array}{cc}
K_{11} & K_{12}^{*} \\
K_{21}^{*} & K_{22}^{*}
\end{array}\right),
$$

onde $K_{12}^{*}=K_{21}^{* \top}=\left[\begin{array}{ll}K_{12} & 0\end{array}\right]$ e $K_{22}^{*}=\operatorname{Diag}\left\{K_{22}, \kappa_{\phi, \phi}\right\}$. Uma vez que

$$
-K_{22}^{*-1} K_{21}^{*}=\left[\begin{array}{rr}
-K_{22}^{-1} & K_{21} \\
0 &
\end{array}\right]
$$

o vetor $\gamma$ de dimensão $p+1$ definido em (1.23) tem a forma

$$
\gamma=\left(\begin{array}{c}
I_{q} \\
-K_{22}^{-1} K_{21} \\
0
\end{array}\right) \xi
$$

e as matrizes $K_{21}$ e $K_{22}^{-1}$ são dadas em (2.25) e (2.26), respectivamente.

Os coeficientes $b_{i j}$ que determinam as expansões assintóticas das funções distribuiçòes das estatísticas $S_{1}, S_{2}$ e $S_{3}$ sob a seqüência de alternativas $H_{n}: \beta_{1}=\beta_{1}^{(0)}+\xi$, podem ser escritos como

$$
b_{i j}=b_{i j, 3}+b_{i j .3 \phi}, \quad i=1,2.3, j=0.1 .2,3,
$$


onde $b_{i j, \beta}$ é $b_{i j}$ obtido na Seçào 4.3.1. considerando o conhecido. e $b_{i j, \beta \phi}$ representa a contribuição adicional devido ao fato de $\dot{o}$ ser desconhecido.

A obtenção de $b_{i j, \beta \phi}$ dependerá de cumulantes conjuntos de derivadas do logaritmo da função de verossimilhança $L(\beta, \phi)$ definida em (2.4) com respeito aos parâmetros $\beta$ e $\phi$. Como $\beta$ e $\phi$ são globalmente ortogonais, as expressões das quantidades $b_{i j}$ se reduzem a

$$
\begin{aligned}
& b_{11, \beta \phi}=\frac{1}{2} \sum_{k=1}^{p}\left(\kappa_{\phi \phi k}+2 \kappa_{\phi, \phi k}\right) a_{\phi \phi} \gamma k . \quad b_{12, \beta \phi}=b_{13, \beta \phi}=0, \\
& b_{21, \beta \phi}=\frac{1}{2} \sum_{k=1}^{p}\left(\kappa_{\phi \phi k}+2 \kappa_{\phi, \phi k}\right) \kappa^{\phi, \phi} \gamma k, \quad b_{22, \beta \phi}=b_{23, \beta \phi}=0, \\
& b_{31, \beta \phi}=b_{11, \beta \phi}, \quad b_{32, \beta \phi}=b_{33, \beta \phi}=0,
\end{aligned}
$$

onde $\gamma_{l}$ é o $l$-ésimo elemento do vetor $\gamma$ definido em $(4.7)$ e $a_{\phi \phi}$ é o $(p+1, p+1)$-ésimo elemento da matriz $A$ definida na Seção 3.3.2, ou seja, $a_{\phi \phi}=\kappa_{\phi, \phi}^{-1}$. Como nos modelos simétricos, $\kappa_{\phi \phi r}=\kappa_{\phi, \phi r}=0$, para $r=1, \ldots, p$, temos em (4.9) - (4.11) que $b_{i j, \beta \phi}=0$, para $i=1,2,3, j=0,1,2,3$. Uma vez que $b_{i j, \beta}=0$ (da Seção 4.3.1), para $i=1,2,3 \mathrm{e}$ $j=1,2,3$, concluímos que da equação (4.\$) $b_{i j}=0, i=1,2,3$ e $j=0,1,2,3$. De forma análoga, temos que o parâmetro de não-centralidade $\lambda$ tem a mesma forma dada em (4.6). Assim, concluímos que, mesmo se $\phi$ for desconhecido, as distribuições de $S_{1}, S_{2}$ e $S_{3}$ sob a sequêencia de alternativas contíguas de Pitman coincidem com a distribuição $\chi_{q, \lambda}^{\prime 2}$, até ordem $n^{-1 / 2}$, o que implica que $S_{1}, S_{2}$ e $S_{3}$ têm o mesmo poder até esta ordem.

Podemos observar também que as distribuições de $S_{1}, S_{2}$ e $S_{3}$ sob $H_{n}$ são idênticas, até ordem $n^{-1 / 2}$, às correspondentes distribuiçôes obtidas no caso onde $\phi$ é conhecido.

\subsubsection{Teste de hipóteses sobre o parâmetro $\phi$}

Para testar a hipótese nula $H_{0}: \phi=\phi^{(0)}$ contra a alternativa $H_{1}: \phi \neq \phi^{(0)}$, podemos utilizar as estatísticas $S_{1}, S_{2}$ e $S_{3}$ definidas em (2.37) - (2.39), respectivamente. Nesta seção desenvolvemos expansões assintóticas para as funções distribuição de $S_{1}, S_{2}$ e $S_{3}$ sob uma seqüencia de hipóteses alternativas de Pitman $H_{n}: \phi=\phi^{(0)}+\xi$, $\operatorname{com} \xi=O\left(n^{-1 / 2}\right)$. Para isto, é necessário obter os termos $b_{i j}$ definidos em (1.24) - (1.26) que envolvem cumulantes conjuntos de derivadas do logaritmo da função de verossimilhança $L(\beta, \phi)$ definida em (2.4) com respeito aos parâmetros $\beta$ e $\phi$.

Utilizando as notações definidas na Seção 3.2 .2 para os cumulantes $\kappa_{r, \phi, \phi}, \kappa_{\phi, \phi, \phi}, \kappa_{\phi, \phi, r}$ etc. e a ortogonalidade global de $\beta$ e $\phi$, temos que o vetor $\gamma$ de dimensão $p+1$, definido em (1.23), é dado por

$$
\gamma=(0, \ldots, 0, \xi)^{\top},
$$

$\operatorname{com} \xi=\phi-\phi^{(0)}$ e que as matrizes $A$ e $M$, definidas na Seção 3.3.2. têm as formas

$$
A=\left(\begin{array}{cc}
0 & 0 \\
0 & K_{\beta, \beta}^{-1}
\end{array}\right) . \quad M=K^{-1}-A=\left(\begin{array}{cc}
\kappa_{\phi, o}^{-1} & 0 \\
0 & 0
\end{array}\right) .
$$

A estrutura de $\gamma$ (envolvendo $p$ elementos nulos) e a de $f$ e $M$ (envolvendo sub-matrizes nulas) simplificam consideravelmente a obtenção dos termos $b_{i j}$. Assim, das equaçòes 
$(1.24)-(1.26) \cdot$ temos

$$
\begin{aligned}
b_{11} & =\frac{1}{2}\left\{\kappa_{\phi, \phi \phi} \xi^{3}+\sum{ }^{\prime} \kappa_{i, j \phi} \kappa^{i, j} \xi\right\}, \\
b_{12} & =\frac{1}{6} \kappa_{\phi, \phi, \phi} \xi^{3}, \quad b_{13}=0, \\
b_{21} & =b_{11}+\frac{1}{2} \kappa_{\phi \phi \phi} \kappa^{\phi, \phi} \xi, \\
b_{22} & =-\frac{1}{2}\left\{\kappa_{\phi, \phi \phi} \xi^{3}+\kappa_{\phi \phi \phi} \kappa^{\phi, \phi} \xi\right\}, \quad b_{23}=-\frac{1}{6} \kappa_{\phi \phi \phi} \xi^{3}, \\
b_{31} & =b_{11}-b_{32}, \quad b_{32}=\frac{1}{2} \kappa_{\phi, \phi, \phi} \kappa^{\phi, \phi} \xi, \\
b_{33} & =b_{12} \\
\mathrm{e} \quad b_{10} & =b_{20}=b_{30}=-\left(b_{11}+b_{12}\right),
\end{aligned}
$$

onde $\sum^{\prime}$ representa o somatório sobre todos os componentes do vetor de parâmetros $\beta$. O parâmetro de não-centralidade $\lambda$ é dado por

$$
\lambda=n\left(\delta_{(2,0,0,0,2)}-1\right) \Delta^{2},
$$

onde

$$
\Delta=\left(\phi-\phi^{(0)}\right) / \phi^{(0)}
$$

é de ordem $n^{-1 / 2}$.

Substituindo os cumulantes conjuntos dos modelos simétricos definidos na Seção 2.3.2 em (4.12) - (4.14), temos

$$
\begin{aligned}
& b_{11}=\frac{1}{2}\left\{n\left(4 \delta_{(0,1,0,0,2)}+\delta_{(0,0,1,0,3)}-2\right) \Delta^{3}+\frac{\left(2 \delta_{(0,1,0,0,0)}+\delta_{(0,0,1,0,1)}\right)}{\delta_{(2,0,0,0,0)}} p \Delta\right\} \\
& b_{12}=\frac{n}{3}\left(\delta_{(1,1,0,0,3)}+1\right) \Delta^{3}, \quad b_{13}=0 \\
& b_{21}=b_{11}-\left(\frac{6 \delta_{(0,1,0,0,2)}+\delta_{(0,0,1,0,3)}-4}{\delta_{(2,0,0,0,2)}-1}\right) \frac{\Delta}{2} \\
& b_{22}=-\frac{1}{2}\left\{n\left(4 \delta_{(0,1,0,0,2)}+\delta_{(0,0,1,0,3)}-2\right) \Delta^{3}-\frac{\left(6 \delta_{(0,1,0,0,2)}+\delta_{(0,0,1,0,3)}-4\right)}{\delta_{(2,0,0,0,2)}-1} \Delta\right\} \\
& b_{23}=\frac{n}{6}\left(6 \delta_{(0,1,0,0,2)}+\delta_{(0,0,1,0,3)}-4\right) \Delta^{3} \\
& b_{31}=b_{11}-b_{32}, \\
& b_{32}=\frac{\left(\delta_{(1,1,0,0,3)}+1\right)}{\left(\delta_{(2,0,0,0,2)}-1\right)} \Delta . \quad b_{33}=b_{12} .
\end{aligned}
$$

A seguir apresentaremos as quantidades $b_{i j}, i=1,2.3, j=1.2,3$, definidas em (4.17) - (4.19), assim como $\lambda$ definida em (4.15), para diversos modelos simétricos. 
(i) Normal: $y_{l} \sim N\left(x_{l}^{\top} 3 . \phi^{2}\right)$;

$$
\begin{aligned}
& b_{11}=-\left(3 n \Delta^{3}+p \Delta\right), \quad b_{12}=\frac{4}{3} n \Delta^{3} . \quad b_{13}=0, \\
& b_{21}=-\left(3 n \Delta^{3}+(p-5 / 2) \Delta\right), \quad b_{22}=3 n \Delta^{3}-\frac{5}{2} \Delta, \\
& b_{23}=-\frac{5}{3} n \Delta^{3}, \\
& b_{31}=-\left(3 n \Delta^{2}+(p+2) \Delta\right), \quad b_{32}=2 \Delta, \quad b_{33}=\frac{4}{3} \Delta^{3}, \\
& \lambda=2 n \Delta^{2} .
\end{aligned}
$$

(ii) Cauchy: $y_{l} \sim C\left(x_{l}^{\top} \beta, \phi^{2}\right)$;

$$
\begin{aligned}
& b_{11}=-\left(\frac{1}{4} n \Delta^{3}+\frac{1}{2} p \Delta\right), \quad b_{12}=b_{13}=0, \\
& b_{21}=-\left(\frac{n}{4} \Delta^{3}+\left(\frac{p}{2}-\frac{3}{2}\right) \Delta\right), \quad b_{22}=\frac{n}{4} \Delta^{3}-\frac{3 \Delta}{2}, \\
& b_{23}=-\frac{n \Delta^{3}}{4}, \\
& b_{31}=-\left(\frac{n \Delta^{3}}{4}+\frac{p}{2} \Delta\right), \quad b_{32}=0, \quad b_{33}=0, \\
& \lambda=\frac{n}{2} \Delta^{2} .
\end{aligned}
$$

(iii) $t$-Student: $y_{l} \sim t\left(x_{l}^{\top} \beta, \phi^{2}, \nu\right), \nu>0$ conhecido;

$$
\begin{aligned}
& b_{11}=-\left(3 n \frac{\nu(\nu+1) \Delta^{3}}{(\nu+3)(\nu+5)}+p\left(\frac{\nu+2}{\nu+5}\right) \Delta\right), \\
& b_{12}=\frac{4}{3} n \frac{\nu(\nu-1)}{(\nu+3)(\nu+5)} \Delta^{3}, \quad b_{13}=0, \\
& b_{21}=-\left(3 n \frac{\nu(\nu+1)}{(\nu+3)(\nu+5)} \Delta^{3}+\left(\frac{2(\nu+2) p-(5 \nu+13)}{2(\nu+5)}\right) \Delta\right), \\
& b_{22}=3 n \frac{\nu(\nu+1)}{(\nu+3)(\nu+5)} \Delta^{3}-\left(\frac{5 \nu+13}{\nu+5}\right) \Delta, \\
& b_{23}=-\frac{n(5 \nu+13) \nu}{3(\nu+3)(\nu+5)} \Delta^{3}, \\
& b_{31}=-\left(3 n \frac{\nu(\nu+1)}{(\nu+3)(\nu+5)} \Delta^{3}+\left(\frac{p(\nu+2)+2(\nu-1)}{(\nu+5)}\right) \Delta\right), \\
& b_{32}=\frac{2(\nu-1)}{(\nu+5)} \Delta, \quad b_{33}=\frac{4 n \nu(\nu-1)}{3(\nu+3)(\nu+5)} \Delta^{3} . \\
& \lambda=\left(\frac{2 n \nu}{\nu+3}\right) \Delta .
\end{aligned}
$$


(iv) $t$-Student generalizada: $y_{l} \sim t G\left(x_{l}^{\top} \beta, \phi^{2}, s, r\right) . r . s>0$ conhecidos;

$$
\begin{aligned}
& b_{11}=-\left(3 n \frac{r(r+1)}{(r+3)(r+5)} \Delta^{3}+\left(\frac{r+2}{r+5}\right) p \Delta\right), \\
& b_{12}=\frac{4}{3} n \frac{r(r-1)}{(r+3)(r+5)} \Delta^{3}, \quad b_{13}=0, \\
& b_{21}=-\left(3 n \frac{r(r+1)}{r(r+3)(r+5)} \Delta^{3}+\left(\frac{2(r+2) p-(5 r+13)}{2(r+5)}\right) \Delta\right), \\
& b_{22}=3 n \frac{r(r+1)}{r(r+3)(r+5)} \Delta^{3}-\frac{(5 r+13)}{(r+5)} \Delta, \\
& b_{23}=-n \frac{r(5 r+13)}{3(r+3)(r+5)} \Delta^{3}, \\
& b_{31}=-\left(3 n \frac{r(r+1)}{(r+3)(r+5)} \Delta^{3}+\left(\frac{p(r+2)+2(r-1)}{(r+5)}\right) \Delta\right), \\
& b_{32}=\frac{2(r-1)}{(r+5)} \Delta, \quad b_{33}=\frac{4 n r(r-1)}{2(r+3)(r+5)} \Delta^{3}, \\
& \lambda=2 n \frac{r}{r+3} \Delta^{2} .
\end{aligned}
$$

(v) Logística I: $y_{l} \sim L I\left(x_{l}^{\top} \beta, \phi^{2}\right)$;

$$
\begin{aligned}
& b_{11} \approx-\left(5,282 n \Delta^{3}+1,4329 p \Delta\right), \quad b_{12} \approx 2,51515 n \Delta^{3}, \quad b_{12}=0, \\
& b_{21} \approx-\left(5,282 n \Delta^{3}+1,4329 p \Delta-2,7526 \Delta\right), \\
& b_{22} \approx 5,282 n \Delta^{3}-2,7526 \Delta, \quad b_{23} \approx-2,76526 n \Delta^{3}, \\
& b_{31} \approx-\left(5,282 n \Delta^{3}+1,4329 p \Delta+2,5036 \Delta\right), \quad b_{32} \approx 2,5036 \Delta, \\
& b_{33} \approx 2,51515 n \Delta^{3}, \quad \lambda \approx 3,01378 n \Delta^{2} .
\end{aligned}
$$

(vi) Logística II: $y_{l} \sim \operatorname{LII}\left(x_{l}^{\top} \beta, \phi^{2}\right)$;

$$
\begin{aligned}
& b_{11} \approx-\left(1,53745 n \Delta^{3}+0,75 p \Delta\right), \quad b_{12} \approx 0,54831 n \Delta^{3}, \quad b_{13}=0, \\
& b_{21} \approx-\left(1,53745 n \Delta^{3}+0,75 p \Delta-2,05643 \Delta\right), \\
& b_{22} \approx 1,53745 n \Delta^{3}-2,05643 \Delta, \quad b_{23} \approx-0,98914 n \Delta^{3}, \\
& b_{31} \approx-\left(1,53745 n \Delta^{3}+0,75 p \Delta+1,15033 \Delta\right), \quad b_{32} \approx 1,15033 \Delta, \\
& b_{33} \approx 0,54831 n \Delta^{3}, \quad \lambda \approx 1,42996 n \Delta^{2} .
\end{aligned}
$$

(vii) Logística generalizada: $Y_{i} \sim L G\left(x_{l}^{\top} \beta, \phi^{2}, m\right), m>0$ conhecido;

$$
\begin{aligned}
b_{11}= & -\frac{1}{2(2 m+1)(m+1)}\left\{2 n\left(m^{2}(4 m+1) \psi^{\prime}(m+1)+(2 m+1)(m+1)\right) \Delta^{3}\right. \\
& \left.+(2 m+1)^{2} p \Delta\right\}
\end{aligned}
$$




$$
\begin{aligned}
b_{12}= & \frac{n}{3(2 m+1)(m+1)}\left(6 m^{3} \imath^{\prime}(m+1)+(2 m+1)(m+1)\right) \Delta^{3}, \quad b_{13}=0, \\
b_{21}= & -\frac{1}{2(2 m+1)(m+1)}\left\{2 n\left(m^{2}(4 m+1) \psi^{\prime}(m+1)+(2 m+1)(m+1)\right) \Delta^{3}\right. \\
& \left.+\left((2 m+1) p^{2}-\frac{2(2 m+1)^{2}\left(3 m^{2} \psi^{\prime}(m+1)+2(m+1)\right)}{\left(2 m^{2} \psi^{\prime}(m+1)+2 m+1\right)}\right)\right\}, \\
b_{22}= & \frac{1}{(2 m+1)(m+1)}\left\{n\left(m^{2}(4 m+1) \psi^{\prime}(m+1)+(2 m+1)(m+1)\right) \Delta^{3}\right. \\
& -\frac{(2 m+1)^{2}\left(3 m^{2} \psi^{\prime}(m+1)+2(m+1)\right)}{\left(2 m^{2} \psi^{\prime}(m+1)+2 m+1\right)}- \\
b_{23}= & \frac{-n}{3(m+1)}\left(3 m^{2} \psi^{\prime}(m+1)+2(m+1)\right) \Delta^{3}, \\
b_{31}= & -\frac{1}{2(2 m+1)(m+1)}\left\{2 n\left(m^{2}(4 m+1) \psi^{\prime}(m+1)+(2 m+1)(m+1)\right) \Delta^{3}\right. \\
& \left.+(2 m+1)\left((2 m+1) p-\frac{2\left(6 m^{3} \psi^{\prime}(m+1)+(2 m+1)(m+1)\right)}{\left(2 m^{2} \psi^{\prime}(m+1)+2 m+1\right)}\right) \Delta\right\}, \\
b_{32}= & \frac{\left(6 m^{3} \psi^{\prime}(m+1)+(2 m+1)(m+1)\right)}{(m+1)\left(2 m^{2} \psi^{\prime}(m+1)+2 m+1\right)} \Delta^{n}, \\
b_{33}= & \frac{n}{3(2 m+1)(m+1)}\left(6 m^{3} \psi^{\prime}(m+1)+(2 m+1)(m+1)\right) \Delta^{3}, \\
\lambda= & n\left(\frac{2 m+1+2 m^{2} \psi^{\prime}(m+1)}{2 m+1}\right) \Delta,
\end{aligned}
$$

onde $\psi^{\prime}(\cdot)$ é a primeira derivada da função digama. Utilizando a expansão dada em (3.12) para valores de $m$ grandes, obtemos

$$
\begin{aligned}
b_{11}= & -\left(3+\frac{35}{m}+\frac{13}{3 m^{2}}-\frac{14}{3 m^{3}}\right) n \Delta^{3}-\frac{1}{2}\left(\frac{2 m+1}{m+1}\right) p \Delta+O\left(m^{-4}\right) \\
b_{12}= & \frac{1}{3}\left(4-\frac{6}{m}+\frac{8}{m^{2}}-\frac{9}{m^{3}}\right) n \Delta^{3}+O\left(m^{-4}\right), \\
b_{13}= & 0, \\
b_{21}= & -\left[\left(3-\frac{7}{2 m}+\frac{13}{3 m^{2}}-\frac{14}{3 m^{3}}\right) n \Delta^{3}+\frac{1}{2}\left(\frac{2 m+1}{m+1}\right) p \Delta\right. \\
& \left.+\left(-\frac{5}{2}+\frac{1}{m}-\frac{7}{6 m^{2}}+\frac{7}{6 m^{3}}\right) \Delta\right]+O\left(m^{-4}\right), \\
b_{22}= & \left(3-\frac{7}{2 m}+\frac{13}{3 m^{2}}-\frac{14}{3 m^{3}}\right) n \Delta^{3}-\left(\frac{5}{2}-\frac{1}{m}+\frac{7}{6 m^{2}}-\frac{7}{6 m^{3}}\right) \Delta+O\left(m^{-4}\right), \\
b_{23}= & \left(\frac{5}{3}-\frac{3}{2 m}+\frac{5}{3 m^{2}}-\frac{5}{3 m^{3}}\right) n \Delta^{3}+O\left(m^{-4}\right), \\
b_{31}= & -\left[\left(3-\frac{7}{2 m}+\frac{13}{3 m^{2}}-\frac{14}{3 m^{3}}\right) n \Delta^{3}+\frac{1}{2}\left(\frac{2 m+1}{m+1}\right) p \Delta\right. \\
& \left.+\left(2-\frac{2}{m}+\frac{i}{3 m^{2}}-\frac{7}{3 m^{3}}\right) \Delta\right]+O\left(m^{-4}\right),
\end{aligned}
$$




$$
\begin{aligned}
b_{32} & =\left(2-\frac{2}{m}+\frac{i}{3 m^{2}}-\frac{i}{3 m^{3}}\right) \Delta+O\left(m^{-4}\right), \\
b_{33} & =\frac{1}{3}\left(4-\frac{6}{m}+\frac{8}{m^{2}}-\frac{9}{m^{3}}\right) n \Delta^{3}+O\left(m^{-4}\right), \\
\lambda & =2-\frac{1}{m}+\frac{2}{3 m^{2}}-\frac{1}{3 m^{3}}+O\left(m^{-4}\right) .
\end{aligned}
$$

Para valores pequenos de $m$, utilizamos a expansão (3.13) e obtemos

$$
\begin{aligned}
& b_{11}=-\left(1+\frac{\pi^{2}}{6} m^{2}+\left(\frac{\pi^{2}}{6}-2 \zeta(3)\right) m^{3}\right) n \Delta^{3}-\frac{1}{2}\left(\frac{2 m+1}{m+1}\right) p \Delta+O\left(m^{4}\right), \\
& b_{12}=\frac{1}{3}\left(1+\pi^{2} m^{3}\right) n \Delta^{3}+O\left(m^{4}\right) \text {, } \\
& b_{13}=0 \text {, } \\
& b_{21}=-\left[\left(1+\frac{\pi^{2}}{6} m^{2}-\left(2 \zeta(3)-\frac{\pi^{2}}{6}\right) m^{3}\right) n \Delta^{3}\right. \\
& \left.+\frac{1}{2}\left(\frac{2 m+1}{m+1}\right) p \Delta+\left(-2+\frac{\pi^{2}}{6} m-\left(2 \zeta(3)+\frac{5}{6} \pi^{2}\right)\right) \Delta\right]+O\left(m^{4}\right), \\
& b_{22}=\left(1+\frac{\pi^{2}}{6} m^{2}+\left(-2 \zeta(3)+\frac{\pi^{2}}{6}\right) m^{3}\right) n \Delta^{3} \\
& +\left(-2+\frac{\pi^{2}}{6} m^{2}+\left(-2 \zeta(3)+\frac{5}{6} \pi^{2}\right) m^{3}\right) \Delta+O\left(m^{4}\right), \\
& b_{23}=\left(-\frac{2}{3}-\frac{\pi^{2}}{6} m^{2}+\left(2+\frac{\pi^{2}}{6}\right) m^{3}\right) n \Delta^{3}+O\left(m^{4}\right), \\
& b_{31}=-\left[\left(1+\frac{\pi^{2}}{6} m^{2}+\left(-2 \zeta(3)+\frac{\pi^{2}}{6}\right) m^{3}\right) n \Delta^{3}+\frac{1}{2}\left(\frac{2 m+1}{m+1}\right) p \Delta\right. \\
& \left.+\left(1-\frac{\pi^{2}}{3} m^{2}+\left(4 \zeta(3)+\frac{5}{3} \pi^{2}\right) m^{3}\right) \Delta\right]+O\left(m^{4}\right) \\
& b_{32}=\left(1-\frac{\pi^{2}}{3} m^{2}+\left(4 \zeta(3)+\frac{5}{3} \pi^{2}\right) m^{3}\right) \Delta+O\left(m^{4}\right), \\
& b_{33}=\frac{1}{3}\left(1+\pi^{2} m^{3}\right) \Delta^{3}+O\left(m^{4}\right), \\
& \lambda=1+\frac{1}{3} \pi^{2} m^{2}-\left(4 \zeta(3)+\frac{2}{3} \pi^{2}\right) m^{3}+O\left(m^{4}\right) .
\end{aligned}
$$

(viii) Exponencial potência: $y_{l} \sim E P\left(x_{l}^{\top} \beta, \phi^{2}, k\right),-1<k<-1 / 2$. conhecido;

$$
\begin{aligned}
& b_{11}=-\left\{\frac{n(k+3)}{(k+1)^{2}} \Delta+\frac{p}{(k+1)} \Delta\right\}, \quad b_{12}=\frac{n}{3}\left(\frac{4-k-k^{2}}{k+1}\right) \Delta^{3}, \quad b_{13}=0, \\
& b_{21}=-\left\{\frac{n(k+3)}{(k+1)^{2}} \Delta^{3}+\left(\frac{p}{1+k}-\frac{(3 k+5)}{2(k+1)}\right) \Delta\right\} .
\end{aligned}
$$




$$
\begin{aligned}
& b_{22}=n \frac{(k+3)}{(k+1)^{2}} \Delta^{3}-\frac{(3 k+5)}{2(k+1)} \Delta, \\
& b_{23}=-\frac{n(3 k+5)}{3(k+1)^{2}} \Delta^{3}, \\
& b_{31}=-\left\{n \frac{(k+3)}{(k+1)^{2}} \Delta^{3}+\left(\frac{p}{1+k}+\frac{4-k-k^{2}}{2}\right) \Delta\right\}, \\
& b_{32}=\frac{4-k-k^{2}}{2} \Delta, \\
& b_{33}=\frac{n}{3}\left(\frac{4-k-k^{2}}{k+1}\right) \Delta^{3}, \\
& \lambda=\frac{2 n}{k+1} \Delta .
\end{aligned}
$$

\subsubsection{Comparações entre funções de poder}

O objetivo desta seção é comparar as funções de poder dos testes da razão de verossimilhança, de Wald e escore de $H_{0}: \phi=\phi^{(0)}$ na classe dos modelos simétricos sob as alternativas contíguas $H_{n}: \phi=\phi^{(0)}+\xi$, com $\xi=O\left(n^{-1 / 2}\right)$. A expansão assintótica da função distribuição acumulada da estatística $S_{i}, i=1,2,3$, como foi visto em (1.22), tem a forma

$$
P\left(S_{i} \leq x\right)=G_{1, \lambda}(x)+\sum_{j=0}^{3} b_{i j} G_{1+2 j, \lambda}(x)+o\left(n^{-1 / 2}\right),
$$

onde os $b_{i j}$ são apresentados em (4.17) - (4.19), $\lambda$ é definida em (4.15) e $G_{r, \lambda}(x)$ é a função distribuição de uma variável aleatória qui-quadrado não-central com $r$ graus de liberdade e parâmetro de não-centralidade $\lambda$.

Denotaremos por $\bar{P}_{1}, \bar{P}_{2}$ e $\bar{P}_{3}$ as funções de poder dos testes da razão de verossimilhança, de Wald e escore, respectivamente. Para os modelos simétricos, sob alternativas contíguas, tem-se $\bar{P}_{i}=\bar{P}_{i}\left(x_{\alpha}\right)=1-P\left[S_{i} \leq x_{\alpha}\right]$, onde $P\left[S_{i} \leq x_{\alpha}\right]$ é obtido substituindo (4.17) - (4.19) em (4.20) e $x_{\alpha}$ é tal que $P\left[\chi_{q}^{2} \leq x_{\alpha}\right]=1-\alpha$.

Como nosso objetivo é comparar poderes somente até ordem $n^{-1 / 2}$, denotamos por $P_{i}, i=1,2,3$, a soma das parcelas de ordem um e $n^{-1 / 2}$ de $\bar{P}_{i}$. Esta notação é também utilizada por Cordeiro, Botter e Ferrari (1994). Chamaremos de $P_{i}$, daqui em diante, simplesmente de função de poder do teste baseado em $S_{i}$. Estabeleceremos condições através das quais $P_{1}, P_{2}$ e $P_{3}$ podem ser comparadas (até ordem $n^{-1 / 2}$ ), identificando possíveis regiões do espaço paramétrico onde um teste é mais poderoso que os demais. Sejam

$$
m(q, \lambda, x)=G_{q+6, \lambda}(x)-G_{q+4, \lambda}(x),
$$

e

$$
n(q, \lambda, x)=G_{q+4, \lambda}(x)-G_{q+2, \lambda}(x) .
$$

É fácil verificar que para $r$ e $\lambda$ fixos, $m(r, \lambda, x)<0$ e $n(r, \lambda, x)<0$, para $x>0$ (Apêndice D). Das equaçōes $(4.1 \tau)$ - (4.19) segue que a diferença entre duas funçòes de poder quaisquer pode ser escrita como combinação linear de $m(1, \lambda, x)$ e $n(1, \lambda, x)$, ou seja (vide 
Apêndice D),

$$
\begin{aligned}
& P_{1}-P_{3}=k_{1} m(1, \lambda, x)+k_{2} n(1, \lambda, x), \\
& P_{2}-P_{3}=k_{3} m(1, \lambda, x)+k_{4} n(1, \lambda, x), \\
& P_{2}-P_{1}=k_{5} m(1, \lambda, x)+k_{6} n(1, \lambda, x),
\end{aligned}
$$

onde

$$
\begin{aligned}
& k_{1}=\frac{n}{3}\left(1+\delta_{(1,1,0,0,3)}\right) \Delta^{3}, \quad k_{2}=\frac{\left(1+\delta_{(1,1,0,0,3)}\right) \Delta}{\left(\delta_{(2,0,0,0,2)}-1\right)}, \\
& k_{3}=-\frac{n}{2}\left(4 \delta_{(0,1,0,0,2)}+\delta_{(0,0,1,0,3)}-2\right) \Delta^{3}, \quad k_{4}=-\frac{3}{2} \frac{\left(4 \delta_{(0,1,0,0,2)}+\delta_{(0,0,1,0,3)}-2\right) \Delta}{\left(\delta_{(2,0,0,0,2)}-1\right)}, \\
& k_{5}=-\frac{n}{6}\left(6 \delta_{(0,1,0,0,2)}+\delta_{(0,0,1,0,3)}-4\right) \Delta^{3}, \quad k_{6}=-\frac{1}{2} \frac{\left(6 \delta_{(0,1,0,0,2)}+\delta_{(0,0,1,0,3)}-4\right) \Delta}{\left(\delta_{(2,0,0,0,2)}-1\right)} .
\end{aligned}
$$

A seguir apresentamos as quantidades $k_{1}, k_{2}, \ldots k_{6}$ para diversas distribuições simétricas.

(i) Normal: $y_{l} \sim N\left(x_{l}^{\top} \beta, \phi^{2}\right) ; k_{1}=(4 n / 3) \Delta^{3}, k_{2}=2 \Delta, k_{3}=3 n \Delta^{3}, k_{4}=9 \Delta / 2$, - $k_{5}=(5 n / 3) \Delta^{3}, k_{6}=5 \Delta / 2$.

(ii) Cauchy: $y_{l} \sim C\left(x_{l}^{\top} \beta, \phi^{2}\right) ; k_{1}=0, k_{2}=0, k_{3}=(n / 4) \Delta^{3}, k_{4}=3 \Delta / 2, k_{5}=(n / 4) \Delta^{3}$, $k_{6}=3 / \Delta / 2$.

(iii) $t$-Student: $y_{l} \sim t\left(x_{l}^{\top} \beta, \phi^{2}, \nu\right), \nu>0$ conhecido;

$$
\begin{aligned}
& k_{1}=\frac{4 \nu(\nu-1) n \Delta^{3}}{(\nu+3)(\nu+5)}, \quad k_{2}=\frac{2(\nu-1) \Delta}{(\nu+5)}, \quad k_{3}=\frac{3 \nu(\nu+1) n \Delta^{3}}{(\nu+3)(\nu+5)}, \\
& k_{4}=\frac{9}{2} \frac{(\nu+1) \Delta}{(\nu+5)}, \quad k_{5}=\frac{\nu(5 \nu+13) n \Delta^{3}}{3(\nu+3)(\nu+5)}, \quad k_{6}=\frac{5 \nu+13}{2(\nu+5)} \Delta .
\end{aligned}
$$

(iv) $t$-Student generalizada: $y_{l} \sim t G\left(x_{l}^{\top} \beta, \phi^{2}, s, r\right), r, s>0$ conhecidos;

$$
\begin{aligned}
& k_{1}=\frac{4 r(r-1) n \Delta^{3}}{(r+3)(r+5)}, \quad k_{2}=\frac{2(r-1) \Delta}{(r+5)}, \quad k_{3}=\frac{3 r(r+1) n \Delta^{3}}{2(r+5)}, \\
& k_{4}=\frac{9}{2} \frac{(r+1) \Delta}{(r+5)}, \quad k_{5}=\frac{r(5 r+13) n \Delta^{3}}{3(r+3)(r+5)}, \quad k_{6}=\frac{5 r+13}{2(r+5)} \Delta .
\end{aligned}
$$

(v) Logística I: $y_{l} \sim \operatorname{LI}\left(x_{l}^{\top} \beta, \phi^{2}\right)$;

$$
\begin{aligned}
& k_{1} \approx 2.515154 n \Delta^{3}, \quad k_{2} \approx 2,50365 \Delta, \quad k_{3} \approx 5,282 n \Delta^{3}, \\
& k_{4} \approx 5,257848 \Delta, \quad k_{5} \approx 2,76393 n \Delta^{3}, \quad k_{6} \approx 2,751288 \Delta .
\end{aligned}
$$


(vi) Logística II: $y_{l} \sim \operatorname{LII}\left(x_{l}^{\top} 3, \phi^{2}\right)$ :

$$
\begin{aligned}
& k_{1} \approx 0.549 \pi i n \Delta^{3}, \quad k_{2} \approx 1.15339 \Delta, \quad k_{3} \approx 1,5345 n \Delta^{3} . \\
& k_{4} \approx 3.90203 \Delta, \quad k_{5} \approx 0.98914 n \Delta^{3}, \quad k_{6} \approx 2,0.5643 \Delta .
\end{aligned}
$$

(vii) Logística generalizada: $Y_{i} \sim L G\left(x_{l}^{\top} \beta, \phi^{2}, m\right), m>0$ conhecido;

$$
\begin{aligned}
& k_{1}=\frac{n}{3(2 m+1)(m+1)}\left(6 m^{3} \psi^{\prime}(m+1)+(2 m+1)(m+1)\right) \Delta^{3}, \\
& k_{2}=\left(\frac{6 m^{3} \psi^{\prime}(m+1)+(2 m+1)(m+1)}{(m+1)\left(2 m^{2} \psi^{\prime}(m+1)+2 m+1\right)}\right) \Delta, \\
& k_{3}=\frac{n}{(2 m+1)(m+1)}\left(m^{2}(4 m+1) \psi^{\prime}(m+1)+(2 m+1)(m+1)\right) \Delta^{3}, \\
& k_{4}=\frac{3\left(m^{2}(4 m+1) \psi^{\prime}(m+1)+(2 m+1)(m+1)\right)}{(m+1)\left(2 m^{2} \psi^{\prime}(m+1)+2 m+1\right)} \Delta, \\
& k_{5}=\frac{n\left(3 m^{2} \psi^{\prime}(m+1)+2(m+1)\right)}{3(m+1)} \Delta^{3}, \\
& k_{6}=\frac{(2 m+1)\left(3 m^{2} \psi^{\prime}(m+1)+2(m+1)\right)}{(m+1)\left(2 m^{2} \psi^{\prime}(m+1)+2 m+1\right)} \Delta,
\end{aligned}
$$

onde $\psi^{\prime}(\cdot)$ é a primeira derivada da função digama.

Utilizando a expressão dada em (3.12) para valores de $m$ grandes,

$$
\begin{aligned}
& k_{1}=\frac{n}{3}\left(4-\frac{6}{m}+\frac{8}{m^{2}}-\frac{9}{m^{3}}\right) \Delta^{3}+O\left(m^{-4}\right), \\
& k_{2}=\left(2-\frac{2}{m}+\frac{7}{3 m^{2}}-\frac{7}{3 m^{3}}\right) \Delta+O\left(m^{-4}\right), \\
& k_{3}=n\left(3-\frac{7}{2 m}+\frac{13}{3 m^{2}}-\frac{14}{3 m^{3}}\right) \Delta^{3}+O\left(m^{-4}\right), \\
& k_{4}=\frac{1}{2}\left(9-\frac{6}{m}+\frac{7}{m^{2}}-\frac{7}{m^{3}}\right) \Delta+O\left(m^{-4}\right), \\
& k_{5}=n\left(\frac{5}{3}-\frac{3}{2 m}+\frac{5}{3 m^{2}}-\frac{5}{3 m^{3}}\right) \Delta^{3}+O\left(m^{-4}\right), \\
& k_{6}=\left(\frac{5}{2}-\frac{1}{m}+\frac{7}{6 m^{2}}-\frac{7}{6 m^{3}}\right) \Delta+O\left(m^{-4}\right) .
\end{aligned}
$$

Para valores pequenos de $m$, utilizamos a expansão (3.13) e obtemos

$$
\begin{aligned}
& k_{1}=\frac{n}{3}\left(1+\pi^{2} m^{3}\right) \Delta^{3}+O\left(m^{4}\right), \\
& k_{2}=\left(1-\frac{\pi^{2}}{3} m^{2}+\left(4 \zeta(3)+\frac{5 \pi^{2}}{3}\right) m^{3}\right) \Delta+O\left(m^{4}\right), \\
& k_{3}=n\left(1+\frac{\pi^{2}}{6} m^{2}+\left(\frac{\pi^{2}}{6}-2 \zeta(3)\right) m^{3}\right) \Delta^{3}+O\left(m^{4}\right),
\end{aligned}
$$




$$
\begin{aligned}
& k_{4}=\left(3-\frac{\pi^{2}}{2} m^{2}+\left(6 \zeta(3)+\frac{5 \pi^{2}}{2}\right) m^{3}\right) \Delta+O\left(m^{4}\right), \\
& k_{5}=\left(\frac{2}{3}+\frac{\pi^{2}}{6} m^{2}-\left(\frac{\pi^{2}}{6}+2 \zeta(3)\right) m^{3}\right) \Delta^{3}+O\left(m^{4}\right), \\
& k_{6}=\left(2-\frac{\pi^{2}}{6} m^{2}+\left(2 \zeta(3)+\frac{5 \pi^{2}}{6}\right) m^{3}\right) \Delta+O\left(m^{4}\right) .
\end{aligned}
$$

(viii) Exponencial potência: $y_{l} \sim \operatorname{EP}\left(x_{l}^{\top} \beta, \phi^{2}, k\right),-1<k<-1 / 2$, conhecido;

$$
\begin{aligned}
& k_{1}=\frac{n}{3}\left(\frac{4-k-k^{2}}{k+1}\right) \Delta^{3}, \quad k_{2}=\left(\frac{4-k-k^{2}}{2}\right) \Delta, \quad k_{3}=\frac{n(k+3)}{(k+1)^{2}} \Delta^{3}, \\
& k_{4}=\frac{3}{4} \frac{(k+3)}{(k+1)} \Delta, \quad k_{5}=\frac{(3 k+5) n}{3(k+1)^{2}} \Delta^{3}, \quad k_{6}=\frac{(3 k+5)}{2(k+1)} \Delta .
\end{aligned}
$$

Uma análise das equações (4.21) mostra que para as distribuições normal, Cauchy, $t$ Student ( $\operatorname{com} \nu \geq 1), t$-Student generalizada $(r \geq 1)$, logística I e II, logística generalizada e exponencial potência $(-1<k<-1 / 2)$, as funções de poder satisfazem as seguintes desigualdades:

$$
P_{3} \leq P_{1}<P_{2} \text { se } \Delta<0 \quad \text { e } \quad P_{2}<P_{1} \leq P_{3} \text { se } \Delta>0
$$

onde $\Delta$ é definido em (4.16). Assim, se $\Delta<0$, o teste de Wald é mais poderoso que o teste da razão de verossimilhança que, por sua vez, é mais (igualmente) poderoso que o teste escore. No entanto, se $\Delta>0$, o teste escore é mais (igualmente) poderoso que o teste da razão de verossimilhança que, por sua vez, é mais poderoso que o teste de Wald. Observamos que o teste da razão de verossimilhança sempre está "na posição central" e este resultado coincide com o Brajedra, Sutradhad e Roy (1992) que fazem um estudo de simulação examinando o desempenho destas três estatísticas.

É importante ressaltar novamente que todas as comparaçòes entre funções de poder são feitas até ordem $n^{-1 / 2}$ e que, portanto, quando afirmamos que um teste é mais poderoso que outro, esta afirmação é válida somente se termos de ordem inferior a $n^{-1 / 2}$ são ignorados. 


\section{Capítulo 5}

\section{Aperfeiçoamento de testes escore em modelos não lineares da família exponencial}

\subsection{Introdução}

Nos últimos anos, têm sido desenvolvidas correções tipo Bartlett (Cordeiro e Ferrari, 1991) para aperfeiçoar testes escore em diversas classes importantes de modelos de regressão. Cordeiro, Ferrari e Paula (1993) obtêm tais correções para estes coeficientes em modelos lineares generalizados com dispersão conhecida e Cribari-Neto e Ferrari (1995b) obtêm para dispersão desconhecida. Ferrari e Cordeiro (1996) obtêm correções tipo Bartlett para os testes escore em modelos não lineares da família exponencial restrito ao caso do parâmetro de dispersão ser conhecido. Neste capítulo, eliminamos a suposição de que o parâmetro de dispersão é conhecido e desenvolvemos testes escore aperfeiçoados para os modelos não lineares da família exponencial.

Sejam $y_{1}, \ldots, y_{n}$ um conjunto de $n$ variáveis aleatórias independentes sendo que cada $y_{l}$ tem função densidade ou de probabilidade da forma

$$
\pi\left(y ; \theta_{l}, \phi\right)=\exp \left[\phi\left\{y \theta_{l}-b\left(\theta_{l}\right)+c(y)\right\}+a(y, \phi)\right],
$$

onde $a(\cdot, \cdot), b(\cdot)$ e $c(\cdot)$ são funções conhecidas e $\theta_{l}$ e $\phi(\phi>0)$ são parâmetros desconhecidos. Para o modelo (5.1) valem as relações $E\left(y_{l}\right)=\mu_{l}=b^{\prime}\left(\theta_{l}\right), \operatorname{Var}\left(y_{l}\right)=\phi^{-1} V_{l}$ e $V_{l}=\mathrm{d} \mu_{l} / \mathrm{d} \theta_{l} . \quad \phi>0$ é um parâmetro de precisão, sua inversa é conhecida como o parâmetro de dispersão e $V_{l}$ é a função variância. A média de $y_{l}$ está relacionada com o componente sistemático através de uma função de ligação da forma

$$
d\left(\mu_{l}\right)=\eta_{l}=f\left(x_{l} ; \beta\right),
$$

onde $d(\cdot)$ é uma função conhecida monótona e diferenciável, $\beta=\left(\beta_{1}, \ldots, \beta_{p}\right)^{\top}$ é um vetor de $p(p<n)$ parâmetros desconhecidos, $x_{l}=\left(x_{l 1}, \ldots, x_{l p}\right)^{\top}$ é um vetor de dimensão $p$ de covariáveis conhecidas associadas à l-ésima observação e $f(\cdot, \cdot)$ é uma função diferenciável 
com respeito aos componentes de $\beta$ tal que a matriz de derivadas $X^{*}=X^{*}(\beta)=\partial \eta / \partial \beta$ tenha posto $p$ para todo $\beta$. Supomos identificabilidade no sentido de que diferentes $\beta$ s implicam em diferentes $\eta$ 's. A matriz $X^{*}$ tem elementos que são, em geral, funçòes do vetor de parâmetros $\beta$ desconhecidos.

Seja $L=L(\beta, \phi)$ o logaritmo da função de verossimilhança de um modelo não linear na família exponencial definida por (5.1) e (5.2) em algum espaço paramétrico dado o vetor de observações $y=\left(y_{1}, \ldots, y_{n}\right)^{\top}$. Assume-se que a função $L$ seja regular com respeito às derivadas em relação aos parâmetros até quarta ordem (Cox e Hinkley, 1974, Capítulo 9). Suponhamos que $U$ e $K$ denotam a função escore total e a matriz de informação total de Fisher, respectivamente.

Consideremos uma partição para $\beta$ dada por $\beta=\left(\beta_{1}^{\top}, \beta_{2}^{\top}\right)^{\top}$, onde $\beta_{1}=\left(\beta_{1}, \ldots, \beta_{q}\right)^{\top}$ e $\beta_{2}=\left(\beta_{q+1}, \ldots, \beta_{p}\right)^{\top}$, com $q \leq p$. Conseqüentemente, a partição da matriz modelo local correspondente é $X^{*}=\left[X_{1}^{*}, X_{2}^{*}\right]$, onde $X_{1}^{*}$ e $X_{2}^{*}$ são submatrizes $n \times q$ e $n \times(p-q)$, respectivamente. $\mathrm{O}$ problema a considerar consiste em testar uma hipótese nula composta $H_{0}: \beta_{1}=\beta_{1}^{(0)}$ contra uma hipótese alternativa, também composta, $H_{1}: \beta_{1} \neq \beta_{1}^{(0)}$, onde $\beta_{2}$ e $\phi$ são parâmetros de perturbação e $\beta_{1}^{(0)}$ é um vetor especificado de dimensão $q$. Sejam $\widetilde{\beta}_{2}$ e $\tilde{\phi}$ os estimadores de máxima verossimilhança restritos de $\beta_{2}$ e $\phi$ sob $H_{0}$, respectivamente, supondo que existam e sejam únicos estes estimadores. A estatística escore para testar $H_{0}$ contra $H_{1}$ é dada por

$$
S_{R}=\widetilde{s}^{\top} \widetilde{W}^{1 / 2}{\widetilde{X_{1}^{*}}}_{(}\left(\widetilde{R}^{* \top} \widetilde{W} R^{*}\right)^{-1}{\widetilde{X_{1}^{* \top}}}^{* \top} \widetilde{W}^{1 / 2} \widetilde{s}
$$

onde $s=\left(s_{1}, \ldots, s_{n}\right)^{\top}$, com $s_{l}=\phi^{1 / 2}\left(y_{l}-\mu_{l}\right) / V_{l}^{1 / 2}$ o vetor de resíduos de Pearson, $w_{l}=\left(\mathrm{d} \mu_{l} / \mathrm{d} \eta_{l}\right)^{2} / V_{l}, W=\operatorname{Diag}\left\{w_{1}, \ldots, w_{n}\right\}$, e $R^{*}=X_{1}^{*}-X_{2}^{*}\left(X_{2}^{* \top} W X_{2}^{*}\right)^{-1} X_{2}^{* \top} W X_{1}^{*}$. Finalmente, o til indica que as funções são avaliadas nas estimativas de máxima verossimilhança sob $H_{0}$. Na próxima seção, desenvolvemos uma expressão fechada para a correção tipo Bartlett para o teste escore de $H_{0}$ versus $H_{1}$ em modelos não lineares da família exponencial (5.1) e (5.2). Esta correção depende de operações simples com matrizes e vetores. $\mathrm{Na}$ Seção 5.3, apresentamos alguns estudos de simulação e na Seção 5.4 apresentamos uma aplicação dos resultados.

\subsection{Desenvolvimento da correção}

Nesta seção obtemos os coeficientes $A_{1}, A_{2}$ e $A_{3}$ que determinam os testes escore aperfeiçoados supondo $\phi$ desconhecido. Usamos a notação das Seçòes 1.2 e 3.2 .2 para os cumulantes conjuntos das derivadas do logaritmo da função de verossimilhança. É interessante notar que $\kappa_{\phi, i}=0$ e que, portanto, a matriz de informação total de Fisher e sua inversa são bloco diagonais, isto é, são da forma

$$
K=\left(\begin{array}{ccc}
K_{11} & K_{12} & 0 \\
K_{21} & K_{22} & 0 \\
0 & 0 & \kappa_{\phi, \phi}
\end{array}\right) \quad \text { e } K^{-1}=\left(\begin{array}{ccc}
K^{-11} & K^{12} & 0 \\
K^{-21} & K^{22} & 0 \\
0 & 0 & \kappa_{\phi, \phi}^{-1}
\end{array}\right) \text {. }
$$

Em outras palavras, $\beta$ e $\phi$ são globalmente ortogonais no sentido de Cox e Reid (1987). 
Definimos

$$
K_{\beta}^{-1}=\left(\begin{array}{ll}
K^{-11} & K^{-12} \\
K^{21} & K^{22}
\end{array}\right), A_{\beta}=\left(\begin{array}{cc}
0 & 0 \\
0 & K_{22}^{-1}
\end{array}\right), A=\left(\begin{array}{cc}
A_{\beta} & 0 \\
0 & \kappa_{0 . \phi}^{-1}
\end{array}\right) \quad \text { e } M=\left(\begin{array}{cc}
M_{\beta} & 0 \\
0 & 0
\end{array}\right),
$$

onde $M_{\beta}=K_{\beta}^{-1}-A_{\beta}$. Sejam $m_{r \phi}$ e $a_{r \phi}$ os elementos da posição $(r, p+1)$ de $M$ e $A$, respectivamente, e sejam $m_{\phi \phi}$ e $a_{\phi \phi}$ os elementos da posiçào $(p+1, p+1)$ de $M$ e $A$, respectivamente. Tem-se $m_{r \phi}=m_{\phi r}=m_{\phi \phi}=0, a_{r \phi}=a_{\phi r}=0$, para $r=1, \ldots, p$, e $a_{\phi \phi}=\kappa_{\phi, \phi}^{-1}$. Se o parâmetro de dispersão é desconhecido, tem-se $A_{1}=A_{1, \beta}+A_{1, \beta \phi}$, $A_{2}=A_{2, \beta}+A_{2, \beta \phi}$ e $A_{3}=A_{3, \beta}+A_{3, \beta \phi}$, onde $A_{1, \beta}, A_{2, \beta}$ e $A_{3, \beta}$ são os $A$ 's no caso em que o parâmetro de dispersão é conhecido. Estes são obtidos da mesma estrutura aditiva para os $A$ 's como apresentaram Cribari-Neto e Ferrari (1995b) para modelos lineares generalizados, quando tem-se uma estrutura ortogonal entre os parâmetros $\beta$ e $\phi$. As fórmulas gerais para $A_{1, \beta \phi}, A_{2, \beta \phi}$ são dadas em $(3.21)$ e $(3.22)$ e $A_{3, \beta \phi}=0$.

Para a família exponencial de distribuições na forma natural, com parâmetros canôni$\cos \phi$ e $\phi \theta$, o termo $a(y, \phi)$ em (5.1) pode ser escrito como $a(y, \phi)=d_{1}(\phi)+d_{2}(y)$. Sejam $d_{(2)}=d_{(2)}(\phi)=\phi^{2} d_{1}^{\prime \prime}(\phi)$ e $d_{(3)}=d_{(3)}(\phi)=\phi^{3} d_{1}^{\prime \prime \prime}(\phi)$. Os cumulantes em (3.21) e (3.22) que envolvem $\phi$ (Apêndice $\mathrm{E}$ ) são dados por

$$
\begin{aligned}
& \kappa_{\phi \phi i}=\kappa_{\phi, \phi i}=\kappa_{i j, \phi}=\kappa_{i, \phi \phi}=\kappa_{i \phi, \phi}=\kappa_{\phi, \phi \varphi}=0, \\
& \kappa_{\phi \phi \phi}=n d_{1}^{\prime \prime \prime}(\phi), \quad \kappa_{\phi, i, j}=\kappa_{i j \phi}=-\kappa_{i, j \phi}=-\sum \omega_{l}(i, j)_{l}, \\
& \kappa_{i, j, \phi, \phi}=-2 \phi^{-1} \sum \omega_{l}(i, j)_{l}, \quad \kappa_{i, j, \phi \phi}=0,
\end{aligned}
$$

onde $\sum$ denota o somatório sobre as $n$ observações e $(i, j)_{l}=\left(\partial \eta_{l} / \partial \beta_{i}\right)\left(\partial \eta_{l} / \partial \beta_{j}\right)$. ́́ interessante notar que estes cumulantes são os mesmos que foram obtidos para os modelos lineares generalizados (Cribari-Neto e Ferrari, 1995b). Cumulantes que não envolvem $\phi$ podem ser vistos em Ferrari e Cordeiro (1996), alguns dos quais são extraídos de Cordeiro e Paula (1989). Alguns dos cumulantes que não envolvem ó são diferentes do caso dos modelos lineares generalizados, pois são sensíveis à estrutura não linear dos modelos considerados aqui. Porém, todos os termos dos $A$ 's envolvendo tais cumulantes se cancelam e, conseqüentemente, depois de alguma álgebra, obtemos (Apêndice E)

$$
\begin{aligned}
& A_{1, \beta \phi}=\frac{6 q\left\{d_{(3)}-(p-q-2) d_{(2)}\right\}}{n d_{(2)}^{2}}, \\
& A_{2, \beta \phi}=\frac{3 q(q+2)}{n d_{(2)}}
\end{aligned}
$$

que são as mesmas expressòes obtidas por Cribari-Neto e Ferrari (1995b) para modelos lineares generalizados com dispersão desconhecida.

Para os modelos normal e normal inversa, temos $A_{1, \beta 0}=12 p(p-q) / n$ e $A_{2, \beta o}=$ $-6 q(q+2) / n$. Para o modelo gama, os termos $d_{(2)}$ e $d_{(3)}$ em $(5.3)$ e (5.4) são dados por $d_{(2)}=\phi\left\{1-\phi \psi^{\prime}(\phi)\right\}$ e $d_{(3)}=-\phi\left\{1+\phi^{2} \psi^{\prime \prime \prime}(\phi)\right\}$, onde $\psi^{\prime}(\cdot)$ e $\iota^{\prime \prime \prime}(\cdot)$ sào as funçòes trigama e tetragama, respectivamente. Para evitar a avaliaçào das funçôes poligama, usaremos as 
expansões (6.4.6), (6.4.12) e (6.4.13) de Abramowitz e Stegun (1970). obtendo as seguintes aproximações:

$$
\begin{aligned}
d_{(2)} \approx & \phi-1-\frac{\phi^{2}}{\phi+1}\left\{1+\frac{1}{2(\phi+1)}+\frac{1}{6(\phi+1)^{2}}-\frac{1}{30(\phi+1)^{4}}\right. \\
& \left.+\frac{1}{42(\phi+1)^{6}}-\frac{1}{30(\phi+1)^{8}}\right\}
\end{aligned}
$$

e

$$
\begin{aligned}
d_{(3)} \approx & -\phi+2+\frac{\phi^{3}}{(\phi+1)^{2}}\left\{1+\frac{1}{\phi+1}+\frac{1}{2(\phi+1)^{2}}-\frac{1}{6(\phi+1)^{4}}\right. \\
& \left.+\frac{1}{6(\phi+1)^{6}}-\frac{3}{10(\phi+1)^{8}}\right\} .
\end{aligned}
$$

Uma avaliação das duas aproximações para diferentes valores de $\phi$ são dadas na Tabela 5.1. Estas avaliaçòes foram feitas usando o sistema computacional algébrico MATHEMATICA (Wolfram, 1991). Esta tabela mostra que as aproximações são muito boas para os valores de $\phi$ considerados. A vantagem de tais aproximações é que elas nào requerem a avaliação das funções trigama e tetragama.

Tabela 5.1: Aproximações para $d_{(2)}(\phi)$ e $d_{(3)}(\phi)$.

\begin{tabular}{c|cc|cc}
\hline \multirow{2}{*}{$\phi$} & \multicolumn{2}{|c|}{$d_{(2)}$} & \multicolumn{2}{c}{$d_{(3)}$} \\
\cline { 2 - 5 } & exata & aprox. & exata & aprox. \\
\hline 0.01 & -0.9902 & -0.9902 & 1.9900 & 1.9900 \\
0.25 & -0.8248 & -0.8247 & 1.7708 & 1.7708 \\
1.00 & -0.6449 & -0.6449 & 1.4041 & 1.4042 \\
2.00 & -0.5797 & -0.5797 & 1.2329 & 1.2329 \\
3.00 & -0.5544 & -0.5544 & 1.1611 & 1.1611 \\
4.00 & -0.5412 & -0.5412 & 1.1225 & 1.1225 \\
5.00 & -0.5331 & -0.5331 & 1.0987 & 1.0987 \\
10.00 & -0.5166 & -0.5166 & 1.0498 & 1.0498 \\
20.00 & -0.5083 & -0.5083 & 1.0250 & 1.0250 \\
30.00 & -0.5056 & -0.5056 & 1.0167 & 1.0167 \\
\hline
\end{tabular}

Definimos agora $Z^{*}=X^{*}\left(X^{* \top} W X^{*}\right)^{-1} X^{* \top}, Z_{2}^{*}=X_{2}^{*}\left(X_{2}^{* \top} W X_{2}^{*}\right)^{-1} X_{2}^{* \top}($ para $q \leq p)$, $Q_{2}=\operatorname{Diag}\left\{q_{1}, \ldots, q_{n}\right\} \operatorname{com} q_{l}=\operatorname{tr}\left\{\left(X_{2}^{* \top} W X_{2}^{*}\right)^{-1} D_{22}^{(l)}\right\}$, onde

$$
D^{(l)}=\left\{\frac{\partial^{2} \eta_{l}}{\partial 3_{l} \partial \beta_{j}}\right\}=\left(\begin{array}{ll}
D_{11}^{(l)} & D_{12}^{(l)} \\
D_{21}^{(l)} & D_{22}^{(l)}
\end{array}\right) .
$$

Também definimos $C_{2}=\left\{c_{l m}\right\} \cdot \operatorname{com} c_{l m}=x_{2 m}^{\times}\left(X_{2}^{\times \top} W X_{2}^{*}\right)^{-1} D_{22}^{(l)}\left(X_{2}^{\times \top} W X_{2}^{\times *}\right)^{-1} x_{2 m}^{* \top} \cdot x_{2 m}^{*}$ denotando a $m$-ésima linha de $X_{2}^{\times}, J_{2}=\left\{j_{l m}\right\} \cdot \operatorname{com} j_{l m}=\operatorname{tr}\left\{D_{22}^{(l)}\left(X_{2}^{\times \times \top} W X_{2}^{* \times}\right)^{-1} D_{22}^{(l)}\left(X_{2}^{* \top} W X_{2}^{*}\right)^{-1}\right\}$, 
$F=\operatorname{Diag}\left\{f_{1}, \ldots, f_{n}\right\}, G=\operatorname{Diag}\left\{g_{1}, \ldots, g_{n}\right\}, B=\operatorname{Diag}\left\{b_{1}, \ldots, b_{n}\right\}$ e $H=\operatorname{Diag}\left\{h_{1}, \ldots, h_{n}\right\}$ com

$$
\begin{aligned}
f & =\frac{1}{V} \frac{\mathrm{d} \mu}{\mathrm{d} \eta} \frac{\mathrm{d}^{2} \mu}{\mathrm{d} \eta^{2}} \\
g & =\frac{1}{V} \frac{\mathrm{d} \mu}{\mathrm{d} \eta} \frac{\mathrm{d}^{2} \mu}{\mathrm{d} \eta^{2}}-\frac{1}{V^{2}} \frac{\mathrm{d} V}{\mathrm{~d} \mu}\left(\frac{\mathrm{d} \mu}{\mathrm{d} \eta}\right)^{3}, \\
b & =\frac{1}{V^{3}}\left(\frac{\mathrm{d} \mu}{\mathrm{d} \eta}\right)^{4}\left\{\left(\frac{\mathrm{d} V}{\mathrm{~d} \mu}\right)^{2}+V \frac{\mathrm{d}^{2} V}{\mathrm{~d} \mu^{2}}\right\}, \\
h & =\frac{1}{V^{2}} \frac{\mathrm{d} V}{\mathrm{~d} \mu}\left(\frac{\mathrm{d} \mu}{\mathrm{d} \eta}\right)^{2} \frac{\mathrm{d}^{2} \mu}{\mathrm{d} \eta^{2}}+\frac{1}{V^{2}} \frac{\mathrm{d}^{2} V}{\mathrm{~d} \mu^{2}}\left(\frac{\mathrm{d} \mu}{\mathrm{d} \eta}\right)^{4} .
\end{aligned}
$$

Usamos a notação ' ' para denotar o produto de Hadamard (Rao, 1973, p.30) de duas matrizes, ou seja, $Z^{\times(2)}=Z^{*} \odot Z^{*}$, etc. O subíndice $d$ indicará que uma matriz diagonal é obtida da matriz original. Por exemplo, $Z_{d}^{*}=\operatorname{Diag}\left\{\tilde{z}_{11}^{*}, \ldots, z_{n n}^{*}\right\}$. Ferrari e Cordeiro (1996) mostram que

$$
\begin{aligned}
A_{1, \beta}= & \phi^{-1}\left[31^{\top} F Z_{2 d}^{*}\left(Z^{*}-Z_{2}^{*}\right) Z_{2 d}^{*} F 1+61^{\top} F Z_{2 d}^{*} Z_{2}\left(Z^{*}-Z_{2}^{*}\right)_{d}(F-G) \mathbf{1}\right. \\
& -61^{\top}(2 G-F)\left\{Z_{2}^{*(2)} \otimes\left(Z^{*}-Z_{2}^{*}\right)\right\} F 1-61^{\top} H\left(Z^{*}-Z_{2 d}^{*}\right)_{d} Z_{2 d}^{*} 1 \\
& +61^{\top} W\left[Q_{2}\left(Z^{*}-Z_{2}^{*}\right) Z_{2 d}^{*}-\left\{\left(Z^{*}-Z_{2}^{*}\right) \otimes C_{2}\right\}\right] F \mathbf{1} \\
& +31^{\top} W\left[Q_{2}\left(Z^{*}-Z_{2}^{*}\right) Q_{2}-2\left\{\left(Z^{*}-Z_{2}^{\star}\right) \otimes J_{2}\right\}\right] W 1 \\
& +61^{\top} W Q_{2} Z_{2}^{*}\left(Z-Z_{2}^{*}\right)_{d}(F-G) 1-61^{\top}(2 G-F)\left\{\left(Z^{*}-Z_{2}^{*}\right) \otimes C_{2}^{\top}\right\} W \mathbf{1} \\
& \left.-61^{\top}(F-G) Q_{2}\left(Z^{*}-Z_{2}^{*}\right)_{d} 1\right] \\
A_{2, \beta}= & \phi^{\prime}\left[-31^{\top}(F-G)\left(Z^{*}-Z_{2}^{*}\right)_{d} Z_{2}^{*}\left(Z^{*}-Z_{2}^{*}\right)_{d}(F-G) 1\right. \\
& -61^{\top} F Z_{2 d}^{*}\left(Z-Z_{2}^{*}\right)\left(Z^{*}-Z_{2}^{*}\right)_{d}(F-G) 1 \\
& -61^{\top}(F-G)\left\{\left(Z-Z_{2}^{*}\right)^{(2)} \otimes Z_{2}^{*}\right\}(F-G) 1 \\
& \left.+31^{\top} B\left(Z^{*}-Z_{2}^{*}\right)_{d}^{(2)} 1-61^{\top} W Q_{2}\left(Z^{*}-Z_{2}^{*}\right)\left(Z^{*}-Z_{2}^{*}\right)_{d}(F-G) \mathbf{1}\right\} \\
A_{3, \beta}= & \phi^{-1}\left\{31^{\top}(F-G)\left(Z^{*}-Z_{2}^{*}\right)_{d}\left(Z^{*}-Z_{2}^{*}\right)\left(Z^{*}-Z_{2}^{*}\right)_{d}(F-G) \mathbf{1}\right. \\
& \left.+21^{\top}(F-G)\left(Z-Z_{2}^{*}\right)^{(3)}(F-G) 1\right\}
\end{aligned}
$$

onde 1 é o vetor de uns. Então, $A_{1}=A_{1, \beta}+A_{1, \beta \phi}, A_{2}=A_{2, \beta}+A_{2, \beta \phi}$ e $A_{3}=A_{3, \beta}$. onde $A_{1, \beta}, A_{2, \beta}$ e $A_{3, \beta}$ em (5.5) - (5.7) são os $A$ 's dados por Ferrari e Cordeiro (1996) para modelos não lineares da família exponencial com parâmetro de dispersão conhecido e $A_{1, \beta \phi}$ e $A_{2, \beta \phi}$ em (5.3) e (5.4) são os dois termos extras dados por Cribari-Neto e Ferrari (1995b) para modelos lineares generalizados com parâmetros de dispersão desconhecida.

As fórmulas dadas acima podem ser usadas para obter correções em amostras finitas para os testes escore em muitos casos especiais. Consideremos, por exemplo, o caso especial de modelos de regressão normal não-linear com ligação identidade. Podemos 
obter facilmente $A_{1}=3 \phi^{-1} 1^{\top}\left[Q_{2}\left(Z^{\star}-Z_{2}^{\times}\right)_{d} Q_{2}-2\left\{\left(Z^{\star}-Z_{2}^{\times}\right) \odot J_{2}\right\}\right] 1+12 q(p-q) / n$, $A_{2}=-6 q(q+2) / n$ e $A_{3}=0$. No caso de modelos de regressão normal linear, nossos resultados reduzem-se ao de Rothenberg (1984) para o caso de homocedasticidade, ou seja, $A_{1}=12 q(p-q) / n, A_{2}=-6 q(q+2) / n$ e $A_{3}=0$. Também os resultados em Ferrari e Cordeiro (1996) podem ser obtidos fazendo $A_{1, \beta_{0}}=A_{2, \beta \phi}=0$. Para obter os resultados em Cribari-Neto e Ferrari (1995b), basta tomar os elementos das matrizes $C_{2}, D, J_{2}$ e $Q_{2}$, todos iguais a zero. e os resultados de Cordeiro, Ferrari e Paula (1993) podem ser obtidos impondo que, além disto, $A_{1, \beta \phi}=A_{2, \beta \phi}=0$.

\subsection{Alguns estudos de simulação}

Desenvolvemos nesta seção quatro experimentos de simulação envolvendo variáveis aleatórias normalmente distribuídas $\operatorname{com} \eta=\beta_{0}+\exp \left(\beta_{3} x_{3}\right)$ (caso $\left.1, p=2\right), \eta=\beta_{0}+\beta_{1} x_{1}+$ $\beta_{2} x_{2}+\exp \left(\beta_{3} x_{3}\right)+\exp \left(\beta_{4} x_{4}\right)($ caso $2, p=5), \eta=\beta_{0}+\beta_{1} x_{1}+\beta_{2} x_{2}+\exp \left(\beta_{3} x_{3}\right)+\exp \left(\beta_{4} x_{4}\right)+$ $\beta_{5} x_{5}+\beta_{6} x_{6}$ (caso $3, p=7$ ) e $\eta=\beta_{0}+\beta_{1} x_{1}+\beta_{2} x_{2}+\exp \left(\beta_{3} x_{3}\right)+\exp \left(\beta_{4} x_{4}\right)+\beta_{5} x_{5}+\beta_{6} x_{6}+$ $\beta_{7} x_{7}+\beta_{8} x_{8}+\beta_{9} x_{9}$ (caso $\left.4, p=10\right)$. Para o primeiro caso, testamos $H_{0}: \beta_{0}=0, \beta_{3}=1$. Para os casos 2 até 4 , testamos $H_{0}: \beta_{3}=\beta_{4}=1$. Os valores para as covariadas são selecionados como se segue. $x_{1}$ é gerada por uma seqüência igualmente espaçada de $n-2$ pontọs de -1 a 1 e pontos finais -4 e 4 , e $x_{2}, \ldots, x_{9}$ são obtidos como amostras aleatórias das seguintes distribuições: normal padrão, $t$-Student com 5 graus de liberdade, uniforme no intervalo $(0,1), F(3,2)$, Cauchy padrão, lognormal padrão, exponencial com média um e qui-quadrado com dois graus de liberdade, respectivamente. Estes valores são mantidos constantes para todas as simulações. Todos os parâmetros de perturbação, incluindo $\phi$, são iguais a 1, e o número de réplicas é de 10.000. Todas as simulações foram desenvolvidas utilizando o programa computacional S-PLUS (Becker, Chambers e Wilks, 1988). As estimativas das probabilidade de rejeição de $H_{0}$ para o teste escore e teste escore corrigido pelo fator de correção tipo Bartlett correspondente aos níveis nominais $\alpha=0,10,0,05$, 0,01 são dados nas Tabelas 5.2 e 5.3 (em porcentagem). As taxas de rejeição para o caso 4 $\operatorname{com} n=10$ não é considerada, já que neste caso temos $n=p=10$, e este resultado não faz sentido. O teste escore no caso 1 subestima o tamanho do teste levemente e superestima nos casos 2 até 4 . Em particular, para amostras finitas, a qualidade do teste escore se deteriora quando o número de parâmetros de perturbação aumenta. Os resultados destas tabelas também mostram que o comportamento da estatística escore corrigida $S_{R}^{*}$ é melhor que o a estatística escore usual $S_{R}$ em quase todos os casos. Notamos que as taxas de rejeição de $S_{R}^{*}$ são próximas dos correspondentes níveis nominais e as taxas de rejeição de $S_{R}$ são sempre superiores aos correspondentes níveis nominais embora, como esperado, aproximem-se deste quando o tamanho da amostra cresce. Em particular, a correção mostra-se efetiva quando o número de parâmetros de perturbação é grande e, neste caso, o teste escore rejeita a hipótese nula com freqüência muito maior que o esperado baseado no nível nominal do teste. 
Tabela 5.2: Valores simulados de $P\left(S_{R} \geq x_{\alpha}\right)$ e $P\left(S_{R}^{\prime x} \geq x_{\alpha}\right)$ (em porcentagem).

\begin{tabular}{c|c|cc|cc}
\hline Tamanho & Nível & \multicolumn{2}{|c|}{$p=q=2$} & \multicolumn{2}{|c}{$p=5, q=2$} \\
\cline { 3 - 6 } da amostra & nominal & \multicolumn{2}{|c}{} & & \\
$n$ & $\alpha$ & $P\left[S_{R} \geq x_{\alpha}\right]$ & $P\left[S_{R}^{\times} \geq x_{\alpha}\right]$ & $P\left[S_{R} \geq x_{\alpha}\right]$ & $P\left[S_{R}^{\times \times} \geq x_{\alpha}\right]$ \\
\hline \multirow{3}{*}{10} & 10 & 8,2 & 8,9 & 21,0 & 11,2 \\
& 5 & 2,7 & 4,0 & 10,0 & 4,6 \\
& 1 & 0,0 & 0,3 & 0,1 & 0,0 \\
\multirow{3}{*}{15} & 10 & 9,2 & 9,7 & 16,5 & 10,9 \\
& 5 & 3,9 & 4,9 & 8,1 & 5,2 \\
& 1 & 0,3 & 0,6 & 0,8 & 0,7 \\
20 & 10 & 9,4 & 9,8 & 14,0 & 10,5 \\
& 5 & 4,1 & 4,9 & 6,9 & 5,0 \\
& 1 & 0,4 & 0,9 & 0,9 & 0,9 \\
25 & 10 & 9,6 & 9,8 & 13,0 & 10,0 \\
& 5 & 4,4 & 5,0 & 6,4 & 5,0 \\
& 1 & 0,4 & 0,7 & 1,1 & 1,0 \\
30 & 10 & 10,2 & 10,5 & 12,5 & 10,0 \\
& 5 & 4,4 & 4,9 & 6,3 & 5,2 \\
& 1 & 0,7 & 1,0 & 1,1 & 1,0 \\
\hline
\end{tabular}

Tabela 5.3: Valores simulados $P\left(S_{R} \geq x_{\alpha}\right)$ e $P\left(S_{R}^{*} \geq x_{\alpha}\right)$ (em porcentagem).

\begin{tabular}{c|c|cc|cc}
\hline Tamanho & Nível & \multicolumn{2}{|c|}{$p=7, q=2$} & \multicolumn{2}{|c}{$p=10, q=2$} \\
\cline { 3 - 6 } da amostra & nominal & & & & \\
\cline { 3 - 6 } & $\alpha$ & $P\left[S_{R} \geq x_{\alpha}\right]$ & $P\left[S_{R}^{* *} \geq x_{\alpha}\right]$ & $P\left[S_{R} \geq x_{\alpha}\right]$ & $P\left[S_{R}^{* *} \geq x_{\alpha}\right]$ \\
\hline \multirow{3}{*}{10} & 10 & 39,7 & 16,4 & - & - \\
& 5 & 25,5 & 6,9 & - & - \\
& 1 & 2,3 & 0,0 & - & - \\
\multirow{3}{*}{15} & 10 & 23,3 & 13,6 & 39,7 & 15,7 \\
& 5 & 13,6 & 6,0 & 27,8 & 8,4 \\
& 1 & 2,4 & 1,0 & 8,9 & 1,2 \\
20 & 10 & 18,1 & 10,8 & 27,5 & 12,7 \\
& 5 & 9,7 & 5,4 & 17,0 & 6,7 \\
& 1 & 1,9 & 1,1 & 4,6 & 1,5 \\
25 & 10 & 16,5 & 10,7 & 21,7 & 11,3 \\
& 5 & 8,6 & 5,1 & 12.9 & 5,7 \\
& 1 & 1,5 & 1.0 & 3,0 & 1,0 \\
30 & 10 & 14,6 & 10,4 & 19,0 & 10,4 \\
& 5 & 7,7 & 5,3 & 10,5 & 5,5 \\
& 1 & 1,5 & 0.9 & 2.5 & 1.2 \\
\hline
\end{tabular}


Tabela 5.4: Primeiro e segundo cumulantes de $S_{R} . S_{R}^{\mathrm{x}}$ e $\lambda_{2}^{2}$.

\begin{tabular}{c|c|c|c|c}
\hline caso & cumulantes & $S_{R}^{\prime}$ & $S_{R}^{\prime \times}$ & $\backslash_{2}^{2}$ \\
\hline 1 & média & 2.0 & 2,0 & 2,0 \\
& variância & 3.4 & 3.8 & 4,0 \\
\hline \multirow{2}{*}{2} & média & 2.3 & 2,0 & 2,0 \\
& variância & 4.4 & 4,0 & 4,0 \\
\hline \multirow{2}{*}{3} & média & 2,5 & 2,0 & 2,0 \\
& variância & 5.1 & 4,0 & 4,0 \\
\hline \multirow{2}{*}{4} & média & 3.0 & 2,1 & 2,0 \\
& variância & 6.9 & 4,4 & 4,0 \\
\hline
\end{tabular}

Na Tabela 5.4 apresentamos as comparações da média e variância de $S_{R}, S_{R}^{*}$ e $\chi_{2}^{2}$ para os quatro casos tomando $n=25$. Os resultados desta tabela mostram que a média e a variância de $S_{R}^{*}$ são bem próximas da média e da variância da variável $\chi_{2}^{2}$. Por outro lado, as medidas de $S_{R}$ excedem às da distribuição $\chi_{2}^{2}$, exceto quando $p=q$ e neste caso a média coincide com a média da variável $\chi_{2}^{2}$ e a variância de $S_{R}$ é menor que a variância da variável $\chi_{2}^{2}$.

Concluindo, os resultados das simulações desta seção sugerem que a estatística escore usual utilizada em conjunto com uma aproximação por $\chi_{2}^{2}$ pode produzir inferências inadequadas com amostras pequenas ou de tamanhos moderados. A estatística escore corrigida pelo fator de correção tipo Bartlett pode ser então utilizada para obter testes com mais confiança em tamanhos de amostras finitos.

\subsection{Uma aplicação}

Nesta seção apresentamos um aplicação dos resultados da Seção 5.2. Consideramos os dados sobre volumes de árvores discutidos por Aitkinson (1982) e Aitkin, Anderson, Francis e Hinde (1989). O volume de madeira utilizável y (em pés cúbicos) depende da altura $h$ (em pés) e do diâmetro $d$ (em polegadas) de uma árvore. O conjunto de dados consiste de 31 observações de plantas de cereja e está apresentado na Tabela 5.5. Assumimos que a variável resposta é normalmente distribuída e que a média $\mu$ da distribuição está relacionada com um preditor linear $\eta$ através de uma ligação potência, ou seja, $\mu^{1 / \lambda}=\eta$. Aitkin, Anderson, Francis e Hinde (1989) sugerem tomar $\lambda=3$. Deve ser notado que um teste escore (corrigido) sobre $\lambda$ pode ser realizado com os resultados aqui obtidos. Isto é feito escrevendo o modelo como um modelo não linear com ligação identidade, ou seja,

$$
\mu_{l}=\eta_{l}=\left(\beta_{0}+\beta_{1} h_{l}+\beta_{2} d_{l}\right)^{\lambda}
$$

e assumindo ainda a independência das observaçôes e a homogeneidade de variâncias.

Realizamos, inicialmente, um teste de $\mathrm{H}_{0}: \lambda=1$. Para este teste, $S_{R}=17,485 \mathrm{e}$ $S_{R}^{\times}=20,442$. Ou seja. a hipótese nula é rejeitada fortemente por ambas as estatísticas. 
Considerando agora um teste de $\mathrm{H}_{0}: \lambda=3$, obtemos $S_{R}=0.308$ e $S_{R}^{* \times}=0,276$. e, portanto, os valores de ambas as estatísticas nos levam a não rejeitar $H_{0}$ aos níveis de significância usuais. Em ambos os casos, a concordância do teste escore com sua correspondente versào corrigida nos dá mais confiança no resultado já que a possibilidade de erro devido a uma má aproximação da estatística escore por qui-quadrado é reduzida.

Agora, estamos interessados em testar se o volume médio utilizável de madeira é um múltiplo do diâmetro da árvore, não dependendo então da sua altura após seu diâmetro ter sido considerado. Ou seja, desejamos testar $\mathrm{H}_{0}: \beta_{0}=\beta_{1}=0$ contra uma alternativa bilateral. A estatística escore e a estatística escore corrigida usando $\lambda=3$ são $25,494 \mathrm{e}$ 33,510. Ambas conduzem à rejeição da hipótese nula mesmo a níveis de significância bem baixos uma vez que o quantil de ordem 99\% da distribuição $\chi_{2}^{2}$ é 9.210. Para analisar o efeito da correção em pequenas amostras, retiramos uma amostra de 10 observações do conjunto original de dados e repetimos o teste para esta subamostra. Isto foi feito utilizando amostragem aleatória com reposição, e as observações selecionadas foram: 1, $6,28,17,11,12,29,15,21$. 20. As estatísticas do teste são $S_{R}=8,635$ e $S_{R}^{\times}=9,773$ que correpondem a niveis descritivos de 0.013 e 0.0008 , respectivamente. Assim, a estatística escore não leva à rejeição de $\mathrm{H}_{0}$ ao nível de significância de $1 \%$ enquanto que a estatística escore corrigida leva à rejeiçào de tal hipótese.

Tabela 5.5: Medidas das árvores de cereja

\begin{tabular}{cccc}
\hline Observação & Diametro & Altura & Volume \\
$l$ & $d_{l}$ & $h_{l}$ & $y_{l}$ \\
\hline 1 & 8.3 & 70 & 10.3 \\
2 & 8.6 & 65 & 10.3 \\
3 & 8.8 & 63 & 10.2 \\
4 & 10.5 & 72 & 16.4 \\
5 & 10.7 & 81 & 18.8 \\
6 & 10.8 & 83 & 19.7 \\
7 & 11.0 & 66 & 15.6 \\
8 & 11.0 & 75 & 18.2 \\
9 & 11.1 & 80 & 22.6 \\
10 & 11.2 & 75 & 19.9 \\
11 & 11.3 & 79 & 24.2 \\
12 & 11.4 & 76 & 21.0 \\
13 & 11.4 & 76 & 21.4 \\
14 & 11.7 & 69 & 21.3 \\
15 & 12.0 & 75 & 19.1 \\
& & & \\
\hline
\end{tabular}

\begin{tabular}{cccc}
\hline Observação & Diametro & Altura & Volume \\
$l$ & $d_{l}$ & $h_{l}$ & $y_{l}$ \\
\hline 16 & 12.9 & 74 & 22.2 \\
17 & 12.9 & 85 & 33.8 \\
18 & 13.3 & 86 & 27.4 \\
19 & 13.7 & 71 & 25.7 \\
20 & 13.8 & 64 & 24.9 \\
21 & 14.0 & 78 & 34.5 \\
22 & 14.2 & 80 & 31.7 \\
23 & 14.5 & 74 & 36.3 \\
24 & 16.0 & 72 & 38.3 \\
25 & 16.3 & 77 & 42.6 \\
26 & 17.3 & 81 & 55.4 \\
27 & 17.5 & 82 & 55.7 \\
28 & 17.9 & 80 & 58.3 \\
29 & 18.0 & 80 & 51.5 \\
30 & 18.0 & 80 & 51.0 \\
31 & 20.6 & 87 & 77.0 \\
\hline
\end{tabular}

Fonte: Aitkinson (1982). 


\section{Capítulo 6}

\section{Aperfeiçoamento de testes escore para modelos da família exponencial uniparamétrica}

\subsection{Introdução}

O propósito deste capítulo é fornecer uma expressão geral e em forma fechada para a correção tipo Bartlett da estatística escore para as distribuições da família exponencial uniparamétrica. Correções de Bartlett para o teste da razào de verossimilhança nesta classe de modelos foram obtidas por Cordeiro, Cribari-Neto, Aubin e Ferrari (1995). Nossa fórmula é facilmente aplicável e envolve somente operações triviais com certas funções e suas derivadas.

Consideremos um conjunto de $n$ variáveis aleatórias independentes e identicamente distribuídas com função densidade ou de probabilidade da forma

$$
\pi(y ; \theta)=\frac{1}{\zeta(\theta)} \exp \{-\alpha(\theta) d(y)+v(y)\}
$$

onde $\theta$ é um parâmetro escalar, $\zeta(\cdot), \alpha(\cdot), d(\cdot)$ e $v(\cdot)$ são funções conhecidas e $\zeta(\cdot)$ é positiva. Assume-se que o suporte de $\pi(y ; \theta)$ é independente de $\theta$ e que $\alpha(\cdot)$ e $\zeta(\cdot)$ têm as quatro primeiras derivadas contínuas com respeito a $\theta$, com $\mathrm{d} \alpha(\theta) / \mathrm{d} \theta \neq 0$ e $\mathrm{d} \beta(\theta) / \mathrm{d} \theta \neq 0$ para todo $\theta$ no espaço paramétrico. Aqui, $\beta(\theta)$ é definido como

$$
\beta(\theta)=\frac{\zeta^{\prime}(\theta)}{\zeta(\theta) \alpha^{\prime}(\theta)},
$$

onde $\zeta^{\prime}(\theta)$ e $\alpha^{\prime}(\theta)$ denotam derivadas com respeito a $\theta$. Estas quantidades têm papel importante no desenvolvimento de correções. Como se sabe. a família exponencial permite dar tratamento unificado para muitas distribuições importantes e goza de propriedades interessantes (Bickel e Doksum, 197T).

Na próxima seção, desenvolvemos uma expressão simples para a correção tipo Bartlett para o teste escore na família exponencial (6.1). Esta expressào envolve somente as funçòes 
$\alpha(\cdot)$ e $\beta(\cdot)$. com suas três primeiras derivadas e pode ser facilmente implementada em sistemas computacionais algébricos como MATHEMATICA (Wolfram, 1991) ou MAPLE V (Abell e Baselton, 1994). Na Seção 6.3, apresentamos uma série de casos especiais. Na Seção 6.4, apresentamos uma análise gráfica examinando como a correção varia $\operatorname{com} \theta$. $\mathrm{Na}$ Seção 6.j. sào consideradas correções tipo Bartlett para a família exponencial natural com alguns tipos de funçòes de variância.

\subsection{Correção para o teste escore}

Sejam $y_{1}, \ldots, y_{n}$ um conjunto de $n$ variáveis aleatórias independentes e identicamente distribuídas com função densidade (ou de probabilidade) dada por

$$
\pi(y ; \theta)=\exp \{t(y ; \theta)\}
$$

onde $\theta$ é um parâmetro escalar e definimos $v_{(r)}=E\left\{\left(t^{\prime}(y ; \theta)\right)^{r}\right\}$ e $v_{r}=E\left\{t^{(r)}(y ; \theta)\right\}$, para $r=1, \ldots, 4, \operatorname{com}^{\prime}(y ; \theta)=\partial t(y ; \theta) / \partial \theta$ e $t^{(r)}(y ; \theta)=\partial^{r} t(y ; \theta) / \partial \theta^{r}$. Os $v$ 's satisfazem certas relações de regularidade tais como $v_{1}=0 . v_{(2)}=-v_{2}, v_{(3)}=2 v_{3}-3 v_{2}^{\prime}$ e $v_{(4)}=$ $-3 v_{4}+8 v_{3}^{\prime}-6 v_{2}^{\prime \prime}+3 v_{2(2)}$, onde $v_{2(2)}=\mathrm{E}\left\{\left(t^{\prime \prime}(y ; \theta)\right)^{2}\right\}$ (vide Lawley, 1956).

A estatística escore para testar a hipótese $H_{0}: \theta=\theta^{(0)}$ contra $H_{1}: \theta \neq \theta^{(0)}$, sendo $\theta^{(0)}$ um valor especificado, pode ser escrita como $S_{R}=\left(\sum_{l=1}^{n} t^{\prime}\left(y_{l} ; \theta^{(0)}\right)\right)^{2} /\left(n v_{(2)}\right)$, onde $v_{(2)}$ é avaliada em $\theta^{(0)}$. É claro que o cálculo de $S_{R}$ não envolve qualquer estimação, já que depende somente do valor de $\theta$ fixado sob $H_{0}$. Sob a hipótese nula, $S_{R}$ tem uma distribuição $\chi_{1}^{2}$. Para modelos com um único parâmetro (escalar) a estatística escore modificada tem distribuição $\lambda_{1}^{2}$ até ordem $n^{-1}$ (vide Seção 1.2) e tem a forma

$$
S_{R}^{*}=S_{R}\left\{1-\frac{1}{n}\left(c+b S_{R}+a S_{R}^{2}\right)\right\},
$$

onde

$$
a=\frac{\gamma_{1}^{2}}{36}, \quad b=\frac{3 \gamma_{2}-10 \gamma_{1}^{2}}{36}, \quad c=\frac{5 \gamma_{1}^{2}-3 \gamma_{2}}{12}
$$

(vide Cordeiro e Ferrari, 1991, Seção 4).

Aqui, $\gamma_{1}=v_{(3)} / v_{(2)}^{3 / 2}$ e $\gamma_{2}=\left(v_{(4)}-3 v_{(2)}^{2}\right) / v_{(2)}^{2}$ são medidas usuais de assimetria e curtose da função escore para uma única observação.

Consideremos agora $\pi(y ; \theta)$ definida como em $(6.1), \operatorname{com} t(y ; \theta)=-\log \zeta(\theta)-\alpha(\theta) d(y)$ $+v(y)$. Das quatro primeiras derivadas de $t(y ; \theta) \operatorname{com}$ respeito a $\theta$ e usando as relações acima para os $v$ 's, temos que

$$
\begin{aligned}
& \mathrm{E}\{d(y)\}=-\beta, \quad \operatorname{Var}\{d(y)\}=\beta^{\prime} / \alpha^{\prime}, \quad v_{2}=-\alpha^{\prime} \beta^{\prime}, \quad v_{3}=-2 \alpha^{\prime \prime} \beta^{\prime}-\alpha^{\prime} \beta^{\prime \prime}, \\
& v_{4}=-3\left(\alpha^{\prime \prime \prime} \beta^{\prime}+\alpha^{\prime \prime} \beta^{\prime \prime}\right)-\alpha^{\prime} \beta^{\prime \prime \prime} \text { e } v_{2(2)}=\alpha^{\prime \prime 2} \beta^{\prime} / \alpha^{\prime}+\alpha^{\prime 2} \beta^{\prime 2},
\end{aligned}
$$

onde $\beta$ é definida em (6.2) e as linhas denotam derivadas em relação a $\theta$. Então. a estatística escore para testar $H_{0}$ versus $H_{1}$ tem uma forma simples dada por $S_{R}=n \alpha^{\prime}(\beta+$ 
$\bar{d})^{2} / \beta^{\prime}$, onde $\bar{d}=n^{-1} \sum d\left(y_{l}\right)$ e 3 e $\beta^{\prime}$ são avaliadas em $\theta^{(0)}$. Finalmente, usando as relações acima para os $v$ 's e substituindo-os nas expressões para $a, b$ e $c$ em (6.4), encontramos

$$
\begin{aligned}
a & =\frac{\left(\beta^{\prime} \alpha^{\prime \prime}-\alpha^{\prime} 3^{\prime \prime}\right)^{2}}{36 \alpha^{\prime 3} \beta^{\prime 3}}, \\
b & =\frac{-\beta^{\prime 2} \alpha^{\prime \prime 2}+11 \alpha^{\prime} \beta^{\prime} \alpha^{\prime \prime} \beta^{\prime \prime}-10 \alpha^{\prime 2} \beta^{\prime \prime 2}-3 \alpha^{\prime} \beta^{\prime 2} \alpha^{\prime \prime \prime}+3 \alpha^{\prime 2} \beta^{\prime} \beta^{\prime \prime \prime}}{36 \alpha^{\prime 3} \beta^{\prime 3}}, \\
c & =\frac{-4 \beta^{\prime 2} \alpha^{\prime \prime 2}-\alpha^{\prime} \beta^{\prime} \alpha^{\prime \prime} \beta^{\prime \prime}+5 \alpha^{\prime 2} \beta^{\prime \prime 2}+3 \alpha^{\prime} \beta^{\prime 2} \alpha^{\prime \prime \prime}-3 \alpha^{\prime 2} \beta^{\prime} \beta^{\prime \prime \prime}}{12 \alpha^{\prime 3} \beta^{\prime 3}} .
\end{aligned}
$$

Substituindo (6.5) - (6.7) em (6.3) e avaliando $\alpha^{\prime}, \alpha^{\prime \prime}, \alpha^{\prime \prime \prime}, \beta^{\prime}, \beta^{\prime \prime}$ e $\beta^{\prime \prime \prime}$ em $\theta^{(0)}$, obtemos a correção tipo Bartlett da estatística escore para testar $H_{0}$ contra $H_{1}$ nos modelos da família exponencial uniparamétrica.

Duas observações interessantes sobre as equações (6.5) - (6.7) merecem ser feitas. Primeiro, $c$ é igual ao coeficiente $\rho$ dividido por 12 da correção de Bartlett da estatística da razão de verossimilhança $R V$ (vide eq. (4) de Cordeiro, Cribari-Neto, Aubin e Ferrari, 1995). Segundo, $a, b$ e $c$ dependem do modelo (6.1) somente através das funções $\alpha$ e $\beta$ com suas três primeiras derivadas. Os coeficientes $a, b$ e $c$ podem também ser expressos em termos das primeiras quatro derivadas de $\alpha$ e $\zeta$, embora as equações (6.5) - (6.7) sejam mais simples de serem utilizadas. Da mesma forma que a correção de Bartlett para a estatística $R V$, a correção da estatística escore pode ser facilmente obtida para uma grande variedade de distribuições importantes. A obtenção da correção fica bastante simples usando-se um sistema computacional algébrico como MATHEMATICA (Wolfram, 1991).

\subsection{Casos especiais}

Nesta seção, apresentamos as quantidades $a, b$ e $c$ (dadas em (6.5) - (6.7)) necessárias à obtenção da correção tipo Bartlett para a estatística escore em diversas distribuições da família exponencial uniparamétrica (6.1). Os resultados foram obtidos usando os sistemas computacionais MATHEMATICA e MAPLE V. Os casos (i) - (viii) envolvem distribuições discretas enquanto (ix) - (xxiv) envolvem distribuições contínuas. É necessário observar que, nos casos discretos, não há garantia de que as correspondentes estatísticas corrigidas tenham distribuição $\chi_{1}^{2}$ até ordem $n^{-1}$ (Frydemberg e Jensen, 1989). O desempenho da correção para alguns modelos discretos será analisado através de simulação em trabalho futuro.

Os casos especiais considerados nesta seção envolvem distribuições de grande importância em aplicações práticas em várias áreas tais como engenharia, biologia. medicina, economia, zoologia, entre outras (Johnson e Kotz, 1970a, 1970b, Johnson, Kotz e Kemp, 1992). Aubin (1995) apresenta algumas considerações e particularidades que caracterizam as distribuições que estudamos. Entretanto, o leitor que necessitar de maiores detalhes e propriedades de todas as distribuições que consideramos, poderá consultar os nove volu- 
mes da "Encyclopedia of Statistical Sciences" (John Wiley and Sons, 198S, editada por S. Kotz e N.L. Johnson).

As distribuições que consideramos aqui serão caracterizadas pelas funções $\alpha(\cdot), \zeta(\cdot)$, $d(\cdot)$ e $v(y)$ e pelo espaço paramétrico, que juntos podem reproduzir, através de $(6.1)$, a função densidade (ou de probabilidade) correspondente.

As seguintes distribuições são consideradas:

(i) Binomial: $(0<\theta<1, m \in I N, m$ conhecido, $y=0,1,2, \ldots, m)$ : $\alpha(\theta)=$ $-\log \{\theta /(1-\theta)\}, \zeta(\theta)=(1-\theta)^{-m}, d(y)=y, v(y)=\log \left(\begin{array}{c}m \\ y\end{array}\right)$,

$$
a=\frac{(2 \theta-1)^{2}}{36 m \theta(1-\theta)}, \quad b=-\frac{22 \theta(\theta-1)+7}{36 m \theta(1-\theta)}, \quad c=-\frac{\theta(1-\theta)-1}{6 m \theta(1-\theta)} .
$$

(ii) Binomial negativa: $(0<\theta<1, \gamma>0, \gamma$ conhecido, $y=0,1,2, \ldots)$ : $\alpha(\theta)=$ $-\log \theta, \zeta(\theta)=(1-\theta)^{-\gamma}, d(y)=y, v(y)=\log \left(\begin{array}{c}\gamma+y-1 \\ y\end{array}\right)$,

$$
a=\frac{(\theta+1)^{2}}{36 \gamma \theta}, \quad b=-\frac{7 \theta^{2}+8 \theta+7}{36 \gamma \theta}, \quad c=\frac{1-\theta(1-\theta)}{6 \gamma \theta} .
$$

(iii) Poisson: $(\theta>0, y=0,1,2, \ldots): \alpha(\theta)=-\log (\theta), \zeta(\theta)=\exp \{\theta\}, d(y)=y$, $v(y)=-\log (y !), a=1 /(36 \theta), b=-7 /(36 \theta), c=1 /(6 \theta)$.

(iv) Poisson truncada: $(\theta>0, y=1,2, \ldots)$ : $\alpha(\theta)=-\log (\theta), \zeta(\theta)=e^{\theta}\left(1-e^{-\theta}\right)$, $d(y)=y, v(y)=-\log (y !)$,

$$
\begin{aligned}
a= & -\left\{\theta^{2}+3 \theta+1+e^{\theta}\left(\theta^{2}-3 \theta-2\right)+e^{2 \theta}\right\}^{2} /\left\{36 \theta e^{\theta}\left(1+\theta-e^{\theta}\right)^{3}\right\}, \\
b= & \left\{7+36 \theta+71 \theta^{2}+39 \theta^{3}+7 \theta^{4}+e^{\theta}\left(-28-108 \theta-140 \theta^{2}-9 \theta^{3}+8 \theta^{4}\right)\right. \\
& \left.+e^{2 \theta}\left(42+108 \theta+67 \theta^{2}-33 \theta^{3}+7 \theta^{4}\right)+e^{3 \theta}\left(-28-36 \theta+2 \theta^{2}+3 \theta^{3}\right)+7 e^{4 \theta}\right\} / \\
& \left\{36 \theta e^{\theta}\left(1+\theta-e^{\theta}\right)^{3}\right\}, \\
c= & -\left\{2+6 \theta+16 \theta^{2}+9 \theta^{3}+2 \theta^{4}+e^{\theta}\left(-8-18 \theta-40 \theta^{2}-9 \theta^{3}-2 \theta^{4}\right)\right. \\
& \left.+e^{2 \theta}\left(12+18 \theta+32 \theta^{2}-3 \theta^{3}+2 \theta^{4}\right)+e^{3 \theta}\left(-8-6 \theta-8 \theta^{2}+3 \theta^{3}\right)+2 e^{4 \theta}\right\} / \\
& \left\{12 \theta e^{\theta}\left(1+\theta-e^{\theta}\right)^{3}\right\} .
\end{aligned}
$$

(v) Série logarítmica: $(0<\theta<1, y=1,2, \ldots)$ : $\alpha(\theta)=-\log (\theta), \zeta(\theta)=-\log (1-\theta)$, $d(y)=y, v(y)=-\log (y)$,

$$
\begin{aligned}
a= & -\left\{\log (1-\theta)(3 \theta+\theta \log (1-\theta)+\log (1-\theta))+2 \theta^{2}\right\}^{2} /\left\{36 \theta(\theta+\log (1-\theta))^{3}\right\}, \\
b= & {\left[22 \theta^{3}(\theta+3 \log (1-\theta))+\theta^{2}\{\log (1-\theta)\}^{2}(28 \theta+73)\right.} \\
& \left.+3 \theta\{\log (1-\theta)\}^{3}\left(12 \theta+12-\theta^{2}\right)+\{\log (1-\theta)\}^{4}\left(7+8 \theta+7 \theta^{2}\right)\right] / \\
& \left\{36 \theta(\theta+\log (1-\theta))^{3}\right\}, \\
c= & -\left[2 \theta^{3}(\theta+3 \log (1-\theta))+8 \theta^{2}\{\log (1-\theta)\}^{2}(1+\theta)\right. \\
& \left.+3 \theta\{\log (1-\theta)\}^{3}\left(2+2 \theta-\theta^{2}\right)+2\{\log (1-\theta)\}^{4}\left(\theta^{2}-\theta+1\right)\right] / \\
& \left\{12 \theta(\theta+\log (1-\theta))^{3}\right\} .
\end{aligned}
$$


(vi) Série de potências: $\left(\theta>0, a_{y} \geq 0, y=0.1,2 \ldots\right)$ : $\alpha=-\log (\theta), \zeta(\theta)=$ $\sum_{y=0}^{\infty} a_{y} \theta^{y} \cdot d(y)=y, v(y)=\log \left(a_{y}\right)$

$$
\begin{aligned}
& a=\frac{\left(g+3 \theta g^{\prime}+\theta^{2} g^{\prime \prime}\right)^{2}}{36 \theta\left(g+\theta g^{\prime}\right)^{3}}, \\
& b=-\frac{i g^{2}+36 \theta g g^{\prime}+\theta^{2}\left(69 g^{\prime 2}+2 g g^{\prime \prime}\right)+3 \theta^{3}\left(14 g^{\prime} g^{\prime \prime}-g g^{\prime \prime \prime}\right)+\theta^{4}\left(10 g^{\prime \prime 2}-3 g^{\prime} g^{\prime \prime \prime}\right)}{36 \theta\left(g+\theta g^{\prime}\right)^{3}}, \\
& c=\frac{2 g^{2}+6 \theta g g^{\prime}+8 \theta^{2}\left(3 g^{\prime 2}-g g^{\prime \prime}\right)+3 \theta^{3}\left(g^{\prime} g^{\prime \prime}-g g^{\prime \prime \prime}\right)+\theta^{4}\left(5 g^{\prime \prime 2}-3 g g^{\prime \prime \prime}\right)}{12 \theta\left(g+\theta g^{\prime}\right)^{3}},
\end{aligned}
$$

onde $g=g(\theta)=\mathrm{d} \log \zeta(\theta) / \mathrm{d} \theta$. Note que os casos (iii), (iv) e (v) podem ser obtidos deste caso por simples especificação da função $g(\cdot)$.

(vii) Zeta: $(\theta>0, y=1,2,3, \ldots): \alpha(\theta)=\theta+1, \zeta(\theta)=\operatorname{Zeta}(\theta+1), d(y)=\log (y)$, $v(y)=0$,

$$
a=\frac{g^{\prime \prime 2}}{36 g^{\prime 3}}, b=-\frac{10 g^{\prime \prime 2}-3 g^{\prime} g^{\prime \prime \prime}}{36 g^{\prime 3}}, c=\frac{5 g^{\prime 2}-3 g^{\prime} g^{\prime \prime \prime}}{12 g^{\prime 3}},
$$

onde $\zeta$ é a função zeta de Riemann, isto é, $\zeta(\theta)=\operatorname{Zeta}(\theta+1)=\sum_{i=1}^{\infty} i^{-(\theta+1)}$ (vide Patterson, 1988) e $g=g(\theta)=\mathrm{d} \log \operatorname{Zeta}(\theta+1) / \mathrm{d} \theta$.

(viii) Hipergeométrica não-central: $\left(\theta>0, m_{1}, m_{2}, r\right.$ são inteiros positivos conhecidos, $\left.k_{1}=\max \left\{0, r-m_{2}\right\} \leq y \leq \min \left\{m_{1}, r\right\}=k_{2}\right): \alpha(\theta)=\theta, \zeta(\theta)=D_{0}(\theta)$, $d(y)=-y, v(y)=\log \left\{\left(\begin{array}{c}m_{1} \\ y\end{array}\right)\left(\begin{array}{c}m_{2} \\ r-y\end{array}\right)\right\}$,

$$
\begin{aligned}
a= & \left(D_{0}^{2} D_{3}-3 D_{0} D_{1} D_{2}+2 D_{1}^{3}\right)^{2} /\left\{36\left(D_{1}^{2}-D_{0} D_{2}\right)^{3}\right\} \\
b= & \left(10 D_{0}^{4} D_{3}^{2}-48 D_{0}^{3} D_{1} D_{2} D_{3}+28 D_{0}^{2} D_{1}^{3} D_{3}+45 D_{0}^{2} D_{1}^{2} D_{2}^{2}-66 D_{0} D_{1}^{4} D_{2}\right. \\
& \left.+22 D_{1}^{6}-3 D_{0}^{4} D_{2} D_{4}+9 D_{0}^{3} D_{2}^{3}+3 D_{0}^{3} D_{1}^{2} D_{4}\right) /\left\{36\left(D_{1}^{2}-D_{0} D_{2}\right)^{3}\right\} \\
c= & \left(-5 D_{0}^{4} D_{3}^{2}+18 D_{0}^{3} D_{1} D_{2} D_{3}-8 D_{0}^{2} D_{1}^{3} D_{3}+6 D_{0} D_{1}^{4} D_{2}-2 D_{1}^{6}+3 D_{0}^{4} D_{2} D_{4}\right. \\
& \left.-9 D_{0}^{3} D_{2}^{3}-3 D_{0}^{3} D_{1}^{2} D_{4}\right) /\left\{12\left(D_{1}^{2}-D_{0} D_{2}\right)^{3}\right\},
\end{aligned}
$$

onde $D_{p}=D_{p}(\theta)=\sum_{y=k_{1}}^{k_{2}} y^{p}\left(\begin{array}{c}m_{1} \\ y\end{array}\right)\left(\begin{array}{c}m_{2} \\ r-y\end{array}\right) \exp \{\theta y\}, p=0,1,2,3,4$.

(ix) Maxwell: $(\theta>0, y>0): \alpha(\theta)=\left(2 \theta^{2}\right)^{-1}, \zeta(\theta)=\theta^{3}, d(y)=y^{2}, v(y)=$ $\log \left(y^{2} \sqrt{2 / \pi}\right), a=2 / 27, b=-11 / 27, c=1 / 9$.

(x) Gama: $(k>0, \theta>0, y>0)$ :

(a) $k$ conhecido: $\alpha(\theta)=\theta, \zeta(\theta)=\theta^{-k}, d(y)=y, v(y)=(k-1) \log (y)-\log \{\Gamma(k)\}$, $a=1 /(9 k), \quad b=-11 /(18 k), c=1 /(6 k)$.

(b) $\theta$ conhecido: $a(k)=1-k, \zeta(k)=\theta^{-k} \Gamma(k) \cdot d(y)=\log (y), v(y)=-\theta y$, $a=\frac{\psi^{\prime \prime}(k)^{2}}{36 \psi^{\prime}(k)^{3}} . \quad b=\frac{-10 \psi^{\prime \prime}(k)^{2}+3 \psi^{\prime}(k) \psi^{\prime \prime \prime}(k)}{36 \psi^{\prime}(k)^{3}}, \quad c=\frac{5 \psi^{\prime \prime}(k)^{2}-3 \psi^{\prime}(k) \psi^{\prime \prime \prime}(k)}{12 \psi^{\prime}(k)^{3}}$, onde $\Gamma(\cdot)$ e $\psi(\cdot)$ são as funções gama e digama, respectivamente. 
(xi) Sistema Burr de distribuições : $(\theta>0, b>0, b$ conhecido, $y>0)$ (Burr. 1942): $\alpha(\theta)=\theta, \zeta(\theta)=r^{\theta} / \theta \cdot d(y)=-\log G(y), v(y)=\log \left\{ \pm G(y)^{-1} \cdot \mathrm{d} G(y) / \mathrm{d} y\right\}$, $a=1 / 9, b=-11 / 18, c=1 / 6$. onde as funções $r^{\hat{\theta}}$ e $G(\cdot)$ assumem valores positivos e o sinal de $v(y)$ é $+(-)$ se $\mathrm{d} G(y) / \mathrm{d} y>0(<0)$. Diferentes escolhas de $r$ e $G(y)$ conduzem a diferentes distribuiçòes neste sistema. As distribuições Burr I e Burr IX não pertencem à família exponencial uniparamétrica.

(xii) Rayleigh: $(\theta>0, y>0): \alpha(\theta)=\theta^{-2}, \zeta(\theta)=\theta^{2}, d(y)=y^{2}, v(y)=\log (2 y)$, $a=1 / 9, \quad b=-11 / 18, c=1 / 6$.

(xiii) Pareto: $(\theta>0, k>0, k$ conhecido, $y>k): \alpha(\theta)=\theta+1, \zeta(\theta)=\left(\theta k^{\theta}\right)^{-1}$, $d(y)=\log (y), v(y)=0, a=1 / 9, b=-11 / 18, c=1 / 6$.

(xiv) Weibull: $(\theta>0, \phi>0, \phi$ conhecido, $y>0): \alpha(\theta)=\theta^{-\phi}, \zeta(\theta)=\theta^{\phi}, d(y)=y^{\phi}$, $v(y)=\log (\phi)+(\phi-1) \log (y), a=1 / 9, b=-11 / 18, c=1 / 6$.

(xv) Potência: $(\theta>0, \phi>0,0$ conhecido, $y>\phi): \alpha(\theta)=1-\theta, \zeta(\theta)=\theta^{-1} \phi^{\theta}$, $d(y)=\log (y), v(y)=0, a=1 / 9, b=-11 / 18, c=1 / 6$.

(xvi) Laplace: $(\theta>0,-\infty<k<\infty, k$ conhecido, $y>0): \alpha(\theta)=\theta^{-1}, \zeta(\theta)=2 \theta$, $d(y)=|y-k|, v(y)=0, a=1 / 9, b=-11 / 18, c=1 / 6$.

(xvii) Valor extremo: $(-\infty<\theta<\infty, \phi>0, \phi$ conhecido, $-\infty<y<\infty)$ : $\alpha(\theta)=$ $\exp \{\theta / \phi\}, \zeta(\theta)=\phi \exp \{-\theta / \phi\}, d(y)=\exp \{-y / \phi\}, v(y)=-y / \phi, a=1 / 9, b=$ $-11 / 18, c=1 / 6$.

(xviii) Valor extremo truncado: $(\theta>0, y>0): \alpha(\theta)=\theta^{-1}, \zeta(\theta)=\theta, d(y)=$ $\exp \{y\}-1, v(y)=y, a=1 / 9, b=-11 / 18, c=1 / 6$.

(xix) Lognormal: $(\theta>0,-\infty<\mu<\infty, \mu$ conhecido, $y>0)$ : $\alpha(\theta)=\theta^{-2}, \zeta(\theta)=\theta$, $d(y)=(\log y-\mu)^{2} / 2, v(y)=-\log y-\{\log (2 \pi)\} / 2, a=2 / 9, b=-11 / 9, c=1 / 3$.

(xx) Normal: $(\theta>0,-\infty<\mu<\infty,-\infty<y<\infty)$ :

(a) $\mu$ conhecido: $\alpha(\theta)=(2 \theta)^{-1}, \zeta(\theta)=\theta^{1 / 2}, d(y)=(y-\mu)^{2}, v(y)=-\{\log (2 \pi)\} / 2$, $a=2 / 9, \quad b=-11 / 9, c=1 / 3$.

(b) $\theta$ conhecido: $\alpha(\mu)=-\mu / \theta, \zeta(\mu)=\exp \left\{\mu^{2} /(2 \theta)\right\}, d(y)=y, v(y)=-\left\{y^{2}+\right.$ $\log (2 \pi \theta)\} / 2, \quad a=0, \quad b=0, c=0$.

(xxi) Normal inversa: $(\theta>0, \mu>0, y>0)$ :

(a) $\mu$ conhecido: $\alpha(\theta)=\theta, \zeta(\theta)=\theta^{-1 / 2}, d(y)=(y-\mu)^{2} /\left(2 \mu^{2} y\right), v(y)=-\left\{\log \left(2 \pi y^{3}\right)\right\} / 2$, $a=2 / 9, b=-11 / 9, c=1 / 3$.

(b) $\theta$ conhecido: $\alpha(\mu)=\theta /\left(2 \mu^{2}\right), \zeta(\mu)=\exp \{-\theta / \mu\}, d(y)=y, v(y)=-\theta /(2 y)+$ $\left[\log \left\{\theta /\left(2 \pi y^{3}\right)\right\}\right] / 2, a=\mu /(4 \theta), b=-5 \mu /(4 \theta), c=0$. 
(xxii) McCullagh: $(\theta>-1 / 2,-1 \leq \mu \leq 1, \mu$ conhecido, $0<y<1): \alpha(\theta)=-\theta, \zeta(\theta)=$ $4^{-\theta} \mathrm{B}(\theta+1 / 2,1 / 2), d(y)=\log \left[y(1-y) /\left\{(1+\mu)^{2}-4 \mu y\right\}\right] . \imath(y)=-[\log \{y(1-y)\}] / 2$,

$$
\begin{gathered}
a=\frac{\left(\psi^{\prime \prime}(\theta+1)-\psi^{\prime \prime}(\theta+0.5)\right)^{2}}{36\left(\psi^{\prime}(\theta+0.5)-\psi^{\prime}(\theta+1)\right)^{3}}, \\
b=\frac{-10\left(\psi^{\prime \prime}(\theta+1)-\psi^{\prime \prime}(\theta+0.5)\right)^{2}+3\left(\psi^{\prime}(\theta+1)-\psi^{\prime}(\theta+0.50)\right)\left(\psi^{\prime \prime \prime}(\theta+1)-\psi^{\prime \prime \prime}(\theta+0.5)\right)}{36\left(\psi^{\prime}(\theta+0.5)-\psi^{\prime}(\theta+1)\right)^{3}}, \\
c=\frac{5\left(\psi^{\prime \prime}(\theta+1)-\psi^{\prime \prime}(\theta+0.5)\right)^{2}-3\left(\psi^{\prime}(\theta+1)-\psi^{\prime}(\theta+0.5)\right)\left(\psi^{\prime \prime \prime}(\theta+1)-\psi^{\prime \prime \prime}(\theta+0.5)\right)}{12\left(\psi^{\prime}(\theta+0.5)-\psi^{\prime}(\theta+1)\right)^{3}},
\end{gathered}
$$

onde $\mathrm{B}(\cdot, \cdot)$ é a função beta (vide McCullagh, 1989).

(xxiii) von Mises: $(\theta>0,0<\mu<2 \pi, \mu$ conhecido, $0<y<2 \pi)$ : $\alpha(\theta)=-\theta$, $\zeta(\theta)=2 \pi I_{0}(\theta), d(y)=\cos (y-\mu), v(y)=0$,

$$
a=\frac{r^{\prime \prime}(\theta)^{2}}{36 r^{\prime}(\theta)^{3}}, \quad b=\frac{-10 r^{\prime \prime}(\theta)^{2}+3 r^{\prime}(\theta) r^{\prime \prime \prime}(\theta)}{36 r^{\prime}(\theta)^{3}}, \quad c=\frac{5 r^{\prime \prime}(\theta)^{2}-3 r^{\prime}(\theta) r^{\prime \prime \prime}(\theta)}{12 r^{\prime}(\theta)^{3}} .
$$

onde $I_{\nu}(\cdot)$ é a função Bessel modificada de primeira espécie e $\nu$-ésima ordem, e $r(\theta)=I_{0}^{\prime}(\theta) / I_{0}(\theta)$.

(xxiv) Secante hiperbólica generalizada: $(-\pi / 2 \leq \theta \leq \pi / 2, r>0, r$ conhecido, $0<y<1): \alpha(\theta)=\theta, \zeta(\theta)=\pi(\sec \theta)^{r}, d(y)=-\log \{y /(1-y)\} / \pi, v(y)=$ $-\log \{y(1-y)\} / 2$,

$$
a=\frac{1-(\cos \theta)^{2}}{9 r}, \quad b=-\frac{11-14(\cos \theta)^{2}}{18 r}, \quad c=\frac{1-4(\cos \theta)^{2}}{6 r} .
$$

Entre os casos estudados aqui, as seguintes distribuições foram previamente consideradas por Cordeiro, Ferrari e Paula (1993): binomial, Poisson, gama (caso a), normal inversa (caso b) e normal (caso b). O último caso, correspondente a testar a média de uma distribuição normal com variância conhecida, é o único para o qual $a=b=c=0$, que concorda com o fato de que $S_{R}$ tem uma distribuição exata $\chi_{1}^{2}$ sob a hipótese nula. Um resultado interessante obtido aqui é que vários casos correspondem às seguintes constantes: $a=1 / 9, b=-11 / 18$ e $c=1 / 6$. Isto implica que $S_{R}^{*}=S_{R}\left\{1-\left(3-11 S_{R}+2 S_{R}^{2}\right) /(18 n)\right\}$. Como $a, b$ e $c$ determinam a expansão da função distribuição da estatística escore até ordem $O\left(n^{-1}\right)$ sob a hipótese nula (vide Cordeiro e Ferrari, 1991), uma conclusão é que a estatística escore para tais exemplos têm a mesma distribuição até ordem $O\left(n^{-1}\right)$ e não somente até ordem $O\left(n^{0}\right)$. É fácil verificar que $a, b$ e $c$ são iguais às constantes dadas acima se uma das seguintes condições é verdadeira: (a1) $\alpha(\theta) \zeta(\theta)=c_{1}$ ou (a2) $\alpha(\theta)=c_{1} \theta+c_{2}$, $\left(c_{1}\right.$ e $c_{2}$ são usualmente iguais a 1 ou -1$)$ e $\zeta(\theta)=c_{3} /\left(\theta c_{4}^{\theta}\right)$, onde $c_{1}, \ldots, c_{4}$ são escalares conhecidos. Estas condições são individualmente suficientes mas não necessárias. As condições (a1) e (a2) sào equivalentes a (a3) $\operatorname{Var}(d(y))=\beta^{2}$ ou $(\mathrm{a} 4) \operatorname{Var}(d(y))=(\beta+r)^{2}$, onde $r$ é uma constante qualquer. As formas (a1) e (a2) acima são mais fáceis de serem verificadas na prática, pois evita o cálculo da variância de $d(y)$. 
Para alguns dos casos especiais considerados aqui, a correçào tem uma forma muito simples e para alguns a correção nem mesmo depende de $\theta$. Porém. em algumas situaçòes. as correçòes são funções complicadas de $\theta$ (por exemplo as distribuições de McCullagh. von Mises e zeta) que requerem a avaliação das funções de Bessel, poligama ou zeta. Com a finalidade de simplificar o cálculo da correção tipo Bartlett em tais casos, desenvolveremos aproximaçòes simples para $a, b$ e $c$.

Consideremos primeiramente as correções que envolvem a função poligama. Utilizando a expansão dada em (3.12) obtemos, para a distribuição gama ( $\theta$ conhecido),

$$
a=\frac{1}{36 k}+\frac{1}{72 k^{2}}+O\left(k^{-4}\right), \quad b=-\frac{1}{9 k}-\frac{1}{18 k^{2}}-\frac{1}{72 k^{3}}+O\left(k^{-4}\right)
$$

$\mathrm{e}$

$$
c=-\frac{1}{12 k}-\frac{1}{24 k^{2}}+\frac{1}{24 k^{3}}+O\left(k^{-4}\right) .
$$

Para a distribuição McCullagh, usando a equação

$$
\psi^{\prime}(z+1)-\psi^{\prime}(z+1 / 2)=2 \psi^{\prime}(z)-4 \psi^{\prime}(2 z)-z^{-2},
$$

obtemos para valores grandes de $\theta$ as seguintes expansões assintóticas:

$$
a=\frac{2}{9}-\frac{1}{24 \theta^{2}}+O\left(\theta^{-3}\right), \quad b=-\frac{11}{9}+\frac{1}{6 \theta^{2}}+O\left(\theta^{-3}\right) \quad \text { e } \quad c=\frac{1}{3}+\frac{1}{8 \theta^{2}}+O\left(\theta^{-3}\right) .
$$

Estes resultados fornecem boas aproximações quando $\theta$ é grande.

Para valores pequenos de $k$ podemos usar a expansão assintótica dada em (3.13). Então, para a distribuição gama ( $\theta$ conhecido) temos as seguintes expansões

$$
\begin{aligned}
& a=\frac{1}{9}-\frac{\pi^{2}}{18} k^{2}+\frac{8}{9} \zeta(3) k^{3}+O\left(k^{4}\right) . \\
& b=-\frac{11}{18}+\frac{7 \pi^{2}}{8} k^{2}-\frac{69}{9} \zeta(3) k^{3}+O\left(k^{4}\right), \\
& c=\frac{1}{6}-\frac{\pi^{2}}{3} k^{2}+\frac{22}{3} \zeta(3) k^{3}+O\left(k^{4}\right) .
\end{aligned}
$$

Para a distribuição $\mathrm{McCullagh}$ obtemos, para valores pequenos de $\theta$, usando o sistema MAPLE V, as seguintes aproximações numéricas:

$$
\begin{aligned}
a \approx & 0,1623209489+0,889901795 \theta-0,7016232778 \theta^{2} \\
& +0,01725636111 \theta^{3}+0,45005 \theta^{4} \\
b \approx & -0,923209489-0,4998371028 \theta+0.4594028611 \theta^{2} \\
& -0,1943055556 \theta^{3}+0,1795111111 \theta^{4} . \\
c \approx & 0,3348142337+0,1646586192 \theta-0.3257735583 \theta^{2} \\
& +0,3240720833 \theta^{3}-0,1365358333 \theta^{4} .
\end{aligned}
$$

Estas expressões são muito convenientes, já que não requerem a avaliação de funções poligama. 
Para o caso da distribuição de von Mises, a correção envolve a função $r(\cdot)$ e suas três primeiras derivadas. Para valores grandes de $\theta$, podemos escrever $r(\theta)$ como (Abramowitz e Stegun, 1970, pp.416-421)

$$
r(\theta)=1-\frac{1}{2 \theta}-\frac{1}{8 \theta^{2}}-\frac{25}{128 \theta^{4}}-\frac{13}{32 \theta^{5}}+\cdots
$$

Usando esta expansão, temos

$$
\begin{aligned}
a & =\frac{2}{9}+\frac{1}{8 \theta^{2}}+\frac{23}{48 \theta^{3}}+O\left(\theta^{-4}\right), \\
b & =-\frac{11}{9}-\frac{1}{2 \theta^{2}}-\frac{77}{48 \theta^{3}}+O\left(\theta^{-4}\right), \\
c & =\frac{1}{3}-\frac{3}{8 \theta^{2}}-\frac{19}{8 \theta^{3}}+O\left(\theta^{-4}\right)
\end{aligned}
$$

para $\theta$ grande. Para valores pequenos de $\theta$ podemos usar o fato de que (Mardia, 1972, p.63)

$$
r(\theta)=\frac{\theta}{2}\left\{1-\frac{\theta^{2}}{8}+\frac{\theta^{4}}{48}-\cdots\right\}
$$

de onde segue que

$$
\begin{aligned}
& a=\frac{\theta^{2}}{32}+O\left(\theta^{4}\right), \\
& b=-\frac{1}{8}-\frac{19 \theta^{2}}{96}+O\left(\theta^{4}\right), \\
& c=\frac{3}{8}+\frac{\theta^{2}}{8}+O\left(\theta^{4}\right) .
\end{aligned}
$$

Vejamos, agora, a distribuição série logarítimica. Para valores pequenos de $\theta$, temos que

$$
\log (1-\theta)=-\theta-\frac{\theta^{2}}{2}-\frac{\theta^{3}}{3}-\frac{\theta^{4}}{4}-\frac{\theta^{5}}{5}-\frac{\theta^{6}}{6}+O\left(\theta^{7}\right)
$$

Usando este resultado temos as seguintes expansões:

$$
\begin{aligned}
& a=\frac{1}{18 \theta}+\frac{1}{54}+O(\theta), \\
& b=-\frac{7}{18 \theta}-\frac{10}{57}+O(\theta), \\
& c=\frac{1}{3 \theta}-\frac{5}{18}+O(\theta) .
\end{aligned}
$$

Para a distribuição de Poisson truncada fazemos uso da expansão em série da função exponencial

$$
\epsilon^{\theta}=1+\theta+\frac{\theta^{2}}{2}+\frac{\theta^{3}}{6}+\frac{\theta^{4}}{23}+\cdots
$$


para obter as seguintes expansòes para $\theta$ pequeno:

$$
\begin{aligned}
& a=\frac{1}{18 \theta}+\frac{1}{54}+O(\theta), \\
& b=-\frac{\tau}{18 \theta}-\frac{2}{2 \tau}+O(\theta) . \\
& c=\frac{1}{3 \theta}-\frac{1}{18}+O(\theta) .
\end{aligned}
$$

Finalmente, consideramos a distribuição zeta. Para encontrar uma expressão aproximada para $a, b$ e $c$, usamos a seguinte expansão da função zeta de Riemann, que denotamos por $\zeta(\cdot)$, dacla em Abramowitz e Stegun (1970, p.807),

$$
\zeta(\theta+1)=\frac{1}{\theta}+\sum_{n=0}^{\infty} \frac{(-1)^{n}}{n !} \gamma_{n} \theta^{n},
$$

onde os coeficientes $\gamma_{n}$ são dados por

$$
\gamma_{n}=\lim _{m \rightarrow \infty}\left\{\sum_{k=1}^{m} \frac{(\log k)^{n}}{k}-\frac{(\log m)^{n+1}}{n+1}\right\},
$$

$n=0,1,2,3$, sendo $\gamma_{0}$ a constante de Euler. A expansão acima implica que

$$
\begin{aligned}
\zeta^{\prime}(\theta+1)= & -\frac{1}{\theta^{2}}-\gamma_{1}+\gamma_{2} \theta-\frac{\gamma_{3} \theta^{2}}{2}+\frac{\gamma_{4} \theta^{3}}{6}-\frac{\gamma_{5} \theta^{4}}{24}+\frac{\gamma_{6} \theta^{5}}{120} \\
& -\frac{\gamma_{7} \theta^{6}}{720}+O\left(\theta^{\tau}\right) .
\end{aligned}
$$

Como $g(\theta)=\mathrm{d} \log \zeta(\theta+1) / \mathrm{d} \theta=\zeta^{\prime}(\theta+1) / \zeta(\theta+1)$, segue que

$$
\begin{aligned}
g(\theta)= & -\frac{1}{\theta}+\gamma_{0}-\left(2 \gamma_{1}+\gamma_{0}^{2}\right) \theta+\left(\gamma_{0}^{3}+3 \gamma_{0} \gamma_{1}+\frac{3}{2} \gamma_{2}\right) \theta^{2} \\
& -\left(\gamma_{0}^{4}+4 \gamma_{0}^{2} \gamma_{1}+2 \gamma_{0} \gamma_{1}+2 \gamma_{1}^{2}+\frac{2}{3} \gamma_{3}\right) \theta^{3}+O\left(\theta^{4}\right) .
\end{aligned}
$$

Tomando as três primeiras derivadas da expansão acima para $g(\theta)$ e substituindo nas expressões de $a, b$ e $c$ do caso (vii), obtemos, para valores pequenos de $\theta$,

$$
\begin{aligned}
a= & \frac{1}{9}+\frac{1}{3}\left(\gamma_{0}^{2}+2 \gamma_{1}\right) \theta^{2}-\frac{4}{9}\left(2 \gamma_{0}^{3}+6 \gamma_{0} \gamma_{1}+3 \gamma_{2}\right) \theta^{3} \\
& +\frac{1}{9}\left(21 \gamma_{0}^{4}+84 \gamma_{0}^{2} \gamma_{1}+54 \gamma_{1}^{2}+30 \gamma_{0} \gamma_{2}+10 \gamma_{3}\right) \theta^{4}+O\left(\theta^{5}\right), \\
b= & -\frac{11}{18}-\frac{7}{3}\left(\gamma_{0}^{2}+2 \gamma_{1}\right) \theta^{2}+\frac{31}{9}\left(2 \gamma_{0}^{3}+6 \gamma_{0} \gamma_{1}+3 \gamma_{2}\right) \theta^{3} \\
& -\frac{1}{9}\left(174 \gamma_{0}^{4}+696 \gamma_{0}^{2} \gamma_{1}+441 \gamma_{1}^{2}+255 \gamma_{0} \gamma_{2}+85 \gamma_{3}\right) \theta^{4}+O\left(\theta^{5}\right), \\
c= & \frac{1}{6}+2\left(\gamma_{0}^{2}+2 \gamma_{1}\right) \theta^{2}-\frac{11}{3}\left(2 \gamma_{0}^{3}+6 \gamma_{0} \gamma_{1}+3 \gamma_{2}\right) \theta^{3} \\
& +\frac{1}{3}\left(69 \gamma_{0}^{4}+276 \gamma_{0}^{2} \gamma_{1}+171 \gamma_{1}^{2}+105 \gamma_{0} \gamma_{2}+35 \gamma_{3}\right) \theta^{4}+O\left(\theta^{5}\right) .
\end{aligned}
$$


É possível simplificar ainda mais as expressões acima, fazendo uma avaliação numérica dos $\gamma$ 's. Usando o sistema MAPLE V, obtemos

$$
\begin{aligned}
& a \approx \frac{1}{9}+\frac{7099}{113556} \theta^{2}-\frac{15043}{327410} \theta^{3}+\frac{24134}{42607} \theta^{4}, \\
& b \approx-\frac{11}{18}-\frac{18068}{29741} \theta^{2}+\frac{29835}{83788} \theta^{3}-\frac{24407}{62468} \theta^{4}, \\
& c \approx \frac{1}{6}+\frac{7099}{18926} \theta^{2}-\frac{137395}{362472} \theta^{3}+\frac{17629}{39036} \theta^{4} .
\end{aligned}
$$

Assim, quando $\theta \approx 0$, temos $a \approx 1 / 9, b \approx-11 / 8$ e $c \approx 1 / 6$. Uma aproximação de $a$, $b$ e $c$ para valores grandes de $\theta$ será objeto de estudos futuros, uma vez que a expansão apropriada da função zeta de Riemann, neste caso, tem uma expressão extremamente complicada.

Para avaliar a qualidade das aproximações dadas acima, calcularemos os valores exatos e aproximados das quantidades $a, b$ e $c$ para alguns valores de $\theta$. Os resultados apresentados nas Tabelas 6.1 a 6.9 correspondem às distribuições gama ( $k$ pequeno e grande), McCullagh ( $\theta$ pequeno e grande), von Mises ( $\theta$ pequeno e $\theta$ grande), série logarítmica, Poisson truncada e zeta.

Tabela 6.1: Valores exatos e aproximados de $a, b$ e $c$ para a distribuição gama ( $\theta$ conhecido, $k$ grande).

\begin{tabular}{ccccccc}
\hline$k$ & $\begin{array}{c}a \\
\text { exato }\end{array}$ & $\begin{array}{c}a \\
\text { aprox. }\end{array}$ & $\begin{array}{c}b \\
\text { exato }\end{array}$ & $\begin{array}{c}b \\
\text { aprox. }\end{array}$ & $\begin{array}{c}c \\
\text { exato }\end{array}$ & $\begin{array}{c}c \\
\text { aprox. }\end{array}$ \\
\hline 1,0 & 0,03607 & 0,04167 & $-0,16071$ & $-0,18056$ & $-0,05893$ & $-0,08333$ \\
2,0 & 0,01691 & 0,01736 & $-0,07015$ & $-0,07118$ & $-0,04322$ & $-0,04688$ \\
3,0 & 0,01071 & 0,01080 & $-0,04356$ & $-0,04372$ & $-0,02998$ & $-0,23086$ \\
10,0 & 0,00292 & 0,00292 & $-0,01168$ & $-0,01168$ & $-0,00870$ & $-0,00870$ \\
\hline
\end{tabular}

Tabela 6.2: Valores exatos e aproximados de $a, b$ e $c$ para a distribuição de McCullagh ( $\theta$ grande).

\begin{tabular}{ccccccc}
\hline$\theta$ & $\begin{array}{c}a \\
\text { exato }\end{array}$ & $\begin{array}{c}a \\
\text { aprox. }\end{array}$ & $\begin{array}{c}b \\
\text { exato }\end{array}$ & $\begin{array}{c}b \\
\text { aprox. }\end{array}$ & $\begin{array}{c}c \\
\text { exato }\end{array}$ & $\begin{array}{c}c \\
\text { aprox. }\end{array}$ \\
\hline 1,0 & 0,20570 & 0,18056 & $-1,14932$ & $-1,05556$ & 0,36253 & 0,708333 \\
2,0 & 0,21543 & 0,21181 & $-1,19370$ & $-1,18056$ & 0,34960 & 0,42708 \\
3,0 & 0,21866 & 0,21759 & $-1,20755$ & $-1,12037$ & 0,34281 & 0,37500 \\
4,0 & 0,22005 & 0,21962 & $-1,21339$ & $-1,21181$ & 0,33937 & 0,35670 \\
5,0 & 0,22077 & 0,22056 & $-1,21635$ & $-1,21556$ & 0,33747 & 0,34833 \\
10,0 & 0,22183 & 0,22189 & -1.22066 & -1.22056 & 0,33454 & 0,33708 \\
\hline
\end{tabular}


Tabela 6.3: Valores exatos e aproximados de $a, b$ e $c$ para a distribuiçào gama ( $\theta$ conhecido. $k$ pequeno).

\begin{tabular}{ccccccc}
\hline$k$ & $\begin{array}{c}a \\
\text { exato }\end{array}$ & $\begin{array}{c}a \\
\text { aprox. }\end{array}$ & $\begin{array}{c}b \\
\text { exato }\end{array}$ & $\begin{array}{c}b \\
\text { aprox. }\end{array}$ & $\begin{array}{c}c \\
\text { exato }\end{array}$ & $\begin{array}{c}c \\
\text { aprox. }\end{array}$ \\
\hline 0.001 & 0,11111 & 0.11111 & $-0,611107$ & $-0,611107$ & 0,166663 & 0,166663 \\
0,01 & 0,11105 & 0,11105 & $-0,610736$ & -0.610736 & 0,166346 & 0,166346 \\
0,02 & 0,11090 & 0,11090 & $-0,609642$ & $-0,609642$ & 0,165421 & 0,165421 \\
0,03 & 0,11064 & 0,11064 & $-0,607879$ & $-0,607880$ & 0,163940 & 0,163941 \\
0,05 & 0,10987 & 0,10987 & $-0,602534$ & $-0,602551$ & 0,159511 & 0,159544 \\
0,10 & 0,10666 & 0,10669 & $-0,580649$ & $-0,581010$ & 0,141964 & 0,142583 \\
0,20 & 0,09692 & 0,09772 & $-0,515969$ & $-0,523831$ & 0,093993 & 0,105593 \\
0,30 & 0,08571 & 0,09061 & $-0,444150$ & $-0,489258$ & 0,046729 & 0,108586 \\
0,40 & 0,07497 & 0,09176 & $-0,377862$ & $-0,526976$ & 0,009000 & 0,204453 \\
\hline
\end{tabular}

Tabela 6.4: Valores exatos e aproximados de $a, b$ e $c$ para a distribuição McCullagh ( $\theta$ pequeno).

\begin{tabular}{ccccccc}
\hline$k$ & $\begin{array}{c}a \\
\text { exato }\end{array}$ & $\begin{array}{c}a \\
\text { aprox. }\end{array}$ & $\begin{array}{c}b \\
\text { exato }\end{array}$ & $\begin{array}{c}b \\
\text { aprox. }\end{array}$ & $\begin{array}{c}c \\
\text { exato }\end{array}$ & $\begin{array}{c}c \\
\text { aprox. }\end{array}$ \\
\hline 0,001 & 0,162410 & 0,163211 & $-0,923709$ & $-0,923709$ & 0,334979 & 0,333665 \\
0,01 & 0,163204 & 0,171213 & $-0,928162$ & $-0,928162$ & 0,336428 & 0,335109 \\
0,02 & 0,164073 & 0,180091 & $-0,933024$ & $-0,933023$ & 0,337979 & 0,336907 \\
0,03 & 0,164928 & 0,188955 & $-0,937797$ & $-0,937796$ & 0,339469 & 0,338723 \\
0,05 & 0,166597 & 0,206643 & $-0,947078$ & $-0,947078$ & 0,342272 & 0,342568 \\
0,10 & 0,170539 & 0,250631 & $-0,960807$ & $-0,968813$ & 0,348316 & 0,353515 \\
\hline
\end{tabular}

Tabela 6.5: Valores exatos e aproximados de $a, b$ e $c$ para a distribuição de von Mises ( $\theta$ pequeno).

\begin{tabular}{ccccccc}
\hline$\theta$ & $\begin{array}{c}a \\
\text { exato }\end{array}$ & $\begin{array}{c}a \\
\text { aprox. }\end{array}$ & $\begin{array}{c}b \\
\text { exato }\end{array}$ & $\begin{array}{c}b \\
\text { aprox. }\end{array}$ & $\begin{array}{c}c \\
\text { exato }\end{array}$ & $\begin{array}{c}c \\
\text { aprox. }\end{array}$ \\
\hline 0,1 & 0,00031 & 0,00031 & $-0,12698$ & $-0,12698$ & 0,37625 & 0,37625 \\
0,5 & 0,00784 & 0,00781 & $-0,19446$ & $-0,19448$ & 0,40584 & 0,40625 \\
1,0 & 0,03141 & 0,03125 & $-0,32119$ & $-0,32292$ & 0,49238 & 0,50000 \\
1,5 & 0,06962 & 0,07031 & $-0,55172$ & $-0,57031$ & 0.610811 & 0,65625 \\
2.0 & 0,11746 & 0,12500 & $-0,82500$ & $-0,91667$ & 0.71313 & 0,87500 \\
\hline
\end{tabular}


Tabela 6.6: Valores exatos e aproximados de $a . b$ e $c$ para a distribuição de von Mises ( $\theta$ grande).

\begin{tabular}{ccccccc}
\hline$\theta$ & $\begin{array}{c}a \\
\text { exato }\end{array}$ & $\begin{array}{c}a \\
\text { aprox. }\end{array}$ & $\begin{array}{c}b \\
\text { exato }\end{array}$ & $\begin{array}{c}b \\
\text { aprox. }\end{array}$ & $\begin{array}{c}c \\
\text { exato }\end{array}$ & $\begin{array}{c}c \\
\text { aprox. }\end{array}$ \\
\hline 3,0 & 0,20350 & 0,25386 & $-1,28562$ & $-1,33719$ & 0,68436 & 0,20370 \\
4,0 & 0,23748 & 0,23752 & $-1,33501$ & -1.27854 & 0,44281 & 0,27279 \\
5,0 & 0,23804 & 0,23106 & $-1,29192$ & -1.25506 & 0,30521 & 0,29933 \\
6,0 & 0,22846 & 0,22617 & $-1,2419.5$ & -1.23710 & 0.29895 & 0,31876 \\
8,0 & 0,22626 & 0,22442 & $-1,23170$ & $-1,23060$ & 0,31961 & 0,32545 \\
10,0 & 0,22431 & 0,22395 & $-1,22953$ & -1.22883 & 0.32401 & 0,32721 \\
\hline
\end{tabular}

Tabela 6.7: Valores exatos e aproximados de $a, b$ e $c$ para a distribuição em série logarítmica ( $\theta$ pequeno).

\begin{tabular}{ccccccc}
\hline$\theta$ & $\begin{array}{c}a \\
\text { exato }\end{array}$ & $\begin{array}{c}a \\
\text { aprox. }\end{array}$ & $\begin{array}{c}b \\
\text { exato }\end{array}$ & $\begin{array}{c}b \\
\text { aprox. }\end{array}$ & $\begin{array}{c}c \\
\text { exato }\end{array}$ & $\begin{array}{c}c \\
\text { aprox. }\end{array}$ \\
\hline 0,05 & 1,20712 & 1,20390 & $-8,16636$ & -8.14815 & 6.39225 & 6,38889 \\
0,10 & 0,65521 & 0,64815 & $-4,29687$ & $-4,25926$ & 3,06253 & 3,05556 \\
0,15 & 0,47390 & 0,46296 & $-3,02129$ & $-2,96296$ & 1,95533 & 1,94444 \\
0,20 & 0,38547 & 0,37037 & $-2,39535$ & $-2,31481$ & 1,40401 & 1,38889 \\
0,25 & 0,33439 & 0,31581 & $-2,03038$ & $-1,92593$ & 1,07529 & 1,05556 \\
0,30 & 0,30219 & 0,27778 & $-1,79699$ & -1.66667 & 0,85812 & 0,83333 \\
\hline
\end{tabular}

Tabela 6.8: Valores exatos e aproximados de $a, b$ e $c$ para a distribuição de Poisson truncada ( $\theta$ pequeno).

\begin{tabular}{ccccccc}
\hline$\theta$ & $\begin{array}{c}a \\
\text { exato }\end{array}$ & $\begin{array}{c}a \\
\text { aprox. }\end{array}$ & $\begin{array}{c}b \\
\text { exato }\end{array}$ & $\begin{array}{c}b \\
\text { aprox. }\end{array}$ & $\begin{array}{c}c \\
\text { exato }\end{array}$ & $\begin{array}{c}c \\
\text { aprox. }\end{array}$ \\
\hline 0,05 & 1,12902 & 1,12963 & $-7,85028$ & $-7,85185$ & 6,61561 & 6,61111 \\
0,10 & 0,57285 & 0,57407 & $-3,95976$ & $-3,96296$ & 3,28681 & 3,27778 \\
0,20 & 0,29398 & 0,29630 & $-2,01090$ & $-2,01852$ & 1,62758 & 1,61111 \\
0,30 & 0,20012 & 0,20370 & $-1,36020$ & $-1,37037$ & 1.07887 & 1,05556 \\
0,40 & 0,15269 & 0,15741 & $-1,03247$ & $-1,04630$ & 0,80714 & 0,77778 \\
0,50 & 0,12381 & 0,12963 & $-0,83432$ & $-0,85185$ & 0,64580 & 0,61111 \\
0,60 & 0,104231 & 0,11111 & $-0,70095$ & -0.72222 & 0,53937 & 0,50000 \\
0,70 & 0,08999 & 0,09788 & $-0,53148$ & -0.56019 & 0,40813 & 0,36111 \\
0,90 & 0,07044 & 0,08025 & $-0,47382$ & -0.50627 & 0,36493 & 0,31482 \\
1.00 & 0.06337 & 0,07407 & $-0,43703$ & -0.46963 & 0,33056 & 0,27778 \\
\hline
\end{tabular}


Tabela 6.9: Valores exatos e aproximados de $a$. $b$ e $c$ para a distribuiçào zeta ( $\theta$ pequeno).

\begin{tabular}{ccccccc}
\hline$\theta$ & $\begin{array}{c}a \\
\text { exato }\end{array}$ & $\begin{array}{c}a \\
\text { aprox. }\end{array}$ & $\begin{array}{c}b \\
\text { exato }\end{array}$ & $\begin{array}{c}b \\
\text { aprox. }\end{array}$ & $\begin{array}{c}c \\
\text { exato }\end{array}$ & $\begin{array}{c}c \\
\text { aprox. }\end{array}$ \\
\hline 0,05 & 0,11126 & 0,11127 & $-0,61216$ & $-0,61259$ & 0.16756 & 0,16756 \\
0,10 & 0.11169 & 0,11175 & $-0,61517$ & $-0,61680$ & 0.17008 & 0,17008 \\
0,20 & 0,11331 & 0,11415 & $-0,62629$ & $-0,63319$ & 0.17924 & 0,17936 \\
0,30 & 0,11580 & 0,12009 & -0.64333 & $-0,65934$ & 0.19298 & 0,19385 \\
0,40 & 0,11907 & 0,13267 & $-0,66555$ & $-0,69553$ & 0.21058 & 0,21398 \\
0.50 & 0,12305 & 0,15640 & $-0,69245$ & $-0,74290$ & 0.23160 & 0,24128 \\
\hline
\end{tabular}

Observando as Tabelas 6.1 - 6.9, verificamos que as expressões aproximadas de $a, b$ e $c$ são bastante satisfatórias em certas regiões do espaço paramétrico, já que seus valores são próximos dos valores exatos. Este desempenho é confirmado graficamente na Seção 6.4 .

\subsection{Análise gráfica}

Dos resultados da seção anterior observamos que para várias distribuições $a, b$ e $c$ nào são constantes, e sim funções de $\theta$, variando, portanto, com estes parâmetros. Também verificamos que em certas regiões do espaço paramétrico, para algumas distribuições, os valores aproximados de $a, b$ e $c$, para $\theta$ pequeno ou grande, estão muito próximos dos valores exatos (vide Figuras 6.1-6.5). Assim, se a aproximação é razoável, a estatística corrigida aproximada e a estatística corrigida exata são aproximadamente iguais. Uma pergunta de interesse é a seguinte: Quão grande ou quão pequeno deve ser $\theta$ para obter uma boa aproximação para $a, b$ e $c$ ? Para ter uma idéia a este respeito, faremos gráficos de $a, b$ e $c$ contra $\theta$ para as distribuições gama ( $\theta$ conhecido), McCullagh, von Mises, série logarítmica e Poisson truncada. Estes gráficos são apresentados nas Figuras 6.16.5 , respectivamente. Para a distribuição gama, nossas aproximações comportam-se bem quando $k \leq 0.2$ e $k \geq 2$ (vide também Tabelas 6.1 e 6.3). Considerando a distribuição de McCullagh, as aproximações são boas quando $\theta \leq 0.2$ e $\theta \geq 4$ (vide também Tabelas 6.2 e 6.4). Para a distribuição de von Mises, a aproximação é razoável, para $\theta \leq 1.5$ e $\theta \geq 5$, para valores pequenos e grandes de $\theta$, respectivamente (vide também Tabelas 6.5 e 6.6). A distribuição zeta requer que $\theta \leq 0,3$ para que a aproximaçào seja razoável (vide Tabela 6.9). Para a distribuição série logarítmica a aproximação é boa quando $\theta \leq 0.2$ (vide também Tabela 6.7). Finalmente para a distribuição Poisson truncada, a aproximação é razoável quando $\theta \leq 1$ (vide também Tabela 6.8 ). 
Figura 6.1: Valores exatos e aproximados de $a . b$ e $c$ para a distribuição gama
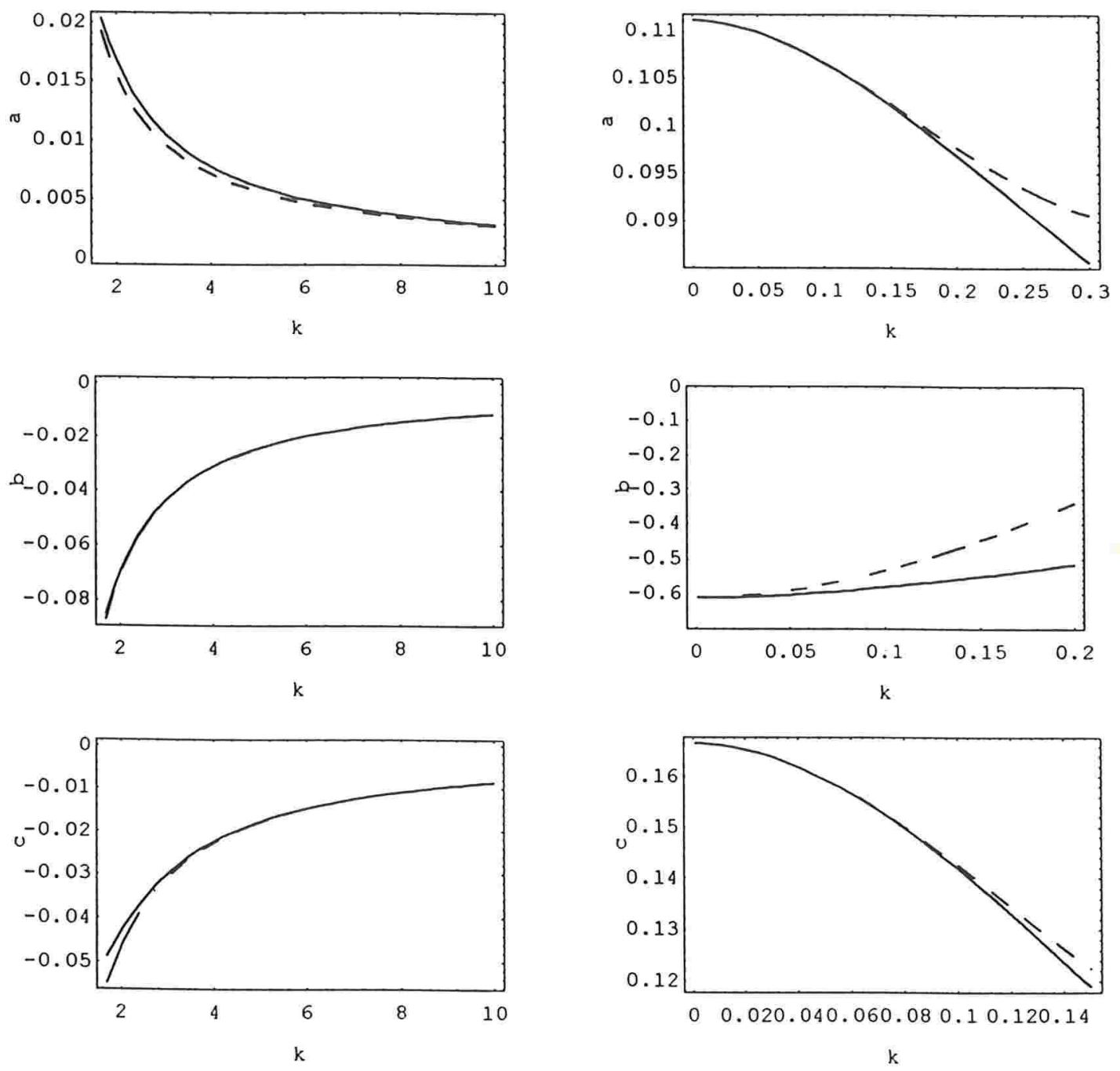

- - . - valores aproximados valores exatos 
Figura 6.2: Valores exatos e aproximados de $a . b$ e c para a distribuiçào de Mc Cullagh
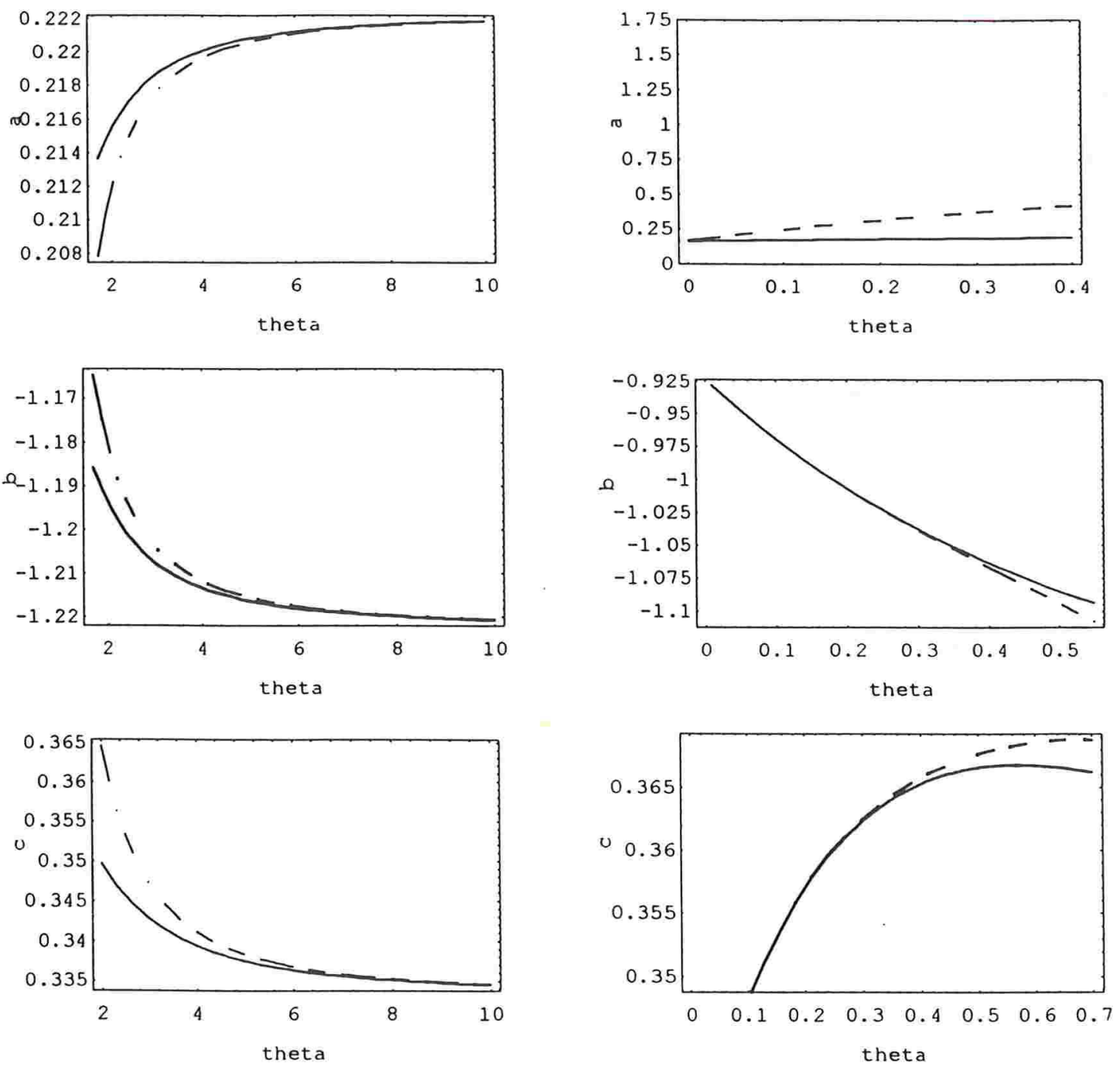
Figura 6.3: Valores exatos e aproximados de $a . b$ e $c$ para a distribuiçào de von Mises
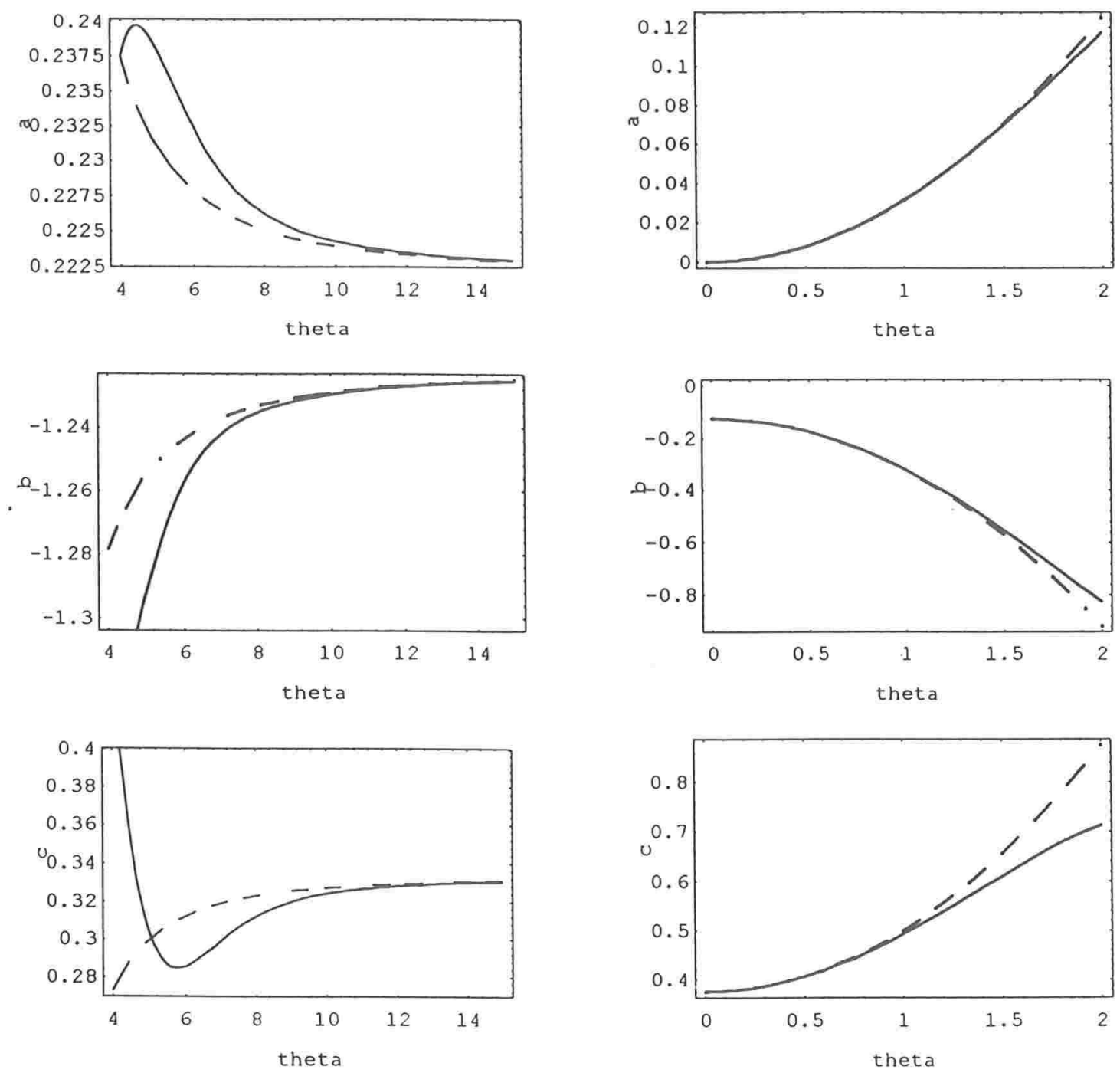

- . - valores aproximados valores exatos 
Figura 6.4: Valores exatos e aproximados de $a . b$ e $c$ para a distribuição em série logarítimica
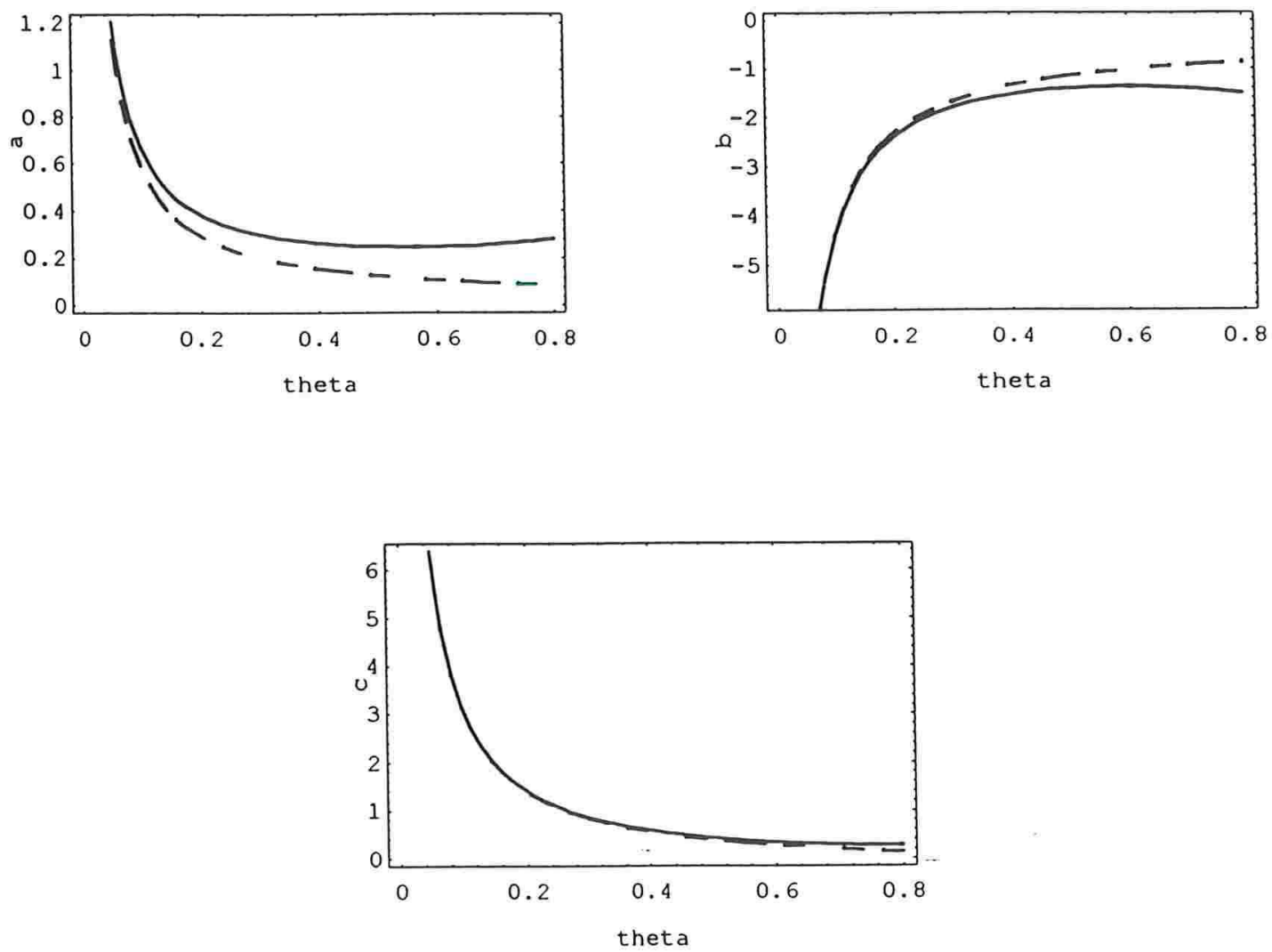

-... valores aproximados valores exatos 
Figura 6.5: Valores exatos e aproximados de $a . b$ e $c$ para a distribuiçào Poisson Truncada
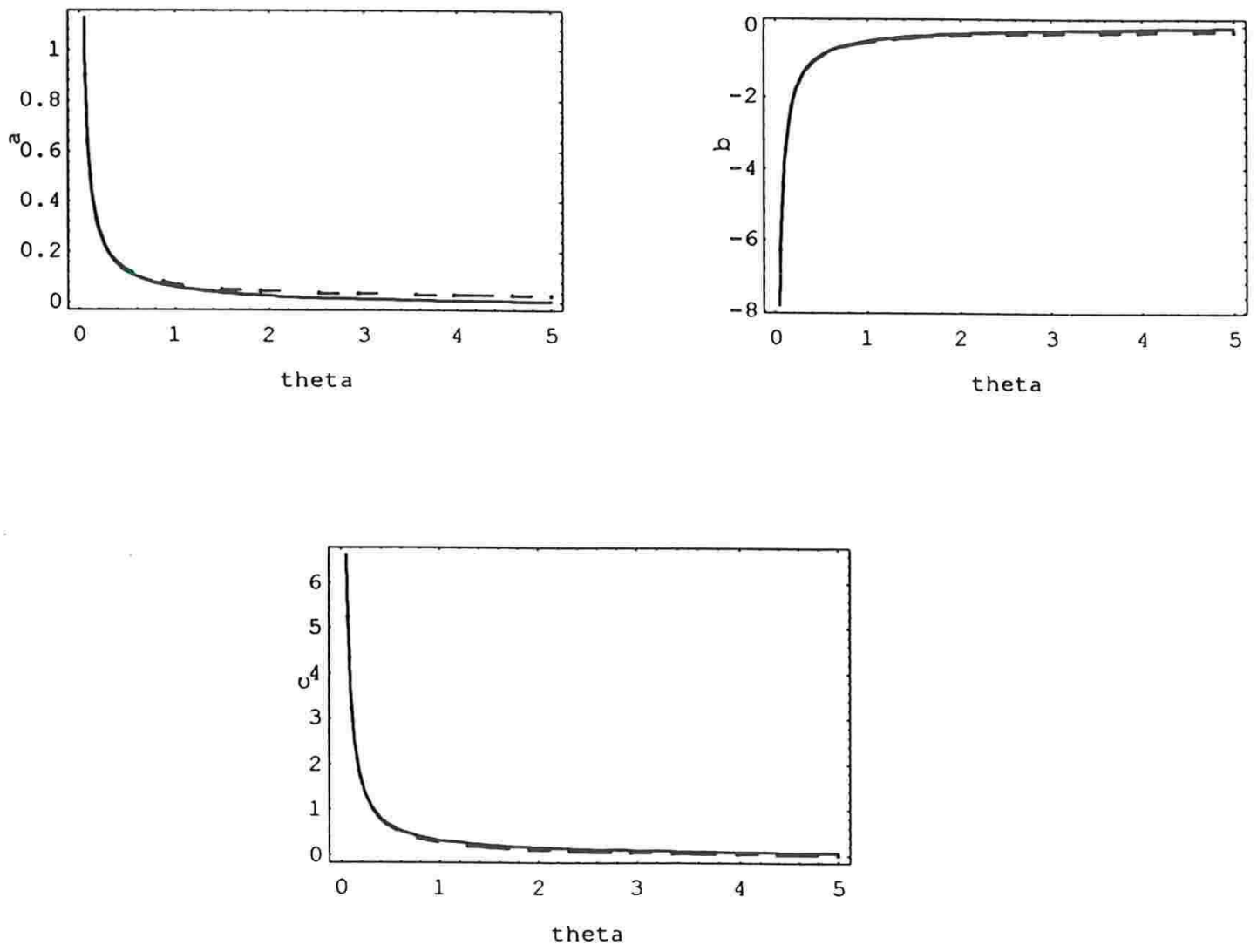

- - - valores aproximados valores exatos 


\subsection{Família exponencial natural}

Na Seção 6.3, apresentamos a correçào tipo Bartlett para a estatística escore na família exponencial uniparamétrica em termos do parâmetro $\theta$ (vide eq. (6.1)). Esta parametrização é conveniente para estudar como a correção varia com $\theta^{(0)}$. Porém, também é interessante escrever a correção para a família exponencial parametrizada na forma natural. Neste caso, podemos escrever os coeficientes $a, b$ e $c$ em forma muito simples, como veremos a seguir.

A família exponencial uniparamétrica na forma natural é escrita como

$$
\pi(y ; \alpha)=\frac{1}{\delta(\alpha)} \exp \{-\alpha d(y)+v(y)\}
$$

onde $\alpha$ é o parâmetro natural e $-d(y)$ é a estatística canônica. Como (6.1) e (6.8) definem uma correspondência um a um entre $\theta$ e $\alpha$, os coeficientes $a, b$ e $c$ dados em (6.5)-(6.T) reduzem-se às correspondentes expressões em (6.4) com $\gamma_{1}=\beta^{\prime \prime 2} / \beta^{\prime 3}$ e $\gamma_{2}=\beta^{\prime \prime \prime} / \beta^{\prime 2}$, onde $\beta=\mathrm{d} \log \{\delta(\alpha)\} / \mathrm{d} \alpha$, com as linhas denotando derivadas com respeito a $\alpha$. Com a finalidade de dar uma interpretação para os coeficientes $a, b$ e $c$, devemos notar que para as distribuições em $(6.8), \log \delta(\alpha)$ é o gerador de cumulantes da função $-d(y)$, isto é, o $r$-ésimo cumulante de $-d(y)$ é $k_{r}=\mathrm{d}^{r} \log \delta(\alpha) / \mathrm{d} \alpha^{r}$, para $r=1,2, \ldots$ Além disto, o $(r+1)$ ésimo cumulante de $-d(y)$ é dado por $\mathrm{d}^{r} \beta / \mathrm{d} \alpha^{r}$, para $r=1,2, \ldots$, onde $\beta(\alpha)=\delta^{\prime}(\alpha) / \delta(\alpha)$. Em particular, a média e variância de $-d(y)$ são $k_{1}=\beta$ e $k_{2}=\mathrm{d} \beta / \mathrm{d} \alpha=\beta^{\prime}$. Aqui, $\gamma_{1}^{2} \mathrm{e}$ $\gamma_{2}$ são o terceiro e quarto cumulantes padronizados de $-d(y)$. Então, $a$ é proporcional a $\gamma_{1}^{2}$, que é a medida usual de assimetria de $-d(y)$ e $c$ é proporcional a $5 \gamma_{1}^{2}-3 \gamma_{2}$ e pode ser visto como uma medida de não normalidade ou não normalidade inversa de $-d(y)$ (vide McCullagh e Cox, 1986). O coeficiente $b$ é uma combinação linear de $a$ e $c$.

Quando a função geradora de cumulantes de $-d(y)$ pode ser escrita como $\log \delta(\alpha)=$ $c_{0}+c_{1} \alpha+c_{2} \alpha^{2}$, onde $c_{0}, c_{1}$ e $c_{2}$ são constantes arbitrárias, temos que $a=b=c=0$, isto é, a correção tipo Bartlett é nula. Este é o caso da distribuição normal com variância conhecida. Por outro lado, se

$$
\delta(\alpha)=\exp \left\{c_{0} \alpha\right\}\left(k \alpha+c_{1}\right)^{-(1 / 9 k)} c_{2},
$$

onde $c_{0}, c_{1}$ e $c_{2}$ são constantes arbitrárias e $k>0$, temos que $a=k, b=-11 k / 2$ e $c=3 k / 2$. Para treze dos casos considerados na Seção $6.3, a, b$ e $c$ são constantes com $a>0$, e é possível mostrar que as correspondentes funções geradoras de cumulantes satisfazem (6.9) e as relações acima se verificam.

Agora, desenvolvemos expressões simples para os coeficientes $a, b$ e $c$ em termos da média $\beta$ da estatística canônica - $d(y)$ para algumas sub-classes de distribuições da família exponencial uniparamétrica. Primeiramente, deve ser notado que, a menos de uma transformação linear, só há uma distribuição em (6.8) com uma função de variância especificada (Jørgensen, 1987a) e logo $\beta$ caracteriza qualquer distribuição em (6.8).

A seguir são consideradas as seguintes famílias de funções de variância $\left(c_{0}, \ldots, c_{3} \in \mathbb{R}\right)$ : 
(1) Função de variância potência: $\beta^{\prime}=\beta^{p} / c_{0}$. para $p \leq 0$ e $p \geq 1, c_{0}>0$ :

$$
a=\frac{p^{2} \beta^{p-2}}{36 c_{0}}, \quad b=-\frac{p(4 p+3) \beta^{p-2}}{36 c_{0}}, \quad c=\frac{p(3-p) \beta^{p-2}}{12 c_{0}} .
$$

Aqui, $p=0,1,2,3$, para as distribuições normal, Poisson, gama e normal inversa, respectivamente. Outras distribuições nesta classe são apresentadas por Jørgensen (1987a).

(2) Função de variância quadrátrica: $\beta^{\prime}=c_{0}+c_{1} \beta+c_{2} \beta^{2}$, $a$, b e $c$ são dadas pelas correspondentes expressões para a função de variância cúbica (ver abaixo) com $c_{3}=0$. Morris (1982) mostra que existem somente as seguintes seis distribuições nesta classe:

(a) Normal $(x x, b): \beta \in \mathbb{R}, c_{0}=\theta, c_{1}=c_{2}=0, \theta>0, \theta$ conhecido; $a=b=c=0$.

(b) Poisson (iii): $\beta>0, c_{0}=c_{2}=0, c_{1}=1$,

$$
a=\frac{1}{36 \beta}, \quad b=-\frac{7}{36 \beta}, \quad c=\frac{1}{6 \beta} .
$$

(c) Binomial $(i): \beta \in(0, m), c_{0}=0, c_{1}=1, c_{2}=-1 / m, m \in \mathbb{N}, m$ conhecido;

$$
a=\frac{(m-2 \beta)^{2}}{36 m \beta(m-\beta)}, \quad b=\frac{-7 m^{2}+22 m \beta-22 \beta^{2}}{36 m \beta(m-\beta)}, \quad c=\frac{m^{2}-m \beta+\beta^{2}}{6 m \beta(m-\beta)} .
$$

(d) Binomial negativa (ii): $\beta>0, c_{0}>0, c_{1}=1, c_{2}=1 / \gamma, \gamma>0, \gamma$ conhecido;

$$
a=\frac{(2 \beta+\gamma)^{2}}{36 \beta \gamma(\beta+\gamma)}, \quad b=\frac{-22 \beta^{2}+22 \beta \gamma+7 \gamma^{2}}{36 \beta \gamma(\beta+\gamma)}, \quad c=\frac{\beta^{2}+\beta \gamma+\gamma^{2}}{12 \beta \gamma(\beta+\gamma)} .
$$

(e) Gama $(x, a): \beta>0, c_{0}=c_{1}=0, c_{2}=1 / k, k>0, k$ conhecido;

$$
a=\frac{1}{9 k}, \quad b=-\frac{11}{18 k}, \quad c=\frac{1}{6 k} .
$$

(f) Secante hiperbólica generalizada (xxiv): $\beta \in \mathbb{R}, c_{0}=r, c_{1}=0, c_{2}=1 / r$, $r>0, r$ conhecido;

$$
a=\frac{\beta^{2}}{9 r\left(\beta^{2}+r^{2}\right)}, \quad b=\frac{-11 \beta^{2}+3 r^{2}}{18 t\left(\beta^{2}+r^{2}\right)}, \quad c=\frac{\beta^{2}-3 r^{2}}{6 r\left(\beta^{2}+r^{2}\right)} .
$$

(3) Função de variância cúbica: $\beta^{\prime}=c_{0}+c_{1} \beta+c_{2} \beta^{2}+c_{3} \beta^{3}$;

$$
\begin{aligned}
a & =\left(36 \beta^{\prime}\right)^{-1}\left\{c_{1}^{2}-4 c_{0} c_{2}+c_{3} \beta^{2}\left(6 c_{1}+8 c_{2} \beta+9 c_{3} \beta^{2}\right)\right\} 4 c_{2}, \\
b & =\left(36 \beta^{\prime}\right)^{-1}\left\{-i\left(c_{1}^{2}-4 c_{0} c_{2}\right)+c_{3} \beta\left(18 c_{0}-24 c_{1} \beta-3 \gamma c_{2} \beta^{2}-45 c_{3} \beta^{3}\right)\right\}-22 c_{2}, \\
c & =\left(6 \beta^{\prime}\right)^{-1}\left\{c_{1}^{2}-4 c_{0} c_{2}-c_{3} \beta\left(9 c_{0}+3 c_{1} \beta+c_{2} \beta^{2}\right)\right\}+2 c_{2} .
\end{aligned}
$$

Letac e Mora (1990) mostram que existem somente as seguintes seis distribuições nesta classe com $c_{3} \neq 0$ : 
(a) Abel ( $p$ conhecido): $\beta>0, y=0,1,2, \ldots c_{0}=0, c_{1}=1, c_{2}=2 / p, c_{3}=1 / p^{2}$;

$$
a=\frac{(3 \beta+p)^{2}}{36 \beta p^{2}}, \quad b=-\frac{45 \beta^{2}+30 \beta p+7 p^{2}}{36 \beta p^{2}}, \quad c=\frac{1}{6 \beta} .
$$

(b) Takács $\left(p>0\right.$ e $m>0$ são conhecidos): $\beta>0, y=0,1,2, \ldots, c_{0}=0, c_{1}=1$, $c_{2}=(2 m+1) /(m p), c_{3}=(m+1) /\left(m p^{2}\right)$,

$$
\begin{aligned}
a= & \frac{\left\{3 \beta^{2}(m+1)+2 \beta p(2 m+1)+m p^{2}\right\}^{2}}{36 m \beta p^{2}(\beta+p)(\beta+m \beta+m p)}, \\
b= & -\left\{45 \beta^{4}(m+1)^{2}+60 \beta^{3} p(m+1)(2 m+1)+2 \beta^{2} p^{2}\left(11+56 m+56 m^{2}\right)\right. \\
& \left.+22 \beta m p^{3}(2 m+1)+7 m^{2} p^{4}\right\} /\left\{36 m \beta p^{2}(\beta+p)(\beta+m \beta+m p)\right\}, \\
c= & \frac{m^{2} p^{2}+m \beta p(2 m+1)+\beta^{2}\left(m^{2}+m+1\right)}{6 m \beta(\beta+p)(\beta+m \beta+m p)} .
\end{aligned}
$$

(c) Arcosseno estrito ( $p$ conhecido): $y=0,1,2, \ldots c_{0}=c_{2}=0, c_{1}=1$, $c_{3}=1 / p^{2}$

$$
a=\frac{\left(3 \beta^{2}+p^{2}\right)^{2}}{36 \beta p^{2}\left(\beta^{2}+p^{2}\right)}, \quad b=-\frac{45 \beta^{4}+24 \beta^{2} p^{2}+7 p^{4}}{36 \beta p^{2}\left(\beta^{2}+p^{2}\right)}, \quad c=\frac{-3 \beta^{2}+p^{2}}{6 \beta\left(\beta^{2}+p^{2}\right)} .
$$

(d) Arcosseno estendido $\left(p, m>0\right.$ conhecidos): $\beta>0, y=0,1,2, \ldots, c_{0}=0$, $c_{1}=1, c_{2}=2 /(m p), c_{3}=\left(m^{2}+1\right) /(m p)^{2}$;

$$
\begin{aligned}
a= & \frac{\left\{3 \beta^{2}\left(m^{2}+1\right)+4 m \beta p+m^{2} p^{2}\right\}^{2}}{36 m^{2} \beta p^{2}\left\{\beta^{2}\left(m^{2}+1\right)+2 m \beta p+m^{2} p^{2}\right\}}, \\
b= & -\left\{45 \beta^{4}\left(1+m^{2}\right)^{2}+120 m \beta^{3} p\left(1+m^{2}\right)+8 m^{2} \beta^{2} p^{2}\left(14+3 m^{2}\right)+44 m^{3} \beta p^{3}\right. \\
& \left.+7 m^{4} p^{4}\right\} /\left\{36 m^{2} \beta p^{2}\left(\beta^{2}\left(m^{2}+1\right)+2 m \beta p+m^{2} p^{2}\right)\right\} \\
c= & \frac{\beta^{2}\left(1-3 m^{2}\right)+2 m p \beta+m^{2} p^{2}}{6 \beta\left\{\beta^{2}\left(m^{2}+1\right)+2 m \beta p+m^{2} p^{2}\right\}} .
\end{aligned}
$$

(e) Ressel $\left(p>0\right.$, conhecido): $\beta>0, y>0, c_{0}=c_{1}=0, c_{2}=1 / p, c_{3}=1 / p^{2}$;

$$
a=\frac{(3 \beta+2 p)^{2}}{36 p^{2}(\beta+p)}, \quad b=-\frac{45 \beta^{2}+60 \beta p+22 p^{2}}{36 p^{2}(\beta+p)}, \quad c=\frac{1}{6(\beta+p)} .
$$

(f) Normal inversa $\left(\theta>0\right.$, conhecido): $\beta>0, c_{0}=c_{1}=c_{2}=0, c_{3}=-1 / \theta$;

$$
a=-\frac{\beta}{4 \theta}, \quad b=\frac{5 \beta}{4 \theta}, \quad c=0 .
$$

(4) Classe de Babel: $\beta^{\prime}=c_{0} \Delta+\left(c_{1} \beta+c_{2}\right) \Delta^{1 / 2}$, onde $\Delta$ é um polinômio de grau menor que 3 , que nào é um quadrado perfeito. Aqui $c_{0} . c_{1}$ e $c_{2}$ são facilmente obtidos usando MATHEMATICA (Wolfram, 1991) mas as expressões resultantes são muito complicadas e não serão apresentadas aqui. 
Finalizando este capítulo. ressaltamos que nosso objetivo foi encontrar expressòes simples e de fácil aplicação prática para os coeficientes do fator de correçào tipo Bartlett para a estatística escore para os modelos da família exponencial uniparamétrica. Pretendemos realizar, futuramente, estudos de simulação para verificar o desempenho dos testes corrigidos. 


\section{Capítulo 7}

\section{Aperfeiçoamento de testes escore para modelos da família exponencial biparamétrica}

\subsection{Introdução}

No Capítulo 6, desenvolvemos um aperfeiçoamento de testes escore para modelos da família exponencial uniparamétrica obtendo fator de correção simples e de fácil aplicação. Nosso objetivo neste capítulo é obter uma expressão geral em forma fechada para a correção tipo Bartlett da estatística escore para as distribuições pertencentes à família exponencial biparamétrica. Correções de Bartlett para o teste da razão de verossimilhança nesta classe de modelos foi obtida por Aubin (1995).

Consideremos $y_{1}, \ldots, y_{n}$ um conjunto de $n$ variáveis aleatórias independentes e identicamente distribuídas com função densidade (ou de probabilidade) da forma

$$
\pi(y ; \mu, \nu)=\exp \left\{\alpha_{1}(\mu, \nu) d_{1}(y)+\alpha_{2}(\mu, \nu) d_{2}(y)-\rho(\mu, \nu)+v(y)\right\}
$$

onde $(\mu, \nu)$ representa o vetor de parâmetros desconhecidos, $\alpha_{1}(\cdot, \cdot), \alpha_{2}(\cdot, \cdot), \rho(\cdot, \cdot), d_{1}(\cdot)$, $d_{2}(\cdot)$ e $v(\cdot)$ são funções conhecidas e considera-se que o suporte de $\pi(y ; \mu, \nu)$ não depende de $\mu$ e $\nu$. Também assumimos que $\alpha_{1}(\cdot, \cdot), \alpha_{2}(\cdot, \cdot)$ e $\rho(\cdot, \cdot)$ têm as quatro primeiras derivadas contínuas em relação aos componentes do vetor de parâmetros $(\mu, \nu)$. O logaritmo da função de verossimilhança total para os parâmetros $\mu$ e $\nu$, dado $\left(y_{1}, \ldots, y_{n}\right)$, é da forma

$$
L=L(\mu, \nu)=\sum_{l=1}^{n} t\left(y_{l} ; \mu, \nu\right)
$$

sendo $t\left(y_{l} ; \mu, \nu\right)=\alpha_{1} d_{1}\left(y_{l}\right)+\alpha_{2} d_{2}\left(y_{l}\right)-\rho+v\left(y_{l}\right)$, onde $\alpha_{1}=\alpha_{1}(\mu, \nu), \alpha_{2}=\alpha_{2}(\mu, \nu)$ e $\rho=\rho(\mu, \nu)$. Assumimos que $L$ é regular (Jensen, 1993) com respeito às derivadas em relação aos componentes de $(\mu, \nu)$ até quarta ordem. Sejam $\alpha_{s}^{(i, j)}=\partial^{i+j} \alpha_{s} / \partial \mu^{i} \partial \nu^{j}$ e $\rho^{(i, j)}=\partial^{i+j} \rho / \partial \mu^{i} \partial \nu^{j}$, para $i, j=0,1,2,3,4$ e $s=1.2$. A partir da primeira derivada 
do logaritmo da função de verossimilhança (7.2) e usando o fato de que $E(\partial L / \partial \mu)=$ $E(\partial L / \partial \nu)=0$, temos

$$
\begin{aligned}
& \alpha_{1}^{(1,0)} \beta_{1}+\alpha_{2}^{(1,0)} \beta_{2}=\rho^{(1,0)}, \\
& \alpha_{1}^{(0,1)} \beta_{1}+\alpha_{2}^{(0,1)} \beta_{2}=\rho^{(0,1)},
\end{aligned}
$$

onde $\beta_{r}=E\left\{d_{r}(y)\right\}, r=1,2$. Assim, resolvendo o sistema de equações acima para $\beta_{1} \mathrm{e}$ $\beta_{2}$, temos

$$
\begin{aligned}
& \beta_{1}=\frac{\rho^{(1,0)} \alpha_{2}^{(0,1)}-\rho^{(0,1)} \alpha_{2}^{(1,0)}}{\alpha_{1}^{(1,0)} \alpha_{2}^{(0,1)}-\alpha_{1}^{(0,1)} \alpha_{2}^{(1,0)}}, \\
& \beta_{2}=\frac{\rho^{(0,1)} \alpha_{1}^{(1,0)}-\rho^{(1,0)} \alpha_{1}^{(0,1)}}{\alpha_{1}^{(1,0)} \alpha_{2}^{(0,1)}-\alpha_{1}^{(0,1)} \alpha_{2}^{(1,0)}},
\end{aligned}
$$

desde que $\alpha_{1}^{(1,0)} a_{2}^{(0,1)}-a_{1}^{(0,1)} a_{2}^{(1,0)} \neq 0$. As funções escore total para $\mu$ e $\nu$ são dadas por

$$
\begin{aligned}
& U_{\mu}=\frac{\partial L}{\partial \mu}=\sum_{j=1}^{n} \sum_{i=1}^{2} \alpha_{i}^{(1,0)}\left(d_{i}\left(y_{j}\right)-\beta_{i}\right), \\
& U_{\nu}=\frac{\partial L}{\partial \nu}=\sum_{j=1}^{n} \sum_{i=1}^{2} \alpha_{i}^{(0,1)}\left(d_{i}\left(y_{j}\right)-\beta_{i}\right) .
\end{aligned}
$$

Definimos $\beta_{r}^{(i, j)}=\left(\partial^{i+j} \beta_{r} / \partial \mu^{i} \partial \nu^{j}\right)$, para $i, j=0,1,2,3,4$ e $r=1,2$. Os cumulantes $\kappa_{\mu, \mu}$, $\kappa_{\mu, \nu}, \kappa_{\nu, \mu}, \kappa_{\nu, \nu}$ têm as seguintes formas, respectivamente:

$$
\begin{aligned}
& \kappa_{\mu, \mu}=E\left\{\left(\frac{\partial L}{\partial \mu}\right)^{2}\right\}=n \sum_{i=1}^{2} \alpha_{i}^{(1,0)} \beta_{i}^{(1,0)}, \\
& \kappa_{\mu, \nu}=E\left\{\frac{\partial L}{\partial \mu} \frac{\partial L}{\partial \nu}\right\}=n \sum_{i=1}^{2} \alpha_{i}^{(1,0)} \beta_{i}^{(0,1)}, \\
& \kappa_{\nu, \mu}=E\left\{\frac{\partial L}{\partial \nu} \frac{\partial L}{\partial \mu}\right\}=n \sum_{i=1}^{2} \alpha_{i}^{(0,1)} \beta_{i}^{(1,0)}, \\
& \kappa_{\nu, \nu}=E\left\{\left(\frac{\partial L}{\partial \nu}\right)^{2}\right\}=n \sum_{i=1}^{2} \alpha_{i}^{(0,1)} \beta_{i}^{(0,1)} .
\end{aligned}
$$

Na Seção 7.2, desenvolvemos uma expressão simples para a correção tipo Bartlett para o teste escore nos modelos da família exponencial biparamétrica (7.1) cujos parâmetros são globalmente ortogonais. Esta correção depende das derivadas de $\alpha_{1}, \alpha_{2}, \beta_{1}$ e $\beta_{2}$ em relação a $\mu$ e $\nu$. Na Seção 7.3, mostramos dois casos particulares deste modelo. Na Seção 7.4, aplicamos os resultados das seções anteriores a distribuições especiais com parâmetros globalmente ortogonais. Finalmente, na Seção 7.5, apresentamos a construção de parâmetros globalmente ortogonais segundo formulação de Cox e Reid (198i) e aplicamos esta metodologia a algumas distribuições cuja densidade tem a forma (7.1). 


\subsection{Correção em modelos com parâmetros globalmen- te ortogonais}

Nesta seção, desenvolvemos a correção de Cordeiro e Ferrari (1991) para a estatística escore na família exponencial biparamétrica supondo que os parâmetros desconhecidos $\mu$ e $\nu$ são globalmente ortogonais segundo Cox e Reid (1987). Neste caso, vale a seguinte relação entre os cumulantes de derivadas do logaritmo da função de verossimilhança em relação a $\mu$ e $\nu: \kappa_{\mu, \nu}=\kappa_{\nu, \mu}=0$. Assim, a matriz de informação total de Fisher é diagonal e tem a forma $K=K(\mu, \nu)=\operatorname{Diag}\left\{\kappa_{\mu, \mu}, \kappa_{\nu, \nu}\right\}$ e sua inversa tem a forma $K^{-1}=K^{-1}(\mu, \nu)=$ $\operatorname{Diag}\left\{\kappa^{\mu \mu}, \kappa^{\nu \nu}\right\}$, onde $\kappa^{\mu \mu}=\kappa_{\mu \mu}^{-1}$ e $\kappa^{\nu \nu}=\kappa_{\nu \nu}^{-1}$. Se a condição $\kappa_{\nu, \mu}=\kappa_{\mu, \nu}=0$ não é satisfeita, podemos, através de reparametrização. obter parâmetros globalmente ortogonais e aplicar os resultados que sâo obtidos nesta seção. Sem perda de generalidade, suponhamos que o parâmetro de interesse seja $\mu$ e $\nu$ seja o parâmetro de perturbação. Assim, considera-se a hipótese nula composta $H_{0}: \mu=\mu^{(0)}$ contra a hipótese alternativa também composta $H_{1}: \mu \neq \mu^{(0)}$, onde $\mu^{(0)}$ é um valor especificado e $\nu$ é o parâmetro de perturbação, e seja $\widetilde{\nu}$ o estimador de máxima verossimilhança de $\nu$ sob $H_{0}$. A estatística escore para testar a hipótese $H_{0}$ é dada por

$$
S_{R}=\widetilde{U}_{\mu}^{2} \widetilde{\kappa}_{\mu, \mu}^{-1},
$$

onde $U_{\mu}$ e $\kappa_{\mu, \mu}$ são definidos em (7.4) e (7.5), sendo que as quantidades assinaladas com til são avaliadas em $\left(\mu^{(0)}, \tilde{\nu}\right)$. Sob $H_{0}, S_{R}$ tem distribuição $\chi_{1}^{2}$ assintoticamente.

A estatística escore aperfeiçoada por um fator de correção tipo Bartlett $\left(S_{R}^{*}\right)$ apresentada em (1.18) depende dos coeficientes $A_{1}, A_{2}$ e $A_{3}$ que, para dois parâmetros $\mu$ e $\nu$ globalmente ortogonais, têm as formas

$$
\begin{aligned}
& A_{1}= \frac{6\left(\kappa_{\mu \mu}^{(\nu)}-2 \kappa_{\mu \mu \nu}\right)\left(\kappa_{\nu \nu \nu}-2 \kappa_{\nu \nu}^{(\nu)}\right)-3 \kappa_{\nu \nu \mu}\left(7 \kappa_{\nu \nu \mu}-4 \kappa_{\nu \nu}^{(\mu)}\right)}{\kappa_{\mu \mu} \kappa_{\nu \nu}^{2}} \\
&+\frac{6\left(2 \kappa_{\nu \mu \mu}-4 \kappa_{\nu \mu \mu}^{(\nu)}-2 \kappa_{\nu \nu \mu}^{(\mu)}+\kappa_{\mu \mu}^{(\nu)}-2 \kappa_{\nu \mu, \nu \mu}\right)}{\kappa_{\mu \mu} \kappa_{\nu \nu}}, \\
& A_{2}= \frac{3 \kappa_{\nu \nu} \kappa_{\mu, \mu, \mu \mu}+6 \kappa_{\mu, \mu, \mu} \kappa_{\nu \nu \mu}+9\left(2 \kappa_{\nu \mu \mu}-\kappa_{\mu \mu}^{(\nu)}\right)^{2}}{\kappa_{\nu \nu} \kappa_{\mu \mu}^{2}}, \\
& A_{3}=-\frac{5 \kappa_{\mu, \mu, \mu}^{2}}{\kappa_{\mu \mu}^{3}},
\end{aligned}
$$

(Ferrari e Cordeiro, 1994). Sob $H_{0}, S_{R}^{*}$ tem uma distribuição $\chi_{1}^{2}$ até ordem $n^{-1}$.

A obtenção dos $A$ s em (7.7) - (7.9) na família exponencial biparamétrica com $\pi(y ; \mu, \nu)$ definida em (7.1) envolve o cálculo de cumulantes mistos até quarta ordem (apresentados no Apêndice F). Substituindo os cumulantes envolvidos e após um longo desenvolvimento algébrico, chega-se às seguintes expressões:

$$
A_{1}=\frac{3}{n}\left\{\left\{2 \left(\sum_{i=1}^{2}\left(\alpha_{i}^{(1,0)} \beta_{i}^{(0,1)}-\alpha_{i}^{(1,1)} \beta_{i}^{(1,0)}\right)\left(\sum_{i=1}^{2} \alpha_{i}^{(0,1)} \beta_{i}^{(0,2)}\right)\right.\right.\right.
$$




$$
\begin{aligned}
& \left.+\left(\sum_{i=1}^{2}\left(3 \alpha_{i}^{(1,1)} \beta_{i}^{(0,1)}-4 \alpha_{i}^{(0,1)} \beta_{i}^{(1,1)}\right)\right)\left(\sum_{i=1}^{2} a_{i}^{(1,1)} \beta_{i}^{(0,1)}\right)\right\} \\
& \left\{\left(\sum_{i=1}^{2} \alpha_{i}^{(1,0)} \beta_{i}^{(1,0)}\right)\left(\sum_{i=1}^{2} \alpha_{i}^{(0,1)} \beta_{i}^{(0,1)}\right)^{-1}\right. \\
& -2\left\{\sum_{i=1}^{2}\left(\alpha_{i}^{(1,0)} \beta_{i}^{(1,2)}-\alpha_{i}^{(1,2)} \beta_{i}^{(1,0)}-2 \alpha_{i}^{(1,1)} \beta_{i}^{(1,1)}\right)+2 \zeta\right\} \\
& \left.\left\{\left(\sum_{i=1}^{2} \alpha_{i}^{(1,0)} \beta_{i}^{(1,0)}\right)\left(\sum_{i=1}^{2} \alpha_{i}^{(0,1)} \beta_{i}^{(0,1)}\right)\right\}^{-1}\right\} \\
\left.A_{2}\right\} & -\frac{3}{n}\left\{\left\{\sum_{i=1}^{2}\left(\alpha_{i}^{(3,0)} \beta_{i}^{(1,0)}+3 \alpha_{i}^{(2,0)} \beta_{i}^{(2,0)}-\alpha_{i}^{(1,0)} \beta_{i}^{(3,0)}\right)-3 \gamma\right\}\right. \\
& \left\{\sum_{i=1}^{2} \alpha_{i}^{(1,0)} \beta_{i}^{(1,0)}\right\}^{-2} \\
+ & \left.\left\{\left(\sum_{i=1}^{2}\left(\alpha_{i}^{(2,0)} \beta_{i}^{(1,0)}-\alpha_{i}^{(1,0)} \beta_{i}^{(2,0)}\right)\right)^{2} \sum_{i=1}^{2} \alpha_{i}^{(1,1)} \beta_{i}^{(0,1)}\right)\right\} \\
A_{3}= & \frac{5}{n}\left\{\sum_{i=1}^{2}\left(\alpha_{i}^{(2,0)} \beta_{i}^{(1,0)}-\alpha_{i}^{(1,0)} \beta_{i}^{(2,0)}\right)\right\}^{2}\left\{\sum_{i=1}^{2} \alpha_{i}^{(1,0)} \beta_{i}^{(1,0)}\right\} \\
& \left\{\left(\sum_{i=1}^{2}\left(\alpha_{i}^{(0,1)} \beta_{i}^{(0,1)}\right)\left(\sum_{i=1}^{2} \alpha_{i}^{(1,0)} \beta_{i}^{(1,0)}\right\}^{-3}\right.\right. \\
+3\left\{\sum_{i=1}^{2}\left(\alpha_{i}^{(1,1)} \beta_{i}^{(1,0)}-\alpha_{i}^{(1,0)} \beta_{i}^{(1,1)}\right\}^{2}\right. & \\
& \left.\left\{\alpha_{i}^{(0,1)} \beta_{i}^{(0,1)}\right)\left(\sum_{i=1}^{2} \alpha_{i}^{(1,0)} \beta_{i}^{(1,0)}\right\}^{-1}\right\}
\end{aligned}
$$

onde

$$
\begin{aligned}
\zeta & =\left(\alpha_{1}^{(1,1)}\right)^{2} V_{1}+\left(\alpha_{2}^{(1,1)}\right)^{2} V_{2}+2 \alpha_{1}^{(1,1)} \alpha_{2}^{(1,1)} V_{12}, \\
\gamma & =\left(\alpha_{1}^{(2,0)}\right)^{2} V_{1}+\left(\alpha_{2}^{(2,0)}\right)^{2} V_{2}+2 \alpha_{1}^{(2,0)} \alpha_{2}^{(2,0)} V_{12},
\end{aligned}
$$

sendo $V_{i}=\operatorname{Var}\left(d_{i}(y)\right)$, para $i=1,2$, e $V_{12}=\operatorname{Cov}\left(d_{1}(y), d_{2}(y)\right)$.

Uma maneira de se obter $V_{1}, V_{2}$ e $V_{12}$ é dada por Johnson, Ladalla e Liu (1979), que mostram que estas quantidades são a solução do seguinte sistema de equações:

$$
\begin{aligned}
& \sum_{i=1}^{2} \alpha_{i}^{(0,1)} \beta_{i}^{(0,1)}=\left(\alpha_{1}^{(0,1)}\right)^{2} V_{1}+\left(\alpha_{2}^{(0,1)}\right)^{2} V_{2}+2 \alpha_{i}^{(0,1)} \alpha_{2}^{(0,1)} V_{12}, \\
& \sum_{i=1}^{2} \alpha_{i}^{(1,0)} \beta_{i}^{(1,0)}=\left(\alpha_{1}^{(1,0)}\right)^{2} V_{1}+\left(\alpha_{2}^{(1,0)}\right)^{2} V_{2}+2 \alpha_{i}^{(1,0)} \alpha_{2}^{(1,0)} V_{12},
\end{aligned}
$$




$$
V_{12}=-\frac{\sum_{i=1}^{2} \alpha_{i}^{(1,0)} \alpha_{i}^{(0,1)} V_{i}}{\left(\alpha_{i}^{(1,0)} \alpha_{2}^{(0,1)}+\alpha_{i}^{(0,1)} \alpha_{2}^{(1,0)}\right)},
$$

sujeito às restrições

$$
\sum_{i=1}^{2} \alpha_{i}^{(1,0)} \beta_{i}^{(0,1)}=0 \quad \text { e } \sum_{i=1}^{2} \alpha_{i}^{(0,1)} \beta_{i}^{(1,0)}=0 .
$$

Observando as expressões (7.10) - (7.12), verificamos que para o teste da hipótese nula $H_{0}: \mu=\mu^{(0)}$, a correção da estatística escore depende de derivadas de $\alpha_{1}, \alpha_{2}, \beta_{1}$ e $\beta_{2}$ (definidas em (7.3)) em relação a $\mu$ e $\nu$, das variâncias das estatísticas canônicas $d_{1}(y)$ e $d_{2}(y)$ e da covariância de $d_{1}(y)$ e $d_{2}(y)$.

\subsection{Casos especiais}

Consideremos agora duas situações particulares de modelos exponenciais biparamétricos, $\operatorname{com} \pi(y ; \mu, \nu)$ definida em (7.1).

Caso 1: $\alpha_{1}(\mu, \nu)=c_{1}(\mu)$ e $\alpha_{2}(\mu, \nu)=c_{2}(\nu)$.

Neste caso, a função $\alpha_{1}$ depende somente do parâmetro $\mu$ e a função $\alpha_{2}$ somente do parâmetro $\nu$. Temos

$$
\begin{gathered}
\alpha_{1}^{(0, s)}=\frac{\partial^{s} \alpha_{1}(\mu, \nu)}{\partial \nu^{s}}=0, \quad \alpha_{2}^{(r, 0)}=\frac{\partial^{r} \alpha_{2}(\mu, \nu)}{\partial \mu^{r}}=0, \\
\text { e } \quad \alpha_{i}^{(r, s)}=\frac{\partial^{s+r} \alpha_{i}(\mu, \nu)}{\partial \mu^{r} \partial \nu^{s}}=0, \quad \text { para } r, s=1,2,3, \quad \text { e } \quad i=1,2,
\end{gathered}
$$

resultando em

$$
\beta_{1}=\frac{\rho^{(1,0)}}{\alpha_{1}^{(1,0)}} \quad \text { e } \quad \beta_{2}=\frac{\rho^{(0,1)}}{\alpha_{2}^{(0,1)}} .
$$

Da ortogonalidade global entre $\mu$ e $\nu$ temos $\rho^{(i, j)}=0$, para $i, j=1,2,3$, levando a $\beta_{1}^{(0, i)}=\beta_{2}^{(j, 0)}=0$ e $\beta_{r}^{(i, j)}=0$, para $r=1,2$, e $i, j=1,2,3$. Utilizando as equações $(7.14)$ obtemos $V_{12}=0, V_{1}=\beta_{1}^{(1,0)} / \alpha_{1}^{(1,0)}$ e $V_{2}=\beta_{2}^{(0,1)} / \alpha_{2}^{(0,1)}$ e, conseqüentemente, de (7.13), temos

$$
\zeta=0 \quad \text { e } \quad \gamma=\frac{\left(\alpha_{1}^{(2,0)}\right)^{2} \beta_{1}^{(1,0)}}{\alpha_{1}^{(1,0)}} .
$$

Logo, as quantidades $A_{1}, A_{2}$ e $A_{3}$ definidas em (7.10) - (7.12), que determinam o fator de correção tipo Bartlett da estatística escore corrigida dada em (1.18), são dadas por

$$
\begin{aligned}
& A_{1}=0 \\
& A_{2}=\frac{-3\left\{\alpha_{1}^{(3,0)} \beta_{1}^{(1,0)}+3 \alpha_{1}^{(2,0)} \beta_{1}^{(2,0)}-\alpha_{1}^{(1,0)} \beta_{1}^{(3,0)}-3\left(\alpha_{1}^{(2,0)}\right)^{2} \beta_{1}^{(1,0)} \alpha_{1}^{(1,0)^{-1}}\right\}}{n\left\{\alpha_{1}^{(1,0)} \beta_{1}^{(1,0)}\right\}^{2}},
\end{aligned}
$$




$$
A_{3}=\frac{5}{n} \frac{\left\{\alpha_{1}^{(2,0)} \beta_{1}^{(1,0)}-\alpha_{1}^{(1,0)} \beta_{1}^{(2,0)}\right\}^{2}}{\left\{\alpha_{1}^{(1,0)} \beta_{1}^{(1,0)}\right\}^{3}} .
$$

Podemos observar que as expressões de $A_{2}$ e $A_{3}\left(A_{1}=0\right)$ em ( 7.16$)$ e $(7.17)$, respectivamente, dependem das três primeiras derivadas de $\alpha_{1}$ e $\beta_{1}$ em relação a $\mu$.

Caso 2: $\alpha_{1}(\mu, \nu)=c_{1}(\mu, \nu)$ e $\alpha_{2}(\mu, \nu)=c_{2}(\nu)$.

Neste caso, a função $\alpha_{2}$ depende somente do parâmetro de perturbação $\nu$ e $\alpha_{1}$ depende dos parâmetros $\mu$ e $\nu$. Isto implica que $\alpha_{2}^{(i, 0)}=\partial^{i} \alpha_{2}(\mu, \nu) / \partial \mu^{i}=0$ e $\alpha_{2}^{(i, j)}=$ $\partial^{i+j} \alpha_{2}(\mu, \nu) / \partial \mu^{i} \partial \nu^{j}=0$, para $i, j=1,2,3$, resultando em

$$
\beta_{1}=\frac{\rho^{(1,0)}}{\alpha_{1}^{(1,0)}}, \quad \beta_{2}=\frac{\rho^{(0,1)} \alpha_{1}^{(1,0)}-\rho^{(1,0)} \alpha_{1}^{(0,1)}}{\alpha_{1}^{(1,0)} \alpha_{2}^{(0,1)}} .
$$

Utilizando as equações de (7.14), obtemos

$$
V_{1}=\frac{\beta_{1}^{(1,0)}}{\alpha_{1}^{(1,0)}}, \quad V_{2}=\frac{\left(\alpha_{1}^{(0,1)}\right)^{2} \beta_{1}^{(1,0)}+\alpha_{1}^{(1,0)} \alpha_{2}^{(0,1)} \beta_{2}^{(0,1)}}{\alpha_{1}^{(1,0)}\left(\alpha_{2}^{(0,1)}\right)^{2}} \text { e } \quad V_{12}=-\frac{\beta_{1}^{(1,0)} \alpha_{1}^{(0,1)}}{\alpha_{1}^{(1,0)} \alpha_{2}^{(0,1)}}
$$

Conseqüentemente, substituindo $V_{1}, V_{2}$ e $V_{12}$ em (7.13), temos

$$
\zeta=\frac{\left(\alpha_{1}^{(1,1)}\right)^{2} \beta_{1}^{(1,0)}}{\alpha_{1}^{(1,0)}} \text { e } \gamma=\frac{\left(\alpha_{1}^{(2,0)}\right)^{2} \beta_{1}^{(1,0)}}{\alpha_{1}^{(1,0)}} .
$$

Assim, as expressões de $A_{1}, A_{2}$ e $A_{3}$ em ( 7.10$)$ - (7.12), respectivamente, que determinam o fator de correção tipo Bartlett da estatística escore, são dadas por

$$
\begin{aligned}
A_{1}= & \frac{3}{n}\left\{\left[2\left(\alpha_{1}^{(1,0)} \beta_{1}^{(1,1)}-\alpha_{1}^{(1,1)} \beta_{1}^{(1,0)}\right)\left(\alpha_{1}^{(0,1)} \beta_{1}^{(0,2)}+\alpha_{2}^{(0,1)} \beta_{2}^{(0,2)}\right)\right.\right. \\
& \left.+\left(\alpha_{1}^{(1,1)} \beta_{1}^{(1,0)}\right)\left(3 \alpha_{1}^{(1,1)} \beta_{1}^{(0,1)}-4 \alpha_{1}^{(0,1)} \beta_{1}^{(1,1)}-4 \alpha_{2}^{(0,1)} \beta_{2}^{(1,1)}\right)\right] \\
& {\left[\left(\alpha_{1}^{(1,0)} \beta_{1}^{(1,0)}\right)\left(\alpha_{1}^{(0,1)} \beta_{1}^{(0,1)} \beta_{2}^{(0,1)}\right)^{2}\right]^{-1}-2\left[\alpha_{1}^{(1,0)} \beta_{1}^{(1,2)}-\alpha_{1}^{(1,2)} \beta_{1}^{(1,0)}-2 \alpha_{1}^{(1,1)} \beta_{1}^{(1,1)}\right.} \\
& \left.\left.+2\left(\alpha_{1}^{(1,1)}\right)^{2} \beta_{1}^{(1,0)} \alpha_{1}^{(1,0)-1}\right]\left[\left(\alpha_{1}^{(1,0)} \beta_{1}^{(1,0)}\right)\left(\alpha_{1}^{(0,1)} \beta_{1}^{(0,1)}+\alpha_{2}^{(0,1)} \beta_{2}^{(0,1)}\right)\right]^{-1}\right\} \\
A_{2}= & -\frac{3}{n}\left\{\left[\alpha_{1}^{(3,0)} \beta_{1}^{(1,0)}+3 \alpha_{1}^{(2,0)} \beta_{1}^{(2,0)}-\alpha_{1}^{(1,0)} \beta_{1}^{(3,0)}-3\left(\alpha_{1}^{(2,0)}\right)^{2} \beta_{1}^{(1,0)} \alpha_{1}^{(1,0)-1}\right]\right. \\
& {\left[\alpha_{1}^{(1,0)} \beta_{1}^{(1,0)}\right]^{-2}+\frac{\left.2\left(\alpha_{1}^{(1,1)} \beta_{1}^{(0,1)}\right)\left(\alpha_{1}^{(2,0)} \beta_{1}^{(1,0)}\right)-\alpha_{1}^{(1,0)} \beta_{1}^{(2,0)}\right)}{\left(\alpha_{1}^{(0,1)} \beta_{1}^{(0,1)}+\alpha_{2}^{(0,1)} \beta_{2}^{(0,1)}\right)\left(\alpha_{1}^{(1,0)} \beta_{1}^{(1,0)}\right)^{2}} } \\
& \left.+\frac{3\left(\alpha_{1}^{(1.1)} \beta_{1}^{(1,0)}-\alpha_{1}^{(1,0)} \beta_{1}^{(1,1)}\right)^{2}}{\left(\alpha_{1}^{(0,1)} \beta_{1}^{(0,1)}+\alpha_{2}^{(0,1)} \beta_{2}^{(0,1)}\right)\left(\alpha_{1}^{(1,0)} \beta_{1}^{(1,0)}\right)^{2}}\right\} \\
& \frac{\left.5\left[\alpha_{1}^{(2,0)} \beta_{1}^{(1,0)}-\alpha_{1}^{(1,0)} \beta_{1}^{(2,0)}\right)\right]^{2}}{n\left[\alpha_{1}^{(1,0)} \beta_{1}^{(1,0)}\right]^{3}} .
\end{aligned}
$$




\subsection{Aplicações}

Nesta seção, utilizamos os resultados obtidos nas Seções 7.2 e 7.3 para a correção tipo Bartlett da estatística escore em alguns modelos que pertencem à família exponencial biparamétrica, cujos parâmetros são globalmente ortogonais. As distribuições consideradas nesta seção são bastante conhecidas e de grande importância nas aplicações práticas (vide Johnson e Kotz, 1970a. 1970b). Os resultados foram obtidos usando os sistemas computacionais MATHEMATICA (Wolfram, 1991) e MAPLE V (Abell e Baselton, 1994). Os programas desenvolvidos são bastante simples. Fornecendo as funções $\alpha_{1}, \alpha_{2}$ e $\rho$, o programa obtém expressões para $A_{1}, A_{2}$ e $A_{3}$ e conseqüentemente os coeficientes $a, b$ e $c$, definidos em (1.19).

Cabe ressaltar que os resultados obtidos para $a, b$ e $c$ para as distribuições normal de média $\theta$ e variância $\phi$, normal inversa com média $\theta$ e variância $\theta^{3} / \phi$, e gama de média $\theta$ e variância $\theta^{2} / \phi$, coincidem com os resultados obtidos por Cordeiro e Ferrari (1991). Além disto, o coeficiente $c$ do fator de correção tipo Bartlett adicionando de uma unidade, ou seja, $1+c$, corresponde ao fator de correção de Bartlett da estatística da razão de verossimilhança (vide Aubin, 1995, Capítulo 4).

As distribuições que são consideradas aqui são caracterizadas pelas funções $\alpha_{1}, \alpha_{2}, \rho$, $d_{1}(\cdot), d_{2}(\cdot), v(\cdot)$ e pelo espaço paramétrico que, juntos, podem reproduzir através de (7.1) a função densidade (ou de probabilidade) correspondente. As seguintes distribuições'são consideradas:

(i) Distribuição normal $N(\theta, \phi)(\phi>0,-\infty<\theta<\infty,-\infty<y<\infty)$ : $\alpha_{1}(\theta, \phi)=$ $\theta / \phi, \alpha_{2}(\theta, \phi)=-1 /\{2 \phi\}, \rho(\theta, \phi)=\theta^{2} /\{2 \phi\}+\log (\phi) / 2, d_{1}(y)=y, d_{2}(y)=y^{2}, v(y)=$ $-\log (2 \pi) / 2, \beta_{1}=\theta, \beta_{2}=\left(\phi+\theta^{2}\right), V_{1}=\phi, V_{2}=2 \phi^{2}+4 \phi \theta^{2}, V_{12}=2 \theta \phi$. Os estimadores de máxima verossimilhança irrestritos para $\theta$ e $\phi$ são, respectivamente,

$$
\hat{\theta}=\bar{y} \quad \text { e } \quad \hat{\phi}=\frac{1}{n} \sum_{i=1}^{n}\left(y_{i}-\bar{y}\right)^{2} .
$$

Consideramos primeiramente o teste de $H_{0}: \theta=\theta^{(0)}$ contra $H_{1}: \theta \neq \theta^{(0)}$, sendo $\theta^{(0)}$ um valor pré-fixado e $\phi$ o parâmetro de perturbação. Identificando $\theta$ e $\phi \operatorname{como} \mu$ e $\nu$, respectivamente, temos que a estatística escore definida em (7.6) tem a forma

$$
S_{R}=\frac{n^{2}\left(\bar{y}-\theta^{(0)}\right)^{2}}{\sum_{i=1}^{n}\left(y_{i}-\theta^{(0)}\right)^{2}} \text {. }
$$

Como a função $\alpha_{2}$ depende apenas do parâmetro $\phi$, utilizaremos os resultados da Seção 7.3 (caso 2) para obter os coeficientes do fator de correção tipo Bartlett. Por (7.18), temos $\zeta=1 / \phi^{3}$ e $\gamma=0$. Logo, as expressões $A_{1}, A_{2}$ e $A_{3}$ são obtidas, respectivamente, de (7.19) - (7.21), resultando em $A_{1}=0, A_{2}=-18 / n$ e $A_{3}=0$. Substituindo estas expressões em (1.19), temos

$$
a=0, \quad b=-\frac{1}{2 n} \quad \text { e } \quad c=\frac{3}{2 n} .
$$


Desta maneira. a estatística escore corrigida definida em (1.18) tem a forma simples

$$
S_{R}^{\times}=S_{R}\left\{1-\frac{\left(3-S_{R}\right)}{2 n}\right\} .
$$

Para verificar que as expressões da estatística escore modificada $S_{R}^{*}$ estão corretas, encontram-se $A_{1}, A_{2}$ e $A_{3}$ a partir de cálculos de momentos de $S_{R}$ definida em (7.22). Ferrari (1991, p.78), considerando o teste da média em um modelo normal, obtém os três primeiros momentos de $S_{R}$ até ordem $n^{-1}$, que são $\mu_{1}^{\prime}=1, \mu_{2}^{\prime}=3-6 / n$ e $\mu_{3}^{\prime}=15-90 / n$. Substituindo estes momentos em (1.17) se comprova que $A_{1}=0, A_{2}=-18 / n$ e $A_{3}=0$.

Se o interesse é testar $H_{0}: \phi=\phi^{(0)}$ contra $H_{1}: \phi \neq \phi^{(0)}$, sendo $\phi^{(0)}$ um valor pré-fixado e $\theta$ o parâmetro de perturbação, identificamos $\phi \operatorname{como} \mu$ e $\theta$ como $\nu$, e obtemos de (7.6) a estatística escore

$$
S_{R}=\frac{1}{2 n}\left[\frac{1}{\phi^{(0)}} \sum_{l=1}^{n}\left(y_{l}-\bar{y}\right)^{2}-n\right]^{2} .
$$

Os coeficientes $A_{1}, A_{2}$ e $A_{3}$ são obtidos de (7.10) - (7.12), sendo que as derivadas envolvidas nestas expressões são em relação ao vetor de parâmetros $(\phi, \theta)$. Então, temos $\zeta=1 / \phi^{3}$ e $\gamma=2 / \phi^{4}$, resultando em $A_{1}=-6 / n, A_{2}=12 / n$ e $A_{3}=40 / n$. Substituindo estas expressões em (1.19), temos

$$
a=\frac{2}{9 n}, \quad b=-\frac{17}{9 n} \quad \text { e } \quad c=\frac{11}{6 n} .
$$

Assim, a estatística escore corrigida definida em (1.18) tem a forma

$$
S_{R}^{*}=S_{R}\left\{1-\frac{1}{3 n}\left(\frac{11}{2}-\frac{17}{3} S_{R}+\frac{2}{3} S_{R}^{2}\right)\right\} .
$$

Para verificar se as expressões $A_{1}, A_{2}$ e $A_{3}$ estão corretas, Ferrari (1991, p.79), considerando o teste de $\phi$, ainda no modelo normal, encontra os três primeiros momentos, até ordem $n^{-1}$, da estatística escore $S_{R}$ dada acima. Estes momentos são, até ordem $n^{-1}$, $\mu_{1}^{\prime}=1-1 /(2 n), \mu_{2}^{\prime}=3+1 / n$ e $\mu_{3}^{\prime}=15+235 /(2 n)$. Agora, substituindo $\mu_{1}^{\prime}, \mu_{2}^{\prime}$ e $\mu_{3}^{\prime}$ em (1.17), chega-se a $A_{1}=-6 / n, A_{2}=12 / n$ e $A_{3}=40 / n$, que conduzem à expressão de $S_{R}^{*}$ dada acima.

(ii) Distribuição normal inversa $N I(\theta, \phi)(\phi>0, \theta>0, y>0): \alpha_{1}(\theta, \phi)=-\phi /\left\{2 \theta^{2}\right\}$, $\alpha_{2}(\theta, \phi)=-\phi / 2, \rho(\theta, \phi)=-(\phi / \theta+\log (\phi) / 2), d_{1}(y)=y, d_{2}(y)=y^{-1}, v(y)=-\log \left(2 \pi y^{3}\right) / 2$, $\beta_{1}=\theta, \beta_{2}=(\theta+\phi) /(\theta \phi), V_{1}=\theta^{3} / \phi, V_{2}=\left\{2 / \phi^{2}+1 /(\phi \theta)\right\}, V_{12}=(\phi-\theta) / \phi$. Os estimadores de máxima verossimilhança irrestritos para $\theta$ e $\phi$ são, respectivamente,

$$
\widehat{\theta}=\bar{y} \quad \text { e } \quad \hat{\phi}=\left(\frac{1}{\bar{y}_{h}}-\frac{1}{\bar{y}}\right)^{-1},
$$

sendo $\bar{y}_{h}$ a média harmônica de $y_{1}, \ldots, y_{n}$, isto é, $\bar{y}_{h}^{-1}=\sum_{i=1}^{n} y_{i}^{-1} / n$. Para testar as hipóteses $H_{0}: \theta=\theta^{(0)}$ contra $H_{1}: \theta \neq \theta^{(0)}$, a estatística escore definida em (7.6) tem a forma

$$
S_{R}=\frac{n^{2}\left(\bar{y}-\theta^{(0)}\right)^{2}}{\theta^{(0)}}\left\{\sum_{l=1}^{n} \frac{\left(y_{l}-\theta^{(0)}\right)^{2}}{y_{l}}\right\}^{-1} .
$$


Como a função $\alpha_{2}$ depende somente do parâmetro 0 . utilizando os resultados da Seção 7.3 (caso 2), temos $\zeta=1 /\left(\phi \theta^{3}\right)$ e $\gamma=9 \phi / \theta^{5}$. Logo, utilizando $(7.19)-(7.21)$, encontramos $A_{1}=0, A_{2}=-9\left(2 \tilde{\phi}-5 \theta^{(0)}\right) /\{n \tilde{\phi}\}$ e $A_{3}=45 \theta^{(0)} /\{n \tilde{\phi}\}$, onde

$$
\tilde{\phi}=n \theta^{(0) 2}\left\{\sum_{i=1}^{n}\left(y_{i}-\theta^{(0)}\right)^{2} / y_{i}\right\}^{-1}
$$

é o estimador de máxima verossimilhança de $\phi$ sob $H_{0}$. Substituindo estas expressões em (1.19), temos

$$
a=\frac{\theta^{(0)}}{4 n \tilde{\phi}}, \quad b=-\frac{1}{4 n}\left\{2+\frac{5 \theta^{(0)}}{\widetilde{\phi}}\right\} \quad \text { e } \quad c=\frac{3}{2 n} .
$$

A estatística escore corrigida, definida em (1.18), tem a forma

$$
S_{R}^{*}=S_{R}\left\{1-\frac{1}{4 n}\left(6-S_{R}\left(2+\frac{5 \theta^{(0)}}{\widetilde{\phi}}\right)+S_{R}^{2} \frac{\theta^{(0)}}{\widetilde{\phi}}\right)\right\} .
$$

Para testar a hipótese nula $H_{0}: \phi=\phi^{(0)}$ contra a alternativa $H_{0}: \phi \neq \phi^{(0)}$, a estatística escore definida em (7.6) tem a forma

$$
S_{R}=\frac{1}{2 n}\left\{n-\frac{\phi^{(0)}}{\bar{y}^{2}} \sum_{i=1}^{n} \frac{\left(y_{l}-\bar{y}\right)^{2}}{y_{l}}\right\}^{2} .
$$

A obtenção das expressões de $A_{1}, A_{2}$ e $A_{3}$ é feita através de (7.10) - (7.12) usando o fato de que $\zeta=1 /\left(\theta^{3} \phi\right)$ e $\gamma=0$. Isto resulta em $A_{1}=-6 / n, A_{2}=12 / n$ e $A_{3}=40 / n$. Substituindo estas expressões em (1.19) chega-se aos coeficientes

$$
a=\frac{2}{9 n}, \quad b=-\frac{17}{9 n} \quad \text { e } \quad c=\frac{11}{6 n}
$$

e à estatística escore corrigida

$$
S_{R}^{*}=S_{R}\left\{1-\frac{1}{18 n}\left(33-34 S_{R}+4 S_{R}^{2}\right)\right\} .
$$

(iii) Distribuição Gama $G(\theta, \phi)(\theta>0, \phi>0, y>0): \alpha_{1}(\theta, \phi)=-\phi / \theta, \alpha_{2}(\theta, \phi)=\phi$, $\rho(\theta, \phi)=\phi \log \theta-\phi \log \phi+\log \Gamma(\phi), d_{1}(y)=y, d_{2}(y)=\log y, v(y)=-\log y, \beta_{1}=\theta$, $\beta_{2}=\log (\theta / \phi)+\psi(\phi), V_{1}=\theta^{2} / \phi, V_{2}=\psi^{\prime}(\phi), V_{12}=\theta / \phi$.

A parametrização considerada aqui é tal que a média é $\theta>0$ e a variância é $\theta^{2} / \phi$, e é muito conveniente, pois faz com que os parâmetros $\theta$ e $\phi$ sejam globalmente ortogonais. O estimador de máxima verossimilhança irrestrito de $\theta$ é $\hat{\theta}=\bar{y}$ e o de $\phi$ pode ser obtido resolvendo-se a equação

$$
\log (\widehat{\phi})-\psi(\widehat{\phi})=\log \left(\bar{y} / \bar{y}_{g}\right),
$$

onde $\bar{y}=n^{-1} \sum_{l=1}^{n} y_{l}$ é a média aritmética e $\bar{y}_{g}=\left(\prod_{l=1}^{n} y_{l}\right)^{1 / n}$ é a média geométrica de $y_{1}, \ldots, y_{n}$. O valor de $\hat{\phi}$ pode ser determinado por interpolação inversa em uma tabela de 
funções $\{\log (\phi)-\psi(\phi)\}$, a partir do valor $\log \left(\bar{y} / \bar{y}_{g}\right)$ (vide Johnson e Kotz, 1970a, p.1S7, e Chapman, 1956, p.500). Se ồ suficientemente grande, podemos usar a aproximação $\psi(\phi)=\log (\phi-1 / 2)$. Assim. a solução da equação acima é $\widehat{\phi} \cong \bar{y} /\left\{2\left(\bar{y}-\bar{y}_{g}\right)\right\}$ (vide Johnson e Kotz, 1970a, p.188).

Para testar $H_{0}: \theta=\theta^{(0)}$ contra $H_{1}: \theta \neq \theta^{(0)}$, a estatística escore é obtida de $(7.6)$ e tem a forma

$$
S_{R}=n \tilde{\phi}\left(\frac{\bar{y}-\theta^{(0)}}{\theta^{(0)}}\right)^{2},
$$

onde $\tilde{\phi}$ é o estimador de máxima verossimilhança de $\phi$ sob $H_{0}$, isto é, a solução da equação não-linear

$$
\log (\tilde{\phi})-\imath \cdot(\tilde{\phi})=\log \left(\frac{\theta^{(0)}}{\bar{y}_{g}}\right)+\left(\frac{\bar{y}-\theta^{(0)}}{\theta^{(0)}}\right) .
$$

De (7.18) obtemos $\zeta=1 /\left\{\theta^{2} \phi\right\}$ e $\gamma=4 \phi /\left\{\theta^{4}\right\}$, e os coeficientes $A_{1}, A_{2}$ e $A_{3}$ são obtidos de $(7.19)-(7.21)$, resultando em

$$
\begin{aligned}
& A_{1}=\frac{6\left\{1-\phi^{2} \psi^{\prime \prime}\left(\phi^{2}\right)-2 \phi \psi^{\prime}(\phi)\right\}}{\left\{n \phi\left(1-\widetilde{\psi}^{\prime}(\phi)\right)\right\}^{2}}, \\
& A_{2}=\frac{\left\{1 \delta+9 /\left(1-\phi \psi^{\prime}(\phi)\right\}\right.}{n \phi} \\
& A_{3}=\frac{20}{n \phi},
\end{aligned}
$$

onde $\psi^{\prime}(\cdot), \psi^{\prime \prime}(\cdot)$ são as duas primeiras derivadas da função digama definida por $\psi(\phi)=$ $\mathrm{d} \log \Gamma(\phi) / \mathrm{d} \phi$. Substituindo as expressões (7.29) em (1.19), temos

$$
\begin{aligned}
& a=\frac{1}{9 n \phi}, \quad b=\frac{1}{36 n \phi}\left\{\frac{9}{\left(1-\phi \psi^{\prime}(\phi)\right)}-22\right\}, \\
& c=\frac{1}{12 n \phi}\left\{\frac{-3\left(2 \phi^{2} \psi^{\prime \prime}(\phi)+\phi \psi^{\prime}(\phi)+1\right)}{\left(1-\phi \psi^{\prime}(\phi)\right)^{2}}+2\right\} .
\end{aligned}
$$

Para testar $H_{0}: \phi=\phi^{(0)}$ contra $H_{1}: \phi \neq \phi^{(0)}$ a estatística escore definida em (7.6) tem a forma

$$
S_{R}=\frac{n \phi^{(0)}\left\{\log \phi^{(0)}-\psi\left(\phi^{(0)}\right)-\log \left(\bar{y} / \bar{y}_{g}\right)\right\}^{2}}{\left(\phi^{(0)} \psi^{\prime}\left(\phi^{(0)}\right)-1\right)} .
$$

As expressões de $A_{1}, A_{2}$ e $A_{3}$ são obtidas, respectivamente, através de (7.10) - (7.12), resultando em

$$
\begin{aligned}
& A_{1}=-\frac{3}{n \phi\left(\phi \psi^{\prime}(\phi)-1\right)} \\
& A_{2}=\frac{3 \phi\left\{\phi \psi^{\prime \prime \prime}(\phi)+2 \psi^{\prime \prime}(\phi)\right\}}{n\left(\phi \psi^{\prime}(\phi)-1\right)^{2}}, \\
& A_{3}=\frac{5\left\{\phi^{2} \psi^{\prime \prime}(\phi)+1\right\}^{2}}{\left\{n \phi\left(\phi \psi^{\prime}(\phi)-1\right)\right\}^{3}} .
\end{aligned}
$$


Desta maneira,

$$
\begin{aligned}
a= & \frac{\left\{\phi^{2} \psi^{\prime \prime \prime}(\phi)+1\right\}^{2}}{36 n\left\{\phi \psi^{\prime}(\phi)-1\right\}^{3}}, \\
b= & \frac{1}{36 n}\left\{\frac{3 \phi\left(\phi \psi^{\prime \prime \prime}(\phi)+2 \psi^{\prime \prime}(\phi)\right)}{\left(\phi \psi^{\prime}(\phi)-1\right)^{2}}-\frac{10\left(\phi^{2} \psi^{\prime \prime}(\phi)+1\right)^{2}}{\phi\left(\phi \psi^{\prime}(\phi)-1\right)^{3}}\right\}, \\
c= & \frac{1}{12 n \phi}\left\{\frac{5\left(\phi^{2} \psi^{\prime \prime}(\phi)+1\right)^{2}}{\left(\phi \psi^{\prime}(\phi)-1\right)^{3}}-\frac{3 \phi^{2}\left(\phi \psi^{\prime \prime \prime}(\phi)+2 \psi^{\prime \prime}(\phi)\right)}{\left(\phi \psi^{\prime}(\phi)-1\right)^{2}}\right. \\
& \left.-\frac{3}{\left(\phi \psi^{\prime}(\phi)-1\right)}\right\} .
\end{aligned}
$$

Observamos que para o cálculo da correção do teste escore para $\theta$, os coeficientes $b$ e $c$ dados em (7.30) envolvem a avaliação das duas primeiras derivadas da função digama, enquanto que para o cálculo da correção do teste aperfeiçoado para $\phi$, os coeficientes $a$, $b$ e $c$ dados em (7.32) envolvem também a terceira derivada da função digama. Com a finalidade de simplificar o cálculo da correção tipo Bartlett em tais casos, desenvolvemos aproximações simples para $a, b$ e $c$. Utilizando a expansão de $\psi^{\prime}(x)$ para valores grandes de $x$, dada em (3.12), obtemos para o teste de $H_{0}: \theta=\theta^{(0)}$ os seguintes coeficientes:

$$
\begin{aligned}
b & =-\frac{1}{n}\left(\frac{1}{2}+\frac{4}{9 \phi}+\frac{1}{18 \phi^{2}}+\frac{2}{135 \phi^{3}}\right)+O\left(\phi^{-4}\right), \\
c & =\frac{1}{n}\left(\frac{3}{2}-\frac{1}{18 \phi^{2}}-\frac{2}{45 \phi^{3}}\right)+O\left(\phi^{-4}\right),
\end{aligned}
$$

e para o teste de $H_{0}: \phi=\phi^{(0)}$, obtemos

$$
\begin{aligned}
a & =\frac{1}{n}\left(\frac{2 \phi}{9}-\frac{1}{54 \phi}-\frac{47}{2430 \phi^{2}}+\frac{22}{1215 \phi^{3}}\right)+O\left(\phi^{-4}\right), \\
b & =\frac{1}{n}\left(-\frac{17}{9}+\frac{1}{9 \phi}+\frac{2}{27 \phi^{2}}+\frac{94}{1215 \phi^{3}}\right)+O\left(\phi^{-4}\right), \\
c & =\frac{1}{n}\left(\frac{11}{6}-\frac{1}{6 \phi}+\frac{7}{162 \phi^{3}}\right)+O\left(\phi^{-4}\right) .
\end{aligned}
$$

Com a finalidade de avaliar a qualidade das aproximações em (7.30) e (7.32), calculamos os valores exatos e aproximados das quantidades $a, b$ e $c$ para alguns valores de $\phi$ (ou $\theta$ ) (vide Tabelas 7.1 e 7.2 ). 
Tabela 7.1: Valores exatos e aproximados de $b$ e $c$ para o teste de $H_{0}: \theta=\theta^{(0)}$, para a distribuiçào gama ( $\phi$ grande).

\begin{tabular}{c|cc|cc}
\hline & $b$ & $b$ & $c$ & $c$ \\
$\phi$ & exato & aprox. & exato & aprox. \\
\hline 0,8 & $-1,136100$ & $-1,171300$ & 1,46339 & 1.32639 \\
1,0 & $-0,998748$ & $-1,01481$ & 1,46691 & 1.40000 \\
1,5 & $-0,821792$ & $-0,825377$ & 1,47869 & 1,46214 \\
2,0 & $-0,736786$ & $-0,737963$ & 1,48628 & 1,48056 \\
3,0 & $-0,654636$ & $-0,65487$ & 1,49337 & 1,49218 \\
4,0 & $-0,614742$ & $-0,614815$ & 1,49621 & 1,49583 \\
5,0 & $-0,59120$ & $-0,59123$ & 1,49757 & 1,49742 \\
10,0 & $-0,545013$ & $-0,545013$ & 1,49941 & 1,49941 \\
20,0 & $-0,522363$ & $-0,522363$ & 1,49986 & 1,49986 \\
\hline
\end{tabular}

Tabela 7.2: Valores exatos e aproximados de $a, b$ e $c$ para o teste de $H_{0}: \phi=\phi^{(0)}$, para a distribuiçào gama ( $\theta$ grande).

\begin{tabular}{c|cc|cc|cc}
\hline & $a$ & $a$ & $b$ & $b$ & $c$ & $c$ \\
$\theta$ & exato & aprox. & exato & aprox. & exato & aprox. \\
\hline 0,8 & 0,158094 & 0,159774 & $-1,65116$ & $-1,48315$ & 1,61701 & 1,70939 \\
1,0 & 0,204153 & 0,202469 & $-1,70380$ & $-1,62634$ & 1,66147 & 1,70988 \\
1,5 & 0,319289 & 0,317756 & $-1,77620$ & $-1,75897$ & 1,72134 & 1,73503 \\
2,0 & 0,433410 & 0,432613 & $-1,81070$ & $-1,80514$ & 1,75029 & 1,75540 \\
3,0 & 0,659254 & 0,659015 & $-1,84182$ & $-1,84076$ & 1,77825 & 1,77938 \\
4,0 & 0,883423 & 0,883333 & $-1,85559$ & $-1,85527$ & 1,79198 & 1,79234 \\
5,0 & 1,106820 & 1,106780 & $-1,86321$ & $-1,86309$ & 1,80020 & 1,80035 \\
10,0 & 2,220200 & 2,220200 & $-1,87697$ & $-1,87696$ & 1,81671 & 1,81674 \\
20,0 & 4,443470 & 4,443470 & $-1,88314$ & $-1,88314$ & 1,82501 & 1,82501 \\
\hline
\end{tabular}

Para valores pequenos de $x$ podemos usar a expansão dada em (3.13), obtendo para o teste de $H_{0}: \theta=\theta^{(0)}$, os seguintes coeficientes

$$
\begin{aligned}
& b=-\frac{1}{n}\left\{\frac{11}{18 \phi}+\frac{1}{4}+\frac{\phi}{4}-\frac{\left(\pi^{2}-6\right) \phi^{2}}{24}-\frac{\left(\pi^{2}-3-6 \xi(3)\right) \phi^{3}}{12}\right\}+O\left(\phi^{4}\right), \\
& c=\frac{1}{n}\left\{\frac{1}{6 \phi}+\frac{3}{4}+\frac{5 \phi}{4}-\frac{7\left(\pi^{2}-6\right) \phi^{2}}{24}-\frac{3\left(\pi^{2}-3-6 \xi(3)\right) \phi^{3}}{4}\right\}+O\left(\phi^{4}\right)
\end{aligned}
$$


e para o teste de $H_{0}: \dot{\phi}=o^{(0)}$. os coeficientes

$$
\begin{aligned}
& a=\frac{1}{n}\left\{\frac{\phi}{9}+\frac{2 \phi^{2}}{9}+\left(\frac{13}{36}-\frac{\pi^{2}}{18}\right) o^{3}\right\}+O\left(\phi^{4}\right), \\
& b=-\frac{1}{n}\left\{\frac{17}{18}+\frac{17 \phi}{9}-\left(\frac{\pi^{2}}{2}-\frac{28}{9}\right) \phi^{2}-\left(\frac{\left.27 \pi^{2}-83-154 \xi(3)\right)}{18}\right) \phi^{3}\right\}+O\left(\phi^{4}\right), \\
& c=\frac{1}{n}\left\{\frac{11}{12}+\frac{25 \phi}{12}+\left(\frac{11}{3}-\frac{5 \pi^{2}}{8}\right) \phi^{2}+\left(\frac{68-23 \pi^{2}+142 \xi(3)}{12}\right) \phi^{3}\right\}+O\left(\phi^{4}\right),
\end{aligned}
$$

onde $\xi$ é a função zeta de Riemann $(\xi(3) \approx 1,2020569)$.

Para avaliar a qualidade das aproximações dadas acima, mostramos nas Tabelas $7.3 \mathrm{e}$ 7.4 os valores exatos e aproximados em (7.30) e (7.32) para alguns valores do parâmetro $\phi$.

Verifica-se nas Tabelas $7.1-7.4$ que em certas regiões do espaço paramétrico os valores aproximados de $(7.30)$ e (7.32), para $\phi$ pequeno (até 0,40$), \theta$ pequeno $(\theta<0,2)$ e $\theta$ grande $(\theta>2)$, e $\phi$ grande $(\phi>2)$, estão muito próximos dos valores exatos.

\begin{tabular}{|c|c|c|c|c|}
\hline$\phi$ & $\begin{array}{c}b \\
\text { exato }\end{array}$ & $\begin{array}{c}b \\
\text { aprox. }\end{array}$ & $\begin{array}{c}c \\
\text { exato }\end{array}$ & $\begin{array}{c}c \\
\text { aprox. }\end{array}$ \\
\hline 0,001 & $-611,361$ & $-611,361$ & 167,418 & 167,418 \\
\hline 0,01 & $-61,3636$ & $-61,3636$ & 17,4291 & 17,4291 \\
\hline 0,02 & $-30,8105$ & $-30,8105$ & 9,10788 & 9,10788 \\
\hline 0,03 & $-20,6277$ & $-20,6277$ & 6,34205 & 6,34205 \\
\hline 0,05 & $-12,4843$ & $-12,4843$ & 4,14305 & 4,14304 \\
\hline 0,10 & $-6,38453$ & $-6,38453$ & 2,53071 & 2,53064 \\
\hline 0,20 & $-3,34943$ & $-3,34933$ & 1,79127 & 1,79024 \\
\hline 0,30 & $-2,34873$ & $-2,3483$ & 1,59025 & 1,58592 \\
\hline 0,40 & $-1,85498$ & $-1,85381$ & 1,511406 & 1,50254 \\
\hline 0,50 & $-1,56296$ & $-1,56048$ & 1,48205 & 1,45831 \\
\hline 0,80 & $-1,1361$ & $-1,12532$ & 1,46339 & 1,36762 \\
\hline 1,00 & $-0,998748$ & $-0,978439$ & 1,46691 & 1,29508 \\
\hline
\end{tabular}

Tabela 7.3: Valores exatos e aproximados de $b$ e $c$ para o teste de $H_{0}: \theta=\theta^{(0)}$, para a distribuição gama ( $\phi$ pequeno). 
Tabela 7.4: Valores exatos e aproximados de $a, b$ e $c$ para o teste de $H_{0}: o=\phi^{(0)}$, para a distribuiçào gama ( $\theta$ pequeno).

\begin{tabular}{c|cc|cc|cc}
\hline & $\begin{array}{c}a \\
\theta\end{array}$ & $\begin{array}{c}a \\
\text { exato }\end{array}$ & $\begin{array}{c}b \\
\text { aprox. }\end{array}$ & $\begin{array}{c}b \\
\text { exato }\end{array}$ & $\begin{array}{c}c \\
\text { aprox. }\end{array}$ & $\begin{array}{c}c \\
\text { exato }\end{array}$ \\
\hline 0,001 & 0,0001133 & 0.00011133 & $-0,946332$ & $-0,946332$ & 0.918747 & 0,918747 \\
0,01 & 0,00113315 & 0.00113315 & $-0,963151$ & $-0,963151$ & 0.937251 & 0,937251 \\
0,02 & 0,0023096 & 0.0023096 & $-0,981494$ & $-0,981493$ & 0.957341 & 0,957341 \\
0,03 & 0,0035282 & 0.0035282 & $-0,999474$ & $-0,999472$ & 0.976943 & 0,976941 \\
0,05 & 0,0060875 & 0.0060877 & $-1,034360$ & $-1,034340$ & 1,01471 & 1,01470 \\
0,10 & 0,0131444 & 0.0131461 & $-1,115410$ & $-1,115190$ & 1,10114 & 1,10096 \\
0,20 & 0,0296236 & 0.0296135 & $-1,253000$ & $-1,250000$ & 1,24336 & 1,24105 \\
0,30 & 0,0484659 & 0.0482789 & $-1,362330$ & $-1,349440$ & 1,352 & 1,34281 \\
0,40 & 0,0689165 & 0.0680192 & $-1,448840$ & $-1,414030$ & 1,43495 & 1,41206 \\
0,50 & 0,090428 & 0.0877111 & $-1,517390$ & $-1,444340$ & 1,49861 & 1,45466 \\
0,80 & 0,158094 & 0.135265 & $-1,651160$ & $-1,334970$ & 1,61701 & 1,48098 \\
1,00 & 0,204153 & 0.146133 & $-1,703800$ & $-1,100610$ & 1,66147 & 1,47243 \\
\hline
\end{tabular}

(iv) Distribuição log-gama $\operatorname{LG}(\theta, \phi)(\phi>0,-\infty<\theta<\infty,-\infty<y<\infty)$ : O logaritmo de uma variável aleatória com distribuição gama com média $\theta$ e índice $\phi$ (vide (iii)) tem distribuição log-gama com função densidade pertencente à família exponencial biparamétrica, sendo: $\alpha_{1}(\theta, \phi)=-\phi e^{-\theta}, \alpha_{2}(\theta, \phi)=\phi, \rho(\theta, \phi)=\phi \theta-\phi \log \phi+\log \{\Gamma(\phi)\}$, $d_{1}(y)=\exp \{y\}, d_{2}(y)=y, v(y)=0, \beta_{1}=\exp \{\theta\}, \beta_{2}=\theta+\psi(\phi)-\log \phi, V_{1}=\exp \{2 \theta\} / \phi$, $V_{2}=\psi^{\prime}(\phi), V_{12}=\exp \{\theta\} / \phi$.

Para testar $H_{0}: \theta=\theta^{(0)}$ contra $H_{1}: \theta \neq \theta^{(0)}$, a estatística escore tem a mesma forma que a estatística escore da distribuição gama dada em (7.28) e os coeficientes $a, b$ e $c$ do fator de correção tipo Bartlett da estatística escore são aqueles do modelo gama, dado em (7.30). Isto deve-se ao fato de que a correção tipo Bartlett é invariante em relação a transformação dos dados, mantida a mesma hipótese nula.

Para testar $H_{0}: \phi=\phi^{(0)}$ contra $H_{1}: \phi \neq \phi^{(0)}$, a estatística escore é definida em (7.31) e, da mesma forma que no teste de $\theta$, os coeficientes $a, b$ e $c$ coincidem com aqueles do modelo gama para esta hipótese, fornecidos em (7.32), conforme esperado pelo motivo exposto acima.

(v) Distribuição gama inversa $G I(\theta, \phi)(\phi>0, \theta>0, y>0)$ : A inversa de uma variável aleatória com distribuição gama com média $\theta$ e índice $\phi$ (vide (iii)) tem distribuição gama inversa com função densidade pertencente à família exponencial biparamétrica, sendo: $\alpha_{1}(\theta, \phi)=-\theta \phi, \alpha_{2}(\theta, \phi)=-\phi, \rho(\theta, \phi)=-\phi \log (\theta \phi)+\log \Gamma(\phi), d_{1}(y)=y^{-1}$, $d_{2}(y)=\log y, v(y)=-\log y, \beta_{1}=\theta^{-1}, \beta_{2}=\log (\theta \phi)-\psi(\phi), V_{1}=1 /\left(\phi \theta^{2}\right), V_{2}=\psi^{\prime}(\phi)$, $V_{12}=-1 /(\theta \phi)$.

Para testar $H_{0}: \theta=\theta^{(0)}$ contra $H_{1}: \theta \neq \theta^{(0)}$, a estatística escore tem a mesma forma da estatística escore da distribuição gama dada em (7.28). 
Os coeficientes $a, b$ e $c$ do fator de correçào tipo Bartlett para a estatística escore também são aqueles do modelo gama dados em (7.30). A justificativa também é a mesma dada para o caso da distribuição log-gama, ou seja. a de que a correção tipo Bartlett é invariante em relação a transformações dos dados. mantida a mesma hipótese nula.

Para testar $H_{0}: \phi=\phi^{(0)}$ contra $H_{1}: \phi=\phi^{(0)}$, a estatística escore tem a forma (7.31) e os coeficientes $a, b$ e $c$ coincidem com as expressões dadas em ( 7.32$)$.

\subsection{Construção de parâmetros globalmente ortogo- nais}

Sejam $\pi(y ; \mu, \nu)$ uma função densidade (ou de probabilidade) que pertence à família exponencial biparamétrica definida em $(7.1)$ e $L(\mu, \nu)$ o logaritmo da função de verossimilhança dada em (7.2). Sejam $\mu$ o parâmetro de interesse e $\nu$ o parâmetro de perturbação. Se $\mu$ e $\nu$ não são globalmente ortogonais, isto é, se a condição (7.15) não é satisfeita, então é necessário determinar uma transformação dos parâmetros $(\mu, \nu)$, para $(\mu, \delta)$ digamos, de tal forma que $\mu$ e $\delta$ sejam globalmente ortogonais. Cox e Reid (1987) mostram que o parâmetro $\delta$, globalmente ortogonal a $\mu$, é dado por qualquer solução do sistema de equações diferenciais parciais

$$
E\left(\frac{\partial^{2} L}{\partial \nu^{2}}\right) \frac{\partial \nu}{\partial \mu}=-E\left(\frac{\partial^{2} L}{\partial \mu \partial \nu}\right)
$$

$\mathrm{Na}$ família exponencial biparamétrica temos

$$
E\left(\frac{\partial^{2} L}{\partial \nu^{2}}\right)=-n \sum_{i=1}^{2} \alpha_{i}^{(0,1)} \beta_{i}^{(0,1)} \text { e } E\left(\frac{\partial^{2} L}{\partial \mu \partial \nu}\right)=-n \sum_{i=1}^{2} \alpha_{i}^{(1,0)} \beta_{i}^{(0,1)}
$$

Assim, encontrar a solução da equação (7.33) é equivalente a encontrar a solução da equação diferencial

$$
\frac{\partial \nu}{\partial \mu}=-\frac{\sum_{i=1}^{2} \alpha_{i}^{(1,0)} \beta_{i}^{(0,1)}}{\sum_{i=1}^{2} \alpha_{i}^{(0,1)} \beta_{i}^{(0,1)}} .
$$

A solução de (7.34) fornece a nova parametrização e, portanto, recai-se no caso de parâmetros globalmente ortogonais, no qual podemos aplicar os resultados das seções anteriores. Cabe ressaltar que a equação (7.34) não tem solução geral e deverá ser resolvida para cada distribuição que pertence à família exponencial biparamétrica em que as equações dadas em ( 7.15$)$ não são satisfeitas. De forma análoga, se o parâmetro de interesse é $\nu$ e $\mu$ for o de perturbação, apenas trocamos $\mu$ por $\nu$ e vice-versa, considerando $(\nu, \mu)$ como vetor paramétrico e utilizamos a equação (7.34) para encontrar a nova parametrização $(\nu, \delta)$, onde $\nu$ e $\delta$ são globalmente ortogonais.

Apresentamos, a seguir. algumas distribuições pertencentes à família exponencial biparamétrica cujos parâmetros nào sào globalmente ortogonais. 
(i) Distribuição normal $N\left(\theta, \theta^{2} \phi^{2}\right)(\theta>0, \phi>0,-\infty<y<\infty)$ : Este modelo normal tem média $\theta$ e coeficiente de variação igual a $\phi$. Sua função densidade tem a forma

$$
\pi(y ; \theta, 0)=\frac{1}{\theta \phi \sqrt{2 \pi}} \exp \left\{-\frac{1}{2 \theta^{2} \phi^{2}}(y-\theta)^{2}\right\} \text {. }
$$

Para esta distribuição, temos $\alpha_{1}(\theta, \phi)=-1 /\left\{2 \theta^{2} \phi^{2}\right\}, \alpha_{2}(\theta, \phi)=1 /\left(\theta \phi^{2}\right), \rho(\theta, \phi)=$ $1 /\left\{2 \phi^{2}\right\}+\log (\theta \phi), d_{1}(y)=y^{2}, d_{2}(y)=y, v(y)=0, \beta_{1}=\theta^{2}\left(\phi^{2}+1\right), \beta_{2}=\theta, V_{1}=$ $2 \theta^{4} \phi^{2}\left(\phi^{2}+2\right), V_{2}=\theta^{2} \phi^{2}$. Através de (7.5), encontramos

$$
E\left(\frac{\partial^{2} L}{\partial \theta^{2}}\right)=-\frac{n\left(2 \phi^{2}+1\right)}{\theta^{2} \phi^{2}}, \quad E\left(\frac{\partial^{2} L}{\partial \phi^{2}}\right)=-\frac{2 n}{\phi^{2}} \quad \text { e } \quad E\left(\frac{\partial^{2} L}{\partial \theta \partial \phi}\right)=-\frac{2 n}{\theta \phi} \neq 0 .
$$

Os estimadores de máxima verossimilhança irrestritos dos parâmetros $\theta$ e $\phi$ são, respectivamente,

$$
\hat{\theta}=\bar{y} \quad \text { e } \quad \widehat{\phi}=\frac{S}{\bar{y}} \quad(\bar{y} \neq 0)
$$

onde $S^{2}=\sum_{i=1}^{n}\left(y_{i}-\bar{y}\right)^{2} / n$.

Consideremos a hipótese nula de interesse $H_{0}: \phi=\phi^{(0)}$ contra a alternativa $H_{1}$ : $\phi=\phi^{(0)}, \operatorname{com} \nu=\phi$ e $\mu=\theta$. Como $\theta$ e $\phi$ não são globalmente ortogonais, a nova parametrização $(\phi, \delta)$, tal que $\phi$ e $\delta$ são ortogonais, pode ser obtida pela solução de (7.34), que resulta na equação diferencial

$$
\frac{\partial \theta}{\partial \phi}=-\frac{2 \theta \phi}{2 \phi^{2}+1}
$$

Desenvolvendo a equação diferencial acima, uma possível solução é $a(\delta)=\theta\left(2 \phi^{2}+1\right)^{1 / 2}$, onde $a(\cdot)$ é uma função arbitrária. Fazendo $a(\delta)=\delta$, temos os parâmetros globalmente ortogonais $\phi$ e $\delta=\theta\left(2 \phi^{2}+1\right)^{1 / 2}$. Considerando a nova parametrização. a função densidade tem a forma

$$
\pi^{*}(y ; \delta, \phi)=\frac{\left(2 \phi^{2}+1\right)^{1 / 2}}{\sqrt{2 \pi} \phi \delta} \exp \left\{-\frac{\left(2 \phi^{2}+1\right)}{\delta^{2} \phi^{2}}\left(y-\frac{\delta}{\left(2 \phi^{2}+1\right)^{1 / 2}}\right)^{2}\right\},
$$

sendo $\alpha_{1}(\delta, \phi)=-\left(2 \phi^{2}+1\right) /\left\{2 \delta^{2} \phi^{2}\right\}, \alpha_{2}(\delta, \phi)=\left(2 \phi^{2}+1\right)^{1 / 2} /\left\{\delta \phi^{2}\right\}, \rho(\delta, \phi)=1 /\left\{2 \phi^{2}\right\}+$ $\log \left\{\delta \phi /\left(2 \phi^{2}+1\right)^{1 / 2}\right\}, d_{1}(y)=y^{2}, d_{2}(y)=y, \beta_{1}=\delta^{2}\left(\phi^{2}+1\right) /\left(2 \phi^{2}+1\right), \beta_{2}=\delta /\left\{\left(2 \phi^{2}+\right.\right.$ $\left.1)^{1 / 2}\right\}, V_{1}=2 \phi^{2}\left(\phi^{2}+2\right) \delta^{4} /\left\{\left(2 \phi^{2}+1\right)^{2}\right\}, V_{2}=\delta^{2} \phi^{2} /\left\{2 \phi^{2}+1\right\}, V_{12}=2 \phi^{2} \delta^{3} /\left\{\left(2 \phi^{2}+\right.\right.$ $\left.1)^{3 / 2}\right\}$. Com estas funções assim definidas, temos $\kappa_{\delta, \phi}=\kappa_{\phi, \delta}=0$. o que demonstra a ortogonalidade global entre $\phi$ e $\delta$. Para calcular a estatística escore $S_{R}$ definida em (7.6), precisamos encontrar a estimativa de máxima verossimilhança de $\theta$ sob $H_{0}$. Essa estimativa $\tilde{\theta}$ é solução da equação $\partial L\left(\tilde{\theta}, \phi^{(0)}\right) / \partial \theta=0$, ou seja,

$$
-\frac{n}{\tilde{\theta}}+\frac{1}{\phi^{(0) 2}}\left\{\frac{1}{\tilde{\theta}^{3}} \sum^{n}\left(y_{l}-\tilde{\theta}\right)^{2}+\frac{1}{\widetilde{\theta}^{2}} \sum^{n}\left(y_{l}-\tilde{\theta}\right)\right\}=0 .
$$


ou, equivalentemente, $\phi^{(0) 2}+\bar{y} \tilde{\theta}-m_{2}=0$, onde $m_{2}=\sum_{i=1}^{n} y_{i}^{2} / n$, resultando em

$$
\tilde{\theta}=-\frac{\bar{y} \pm \sqrt{\bar{y}^{2}+4 \phi^{(0) 2} m_{2}}}{2 \phi^{(0) 2}} .
$$

Aubin (1995, pp.130-131) demonstra qual solução de $\tilde{\theta}$ deve ser considerada tal que maximiza o logaritmo da função de verossimilhança segundo $H_{0}$, chegando à conclusão de que

$$
\tilde{\theta}=\frac{\left(\bar{y}^{2}+4 \phi^{(0) 2} m_{2}\right)^{1 / 2}-\bar{y}}{2 \phi^{(0) 2}}, \text { quando } \bar{y}>0
$$

$\mathrm{e}$

$$
\tilde{\theta}=\frac{-\left(\bar{y}^{2}+4 \phi^{(0) 2} m_{2}\right)^{1 / 2}-\bar{y}}{2 \phi^{(0) 2}}, \text { quando } \bar{y}<0 .
$$

Assim, estas expressões de $\tilde{\theta}$ são usadas para calcular a estatística escore $S_{R}^{\prime}$ definida em (7.6) que tem a forma

$$
S_{R}=\frac{1}{2 n \phi^{(0) 4}}\left\{\sum_{l=1}^{n}\left(\frac{y_{l}-\tilde{\theta}}{\tilde{\theta}}\right)^{2}-n \phi^{(0)}\right\}^{2} .
$$

Das equações dadas em (7.13), obtemos $\zeta=4\left(\phi^{2}+1\right) /\left\{\delta^{2} \phi^{4}\left(2 \phi^{2}+1\right)^{1 / 2}\right\}$ e $\gamma=$ $4\left(3+9 \phi^{2}+4 \phi^{4}\right)^{2} /\left\{\phi^{6}\left(2 \phi^{2}+1\right)^{4}\right\}$. Logo, as expressões de $A_{1}, A_{2}$ e $A_{3}$ são obtidas, repectivamente, de (7.10) - (7.12), resultando em

$$
\begin{aligned}
& A_{1}=\frac{6\left(11 \phi^{4}-2 \phi^{2}-1\right)}{n\left(2 \phi^{2}+1\right)^{3}}, \\
& A_{2}=\frac{12\left(-12 \phi^{6}+18 \phi^{4}+10 \phi^{2}+1\right)}{n\left(2 \phi^{2}+1\right)^{3}}, \\
& A_{3}=\frac{40\left(3 \phi^{2}+1\right)^{2}}{n\left(2 \phi^{2}+1\right)^{3}} .
\end{aligned}
$$

Substituindo estas expressões em (1.19), temos

$$
\begin{aligned}
& a=\frac{2\left(3 \phi^{2}+1\right)^{2}}{9 n\left(2 \phi^{2}+1\right)^{3}}, \\
& b=-\frac{\left(36 \phi^{6}+126 \phi^{4}+90 \phi^{2}+17\right)}{9 n\left(2 \phi^{2}+1\right)^{3}}, \\
& c=\frac{\left(72 \phi^{6}+105 \phi^{4}+54 \phi^{2}+11\right)}{6 n\left(2 \phi^{2}+1\right)^{3}} .
\end{aligned}
$$

Para testar a hipótese $H_{0}: \theta=\theta^{(0)}$ contra $H_{1}: \theta \neq \theta^{(0)}$. sendo $\phi$ o parâmetro de perturbação e $\theta^{(0)}$ um valor pré-fixado, devemos encontrar uma transformação de $(\theta, \phi)$ 
para $(\theta, \delta)$, tal que $\theta$ e $\delta$ sejam globalmente ortogonais. A nova parametrização pode ser obtida da solução de (7.34), que resulta na equaçào diferencial

$$
\frac{\partial \phi}{\partial \theta}=\frac{\phi}{\theta} .
$$

Uma possivel solução da equação acima é $a(\delta)=\theta 0$. onde $a(\cdot)$ é uma função arbitrária. Fazendo $a(\delta)=\delta$, temos os parâmetros globalmente ortogonais $\theta$ e $\delta=\theta \phi$. Considerando a nova parametrização, a função densidade tem a nova forma

$$
\pi^{\star}(y ; \theta, \delta)=\frac{1}{\sqrt{2 \pi} \delta} \exp \left\{-\frac{1}{2 \delta^{2}}(y-\theta)^{2}\right\},
$$

sendo $\alpha_{1}(\theta, \delta)=-1 /\left\{2 \delta^{2}\right\}, \alpha_{2}(\theta, \delta)=\theta / \delta^{2}, \rho(\theta, \delta)=\log \delta+\theta^{2} /\left\{2 \delta^{2}\right\}, \beta_{1}=\delta^{2}+\theta^{2}$, $\beta_{2}=\theta, V_{1}=2 \delta^{4}+4 \theta^{2} \delta^{2}, V_{2}=\delta^{2}$ e $V_{12}=2 \theta \delta^{2}$. Com estas funções assim definidas, temos $\kappa_{\delta, \theta}=\kappa_{\theta, \delta}=0$, o que demonstra a ortogonalidade global entre $\theta$ e $\delta$.

Para o teste do parâmetro $\theta$, a estatística escore é calculada por (7.22) e os coeficientes da correção tipo Bartlett são idênticos àqueles dados em (7.23), isto é, $a=0, b=-1 / 2 n$ e $c=3 / 2 n$. Desta maneira, a estatística escore corrigida tem a forma dada em (7.24). Este resultado é esperado, pois para testar a média da distribuição $N\left(\theta, \theta^{2} \phi^{2}\right)$, as estatísticas escore são iguais, uma vez que esta estatística é invariante sob reparametrização e, assim, as correções de tipo Bartlett para esta hipótese são também idênticas.

(ii) Distribuição normal inversa $N I\left(\theta, \theta^{2} \phi^{2}\right)(\theta>0, \phi>0, y>0)$ : Na parametrização considerada aqui, esta distribuição tem média $\theta$ e coeficiente de variação $\phi$. Sua função densidade tem a forma

$$
\pi(y ; \theta, \phi)=\exp \left\{-\frac{(y-\theta)^{2}}{2 y \theta \phi^{2}}+\frac{1}{2} \log \left(\frac{\theta}{\phi^{2}}\right)-\frac{1}{2} \log \left(2 \pi y^{3}\right)\right\} .
$$

Para esta função densidade, temos $\alpha_{1}(\theta, \phi)=-1 /\left\{2 \theta \phi^{2}\right\}, \alpha_{2}(\theta, \phi)=-\theta /\left\{2 \phi^{2}\right\}, \rho(\theta, \phi)=$ $\log \left(\phi^{2}\right) / 2-\log (\theta) / 2-1 / \phi^{2}, d_{1}(y)=y, d_{2}(y)=y^{-1}, v(y)=-\log \left(2 \pi y^{3}\right) / 2, \beta_{1}=\theta$, $\beta_{2}=\left(\phi^{2}+1\right) / \theta, V_{1}=\theta^{2} \phi^{2}, V_{2}=\phi^{2}\left(2 \phi^{2}+1\right) / \theta^{2}$. Através de (7.5), obtemos

$$
E\left(\frac{\partial^{2} L}{\partial \theta^{2}}\right)=-\frac{n\left(\delta^{2}+2\right)}{2 \theta^{2} \phi^{2}}, \quad E\left(\frac{\partial^{2} L}{\partial \phi^{2}}\right)=-\frac{2 n}{\phi^{2}} \quad \text { e } \quad E\left(\frac{\partial^{2} L}{\partial \theta \partial \phi}\right)=\frac{n}{\theta \phi} \neq 0 .
$$

As estimativas de máxima verossimilhança irrestritas para os parâmetros $\theta$ e $\phi$ são, respectivamente,

$$
\tilde{\theta}=\bar{y} \quad \text { e } \quad \hat{\phi}^{2}=\frac{\bar{y}}{\bar{y}_{h}}-1 .
$$

Consideramos a hipótese nula $H_{0}: \phi=\phi^{(0)}$ a ser testada contra a alternativa $H_{0}: \phi \neq \phi^{(0)}$. Como $\theta$ e $\phi$ não sào globalmente ortogonais, a nova parametrização $(\phi, \delta)$, tal que $\phi$ e $\delta$ sejam ortogonais, pode ser obtida pela solução de (7.34), que resulta na equaçào diferencial

$$
\frac{\partial \theta}{\partial \phi}=\frac{2 \theta \phi}{\phi^{2}+2} \text {. }
$$


Desenvolvendo esta equação diferencial, uma solução possível é $a(\delta)=\theta /\left\{\delta^{2}+2\right\}$, onde $a(\cdot)$ é uma função arbitrária. Tomando $a(\delta)=\delta$. temos os parâmetros globalmente ortogonais $\phi$ e $\delta=\theta /\left\{\phi^{2}+2\right\}$. Considerando a nova parametrização. a função densidade tem a seguinte forma.

$$
\pi^{*}(y ; \delta, \phi)=\exp \left\{-\frac{\left(y-\delta\left(\phi^{2}+2\right)\right)^{2}}{2 y \phi \delta\left(\phi^{2}+2\right)}+\frac{1}{2} \log \left(\frac{\delta\left(\phi^{2}+2\right)}{\phi^{2}}\right)-\frac{1}{2} \log \left(2 \pi y^{3}\right)\right\},
$$

sendo $\alpha_{1}(\delta, \phi)=-1 /\left\{2 \delta \phi^{2}\left(\phi^{2}+2\right)\right\}, \alpha_{2}(\delta, \phi)=-\delta\left(\phi^{2}+2\right) /\left\{2 \phi^{2}\right\}, \rho(\delta, \phi)=\log \left(\phi^{2}\right) / 2-$ $\log \left(\delta\left(\phi^{2}+2\right)\right) / 2-1 / \phi^{2}, \beta_{1}=\delta\left(\phi^{2}+2\right), \beta_{2}=\left(\phi^{2}+1\right) /\left\{\delta\left(\phi^{2}+2\right)\right\}, V_{1}=\delta^{2} \phi^{2}\left(\phi^{2}+2\right)^{2}$, $V_{2}=\phi^{2}\left(2 \phi^{2}+1\right) /\left\{\delta^{2}\left(\phi^{2}+2\right)^{2}\right\}, V_{12}=-\phi^{2}$. Com estas funções assim definidas, temos $\kappa_{\delta, \phi}=\kappa_{\phi, \delta}=0$. Para calcular a estatística escore $S_{R}$ definida em (7.6), precisamos encontrar a estimativa de máxima verossimilhança de $\theta$, sob $H_{0}$. Essa estimativa $\tilde{\theta}$ é solução da equação $\partial L\left(\tilde{\theta}, \phi^{(0)}\right) / \partial \theta=0$, ou seja,

$$
\sum_{l=1}^{n} \frac{\left(y_{l}^{2}-\tilde{\theta}^{2}\right)}{y_{l}}+\frac{n}{\tilde{\theta}}=0
$$

ou, equivalentemente, da equação

$$
\bar{y}_{n}^{-1} \tilde{\theta}^{2}-\phi^{(0) 2} \tilde{\theta}-\bar{y}=0,
$$

resultando em

$$
\tilde{\theta}=\bar{y}_{h}\left\{\frac{\phi^{(0) 2} \pm\left(\phi^{(0) 4}+4 \bar{y} / \bar{y}_{h}\right)^{1 / 2}}{2}\right\} .
$$

Como $\theta>0$, então somente consideramos a solução

$$
\tilde{\theta}=\frac{\bar{y}_{h}}{2}\left\{\phi^{(0) 2}+\left(\phi^{(0) 4}+4 \bar{y} / \bar{y}_{h}\right)^{1 / 2}\right\} .
$$

A estatística escore tem a forma

$$
S_{R}=\frac{n}{2 \widetilde{\theta}^{2} \phi^{(0) 4}}\left\{\bar{y}+\widetilde{\theta}^{2} \bar{y}_{h}^{-1}-\tilde{\theta}\left(\phi^{(0) 2}+2\right)\right\}^{2} .
$$

Das equações dadas em (7.13), obtemos $\zeta=4\left(\phi^{4}+6 \phi^{2}+4\right) /\left\{\delta^{2} \phi^{4}+\left(\phi^{2}+2\right)^{2}\right\}$ e $\gamma=$ $4\left(25 \phi^{6}+78 \phi^{4}+108 \phi^{2}+72\right) /\left\{\phi^{4}\left(\phi^{2}+2\right)^{4}\right\}$. Logo, os coeficientes $A_{1}, A_{2}$ e $A_{3}$ são obtidos, respectivamente, de $(7.10)-(7.12)$, resultando em

$$
\begin{aligned}
& A_{1}=-\frac{24\left(3 \phi^{6}+2 \phi^{4}+4 \phi^{2}+2\right)}{n\left(\phi^{2}+2\right)^{3}}, \\
& A_{2}=\frac{3\left(15 \phi^{8}+30 \phi^{6}+72 \phi^{4}+32 \phi^{2}+32\right)}{n\left(\phi^{2}+2\right)^{3}}, \\
& A_{3}=\frac{5\left(3 \phi^{4}+6 \phi^{2}+8\right)^{2}}{n\left(\phi^{2}+2\right)^{3}} .
\end{aligned}
$$


Substituindo estas expressões em (1.19), temos

$$
\begin{aligned}
& a=\frac{\left(3 \phi^{4}+6 \phi^{2}+8\right)^{2}}{36 n(\phi+2)^{3}} \\
& b=-\frac{\left(45 \phi^{8}+270 \phi^{6}+624 \phi^{4}+864 \phi^{2}+544\right)}{36 n\left(\phi^{2}+2\right)^{3}} \\
& c=\frac{\left(9 \phi^{6}+78 \phi^{4}+144 \phi^{2}+88\right)}{6 n\left(\phi^{2}+2\right)^{3}}
\end{aligned}
$$

Para testar $H_{0}: \theta=\theta^{(0)}$ contra $H_{1}: \theta \neq \theta^{(0)}$, sendo $\phi$ o parâmetro de perturbação e $\theta^{(0)}$ um valor pré-fixado, devemos encontrar uma transformação de $(\theta, \phi)$ para $(\theta, \delta)$, tal que $\theta$ e $\delta$ sejam globalmente ortogonais. A nova parametrização pode ser obtida através da solução da equação diferencial (7.34), que é expressa por

$$
\frac{\partial \phi}{\partial \theta}=\frac{\phi}{2 \theta}
$$

Uma possível solução desta equação é $a(\delta)=\phi \theta^{-1 / 2}$, onde $a(\cdot)$ é uma função arbitrária. Fazendo $a(\delta)=\delta$, temos os parâmetros globalmente ortogonais $\theta$ e $\delta=\phi \theta^{-1 / 2}$. Com esta. nova parametrização, a função densidade tem a forma

$$
\pi^{*}(y ; \theta, \delta)=\exp \left\{-\frac{y}{2 \theta^{2} \delta^{2}}-\frac{1}{2 y \delta^{2}}+\frac{1}{\theta \delta^{2}}-\frac{1}{2} \log \left(\delta^{2}\right)-\frac{1}{2} \log \left(2 \pi y^{3}\right)\right\}
$$

sendo $\alpha_{1}(\theta, \delta)=-1 /\left\{2 \theta^{2} \delta^{2}\right\}, \alpha_{2}(\theta, \delta)=-1 /\left\{2 \delta^{2}\right\}, \rho(\theta, \delta)=\log \left(\delta^{2}\right) / 2-1 /\left\{\theta \delta^{2}\right\}, d_{1}(y)=$ $y, d_{2}(y)=y^{-1}, v(y)=-\frac{1}{2} \log \left(2 \pi y^{3}\right), \beta_{1}=\theta, \beta_{2}=\left(\delta^{2} \theta+1\right) / \theta, V_{1}=\delta^{2} \theta^{3}, V_{2}=$ $\delta^{2}\left(2 \delta^{2} \theta+1\right) / \theta$ e $V_{12}=-\delta^{2} \theta$. Com estas funções assim definidas, temos $\kappa_{\delta, \theta}=\kappa_{\theta, \delta}=0$, o que demonstra a ortogonalidade global entre $\theta$ e $\delta$. Para o teste do parâmetro $\theta$, a estatística escore é calculada por (7.25), e os coeficientes da correção tipo Bartlett são idênticos àqueles dados em (7.26).

Desta maneira, a estatística escore corrigida tem a forma dada em (7.27), pois para testar a média $\theta$ da distribuição $N I(\theta, \phi)$, ou da distribuição $N I\left(\theta, \theta^{2} \phi^{2}\right)$, as estatísticas escore são iguais, uma vez que esta estatística é invariante sob reparametrização e, assim, as correções tipo Bartlett para esta hipótese são também idênticas.

(iii) Distribuição gama $G(\theta, \phi)(\theta>0, \phi>0, y>0)$ : Na parametrização considerada aqui a distribuição tem média $\theta \phi$, sendo $\theta$ o parâmetro de escala e $\phi$ é o parâmetro de forma. Sua função de densidade pode ser escrita como

$$
\pi(y ; \theta, \phi)=\frac{1}{\Gamma(\phi)} \theta^{-\phi} y^{\phi-1} \exp \{-y / \theta\} .
$$

Para esta função densidade temos $\alpha_{1}(\theta, \phi)=-\theta^{-1}, \alpha_{2}(\theta .0)=\phi, \rho(\theta, \phi)=\phi \log \theta+$ $\log \Gamma(\phi), d_{1}(y)=y, d_{2}(y)=\log y, v(y)=-\log y, \beta_{1}=\theta \phi . \beta_{2}=\log \theta+\psi(\phi), V_{1}=\phi \theta^{2}$, $V_{2}=\psi^{\prime}(\phi)$. 
Através de (7.6) encontramos

$$
E\left(\frac{\partial^{2} L}{\partial \theta^{2}}\right)=-\frac{n \phi}{\theta^{2}}, \quad E\left(\frac{\partial^{2} L}{\partial \phi^{2}}\right)=-n \psi^{\prime}(\phi) \quad \text { e } \quad E\left(\frac{\partial^{2} L}{\partial \theta \partial \phi}\right)=-\frac{n}{\theta} \neq 0 .
$$

A estimativa de máxima verossimilhança irrestrita de $\theta$ é $\hat{\theta}=\bar{y} / \widehat{\phi}$ e a de $\phi$ é a solução da equação não-linear

$$
\log \widehat{\phi}-\psi(\widehat{\phi})=\log \left(\frac{\bar{y}}{\bar{y}_{g}}\right) .
$$

Considerando $\phi$ o parâmetro de interesse, a nova parametrização $(\phi, \delta)$, com $\delta$ e $\phi$ globalmente ortogonais, é solução da equação diferencial dada em (7.34) que tem a forma

$$
\left(-\frac{n \phi}{\theta^{2}}\right) \frac{\partial \theta}{\partial \phi}=\frac{n}{\theta}
$$

Uma possível solução desta equação é $a(\delta)=\theta \phi$, onde $a(\cdot)$ é uma função arbitrária. Tomando $a(\delta)=\delta$, vêm os parâmetros globalmente ortogonais 0 e $\delta=\theta \phi$. Com esta nova parametrização, a função densidade é escrita como

$$
\pi^{*}(y ; \delta, \phi)=\exp \left\{-\frac{y \phi}{\delta}+\phi \log y-\log y+\phi \log \left(\frac{\phi}{\delta}\right)-\log \Gamma(\phi)\right\},
$$

sendo $\alpha_{1}(\delta, \phi)=-\phi / \delta, \alpha_{2}(\delta, \phi)=\phi, \rho(\delta, \phi)=-\phi \log (\phi / \delta)+\log \Gamma(\phi), v(y)=-\log y$, $d_{1}(y)=y, d_{2}(y)=\log y, \beta_{1}=\delta, \beta_{2}=\log (\delta / \phi)+\psi(\phi), V_{1}=\delta^{2} / \phi, V_{2}=\psi^{\prime}(\phi), V_{12}=\delta / \phi$. Substituindo as derivadas apropriadas em (7.5) encontramos $\kappa_{\delta, \phi}=\kappa_{\phi, \delta}=0$, o que prova que $\delta$ e $\phi$ são globalmente ortogonais. Para testar $H_{0}: \phi=\phi^{(0)}$ contra $H_{1}: \phi=\phi^{(0)}$, a estatística escore é idêntica à do teste de $H_{0}: \phi=\phi^{(0)}$, com média desconhecida, dado anteriormente em (7.31). Este fato era esperado, pois em ambas as situações, a hipótese nula é a mesma e a estatística escore é invariante sob reparametrização. Assim, os coeficientes da correção tipo Bartlett são aqueles dados em (7.32).

Para testar a hipótese nula $H_{0}: \theta=\theta^{(0)}$ contra a alternativa $H_{1}: \theta \neq \theta^{(0)}$, sendo $\phi$ o parâmetro de perturbação e $\theta^{(0)}$ fixo, a reparametrização $(\theta, \delta)$, com $\theta$ e $\delta$ ortogonais, pode ser obtida solucionando-se a equação diferencial dada em (7.34), que tem a forma

$$
\iota^{\prime}(\phi) \frac{\partial \phi}{\partial \theta}=-\frac{1}{\theta} \text {. }
$$

Uma possível solução desta equação é $a(\delta)=\psi(\phi)+\log \theta$, onde $a(\cdot)$ é uma função arbitrária. Tomando $a(\delta)=\delta$, resulta que $\theta$ e $\delta=\psi(\phi)+\log \theta$ são parâmetros globalmente ortogonais. Usando a fórmula 6.4.1 da derivada da função digama, dada em Abramowitz e Stegun (1970, p.260), temos que $\iota^{\prime}(\phi)=\int_{0}^{\infty} \frac{t e^{-\phi t}}{1-e^{-t}} \mathrm{~d} t>0$, para $\phi>0$, o que implica que $\psi(\phi)$ é uma função contínua crescente para todo $\phi>0$ e conseqüentemente existe sua função inversa $\psi^{-1}(\cdot)$ (que também é contínua e crescente). Definimos $r(u)=\psi^{-1}(u)$, $\operatorname{com} u=u(\theta, \delta)=\delta-\log \theta$. Com esta nova parametrização, a função densidade tem a forma

$$
\pi^{*}(y ; \theta, \delta)=\exp \left\{-\frac{y}{\theta}+r(u) \log y-r(u) \log \theta-\log \Gamma(r(u))-\log y\right\},
$$


sendo

$$
\begin{aligned}
& \alpha_{1}(\theta, \delta)=-\frac{1}{\theta}, \quad \alpha_{2}(\theta, \delta)=r(u), \\
& \rho(\theta, \delta)=r(u) \log \theta+\log \Gamma(r(u)) . \quad d_{1}(y)=y, \\
& d_{2}(y)=\log y, \quad v(y)=-\log y, \quad \beta_{1}=\theta r(u), \quad \beta_{2}=\delta, \\
& V_{1}=\theta^{2} r(u), \quad V_{2}=\left(r^{\prime}(u)\right)^{-1} \quad \text { e } \quad V_{12}=\theta,
\end{aligned}
$$

onde $r^{\prime}(u)=\mathrm{d} r(u) / \mathrm{d} u=1 / \psi^{\prime}(u)$. Com esta função assim definida, temos

$$
\alpha_{1}^{(1,0)}=\frac{1}{\theta^{2}}, \quad \alpha_{2}^{(1,0)}=-\frac{r^{\prime}(u)}{\theta}, \quad \beta_{1}^{(0,1)}=\theta r^{\prime}(u), \quad \beta_{2}^{(0,1)}=1 .
$$

Conseqüentemente, a ortogonalidade entre $\theta$ e $\delta$ é obtida através destes resultados na expressão (7.5). A estatística escore para testar a hipótese nula $H_{0}: \theta=\theta^{(0)}$ contra a alternativa $H_{1}: \theta \neq \theta^{(0)}$ definida em (7.6) é da forma

$$
S_{R}=\frac{\left\{\sum_{i=1}^{n}\left[\left(y_{l} / \theta^{(0)}\right)-r(\tilde{u})-r^{\prime}(\tilde{u})\left(\log \left(y_{i} / \theta^{(0)}\right)-\tilde{u}\right)\right]\right\}^{2}}{n\left(r(\tilde{u})-r^{\prime}(\tilde{u})\right)} .
$$

De (7.13) obtemos $\xi=r^{\prime \prime}(u)^{2} /\left(\theta^{2} r^{\prime}(u)\right)$ e

$$
\gamma=\frac{\left\{4 r(u) r^{\prime}(u)-3 r^{\prime}(u)^{2}-2 r^{\prime}(u) r^{\prime \prime}(u)+r^{\prime \prime}(u)^{2}\right\}}{\theta^{4} r^{\prime}(u)} .
$$

Os coeficientes $A_{1}, A_{2}$ e $A_{3}$ são obtidos de ( 7.10$)$ - (7.12), resultando em

$$
\begin{aligned}
A_{1}= & -\frac{3}{n}\left\{\frac{2 r^{\prime}(u)\left(r^{\prime \prime}(u)-r^{\prime \prime \prime}(u)\right)+r^{\prime \prime}(u)^{2}}{r^{\prime}(u)^{2}\left(r(u)-r^{\prime}(u)\right)}\right\} \\
A_{2}= & -\frac{3}{n}\left\{2 r^{\prime \prime}(u)\left(r^{\prime \prime}(u)+2 r(u)\right)+r^{\prime}(u)\left(11 r^{\prime}(u)+r^{\prime \prime \prime}(u)\right)\right. \\
& \left.-6 r^{\prime}(u)\left(2 r^{\prime \prime}(u)+r(u)\right)\right\}\left\{r^{\prime}(u)\left(r(u)-r^{\prime}(u)\right)^{2}\right\}^{-1}, \\
A_{3}= & \frac{5}{n} \frac{\left\{2 r(u)-3 r^{\prime}(u)+r^{\prime \prime}(u)\right\}^{2}}{\left\{r(u)-r^{\prime}(u)\right\}^{3}},
\end{aligned}
$$

sendo

e

$$
r^{\prime}(u)=\left(\psi^{\prime}(u)\right)^{-1}, \quad r^{\prime \prime}(u)=-\frac{\psi^{\prime \prime}(u)}{\left(\psi^{\prime}(u)\right)^{2}}
$$

$$
r^{\prime \prime \prime}(u)=-\frac{\left.\psi^{\prime \prime \prime}(u) \psi^{\prime}(u)-2 \psi^{\prime \prime}(u)^{2}\right\}}{\left(\psi^{\prime}(u)\right)^{3}} .
$$




\section{Conclusões}

Ao finalizar este trabalho, esperamos ter dado uma contribuição relevante ao estudo de correções de Bartlett para a estatística da razào de verossimilhança e tipo-Bartlett para a estatística escore. Alguns resultados não relacionados com correções de testes foram também obtidos, embora a ênfase do trabalho tenha sido a obtenção de correções. Resumimos, a seguir, os principais resultados obtidos.

(i) Foram estudados propriedades e problemas de estimação e testes de hipóteses em modelos de regressão linear com uma distribuição simétrica para os erros.

(ii) Foram obtidas correções de Bartlett e tipo-Bartlett nos modelos citados em (i). Aplicações foram feitas a diversos casos especiais. Um estudo de simulação mostrou a eficácia das correções nos casos particulares estudados. Este estudo generaliza o trabalho de Ferrari e Arellano-Valle (1996).

(iii) Ainda considerando os modelos citados em (i), foram obtidos os vícios, até ordem $n^{-1}$, dos estimadores de máxima verossimilhança dos parâmetros de regressão e do parâmetro de escala. Dentre outros resultados, foi demonstrado que os estimadores de máxima verossimilhança dos parâmetros de regressão são não viciados até ordem $n^{-1}$.

(iv) Novamente nos modelos citados em (i), foram obtidos e comparados os poderes dos testes da razão de verossimilhança, escore e Wald, sob alternativas de Pitman, até ordem $n^{-1 / 2}$. Mostrou-se que os poderes dos três testes sobre os parâmetros de regressão coincidem até esta ordem mas os testes sobre o parâmetro de escala têm poderes distintos, sendo possível identificar regiões do espaço paramétrico onde um teste é mais poderoso que os demais.

(v) Foi obtida uma correção tipo-Bartlett para a estatística escore do teste dos parâmetros de regressão em modelos não lineares da família exponencial supondo que o parâmetro de dispersão é desconhecido. Este resultado generaliza os trabalhos de Cordeiro, Ferrari e Paula (1993), Cribari-Neto e Ferrari (1995) e Ferrari e Cordeiro (1996).

(vi) Foi obtida uma correção tipo Bartlett para a estatística escore na família exponencial uniparamétrica e foi estudado um grande número de casos especiais. 
(vii) Foi obtida uma correçào tipo Bartlett para a estatística escore na família exponencial biparamétrica e alguns casos especiais foram estudados.

Embora este trabalho tenha englobado uma gama abrangente de problemas, diversos tópicos para pesquisa futura ficam em aberto. Entre eles, citamos:

(a) Estudo de técnicas de diagnóstico nos modelos citados em (i).

(b) Generalização dos resultados em (i)-(iv) para modelos lineares heterocedásticos.

(c) Generalização dos resultados em (i)-(iv) para modelos não lineares.

(d) Estudos de simulação mais abrangentes para avaliar a eficácia das correções em diversos casos especiais. 


\section{Apêndice A}

Neste apêndice apresentamos a obtenção das quantidades $A_{1}, A_{2}$ e $A_{3}$. referentes ao teste $H_{0}: \beta_{1}=\beta_{1}^{(0)}$ versus $H_{1}: \beta_{1} \neq \beta_{1}^{(0)}$ com parâmetro $\phi$ conhecido nos modelos simétricos definidos no Capítulo 2. Inicialmente, obtemos os cumulantes conjuntos das derivadas do logaritmo da função de verossimilhança utilizados na Seção 3.2.1 e envolvidos nas expressões dos $A$ 's.

\section{A.1 Obtenção de alguns cumulantes conjuntos de de- rivadas do logaritmo da função de verossimilhan- ça em modelos simétricos}

O logaritmo da função de verossimilhança total do parâmetro $\beta$, dado o vetor de observações $\left(y_{1}, \ldots, y_{n}\right)$, do modelo simétrico descrito na Seção 2.5 , tem a forma

$$
L(\beta)=-n \log \phi+\sum t\left(z_{l}\right),
$$

onde $t\left(z_{l}\right)=\log h\left(z_{l}^{2}\right)$, com $z_{l}=\left(y_{l}-\mu_{l}\right) / \phi, \mu_{l}=x_{l}^{\top} \beta$ e $\sum$ é o somatório sobre os dados. Para problemas regulares, por simples diferenciação, temos

$$
\begin{aligned}
\frac{\partial L(\beta)}{\partial \beta_{r}} & =\sum \frac{\partial t\left(z_{l}\right)}{\partial \beta_{r}}=\sum \frac{\mathrm{d} t\left(z_{l}\right)}{\mathrm{d} z_{l}} \frac{\partial z_{l}}{\partial \mu_{l}} \frac{\partial \mu_{l}}{\partial \beta_{r}}=-\frac{1}{\phi} \sum t_{l}^{(1)}(r)_{l}, \\
\frac{\partial^{2} L(\beta)}{\partial \beta_{r} \partial \beta_{s}} & =-\frac{1}{\phi} \sum \frac{\partial t_{l}^{(1)}}{\partial \beta_{s}}(r)_{l}=-\frac{1}{\phi} \sum \frac{\mathrm{d} t_{l}^{(1)}}{\mathrm{d} z_{l}} \frac{\partial z_{l}}{\partial \mu_{l}} \frac{\partial \mu_{l}}{\partial \beta_{s}}(r)_{l} \\
& =\frac{1}{\phi^{2}} \sum t_{l}^{(2)}(r, s)_{l} .
\end{aligned}
$$

De forma análoga, temos

$$
\frac{\partial^{3} L(\beta)}{\partial \beta_{r} \partial \beta_{s} \partial \beta_{t}}=-\frac{1}{\phi^{3}} \sum t_{l}^{(3)}(r, s, t)_{l}
$$

e

$$
\frac{\partial^{4} L(\beta)}{\partial \beta_{r} \partial \beta_{s} \partial \beta_{t} \partial \beta_{u}}=-\frac{1}{\phi^{4}} \sum t_{l}^{(4)}(r, s, t, u)_{l},
$$


onde $t_{l}^{(1)}, t_{l}^{(2)}, t_{l}^{(3)}$ e $t_{l}^{(4)}$ denotam as quatro primeiras derivadas de $t$, isto é, $\partial^{r} t\left(z_{l}\right) / \partial z_{l}^{r}$, $(r)_{l}=x_{l r},(r, s)_{l}=x_{l r} x_{l s}=(r)_{l}(s)_{l},(r . s, t)_{l}=x_{l r} x_{l s} x_{l t}=(x)_{l}(s)_{l}(t)_{l}$. etc., sendo que $x_{l r}$ é o elemento $(l, r)$ da matriz $X^{\prime}$.

Considerando a notação $\delta_{(a, b, c, d, e)}=E\left(t^{(1) a} t^{(2) b} t^{(3) c} t^{(4) d} z^{e}\right)$ e tomando as esperanças encontram-se os cumulantes

$$
\begin{aligned}
& \kappa_{r}=-\frac{1}{\phi} \sum E\left(t_{l}^{(1)}\right)(r)_{l}=-\frac{1}{o} \delta_{(1,0,0,0,0)} \Sigma(r)_{l}=0, \\
& \kappa_{r s}=\frac{1}{\phi^{2}} \sum E\left(t_{l}^{(2)}\right)(r, s)_{l}=\frac{1}{\phi^{2}} \delta_{(0,1,0,0,0)} \Sigma(r, s)_{l}
\end{aligned}
$$

$\mathrm{e}$

$$
\begin{aligned}
\kappa_{r, s} & =\frac{1}{\phi^{2}}\left\{\sum E\left(t_{l}^{(1) 2}\right)(r, s)_{l}+\sum_{l \neq l^{\prime}} E\left[t_{l}^{(1)} t_{l^{\prime}}^{(1)}\right](r)_{l}(s)_{l^{\prime}}\right\} \\
& =\frac{1}{\phi^{2}} \delta_{(2,0,0,0,0)} \Sigma(r, s)_{l},
\end{aligned}
$$

mas $\kappa_{r s}=-\kappa_{r, s}$, então $\delta_{(0,1,0,0,0)}=-\delta_{(2,0,0,0,0)}$. Também

$$
\begin{aligned}
\kappa_{r s t} & =-\frac{1}{\phi^{3}} \sum E\left(t_{l}^{(3)}\right)(r, s, t)_{l}=0 \\
\kappa_{r, s t} & =-\frac{1}{\phi^{3}}\left\{\sum E\left(t_{l}^{(1)} t_{l}^{(2)}\right)(r, s, t)_{l}+\sum_{l \neq l^{\prime}} E\left(t_{l}^{(1)} t_{l^{\prime}}^{(3)}\right)(r)_{l}(s, t)_{l^{\prime}}\right\}=0 \\
\kappa_{r, s, t} & =-\frac{1}{\phi^{3}}\left\{\sum E\left(t_{l}^{(1) 3}\right)(r, s, t)_{l}\right\}=0 \\
\kappa_{r s t}^{(u)} & =0 .
\end{aligned}
$$

Devido à estrutura linear da relação de $\mu$ e $\beta$, temos

$$
\kappa_{r s}^{(u)}=\frac{\partial}{\partial \beta_{u}}\left(\frac{1}{\phi^{2}} \delta_{(2,0,0,0,0)} \sum(r, s)_{l}\right)=0,
$$

e conseqüentemente $\kappa_{r s}^{(t u)}=0$. Os demais cumulantes são obtidos de forma análoga. Assim,

$$
\begin{aligned}
\kappa_{r s t u}= & \frac{1}{\phi^{4}} \sum E\left(t_{l}^{(4)}\right)(r, s, t, u)_{l}=\frac{-1}{\phi^{4}} \delta_{(0,0,0,1,0)} \sum(r, s, t, u)_{l} \\
\kappa_{r, s, t, u}= & E\left\{\frac{\partial L(\beta)}{\partial \beta_{r}} \frac{\partial L(\beta)}{\partial \beta_{s}} \frac{\partial L(\beta)}{\partial \beta_{t}} \frac{\partial L(\beta)}{\partial \beta_{u}}\right\} \\
& -\kappa_{r, s} \kappa_{t, u}-\kappa_{r, t} \kappa_{s, u}-\kappa_{r, u} \kappa_{s, t} \\
= & \frac{1}{\phi^{4}} \sum E\left(t_{l}^{(1) 4}\right)(r, s, t, u)_{l}-\frac{3}{\phi^{4}} \delta_{(2,0,0,0,0)}^{2} \sum(r, s, t, u)_{l} \\
= & \frac{1}{\phi^{4}}\left(\delta_{(4,0,0,0,0)}-3 \delta_{(2,0,0,0,0)}^{2}\right) \sum(r, s, t, u)_{l}
\end{aligned}
$$




$$
\begin{aligned}
\kappa_{r, s, t u} & =E\left\{\frac{\partial L(\beta)}{\partial \beta_{r}} \frac{\partial L(\beta)}{\partial \beta_{s}} \frac{\partial^{2} L(\beta)}{\partial \beta_{t} \partial \beta_{u}}\right\}-\kappa_{r, s} \kappa_{t u} \\
& =\frac{1}{\phi^{4}} \sum E\left(t_{l}^{(1) 2} t_{l}^{(2)}\right)(r, s, t, u)_{l}+\frac{1}{\phi^{4}} \delta_{(2,0,0,0,0)}^{2} \sum(r, s, t, u)_{l} \\
& =\frac{1}{\phi^{4}}\left(\delta_{(2,1,0,0,0)}+\delta_{(2,0,0,0,0)}^{2}\right) \sum(r, s, t, u)_{l}, \\
\kappa_{r s, t u} & =E\left\{\frac{\partial^{2} L(\beta)}{\partial \beta_{r} \beta_{s}} \frac{\partial^{2} L(\beta)}{\partial \beta_{t} \partial \beta_{u}}\right\}-\kappa_{r s} \kappa_{t u} \\
& =\frac{1}{\phi^{4}}\left(\sum E\left(t_{l}^{(2) 2}\right)(r, s, t, u)_{l}+\frac{1}{\phi^{4}} \delta_{(0,1,0,0,0)}^{2} \sum(r, s, t, u)_{l}\right. \\
& =\frac{1}{\phi^{4}}\left(\delta_{(0,2,0,0,0)}+\delta_{(0,1,0,0,0)}^{2}\right) \sum(r, s, t, u)_{l} .
\end{aligned}
$$

Da relação entre cumulantes dada na Seção 1.2.1 temos

$$
\kappa_{r, s, t, u}+3 \kappa_{r, s, t u}=\kappa_{r t, s u}+\kappa_{r u, s t}-2 \kappa_{r s, t u} .
$$

Conseqüentemente do resultado acima concluímos que

$$
\kappa_{r, s, t, u}+3 \kappa_{r, s, t u}=0 . \Rightarrow K_{\phi \phi, \phi, \phi}=-\frac{1}{3} K_{\phi, \phi, \phi, \phi}
$$

\section{A.2 Obtenção de $A_{1}, A_{2}$ e $A_{3}$ para o teste de hipóte- ses $H_{0}: \beta_{1}=\beta_{1}^{(0)}$ versus $H_{1}: \beta_{1} \neq \beta_{1}^{(0)}$ em modelos simétricos com $\phi$ conhecido}

As quantidades $A_{1}, A_{2}$ e $A_{3}$ são agora obtidas substituindo os cumulantes encontrados neste apêndice nas expressões (1.13) - (1.15). Assim,

$$
A_{1}=-6 \Sigma^{\prime}\left(\kappa_{i, j, k, r}+\kappa_{i, j, k r}\right) a_{k r} m_{i j} .
$$

Como $\kappa_{i, j, k, r}+3 \kappa_{i, j, k r}=0$, temos

$$
A_{1}=12 \Sigma^{\prime} \kappa_{i, j, k r} a_{k r} m_{i j}=12 \frac{1}{\phi^{4}}\left(\delta_{(2,1,0,0,0)}+\delta_{(2,0,0,0,0)}^{2}\right) \Sigma^{\prime} \Sigma(i, j, k, r)_{l} a_{k r} m_{i j} .
$$

Invertendo a ordem dos somatórios e rearranjando os termos, temos

$$
A_{1}=\frac{12}{\phi^{4}}\left(\delta_{(2,1,0,0,0)}+\delta_{(2,0,0,0,0)}^{2}\right) \Sigma\left(\Sigma^{\prime}(i, j)_{l} m_{i j}\right)\left(\Sigma^{\prime}(k, r)_{l} a_{k r}\right) .
$$

Das definições das matrizes $A, M, Z$ e $Z_{2}$ dadas em (1.16) e (3.1), respectivamente, $A_{1}$ pode ser escrito como

$$
\begin{aligned}
A_{1} & =\frac{12}{\phi^{4}}\left(\delta_{(2,1,0,0,0)}+\delta_{(2,0,0,0,0)}^{2}\right) \Sigma\left(\frac{\phi^{2}}{\delta_{(2,0,0,0,0)}}\left(z_{l l}-z_{2 l l}\right)\right)\left(\frac{\phi^{2}}{\delta_{(2,0,0,0,0)}} z_{2 l l}\right) \\
& =\frac{12\left(\delta_{(2,1,0,0,0)}+\delta_{(2,0,0,0,0)}^{2}\right)}{\delta_{(2,0,0,0,0)}^{2}} \Sigma\left(z_{l l}-z_{2 l l}\right) z_{2 l l} .
\end{aligned}
$$


Em notaşão matricial, $A_{1}$ é dado por

$$
A_{1}=12\left(\frac{\delta_{(2,1,0,0,0)}}{\delta_{(2,0,0,0,0)}^{2}}+1\right) \operatorname{tr}\left(\left(Z-Z_{2}\right)_{d} Z_{2 d}\right) .
$$

Assim, a expressão resultante é escrita como

$$
A_{1}=\frac{12 b_{0}}{n}\left(\rho_{Z Z_{2}}-\rho_{Z_{2} Z_{2}}\right)
$$

sendo $b_{0}=\left(\delta_{(2,1,0,0,0)} / \delta_{(2,0,0,0,0)}^{2}\right)+1, \rho_{Z Z_{2}}=n \operatorname{tr}\left(Z_{d} Z_{2 d}\right)$ e $\rho_{Z_{2} Z_{2}}=n \operatorname{tr}\left(Z_{2 d} Z_{2 d}\right)$, onde a notação utilizada segue a da Seção 3.3.1.

A obtenção de $A_{2}$ é conduzida de maneira análoga. Temos

$$
\begin{aligned}
A_{2} & =3 \Sigma^{\prime} \kappa_{i, j, k, r} m_{i j} m_{k r} \\
& =-9 \Sigma^{\prime} \kappa_{i, j, k r} m_{i j} m_{k r} \\
& =-\frac{9}{\phi^{4}}\left(\delta_{(2,1,0,0,0)}+\delta_{(2,0,0,0,0)}^{2}\right) \Sigma\left(\Sigma^{\prime}(i, j)_{l} m_{i j}\right)\left(\Sigma^{\prime}(k, r)_{l} m_{k r}\right) \\
& =-\frac{9}{\phi^{4}}\left(\delta_{(2,1,0,0,0)}+\delta_{(2,0,0,0,0)}^{2}\right) \Sigma\left(\frac{\phi^{2}}{\delta_{(2,0,0,0,0)}}\left(z_{l l}-z_{2 l l}\right)\right)^{2} \\
& =-9\left(\frac{\delta_{(2,1,0,0,0)}}{\delta_{(2,0,0,0,0)}^{2}}+1\right) \Sigma\left(z_{l l}-z_{2 l l}\right)^{2}
\end{aligned}
$$

que, em forma matricial, se reduz a

$$
A_{2}=-9 b_{0} \operatorname{tr}\left(\left(Z-Z_{2}\right)_{d}^{2}\right)
$$

Assim, a expressão $A_{2}$ resultante é escrita como

$$
A_{2}=-\frac{9 b_{0}}{n}\left(\rho_{Z Z}-2 \rho_{Z Z_{2}}+\rho_{Z_{2} Z_{2}}\right)
$$

sendo $\rho_{Z Z}=n \operatorname{tr}\left(Z_{d} Z_{d}\right), \rho_{Z Z_{2}}$ e $\rho_{Z_{2} Z_{2}}$ dados acima.

Finalmente, substituindo os cumulantes em $A_{3}$, temos

$$
\begin{aligned}
A_{3}=0 . & \\
z z_{2}=\quad\left(z-z_{2}\right)^{2} & =\left(z-z_{2}\right)\left(z-z_{2}\right) \\
& =z^{2}-z_{2}-z_{2} z+z_{2}^{2} \\
& =z^{2}-2 z z_{2}+z_{2}^{2}
\end{aligned}
$$




\section{Apêndice B}

Neste apêndice obtemos as quantidades que determinam os testes da razão de verossimilhança e escore aperfeiçoados em problemas que envolvem a família simétrica considerando o parâmetro $\phi$ desconhecido (vide Capítulo 3). Inicialmente, obtemos os cumulantes conjuntos de derivadas do logaritmo da função de verossimilhança envolvidos nas expressões que, juntamente com os cumulantes obtidos no Apêndice $A$, determinam os fatores de correção Bartlett e tipo Bartlett.

\section{B.1 Obtenção de alguns cumulantes conjuntos de de- rivadas do logaritmo da função de verossimilhan- ça em modelos simétricos}

Seja $L=L(\theta)$ o logaritmo da função de verossimilhança descrito em (2.4) de um problema regular dependendo dos parâmetros $\beta=\left(\beta_{1}, \ldots, \beta_{p}\right)^{\top}$ e $\phi$, ambos desconhecidos.

Os cumulantes de derivadas do logaritmo da funçào de verossimilhança em relação a $\beta$ foram obtidos no Apêndice A. Diferenciando $L(\theta)$ temos os seguintes resultados:

$$
\begin{aligned}
\frac{\partial L}{\partial \phi} & =-\frac{1}{\phi} \Sigma\left(1+t_{l}^{(1)} z_{l}\right), \\
\frac{\partial^{2} L}{\partial \phi^{2}} & =\frac{1}{\phi^{2}} \Sigma\left(1+2 t_{l}^{(1)} z_{l}+t_{l}^{(2)} z_{l}^{2}\right), \\
\frac{\partial^{2} L}{\partial \beta_{r} \partial \phi} & =\frac{1}{\phi^{2}} \Sigma\left(t_{l}^{(1)}+t_{l}^{(2)} z_{l}\right)(r)_{l}, \\
\frac{\partial^{3} L}{\partial \phi^{3}} & =-\frac{1}{\phi^{3}} \Sigma\left(2+6 t_{l}^{(1)} z_{l}+6 t_{l}^{(2)} z_{l}^{2}+t_{l}^{(3)} z_{l}^{3}\right), \\
\frac{\partial^{3} L}{\partial \beta_{r} \partial 3_{s} \partial \phi} & =-\frac{1}{\phi^{3}} \Sigma\left(t_{l}^{(3)} z_{l}+2 t_{l}^{(2)}\right)(r, s)_{l}, V \\
\frac{\partial^{3} L}{\partial \beta_{r} \partial \phi^{2}} & =-\frac{1}{\phi^{3}} \Sigma\left(2 t_{l}^{(1)}+4 t_{l}^{(2)} z_{l}+t^{(3)} z_{l}^{2}\right)(r)_{l}, \\
\frac{\partial^{4} L}{\partial \phi^{2} \partial \beta_{r} \partial \beta_{s}} & =\frac{1}{\phi^{4}} \Sigma\left(6 t_{l}^{(2)}+t_{l}^{(4)} z_{l}^{2}+6 t_{l}^{(3)} z_{l}\right)(r, s)_{l} .
\end{aligned}
$$


Tomando esperança, encontram-se os cumulantes

$$
\begin{aligned}
& \kappa_{\phi}=E\left(\frac{\partial L}{\partial \phi}\right)=0 \\
& \kappa_{\phi \phi}=E\left(\frac{\partial^{2} L}{\partial \phi^{2}}\right)=\frac{1}{\phi^{2}} \Sigma\left(1+2 E\left(t_{l}^{(1)} z_{l}\right)+E\left(t_{l}^{(2)} z_{l}^{2}\right)\right) \\
& \begin{aligned}
& =\frac{n}{\phi^{2}}\left(\delta_{(0,1,0,0,2)}-1\right)=-\frac{n}{\phi^{2}}\left(\delta_{(2,0,0,0,2)}-1\right)=-\kappa_{\phi, \phi}, \Rightarrow \\
\kappa_{r \phi} & =E\left(\frac{\partial^{2} L}{\partial \beta_{r} \partial \phi}\right)=0, \quad \downarrow 2-\delta_{01002}
\end{aligned} \\
& \kappa_{\phi \phi \phi}=-\frac{n}{\phi^{3}}\left(6 \delta_{(0,1,0,0,2)}+\delta_{(0,0,1,0,3)}-4\right), \\
& \kappa_{\phi \phi r}=0 \text {, } \\
& \gg \underbrace{}_{\text {rs } \phi}=-\frac{1}{\phi^{3}}\left(\delta_{(0,0,1,0,1)}+2 \delta_{(0,1,0,0,0)}\right) \Sigma(r, s)_{l}, \\
& \kappa_{\phi \phi r s}=\frac{1}{\phi^{4}}\left(6 \delta_{(0,1,0,0,0)}+6 \delta_{(0,0,1,0,1)}+\delta_{(0,0,0,1,2)}\right) \Sigma(r, s)_{l}, \\
& \kappa_{\phi \phi}^{(\phi)}=\frac{\partial \kappa_{\phi \phi}}{\partial \phi}=-\frac{2 n}{\phi^{3}}\left(\delta_{(0,1,0,0,2)}-1\right) \text {. pu } \frac{2 n}{\phi^{3}}\left(\delta_{2000,-1}\right)
\end{aligned}
$$

Como $\kappa_{\phi \phi}^{(\phi)}=\kappa_{\phi \phi \phi}+\kappa_{\phi \phi, \phi}$, temos $=-\frac{2 \eta}{\phi^{5}}\left(\delta_{01002}-1\right)+\frac{\eta}{\phi^{3}}\left(6 \delta 01002+\delta_{00103}-4\right)=-\frac{\eta}{\phi^{3}}\left[+2 \delta_{01002}-2-6 \delta_{01002}-\right.$

$$
\kappa_{\phi \phi, \phi}=+\frac{n}{\phi^{3}}\left(\delta_{(0,0,1,0,3)}+4 \delta_{(0,1,0,0,2)}-2\right) .
$$

De $\kappa_{\phi, \phi, \phi}=2 \kappa_{\phi \phi \phi}-3 \kappa_{\phi \phi}^{(\phi)}$, temos

$$
\kappa_{\phi, \phi, \phi}=\frac{2 n}{\phi^{3}}\left(1+\frac{\left.\delta_{(1,1,0,0,3)}\right)}{-3 \delta_{01002}-\delta_{00103}}\right.
$$

ou, equivalentemente,

$$
\kappa_{\phi, \phi, \phi}=\frac{2 n}{\phi^{3}}\left(1-3 \delta_{(0,1,0,0,2)}-\delta_{(0,0,1,0,3)}\right) .
$$

Temos também

$$
\begin{aligned}
\kappa_{r s, \phi} & =E\left(\frac{\partial^{2} L}{\partial \beta_{r} \partial \beta_{s}} \cdot \frac{\partial L}{\partial \phi}\right) \\
& =-\frac{1}{\phi^{3}} E\left[\sum_{l}\left(t_{l}^{(2)}+t_{l}^{(1)} t_{l}^{(2)} z_{l}\right)(r, s)_{l}+\sum_{l \neq m}\left(t_{l}^{(3)}\left(1+t_{m}^{(1)} z_{m}\right)(r, s)_{l}\right]\right.
\end{aligned}
$$

e, assim,

$$
\kappa_{r s, \phi}=-\frac{1}{\phi^{3}}\left(\delta_{(1,1,0,0,1)}+\delta_{(0,1,0,0,0)}\right) \Sigma(r, s)_{l} .
$$


Como $\kappa_{r \phi}^{(\phi)}=0$ e $\kappa_{r \phi}^{(t)}=0$, temos, segundo as relaçòes de Bartlett, $\kappa_{r \phi}^{(\phi)}=\kappa_{r \phi \phi}+\kappa_{r \phi, \phi} \mathrm{e}$ $\kappa_{r \phi}^{(t)}=\kappa_{r t \phi}+\kappa_{r \phi, t}$. que implica em $\kappa_{\phi r, o}=0$ e $\kappa_{r \phi, t}=-\kappa_{r t o}$. Agora,

$$
\kappa_{\phi, \phi, \phi, \phi}=E\left[\left(\frac{\partial L}{\partial \phi}\right)^{4}\right]-3 \kappa_{\phi, \odot}^{2}
$$

e como

$$
\begin{aligned}
E\left[\left(\frac{\partial L}{\partial \phi}\right)^{4}\right] ? & \frac{1}{\phi^{4}} E\left[\sum_{l}\left(1+t_{l}^{(1) 4} z_{l}^{4}+4 t_{l}^{(1) 3} z_{l}^{3}+6 t_{l}^{(1) 2} z_{l}^{2}+4 t_{l}^{(1)} z_{l}\right)\right. \\
& +\sum_{l \neq m}\left(1+t_{l}^{(1)} z_{l}\right)^{3}\left(1+t_{m}^{(1)} z_{m}\right)+3 \sum_{l \neq m}\left(1+t_{l}^{(1)} z_{l}\right)^{2}\left(1+t_{m}^{(l)} z_{m}\right)^{2} \\
& +6 \sum_{l \neq m \neq v}\left(1+t_{l}^{(1)} z_{l}\right)^{2}\left(1+t_{v}^{(1)} z_{v}\right)\left(1+t_{m}^{(1)} \tilde{z}_{m}\right) \\
& \left.+\sum_{l \neq m \neq v \neq s}\left(1+t_{l}^{(1)} z_{l}\right)\left(1+t_{m}^{(1)} \tilde{z}_{m}\right)\left(1+t_{v}^{(1)} \tilde{z}_{v}\right)\left(1+t_{s}^{(1)} z_{s}\right)\right] \\
= & \frac{n}{\phi^{4}}\left(\delta_{(4,0,0,0,4)}+4 \delta_{(3,0,0,0,3)}+6 \delta_{(2,0,0,0,2)}-3\right)+\frac{3 n(n-1)}{\phi^{4}}\left(\delta_{(2,0,0,0,0)}-1\right)^{2}
\end{aligned}
$$

$\mathrm{e}$

$$
\kappa_{\phi, \phi}^{2}=\frac{n^{2}}{\phi^{4}}\left(\delta_{(2,0,0,0,2)}-1\right)^{2}
$$

temos

$$
\kappa_{\phi, \phi, \phi, \phi}=\frac{n}{\phi^{4}}\left(\delta_{(4,0,0,0,4)}+4 \delta_{(3,0,0,0,3)}+12 \delta_{(2,0,0,0,2)}-3 \delta_{(2,0,0,0,2)}^{2}-6\right) .
$$

Temos também

$$
\begin{aligned}
\kappa_{r, s \phi \phi}= & E\left(\frac{\partial L}{\partial \beta_{r}} \frac{\partial^{3} L}{\partial \beta_{s} \partial \phi^{2}}\right) \\
= & \frac{1}{\phi^{4}} E\left\{\sum_{l}\left(t_{l}^{(1)} t^{(3)} z_{l}^{2}+4 t_{l}^{(1)} t_{l}^{(2)} z_{l}+2 t_{l}^{(1) 2}\right)(r, s)_{l}\right. \\
& \left.+\sum_{l \neq m}\left(t_{l}^{(1)}\right)\left(t_{m}^{(3)} z_{m}^{2}+4 t_{m}^{(2)} \tilde{\sim}_{m}+2 t_{m}^{(1)}\right)(r)_{l}(s)_{m}\right\} \\
= & \frac{1}{\phi^{4}}\left(\delta_{(1,0,1,0,2)}+4 \delta_{(1,1,0,0,1)}+2 \delta_{(2,0.0 .0,0)}\right) \sum(r, s)_{l} .
\end{aligned}
$$

Como

$$
\kappa_{r s, \phi, \phi} \stackrel{?}{=} E\left(\frac{\partial^{2} L}{\partial \beta_{r} \partial \beta_{s}} \frac{\partial L}{\partial \phi} \frac{\partial L}{\partial \phi}\right)-\kappa_{r s} \kappa_{\phi \phi}
$$

e

$$
E\left(\frac{\partial^{2} L}{\partial \beta_{r} \partial \beta_{s}} \frac{\partial L}{\partial \phi} \frac{\partial L}{\partial \phi}\right)=\frac{1}{\phi^{4}} E\left\{\sum_{l}\left(1+t^{(1) 2} z_{l}^{2}+2 t_{l}^{(1)} z_{l}\right) t_{l}^{(2)}(r, s)_{l}\right.
$$




$$
\begin{aligned}
& +\sum_{l \neq v}\left(1+t_{l}^{(1)} z_{l}\right)^{2} t_{v}^{(2)}(r . s)_{v}+2 \sum_{l \neq m}\left(1+t_{l}^{(1)}\right) t_{l}^{(2)}\left(1+t_{m}^{(1)} z_{m}\right) \\
& \left.+\sum_{l \neq m \neq v}\left(1+t_{l}^{(1)} z_{l}\right)\left(1+t_{m}^{(1)} z_{m}\right) t_{v}^{(2)}(r . s)_{v}\right\} \\
& =\frac{1}{\phi^{4}}\left(\delta_{(1,0,0,0,2)}+2 \delta_{(1,1,0,0,1)}+\delta_{(0,1,0,0.0)}\right) \sum(r, s)_{l} \\
& +\frac{(n-1)}{\phi^{4}} \delta_{(0,1,0,0,0)}\left(\delta_{(2,0,0,0,2)}-1\right) \sum(r, s)_{l},
\end{aligned}
$$

temos

$$
\kappa_{r s, \phi, \phi}=\frac{1}{\phi^{4}}\left(2 \delta_{(1,1,0,0,1)}+\delta_{(0,1,0,0,0)} \delta_{(0,1,0,0,2)}+\stackrel{\delta_{10002} ?}{\left.\delta_{(2,1,0,0,2)}\right) \sum(r, s)_{l} .}\right.
$$

Das relações dos cumulantes apresentadas na Seção 1.2.1, obtemos as seguintes relações dos $\delta$ 's que facilitam seus cálculos:

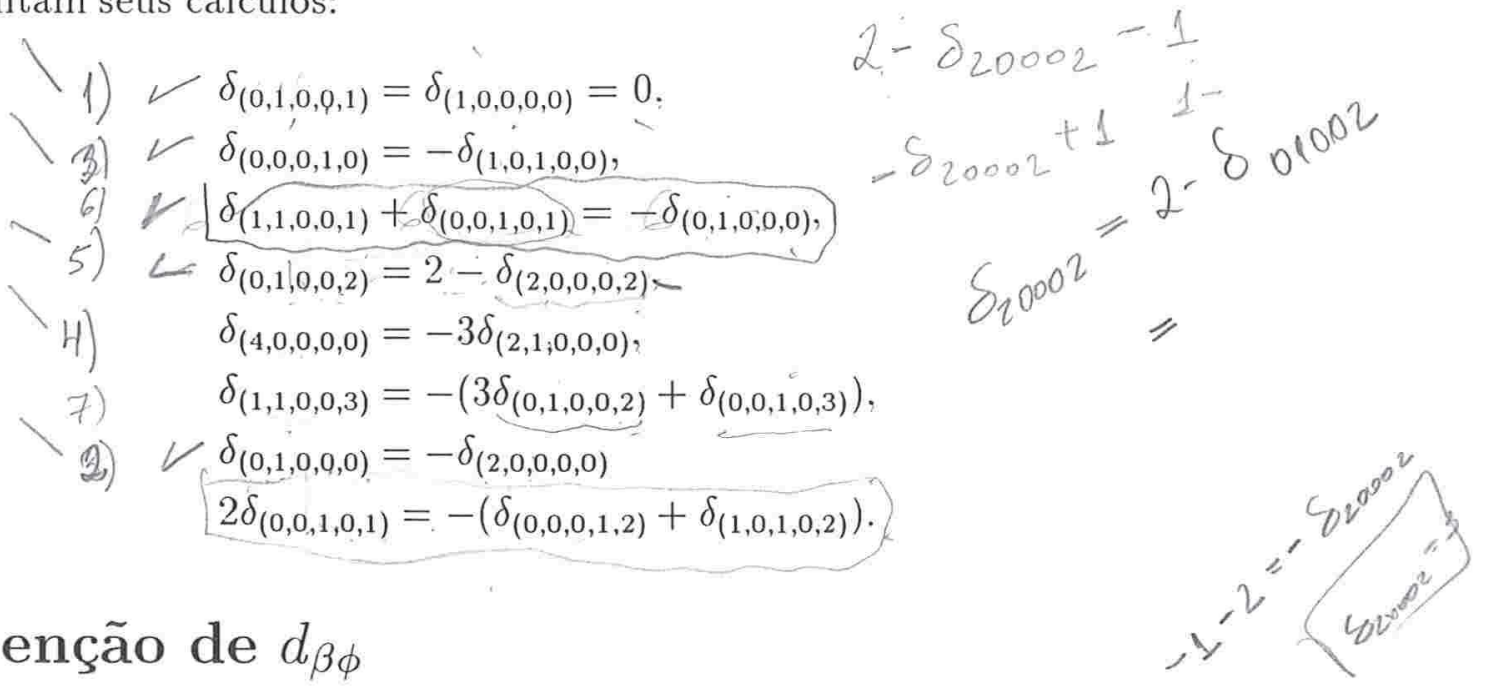

\section{B.2 Obtenção de $d_{\beta \phi}$}

A quantidade $d_{\beta \phi}$ definida na Seção 3.2 .2 para o teste $H_{0}: \beta_{1}=\beta_{1}^{(0)}$ versus $H_{1}: \beta_{1} \neq \beta_{1}^{(0)}$ com parâmetro de escala $\phi$ desconhecido tem a forma

$$
d_{\beta \phi}=q^{-1}\left(\sum_{\beta, \phi}^{\prime}\left(\ell_{r s t u}-\ell_{r s t u v w}\right)-\sum_{\beta, \phi}^{\prime \prime}\left(\ell_{r s t u}-\ell_{r s t u v w}\right)\right)
$$

onde $\sum_{\beta, \phi}^{\prime}$ representa o somatório sobre todas as combinações dos parâmetros $\beta$ e $\phi$, mas com pelo menos um índice igual a $\phi$ e $\sum_{\beta, \phi}^{\prime \prime}$ representa o somatório sobre todas as combinações de parâmetros $\left\{\beta_{q+1}, \ldots, \beta_{p} ; \phi\right\}$ com pelo menos um índice igual a $\phi . \ell_{r s t u} \mathrm{e}$

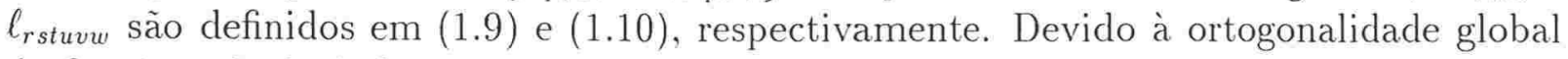
de $\beta$ e $\phi$ e substituindo os cumulantes deste apêndice na expressão (B.1), temos

$$
\begin{aligned}
\sum_{\beta, \phi}{ }^{\prime} \ell_{r s t u}= & \Sigma^{\prime}\left\{\kappa^{\phi \phi}\left[\frac{1}{4} \kappa^{t u} \kappa_{\phi \phi t u}+\kappa^{r s}\left(\frac{1}{4} \kappa_{r s \phi \phi}-\kappa_{r s \phi}^{(\phi)}\right)\right]\right\} \\
& +\kappa^{\phi \phi^{2}}\left(\frac{1}{4} \kappa_{\phi \phi \phi \phi}-\kappa_{\phi \phi O}^{(\phi)}+\kappa_{\phi \phi}^{(\phi \phi)}\right)
\end{aligned}
$$




$$
\begin{aligned}
\sum_{\beta, \phi}^{\prime} \ell_{r s t u v u^{\prime}}= & \Sigma^{\prime}\left\{\frac{1}{4} \kappa^{\phi \phi^{2}} \kappa^{v w} \kappa_{\phi \phi \phi} \kappa_{\phi v w}\right. \\
& +\kappa^{\phi \phi^{2}} \kappa^{t u}\left(\frac{1}{4} \kappa_{\phi t u} \kappa_{\phi \phi \phi}-\kappa_{\phi t u} \kappa_{\phi \phi}^{(\phi)}\right) \\
& +\kappa^{\phi \phi} \kappa^{t u} \kappa^{v w}\left(\frac{1}{6} \kappa_{\phi t v} \kappa_{\phi u w}+\frac{1}{4} \kappa_{\phi t u} \kappa_{\phi s w}\right) \\
& +\kappa^{\phi \phi} \kappa^{r s} \kappa^{v w}\left(\frac{1}{6} \kappa_{r \phi v} \kappa_{s \phi w}-\kappa_{r \phi v} \kappa_{s w}^{(\phi)}\right) \\
& \left.+\frac{1}{6} \kappa^{\phi \phi} \kappa^{r s} \kappa^{t u} \kappa_{r t \phi} \kappa_{s u \phi}\right\} \\
& +\kappa^{\phi \phi^{3}}\left\{\frac{1}{6} \kappa_{\phi \phi \phi}^{2}+\frac{1}{4} \kappa_{\phi \phi \phi}^{2}-2 \kappa_{\phi \phi \phi} \kappa_{\phi \phi}^{(\phi)}+2 \kappa_{\phi \phi}^{(\phi)^{2}}\right\}
\end{aligned}
$$

onde $\Sigma^{\prime}$ é o somatório sobre todos os componentes de $\beta$. De forma similar, $\Sigma_{\beta, \phi}^{\prime \prime}\left(\ell_{r s t u}-\right.$ $\ell_{\text {rstuvw }}$ ) vem de (B.2) e (B.3), mas com $\Sigma^{\prime \prime}$ representando o somatório sobre $\left\{\beta_{q+1}, \ldots, \beta_{p}\right\}$. Consideramos inicialmente a obtenção de $\Sigma^{\prime} \ell_{r s t u}$. Assim, substituindo os cumulantes e rearranjando os somatórios, temos

$$
\begin{aligned}
\Sigma^{\prime} \ell_{r s t u}= & \ell_{\phi \phi \phi \phi}+\frac{\phi^{2}}{n\left(\delta_{(0,1,0,0,2)}-1\right)} \sum\left\{\frac{1}{4 \phi^{4}}\left(\delta_{(0,0,0,1,2)}-6 \delta_{(1,1,0,0,1)}\right)\right. \\
& \Sigma^{\prime} \kappa^{t u}(t, u)_{l}+\frac{1}{4 \phi^{2}}\left(\delta_{(0,0,0,1,2)}-6 \delta_{(1,1,0,0,1)}\right) \Sigma^{\prime} \kappa^{r s}(r, s)_{l} \\
& \left.-\frac{3}{\phi^{4}}\left(\delta_{(0,0,1,0,1)}+2 \delta_{(0,1,0,0,0)}\right) \Sigma^{\prime} \kappa^{r s}(r, s)_{l}\right\} .
\end{aligned}
$$

Como $-\Sigma^{\prime} \kappa^{r s}(r, s)_{l}$ é o elemento $(l, l)$ da matriz $Z \phi^{2} / \delta_{(2,0,0,0,0)}$, temos

$$
\begin{aligned}
\Sigma^{\prime} \ell_{\text {rstu }}= & \ell_{\phi \phi \phi \phi}+\frac{\left(\delta_{(0,0,0,1,2)}-6 \delta_{(1,1,0,0,1)}\right)}{2 n \phi^{2}\left(\delta_{(0,1,0,0,2)}-1\right)} \\
& \Sigma\left(-\frac{\phi^{2}}{\delta_{(2,0,0,0,0)}} z_{l l}\right)-\frac{3\left(\delta_{(0,0,1,0,1)}+2 \delta_{(0,1,0,0,0)}\right)}{n \phi^{2}\left(\delta_{(0,1,0,0,2)}-1\right)} \Sigma\left(-\frac{\phi^{2}}{\delta_{(2,0,0,0,0)}} z_{l l}\right) .
\end{aligned}
$$

Escrevendo em notação matricial, vem

$$
\Sigma^{\prime} \ell_{r s t u}=\ell_{\phi \phi \phi \phi}-\frac{m_{4}}{2 n m_{1}} \operatorname{tr}(Z)+3 \frac{m_{3}}{n m_{1}} \operatorname{tr}(Z)
$$

onde

$$
\begin{aligned}
m_{1} & =\delta_{(0,1,0,0,2)}-1 \\
m_{2} & =4-\delta_{(0,0,1,0,3)}-6 \delta_{(0,1,0,0,2)} \\
m_{3} & =\left(\delta_{(0,0,1,0,1)}+2 \delta_{(0,1,0,0,0)}\right) / \delta_{(2,0,0,0,0)} \\
\mathrm{e} \quad m_{4} & =\left(\delta_{(0,0,0,1,2)}-6 \delta_{(1,1,0,0,1)}\right) / \delta_{(2,0,0,0,0)}
\end{aligned}
$$


Comotr $(Z)=\operatorname{tr}\left(X^{X}\left(X^{\top} X\right)^{-1} X^{\top}\right)=\operatorname{tr}\left(I_{p}\right)=p$, sendo $p$ o posto da matriz $X$ e $I_{p}$ a matriz identidade $p \times p$. Assim, temos

$$
\Sigma^{\prime} \ell_{r s t u}=\ell_{\phi \phi \phi \phi}+\left(\frac{6 m_{3}-m_{4}}{2 n m_{1}}\right) p
$$

De forma análoga,

$$
\begin{aligned}
\Sigma^{\prime \prime} \ell_{r s t u} & =\ell_{\phi \phi \phi \phi}+\left(\frac{6 m_{3}-m_{4}}{2 n m_{1}}\right) \operatorname{tr}\left(Z_{d}-Z_{2 d}\right) \\
& =\ell_{\phi \phi \phi}+\left(\frac{6 m_{3}-m_{4}}{2 n m_{1}}\right)(p-q)
\end{aligned}
$$

Para obter $\Sigma^{\prime} \ell_{\text {rstuvw }}$ temos, de (B.3), que

$$
\begin{aligned}
& \Sigma_{\beta, \phi}^{\prime} \ell_{r s t u v w}=\ell_{\phi \phi \phi \phi \phi \phi} \\
& +\frac{1}{4} \kappa_{\phi \phi \phi} \kappa^{\phi \phi^{2}}\left(-\frac{1}{\phi^{3}}\right)\left(\delta_{(0,0,1,0,1)}+2 \delta_{(0,1,0,0,0)}\right) \Sigma\left(\Sigma^{\prime} \kappa^{v w}(v, w)_{l}\right) \\
& +\frac{1}{4} \kappa_{\phi \phi \phi} \kappa^{\phi \phi^{2}}\left(-\frac{1}{\phi^{3}}\right)\left(\delta_{(0,0,1,0,1)}+2 \delta_{(0,1,0,0,0)}\right) \Sigma\left(\Sigma^{\prime} \kappa^{t u}(t, u)_{l}\right) \\
& -\kappa^{\phi \phi^{2}} \kappa_{\phi \phi}^{(\phi)}\left(-\frac{1}{\phi^{3}}\right)\left(\delta_{(0,0,1,0,1)}+2 \delta_{(0,1,0,0,0)}\right) \Sigma\left(\Sigma^{\prime} \kappa^{t u}(t, u)_{l}\right) \\
& +\frac{1}{6} \kappa^{\phi \phi}\left(-\frac{1}{\phi^{3}}\right)^{2} k_{l}\left(\delta_{(0,0,1,0,1)}+2 \delta_{(0,1,0,0,0)}\right)^{2} \Sigma\left(\Sigma^{\prime} \kappa^{t u}(t)_{l}(u)_{m}\right)\left(\Sigma^{\prime} \kappa^{v w}(v)_{l}(w)_{m}\right) \\
& +\frac{1}{4} \kappa^{\phi \phi}\left(-\frac{1}{\phi^{3}}\right)^{2}\left(\delta_{(0,0,1,0,1)}+2 \delta_{(0,1,0,0,0)}\right)^{2} \Sigma\left(\Sigma^{\prime} \kappa^{t u}(t, u)_{l}\right)\left(\Sigma^{\prime} \kappa^{v w}(v, w)_{m}\right) \\
& +\frac{1}{6} \kappa^{\phi \phi}\left(-\frac{1}{\phi^{3}}\right)^{2}\left(\delta_{(0,0,1,0,1)}+2 \delta_{(0,1,0,0,0)}\right)^{2} \Sigma\left(\Sigma^{\prime} \kappa^{r s}(r)_{l}(s)_{m}\right)\left(\Sigma^{\prime} \kappa^{v w}(v)_{l}(w)_{m}\right) \\
& -\kappa^{\phi \phi}\left(-\frac{1}{\phi^{3}}\right)\left(\delta_{(0,0,1,0,1)}+2 \delta_{(0,1,0,0,0)}\right)\left(\frac{2}{\phi^{3}}\right) \delta_{(2,0,0,0,0)} \Sigma\left(\Sigma^{\prime} \kappa^{r s}(r)_{l}(s)_{m}\right)\left(\Sigma^{\prime} \kappa^{v w}(v)_{l}(w)_{m}\right) \\
& +\frac{1}{6} \kappa^{\phi \phi}\left(-\frac{1}{\phi^{3}}\right)^{2}\left(\delta_{(0,0,1,0,1)}+2 \delta_{(0,1,0,0,0)}\right) \Sigma\left(\Sigma^{\prime} \kappa^{r s}(r)_{l}(s)_{m}\right)\left(\Sigma^{\prime} \kappa^{t u}(t)_{l}(u)_{m}\right)
\end{aligned}
$$

Como

$$
\begin{aligned}
& \delta_{01002}=2-\delta_{20002} \\
& \kappa^{\phi \phi}=\kappa_{\phi \phi}^{-1}=\frac{\phi^{2}}{n}\left(\delta_{(0,1,0,0,2)}-1\right)^{-1},=\frac{\phi^{2}}{m}\left(2-\delta_{20002}-1\right)^{-1}= \\
& \kappa_{\phi \phi}^{(\phi)}=-\frac{2}{\phi} \kappa_{\phi \phi}, \\
& \kappa_{\phi \phi \phi}=-\frac{n}{\phi^{3}}\left(6 \delta_{(0,1,0,0,2)}+\delta_{(0,0,1,0,3)}-4\right) \frac{\phi^{2}}{m}\left(1-\delta_{20002}\right) \\
& \frac{-\phi^{2}}{m}\left(\delta_{20002}-1\right)
\end{aligned}
$$


e $-\Sigma r^{r s}(r, s)_{l}$ é o elmento $(l, l)$ da matriz $Z \phi^{2} / \delta_{(2,0,0,0,0)}$, temos

$$
\begin{aligned}
\Sigma_{\beta, \phi}^{\prime} \ell_{\text {rstuvw }}= & \ell_{\phi \phi \phi \phi \phi} \\
& -\frac{1}{2} \frac{\left(6 \delta_{(0,1,0,0,2)}+\delta_{(0,0,1,0,3)}-4\right)}{n\left(\delta_{(0,1,0,0,2)}-1\right)^{2}}\left(\frac{\delta_{(0,0,1,0,1)}+2 \delta_{(0,1,0,0,0)}}{\delta_{(2,0,0,0,0)}}\right) \Sigma z_{l l}, \\
& +\frac{2}{n}\left(\frac{1}{\delta_{(0,1,0,0,2)}-1}\right)\left(\frac{\delta_{(0,0,1,0,1)}+2 \delta_{(0,1,0,0,0)}}{\delta_{(2,0,0,0,0)}}\right) \Sigma z_{l l}, \\
& +\frac{1}{2 n}\left(\frac{1}{\delta_{(0,1,0,0,2)}-1}\right)\left(\frac{\delta_{(0,0,1,0,1)}+2 \delta_{(0,1,0,0,0)}}{\delta_{(2,0,0,0,0)}}\right)^{2} \Sigma_{z_{l m} z_{m l}} \\
& +\frac{1}{4 n}\left(\frac{1}{\delta_{(0,1,0,0,2)}-1}\right)\left(\frac{\delta_{(0,0,1,0,1)}+2 \delta_{(0,1,0,0,0)}}{\delta_{(2,0,0,0,0)}}\right)^{2} \Sigma z_{l l}^{2} \\
& +\frac{2}{n}\left(\frac{1}{\delta_{(0,1,0,0,2)}-1}\right)\left(\frac{\delta_{(0,0,1,0,1)}+2 \delta_{(0,1,0,0,0)}}{\delta_{(2,0,0,0,0)}}\right) \Sigma z_{l m} z_{m l} .
\end{aligned}
$$

Escrevendo em forma matricial vem

$$
\begin{aligned}
\Sigma_{\beta, \phi}^{\prime} \ell_{\text {rstuvw }}= & \ell_{\phi \phi \phi \phi \phi}+\frac{1}{2 n} \frac{m_{2} m_{3}}{m_{1}^{2}} \operatorname{tr}\left(Z_{d}\right)+\frac{2}{n} \frac{m_{3}}{m_{1}} \operatorname{tr}\left(Z_{d}\right)+\frac{1}{2 n} \frac{m_{3}^{2}}{m_{1}} \operatorname{tr}(Z Z) \\
& +\frac{1}{4 n} \frac{m_{3}^{2}}{m_{1}} \operatorname{tr}(Z)^{2}+\frac{2}{n} \frac{m_{3}}{m_{1}} \operatorname{tr}(Z Z) .
\end{aligned}
$$

Mas

$$
\begin{aligned}
\operatorname{tr}(Z) & =\operatorname{tr}\left(X\left(X^{\top} \not\right)^{-1} X^{\top}\right)=\operatorname{tr}\left(I_{p}\right)=p \\
\mathrm{e} \operatorname{tr}(Z Z) & =\operatorname{tr}\left(X\left(X^{\top} X^{\top}\right)^{-1} X^{\top} X\left(X^{\top} X^{\top}\right)^{-1} X^{\top}\right)=\operatorname{tr}\left(I_{p}\right)=p
\end{aligned}
$$

sendo $p$ o posto da matriz $X$. Assim, temos

$$
\Sigma_{\beta, \phi}^{\prime} \ell_{r s t u v w}=\ell_{\phi \phi \phi \phi \phi}+\frac{1}{2 n} \frac{m_{2} m_{3}}{m_{1}^{2}} p+\frac{4}{n} \frac{m_{3}}{m_{1}} p+\frac{1}{2 n} \frac{m_{3}^{2}}{m_{1}} p+\frac{1}{2} \frac{m_{3}^{2}}{m_{1}} \frac{p^{2}}{2} .
$$

De forma análoga,

$$
\begin{aligned}
\Sigma_{\beta, \phi}^{\prime \prime} \ell_{r s t u v w}= & \ell_{\phi \phi \phi \phi \phi \phi}+\frac{1}{2 n} \frac{m_{2} m_{3}}{m_{1}^{2}}(p-q) \\
& +\frac{4}{n} \frac{m_{3}}{m_{1}}(p-q)+\frac{1}{2 n} \frac{m_{3}^{2}}{m_{1}}(p-q)+\frac{1}{2 n} \frac{m_{3}^{2}}{m_{1}} \frac{(p-q)^{2}}{2} .
\end{aligned}
$$

Assim, substituindo em (B.1), temos

$$
d_{\beta \phi}=\frac{1}{n}\left\{d_{1}+\frac{(2 p-q)}{2} d_{2}\right\}
$$

onde

$$
\begin{aligned}
d_{1} & =-\frac{m_{3}}{2 m_{1}^{2}}\left(m_{2}+m_{1}\left(2+m_{3}\right)\right)-\frac{m_{4}}{2 m_{1}} \\
\text { e } \quad d_{2} & =-\frac{m_{3}^{2}}{2 m_{1}}
\end{aligned}
$$




\section{B.3 Obtenção de $A_{1, \beta \phi}$ e $A_{2, \beta \phi}$}

As quantidades $A_{1, \beta \phi}$ e $A_{2, \beta}\left(A_{3, \beta}=0\right)$ para o teste de $H_{0}: 3_{1}=\beta_{1}^{(0)}$ versus $H_{1}: \beta_{1} \neq 3_{1}^{(0)}$ com parâmetro de escala $\phi$ desconhecido para modelos simétricos univariados são agora obtidas substituindo os cumulantes obtidos neste apêndice nas expressões (3.21) e (3.22). Consideremos, inicialmente, a obtenção de $A_{1, \beta \phi}$. De (3.21) vem

$$
\begin{aligned}
A_{1, \beta \phi}= & -6 \Sigma^{\prime}\left\{-\frac{1}{\phi^{3}}\left(\delta_{(1,1,0,0,1)}-\delta_{(0,1,0,0,0)}\right)\left(\Sigma(i, j)_{l} a_{i j}\right)\right. \\
& \left.\left(\frac{2}{\phi^{3}}\right) \delta_{(1,1,0,0,1)} \Sigma(s, t)_{k} m_{s t}\right\} a_{\phi \phi} \\
& -6 \Sigma^{\prime}\left\{\frac{n}{\phi^{3}}\left(2 \delta_{(0,1,0,0,2)}+\delta_{(0,0,1,0,3)}\right) \frac{2}{\phi_{3}} \delta_{(1,1,0,0,1)} \Sigma(s, t)_{l} m_{s i t}\right\} a_{\phi \phi}^{2} \\
& +6 \Sigma^{\prime}\left\{-\frac{1}{\phi^{3}}\left(3 \delta_{(1,1,0,0,1)}-\delta_{(0,1,0,0,0)}\right) \Sigma(i, k)_{l}\right\} \\
& \left\{-\frac{1}{\phi^{3}}\left(\delta_{(1,1,0,0,1)}-\delta_{(0,1,0,0,0)}\right) \Sigma(t, r)_{m}\right\} a_{\phi \phi} a_{k t} m_{l r}- \\
& +6 \Sigma^{\prime}\left\{-\frac{1}{\phi^{3}}\left(3 \delta_{(1,1,0,0,1)}-\delta_{(0,1,0,0,0)}\right) \Sigma(i, j)_{l}\right\} \\
& \left\{-\frac{1}{\phi^{3}}\left(2 \delta_{(0,1,0,0,0)}-\delta_{(0,0,1,0,1)}\right) \Sigma(r, s)_{m}\right\} a_{\phi \phi} a_{\underbrace{\prime}_{k t}} a_{\underbrace{}_{j s} m_{l r}} \\
& +6 \Sigma^{\prime}\left\{\frac { 1 } { \phi ^ { 4 } } \left(4 \delta_{(3,0,0,0,1)}+\delta_{(4,0,0,0,2)}+\delta_{(2,1,0,0,2)}\right.\right. \\
& \left.-2 \delta_{(0,1,0,0,0)} \Sigma(i, j)_{l} m_{i j}\right\} a_{\phi \phi},
\end{aligned}
$$

onde $\Sigma^{\prime}$ indica o somatório sobre todos os $\beta$ 's e $a_{i j}, m_{i j}$ são os elementos $(i, j)$ das matrizes $A$ e $M$, respectivamente, com estas matrizes dadas na Seção 3.3.2.

Invertendo a ordem dos somatórios $\Sigma^{\prime}$ e $\Sigma$ e rearranjando os termos, tem-se

$$
\begin{aligned}
A_{1, \beta \phi}= & \frac{12 \phi^{-4}}{n\left(\delta_{(2,0,0,0,2)}-1\right)}\left\{\left(\delta_{(1,1,0,0,1)}-\delta_{(0,1,0,0,0)}\right) \delta_{(1,1,0,0,1)}\right\} \\
& \Sigma\left(\Sigma^{\prime}(i, j)_{l} a_{i j}\right) \Sigma\left(\Sigma^{\prime}(s, t)_{m} m_{s t}\right) \\
& -\frac{12 \phi^{-2}}{n\left(\delta_{(2,0,0,0,2)}-1\right)^{2}}\left\{\delta_{(1,1,0,0,1)}\left(2 \delta_{(0,1,0,0,2)}+\delta_{(0,0,1,0,3)}\right)\right\} \Sigma\left(\Sigma^{\prime}(s, t)_{l} m_{s t}\right) \\
& -\frac{6 \phi^{-4}}{n\left(\delta_{(2,0,0,0,2)}-1\right)}\left\{\left(3 \delta_{(1,1,0,0,1)}-\delta_{(0,1,0,0,0)}\right)\left(\delta_{(1,1,0,0,1)}-\delta_{(0,1,0,0,0)}\right)\right\} \\
& \Sigma\left(\Sigma^{\prime}(i)_{l}(r)_{m} m_{i r}\right)\left(\Sigma^{\prime}(k)_{l}(t)_{m} a_{k t}\right)
\end{aligned}
$$




$$
\begin{aligned}
& +\frac{6 \phi^{-4}}{n\left(\delta_{(2,0,0.0,2)}-1\right)}\left\{\left(3 \delta_{(1.1,0,0,1)}-\delta_{(0,1,0,0,0)}\right)\left(\delta_{(0,0,1,0.1)}-2 \delta_{(0.1,0,0,0)}\right)\right\} \\
& \Sigma\left(\Sigma^{\prime}(i)_{l}(r)_{m} m_{i r}\right)\left(\Sigma^{\prime}(j)_{l}(s)_{m} a_{j s}\right) \\
& -\frac{6 \phi^{-4}}{n\left(\delta_{(2,0,0,0,2)}-1\right)}\left\{4 \delta_{(3,0,0,0,1)}+\delta_{(4,0,0,0,2)}+\delta_{(2,1,0,0,2)}-2 \delta_{(0,1,0,0,0)}\right\} \\
& \Sigma\left(\Sigma^{\prime}(i, j)_{l} m_{i j}\right) .
\end{aligned}
$$

Agora, podemos escrever os termos $\Sigma^{\prime}(i)_{l}(j)_{l} a_{i j}$ e $\Sigma^{\prime}(s)_{l}(t)_{l} m_{s t}$ como os elementos $(l, l)$ das matrizes $\phi^{2} Z_{2} / \delta_{(2,0,0,0,0)}$ e $\phi^{2}\left(Z-Z_{2}\right) / \delta_{(2,0,0,0,0)}$, respectivamente. Assim,

$$
\begin{aligned}
A_{1, \beta \phi}= & \frac{12 \phi^{-2}}{n\left(\delta_{(2,0,0,0,2)}-1\right) \delta_{(2,0,0,0,0)}^{2}}\left\{\delta_{(1,1,0,0,1)}\left(\delta_{(1,1,0,0,1)}-\delta_{(0,1,0,0,0)}\right)\right\} \\
& \left(\Sigma z_{2 l l}\right)\left(\Sigma\left(z_{m m}-z_{2 m m}\right)\right) \\
& -\frac{12 \delta_{(1,1,0,0,1)}}{n\left(\delta_{(2,0,0,0,2)}-1\right)^{2} \delta_{(2,0,0,0,0)}}\left(2 \delta_{(0,1,0,0,2)}+\delta_{(0,0,1,0,3)}\right) \Sigma\left(z_{l l}-z_{2 l l}\right) \\
& +\frac{6\left(3 \delta_{(1,1,0,0,1)}-\delta_{(0,1,0,0,0)}\right)\left(\delta_{(1,1,0,0,1)}-\delta_{(0,1,0,0,0)}\right)}{n\left(\delta_{(2,0,0,0,2)}-1\right) \delta_{(2,0,0,0,0)}^{2}} \Sigma\left(z_{l m}-z_{2 l m}\right) z_{2 l m} \\
& -\frac{6\left(3 \delta_{(1,1,0,0,1)}-\delta_{(0,1,0,0,0)}\right)\left(\delta_{(0,0,1,0,1)}-2 \delta_{(0,1,0,0,0)}\right)}{n\left(\delta_{(2,0,0,0,2)}-1\right) \delta_{(2,0,0,0,0)}^{2}} \Sigma\left(z_{l m}-z_{2 l m}\right) z_{2 l m} \\
& -\frac{6\left(4 \delta_{(3,0,0,0,1)}+\delta_{(4,0,0,0,2)}+\delta_{(2,1,0,0,2)}-2 \delta_{(0,1,0,0,0)}\right)}{n\left(\delta_{(2,0,0,0,2)}-1\right) \delta_{(2,0,0,0,0)}} \Sigma\left(z_{l l}-z_{2 l l}\right) .
\end{aligned}
$$

Como $\Sigma z_{2 l l}=\operatorname{tr}\left(Z_{2}\right)=p-q, \Sigma\left(z_{l l}-z_{2 l l}\right)=\operatorname{tr}\left(Z_{d}-Z_{2 d}\right)=q$ e $\Sigma\left(z_{l m}-z_{2 l m}\right) z_{2 l m}=$ $\operatorname{tr}\left(Z Z_{2}\right)-\operatorname{tr}\left(Z_{2}\right)=0$, temos

$$
\begin{aligned}
A_{1, \beta \phi}= & \frac{12}{n} \frac{\left(\delta_{(1,1,0,0,1)}\right)\left(\delta_{(1,1,0,0,1)}-\delta_{(0,1,0,0,0)}\right)}{\left(\delta_{(2,0,0,0,2)}-1\right) \delta_{(2,0,0,0,0)}^{2}}(p-q) q \\
& -\frac{12}{n} \frac{\left(\delta_{(1,1,0,0,1)}\right)\left(2 \delta_{(0,1,0,0,2)}+\delta_{(0,0,1,0,3)}\right)}{\left(\delta_{(2,0,0,0,2)}-1\right)^{2} \delta_{(2,0,0,0,0)}} q \\
& -\frac{6}{n} \frac{\left(4 \delta_{(3,0,0,0,1)}+\delta_{(4,0,0,0,2)}+\delta_{(2,1,0,0,2)}-2 \delta_{(0,1,0,0,0)}\right)}{\left(\delta_{(2,0,0,0,2)}-1\right) \delta_{(2,0,0,0,0)}} q .
\end{aligned}
$$

A obtenção de $A_{2, \beta}$ é conduzida de maneira análoga. De (3.22) vem

$$
\begin{aligned}
A_{2, \beta \phi}= & -12 \Sigma^{\prime} \frac{1}{\phi^{6}} \delta_{(1,1,0,0,1)}^{2}\left(\Sigma(i, j)_{l}\left(\Sigma(s, t)_{m}\right) m_{i j} m_{s t} a_{\phi \phi}^{\prime}\right. \\
& -24 \Sigma^{\prime} \frac{1}{\phi^{6}} \delta_{(1,1,0,0,1)}^{2}\left(\Sigma(i, j)_{l}\left(\Sigma(r, s)_{m}\right) m_{i r} m_{j s} a_{\phi \phi}\right. \\
= & -\frac{12 \phi^{-4}}{n\left(\delta_{(2,0,0,0,0)}-1\right)} \delta_{(1,1,0,0,1)}^{2}\left\{\Sigma\left(\Sigma^{\prime}(i, j)_{l} m_{i j}\right) \Sigma\left(\Sigma^{\prime}(s, t)_{m} m_{s t}\right)\right.
\end{aligned}
$$




$$
\begin{aligned}
& \left.+2 \Sigma\left(\Sigma^{\prime}(i)_{l}(r)_{m} m_{l r}\right)\left(\Sigma^{\prime}(j)_{l}(s)_{m} m_{j s}\right)\right\} \\
= & -\frac{12 \delta_{(1.1,0,0,1)}^{2}}{n\left(\delta_{(2.0 .0,0,2)}-1\right) \delta_{(2.0,0,0,0)}^{2}}\left\{\left(\Sigma ( z _ { l l } - z _ { 2 l l } ) \left(\Sigma\left(z_{m m}-z_{2 m m}\right)\right.\right.\right. \\
& \left.+2 \Sigma\left(z_{l m}-z_{2 l m}\right)\left(z_{l m}-z_{2 l m}\right)\right\}
\end{aligned}
$$

que se reduz a

$$
\begin{aligned}
A_{2, \beta \phi} & =-\frac{12 \delta_{(1,1,0,0 ; 1)}^{2}}{n\left(\delta_{(2,0,0,0,2)}-1\right) \delta_{(2,0,0,0,0)}^{2}}\left\{\left(\operatorname{tr}\left(Z_{d}-Z_{2 d}\right)\right)^{2}+2 \operatorname{tr}\left(Z_{d}-Z_{2 d}\right)\right\} \\
& =-\frac{12 \delta_{(1,1,0,0,1)}^{2}}{n \delta_{(2,0,0,0,0)}^{2}\left(\delta_{(2,0,0,0,2)}-1\right)}\left(q^{2}+2 q\right) .
\end{aligned}
$$

\section{B.4 Obtenção de $d_{3}$}

A quantidade $d_{3}$ definida na Seção 3.2 .3 para o teste $H_{0}: \phi=\phi^{(0)}$ versus $H_{1}: \phi \neq \phi^{(0)}$ depende de $\varepsilon_{\phi}$. Desta maneira, segundo o desenvolvimento feito em (3.7), $\varepsilon_{\phi}$ é escrito como

$$
\varepsilon_{\phi}=\ell_{\phi \phi \phi \phi}-\ell_{\phi \phi \phi \phi \phi \phi},
$$

onde

e

$$
\ell_{\phi \infty \circ \circ}=\left(\kappa^{\phi \phi}\right)^{2}\left\{\frac{1}{4} \kappa_{\phi \phi \phi \phi}-\kappa_{\phi \phi \phi}^{(\phi)}+\kappa_{\phi \phi}^{\phi \phi}\right\}
$$

$$
\ell_{\phi \phi \phi \phi \phi \phi}=\left(\kappa^{\phi \phi}\right)^{3}\left\{\frac{5}{12} \kappa_{\phi \phi \phi}^{2}-2 \kappa_{\phi \phi \phi} \kappa_{\phi \phi}^{(\phi)}+2\left(\kappa_{\phi \phi}^{(\phi)}\right)^{2}\right\} .
$$

Substituindo cumulantes dos modelos simétricos em $\ell_{\phi \phi \phi \phi}$ temos

$$
\begin{aligned}
\ell_{\phi \phi \phi \phi}= & \frac{\phi^{4}}{n\left(\delta_{(0,1,0,0,2)}-1\right)^{2}}\left\{\frac{1}{4 \phi^{4}}\left(\delta_{(0,0,0,1,4)}+12 \delta_{(0,0,1,0,3)}+36 \delta_{(0,1,0,0,2)}-8\right)\right. \\
& \left.-\frac{3}{\phi^{4}}\left(\delta_{(0,1,0,0,2)}+\delta_{(0,0,1.0,3)}-4\right)+\frac{6}{\phi^{4}}\left(\delta_{(0,1,0,0,2)}-1\right)\right\} .
\end{aligned}
$$

Utilizando algumas relações entre $\delta$ 's, resulta

$$
\ell_{\phi \phi \phi \phi}=\frac{1}{4 n\left(\delta_{(2,0,0,0,2)}-1\right)^{2}}\left\{\delta_{(0,0,0,1,4)}-12 \delta_{(0,1,0,0,2)}+6\right\} .
$$

Por outro lado,

$$
\begin{aligned}
\ell_{\phi \phi \phi \phi \phi \phi}= & \frac{\phi^{6}}{n\left(\delta_{(0,1,0,0,2)}-1\right)^{3}}\left\{\frac{5}{12} \frac{1}{\phi^{6}}\left(6 \delta_{(0,1,0,0,2)}+\delta_{(0,0,1,0,3)}-4\right)^{2}\right. \\
& -2\left(\frac{1}{\phi^{6}}\right)\left(6 \delta_{(0,1,0,0,2)}+\delta_{(0,0,1,0,3)}-4\right)\left(\delta_{(0,1,0,0,2)}-1\right) \\
& \left.+\frac{\delta}{\delta^{6}}\left(\delta_{(0,1,0,0,2)}-1\right)^{2}\right\} .
\end{aligned}
$$


Assim.

$$
\begin{aligned}
\ell_{\phi \phi \phi \phi \phi \phi}= & \frac{1}{4 n\left(\delta_{(0,1,0,0,2)}-1\right)^{3}}\left\{\frac{5}{3}\left(6 \delta_{(0,1,0,0.2)}+\delta_{(0,0,1,0,3)}-4\right)^{2}\right. \\
& \left.-16\left(\delta_{(0,1,0,0,2)}-1\right)\left(4 \delta_{(0,1,0,0,2)}+\delta_{(0,0,1,0,3)}-2\right)\right\} .
\end{aligned}
$$

Logo,

$$
\varepsilon_{\phi}=d_{3} / n,
$$

onde

$$
\begin{aligned}
d_{3}= & \frac{1}{4\left(\delta_{(0,0,1,0,2)}-1\right)^{3}}\left\{( \delta _ { ( 0 , 1 , 0 , 0 , 2 ) } - 1 ) \left(\delta_{(0,0,0,1,4)}+16 \delta_{(0,0,1,0,3)}\right.\right. \\
& \left.+26\left(\delta_{(0,1,0,0,2)}-1\right)-\frac{5}{3}\left(6 \delta_{(0,1,0,0,2)}+\delta_{(0,0,1,0,3)}-4\right)^{2}\right\} .
\end{aligned}
$$

\section{B.5 Obtenção de $A_{1}, A_{2}$ e $A_{3}$}

O desenvolvimento de fórmulas para $A_{1}, A_{2}$ e $A_{3}$ para o teste de $H_{0}: \phi=\phi^{(0)}$ versus $H_{1}: \phi \neq \phi^{(0)}$ é baseado nas fórmulas de $A$ 's dadas na Seção 3.3.3. Assim, de

$$
\begin{aligned}
A_{1}= & \kappa_{\phi \phi}^{-1}\left\{-3\left(\Sigma^{\prime} \kappa_{i j \phi} \kappa^{i j}\right)^{2}-6 \Sigma^{\prime} \kappa_{i j \phi}\left(3 \kappa_{i s t}-2 \kappa_{i s}^{(\phi)}\right) \kappa^{i j} \kappa^{s t}\right. \\
& \left.+12 \Sigma^{\prime}\left(\kappa_{i j \phi \phi}-\kappa_{i j \phi}^{(\phi)}-\kappa_{i \phi, j \phi}\right) \kappa^{i j}\right\}
\end{aligned}
$$

e utilizando algumas propriedades dos $\delta$ 's, vem

$$
\begin{aligned}
A_{1}= & \frac{\phi^{2}}{n\left(\delta_{(0,1,0,0,2)}-1\right)}\left\{-3\left(-\frac{1}{\phi^{3}}\right)^{2}\left(\delta_{(0,0,1,0,1)}+2 \delta_{(0,1,0,0,0)}\right)^{2}\left(\Sigma\left(\Sigma^{\prime}(i, j)_{l} \kappa^{i j}\right)\right)^{2}\right. \\
& \left.-6\left(\left(-\frac{1}{\phi^{3}}\right)\left(\delta_{(0,0,1,0,1)}+2 \delta_{(0,1,0,0,0)}\right)\right)\left(3 \delta_{(0,0,1,0,1)}+2 \delta_{(0,1,0,0,0)}\right)\right) \\
& \Sigma\left(\Sigma^{\prime}(i)_{m}(j)_{l} \kappa^{i j}\right)\left(\Sigma^{\prime}(t)_{l}(s)_{m} \kappa^{s t}\right) \\
& +\frac{12}{\phi^{4}}\left(\delta_{(0,0,0,1,2)}-\delta_{(0,2,0,0,2)}+3 \delta_{(1,1,0,0,1)}-2 \delta_{(0,1,0,0,0)}\right. \\
& \left.-8 \delta_{(1,1,0,0,1)}\right) \Sigma\left(\Sigma^{\prime}(i, j)_{l} \kappa^{i j}\right) .
\end{aligned}
$$

Agora, podemos escrever o termo $\Sigma^{\prime}(l)_{l}(j)_{m} \kappa^{i j}$ como o elemento $(l, m)$ da matriz $\phi^{2} Z / \delta_{(0,1,0,0,0)}$ ou $-\phi^{2} Z / \delta_{(2,0,0,0,0)}$ e obtemos

$$
\begin{aligned}
A_{1}= & \frac{\phi^{2}}{n\left(\delta_{(0,1,0,0,2)}-1\right)}\left\{-\frac{3 \phi^{-2}}{\delta_{(2,0,0,0,0)}^{2}}\left(\delta_{(0,0,1,0,1)}+2 \delta_{(0,1,0,0,0)}\right)^{2}\left(\Sigma z_{l l}\right)^{2}\right. \\
& -\frac{6 \phi^{-2}}{\delta_{(2,0,0,0,0)}^{2}}\left(\delta_{(0,0,1,0,1)}+2 \delta_{(0,1,0,0,0)}\right)\left(3 \delta_{(0,0,1,0,1)}+2 \delta_{(0,1,0,0,0)}\right) \Sigma z_{l m} z_{m l} \\
& \left.-\frac{12 \phi^{-2}}{\delta_{(2,0,0,0,0)}}\left(\delta_{(0,2,0,0,2)}-\delta_{(0,0,0,1,2)}+5 \delta_{(1,1,0,0,1)}+2 \delta_{(0,1,0,0,0)}\right) \Sigma z_{l l}\right\}
\end{aligned}
$$


Como $\Sigma z=\operatorname{tr}(Z)=p$. temos

$$
\begin{aligned}
A_{1}= & -\frac{3}{n \delta_{(2,0,0,0,0)}^{2}\left(\delta_{(0,1,0,0,2)}-1\right)}\left\{\left(\delta_{(0,0,1,0,0)}+2 \delta_{(0,1,0,0,0)}\right)^{2} p^{2}\right. \\
& +2\left(\delta_{(0,0,1,0,1)}+2 \delta_{(0.1,0,0,0)}\right)\left(3 \delta_{(0,0,1.0 .1)}+2 \delta_{(0,1,0,0,0)}\right) p \\
& \left.-4 \delta_{(2,0,0,0,0)}\left(\delta_{(0,2,0,0,2)}-\delta_{(0,0,0,1,2)}+5 \delta_{(1,1,0,0,1)}+2 \delta_{(0,1,0,0,0)}\right) p\right\} .
\end{aligned}
$$

A obtenção de $A_{2}$ e $A_{3}$ é conduzida de maneira análoga. Assim, de

$$
A_{2}=\kappa_{\phi \phi}^{-2}\left\{\Sigma^{\prime}\left(6 \kappa_{\phi, \phi, \phi} \kappa_{i j \phi}\right) \kappa^{i j}+3 \kappa_{\phi, \phi, \phi, \phi}\right\}
$$

vem

$$
\begin{aligned}
A_{2}= & \frac{\phi^{4}}{\left.n \delta_{(0,1,0,0,2)}-1\right)^{2}}\left\{-\frac{12}{\phi^{6}}\left(1+\delta_{(1,1,0,0,3)}\right)\left(\delta_{(0,0,1,0,1)}+2 \delta_{(0,1,0,0,0)}\right) \Sigma\left(\Sigma^{\prime}(i, j)_{l} \kappa^{i j}\right)\right. \\
& \left.+\frac{3}{\phi^{4}}\left(\delta_{(4,0,0,0,4)}+4 \delta_{(3,0,0,0,3)}+12 \delta_{(2,0,0,0,0)}-3 \delta_{(2,0,0,0,0)}^{2}-6\right)\right\} .
\end{aligned}
$$

Assim,

$$
\begin{aligned}
A_{2}= & \frac{3}{n\left(\delta_{(0,1,0,0,2)}-1\right)^{2} \delta_{(2.0,0,0,0)}}\left\{4\left(1+\delta_{(1,1,0,0,3)}\right)\left(\delta_{(0,0,1,0,1)}+2 \delta_{(0,1,0,0,0)}\right) p\right. \\
& \left.+\delta_{(2,0,0,0,0)}\left(\delta_{(4,0,0,0,4)}+4 \delta_{(3,0,0,0,3)}+12 \delta_{(2,0,0,0,0)}-3 \delta_{(2,0,0,0,0)}^{2}-6\right)\right\} .
\end{aligned}
$$

Finalmente, de

$$
A_{3}=-\frac{5 \kappa_{\phi, \phi, \phi}^{2}}{\kappa_{\phi \phi}^{3}},
$$

vem

$$
A_{3}=-\frac{20\left(1+\delta_{(1,1,0,0,3)}\right)^{2}}{n\left(\delta_{(0,1,0,0,2)}-1\right)^{3}}
$$




\section{Apêndice C}

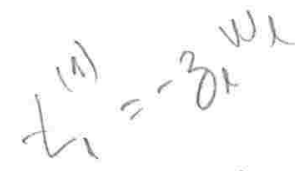

Neste apêndice, encontraremos os valores dos $\delta$ 's associados à correção de Bartlett e tipo Bartlett para cada distribuição na classe dos modelos simétricos. A notação para $t^{(r)} \mathrm{e}$ $\delta_{(a, b, c, d, e)}$ segue da Seção 2.5. Além disso. $z_{l}=\left(y_{l}-x_{l}^{\top} \beta\right) / \phi$.

\section{C.1 Distribuição normal}

Seja $y_{l} \sim N\left(x_{l}^{\top} \beta, \phi^{2}\right)$ com função densidade da forma

$$
\pi\left(y_{l}, \beta, \phi\right)=\frac{1}{\phi} \frac{1}{\sqrt{2 \pi}} \exp \left\{-\frac{1}{2}\left(\frac{y_{l}-x_{l}^{\top} \beta}{\phi}\right)^{2}\right\}, y \in \mathbb{R},
$$

onde $\phi>0, \beta \in \mathbb{R}^{p}$. O logaritmo da função de verossimilhança é da forma

$$
L(\beta, \phi)=-n \log \phi+\sum_{l=1}^{n} t\left(z_{l}\right),
$$

onde $t_{l}=-z_{l}^{2} / 2$. Como $t^{(r)}=\partial^{r} t / \partial z^{r}$, temos

$$
t^{(1)}=-z, \quad t^{(2)}=-1, \quad t^{(3)}=t^{(4)}=0 .
$$

Já que $z \sim N(0,1)$, temos que

$$
\begin{array}{lll}
\delta_{(2,0,0,0,0)}=1, & \delta_{(0,0,0.1,0)}=0, & \delta_{(4,0,0,0,0)}=3, \\
\delta_{(2,1,0,0,0)}=-1, & \delta_{(0,0,0,1,2)}=0, & \delta_{(2,0,0,0,2)}=3, \\
\delta_{(0,0,1,0,1)}=0, & \delta_{(0,1,0,0,0)}=-1, & \delta_{(0,1,0,0,2)}=-1, \\
\delta_{(0,0,1,0,3)}=0, & \delta_{(1,1,0,0,1)}=1, & \delta_{(3,0,0,0,1)}=-3, \\
\delta_{(4,0,0,0,2)}=15, & \delta_{(0,0,0,1,4)}=0, & \delta_{(0,2,0,0,2)}=1, \\
\delta_{(1,1,0,0,3)}=3, & \delta_{(4,0,0,0,4)}=105, & \delta_{(3,0,0,0,3)}=-15, \\
\delta_{(2,1,0,0,2)}=3,-3 & \delta_{(1,0.1,0,0)}=0 . &
\end{array}
$$

\section{C.2 Distribuição de Cauchy}

$$
\begin{aligned}
\delta_{20002} & =E\left(t^{(1)^{2}} \cdot z^{2}\right) \\
& =E\left(z^{2} \cdot z^{2}\right)=E\left(z^{2}\right)=0
\end{aligned}
$$

Seja $y_{l} \sim C\left(x_{l}^{\top} \beta, \phi^{2}\right)$ com funçào densidade da forma

$$
\pi\left(y_{l} ; \beta, \phi\right)=\frac{1}{\pi \phi}\left[1+\left(\frac{y_{l}-x_{l}^{\top} \beta}{\phi}\right)^{2}\right]^{-1}, y_{l} \in \mathbb{R} .
$$


onde $\phi>0$ e $\beta \in \mathbb{R}^{p}$. O logaritmo da função de verossimilhança tem a forma

$$
L(\beta, \phi)=-n \log \phi+\sum_{l=1}^{n} t\left(z_{l}\right) .
$$

onde

$$
t_{l}=t\left(z_{l}\right)=\log \left(\frac{1}{\pi}\left[1+z_{l}^{2}\right]^{-1}\right) .
$$

Assim, temos $t^{(1)}=2 z w$, onde $w=-1 /\left(1+z^{2}\right), t^{(2)}=2 w+4 z^{2} w^{2}, t^{(3)}=12 z w^{2}+16 z^{3} w^{3}$ e $t^{(4)}=12 w^{2}+96 z^{2} w^{2}+96 z^{4} w^{4}$. Considerando que $z \sim C(0,1)$, então $z^{2} \sim F(1,1) \mathrm{e}$ assim:

$$
\begin{array}{lll}
\delta_{(2,0,0,0,0)}=1 / 2, & \delta_{(0,0,0,1,0)}=3 / 4, & \delta_{(4,0.000,0)}=3 / 8, \\
\delta_{(2,1,0,0,0)}=-1 / 8, & \delta_{(0,0,0,1,2)}=-3 / 4, & \delta_{(2,0.0 .0,2)}=3 / 2, \\
\delta_{(0,0,1,0,1)}=1 / 2, & \delta_{(0,1,0,0,0)}=-1 / 2, & \delta_{(0,1,0.0,2)}=1 / 2, \\
\delta_{(0,0,1,0,3)}=-1 / 2, & \delta_{(1,1,0,0,1)}=0, & \delta_{(3,0.0,0,1)}=-1 / 2, \\
\delta_{(4,0,0,0,2)}=5 / 8, & \delta_{(0,0,0,1,4)}=3 / 4, & \delta_{(0,2,0.0,2)}=1 / 8, \\
\delta_{(1,1,0,0,3)}=-1, & \delta_{(4,0,0,0,4)}=35 / 8, & \delta_{(3,0.0 .0,3)}=-5 / 2, \\
\delta_{(1,0,1,0,0)}=-3 / 4, & \delta_{(2,1,0,0,2)}=1 / 8 . &
\end{array}
$$

\section{C.3 Distribuição t-Student}

Seja $y_{l} \sim t\left(x_{l}^{\top} \beta, \phi^{2}, \nu\right)$ com função densidade da forma

$$
\pi\left(y_{l} ; \beta, \phi, \nu\right)=\frac{1}{\phi} \frac{\nu^{\nu / 2}}{B(1 / 2, \nu / 2)}\left[\nu+\left(\frac{y_{l}-x_{l}^{\top} \beta}{\phi}\right)^{2}\right]^{-\frac{\nu+1}{2}}, y_{l} \in \mathbb{R},
$$

onde $\phi>0, \nu>0, \beta \in \mathbb{R}^{p}$. O logaritmo da função de verossimilhança tem a forma

$$
L(\beta, \phi)=-n \log \phi+\sum_{l=1}^{n} t\left(z_{l}\right),
$$

onde

$$
t_{l}=t(z l)=\log \left(\frac{\nu^{\nu / 2}}{B(1 / 2, \nu / 2)}\left[\nu+z_{l}^{2}\right]^{-\frac{\nu+1}{2}}\right) .
$$

Derivando $t_{l}$ com respeito a $z$, temos $t^{(1)}=2 z w$, onde $w=-(\nu+1) /\left[2\left(\nu+z^{2}\right)\right]$,

$$
\begin{aligned}
& t^{(2)}=2 w+\frac{8 z^{2} w^{2}}{(\nu+1)}, \quad t^{(3)}=\frac{24}{(\nu+1)} z w^{2}+\frac{64}{(\nu+1)^{2}} z^{3} w^{3}, \\
& t^{(4)}=\frac{24}{(\nu+1)} w^{2}+\frac{384}{(\nu+1)^{2}} z^{2} w^{3}+\frac{768}{(\nu+1)^{3}} z^{4} w^{4} .
\end{aligned}
$$

Considerando que $z \sim t(0,1, \nu)$, temos $z^{2} \sim F(1, \nu)$. Logo,

$$
\begin{gathered}
E\left[z^{2 k}, w^{m}\right]=\left(-\frac{\nu+1}{2}\right)^{m} \frac{1}{\nu^{m-k}} \frac{B\left(\frac{1+2 k}{2}, \frac{\nu+2(m-k)}{2}\right)}{B(1 / 2 \cdot \nu / 2)}, \\
E\left(\left(3^{2}\right)^{k}\right. \\
183
\end{gathered}
$$


para $m=0,1,2, \ldots$ Assim, os $\delta$ 's são dados por

$$
\begin{aligned}
& \begin{array}{ll}
\left.\delta_{(2,0,0,0,0)}=\frac{\nu+1}{\nu+3}, j\right) \backslash & \delta_{(0,0,0,1,0)}=\frac{6(\nu+1)(\nu+2)}{\nu(\nu+5)(\nu+7)}, \quad 0 K ! \\
\delta_{(4,0,0,0,0)}=\frac{3(\nu+1)^{3}(\nu+2)}{\nu(\nu+3)(\nu+5)(\nu+7)}, & \delta_{(2,1,0,0,0)}=-\frac{(\nu+1)^{3}(\nu+2)}{\nu(\nu+3)(\nu+5)(\nu+7)},
\end{array} \\
& \left(\delta_{(0,0,0,1,2)}=\frac{6}{(\nu+3)}\left(\frac{\nu-19}{\nu+5}+\frac{120}{(\nu+5)(\nu+7)}\right), \delta_{(2,0,0,0,2)}=\frac{3(\nu+1)}{(\nu+3)}\right. \\
& \delta_{(0,0,1,0,1)}=\frac{6(\nu+1)}{(\nu+3)(\nu+5)}, \quad 0 K^{\prime} \cdot \quad \delta_{(0,1,0,0,0)}=-\frac{(\nu+1)}{(\nu+3)} \quad 0 K ! \\
& \delta_{(0,1,0,0,2)}=\frac{3-\nu}{(\nu+3)}, \\
& \delta_{(0,0,1,0,3)}=\frac{6(3 \nu-5)}{(\nu+3)(\nu+5)} \\
& \delta_{(1,1,0,0,1)}=\frac{(\nu+1)(\nu-1)}{(\nu+3)(\nu+5)} \\
& \delta_{(4,0,0,0,2)}=\frac{15(\nu+1)^{3}}{(\nu+3)(\nu+5)(\nu+7)} \\
& \delta_{(3,0,0,0,1)}=-\frac{3(\nu+1)^{2}}{(\nu+3)(\nu+5)}, \\
& \delta_{(0,0,0,1,4)}=\frac{18\left(\nu^{2}-28 \nu+35\right)}{(\nu+3)(\nu+5)(\nu+7)}, \\
& \delta_{(0,2,0,0,2)}=\frac{(\nu+1)\left(\nu^{2}+11\right)}{(\nu+3)(\nu+5)(\nu+7)}, \\
& \delta_{(1,1,0,0,3)}=\frac{3(\nu+1)(\nu-5)}{(\nu+3)(\nu+5)}, \\
& \delta_{(4,0,0,0,4)}=\frac{105(\nu+1)^{3}}{(\nu+3)(\nu+5)(\nu+7)}, \\
& \delta_{(3,0,0,0,3)}=-\frac{15(\nu+1)^{2}}{(\nu+3)(\nu+5)}, \\
& \delta_{(2,1,0,0,2)}=\frac{3(\nu+1)^{2}(3-\nu)}{(\nu+3)(\nu+5)(\nu+7)}, \\
& \delta_{(1,0,1,0,0)}=-\frac{6(\nu+1)(\nu+2)}{\nu(\nu+5)(\nu+7)} .
\end{aligned}
$$

\section{C.4 Distribuição t-Student generalizada}

Seja $y_{l} \sim t G\left(x_{l}^{\top} \beta, \phi^{2}, r, s\right)$ com funçào densidade da forma.

$$
\pi\left(y_{l} ; \beta, \phi, r, s\right)=\frac{1}{\phi} \frac{s^{r / 2}}{B(1 / 2, r / 2)}\left[s+\left(\frac{y_{l}-x_{l}^{\top} \beta}{\phi}\right)^{2}\right]^{-\frac{r+1}{2}}, y_{l} \in \mathbb{R},
$$

onde $s, r>0, \phi>0, \beta \in \mathbb{R}^{p}$. O logaritmo da função de verossimilhança tem a forma

$$
L(\beta, \phi)=-n \log \phi+\sum_{l=1}^{n} t\left(z_{l}\right)
$$


onde

$$
t_{l}=t\left(z_{l}\right)=\log \left(\frac{s^{r / 2}}{B(1 / 2 \cdot r / 2)}\left[s+z_{l}^{2}\right]^{-\frac{r+1}{2}}\right) .
$$

Derivando $t_{l}$ com respeito a $z$, temos $t^{(1)}=2 z w$, onde $w=-(r+1) /\left[2\left(s+z^{2}\right)\right]$,

$$
\begin{aligned}
& t^{(2)}=2 w+\frac{8}{(r+1)} z^{2} w^{2}, \quad t^{(3)}=\frac{24}{(r+1)} z w^{2}+\frac{64}{(r+1)^{2}} z^{3} w^{3}, \\
& t^{(4)}=\frac{24}{(r+1)} w^{2}+\frac{384}{(r+1)^{2}} z^{2} w^{3}+\frac{768}{(r+1)^{3}} z^{4} w^{4} .
\end{aligned}
$$

Considerando que $z \sim t G(0,1, s, r)$, temos

$$
u=\frac{\sqrt{r}}{\sqrt{s}} z \sim t(0,1, s) \quad \text { e } \quad v=\frac{r}{s} z^{2} \sim F(1, r) .
$$

Logo,

$$
E\left[z^{2 k} w^{m}\right]=\frac{\left(-\frac{r+1}{2}\right)^{m}}{s^{m-k} B(1 / 2, r / 2)} B\left(\frac{2 k+1}{2}, \frac{r+k(m-k)}{2}\right), \quad \text { para } m=0,1,2, \ldots
$$

Assim, os $\delta$ 's são dados por

$$
\begin{array}{ll}
\delta_{(2,0,0,0,0)}=\frac{r(r+1)}{s(r+3)}, & \delta_{(0,0,0,1,0)}=\frac{6(r+1)(r+2)}{s^{2}(r+5)(r+7)}, \\
\delta_{(4,0,0,0,0)}=\frac{3 r(r+1)^{3}(r+2)}{s^{2}(r+3)(r+5)(r+7)}, & \delta_{(2,1,0,0,0)}=-\frac{r(r+1)^{3}(r+2)}{3 s^{2}(r+3)(r+5)(r+7)}, \\
\delta_{(0,0,0,1,2)}=\frac{6 r((r-19)(r+7)+120)}{s(r+3)(r+5)(r+7)}, & \delta_{(2,0,0,0,2)}=\frac{3(r+1)}{(r+3)}, \\
\delta_{(0,0,1,0,1)}=\frac{6 r(r+1)}{s(r+3)(r+5)}, & \delta_{(0,1,0,0,0)}=-\frac{r(r+1)}{s(r+3)}, \\
\delta_{(0,1,0,0,2)}=\frac{3-r}{r+3}, & \delta_{(0,0,1,0,3)}=\frac{6(3 r-5)}{(r+3)(r+5)} \\
\delta_{(4,0,0,0,2)}=\frac{15 r(r+1)^{3}}{s(r+3)(r+5)(r+7)}, & \delta_{(0,0,0,1,4)}=\frac{18\left(r^{2}-28 r+35\right)}{(r+3)(r+5)(r+7)}, \\
\delta_{(0,2,0,0,2)}=\frac{r(r+1)\left(r^{2}+11\right)}{s(r+3)(r+5)(r+7)}, & \delta_{(1,1,0,0,3)}=\frac{3(r+1)(r-5)}{(r+3)(r+5)},
\end{array}
$$




$$
\begin{array}{ll}
\delta_{(4,0,0,0,4)}=\frac{105(r+1)^{3}}{(r+3)(r+5)(r+7)}, & \delta_{(3,0,0,0,3)}=\frac{-15(r+1)^{2}}{(r+3)(r+5)}, \\
\delta_{(2,1,0,0,2)}=\frac{-3 r(r+1)^{2}(r-3)}{s(r+3)(r+5)(r+7)}, & \delta_{(1,1,0,0,1)}=\frac{r\left(r^{2}-1\right)}{s(r+3)(r+5)}, \\
\delta_{(3,0,0,0,1)}=\frac{-3 r(r+1)^{2}}{s(r+3)(r+5)}, & \delta_{(1,0,1,0,0)}=-\frac{6(r+1)(r+2)}{s^{2}(r+5)(r+7)} .
\end{array}
$$

\section{C.5 Distribuição Logística I}

Seja $y_{l} \sim L I\left(x_{l}^{\top} \beta, \phi^{2}\right)$ com função densidade da forma

$$
\pi\left(y_{l} ; \beta, \phi\right)=\frac{c}{\phi} \frac{\exp \left\{-\left(\frac{y_{l}-x_{l}^{\top} \beta}{\phi}\right)^{2}\right\}}{\left(1+\exp \left\{-\left(\frac{y-x_{l}^{\top} \beta}{\varphi}\right)^{2}\right\}\right)^{2}}, \quad y \in \mathbb{R},
$$

onde $c \approx 1,484300029, \beta \in \mathbb{R}^{p}$ e $\phi>0$. O logaritmo da função de verossimilhança tem a forma

$$
L(\beta, \phi)=-n \log \phi+\sum_{l=1}^{n} t\left(z_{l}\right)
$$

onde

$$
t_{l}=t\left(z_{l}\right)=\log \left(\frac{e^{-z_{l}^{2}}}{1+e^{-z_{l}^{2}}}\right) .
$$

As quatro primeiras derivadas de $t$ com respeito a $z$ são $t^{(1)}=2 z u_{\text {r }}$ onde $w=\left(e^{-z^{2}}-\right.$ 1)/(1+ $\left.e^{-z^{2}}\right)$,

$$
\begin{aligned}
& t^{(2)}=2 w+2 z^{2}\left(w^{2}-1\right), \quad t^{(3)}=6 z\left(w^{2}-1\right)+4 z^{3} w\left(w^{2}-1\right), \\
& t^{(4)}=6\left(w^{2}-1\right)+24 w z^{2}\left(w^{2}-1\right)+4 z^{4}\left(w^{2}-1\right)\left(3 w^{2}-1\right) .
\end{aligned}
$$

Considerando que $\approx \sim L I(0,1)$ e

$$
E\left[z^{2 r} w^{m}\right]=(-1)^{m} \frac{c}{2} \int_{0}^{1}\left(\log \left(\frac{1+s}{1-s}\right)\right)^{\frac{2 r-1}{2}} s^{m} \mathrm{~d} s, \text { para } m=0,1,2, \ldots
$$


onde $s=1-e^{z^{2}} /$ fy $_{1}+e^{-z^{2} z^{2}}$. Assim, os $\delta$ 's são dados por

$$
\begin{aligned}
& \delta_{(2,0,0,0,0)} \approx 1.477240176, \quad \delta_{(0,0,0,1,0)} \approx 4,259052264 . \\
& \delta_{(4,0,0,0,0)} \approx 12,46141962, \quad \delta_{(2,1,0,0,0)} \approx 4,153806544, \quad-4.153806544 \\
& V \delta_{(0,0,0,1,2)} \approx 2.65931983, \quad \delta_{(2,0,0,0,2)} \approx 4,013783934 \\
& \sqrt{\delta_{(0,0,1,0,1)}} \approx-1,27916363, \quad \delta_{(0,1,0,0,0)} \approx-1,477240176, \\
& \bigvee \delta_{(0,1,0,0,2)} \approx-2,013783934, \quad \delta_{(0,0,1,0,3)} \approx-0,508877866, \\
& \delta_{(1,1,0,0,1)} \approx 2,756409976, \quad \delta_{(3,0,0,0,1)} \approx 46,76577386,-6.990064 \\
& V \delta_{(4,0,0,0,2)} \approx 46,76577386, \quad \delta_{(0,0,0,1,4)} \approx 4,135810 \\
& \delta_{(0,2,0,0,2)} \approx-26,69177514, \quad \delta_{(1,1,0,0,3)} \approx 6,545463516 \\
& \delta_{(4,0,0,0,4)} \approx 206,1514675, \quad \delta_{(3,0,0,0,3)} \approx-25,12989577, \\
& \delta_{(2,1,0,0,2)} \approx-10,92854975, \quad \delta_{(1,0,1,0,0)} \approx-4,259052264
\end{aligned}
$$

\section{C.6 Distribuição Logística II}

Seja $y_{l} \sim L I I\left(x_{l}^{\top} \beta, \phi^{2}\right)$ com função densidade da forma

$$
\pi\left(y_{l} ; \beta, \phi\right)=\frac{1}{\phi} \frac{\exp \left\{\left(\frac{y_{l}-x_{l}^{\top} \beta}{\phi}\right)\right\}}{\left(1+\exp \left\{\left(\frac{y-x_{l}^{\top} \beta}{\phi}\right)\right\}\right)^{2}}, \quad y_{l} \in \mathbb{R},
$$

onde $\phi>0$ e $\beta \in \mathbb{R}^{p}$. O logaritmo da função de verossimilhança tem a forma

$$
L(\beta, \phi)=-n \log \phi+\sum_{l=1}^{n} t\left(z_{l}\right)
$$

onde

$$
t_{l}=t\left(z_{l}\right)=\log \left(\frac{e^{z_{l}}}{\left(1+e^{z_{l}}\right)^{2}}\right)=\log z_{l}-2 \log \left(1+e^{z_{l}}\right)
$$

As primeiras quatro derivadas da função $t$ com respeito a $z$ são

$$
t^{(1)}=\frac{1-e^{z}}{1+e^{z}}, \quad t^{(2)}=\frac{-2 e^{z}}{\left(1+e^{z}\right)^{2}}, \quad t^{(3)}=\frac{2\left(e^{2 z}-e^{z}\right)}{\left(1+e^{z}\right)^{3}} \quad \text { e } \quad t^{(4)}=\frac{-2\left(e^{3 z}-4 e^{2 z}+e^{z}\right)}{\left(1+e^{z}\right)^{4}} .
$$


Expressões para os $\delta$ 's podem ser obtidas dos resultados desenvolvidos abaixo para a distribuição logística generalizada $\operatorname{com} m=1$ e $a=1$. Temos

$$
\begin{aligned}
& \text { f } \delta(2,0,0.0,0)=1 / 3 . \quad \delta(0,0.0 .1 .0)=1 / 15,1 \\
& \delta_{(4,0,0,0,0)}=1 / 5, \quad \delta_{(2,1,0,0.0)}=-1 / 15, \quad 10 \\
& d \delta_{(0,0,0,1,2)} \approx-0,11401, \delta_{(2,0,0.0,2)} \approx 2,42996, \& \\
& H \delta_{(0,0,1,0,1)}=1 / 6, \quad \delta_{(0,1,0,0,0)}=-1 / 3,6 \\
& \mathcal{J} \delta_{(0,1,0,0,2)} \approx-0,42996, \delta_{(0,0,1,0,3)} \approx 0,64493,3 \\
& 5 \delta_{(1,1,0,0,1)}=1 / 6, \quad \delta_{(3,0,0,0,1)}=-2 / 3, \| \\
& 12 \delta_{(4,0,0,0,2)} \approx 1,99131 \quad \delta_{(0,0,0.1,4)} \approx-1,14923, \\
& \delta_{(0,2,0,0,2)} \approx 0,10532, \quad \delta_{(1,1,0.0,3)} \approx 0,64493, \\
& \delta_{(4,0,0,0,4)} \approx 38,61046, \quad \delta_{(3,0,0,0,3)} \approx-8,57974, \\
& 13 \delta_{(2,1,0,0,2)} \approx-0,21932, \delta_{(1,0,1,0,0)}=-1 / 15 .
\end{aligned}
$$

\section{C.7 Distribuição Logística Generalizada}

Seja $y_{l} \sim L G\left(x_{l}^{\top} \beta, \phi^{2}, m\right)$ com função densidade da forma

$$
\pi\left(y_{l} ; \beta, \phi\right)=\frac{1}{\phi} \frac{\alpha}{B(m, m)}\left(\frac{\exp \left\{\alpha\left(\frac{y_{l}-x_{l}^{\top} \beta}{\phi}\right)\right\}}{\left(1+\exp \left\{\alpha\left(\frac{y_{l}-x_{l}^{\top} \beta}{\phi}\right)\right\}\right)^{2}}\right)^{m}, \quad y_{l} \in \mathbb{R}
$$

onde $\alpha=\alpha(m), \alpha(m)>0$ para $m>0, B(m, m)$ é a função Beta, $\phi>0$ e $\beta \in \mathbb{R}^{p}$. O logaritmo da função de verossimilhança é da forma

$$
L(\beta, \phi)=-n \log \phi+\sum_{l=1}^{n} t\left(z_{l}\right),
$$

onde

$$
t_{l}=t(\tilde{z})=\log \alpha-\log B(m, m)+m \log \frac{e^{\alpha z_{l}}}{\left(1+e^{\alpha z_{l}}\right)^{2}} .
$$

As quatro primeiras derivadas da função $t$ com respeito a $z$ são

$$
t^{(1)}=\alpha m \frac{\left(1-e^{\alpha z}\right)}{1+e^{\alpha z}}, \quad t^{(2)}=\frac{-2 \alpha^{2} m e^{\alpha z}}{\left(1+e^{\alpha z}\right)^{2}},
$$




$$
\begin{aligned}
t^{(3)} & =2 a^{3} m \frac{\left(e^{2 \alpha z}-e^{\alpha z}\right)}{\left(1+e^{\alpha z}\right)^{3}} \\
\text { e } \quad t^{(4)} & =2 a^{-4} m \frac{\left(4 e^{2 \alpha z}-e^{3 \alpha z}-e^{\alpha z}\right)}{\left(1+e^{\alpha z}\right)^{4}} .
\end{aligned}
$$

Fazendo $w=e^{\alpha z} /\left(1+e^{\alpha z}\right)$ temos $\omega \sim B(m, m)$. Como $\alpha z=\log \left(\frac{w}{1-w}\right)$, então a função geradora de momentos de $\alpha z$ é

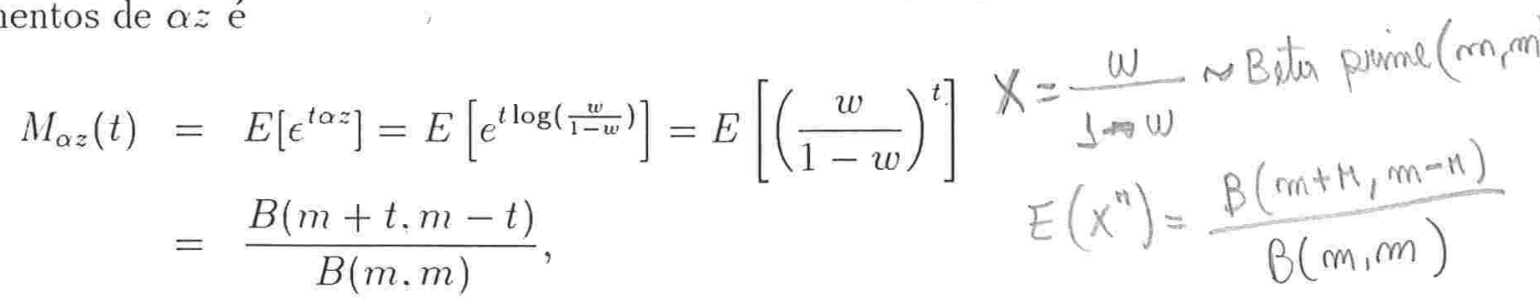

$\log$,

$$
E\left[\omega^{r}\left(\log \left(\frac{\omega}{1-\omega}\right)\right)^{s}\right]=\left.\frac{B^{(s)}(m+r+t, m-t)}{B(m, m)}\right|_{t=0},
$$

para $r=0,1,2,3,4, s=1.2,3,4$. onde $B^{(s)}(\cdot, \cdot)$ é a $s$-ésima derivada da funçào Beta. Utilizando este artifício, obtemos

$$
\begin{aligned}
& \delta_{(2,0,0,0,0)}=\frac{\alpha^{2} m^{2}}{(2 m+1)}, \quad \delta_{(0,0,0,1,0)}=\frac{\alpha^{4} m^{3}}{(2 m+3)(2 m+1)}, \\
& \delta_{(4,0,0,0,0)}=\frac{3 \alpha^{4} m^{4}}{(2 m+3)(2 m+1)}, \quad \delta_{(2,1,0,0,0)}=-\frac{\alpha^{4} m^{4}}{(2 m+3)(2 m+1)} \\
& \delta_{(0,0,0,1,2)}=\frac{2 \alpha^{2} m\left(m^{2}(m+1) \iota^{\prime}(m)-4 m-1\right)}{(2 m+3)(2 m+1)(m+1)}, \quad \delta_{(2,0,0,0,2)}=\frac{2 m\left(2+m^{2} \psi^{\prime}(m)\right)}{(2 m+1)} \\
& \delta_{(0,0,1,0,1)}=\frac{\alpha^{2} m^{2}}{(2 m+1)(m+1)} . \quad \delta_{(0,1,0,0,0)}=-\frac{\alpha^{2} m^{2}}{(2 m+1)} \\
& \delta_{(0,1,0,0,2)}=\frac{2\left(1-m^{2} \psi^{\prime}(m)\right)}{(2 m+1)}, \quad \delta_{(0,0,1,0,3)}=\frac{6\left(m^{2} \psi^{\prime}(m)-1\right)}{(2 m+1)(m+1)} \\
& \delta_{(1,1,0,0,1)}=\frac{\alpha^{2} m^{3}}{(2 m+1)(m+1)}, \quad \delta_{(3,0,0,0,1)}=\frac{-\alpha^{2} m^{2}(3 m+1)}{(2 m+1)(m+1)} \\
& \delta_{(0,0,0,1,4)}=\frac{2\left(6 m^{3}(m+1) \psi^{\prime}(m)^{2}-12 m(m+1) \psi^{\prime}(m)+m^{3}(m+1) \psi^{\prime \prime \prime}(m)+36\right)}{(2 m+3)(2 m+1)(m+1)} \\
& \delta_{(0,2,0,0,2)}=\frac{2 \alpha^{2}\left(m^{2}(m+1)^{2} \iota^{\prime}(m)-2 m^{2}-2 m-1\right)}{(2 m+3)(2 m+1)(m+1)} \\
& \delta_{(1,1,0,0,3)}=\frac{6 m\left(m^{2} \psi^{\prime}(m)-1\right)}{(2 m+1)(m+1)} .
\end{aligned}
$$




$$
\begin{aligned}
& \delta_{(4,0,0,0,4)}=\frac{6 m^{3}\left(6 m(m+1) \psi^{\prime}(m)^{2}+16 m(3 m+2) \psi^{\prime \prime}(m)+m(m+1) \psi^{\prime \prime \prime \prime}(m)+16\right)}{(2 m+3)(2 m+1)(m+1)} . \\
& \delta_{(3,0,0,0,3)}=\frac{-6 m^{2}\left(2+(3 m+1) \psi^{\prime}(m)\right)}{(2 m+1)(m+1)}, \quad \delta_{(1,0,1,0,0)}=-\frac{\alpha^{4} m^{3}}{(2 m+3)(2 m+1)}, \\
& \delta_{(2,1,0,0,2)}=-\frac{2 \alpha^{2} m^{2}\left(m^{2}(m+1) \psi^{\prime}(m)+2 m^{2}-m-1\right)}{(2 m+3)(2 m+1)(m+1)} . \\
& \delta_{(4,0,0,0,2)}=\frac{2 \alpha^{2} m^{3}\left(3 m(m+1) \psi^{\prime}(m)+4(3 m+2)\right)}{(2 m+3)(2 m+1)(m+1)} .
\end{aligned}
$$

\section{C.8 Exponencial potência}

Seja $y_{l} \sim E P\left(x_{l}^{\top} \beta, \phi^{2}, k\right)$ com função densidade da forma

$$
\pi\left(y_{l} ; \beta, \phi, \underset{, \sim, s}{K}\right)=\frac{C(k)}{\phi} \exp \left\{-\frac{1}{2}\left[\left|\frac{y_{l}-x_{l}^{\top} \beta}{\phi}\right|\right]^{2 /(1+k)}\right\}, y_{l} \in \mathbb{R},
$$

onde $C(k)^{-1}=\Gamma\left(1+\frac{1+k}{2}\right) 2^{1+(1+k) / 2}, \phi>0,-1<k \leq 1, \beta \in \mathbb{R}^{p}$. O logaritmo da função de verossimilhança tem a forma

$$
L(\beta, \phi)=-n \log C(k)-n \log \phi+\sum_{l=1}^{n} t\left(z_{l}\right)
$$

onde

$$
t_{l}=t\left(z_{l}\right)=-\frac{1}{2}\left|z_{l}\right|^{2 /(1+k)}
$$

As quatro primeiras derivadas da função $t$ com respeito a $z$ são

$$
\begin{aligned}
& t^{(1)}=-\frac{1}{(1+k)}|z|^{\frac{1-k}{1+k}} \operatorname{sinal}(z), \text { se }-1<k<1, \\
& \left.t^{(2)}=-\frac{(1-k)}{(1+k)^{2}}|z|\right)^{-\frac{2 k}{1+k}}, \text { se } k \leq 0, \\
& t^{(3)}=\frac{2(1-k) k}{(1+k)^{3}}|z|^{-\frac{1+3 k}{1+k}} \operatorname{sinal}(z), \text { se } k<-1 / 3, \text { e pow pusina nul } \\
& t^{(4)}=-\frac{2(1-k)(1+3 k) k}{(1+k)^{4}}|z|^{-\frac{2(1+2 k)}{(1+k)}}, \text { se } k<-1 / 2 .
\end{aligned}
$$

Considerando $z \sim E P(0,1, k)$

$$
E\left[z^{2 r}\right]=\left\{2^{r(1+k)} \Gamma\left(\left(\frac{1+k}{2}\right)(1+2 r)\right) / \Gamma\left(\frac{1+k}{2}\right) . \quad \text { se } r>-1 / 2 .\right.
$$


Os $\delta$ 's, para $-1<k<-1 / 2$. são dados da seguinte forma:

$$
\begin{aligned}
& \delta_{(2,0,0,0,0)}=\frac{\Gamma\left(\frac{3-k}{2}\right)}{2^{k-1}(1+k)^{2} \Gamma\left(\frac{k+1}{2}\right)}, \quad \delta_{(0,0,0,1,0)}=\frac{k(1-k) \Gamma\left(\frac{1-3 k}{2}\right)}{2^{2 k-1}(1+k)^{4} \Gamma\left(\frac{k+1}{2}\right)}, \\
& \delta_{(2,1,0,0,0)}=-\frac{(1-k)}{2^{2 k-1}(1+k)^{4}} \frac{\Gamma\left(\frac{3(1-k)}{2}\right)}{\Gamma\left(\frac{1+k}{2}\right)}, \sqrt{\delta_{(0,0,0,1,2)}}=-\frac{k(1+3 k)}{2^{k-2}(1+k)^{4}} \frac{\Gamma\left(\frac{3-k}{2}\right)}{\Gamma\left(\frac{k+1}{2}\right)}, \\
& \delta_{(2,0,0,0,2)}=\frac{k+3}{k+1}, V_{(0,0,1,0,1)}=\frac{k}{2^{k-2}(1+k)^{3}} \frac{\dot{\Gamma}\left(\frac{3-k}{2}\right)}{\Gamma\left(\frac{k+1}{2}\right)}, \\
& \delta_{(0,1,0,0,0)}=-\frac{\Gamma\left(\frac{3-k}{2}\right)}{2^{k-1}(1+k)^{2} \Gamma\left(\frac{k+1}{2}\right)}, \delta_{(0,1,0,0,2)}=-\frac{(1-k)}{(1+k)}, \\
& \delta_{(0,0,1,0,3)}=\frac{2 k(1-k)}{(1+k)^{2}}, \delta_{(1,1,0,0,1)}=\frac{(1-k)}{2^{k-1}(1+k)^{3}} \frac{\Gamma\left(\frac{3-k}{2}\right)}{\Gamma\left(\frac{1+k}{2}\right)}, \\
& \delta_{(4,0,0,0,2)}=\frac{1}{2^{k-3}(1+k)^{4}} \frac{\Gamma\left(\frac{1}{2}(7-k)\right)}{\Gamma\left(\frac{1+k}{2}\right)}, \delta_{(3,0,0,0,1)}=-\frac{1}{2^{k-2}(1+k)^{3}} \frac{\Gamma\left(\frac{5-k}{2}\right)}{\Gamma\left(\frac{1+k}{2}\right)}, \\
& \delta_{(0,0,0,1,4)}=-\frac{2(1-k) k(1+3 k)}{(1+k)^{3}}, \quad \delta_{(0,2,0,0,2)}=\frac{(1-k)^{2}}{2^{k-1}(1+k)^{2}} \frac{\Gamma\left(\frac{3-k}{2}\right)}{\Gamma\left(\frac{1+k}{2}\right)}, \\
& \delta_{(1,1,0,0,3)}=\frac{(1-k)(3+k)}{(1+k)}, \quad \delta_{(4,0,0,0,4)}=\frac{16}{(1+k)^{4}} \frac{\Gamma\left(\frac{9+k}{2}\right)}{\Gamma\left(\frac{1+k}{2}\right)} \text {, } \\
& \delta_{(3,0,0,0,3)}=-\frac{8}{(1+k)^{3}} \frac{\Gamma\left(\frac{7+k}{2}\right)}{\Gamma\left(\frac{1+k}{2}\right)}, \quad \delta_{(2,1,0,0,2)}=\frac{(k-1)}{2^{k-2}(1+k)^{4}} \frac{\Gamma\left(\frac{5-k}{2}\right)}{\Gamma\left(\frac{1+k}{2}\right)}, \\
& \delta_{(4,0,0,0,0)}=\frac{3(1-k)}{2^{2 k-1}(1+k)^{4}} \frac{\Gamma\left(\frac{3(1-k)}{2}\right)}{\Gamma\left(\frac{1+k}{2}\right)}, \quad \delta_{(1,0,1,0,0)}=\frac{k(k-1)}{2^{2 k-1}(1+k)^{4}} \frac{\Gamma\left(\frac{1-3 k}{2}\right)}{\Gamma\left(\frac{k+1}{2}\right)} .
\end{aligned}
$$




\section{Apêndice D}

Neste apêndice obtemos o vício até ordem $n^{-1}$ para o estimador de máxima verossimilhança $\hat{\phi}$ de $\phi$, utilizando a expressão geral de Cox e Snell (1968), apresentada na Seção 1.3. Também obtemos as diferenças entre as funções poder de teste da razão de verossimilhança, escore e de Wald para realizar as comparações entre funções de poder, sob a seqüência de hipóteses alternativas de Pitman definida na Seção 4.3.3.

\section{D.1 Vício de ordem $n^{-1}$ para $\widehat{\phi}$}

Da expressão geral de Cox e Snell apresentada na Seção 1.3 para o vício de ordem $n^{-1}$ dos estimadores de máxima verossimilhança pode ser escrito

$$
\left.B_{\widehat{\phi}}(\theta)=-\frac{1}{2} \kappa^{\phi \phi} \Sigma^{\prime} \kappa^{r s} \kappa_{\phi r s}\right)+\kappa^{\phi \phi^{2}}\left(\kappa_{\phi \phi}^{(\phi)}-\frac{1}{2} \kappa_{\phi \phi \phi}\right) .
$$

Substituindo os cumulantes dos modelos simétricos, temos

$$
\begin{aligned}
B_{\widehat{\phi}}(\theta)= & -\frac{1}{2 n\left(\delta_{(0,1,0,0,2)}-1\right)}\left(-\frac{1}{\phi}\right)(\delta_{(0,1,0,0,2)}+\underbrace{\left.2 \delta_{(0,1,0,0,0)}\right)} \\
& \Sigma\left(\Sigma^{\prime} \kappa^{r s}(r, s)_{l}\right) \\
& +\frac{\phi}{n\left(\delta_{(0,1,0,0,2)}-1\right)^{2}}\left(-2\left(\delta_{(0,1,0,0,2)}-1\right)+\frac{1}{2}\left(6 \delta_{(0,1,0,0,2)}+\delta_{(0,0,1,0,3)}-4\right)\right) .
\end{aligned}
$$

Como $-\Sigma^{\prime} \kappa^{r s}(r, s)_{l}$ é o elemento $(l, l)$ da matriz $\phi^{2} Z / \delta_{(2,0,0,0,0)}$, temos

$$
\begin{array}{rlr}
B_{\widehat{\phi}}(\theta)= & -\frac{\phi}{n}\left(\frac{\delta_{(0,1,0,0 ; 2)}+2 \delta_{(0,1,0,0,0)}}{2 \delta_{(2,0,0,0,0)}\left(\delta_{(0,1,0,0,2)}-1\right)}\right) \Sigma z_{l l} & \delta_{01000}=-\delta_{20000} \\
& +\frac{\phi}{n}\left(\frac{2 \delta_{(0,1,0,0,0)}+2 \delta_{(0,0,1,0,3)}}{\left(2 \delta_{(0,1,0,0,2)}-1\right)^{2}}\right) . &
\end{array}
$$

Como $\Sigma z l l=\operatorname{tr}(Z)=\operatorname{tr}\left(X\left(X^{\top} X^{\top}\right)^{-1} X^{\top}\right)=\operatorname{tr}\left(I_{p}\right)=p$, sendo $p$ o posto da matriz $X$ e $I_{p}$ a matriz identidade de ordem $p$. Assim, temos

$$
B_{\widehat{\phi}}(\theta)=\frac{\phi}{n}\left(r_{1}-r_{2} p\right)
$$


onde

$$
r_{1}=\frac{2 \delta_{(0,1,0,0,0)}+\delta_{(0.0 .1 .0,3)}}{\left(2 \delta_{(0,1,0,0,2)}-1\right)^{2}}
$$

$\mathrm{e}$

$$
r_{2}=\frac{\delta_{(0,1,0,0,2)}+2 \delta_{(0,1,0,0,0)}}{2 \delta_{(2,0,0,0,0)}\left(\delta_{(0,1,0,0,2)}-1\right)} .
$$

\section{D.2 Comparações entre funções de poder}

Com o objetivo de comparar as funções poder de testes definidos na Seção 4.3 .3 até ordem $n^{-1 / 2}$, sob uma sequência de alternativas de Pitman, chamamos de $P_{i}$ a soma das parcelas de ordem um e $n^{-1 / 2}$ da função poder $\bar{P}_{i}$, isto é,

$$
P_{i}=1-\left\{G_{1, \lambda}(x)+\sum_{j=0}^{3} b_{i j} G_{1+2 j, \lambda}(x)\right\} \text {, }
$$

para $i=1,2$ e 3 . Considerando os $b_{i j}$ definidos em (4.12) - (4.14), temos

$$
\begin{aligned}
P_{1}-P_{3}= & \left(b_{30}-b_{10}\right) G_{1, \lambda}(x)+\left(b_{31}-b_{11}\right) G_{3, \lambda}(x) \\
& +\left(b_{32}-b_{12}\right) G_{5, \lambda}(x)+b_{33} G_{7, \lambda}(x) .
\end{aligned}
$$

De $(4.12)$ - (4.14), temos $b_{30}=b_{10}, b_{31}=b_{11}-b_{32}$ e $b_{33}=b_{12}$ e, conseqüentemente,

$$
\begin{aligned}
P_{1}-P_{3} & =-b_{32} G_{3, \lambda}(x)+\left(b_{32}-b_{12}\right) G_{5, \lambda}(x)+b_{12} G_{7, \lambda}(x) \\
& =b_{12}\left(G_{7, \lambda}(x)-G_{5, \lambda}(x)\right)+b_{32}\left(G_{5, \lambda}(x)-G_{3, \lambda}(x)\right) .
\end{aligned}
$$

Chamemos de

$$
\begin{aligned}
& m(1, \lambda, x)=G_{7, \lambda}(x)-G_{5, \lambda}(x), \\
& n(1, \lambda, x)=G_{5, \lambda}(x)-G_{3, \lambda}(x), \\
& k_{1}=b_{12}=\frac{n}{3}\left(\delta_{(1,1,0,0,3)}+1\right) \Delta^{3} \mathrm{e} \\
& k_{2}=b_{32}=\frac{\left.\delta_{(1,1,0,0,3)}+1\right) \Delta}{\left(\delta_{(2,0,0,0,2)}-1\right)} .
\end{aligned}
$$

Assim,

$$
P_{1}-P_{3}=k_{1} m(1, \lambda, x)+k_{2} n(1, \lambda, x)
$$

De forma análoga,

$$
\begin{aligned}
P_{2}-P_{3}= & \left(b_{30}-b_{20}\right) G_{1, \lambda}(x)+\left(b_{31}-b_{21}\right) G_{3, \lambda}(x) \\
& +\left(b_{32}-b_{22}\right) G_{5, \lambda}(x)+\left(b_{33}-b_{23}\right) G_{7, \lambda}(x) .
\end{aligned}
$$


De (4.12) - (4.14) temos que

$$
\begin{aligned}
& b_{30}=b_{20}, \quad b_{31}-b_{2}=-\frac{1}{2} \kappa^{\phi, \phi}\left(\kappa_{\phi, \phi, \phi}+\kappa_{\phi \phi \phi}\right) \xi, \\
& b_{32}-b_{22}=\frac{1}{2}\left(\kappa_{\phi, \phi, \phi} \kappa^{\phi, \phi} \xi+\kappa_{\phi \phi \phi} \kappa^{\phi, \phi} \xi+\kappa_{\phi, \phi \phi} \xi^{3}\right) \\
& =\frac{1}{2}\left(-3 \kappa_{\phi, \phi \phi} \kappa^{\phi \phi} \xi+\kappa_{\phi, \phi \phi} \xi^{3}\right) \quad \mathrm{e} \\
& b_{33}-b_{23}=\frac{1}{6}\left(\kappa_{\phi, \phi, \phi}+\kappa_{\phi \phi \phi}\right) \xi^{3}=-\frac{1}{2} \kappa_{\phi, \phi \phi} \xi^{3} .
\end{aligned}
$$

Logo

$$
\begin{aligned}
P_{2}-P_{3}= & \left(\frac{3}{2} \kappa_{\phi, \phi \phi} \kappa^{\phi, \phi} \xi\right) G_{3, \lambda}(x) \\
& +\frac{1}{2}\left(\kappa_{\phi, \phi \phi} \xi^{3}-3 \kappa_{\phi, \phi \phi} \kappa^{\phi, \phi} \xi\right) G_{5, \lambda}(x)-\frac{1}{2} \kappa_{\phi, \phi \phi} \xi^{3} G_{7, \lambda}(x) .
\end{aligned}
$$

Assim

$$
\begin{aligned}
P_{2}-P_{3}= & -\frac{11}{2} \kappa_{\phi, \phi \phi} \xi^{3}\left(G_{7, \lambda}(x)-G_{5, \lambda}(x)\right) \\
& -\frac{3}{2} \kappa_{\phi, \phi \phi} \kappa^{\phi, \phi} \xi\left(G_{5, \lambda}(x)-G_{3, \lambda}(x)\right) .
\end{aligned}
$$

Conseqüentemente,

$$
P_{2}-P_{3}=k_{3} m(1, \lambda, x)+k_{4} n(1, \lambda, x),
$$

onde

$$
\begin{aligned}
& k_{3}=-\frac{n}{2}\left(4 \delta_{(0,1,0,0,2)}+\delta_{(0,0,1,0,3)}-2\right) \Delta^{3} \mathrm{e} \\
& k_{4}=-\frac{3}{2} \frac{\left(4 \delta_{(0,1,0,0,2)}+\delta_{(0,0,1,0,3)}-2\right) \Delta}{\left(\delta_{(2,0,0,0,2)}-1\right)} .
\end{aligned}
$$

Finalmente,

$$
\begin{aligned}
P_{2}-P_{1}= & \left(b_{10}-b_{20}\right) G_{1, \lambda}(x)+\left(b_{11}-b_{21}\right) G_{3, \lambda}(x) \\
& +\left(b_{12}-b_{22}\right) G_{5, \lambda}^{\prime}(x)-b_{23} G_{7, \lambda}(x) .
\end{aligned}
$$

\section{Como}

$$
\begin{aligned}
& b_{10}=b_{20}, \quad b_{21}=b_{11}+\frac{1}{2} \kappa_{\phi \phi \phi} \kappa^{\phi, \phi} \xi, \\
& b_{12}-b_{22}=\frac{1}{6}\left(3 \kappa_{\phi \phi \phi} \kappa^{\phi, \phi} \xi-\kappa_{\phi \phi \phi} \xi^{3}\right) \quad \mathrm{e} \\
& b_{23}=-\frac{1}{6} \kappa_{\phi \phi \phi} \xi^{3},
\end{aligned}
$$


temos

$$
\begin{aligned}
P_{2}-P_{1}= & -\frac{1}{2} \kappa_{\phi \phi \phi} \kappa^{\phi, \phi} G_{3 . \lambda}(x) \\
& +\frac{1}{6}\left(3 \kappa_{\phi \phi \phi} \kappa^{\phi, \phi} \xi-\kappa_{\phi \phi \phi} \xi^{3}\right) G_{5, \lambda}(x) \\
& +\frac{1}{6} \kappa_{\phi \phi \phi} \xi^{3} G_{\bar{\tau}, \lambda}(x)
\end{aligned}
$$

Assim

$$
P_{2}-P_{1}=k_{5} m(1, \lambda, x)+k_{6} n(1, \lambda, x),
$$

onde

$$
\begin{aligned}
& k_{5}=-\frac{n}{6}\left(6 \delta_{(0,1,0,0,2)}+\delta_{(0,0,1,0,3)}-4\right) \Delta^{3} \mathrm{e} \\
& k_{6}=-\frac{1}{2} \frac{\left(6 \delta_{(0,1,0,0.2)}+\delta_{(0,0,1,1,3)}-4\right) \Delta}{\left(\delta_{(2,0,0,0,2)}-1\right)} .
\end{aligned}
$$

A seguir, determinamos o sinal de $m(q, \lambda, x)$ e $n(q, \lambda, x)$ para $\lambda$ definido em (4.15) e $q$ fixos.

Seja $G_{r, \lambda}(x)$ a função distribuição de uma variável aleatória qui-quadrado não-central com $r$ graus de liberdade e parâmetro de não-centralidade $\lambda$. Para $r$ e $\lambda$ fixos é possível expressar $G_{r, \lambda}(x)$, para $x>0$, na forma (Johnson e Kotz, 1970b, p.132)

$$
G_{r, \lambda}(x)=\left[\sum_{j=0}^{\infty} \frac{\left(\frac{1}{2} \lambda\right)^{j}}{j !} e^{-\frac{1}{2} \lambda}\right] G_{r+2 j}(x)
$$

onde $G_{r+2 j}(x)$ é a função distribuição de uma variável aleatória $\chi^{2}$ central com $r+2 j$ graus de liberdade. Como $m(q, \lambda, x)=G_{q+6, \lambda}(x)-G_{q+4, \lambda}(x)$, para todo $x>0$, com $\lambda$ e $q$ fixos, temos que

$$
m(q, \lambda, x)=\sum_{j=0}^{\infty}\left(\frac{\left(\frac{1}{2} \lambda\right)^{j}}{j !} e^{-\frac{1}{2} \lambda}\right)\left\{G_{q+2 j+6}(x)-G_{q+2 j+4}(x)\right\} .
$$

Como $\frac{\left(\frac{1}{2} \lambda\right)^{j}}{j !} e^{-\frac{1}{2} \lambda}>0$, para todo $j>0$, basta observar o sinal de $G_{q+2 j+6}(x)-G_{q+2 j+4}(x)$ para determinar o sinal de $m(q, \lambda, x)$. Usando a relação de recorrência $G_{m+2}(x)=G_{m}(x)-$ $\frac{2 x}{m} g_{m}(x)$ temos

$$
G_{q+2 j+6}(x)-G_{q+2 j+4}(x)=-\frac{2 x}{q+2 j+4} g_{q+2 j+4}(x),
$$

para todo $x>0, \lambda$ e $q$ fixos. Como $g_{q+2 j+4}(x)>0$, para todo $x>0$ e $j>0$, temos $G_{q+2 j+6}(x)-G_{q+2 j+4}(x)<0$, para todo $x>0$. De forma análoga, temos que para todo 
$x>0, \lambda$ e $q$ fixos.

$$
\begin{aligned}
n(q \cdot \lambda, x) & =G_{q+4, \lambda}(x)-G_{q+2, \lambda}(x) \\
& =\sum_{j=0}^{\infty}\left(\frac{\left(\frac{1}{2} \lambda\right)^{j}}{j !} e^{-\frac{1}{2}}\right)\left\{G_{q+2 j+4}(x)-G_{q+2 j+2}(x)\right\} \\
& =-\sum_{j=0}^{\infty}\left(\frac{\left(\frac{1}{2} \lambda\right)^{j}}{j !} e^{-\frac{1}{2} \lambda}\right)\left\{\frac{2 x}{q+2 j+2} g_{q+2 j+2}(x)\right\} .
\end{aligned}
$$

Estes dois resultados demonstram que $m(q, \lambda, x)<0$ e $n(q, \lambda, x)<0$ para todo $x>0, \lambda$ e $q$ fixos. 


\section{Apêndice E}

Neste apêndice apresentamos a obtenção das quantidades $A_{1, \beta \phi}$ e $A_{2, \beta \phi}$ referentes ao teste da hipótese $H_{0}: \beta=\beta^{(0)}$ versus $H_{1}: \beta \neq \beta^{(0)}$ em modelos não lineares da família exponencial, com parâmetros de dispersão $\phi^{-1}$ desconhecido mas o mesmo para todas observações (vide Capítulo 5).

\section{E.1 Obtenção de alguns cumulantes conjuntos de de- rivadas do logaritmo da função de verossimilhan- ça em modelos não lineares da família exponen- cial}

O logaritmo da função de verossimilhança total do vetor de parâmetros $\left(\beta^{\top}, \phi\right)$ dos modelos não lineares na família exponencial na Seção 5.1 tem a forma

$$
L=L(\beta, \phi)=\sum_{l}\left(\phi\left\{y_{l} \theta_{l}-b\left(\theta_{l}\right)+c\left(y_{l}\right)\right\}+a\left(y_{l}, \phi\right)\right),
$$

onde $\Sigma$ indica o somatório sobre os dados. Assumindo que $a\left(y_{l}, \phi\right)=d_{1}(\phi)+d_{2}\left(y_{l}\right)$, temos que as três primeiras derivadas do logaritmo da função de verossimilhança em relação a $\beta$ são

$$
\begin{aligned}
\frac{\partial L}{\partial \beta_{r}}= & \phi \sum_{l}\left\{(y-\mu) w \frac{\mathrm{d} \eta}{\mathrm{d} \mu}\right\}_{l}(r)_{l} \\
\frac{\partial^{2} L}{\partial \beta_{r} \partial \beta_{s}}= & \phi \sum_{l}\left\{\left[(y-\mu) \frac{\mathrm{d}^{2} \theta}{\mathrm{d} \eta^{2}}-w\right](r, s)+\left(y_{l}-\mu\right) w \frac{\mathrm{d} \eta}{\mathrm{d} \mu}(r s)\right\}_{l}, \\
\frac{\partial^{3} L}{\partial \beta_{r} \partial \beta_{s} \partial \beta_{t}}= & \phi \sum_{l}\left\{\left[(y-\mu) \frac{\mathrm{d}^{3} \theta}{\mathrm{d} \eta^{3}}-2 \frac{\mathrm{d}^{2} \theta}{\mathrm{d} \eta^{2}} \frac{\mathrm{d} \mu}{\mathrm{d} \eta}-\frac{\mathrm{d}^{2} \mu}{\mathrm{d} \eta^{2}} \frac{\mathrm{d} \eta}{\mathrm{d} \mu}\right]\right. \\
& +\left[(y-\mu) \frac{\mathrm{d}^{2} \theta}{\mathrm{d} \eta^{2}}-w\right][(r, s t)+(s, r t)+(t, r s)] \\
& \left.+\left[(y-\mu) \frac{\mathrm{d} \theta}{\mathrm{d} \eta}(r s t)\right]\right\}_{l}
\end{aligned}
$$


onde $w=(\mathrm{d} \mu / \mathrm{d} \eta)^{2} V^{-1}, \operatorname{com} V=\mathrm{d} \mu / \mathrm{d} \theta$, e

$$
\begin{aligned}
(r)_{l} & =\frac{\partial \eta_{l}}{\partial \beta_{r}}, \quad(r, s)_{l}=\left(\frac{\partial \eta_{l}}{\partial \beta_{r}}\right)\left(\frac{\partial \eta_{l}}{\partial \beta_{s}}\right) . \\
(r s)_{l} & =\frac{\partial^{2} \eta_{l}}{\partial \beta_{r} \partial \beta_{s}}, \quad(r, s t)_{l}=\left(\frac{\partial \eta_{l}}{\partial \beta_{r}}\right)\left(\frac{\partial^{2} \eta_{l}}{\partial \beta_{s} \partial \beta_{t}}\right) \\
\mathrm{e} \quad(r s t)_{l} & =\frac{\partial^{3} \eta_{l}}{\partial \beta_{r} \partial \beta_{s} \partial \beta_{t}} .
\end{aligned}
$$

Tomando as esperanças das duas últimas expressões, encontram-se os cumulantes

$$
\begin{aligned}
& \kappa_{r s}=-\kappa_{r, s}=-\phi \sum_{l} w_{l}(r, s)_{l}, \\
& \kappa_{r s t}=-\phi \sum_{l}\{(f+2 g)(r, s, t)+w[(r, s t)+(s, r t)+(t, r s)]\}_{l},
\end{aligned}
$$

onde

$$
f=\frac{1}{V} \frac{\mathrm{d}^{2} \mu}{\mathrm{d} \eta^{2}} \cdot \frac{\mathrm{d} \mu}{\mathrm{d} \eta}
$$

$\mathrm{e}$

$$
g=\frac{1}{V} \frac{\mathrm{d}^{2} \mu}{\mathrm{d} \eta^{2}} \frac{\mathrm{d} \mu}{\mathrm{d} \eta}-\frac{1}{V^{2}} \frac{\mathrm{d} V}{\mathrm{~d} \mu}\left(\frac{\mathrm{d} \mu}{\mathrm{d} \eta}\right)^{3} .
$$

Agora,

$$
\begin{aligned}
\kappa_{r, s t}= & E\left\{\frac{\partial L}{\partial \beta_{r}} \frac{\partial^{2} L}{\partial \beta_{s} \partial \beta_{t}}\right\} \\
= & \phi^{2} E\left\{\sum _ { l } [ ( y - \mu ) w \frac { \mathrm { d } \eta } { \mathrm { d } \mu } ] _ { l } ( r ) _ { l } \sum _ { j } \left\{\left[(y-\mu) \frac{\mathrm{d}^{2} \theta}{\mathrm{d} \eta^{2}}-w\right](s, t)\right.\right. \\
& \left.\left.+(y-\mu) w \frac{\mathrm{d} \eta}{\mathrm{d} \mu}(s t)\right\}_{j}\right\} \\
= & \phi \sum_{l}\left\{\frac{\mathrm{d} \mu}{\mathrm{d} \eta} \frac{\mathrm{d}^{2} \theta}{\mathrm{d} \eta^{2}}(r, s, t)+w(r, s t)\right\}_{l} .
\end{aligned}
$$

Assim, chega-se a

$$
\kappa_{r, s t}=o \sum_{l}\{g(r, s, t)+w(r, s t)\}_{l} .
$$

De forma análoga, vem

$$
\begin{aligned}
\kappa_{r, s, t} & =E\left\{\frac{\partial L}{\partial \beta_{r}} \frac{\partial L}{\partial \beta_{s}} \frac{\partial L}{\partial \beta_{t}}\right\} \\
& =\phi^{3} E\left(\sum_{l}\left\{(y-\mu)^{3} w^{3}\left(\frac{\mathrm{d} \eta}{\mathrm{d} \mu}\right)^{3}(r, s, t)\right\}_{l}\right)
\end{aligned}
$$


Como na família exponencial. $E\left((y-\mu)^{3}\right)=V / \phi^{2}(\mathrm{~d} V / \mathrm{d} \mu)$, temos

$$
\kappa_{r, s, t}=\varnothing \sum_{l}(f-g)_{l}(r, s, t)_{l}
$$

$\mathrm{e}$

$$
\kappa_{r, s, t, u}=\phi \sum_{l} b_{l}(r, s, t, u)_{l},
$$

onde

$$
b=\frac{1}{V^{3}}\left(\frac{\mathrm{d} \mu}{\mathrm{d} \eta}\right)^{4}\left[\left(\frac{\mathrm{d} V}{\mathrm{~d} \mu}\right)^{2}+V \frac{\mathrm{d}^{2} V}{\mathrm{~d} \mu^{2}}\right] .
$$

Para obter os cumulantes para parâmetros em relação a $\phi$ temos que

$$
\begin{aligned}
& \frac{\partial L}{\partial \phi}=\sum_{l}\left\{y_{l} \theta_{l}-b\left(\theta_{l}\right)+c\left(y_{l}\right)\right\}+n d_{1}^{\prime}(\phi), \\
& \frac{\partial^{2} L}{\partial \phi^{2}}=n d_{1}^{\prime \prime}(\phi) \quad \text { e } \quad \frac{\partial^{3} L}{\partial \phi^{3}}=n d_{1}^{\prime \prime \prime}(\phi),
\end{aligned}
$$

onde $d_{1}^{r}(\phi)=\partial^{r} \mathrm{~d}(\phi) / \partial \phi^{r}$. Tomando as esperanças das duas últimas expressões, encontram-se os cumulantes

$$
\kappa_{\phi \phi}=-\kappa_{\phi, \phi}=n d_{1}^{\prime \prime}(\phi) \quad \text { e } \quad \kappa_{\phi \phi \phi}=n d_{1}^{\prime \prime \prime}(\phi) .
$$

Agora, os cumulantes mistos são

$$
\begin{aligned}
\kappa_{r \phi} & =E\left[\frac{\partial^{2} L}{\partial \beta_{r} \partial \phi}\right]=0 \\
\kappa_{r \phi \phi} & =E\left[\frac{\partial^{3} L}{\partial \beta_{r} \partial \phi^{2}}\right]=E\left[\frac{\partial}{\partial \beta_{r}}\left(n d_{1}^{\prime \prime}(\phi)\right)\right]=0 \quad \mathrm{e} \\
\kappa_{\phi \mu r} & r_{\text {r. } \phi \phi}=E\left[\frac{\partial^{3} L}{\partial \phi \partial \beta_{r} \partial \beta_{s}}\right]=-\sum w_{l}(r, s)_{l} .
\end{aligned}
$$

É fácil ver que $\kappa_{\phi \phi}^{(\phi)}=\partial \kappa_{\phi \phi} / \partial \phi=n d_{1}^{\prime \prime \prime}(\phi), \kappa_{r s}^{(\phi)}=\partial \kappa_{r s} / \partial \phi=-\Sigma w_{l}(r, s)_{l}$ e $\kappa_{\phi \phi}^{(t)}=0$.

Os cumulantes satisfazem certas relações dadas na Seção 1.2.1, como $\kappa_{\phi \phi}^{(\phi)}=\kappa_{\phi \phi \phi}+$ $\kappa_{\phi \phi, \phi}, \kappa_{r s}^{(\phi)}=\kappa_{r s \phi}+\kappa_{r s, \phi}, \kappa_{\phi \phi}^{(t)}=\kappa_{\phi \phi t}+\kappa_{\phi \phi, t}$ que facilitam a obtenção de cumulantes. Chegamos a

$$
\begin{array}{ll} 
& \kappa_{\phi \phi, \phi}=\kappa_{r s, \phi}=\kappa_{\phi \phi, t}=\kappa_{r \phi}^{(\phi)}=\kappa_{r \phi}^{(r)}=\kappa_{r \phi, \phi}=0 \\
\text { e } \quad \kappa_{r \phi, t}=\sum w_{l}(r, t)_{l} .
\end{array}
$$

Considerando outras relações definidas na Seção 1.2.1, temos

$$
\begin{aligned}
\kappa_{\phi, r, s} & =2 \kappa_{r s \phi}-\kappa_{r s}^{(\phi)}=-\Sigma w_{l}(r, s)_{l}, \\
\kappa_{\phi, \phi t} & =2 \kappa_{t \phi \phi}-\kappa_{\phi \phi}^{(t)}=0,
\end{aligned}
$$




$$
\begin{aligned}
\kappa_{r s \phi \phi}= & E\left[\frac{\partial^{4} L}{\partial \beta_{r} \partial \beta_{s} \partial^{2} \phi}\right] \\
= & E\left[\frac{\partial}{\partial \phi}\left(\sum\left\{\left((y-\mu) \frac{\partial^{2} \phi}{\partial \eta}-w\right)(r, s)+(y-\mu) w \frac{\partial \eta}{\partial \mu}(r s)\right\}\right)\right]=0, \\
\kappa_{r s, \phi \phi}= & E\left[\frac{\partial^{2} L}{\partial \beta_{r} \partial \beta_{s}} \frac{\partial^{2} L}{\partial \phi^{2}}\right]-\kappa_{r s} \kappa_{\phi \phi} \\
= & E\left[\phi \sum\left\{\left((y-\mu) \frac{\partial^{2} \theta}{\partial \eta^{2}}-w\right)(r, s)+(y-\mu) w \frac{\partial \eta}{\partial \mu}(r s)\right\}_{l}\left(n l_{1}^{\prime \prime}(\phi)\right)\right] \\
& -n d_{1}^{\prime \prime}(\phi)\left(-\phi \sum w_{l}(r, s)_{l}\right)=0, \\
\mathrm{e} \quad \kappa_{r, s, \phi \phi}= & \kappa_{r s \phi \phi}-\kappa_{\phi \phi s}^{(r)}-\kappa_{\phi \phi r}^{(s)}+\kappa_{\phi \phi}^{(r s)}-\kappa_{\phi \phi, r s}=0, \\
2 \kappa_{r \phi, s \phi}= & -3 \kappa_{r s \phi \phi}+2 \Sigma_{(4)} \kappa_{r \phi \phi}^{(s)}-\kappa_{r s}^{(\phi \phi)}-\kappa_{\phi \phi}^{(r s)}+\Sigma_{(3)} \kappa_{r s, \phi \phi} .
\end{aligned}
$$

Como $\kappa_{r \phi, s \phi}=E\left[\partial^{2} L / \partial \beta_{r} \partial \phi \partial^{2} L / \partial \beta_{s} \partial \phi\right]-\kappa_{r \phi} \kappa_{s \phi}=\phi^{-1} \sum w_{l}(r, s)_{l}$, temos

$$
\kappa_{r, s, \phi, \phi}=2 \phi^{-1} \sum w_{l}(r, s)_{l} .
$$

E.2 Obtenção de $A_{1, \beta \phi}$ e $A_{2, \beta \phi}$ para o teste das hipóteses $H_{0}: \beta_{1}=\beta_{1}^{(0)}$ versus $H_{1}: \beta_{1} \neq \beta_{1}^{(0)}$ em modelos não lineares de família exponencial

Devido à ortogonalidade global de $\beta$ e $\phi$, as quantidades $A_{1, \beta \phi}$ e $A_{2, \beta \phi}$ referentes ao teste da hipótese $H_{0}: \beta_{1}=\beta_{1}^{(0)}$ versus $H_{1}: \beta_{1} \neq \beta_{1}^{(0)}$ em modelos não lineares da família exponencial podem ser obtidas dos resultados da Seção 3.3.2. De (3.21) e (3.22) vem

$$
\begin{aligned}
A_{1, \beta \phi}= & -6 \Sigma^{\prime} \kappa_{i j \phi} \kappa_{\phi, s, t} a_{\phi \phi} a_{i j} m_{s t} \\
& -6 \Sigma^{\prime} \kappa_{\phi \phi \phi} \kappa_{\phi, s, t} a_{\phi \phi}^{2} m_{s t} \\
& +12 \Sigma^{\prime} \kappa_{i, \phi, k} \kappa_{r \phi t} a_{\phi \phi} a_{k t} m_{i r} \\
& -12 \Sigma^{\prime} \kappa_{i, j, \phi} \kappa_{r s \phi} a_{\phi \phi} a_{j s} m_{i r} \\
& -6 \Sigma^{\prime} \kappa_{i, j, \phi, \phi} a_{\phi \phi} m_{i j}
\end{aligned}
$$

e

$$
\begin{aligned}
A_{2, \beta \phi}= & -3 \Sigma^{\prime} \kappa_{i, j, \phi} \kappa_{\phi, s, t} a_{\phi \phi} m_{i j} m_{s t} \\
& -6 \Sigma^{\prime} \kappa_{i, j, \phi} \kappa_{r, s, \phi} a_{\phi \phi} m_{i r} m_{j s} .
\end{aligned}
$$

Consideremos inicialmente, a obtenção de $A_{1, \beta \phi}$. Invertendo a ordem das somas e rearranjando os termos, obtemos de (E.1)

$$
A_{1, \beta \phi}=-\frac{6}{n d_{1}^{\prime \prime}(\phi)} \Sigma\left(-\Sigma^{\prime} w_{l}(i)_{l}(j)_{l} a_{i j}\right)\left(-\Sigma^{\prime} w_{k}(s)_{k}(t)_{k} m_{s t}\right)
$$




$$
\begin{aligned}
& -\frac{6}{n^{2} d_{1}^{\prime \prime}(\phi)^{2}} n d_{1}^{\prime \prime \prime}(\phi) \Sigma\left(-\Sigma^{\prime} w_{l}(s)_{l}(t)_{l} m_{s t}\right) \\
& -\frac{12}{n d_{1}^{\prime \prime}(\phi)} \Sigma\left(-\Sigma^{\prime} w_{l}(i)_{l}(r)_{k} m_{i r}\right)\left(-\Sigma^{\prime} w_{k}(k)_{l}(t)_{k} a_{k t}\right) \\
& +\frac{12}{n d_{1}^{\prime \prime}(\phi)} \Sigma\left(-\Sigma^{\prime} w_{l}(i)_{l}(r)_{k} m_{i r}\right)\left(-\Sigma^{\prime} w_{k}(j)_{l}(s)_{k} a_{j s}\right) \\
& +\frac{12}{\phi n d_{1}^{\prime \prime}(\phi)} \Sigma\left(\Sigma^{\prime} w_{l}(i)_{l}(j)_{l} m_{i j}\right) .
\end{aligned}
$$

Sejam as matrizes $Z^{*}=X^{*}\left(X^{*} W X^{* \top}\right)^{-1} X^{* \top}$ e $Z_{2}^{*}=X_{2}^{*}\left(X_{2}^{*} W X_{2}^{* \top}\right)^{-1} X_{2}^{* \top}$. O termo da forma $\Sigma^{\prime} m_{i j}(i)_{l}(j)_{k}$ é o elemento $(l, k)$ da matriz $X^{*} M X^{* \top}$. Como $M=K^{-1}-A$ (vide Seção 5.2), temos

$$
X^{\top} M X^{* \top}=X^{*}\left(K^{-1}-A\right) X^{* \top}=X^{*} K^{-1} X^{* \top}-X^{*} A^{* \top} X^{*^{\top}}=\frac{1}{\phi}\left(Z^{*}-Z_{2}^{*}\right) .
$$

Assim,

$$
\Sigma^{\prime} m_{i j}(i)_{l}(j)_{l}=\frac{1}{\phi}\left(z_{l l}^{*}-z_{2 l l}^{*}\right)
$$

$\mathrm{e}$

$$
\Sigma^{\prime} a_{i j}(i)_{l}(j)_{l}=\frac{1}{\phi} z_{2 l l}^{*}
$$

Substituindo em (E.3) reduz a

$$
\begin{aligned}
A_{1, \beta \phi}= & -\frac{6}{n d_{(2)}} \sum w_{l}\left(z_{l l}^{*}-z_{2 l l}^{*}\right) w_{k} z_{2 k k}^{*} \\
& +\frac{6 d_{(3)}}{n d_{(2)}^{2}} \sum w_{l}\left(z_{l l}^{*}-z_{2 l l}^{*}\right)+\frac{12}{n d_{(2)}} \Sigma w_{l}\left(z_{l l}^{*}-z_{2 l l}^{*}\right),
\end{aligned}
$$

onde $d_{(2)}$ e $d_{(3)}$ estão definidos na Seção 5.2. Escrevendo em notação matricial, vem

$$
\begin{aligned}
A_{1, \beta \phi}= & -\frac{6}{n d_{(2)}} \operatorname{tr}\left(W\left(Z^{*}-Z_{2}^{*}\right)\right) \operatorname{tr}\left(W Z_{2}^{*}\right)+\frac{6 d_{(3)}}{n d_{(2)}^{2}} \operatorname{tr}\left(W\left(Z^{*}-Z_{2}^{*}\right)\right) \\
& +\frac{12}{n d_{(2)}} \operatorname{tr}\left(W\left(Z^{*}-Z_{2}^{*}\right)\right),
\end{aligned}
$$

mas

$$
\begin{aligned}
\operatorname{tr}\left(W Z_{2}^{*}\right) & =\operatorname{tr}\left(W X_{2}^{*}\left(X_{2}^{*} W X_{2}^{* \top}\right)^{-1} X_{2}^{* \top}\right) \\
& =\operatorname{tr}\left(X_{2}^{* \top} W X_{2}^{*}\left(X_{2}^{*} W X_{2}^{* \top}\right)^{-1}\right)=\operatorname{tr}\left(I_{p-q}\right)=p-q
\end{aligned}
$$

sendo $(p-q)$ o posto da matriz $X_{2}^{*}$ e $I_{p-q}$ a matriz identidade de ordem $p-q$. Da mesma foma, nota-se também que $\operatorname{tr}\left(W\left(Z^{*}-Z_{2}^{*}\right)\right)=q$. Assim, temos

$$
A_{1,3 \phi}=\frac{6 q}{n d_{(2)}}\left(d_{(3)}-(p-q-2) d_{(2)}\right) .
$$


A obtenção de $A_{2, \beta}$ é conduzida de maneira análoga. De (E.2) vem

$$
\begin{aligned}
A_{2, \beta \phi}= & \frac{3}{n d_{(2)}}\left\{\left(\Sigma w_{l}\left(z_{l l}^{*}-z_{2 l l}^{*}\right)\right)^{2}\right. \\
& \left.+2 \Sigma\left(w_{l} w_{k}\left(z_{l k}^{*}-z_{2 l k}^{*}\right)\left(z_{l k}^{*}-z_{2 l k}^{*}\right)\right)\right\} .
\end{aligned}
$$

Escrevendo em notação matricial,

$$
A_{2, \beta \phi}=\frac{3}{n d_{(2)}}\left\{\operatorname{tr}\left(W\left(Z^{*}-Z_{2}^{*}\right)\right)^{2}+2 \operatorname{tr}\left(W\left(Z^{*}-Z_{2}^{*}\right) W\left(Z^{*}-Z_{2}^{*}\right)\right)\right\} .
$$

Mas $\operatorname{tr}\left(W\left(Z^{*}-Z_{*}^{2}\right) W\left(Z^{*}-Z_{2}^{*}\right)\right)=q$. Assim, temos

$$
A_{2, \beta \phi}=\frac{3 q}{n d_{(2)}}(q+2) .
$$




\section{Apêndice F}

Neste apêndice detalhamos a obtenção dos cumulantes conjuntos de derivadas do logaritmo da função de verossimilhança para os modelos da família exponencial biparamétrica. Estes cumulantes estão envolvidos na construção dos coeficientes $A_{1}, A_{2}$ e $A_{3}$, definidos na Seção 1.2.1 para a obtenção do fator de correção tipo Bartlett para a estatística escore.

\section{F.1 Obtenção de alguns cumulantes conjuntos de de- rivadas do logaritmo da função de verossimilhança em modelos exponenciais biparamétricos}

O logaritmo da função de verossimilhança total para o vetor de parâmetros $\theta=(\mu, \nu)$ para modelos da família exponencial biparamétrica introduzido na Seção 7.1, dados $y_{1}, \ldots, y_{n}$, é da forma

$$
L(\theta)=\sum_{l=1}^{n} t\left(y_{l} ; \mu, \nu\right)
$$

onde

$$
t_{l}=t\left(y_{l} ; \mu, \nu\right)=\alpha_{1} d_{1}\left(y_{l}\right)+\alpha_{2} d_{2}\left(y_{l}\right)-\rho+v\left(y_{l}\right),
$$

para $l=1,2, \ldots, n$, sendo $\alpha_{1}=\alpha_{1}(\mu, \nu), \alpha_{2}=\alpha_{2}(\mu, \nu)$ e $\rho=\rho(\mu, \nu)$. Consideramos a seguinte notação para as derivadas das funções $\alpha_{1}, \alpha_{2}$ e $\rho$ :

$$
\alpha_{r}^{(i, j)}=\frac{\partial^{i+j} \alpha_{r}}{\partial \mu^{i} \partial \nu^{j}} \quad \text { e } \quad \rho^{(i, j)}=\frac{\partial^{i+j} \rho}{\partial \mu^{i} \partial \nu^{j}},
$$

para $i, j=0,1,2,3,4$, e $r=1,2$. As derivadas em relação a $\mu$ e $\nu$ da função $L(\theta)$ são dadas, respectivamente, por

$$
U_{\mu}=\sum_{l=1}^{n} \frac{\partial t}{\partial \mu} \quad \text { e } \quad U_{\nu}=\sum_{l=1}^{n} \frac{\partial t}{\partial \nu}
$$

onde

$$
\begin{aligned}
& \frac{\partial t}{\partial \mu}=a_{1}^{(1,0)} d_{1}(y)+\alpha_{2}^{(1,0)} d_{2}(y)-\rho^{(1,0)} \mathrm{e} \\
& \frac{\partial t}{\partial \nu}=a_{1}^{(0,1)} d_{1}(y)+\alpha_{2}^{(0,1)} d_{2}(y)-\rho^{(0,1)}
\end{aligned}
$$


Por condições de regularidade. temos $E(\partial t / \partial \mu)=0$ e $E(\partial t / \partial \nu)=0$, de onde vem:

$$
\rho^{(1,0)}=a_{1}^{(1,0)} \beta_{1}+a_{2}^{(1,0)} \beta_{2} \text { e } \rho^{(0,1)}=a_{1}^{(0,1)} \beta_{1}+a_{2}^{(0,1)} \beta_{2},
$$

onde $\beta_{r}=E\left(d_{r}(y)\right)$, para $r=1,2$. Conseqüentemente,

$$
\beta_{1}=\frac{\rho^{(1,0)} \alpha_{2}^{(0,1)}-\rho^{(0,1)} \alpha_{2}^{(1,0)}}{\alpha_{1}^{(1,0)} \alpha_{2}^{(0,1)}-\alpha_{1}^{(0,1)} \alpha_{2}^{(1,0)}} \quad \text { e } \quad \beta_{2}=\frac{\rho^{(0,1)} \alpha_{1}^{(0,1)}-\rho^{(1,0)} \alpha_{1}^{(0,1)}}{\alpha_{1}^{(1,0)} \alpha_{2}^{(0,1)}-\alpha_{1}^{(0,1)} \alpha_{2}^{(1,0)}} .
$$

Logo,

$$
\begin{aligned}
& \frac{\partial t}{\partial \mu}=\sum_{i=1}^{2} \alpha_{i}^{(1,0)}\left(d_{i}(y)-\beta_{i}\right), \\
& \frac{\partial t}{\partial \nu}=\sum_{i=1}^{2} \alpha_{i}^{(0,1)}\left(d_{i}(y)-\beta_{i}\right)
\end{aligned}
$$

e

$$
\frac{\partial^{2} t}{\partial \mu \partial \nu}=\sum_{i=1}^{2}\left(\alpha_{i}^{(1,1)}\left(d_{i}(y)-\beta_{i}\right)-\alpha_{i}^{(1,0)} \beta_{i}^{(0,1)}\right) .
$$

Estes fatos implicam que

$$
\kappa_{\mu \nu}=-n \sum_{i=1}^{2} \alpha_{i}^{(0,1)} \beta_{i}^{(1,0)} .
$$

Se $\mu$ e $\nu$ são ortogonais, temos $\kappa_{\mu \nu}=0$.

Obtemos a seguir os cumulantes necessários ao desenvolvimento da estatística escore corrigida $S_{R}^{*}$ no caso dos parâmetros $\mu$ e $\nu$ serem ortogonais. Como

$$
\begin{aligned}
& \frac{\partial^{2} t}{\partial \mu^{2}}=\sum_{i=1}^{2}\left(\alpha_{i}^{(2,0)}\left(d_{i}(y)-\beta_{i}\right)-\alpha_{i}^{(1,0)} \beta_{i}^{(1,0)}\right), \\
& \frac{\partial^{2} t}{\partial \nu^{2}}=\sum_{i=1}^{2}\left(\alpha_{i}^{(0,2)}\left(d_{i}(y)-\beta_{i}\right)-\alpha_{i}^{(0,1)} \beta_{i}^{(0,1)}\right),
\end{aligned}
$$

temos

$$
\kappa_{\mu \mu}=-n \sum_{i=1}^{2} \alpha_{i}^{(1,0)} \beta_{i}^{(1,0)} \text { e } \kappa_{\nu \nu}=-n \sum_{i=1}^{2} \alpha_{i}^{(0,1)} \beta_{i}^{(0,1)} .
$$

Diferenciando $\kappa_{\mu \mu}$ e $\kappa_{\nu \nu}$ em relaçào a $\mu$ e $\nu$, chegamos a

$$
\begin{aligned}
\kappa_{\mu \mu}^{(\mu)} & =-n \sum_{i=1}^{2}\left(\alpha_{i}^{(2,0)} \beta_{i}^{(1,0)}+\alpha_{i}^{(1,0)} \beta_{i}^{(2,0)}\right) \\
\kappa_{\mu \mu}^{(\mu \mu)} & =-n \sum_{i=1}^{2}\left(\alpha_{i}^{(3,0)} \beta_{i}^{(1,0)}+2 \alpha_{i}^{(2,0)} \beta_{i}^{(2,0)}+\alpha_{i}^{(1,0)} \beta_{i}^{(3,0)}\right) \\
\kappa_{\mu \mu}^{(\nu)} & =-n \sum_{i=1}^{2}\left(\alpha_{i}^{(1,1)} \beta_{i}^{(1,0)}+\alpha_{i}^{(1,0)} \beta_{i}^{(1,1)}\right)
\end{aligned}
$$




$$
\begin{aligned}
\kappa_{\mu \mu}^{(\nu \nu)} & =-n \sum_{i=1}^{2}\left(\alpha_{i}^{(1,2)} \beta_{i}^{(1,0)}+2 \alpha_{i}^{(1,1)} \beta_{i}^{(1,1)}+\alpha_{i}^{(1,0)} \beta_{i}^{(1,2)}\right), \\
\kappa_{\nu \nu}^{(\nu)} & =-n \sum_{i=1}^{2}\left(\alpha_{i}^{(0,2)} \beta_{i}^{(0,1)}+\alpha_{i}^{(0,1)} \beta_{i}^{(0,2)}\right), \\
\kappa_{\nu \nu}^{(\nu \nu)} & =-n \sum_{i=1}^{2}\left(\alpha_{i}^{(0,3)} \beta_{i}^{(0,1)}+2 \alpha_{i}^{(0,2)} \beta_{i}^{(0,2)}+\alpha_{i}^{(0,1)} \beta_{i}^{(0,3)}\right), \\
\kappa_{\nu \nu}^{(\mu)} & =-n \sum_{i=1}^{2}\left(\alpha_{i}^{(1,1)} \beta_{i}^{(0,1)}+\alpha_{i}^{(0,1)} \beta_{i}^{(1,1)}\right), \\
\kappa_{\nu \nu}^{(\mu \mu)} & =-n \sum_{i=1}^{2}\left(\alpha_{i}^{(2,1)} \beta_{i}^{(0,1)}+2 \alpha_{i}^{(1,1)} \beta_{i}^{(1,1)}+\alpha_{i}^{(0,1)} \beta_{i}^{(2,1)}\right) .
\end{aligned}
$$

Como estamos supondo que $\kappa_{\mu \nu}=0$, temos

$$
\begin{aligned}
& \kappa_{\mu \nu}=-n \sum_{i=1}^{2} \alpha_{i}^{(0.1)} \beta_{i}^{(1,0)}=0, \\
& \kappa_{\nu \mu}=-n \sum_{i=1}^{2} \alpha_{i}^{(1,0)} \beta_{i}^{(0,1)}=0, \\
& \kappa_{\mu \nu}^{(\mu)}=-n \sum_{i=1}^{2}\left(\alpha_{i}^{(2,0)} \beta_{i}^{(0,1)}+\alpha_{i}^{(1,0)} \beta_{i}^{(1,1)}\right)=0, \\
& \kappa_{\mu \nu}^{(\nu)}=-n \sum_{i=1}^{2}\left(\alpha_{i}^{(1,1)} \beta_{i}^{(0,1)}+\alpha_{i}^{(1,0)} \beta_{i}^{(0,2)}\right)=0 .
\end{aligned}
$$

Agora,

$$
\begin{aligned}
\frac{\partial^{3} t}{\partial \mu^{2} \partial \nu} & =\sum_{i=1}^{2}\left(\alpha_{i}^{(2,1)}\left(d_{i}(y)-\beta_{i}\right)-\alpha_{i}^{(2,0)} \beta_{i}^{(0,1)}-\alpha_{i}^{(1,1)} \beta_{i}^{(1,0)}-\alpha_{i}^{(1,0)} \beta_{i}^{(1,1)}\right), \\
\frac{\partial^{3} t}{\partial \nu^{2} \partial \mu} & =\sum_{i=1}^{2}\left(\alpha_{i}^{(1,2)}\left(d_{i}(y)-\beta_{i}\right)-2 \alpha_{i}^{(1,1)} \beta_{i}^{(0,1)}-\alpha_{i}^{(1,0)} \beta_{i}^{(0,2)}\right), \\
\frac{\partial^{3} t}{\partial \mu^{3}} & =\sum_{i=1}^{2}\left(\alpha_{i}^{(3,0)}\left(d_{i}(y)-\beta_{i}\right)-2 \alpha_{i}^{(2,0)} \beta_{i}^{(1,0)}-\alpha_{i}^{(1,0)} \beta_{i}^{(2,0)}\right), \\
\frac{\partial^{3} t}{\partial \nu^{3}} & =\sum_{i=1}^{2}\left(\alpha_{i}^{(0,3)}\left(d_{i}(y)-\beta_{i}\right)-2 \alpha_{i}^{(0,2)} \beta_{i}^{(0,1)}-\alpha_{i}^{(1,0)} \beta_{i}^{(0,2)}\right)
\end{aligned}
$$

e, conseqüentemente. temos

$$
\begin{aligned}
\kappa_{\mu \mu \nu} & =-n \sum_{i=1}^{2} \alpha_{i}^{(1,1)} \beta_{i}^{(1,0)}, \\
\kappa_{\nu \nu \mu} & =-n \sum_{i=1}^{2} \alpha_{i}^{(1,1)} \beta_{i}^{(0,1)},
\end{aligned}
$$




$$
\begin{aligned}
& \kappa_{\mu \mu \mu}=-n \sum_{i=1}^{2}\left(2 \alpha_{i}^{(2,0)} \beta_{i}^{(1,0)}+\alpha_{i}^{(1,0)} \beta_{i}^{(2,0)}\right), \\
& \kappa_{\nu \nu \nu}=-n \sum_{i=1}^{2}\left(2 \alpha_{i}^{(0,2)} \beta_{i}^{(0,1)}+\alpha_{i}^{(0,1)} \beta_{i}^{(0,2)}\right) .
\end{aligned}
$$

Diferenciando $\kappa_{\nu \mu \mu}$ em relação a $\nu, \kappa_{\mu \nu \nu}$ em relação a $\mu$ e $\kappa_{\mu \mu \mu}$ em relação a $\mu$, verificamos que

$$
\begin{aligned}
& \kappa_{\nu \mu \mu}^{(\nu)}=-n \sum_{i=1}^{2}\left(\alpha_{i}^{(1,2)} \beta_{i}^{(1,0)}+\alpha_{i}^{(1,1)} \beta_{i}^{(1,1)}\right), \\
& \kappa_{\mu \nu \nu}^{(\mu)}=-n \sum_{i=1}^{2}\left(\alpha_{i}^{(2,1)} \beta_{i}^{(0,1)}+\alpha_{i}^{(1,1)} \beta_{i}^{(1,1)}\right)
\end{aligned}
$$

e

$$
\kappa_{\mu \mu \mu}^{(\mu)}=-n \sum_{i=1}^{2}\left(2 \alpha_{i}^{(3,0)} \beta_{i}^{(1,0)}+3 \alpha_{i}^{(2,0)} \beta_{i}^{(2,0)}+\alpha_{i}^{(1,0)} \beta_{i}^{(3,0)}\right)
$$

Da relação $\kappa_{\mu, \mu, \mu}=2 \kappa_{\mu \mu \mu}-3 \kappa_{\mu \mu}^{(\mu)}$, temos que

$$
\kappa_{\mu, \mu, \mu}=n \sum_{i=1}^{2}\left(\alpha_{i}^{(1,0)} \beta_{i}^{(2,0)}-\alpha_{i}^{(2,0)} \beta_{i}^{(1,0)}\right) .
$$

Agora, derivando $t$ novamente em relação a $\mu$, vem

$$
\frac{\partial^{4} t}{\partial \mu^{4}}=\sum_{i=1}^{2}\left(\alpha_{i}^{(4,0)}\left(d_{i}(y)-\beta_{i}\right)-3 \alpha_{i}^{(3,0)} \beta_{i}^{(1,0)}-3 \alpha_{i}^{(2,0)} \beta_{i}^{(2,0)}-\alpha_{i}^{(1,0)} \beta_{i}^{(3,0)}\right),
$$

implicando que

$$
\kappa_{\mu \mu \mu \mu}=-n \sum_{i=1}^{2}\left(3 \alpha_{i}^{(3,0)} \beta_{i}^{(1,0)}+3 \alpha_{i}^{(2,0)} \beta_{i}^{(2,0)}+\alpha_{i}^{(1,0)} \beta_{i}^{(3,0)}\right) .
$$

Temos também

$$
\begin{aligned}
\frac{\partial^{4} t}{\partial \mu^{2} \partial \nu^{2}}= & \sum_{i=1}^{2}\left(\alpha_{i}^{(2,2)}\left(d_{i}(y)-\beta_{i}\right)-\alpha_{i}^{(1,2)} \beta_{i}^{(1,0)}\right. \\
& \left.-2 \alpha_{i}^{(2,1)} \beta_{i}^{(0,1)}-2 \alpha_{i}^{(1,1)} \beta_{i}^{(1,1)}-\alpha_{i}^{(2,0)} \beta_{i}^{(0,2)}-\alpha_{i}^{(1,0)} \beta_{i}^{(1,2)}\right),
\end{aligned}
$$

e como $\kappa_{\mu \nu}=0$, temos $\kappa_{\mu \nu}^{(\mu \nu)}=0$, isto é,

$$
\sum_{i=1}^{2}\left(\alpha_{i}^{(2,1)} \beta_{i}^{(0,1)}+\alpha_{i}^{(2,0)} \beta_{i}^{(0,2)}+\alpha_{i}^{(1,1)} \beta_{i}^{(1,1)}+\alpha_{i}^{(1,0)} \beta_{i}^{(1,0)}\right)=0 .
$$

Assim,

$$
\kappa_{\mu \mu \nu \nu}=-n \sum_{i=1}^{2}\left(\alpha_{i}^{(1,2)} \beta_{i}^{(1,0)}+\alpha_{i}^{(2,1)} \beta_{i}^{(0,1)}+\alpha_{i}^{(1,1)} \beta_{i}^{(1,1)}\right) .
$$


Da definição dos cumulantes apresentados na Seção 1.2.1. depois de algumas manipulações algébricas, temos

$$
\begin{aligned}
& \kappa_{\mu \mu, \mu \mu}=n \operatorname{Var}\left(\sum_{i=1}^{2} a_{i}^{(2,0)} d_{i}(y)\right)=n \gamma, \\
& \kappa_{\nu \mu, \nu \mu}=n \operatorname{Var}\left(\sum_{i=1}^{2} a_{i}^{(1,1)} d_{i}(y)\right)=n \xi,
\end{aligned}
$$

onde $\gamma$ e $\xi$ estão definidos em (7.13). Finalmente, da relação $\kappa_{\mu, \mu, \mu, \mu}=-3 \kappa_{\mu \mu \mu \mu}+\delta \kappa_{\mu \mu \mu}^{(\mu)}-$ $6 \kappa_{\mu \mu}^{(\mu \mu)}+3 \kappa_{\mu \mu, \mu \mu}$, temos

$$
\kappa_{\mu, \mu, \mu, \mu}=-n\left\{\sum_{i=1}^{2}\left(\alpha_{i}^{(3,0)} \beta_{i}^{(1,0)}+3 \alpha_{i}^{(2.0)} \beta_{i}^{(2,0)}-\alpha_{i}^{(1,0)} \beta_{i}^{(3,0)}\right)-3 \gamma\right\} .
$$




\section{Bibliografia}

ABELL, M.L., BASELTON, J.P. (1994). The MAPLE $V$ Handbook. New York: AP Professional.

ABRAMOWITZ, M., STEGUN, I.R. (1970). Handbook of Mathematical Functions. Washington, D.C.: National Bureau of Stadards.

AITKIN, M., ANDERSON, D., FRANCIS, B. and HINDE, J. (1989). Statistical Modelling in GLIM. Oxford University Press, New York, NY.

AITKINSON, A.C. (1982). Regression diagnostics, transformations and constructed variables (with discussion). Journal of the Royal Statistical Society B, 44, 1-36.

ALBERT, J., DELAMPADY, M., POLASEK, W. (1991). A class of distributions for robustness studies. Journal of Statistical Planning and Inference, 28, 291-304.

ANDERSON, T.W., FANG, K.T. (1987). Cochran's theorem for elliptically contourned distributions. Sankhya A, 49, 305-315.

ARELlanO-VAlLE, R.B. (1994). Distribuições Elípticas: Propriedades, Inferência e Aplicações a Modelos de Regressão. Tese (Doutorado) - IME-USP.

ARNOLD, S.F. (1981). The Theory of Linear Models and Multivariate Analysis. New York: Wiley.

AUBIN, E.C.Q. (1995). Aperfeiçoamento de Testes da Razão de Verossimilhança em Modelos Especiais, com algumas Aplicações e Extensões. Tese de Doutorado. IMEUSP.

AUBIN, E.C.Q., CORDEIRO, G.M. (1997). Bias in linear regression models with unknown covariance matrix. Communications in Statistics - Simulation and Computation. A aparecer.

BARNDORFF-NIELSEN, O.E., BLAESILD, P. (1986). A note on the calculation of Bartlett adjustments. Journal of the Royal Statistical Society B, 46, 48.3-495.

BARNDORFF-NIELSEN, O.E., COX, D.R. (1984). Bartlett adjustments to the likelihood ratio statistics and the distribution of the maximum likelihood estimator. Journal fo the Royal Statistical Society B, 46, 484-49. 
BARTLETT, M.S. (1953a). Approximate confidence intervals I. Biometrika. 40, 12-19.

BARTLETT, M.S. (1953b). Approximate confidence intervals II. More than one parameter. Biometrika, 40, 306-317.

BARTLETT, M.S. (1937). Properties of sufficiency and statistical tests. Proceedings of the Royal Society A, 160. 268-282.

BECKER, R.A., CHAMBERS, J.M., WILKS, A.R. (1988). The new S language: a programming environment for data analysis and graphics. (Wadsworth \& Brooks/Cole, Pacific Grove. CA).

BERKANE, M., BENTLER. P.M. (1986). Moments of elliptically distributed random variates. Statistics and Probability Letters, 4, 333-335.

BICKEL, P.L., DOKSUM, K.A. (1977). Properties of sufficiency and statistical tests. Proceedings of Royal Society A, 160, 268-282.

BIRKES, D., DODGE, Y. (1993). Alternative Methods of Regression. New York: John Wiley.

BOX, G.E.P., TIAO, G.C. (1973). Bayesian Inference in Statistical Analysis. London: Addison-Wesley.

BRAJENDRA, C., SUTRADHAR, ROY F. BARTLETT (1992). Monte Carlo comparison of Wald's, likelihood ration and Rao's tests. Journal of Statistical Computation and Simulation, 46, 23-33.

BURR, I.W. (1942). Cumulative frequency functions. Annals of Mathematical Statistics, 13, 215-232.

BUSTOS, O. (1981). Estimaçâo Robusta no Modelo de Posição. $13^{\circ}$ Colóquio Brasileiro de Matemática. IMPA.

CAMBANIS, S., HUANG, S., SIMONS, G. (1981). On the theory of ellipatically contoured distributions. Journal of Multivariate Analysis, 11, 368-385.

CHANDRA, T.K. (1985). Asymptotic expansions of perturbed chi-squared variables. Sankhyā A, 47, 100-110.

CHANDRA, T.K., MUKERJEE, R. (1991). Bartlett-type modification for Rao's efficient score statistic. Journal of Multivariate Analysis, 36, 103-112.

CHAPMAN, D.G. (1956). Estimating the parameters of a truncated gamma distribution. Annals of Mathematical Statistics, 27, 498-506.

CHESHER, A., SMITH, R. (1995). Bartlett corrections to likelihood ratio tests. Biometrika, 82, 43:3-436. 
CORDEIRO, G.M. (1983). Improved likelihood ratio statistics for generalized linear models. Journal of the Royal Statistical Society B. 45, 404-413.

CORDEIRO, G.M. (1985). The null expected deviance for an extended class of generalized linear models. Lecture Notes in Statistics, 32, $27-34$.

CORDEIRO, G.M. (1987). On the corrections to the likelihood ratio statistics. Biometrika, 74, 265-274.

CORDEIRO, G.M. (1993). Bartlett corrections and bias correction for two heteroscedastic regression models. Communications in Statistics A, 22, 169-188.

CORDEIRO, G.M., BOTTER, D.A., FERRARI, S.L.P. (1994). Nonull asymptotic distributions of three classic criteria in generalized linear models. Biometrika, 81, $709-720$.

CORDEIRO, G.M., CRIBARI-NETO, F. (1993). On Bartlett corrections and bias reduction and a new class of transformations. Brazilian Journal of Probability and Statistics, 7, 197-200.

CORDEIRO, G.M., CRIBARI-NETO, F., AUBIN, E.C.Q., FERRARI, S.L.P. (1995).

- Bartlett correction for one-parameter exponential family models. Journal of Statistical Computation and Simulation, 53, 211-231.

CORDEIRO, G.M., FERRARI, S.L.P. (1991). A modified score test statistic having chi-squared distribution to order $n^{-1}$. Biometrika, 78, 573-582.

CORDEIRO, G.M., FERRARI, S.L.P., PAULA, G.A. (1993). Improved score tests for generalized linear models. Journal of the Royal Statistical Society B, 55, 661-674.

CORDEIRO, G.M., KLEIN, R. (1994). Bias correction in ARMA models. Statistic and Probability Letters, 19, 169-176.

CORDEIRO, G.M., McCULLAGH, P. (1991). Bias correction in generalized linear models. Journal of the Royal Statistical Society B, 53, 629-643.

CORDEIRO, G.M., PAULA, G.A. (1989). Improved likelihood ratio statistics for exponential family nonlinear models. Biometrika, 76, 93-100.

CORDEIRO, G.M., PAULA, G.A., BOTTER, D.A. (1994). Improved likelihood ratio tests for dispersion models. International Statistical Review, 62, 257-276.

COX, D.R., HINkLEY, D.V. (1974). Theoretical Statistics. London: Chapman and Hall.

COX, D.R., REID. X. (198T). Parameter orthogonality and approximate conditional inference (with discussion). Journal of the Royal Statistical Society B, 49, 1-39. 
COX, D.R., SNELL, E.J. (1968). A general definition of residuals (with discussion). Journal of the Royal Statistical Society B, 30, 248-278.

CRIBARI-NETO, F., BOTTER, D.A.. CORDEIRO, G.M., FERRARI, S.L.P. (1995). Bias reduction in one-parameter exponential family models. Relatório Técnico RT-MAE-9532, Departamento de Estatística, Universidade de São Paulo.

CRIBARI-NETO, F., CORDEIRO, G.M. (1997). On Bartlett and Bartlett-type corrections. Econometrics Reviews, 15, 339-367.

CRIBARI-NETO, F., FERR.ARI, S.L.P. (1995a). Bartlett corrected tests for heteroskedastic linear models. Economics Letters, 48, 113-118.

CRIBARI-NETO, F., FERRARI, S.L.P. (1995b). Second order asymptotics for score tests in generalized linear models. Biometrika, 82, 426-432.

CRIBARI-NETO, F., FERR.ARI, S.L.P. (1995c). An improved Lagrange multiplier test of heteroskedasticity. Communications in Statistic - Simulation and Computation, $24,31-44$.

CRIBARI-NETO, F., ZARKOS, S. (1995). Improved test statistics for multivariate regression. Economics Letters, 49, 113-120.

DEMPSTER, A., LAIRD, N.. RUBIN, D.B. (1977). Maximum likelihood estimator from incomplete data via the EM algorithm (with discussion). Journal of the Royal Statistical Society B, 39, 1-38.

DEVLIN, S.J. GNANADESIKAN, R., KETTENRING, J.R. (1976). Some multivariate applications of elliptical distributions. Essays in Probability and Statistics, 24, 365393. Tokyo: Shinko Tsusho Co.

DEVROYE, L. (1986). Non-Uniform Random Variate Generator. New York: SpringerVerlag.

DICICCIO, T.J. (1986). Approximate conditional inference for location families. The Canadian Journal of Statistical, 14, 1, 5-18.

DICKEY, J.M. (1967). Multivariate generalizations of the multivariate $t$ distribution and the inverted multivariate $t$ distribution. Annals of Mathematical Statistics, 38, $511-518$.

DRAPER, N.R., STONEMAX. D.M. (1966). Testing for the inclusion of variables in linear regression by a randomization technique. Technometrics, 8, 695-699.

FANG, K.T., KOTZ, S., NG. K.W. (1990). Symmetric Multivariate and Related Distributions. London: Chapman and Hall. 
FANG, K.T., ZHANG. Y.T. (1990). Generalized Multivariate Analysis. London: Springer-Verlag.

FERRARI, S.L.P. (1991). Aperfeiçoamento de Testes Escort. Aplicações e Extensōes. São Paulo. 18.5p. Tese (Doutorado) - Instituto de Matemática e Estatística - Universidade de São Paulo.

FERRARI, S.L.P., ARELLANO-VALLE, R.B. (1996). Bartlett corrected tests for regression models with Student-t independent errors. Revista Brasileira de Probabilidade e Estatística, 10, 15-33.

FERRARI, S.L.P., BOTTER, D.A., CORDEIRO, G.M., CRIBARI-NETO, F. (1996). Second and third order bios reduction for one-parameter family models. Statistics and Probability Letters, 30, 339-345.

FERRARI, S.L.P., BOTTER, D.A., CRIBARI-NETO, F. (1997). Local power of three classic criteria in generalised linear models with unknown dispersion. Biometrika. A aparecer.

FERRARI, S.L.P., CORDEIRO. G.M. (1994). Matrix formulae for computing improved score tests. Journal of Statistical Computation and Simulation, 49, 196-206.

FERRARI, S.L.P., CORDEIRO, G.M. (1996). Corrected score tests for exponential family non-linear models. Statistics and Probability Letters, 26, 7-12.

FRYDEMBERG, M., JENSEN, J.L. (1989). Is the 'improved likelihood ratis statistic' really improved in discrete case? Biometrika, 76(4), 655-661.

GUMBEL, E. (1944). Ranges and midranges. Annals of Mathematical Statistics, 15, $414-422$.

HAMPET, F.R. (1971). A general qualitative definition of robustness. Annals of Mathematical Statistics, 42, 1887-1896.

HARRIS, P. (1985). An asymptotic expansion for the null distribution of the efficient score statistic. Biometrika. 72, 653-9. (Errata no volume 74, p.669.)

HARRIS, P. (1986). A note on Bartlett adjustments to likelihood ratio tests. Biometrika, $73,735-737$.

HARRIS, P., PEERS, H.W. (1980). The local power of the efficient scores test statistic. Biometrika, 67, 525-529.

HASTINGS, N.A.J., PEACOCK. J.B. (1975). Statistical distributions. New York: Wiley.

HAYAKAWA, T. (1975). The likelihood ratio criterion for a composite hypothesis under a local alternative. Biometrika. 62, 451-460. 
HAYAKAWA, T. (1977). The likelihood ratio criterion and the asymptotic expansion of its distribution. Annals of the Institute of Statistical Mathematics A. 29, 359-378.

HAYAKAWA, T. (1987). Correction to "The likelihood ratio criterion and the asymptotic expansion of its distribution." Annals of the Institute of Statistical Mathematics A. 39, 681 .

HUBER, P.J. (1973). Robust regression: asymptotic conjectures and Monte Carlo. Annals of Statistics, 1, 799-821.

JENSEN, J.L. (1993). A historical sketch and some new results on the improved likelihood ratio statistic. Scandinavian Journal of Statistics, 20, 1-15.

JOHnSOn, R., KOTZ, S. (1970a). Continuous Univariate Distributions. v.1. Boston: Houghton Mifflin.

JOHNSON, R., KOTZ, S. (1970b). Continuous Univariate Distributions. v.2. Boston: Houghton Mifflin.

JOHNSON, R., KOTZ, S., KEMP, A.W. (1992). Univariate Discrete Distributions. 2.ed. New York: Wiley.

JOHNSON, R., LADALLA, J., LIU, S.T. (1979). Differential relations, in original parameters, which determine the first two moments of the multiparameter exponential family. The Annals of Statistics, 7, 1, 232-235.

JØRGENSEN, B. (1983). Maximum likelihood estimation and large-sample inference for generalized linear and nonlinear regression models. Biometrika, 70, 19-28.

JØRGENSEN, B. (1987a). Exponential dispersion models. Journal of the Royal Statistical Society B, 49, 127-162.

JØRGENSEN, B. (1987b). Small dispersion asymptotic. Revista Brasileira de Probabilidade e Estatística, 1, 59-90.

KELKER, D. (1970). Distribution theory of spherical distributions and a location-scale parameter generalization. Sankhȳa A, 32, 419-430.

KOTZ, S. (1975). Multivariate distributions at a cross-road. Em Statistical Distributions in Scientific Work, 1, Ed. G.P. Patil, S. Fiotz and J.K. Ord., 24i-270. Dordrecht, Reidel.

LANGE. K.L., LITTLE, R.J., TAYLOR, J. (1989). Robust statistical modeling using the $t$-distribution. Journal of the American Statistical Association, 84, 881-896.

LAWLEY, D.N. (1956). A general method for approximating to the distribution of the likelihood ratio criteria. Biometrika, 71, 233-244. 
LETAC, C., MORA, M. (1990). Natural real exponential families with cubic variance functions. Annals of Statistics, 18, 1-37.

LITTLE, R.J.A (1988). Robust estimation of the mean and covariance matrix from data with missing values. Appl. Statist., 37, 1, 23-39.

LITTLE, R.J.A., RUBIN, D.B. (1987). Statistical Analysis with Missing Data. New York: Wiley.

MANOUKIAN, E.B. (1985). Modern Concepts and Theorems of Mathematical Statistics. New York: Springer-Verlag.

MARDIA, K.V. (1972). Statistics of Directional Data. London: Academic Press.

McCULLAGH, P. (1984). Tensor notation and cumulants of polynomials. Biometrika, $71,461-476$.

McCULLAGH, P. (1985). On the asymptotic distribution of Pearson's statistic in linear exponential family models. International Statistical Revieu, 53, 61-67.

McCUlLAGH, P. (1987). Tensor Methods in Statistics. London: Chapman and Hall. $285 \mathrm{p}$.

McCULLAGH, P. (1989). Some statistical properties of a family of continuous univariate distributions. Journal of the American Statistical Association, 84, 125-141.

McCULLAGH, P., COX, DR. (1986). Invariants and likelihood ratio statistics. Annals of Statistics, 14, 1419-1430.

MENG, X.L., RUBIN, D.B. (1993). Maximum likelihood estimation via the ECM algorithm: a general framework. Biometrika, 80, 267-278.

MORRIS, C.N. (1982). Natural exponential families with quadratic variance functions. Annals of Statistics, 10, 65-80.

MOULTON, L.H., WEISSFELD, L.A., LAURENT, R.T. (1993). Bartlett correction factors in logistic regression models. Computational Statistic and Data Analysis, 15, 1-11.

MUIRHEAD, R. (1980). The effects of elliptical distributions on some standard procedures involving correlation coefficients. Em Multivariate Statistical Analysis (ed. R.P. Gupta), North-Holland, 143-159.

MUIRHEAD, R. (1982). Aspects of Multivariate Statistical Theory. New York: John Wiley.

MUKERJEE, R. (1992). Parametric orthogonality and Bartlett-type modification for Rao's statistic in the presence of a nuisance parameter. Statistic and Probability Letters, 13, 397-400. 
NARUla, S.C., STANGEnhaUS, G. (1988). Análise de Regressão. VIII SINAPEIMPA.

PATTERSON, S.J. (1988). An Introduction to the Theory of the Riemann ZetaFunction. New York: Cambridge University Press.

RAO, B.L.S.P. (1990). Remarks on univariate elliptical distributions. Statistics and Probability Letters, 10, 307-315.

RAO, C.R. (1947). Large sample tests of statistical hypotheses concerning several parameters with applications to problems of estimation. Proceedings of the Cambridge Philosophical Society, 44, 50-57.

RAO, C.R. (1973). Linear Statistical Inference and its Applications. 2.ed. New York: John Wiley.

ROTHENBERG, T. (1984). Hypothesis testing in linear models when erro covariance matrix is nonscalar. Econometrica, 52, $827-842$.

SELF, S., MAURITSEN, R.H. (1988). Power/Sample size calculations for generalized linear model. Biometrics, 44, 79-86.

SELF, S., MAURITSEN, R.H., OHARA, J. (1992). Power calculations for likelihood ratio tests in generalized linear models. Biometrics, 48, 31-39.

SEN, P.K., SINGER, J.M. (1993). Large Sample Methods in Statistics: an Introduction with Applications. New York: Chapman and Hall.

TAYLOR, J.M.G. (1992). Properties of modeling the errors distribution with an extra shape parameter. Computational Statistical \& Data Analysis, 13, 33-46.

WILLIAMS, D.A. (1976). Improved likelihood ratio test for complete contingency tables. Biometrika, 63, 33-37.

WOLFRAM, S. (1991). Mathematica: a System for Doing Mathematics by Computer. 2.ed. New York: Addison-Wesley. 